Prepared for the U.S. Department of Energy under Contract DE-AC05-76RL01830

\title{
Calculation of Ambient $\left(H^{*}(10)\right)$ and Personal $\left(H_{p}(10)\right)$ Dose Equivalent from a ${ }^{252} \mathrm{Cf}$ Neutron Source
}

RJ Traub

March 2010

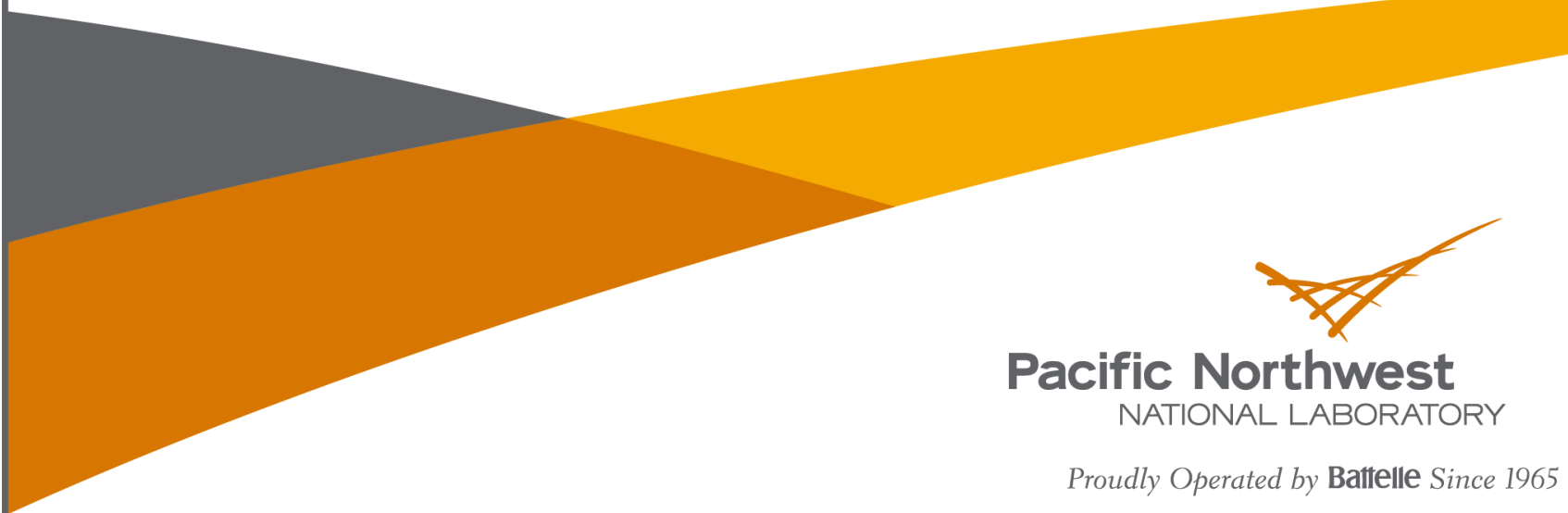




\section{DISCLAIMER}

This report was prepared as an account of work sponsored by an agency of the United States Government. Neither the United States Government nor any agency thereof, nor Battelle Memorial Institute, nor any of their employees, makes any warranty, express or implied, or assumes any legal liability or responsibility for the accuracy, completeness, or usefulness of any information, apparatus, product, or process disclosed, or represents that its use would not infringe privately owned rights. Reference herein to any specific commercial product, process, or service by trade name, trademark, manufacturer, or otherwise does not necessarily constitute or imply its endorsement, recommendation, or favoring by the United States Government or any agency thereof, or Battelle Memorial Institute. The views and opinions of authors expressed herein do not necessarily state or reflect those of the United States Government or any agency thereof.

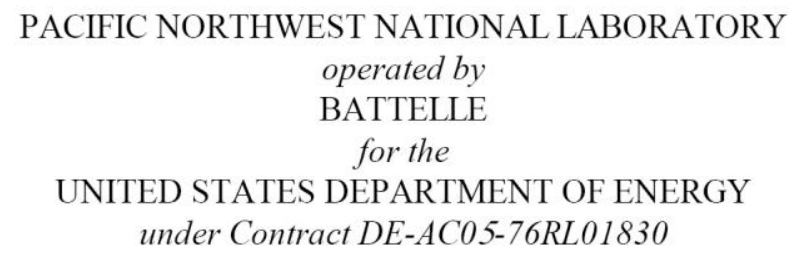

Printed in the United States of America

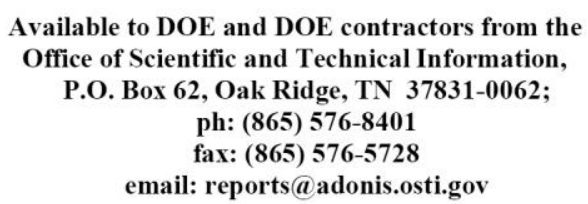

Available to the public from the National Technical Information Service, U.S. Department of Commerce, 5285 Port Royal Rd., Springfield, VA 22161 ph: (800) 553-6847 fax: (703) 605-6900

email: orders@ntis.fedworld.gov online ordering: http://www.ntis.gov/ordering.htm 


\section{Calculation of Ambient $\left(H^{*}(10)\right)$ and Personal $\left(H_{p}(10)\right)$ Dose Equivalent from a ${ }^{252}$ Cf Neutron Source}

RJ Traub

March 2010

Prepared for

the U.S. Department of Energy

under Contract DE-AC05-76RL01830

Pacific Northwest National Laboratory

Richland, Washington 99352 



\section{Executive Summary}

The neutron emission rate from radioisotope neutron sources such as ${ }^{252} \mathrm{Cf}$ sources can be calibrated by the National Institute of Standards and Technology (NIST) by the manganous sulfite bath method. This calibration method does not compute the dose or dose equivalent rate from the source. Because there is no accepted transfer standard for neutron dose measurements, secondary calibration facilities are responsible for determining their own neutron dose factors.

This report describes a neutron source and irradiation apparatus in use at the Pacific Northwest National Laboratory Calibration Facility. The neutron source is a SR-Cf-3000 design ${ }^{252} \mathrm{Cf}$ neutron source. The neutron source is contained in an aluminum transfer capsule that is used to transport the neutron source between its storage location and it's irradiation location. During irradiations, the source is located in a transfer tube assembly that consists of two concentric aluminum tubes. When moderated neutrons are desired, the transfer tube assembly is surrounded by a sphere that is filled with $\mathrm{D}_{2} \mathrm{O}$ as a moderating medium.

Ambient dose equivalent $\left(\mathrm{H}^{*}(10)\right)$ and Personal dose equivalent $\left(\mathrm{H}_{\mathrm{p}}(10)\right)$ dose rates and dose conversion factors were calculated for moderated and umoderated neutron spectra based on the data provided in ICRP Publication 74. The neutron fluence-to-dose-equivalent conversion factors calculated for this report are summarized and compared to published values in Table 47. The calculated coefficients for the unmoderated ${ }^{252} \mathrm{Cf}$ spectra agree very well with published reference values, within about $2 \%$. The calculated coefficients for the $\mathrm{D}_{2} \mathrm{O}$ moderated ${ }^{252} \mathrm{Cf}$ spectra, however, differ from published reference values by about $22 \%$. The PNNL coefficients are higher than the published values which could be interpreted to mean that the PNNL $\mathrm{D}_{2} \mathrm{O}$ moderated spectrum is harder, i.e. higher average energy, than the spectrum published by the ISO (2000), which formed the basis for the published coefficients. The $\mathrm{D}_{2} \mathrm{O}$ moderating sphere in use at PNNL contains less $\mathrm{D}_{2} \mathrm{O}$ moderator than did the $\mathrm{D}_{2} \mathrm{O}$ moderating sphere that was used to calculate the ISO spectrum and this is the likely reason for much of the spectral differences.

The exact location of the ${ }^{252} \mathrm{Cf}$ activity in the source encapsulation is unknown. Preliminary measurements, not described in this report, indicate that the activity may be offset from the location that has been assumed in the past. Corrections for the offset are being proposed and will be the subject of another report.

This report was written in a calculation package format to help meet various Quality Assurance requirements. 


\section{Acknowledgments}

This work was prepared for the U.S. Department of Energy under Contract DE-AC05-76RL01830. 


\section{Contents}

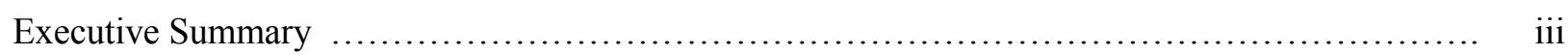

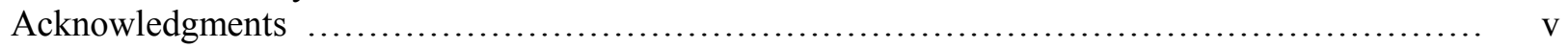

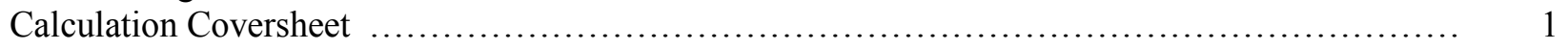

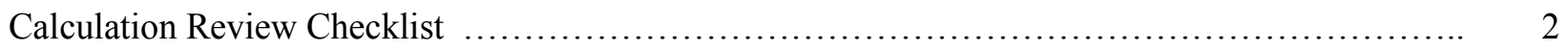

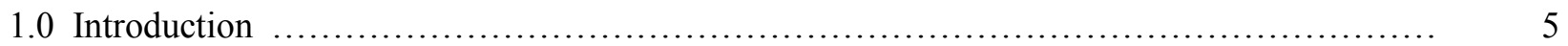

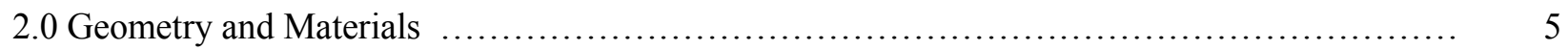

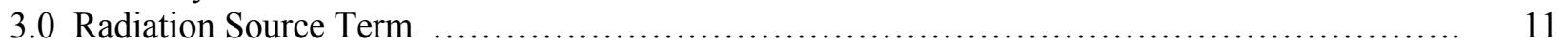

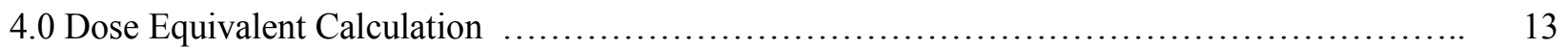

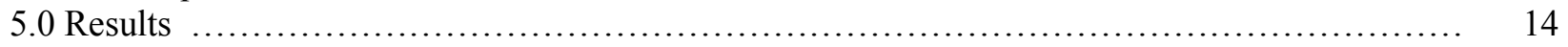

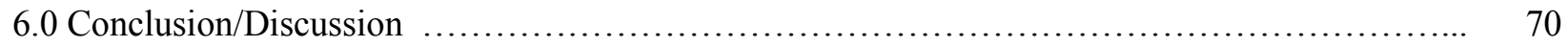

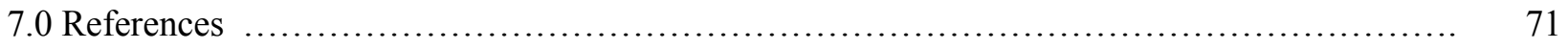

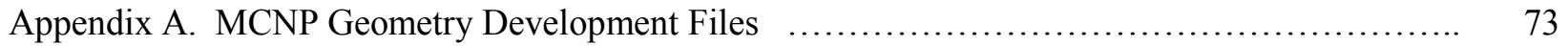

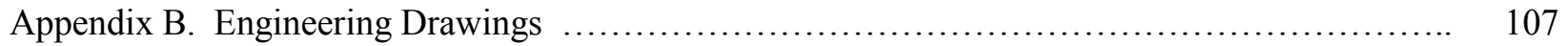

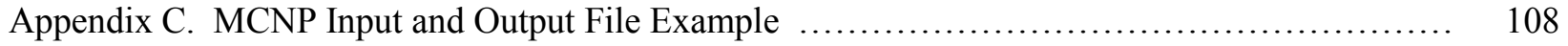





\section{Figures}

1.a The primary encapsulation of the Sr-Cf-3000 source. Sr3000_Primary.mcnp.inp ........ 7

1.b The primary encapsulation in the inverted geometry. Sr3000_Primary_flip.mcnp.inp... 7

2.a The secondary encapsulation. Sr3000_Secondary.mcnp.inp .............................. 8

2.b The secondary encapsulation with the primary encapsulation.

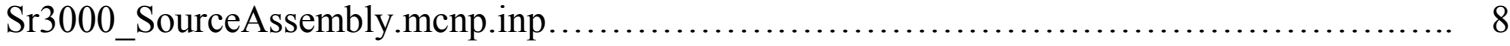

3.a The pneumatic transfer capsule. Sr3000_TransferCapsule.mcnp.inp ............................ 9

3.b The Sr-Cf-3000 transfer capsule assembly. Sr3000_TransferCapsuleAssembly.mcnp.inp. 9

4.a Transfer Tube. LSR_TransferTubeAssembly.mcnp.inp ..................................... 10

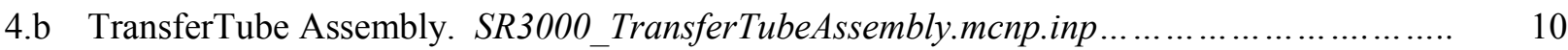

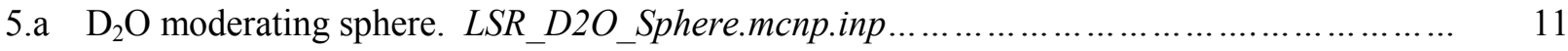

5.b Cf-Sr-3000 moderating sphere assembly. SR3000_D2OSphere.mcnp.inp ..................... 11

6. Neutron fluence $\left(\mathrm{n} \cdot \mathrm{cm}^{-2} \cdot \mathrm{s}^{-1}\right)$ at $50 \mathrm{~cm}$ from the ${ }^{252} \mathrm{Cf}$ source. The horizontal line represents the equator of the reference sphere. The dashed line, labeled XCAP average represents the average neutron fluence at $50 \mathrm{~cm}$ from the transfer capsule assembly. The plot on the left side represents the neutron fluence at $50 \mathrm{~cm}$ when the source encapsulation is in the $\mathrm{D}_{2} \mathrm{O}$ moderating sphere. The plot on the right side represents the neutron fluence at $50 \mathrm{~cm}$ when the source encapsulation is in the transfer tube assembly. The ${ }^{252} \mathrm{Cf}$ activity is assumed to be

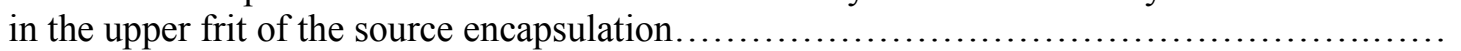

7. Neutron fluence $\left(\mathrm{n} \cdot \mathrm{cm}^{-2} \cdot \mathrm{s}^{-1}\right)$ at $50 \mathrm{~cm}$ from the ${ }^{252} \mathrm{Cf}$ source. The horizontal line represents the equator of the reference sphere. The dashed line, labeled XCAP average, represents the average neutron fluence at $50 \mathrm{~cm}$ from the transfer capsule assembly. The plot on the left side represents the neutron fluence at $50 \mathrm{~cm}$ when the source encapsulation is in the $\mathrm{D}_{2} \mathrm{O}$ moderating sphere. The plot on the right side represents the neutron fluence at $50 \mathrm{~cm}$ when the source encapsulation is in the transfer tube assembly. The ${ }^{252} \mathrm{Cf}$ activity is assumed to be in the void between the frits of the source encapsulation

8. Neutron fluence $\left(\mathrm{n} . \mathrm{cm}^{-2} \cdot \mathrm{s}^{-1}\right)$ at $50 \mathrm{~cm}$ from the ${ }^{252} \mathrm{Cf}$ source. The horizontal line represents the equator of the reference sphere. The dashed line, labeled XCAP average, represents the average neutron fluence at $50 \mathrm{~cm}$ from the transfer capsule assembly. The plot on the left side represents the neutron fluence at $50 \mathrm{~cm}$ when the source encapsulation is in the $\mathrm{D}_{2} \mathrm{O}$ moderating sphere. The plot on the right side represents the neutron fluence at $50 \mathrm{~cm}$ when the source encapsulation is in the transfer tube assembly. The ${ }^{252} \mathrm{Cf}$ activity is assumed to be in the lower frit of the source encapsulation.

9. Neutron spectrum (lethargy plots) at $50 \mathrm{~cm}$ from the center of the $\mathrm{D}_{2} \mathrm{O}$ moderating sphere. The three spectra were taken on the equator of the moderating sphere and $10^{\circ}$ above the 
equator and $10^{\circ}$ below the equator. The ${ }^{252} \mathrm{Cf}$ activity is assumed to be in the upper frit of the

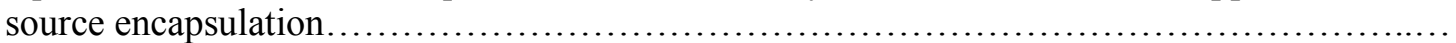

10. Neutron spectrum (lethargy plots) at $50 \mathrm{~cm}$ from the center of the $\mathrm{D}_{2} \mathrm{O}$ moderating sphere. The three spectra were taken on the equator of the moderating sphere and $10^{\circ}$ above the equator and $10^{\circ}$ below the equator. The ${ }^{252} \mathrm{Cf}$ activity is assumed to be in the void between

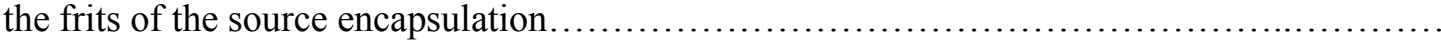

11. Neutron spectrum (lethargy plots) at $50 \mathrm{~cm}$ from the center of the $\mathrm{D}_{2} \mathrm{O}$ moderating sphere. The three spectra were taken on the equator of the moderating sphere and $10^{\circ}$ above the equator and $10^{\circ}$ below the equator. The ${ }^{252} \mathrm{Cf}$ activity is assumed to be in the lower frit of the source encapsulation.

12. Neutron spectrum (lethargy plots) at $50 \mathrm{~cm}$ from the center of the $\mathrm{D}_{2} \mathrm{O}$ moderating sphere. The ASIS spectrum represents the spectrum on the equator of the $\mathrm{D}_{2} \mathrm{O}$ moderating sphere. The ${ }^{252} \mathrm{Cf}$ activity is assumed to be in the lower frit of the source encapsulation. The ISO standard spectrum was obtained from ISO 8529-1:2000(E). 


\section{Tables}

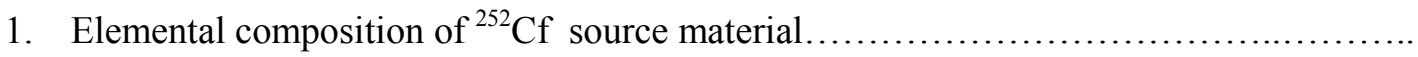

2. Neutron dose rate $\left(\right.$ rem. $\left.h^{-1}\right)$ at increments of $10^{\circ}$ latitude above and below the equator of the reference sphere. The source encapsulation is in the upright configuration in the pneumatic transfer tube assembly. The activity is on the upper frit. The reference date is March 3, 2003. The emission rate is $4.85 \mathrm{e} 9 \mathrm{n} . \mathrm{s}^{-1}$.

3. Neutron dose factors $\left(\mathrm{pSv} \cdot \mathrm{cm}^{2}\right)$ at increments of $10^{\circ}$ latitude above and below the equator of the reference sphere. The source encapsulation is in the upright configuration in the pneumatic transfer tube assembly. The activity is on the upper frit. The reference date is March 3, 2003. The emission rate is $4.85 \mathrm{e} 9 \mathrm{n} . \mathrm{s}^{-1}$.

4. Neutron dose rate $\left(\right.$ rem. $\left.h^{-1}\right)$ at increments of $10^{\circ}$ latitude above and below the equator of the reference sphere. The source encapsulation is in the upright configuration in the pneumatic transfer tube assembly. The activity is in the void region between the frits. The reference date is March 3, 2003. The emission rate is $4.85 \mathrm{e} 9 \mathrm{n} . \mathrm{s}^{-1}$....

5. Neutron dose factors $\left(\mathrm{pSv} \cdot \mathrm{cm}^{2}\right)$ at increments of $10^{\circ}$ latitude above and below the equator of the reference sphere. The source encapsulation is in the upright configuration in the pneumatic transfer tube assembly. The activity is in the void region between the frits. The reference date is March 3, 2003. The emission rate is $4.85 \mathrm{e} 9 \mathrm{n} . \mathrm{s}^{-1}$.

6. Neutron dose rate $\left(\mathrm{rem} \cdot \mathrm{h}^{-1}\right)$ at increments of $10^{\circ}$ latitude above and below the equator of the reference sphere. The source encapsulation is in the upright configuration in the pneumatic transfer tube assembly. The activity is on the lower frit. The reference date is March 3, 2003. The emission rate is $4.85 \mathrm{e} 9 \mathrm{n} . \mathrm{s}^{-1}$....

7. Neutron dose factors $\left(\mathrm{pSv} . \mathrm{cm}^{2}\right)$ at increments of $10^{\circ}$ latitude above and below the equator of the reference sphere. The source encapsulation is in the upright configuration in the pneumatic transfer tube assembly. The activity is on the lower frit. The reference date is March 3, 2003. The emission rate is $4.85 \mathrm{e} 9$ n.s ${ }^{-1}$....

8. Neutron dose rate $\left(\right.$ rem. $\left.h^{-1}\right)$ at increments of $10^{\circ}$ latitude above and below the equator of the reference sphere. The ${ }^{252} \mathrm{Cf}$ source is in the $\mathrm{D}_{2} \mathrm{O}$ Moderating Sphere. The activity is on the upper frit. The reference date is March 3, 2003. The emission rate is $4.85 \mathrm{e} 9$ n. s $^{-1}$

9. Neutron dose factors $\left(\mathrm{pSv} . \mathrm{cm}^{2}\right)$ at increments of $10^{\circ}$ latitude above and below the equator of the reference sphere. The ${ }^{252} \mathrm{Cf}$ source is in the $\mathrm{D}_{2} \mathrm{O}$ Moderating Sphere. The activity is on the upper frit. The reference date is March 3, 2003. The emission rate is $4.85 \mathrm{e} 9 \mathrm{n} . \mathrm{s}^{-1}$.....

10. Neutron dose rate $\left(\mathrm{rem} \cdot \mathrm{h}^{-1}\right)$ at increments of $10^{\circ}$ latitude above and below the equator of the reference sphere. The ${ }^{252} \mathrm{Cf}$ source is in the $\mathrm{D}_{2} \mathrm{O}$ Moderating Sphere. The activity is 
in the void between frits. The reference date is March 3, 2003. The emission rate is $4.85 \mathrm{e} 9 \mathrm{n} \cdot \mathrm{s}^{-1}$

11. Neutron dose factors $\left(\mathrm{pSv} \cdot \mathrm{cm}^{2}\right)$ at increments of $10^{\circ}$ latitude above and below the equator of the reference sphere. The ${ }^{252} \mathrm{Cf}$ source is in the $\mathrm{D}_{2} \mathrm{O}$ Moderating Sphere. The activity is in the void between frits. The reference date is March 3, 2003. The emission rate is $4.85 \mathrm{e} 9 \mathrm{n} . \mathrm{s}^{-1}$....

12. Neutron dose rate $\left(\mathrm{rem} \cdot \mathrm{h}^{-1}\right)$ at increments of $10^{\circ}$ latitude above and below the equator of the reference sphere. The ${ }^{252} \mathrm{Cf}$ source is in the $\mathrm{D}_{2} \mathrm{O}$ Moderating Sphere. The activity is on the lower frit. The reference date is March 3, 2003. The emission rate is 4.85e9 n.s. ${ }^{-1}$

13. Neutron dose factors (pSv. $\left.\mathrm{cm}^{2}\right)$ at increments of $10^{\circ}$ latitude above and below the equator of the reference sphere. The ${ }^{252} \mathrm{Cf}$ source is in the $\mathrm{D}_{2} \mathrm{O}$ Moderating Sphere. The activity is on the lower frit. The reference date is March 3, 2003. The emission rate is $4.85 \mathrm{e} 9 \mathrm{n} \cdot \mathrm{s}^{-1}$

14. Neutron fluence at the surface of a $10-\mathrm{cm} \times 10-\mathrm{cm}$ rectangle $50 \mathrm{~cm}$ and $100 \mathrm{~cm}$ from the center of the reference sphere. The activity is on the upper frit. The reference date is March 3, 2003. The emission rate is $4.85 \mathrm{e} 9 \mathrm{n} . \mathrm{s}^{-1}$.

15. Neutron fluence at the surface of a $10-\mathrm{cm} \times 10-\mathrm{cm}$ rectangle $50 \mathrm{~cm}$ and $100 \mathrm{~cm}$ from the center of the reference sphere. The activity is in the void between the frits. The reference date is March 3, 2003. The emission rate is $4.85 \mathrm{e} 9 \mathrm{n} . \mathrm{s}^{-1}$.

16. Neutron fluence at the surface of a $10-\mathrm{cm} \times 10-\mathrm{cm}$ rectangle $50 \mathrm{~cm}$ and $100 \mathrm{~cm}$ from the center of the reference sphere. The activity is on the lower frit. The reference date is March 3, 2003. The emission rate is $4.85 \mathrm{e} 9 \mathrm{n} . \mathrm{s}^{-1}$....

17. Neutron Dose Factors at the surface of a $10-\mathrm{cm} \times 10-\mathrm{cm}$ rectangle $50 \mathrm{~cm}$ and $100 \mathrm{~cm}$ from the center of the reference sphere. The ${ }^{252} \mathrm{Cf}$ source is in the $\mathrm{D}_{2} \mathrm{O}$ moderating sphere. The activity is on the upper frit. The reference date is March 3, 2003. The emission rate is $4.85 \mathrm{e} 9 \mathrm{n} . \mathrm{s}^{-1}$....

18. Neutron Dose Factors at the surface of a $10-\mathrm{cm} \times 10-\mathrm{cm}$ rectangle $50 \mathrm{~cm}$ and $100 \mathrm{~cm}$ from the center of the reference sphere. The ${ }^{252} \mathrm{Cf}$ source is in the $\mathrm{D}_{2} \mathrm{O}$ moderating sphere. The activity is in the void between the frits. The reference date is March 3, 2003. The emission rate is $4.85 \mathrm{e} 9 \mathrm{n} . \mathrm{s}^{-1}$

19 Neutron Dose Factors at the surface of a $10-\mathrm{cm} \times 10-\mathrm{cm}$ rectangle $50 \mathrm{~cm}$ and $100 \mathrm{~cm}$ from the center of the reference sphere. The ${ }^{252} \mathrm{Cf}$ source is in the $\mathrm{D}_{2} \mathrm{O}$ moderating sphere. The activity is on the lower frit. The reference date is March 3, 2003. The emission rate is $4.85 \mathrm{e} 9 \mathrm{n} . \mathrm{s}^{-1}$

20. Neutron Dose Factors at the surface of a $10-\mathrm{cm} \times 10-\mathrm{cm}$ rectangle $50 \mathrm{~cm}$ and $100 \mathrm{~cm}$ from the center of the reference sphere. The ${ }^{252} \mathrm{Cf}$ source is in the pneumatic transfer assembly. The activity is on the upper frit. The reference date is March 3, 2003. The emission rate is $4.85 \mathrm{e} 9 \mathrm{n} . \mathrm{s}^{-1}$

21. Neutron Dose Factors at the surface of a $10-\mathrm{cm} \times 10-\mathrm{cm}$ rectangle $50 \mathrm{~cm}$ and $100 \mathrm{~cm}$ 
from the center of the reference sphere. The ${ }^{252} \mathrm{Cf}$ source is in the pneumatic transfer assembly. The activity is in the void between the frits. The reference date is March 3, 2003. The emission rate is $4.85 \mathrm{e} 9 \mathrm{n} \cdot \mathrm{s}^{-1}$

22. Neutron Dose Factors at the surface of a $10-\mathrm{cm} \times 10-\mathrm{cm}$ rectangle $50 \mathrm{~cm}$ and $100 \mathrm{~cm}$ from the center of the reference sphere. The ${ }^{252} \mathrm{Cf}$ source is in the pneumatic transfer assembly. The activity is on the lower frit. The reference date is March 3, 2003. The emission rate is $4.85 \mathrm{e} 9 \mathrm{n} . \mathrm{s}^{-1}$

23. Neutron dose rate at the surface of a $10-\mathrm{cm} \times 10-\mathrm{cm}$ rectangle $50 \mathrm{~cm}$ and $100 \mathrm{~cm}$ from the center of the reference sphere. The ${ }^{252} \mathrm{Cf}$ source is in the $\mathrm{D}_{2} \mathrm{O}$ moderating sphere. The activity is in the upper frit. The reference date is March 3, 2003. The emission rate is $4.85 \mathrm{e} 9 \mathrm{n} \cdot \mathrm{s}^{-1}$.

24. Neutron dose rate at the surface of a $10-\mathrm{cm} \times 10-\mathrm{cm}$ rectangle $50 \mathrm{~cm}$ and $100 \mathrm{~cm}$ from the center of the reference sphere. The ${ }^{252} \mathrm{Cf}$ source is in the $\mathrm{D}_{2} \mathrm{O}$ moderating sphere. The activity is in the void between the frits. The reference date is March 3, 2003. The emission rate is $4.85 \mathrm{e} 9 \mathrm{n} . \mathrm{s}^{-1}$....

25. Neutron dose rate at the surface of a $10-\mathrm{cm} \times 10-\mathrm{cm}$ rectangle $50 \mathrm{~cm}$ and $100 \mathrm{~cm}$ from the center of the reference sphere. The ${ }^{252} \mathrm{Cf}$ source is in the $\mathrm{D}_{2} \mathrm{O}$ moderating sphere. The activity is in the lower frit. The reference date is March 3, 2003. The emission rate is $4.85 \mathrm{e} 9 \mathrm{n} . \mathrm{s}^{-1}$....

26. Neutron dose equivalent rate at the surface of a $10-\mathrm{cm} \times 10-\mathrm{cm}$ rectangle $50 \mathrm{~cm}$ and 100 $\mathrm{cm}$ from the center of the reference sphere. The ${ }^{252} \mathrm{Cf}$ source is in the pneumatic transfer tube. The activity is in the upper frit. The reference date is March 3, 2003. The emission rate is $4.85 \mathrm{e} 9 \mathrm{n} . \mathrm{s}^{-1}$...

27. Neutron dose equivalent rate at the surface of a $10-\mathrm{cm} \times 10-\mathrm{cm}$ rectangle $50 \mathrm{~cm}$ and 100 $\mathrm{cm}$ from the center of the reference sphere. The ${ }^{252} \mathrm{Cf}$ source is in the pneumatic transfer tube. The activity is in the void between the frits. The reference date is March 3, 2003. The emission rate is $4.85 \mathrm{e} 9 \mathrm{n} . \mathrm{s}^{-1}$

28. Neutron dose equivalent rate at the surface of a $10-\mathrm{cm} \times 10-\mathrm{cm}$ rectangle $50 \mathrm{~cm}$ and 100 $\mathrm{cm}$ from the center of the reference sphere. The ${ }^{252} \mathrm{Cf}$ source is in the pneumatic transfer tube. The activity is in the lower frit. The reference date is March 3, 2003. The emission rate is $4.85 \mathrm{e} 9 \mathrm{n} . \mathrm{s}^{-1}$....

29. Neutron fluence at the surface of a $10-\mathrm{cm} \times 10-\mathrm{cm}$ rectangle $50 \mathrm{~cm}$ and $100 \mathrm{~cm}$ from the center of the reference sphere. The dose rectangle has been elevated $1 \mathrm{~cm}$. relative to the equator of the reference sphere. The activity is on the upper frit. The reference date is March 3, 2003. The emission rate is $4.85 \mathrm{e} 9 \mathrm{n} . \mathrm{s}^{-1}$....

30. Neutron dose factors at the surface of a $10-\mathrm{cm} \times 10-\mathrm{cm}$ rectangle $50 \mathrm{~cm}$ and $100 \mathrm{~cm}$ from the center of the reference sphere. The ${ }^{252} \mathrm{Cf}$ source is in the pneumatic transfer tube assembly. The dose rectangle has been elevated $1 \mathrm{~cm}$. relative to the equator of the reference sphere. The activity is on the upper frit. The reference date is March 3, 2003. The emission rate is $4.85 \mathrm{e} 9 \mathrm{n} . \mathrm{s}^{-1}$....

31. Neutron dose rates at the surface of a $10-\mathrm{cm} \times 10-\mathrm{cm}$ rectangle $50 \mathrm{~cm}$ and $100 \mathrm{~cm}$ from 
the center of the reference sphere. The ${ }^{252} \mathrm{Cf}$ source is in the pneumatic transfer tube assembly. The activity is on the upper frit. The dose rectangle has been elevated $1 \mathrm{~cm}$. relative to the equator of the reference sphere. The reference date is March 3, 2003. The emission rate is $4.85 \mathrm{e} 9 \mathrm{n} . \mathrm{s}^{-1}$

32. Neutron fluence at the surface of a $10-\mathrm{cm} \times 10-\mathrm{cm}$ rectangle $50 \mathrm{~cm}$ and $100 \mathrm{~cm}$ from the center of the reference sphere. The ${ }^{252} \mathrm{Cf}$ source is in the pneumatic transfer tube assembly. The dose rectangle has been elevated $1 \mathrm{~cm}$. relative to the equator of the reference sphere. The activity is in the void between the frits. The reference date is March 3, 2003. The emission rate is $4.85 \mathrm{e} 9 \mathrm{n} \cdot \mathrm{s}^{-1}$

33. Neutron dose factors at the surface of a $10-\mathrm{cm} \times 10-\mathrm{cm}$ rectangle $50 \mathrm{~cm}$ and $100 \mathrm{~cm}$ from the center of the reference sphere. The ${ }^{252} \mathrm{Cf}$ source is in the pneumatic transfer tube assembly. The dose rectangle has been elevated $1 \mathrm{~cm}$. relative to the equator of the reference sphere. The activity is in the void between the frits. The reference date is March 3, 2003. The emission rate is $4.85 \mathrm{e} 9 \mathrm{n} \cdot \mathrm{s}^{-1}$.

34. Neutron dose rates at the surface of a $10-\mathrm{cm} \times 10-\mathrm{cm}$ rectangle $50 \mathrm{~cm}$ and $100 \mathrm{~cm}$ from the center of the reference sphere. The ${ }^{252} \mathrm{Cf}$ source is in the pneumatic transfer tube assembly. The activity is in the void between the frits. The dose rectangle has been elevated $1 \mathrm{~cm}$. relative to the equator of the reference sphere. The reference date is March 3, 2003. The emission rate is $4.85 \mathrm{e} 9 \mathrm{n} . \mathrm{s}^{-1}$.

35. Neutron fluence at the surface of a $10-\mathrm{cm} \times 10-\mathrm{cm}$ rectangle $50 \mathrm{~cm}$ and $100 \mathrm{~cm}$ from the center of the reference sphere. The ${ }^{252} \mathrm{Cf}$ source is in the pneumatic transfer tube assembly. The dose rectangle has been elevated $1 \mathrm{~cm}$. relative to the equator of the reference sphere. The activity is on the lower frit. The reference date is March 3, 2003. The emission rate is $4.85 \mathrm{e} 9 \mathrm{n} . \mathrm{s}^{-1}$

36. Neutron dose factors at the surface of a $10-\mathrm{cm} \times 10-\mathrm{cm}$ rectangle $50 \mathrm{~cm}$ and $100 \mathrm{~cm}$ from the center of the reference sphere. The ${ }^{252} \mathrm{Cf}$ source is in the pneumatic transfer tube assembly. The dose rectangle has been elevated $1 \mathrm{~cm}$. relative to the equator of the reference sphere. The activity is on the lower frit. The reference date is March 3, 2003. The emission rate is $4.85 \mathrm{e} 9 \mathrm{n} . \mathrm{s}^{-1}$

37 Neutron dose rates at the surface of a $10-\mathrm{cm} \times 10-\mathrm{cm}$ rectangle $50 \mathrm{~cm}$ and $100 \mathrm{~cm}$ from the center of the reference sphere. The ${ }^{252} \mathrm{Cf}$ source is in the pneumatic transfer tube assembly. The activity is on the lower frit. The dose rectangle has been elevated $1 \mathrm{~cm}$. relative to the equator of the reference sphere....

38 Neutron fluence at the surface of a $10-\mathrm{cm} \times 10-\mathrm{cm}$ rectangle $50 \mathrm{~cm}$ and $100 \mathrm{~cm}$ from the center of the reference sphere. The ${ }^{252} \mathrm{Cf}$ source is in the pneumatic transfer tube assembly. The dose rectangle has been elevated $1.864 \mathrm{~cm}$. relative to the equator of the reference sphere. The activity is on the upper frit. The reference date is March 3, 2003. The emission rate is $4.85 \mathrm{e} 9 \mathrm{n} \cdot \mathrm{s}^{-1}$

39. Neutron dose rates at the surface of a $10-\mathrm{cm} \times 10-\mathrm{cm}$ rectangle $50 \mathrm{~cm}$ and $100 \mathrm{~cm}$ from the center of the reference sphere. The ${ }^{252} \mathrm{Cf}$ source is in the pneumatic transfer tube assembly. The activity is on the upper frit. The dose rectangle has been elevated 1.864 $\mathrm{cm}$. relative to the equator of the reference sphere. The reference date is March 3, 2003. The emission rate is $4.85 \mathrm{e} 9 \mathrm{n} . \mathrm{s}^{-1}$ 
40. Neutron dose factors at the surface of a $10-\mathrm{cm} \times 10-\mathrm{cm}$ rectangle $50 \mathrm{~cm}$ and $100 \mathrm{~cm}$ from the center of the reference sphere. The ${ }^{252} \mathrm{Cf}$ source is in the pneumatic transfer tube assembly. The dose rectangle has been elevated $1.864 \mathrm{~cm}$. relative to the equator of the reference sphere. The activity is on the upper frit. The reference date is March 3, 2003. The emission rate is $4.85 \mathrm{e} 9 \mathrm{n} . \mathrm{s}^{-1}$....

41. Neutron fluence at the surface of a $10-\mathrm{cm} \times 10-\mathrm{cm}$ rectangle $50 \mathrm{~cm}$ and $100 \mathrm{~cm}$ from the center of the reference sphere. The ${ }^{252} \mathrm{Cf}$ source is in the pneumatic transfer tube assembly. The dose rectangle has been elevated $1.864 \mathrm{~cm}$. relative to the equator of the reference sphere. The activity is in the void between the frits. The reference date is March 3, 2003. The emission rate is $4.85 \mathrm{e} 9 \mathrm{n} . \mathrm{s}^{-1}$...

42. Calculated dose rates at the surface of a $10-\mathrm{cm} \times 10-\mathrm{cm}$ rectangle $50 \mathrm{~cm}$ and $100 \mathrm{~cm}$ from the center of the reference sphere. The ${ }^{252} \mathrm{Cf}$ source is in the pneumatic transfer tube assembly. The activity is in the void between the frits. The dose rectangle has been elevated $1.864 \mathrm{~cm}$. relative to the equator of the reference sphere. The reference date is March 3, 2003. The emission rate is $4.85 \mathrm{e} 9 \mathrm{n} . \mathrm{s}^{-1}$.

43. Neutron dose factors at the surface of a $10-\mathrm{cm} \times 10-\mathrm{cm}$ rectangle $50 \mathrm{~cm}$ and $100 \mathrm{~cm}$ from the center of the reference sphere. The ${ }^{252} \mathrm{Cf}$ source is in the pneumatic transfer tube assembly. The dose rectangle has been elevated $1.864 \mathrm{~cm}$. relative to the equator of the reference sphere. The activity is in the void between the frits. The reference date is March 3, 2003. The emission rate is $4.85 \mathrm{e} 9 \mathrm{n} . \mathrm{s}^{-1}$.

44. Neutron fluence at the surface of a $10-\mathrm{cm} \times 10-\mathrm{cm}$ rectangle $50 \mathrm{~cm}$ and $100 \mathrm{~cm}$ from the center of the reference sphere. The ${ }^{252} \mathrm{Cf}$ source is in the pneumatic transfer tube assembly. The dose rectangle has been elevated $1.864 \mathrm{~cm}$. relative to the equator of the reference sphere. The activity is on the lower frit. The reference date is March 3, 2003. The emission rate is $4.85 \mathrm{e} 9 \mathrm{n} . \mathrm{s}^{-1}$

45. Calculated dose rates at the surface of a $10-\mathrm{cm} \times 10-\mathrm{cm}$ rectangle $50 \mathrm{~cm}$ and $100 \mathrm{~cm}$ from the center of the reference sphere. The ${ }^{252} \mathrm{Cf}$ source is in the pneumatic transfer tube assembly. The activity is on the lower frit. The dose rectangle has been elevated $1.864 \mathrm{~cm}$. relative to the equator of the reference sphere. The reference date is March 3 , 2003. The emission rate is $4.85 \mathrm{e} 9 \mathrm{n} \cdot \mathrm{s}^{-1}$

46. Neutron dose factors at the surface of a $10-\mathrm{cm} \times 10-\mathrm{cm}$ rectangle $50 \mathrm{~cm}$ and $100 \mathrm{~cm}$ from the center of the reference sphere. The ${ }^{252} \mathrm{Cf}$ source is in the pneumatic transfer tube assembly. The dose rectangle has been elevated $1.864 \mathrm{~cm}$. relative to the equator of the reference sphere. The activity is on the lower frit. The reference date is March 3, 2003. The emission rate is $4.85 \mathrm{e} 9 \mathrm{n} . \mathrm{s}^{-1}$

47. Comparison of neutron fluence-to-dose-equivalent conversion factors published by international agencies and those prepared for this report. 


\begin{tabular}{|c|c|}
\hline & SHEET 1 OF 246 \\
\hline CALCULATION COVER SHEET & $\frac{\text { CALCULATION NO: }}{\text { PNNL-19273 Rev.0 }}$ \\
\hline
\end{tabular}

TITLE:

Neutron Fluence, dose equivalent, personal dose equivalent, and ambient dose equivalent calculations for the SR-Cf3000 neutron source in the PNNL Low Scatter Room

\section{PURPOSE AND OBJECTIVE:}

The purpose of this calculation is to calculate the neutron dose factors for the Sr-Cf-3000 neutron source that is located in the 318 low scatter room (LSR). The dose factors were based on the dose conversion factors published in ICRP-21 Appendix 6, and the Ambient dose equivalent $\left(\mathrm{H}^{*}(10)\right)$ and Personal dose equivalent $\left(\mathrm{H}_{\mathrm{p}}(10)\right)$ dose factors published in ICRP Publication 74.

\begin{tabular}{|c|c|c|c|c|}
\hline $\begin{array}{l}\text { REV. } \\
\text { No. }\end{array}$ & $\begin{array}{l}\text { REVISION DESCRIPTION } \\
\text { (ADD CoNTINUATION SHEET I REQUURED) }\end{array}$ & $\begin{array}{l}\text { PREPARED BY } \\
\text { NAME/DATE }\end{array}$ & $\begin{array}{l}\text { REVIEWED BY } \\
\text { NAME/DATE }\end{array}$ & $\begin{array}{c}\text { PM/TL } \\
\text { APPROVAL/DATE }\end{array}$ \\
\hline 0 & Original & $\begin{array}{c}\text { R.J. Traub } \\
2 / 8 / 2010\end{array}$ & $\begin{array}{l}\text { R.J. McConn } \\
3 / 21 / 2010\end{array}$ & $\begin{array}{l}\text { R.J. Traub } \\
3 / 21 / 2010\end{array}$ \\
\hline
\end{tabular}




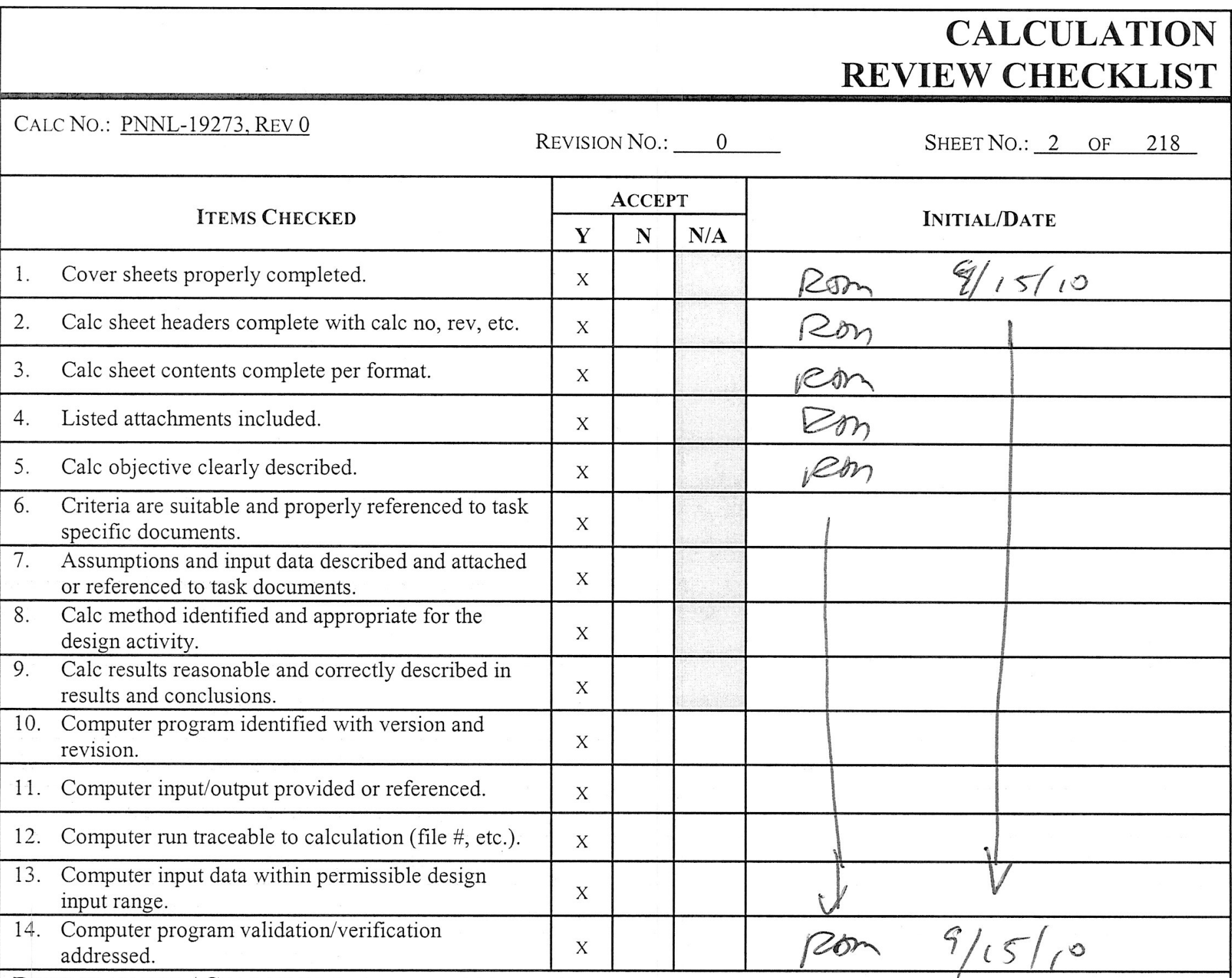

DISCREPANCIES / COMMENTS
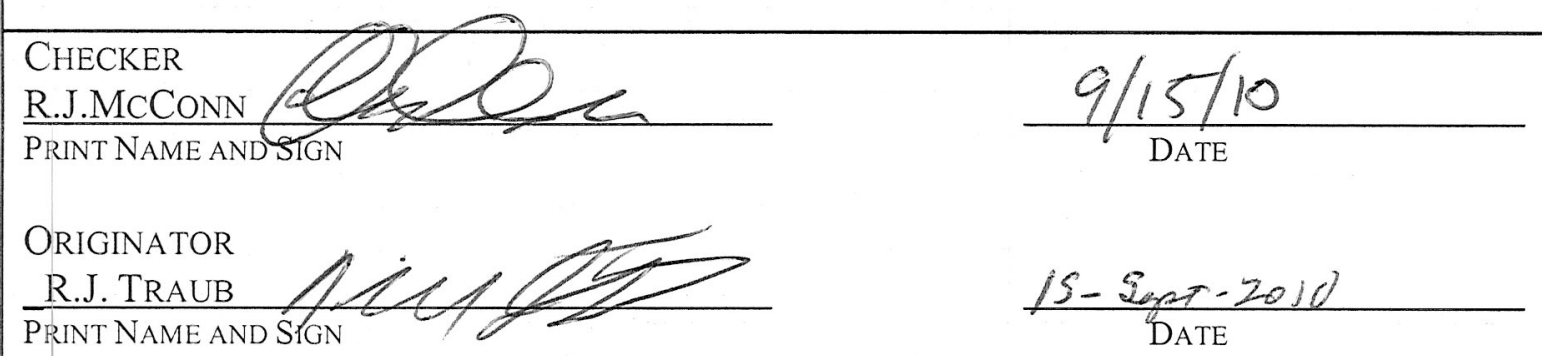

Signatures obtained only after discrepancies are corrected and comments are resolved. 


\section{CALCULATION SHEET}

\section{PROJECT No: \\ CALC NO: \\ REVISION NO.: $\underline{0}$ \\ SHEET NO.: $\underline{3 / 246}$ \\ PNNL-19273 \\ SubJeCt: CALCULATIONS THE SR-CF-3000 ${ }^{252}$ CF NEUTRON SOURCE IN 318LOW SCATTER RoOM \\ PREPARED BY: R.J. TRAUB DATE: $\underline{02 / 8 / 10}$ REVIEWED BY: R.J.MCCONN \\ DATE: $\underline{03 / 21 / 10}$ \\ Objective: \\ The purpose of the calculations described in this report is to provide the user with neutron dose rates from the SR-Cf- 3000 neutron source located in $318 \mathrm{Rm} .106$ that are based on the ambient dose equivalent and personal dose equivalent tables published in ICRP Publication 74 (ICRP 1986).}

\section{Design Input References:}

References are located in section 7.0.

\section{Method:}

Simplified geometries of the Sr-Cf-3000 neutron source encapsulation, the pneumatic transfer mechanism and the $\mathrm{D}_{2} \mathrm{O}$ moderating sphere were defined for the input to the computer codes used to calculate radiation doses.

All calculations were performed using MCNP5 Version 1.4, Los Alamos National Laboratory, Los Alamos, NM. MCNP5 is a general purpose Monte Carlo radiation transport code that can be used for neutron, photon, electron, or coupled neutron/photon/electron transport. The code is used for arbitrary three dimensional materials bounded by first- and second-degree surfaces and fourth-degree elliptical tori. This code was chosen because the problem required the calculation of radiation dose from multiply scattered neutrons in a complex geometry. Upon installation of the code on each machine on which it was run, the program was validated by running appropriate test cases provided by Los Alamos National Laboratory. Results were acceptable and copies of the validation reports are included in the project files.

The results of the MCNP5 calculations were consolidated by the Excel spreadsheet program. The spreadsheet program was used to extract the appropriate data from the MCNP5 output, to sum the dose equivalent rates from the separate MCNP5 calculations, and to propagate the Monte Carlo associated error of the calculations.

\section{Assumptions:}

1.) The neutron emission rate from the neutron source was $9.85 \times 10^{9} \mathrm{n} \cdot \mathrm{s}^{-1}$ on a calibration date of 3-March-2003.

2.) The engineering drawings available to us adequately describe the geometry of the source encapsulation.

\section{Conclusions/Remarks:}

The exact location of the ${ }^{252} \mathrm{Cf}$ source material in the source encapsulation could not be determined from the engineering drawings. Measurements, not included in this report, indicate that the ${ }^{252} \mathrm{Cf}$ activity might be associated with the upper or lower frit of the primary encapsulation. The impact of this observation is that the center of the ${ }^{252} \mathrm{Cf}$ neutron source apparently is not collocated with the center of the $\mathrm{D}_{2} \mathrm{O}$ filled sphere that is used to moderate neutrons for certain irradiations. Other measurements could be interpreted to mean that the ${ }^{252} \mathrm{Cf}$ activity is in two separate locations at the upper and lower boundary of the central void region of the primary source encapsulation. The numeric models implemented in MCNP5, which are based on engineering drawings and discussed in section 1, indicate that the ${ }^{252} \mathrm{Cf}$ activity is located above the center of the $\mathrm{D}_{2} \mathrm{O}$ moderating sphere and the distance above the 


\section{CALCULATION SHEET}

PROJECT NO.:

CALC NO.:

REVISION NO.: $\underline{0}$

SHEET NO.: $\underline{4 / 246}$

PNNL-19273

SubJect: CAlCulations THE SR-CF-3000 ${ }^{252}$ CF NEUTRON SOURCE IN 318Low SCATtER RoOM

PREPARED BY: R.J. TRAUB DATE: $\underline{02 / 8 / 10}$ REVIEWED BY: R.J.MCCONN

DATE: $\underline{03 / 21 / 10}$

equator can range from $0.92 \mathrm{~cm}$ to $2.80 \mathrm{~cm}$ above the equator of the sphere.

The result of the offset from the center of the moderating sphere is that the moderated neutron fluence and dose rates appear to be highly asymmetrical with respect to the midplane (equator) of the $\mathrm{D}_{2} \mathrm{O}$ moderating sphere. Previously, the moderated neutron fluence and dose rates were assumed to be symmetric about the midplane (equator) of the $\mathrm{D}_{2} \mathrm{O}$ moderating sphere.

This calculation package presents fluence rates and ambient dose equivalent and personal dose equivalent rates from the Sr-Cf-3000 neutron source in the 318 low scatter room for moderated and unmoderated neutrons. 


\subsection{INTRODUCTION}

The Monte Carlo N-Particle (MCNP), version 5.14, radiation transport code, developed at Los Alamos National Laboratory (X-5 Monte Carlo Team 2003) was used to perform the calculations described in this report. The neutron fluence was used to assess the influence of the source encapsulation. All calculated neutron fluences were binned into 53 energy groups. The binning structure was the 52-group structure used in ISO-8529 that was extended down to $10^{-11} \mathrm{MeV}$ from $0.414 \times 10^{-7} \mathrm{MeV}$ to maintain consistency with previous work (Hertel and McDonald 1991).

The fluences were converted into dose equivalent rates using the dose factors of ICRP Publication 21 (ICRP 1973) and the ICRP Publication 74 (ICRP 1996) $\left(H_{p}(10)\right.$ and $\left.H^{*}(10)\right)$. The application of the dose factors was performed using functions built into MCNP that used log-log interpolation of the published dose conversion factors. The factors from ICRP Publication 21 (ICRP 1973) were included to show consistency with previous calculations.

\subsection{Geometry and Materials}

The geometry is based on the geometry that exists in the Low Scatter Room of the 318 Building. When not in use, radiation sources are housed in a shielded location. The sources are transported from the shielded location to the irradiation location by means of a pneumatic transfer system. Two types of neutron irradiations are possible, moderated and unmoderated neutrons.

In the case of unmoderated neutron irradiations, the ${ }^{252} \mathrm{Cf}$ source material is encased in a primary and secondary sealed encapsulation. The secondary encapsulation is encased in an aluminum transfer capsule that is designed to function with a pneumatic transfer system. The radiation source transfer capsule assembly is located in the irradiation position of the pneumatic transfer system which consists of two concentric tubes, base plate, and a spring to dampen the force associated with the source landing in the irradiation position. Radiation detectors are then positioned at selected distances from the ${ }^{252} \mathrm{Cf}$ source for calibration irradiations.

In the case of moderated neutron irradiations, a15-cm radius $\mathrm{D}_{2} \mathrm{O}$ filled, stainless steel sphere with a $0.0508-\mathrm{cm}$ thick cadmium outer layer is positioned around the transfer tubing. A second moderating sphere without a $0.05-\mathrm{cm}(0.02$-inch) thick cadmium layer is available at the 318 low scatter room but was not modeled for these calculations.

The intent of the design of the irradiation facility is that the center of the $\mathrm{D}_{2} \mathrm{O}$ filled moderating sphere should be collocated with the center of the ${ }^{252} \mathrm{Cf}$ radiation source. For that reason, the midplane (equator) of the $\mathrm{D}_{2} \mathrm{O}$ moderating sphere is a reference position for the irradiations in that irradiations are planned so that approximately one-half of the sensitive volume of a radiation detector or dosimeter is located above the equator of the moderating sphere and one-half of the sensitive volume is located below the equator of the moderating sphere.

During the evolution of these calculations, it was determined that when the SR-Cf-3000 source is in its irradiation location the ${ }^{252} \mathrm{Cf}$ activity is not located at the center of the $\mathrm{D}_{2} \mathrm{O}$ moderating sphere but could 


\section{CALCULATION SHEET}

\begin{tabular}{|c|c|c|c|}
\hline \multicolumn{4}{|c|}{$\begin{array}{l}\text { CALC NO.: } \\
\text { PNNL-19273 }\end{array}$} \\
\hline SuBJECt: CALCULATIONS T & $00{ }^{252} \mathrm{CF}$ NEUTRO & E IN 318LOW SCATTER ROOM & \\
\hline PREPARED BY: R.J. TRAUB & DATE: $\underline{02 / 8 / 10}$ & REVIEWED BY: R.J.MCCONN & DATE: $\underline{03 / 21 / 10}$ \\
\hline
\end{tabular}

be located above the equator of the sphere at a distance ranging from $0.9 \mathrm{~cm}$ above the equator of the sphere to $2.8 \mathrm{~cm}$ above the equator of the sphere.

Three geometries were investigated for this report. The first geometry, labeled "lower," places all of the activity $0.9 \mathrm{~cm}$ above the equator of the $\mathrm{D}_{2} \mathrm{O}$ moderating sphere and is thought to be the most likely true geometry. The second geometry, labeled "middle," places all the ${ }^{252} \mathrm{Cf}$ activity in the void region between the upper and lower frits of the primary encapsulation. The last geometry, labeled "upper," places all the activity $2.8 \mathrm{~cm}$ above the equator of the $\mathrm{D}_{2} \mathrm{O}$ moderating sphere.

This section describes the evolution of the MCNP input files for the source encapsulation, the pneumatic transfer capsule and tubing, and the $\mathrm{D}_{2} \mathrm{O}$ moderating sphere. This discussion, along with copies of the source drawings in Appendix A and the MCNP input file stubs in Appendix B are intended to allow future modelers to modify the MCNP input files without difficulty. This also documents what is known about the source encapsulation.

The initial hope was that the ${ }^{252} \mathrm{Cf}$ source material would be located in a small, well defined region. It turns out that the exact location of the ${ }^{252} \mathrm{Cf}$ activity isn't really known. The volume in which the ${ }^{252} \mathrm{Cf}$ activity could be located is about $0.75+/-0.02$ inches long. There is the possibility that some unknown fraction of the activity could be imbedded into one of the frits, most likely the upper frit. Some other unknown fraction of the ${ }^{252} \mathrm{Cf}$ activity could be loose and be drifting from end to end of the void volume. 


\section{CALCULATION SHEET}

\begin{tabular}{|c|c|c|c|}
\hline Project No.: & $\begin{array}{l}\text { CALC NO.: } \\
\text { PNNL-19273 }\end{array}$ & REVISION NO.: $\underline{0}$ & SHEET NO.: $\underline{7 / 246}$ \\
\hline \multicolumn{4}{|c|}{ SUBJECT: CALCULATIONS THE SR-CF-3000 ${ }^{252}$ CF NEUTRON SOURCE IN 318 LOW SCATTER ROOM } \\
\hline PREPARED BY: R.J. TRAUB & DATE: $\underline{02 / 8 / 10}$ & REVIEWED BY: R.J.MCCONN & DATE: $\underline{03 / 21 / 10}$ \\
\hline
\end{tabular}

\section{Source encapsulation}

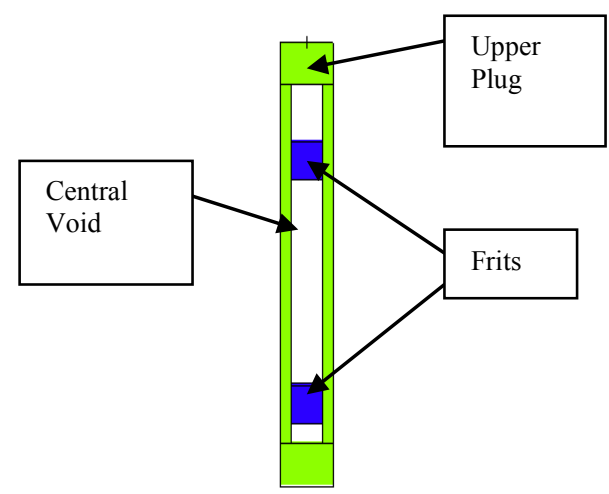

Figure 1.a. The primary encapsulation of the Sr-Cf-3000 source. Sr3000_Primary.menp.inp

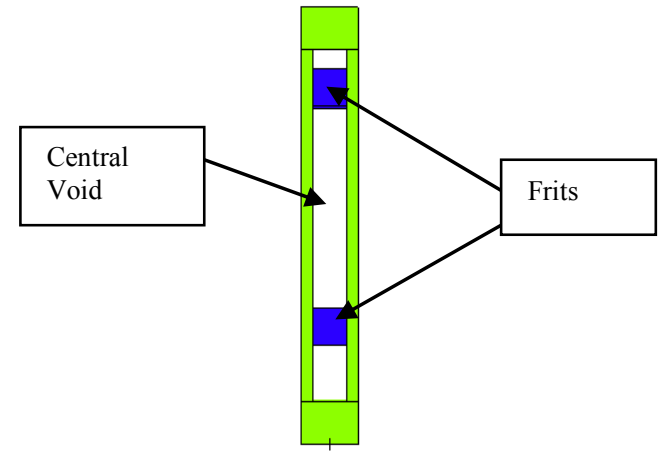

Figure 1.b. The primary encapsulation in the inverted geometry. Sr3000_Primary_flip.mcnp.inp.

The SR-Cf-3000 source is a double encapsulated source. The primary encapsulation is a platinumrhodium alloy that is $90 \%$ platinum, by weight. The primary encapsulation is contained in a secondary encapsulation that was specified in the engineering drawings as either 304L or Zircaloy-2. For these calculations, the authors assumed that the secondary encapsulation material was Zircaloy-2 to maintain consistency with previous work (Hertel and McDonald).

The dimensions of the primary encapsulation were obtained from DWG M-12541-CP-338E. A cross sectional view of the primary encapsulation is shown in Figure 1.a. (Note: the italicized part of the figure name is the name of the MCNP input file that is being shown.) The reference drawing clearly shows a shoulder in the capsule body interior for the placement of the bottom frit which results in the small void region at the lower part of Figure 1.a. The drawing shows the upper frit at the opposite end of the primary capsule body and in contact with the upper plug. Other drawings of the same series source show a void region at both ends of the primary encapsulation. An email from $\{$ LK Felker (Appendix C) $\}$ stated that the circumferential scribes noted in the Primary Capsule drawing describe the upper and lower boundaries of the source volume. According to the drawing, the scribes are 0.755 inches apart which implies that the source volume is 0.755 inches $(1.9177 \mathrm{~cm})$ long. However, the drawing also indicates that shoulder is milled into the inner wall of the primary encapsulation to serve as a stop when pushing the lower frit into position. The tolerances for the frit are such that the length of the frit can range from 0.130 inch to 0.140 inch. If the 0.140 inch length is used then the void between the two frits is 0.74 inches apart. This latter number is what was used for the model.

Also according to $\mathrm{Mr}$. Felker, the ${ }^{252} \mathrm{Cf}$ is introduced to the primary encapsulation as an oxalate precipitate and the lower frit serves as a filter for the material. The upper, second frit is then pressed in with a tool that places the bottom of the frit at the top scribe mark in this configuration The oxalate is 


\section{CALCULATION SHEET}

\begin{tabular}{|c|c|c|c|}
\hline PROJECT NO.: & $\begin{array}{l}\text { CALC NO.: } \\
\text { PNNL-19273 }\end{array}$ & REVISION NO.: $\underline{0}$ & SHEET NO. \\
\hline \multicolumn{4}{|c|}{ SUBJECT: CALCULATIONS THE SR-CF-3000 ${ }^{252}$ CF NEUTRON SOURCE IN 318 LOW SCATTER ROOM } \\
\hline PREPARED BY: R.J. TRAUB & DATE: $\underline{02 / 8 / 10}$ & REVIEWED BY: $\underline{\text { R.J.MCCONN }}$ & DATE: $\underline{03 / 2}$ \\
\hline
\end{tabular}

Appendix B contains the MCNP cell and surface descriptions for the primary encapsulation. The pz type surfaces are the distances from the top end, in agreement with DWG M-12541-CP-338E. When the primary is placed in the secondary, the initial bottom frit end is placed up toward the threaded end of the secondary encapsulation as shown in Figure 1.b. In the MCNP implementation of the primary encapsulation, the inversion of the primary encapsulation is accomplished by reversing the signs of the $\mathbf{p z}$ surfaces both in the surface definition section and the cell definition section.

The distribution of the source material in the void region is unknown. It could range from a single mass somewhere in the source volume to a uniform distribution throughout the entire volume. At the end of the transfer operation from the storage location to the irradiation location in the Low Scatter Room (LSR) the operators hear an audible "knock" as the transport capsule reaches the irradiation position and comes to an abrupt stop. Based on this observation, it seems plausible that most of the unbound source material has been shaken to the bottom of the source void region.

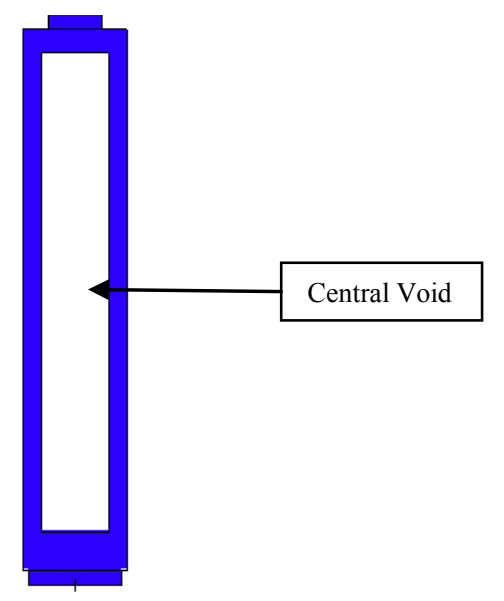

Figure 2.a. The secondary encapsulation. Sr3000_Secondary.mcnp.inp

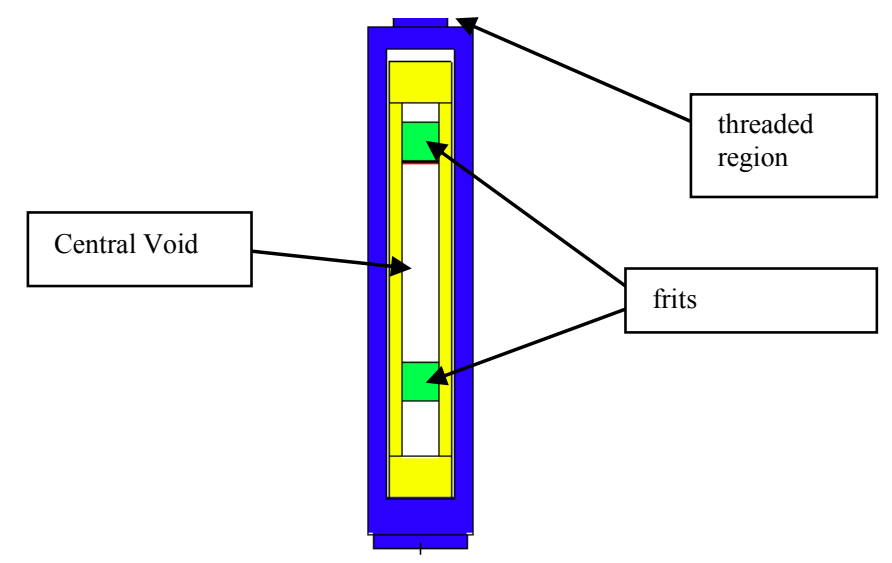

Figure 2.b. The secondary encapsulation with the primary encapsulation. Sr3000_SourceAssembly.mcnp.inp

The inverted primary encapsulation is then placed in a secondary encapsulation. A cross sectional view of the secondary encapsulation is shown in Figure 2.a. Figure 2.b is a cross sectional view of the the secondary encapsulation with the primary encapsulation. The primary encapsulation is somewhat shorter than the interior length of the secondary encapsulation. The top of the secondary encapsulation is threaded. The threaded region is screwed into the cap of the aluminum transfer capsule. 


\section{CALCULATION SHEET}

\begin{tabular}{|c|c|c|}
\hline PROJECT NO.: & $\begin{array}{ll}\text { CALC NO.: } & \text { REVISION NO.: } \underline{0} \\
\text { PNNL-19273 } & \end{array}$ & SHEET NO.: 9 \\
\hline \multicolumn{3}{|c|}{ 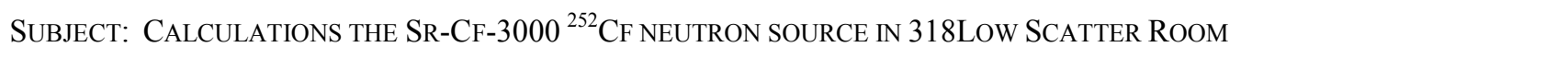 } \\
\hline PREPARED BY: R.J. TRAUB & REVIEWED BY: $\underline{\text { R.J.MCCONN }}$ & DATE: $\underline{03 / 21}$ \\
\hline
\end{tabular}

\section{Pneumatic Transfer System}
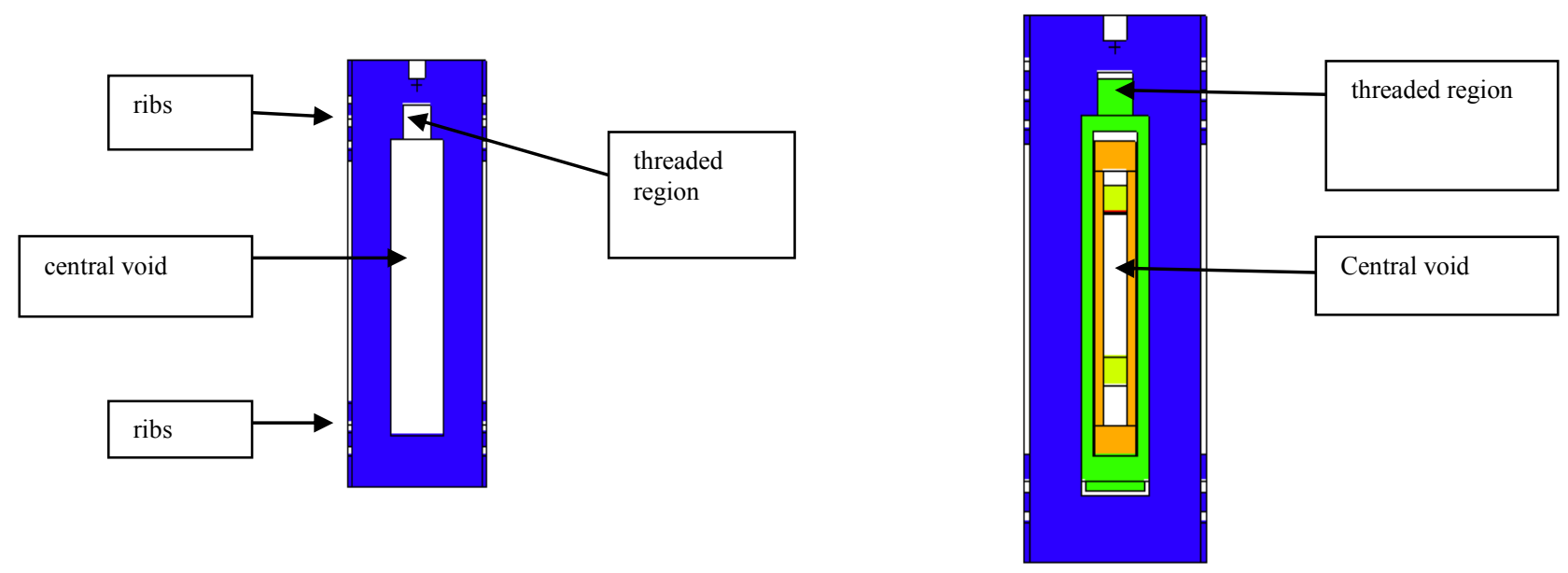

Figure 3.a. The pneumatic transfer capsule. Sr3000_TransferCapsule.mcnp.inp
Figure 3.b. The Sr-Cf-3000 transfer capsule assembly. Sr3000_TransferCapsuleAssembly.mcnp.inp

The source encapsulation is encased in an aluminum transfer enclosure, called a transfer capsule. This item is used by the pneumatic transfer system to transfer the source from the source storage location to the irradiation location. The transfer capsule provides a uniform interface between the pneumatic system and the source encapsulation.

The dimensions of the transfer capsule were obtained from something labeled "Figure 2." A cross sectional view of the transfer capsule is shown in Figure 3.a. The threaded region noted in the figure is tapped into the screw-on cover of the transfer capsule.

Figure 3.b shows a cross sectional view of the transfer capsule with the primary and secondary encapsulations (transfer capsule assembly). The threaded portion of the secondary encapsulation is screwed into the tapped hole of the transfer capsule. The secondary encapsulation is shorter than the cavity in the transfer capsule so there is an air gap under the secondary encapsulation. 


\section{CALCULATION SHEET}

\begin{tabular}{|c|c|c|c|}
\hline \multicolumn{4}{|c|}{$\begin{array}{l}\text { CALC NO.: } \\
\text { PNNL-19273 } \\
\end{array}$} \\
\hline T: CALCULATIONS & $00{ }^{252} \mathrm{CF}$ NeUtro & E IN 318LOW SCATTER ROOM & \\
\hline PREPARED BY: R.J. TRAUB & DATE: $\underline{02 / 8 / 10}$ & REVIEWED BY: R.J.MCCONN & DATE: $\underline{03 / 21 / 10}$ \\
\hline
\end{tabular}

The source is transferred between its storage location and its irradiation position using a pneumatic transfer system. The part of the transfer system included in the modeling was the metal tubing located at the irradiation position. The dimensions of the transfer tube assembly were obtained from measurements of the actual assembly. A cross sectional view of the transfer tube assembly is shown in Figure 4.a.

Figure 4.b shows a cross sectional view of the transfer tube assembly with the loaded transfer capsule in place. For this figure, 4.953 was added to the value of the $\mathbf{p z}$ surfaces of the transfer capsule assembly. This process lowers the transfer capsule assembly so that the lower surface of the transfer capsule assembly is at the upper surface of the spring. The spring was modeled as a solid mass of A228 spring steel at a reduced density of $1.123 \mathrm{~g} . \mathrm{cm}^{-3}$.

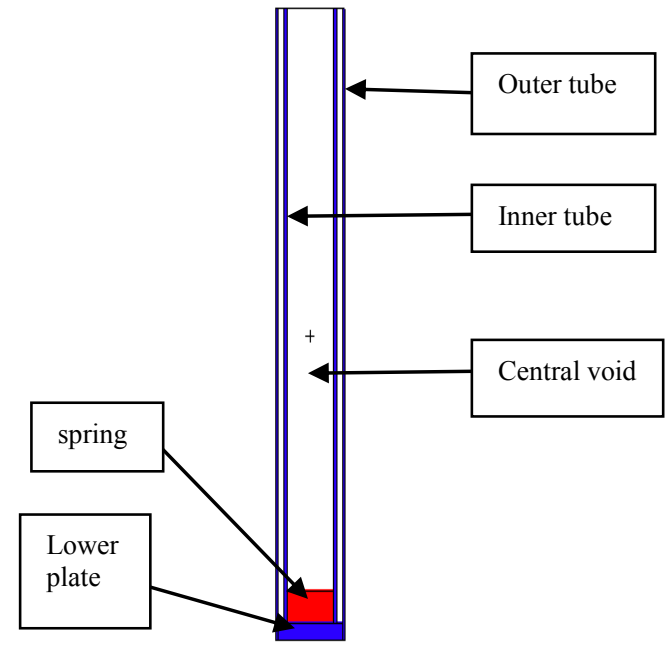

Figure 4.a Transfer Tube LSR_TransferTubeAssembly.mcnp.inp

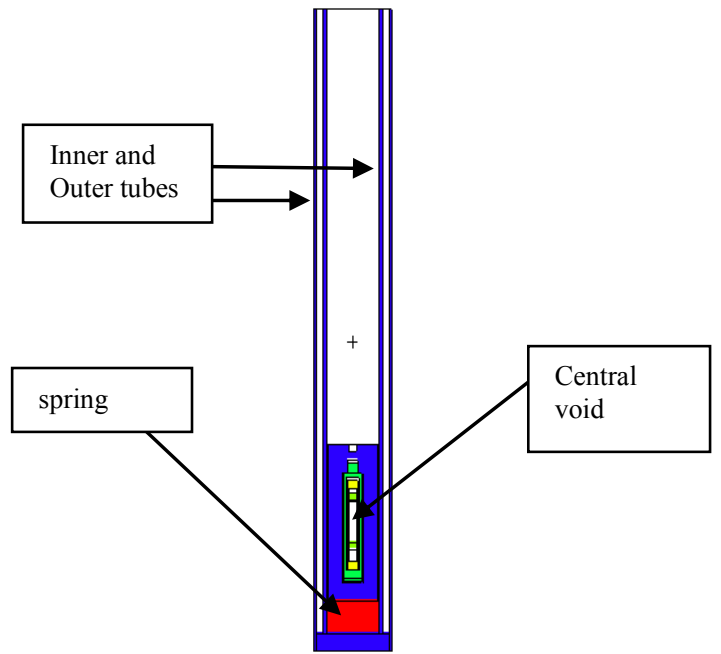

Figure 4.b TransferTube Assembly SR3000_TransferTubeAssembly.mcnp.inp

\section{$\mathrm{D}_{2} \mathrm{O}$ Moderating Sphere}

To moderate the neutrons, the neutron source is surrounded by a $30-\mathrm{cm}$ (I.D.) stainless steel sphere that contains $\mathrm{D}_{2} \mathrm{O}$ as a moderator. The sphere is made up of two hemispheres welded together with the seam running from pole to pole such that the sphere can be positioned without the seam being in the direct path between the source and artifacts being exposed. The MCNP5 model is based on DWG. SK-3-27847. This sphere is surrounded by a $0.0508 \mathrm{~cm}$ thick layer of $\mathrm{Cd}$ to remove the thermal neutrons from the beam. Again, two hemispheres make up the sphere, but in this case, the seam is at the equator and as such forms a slightly thicker region along the direct axis of the source and artifacts being exposed. This seam was not included in the MCNP model. Figure 5.a shows a cross sectional view of the $\mathrm{D}_{2} \mathrm{O}$ moderating sphere. The outer shell consists of a 0.03 inch $(0.0762 \mathrm{~cm})$ layer of 304L steel and an outer 0.02 inch $(0.508 \mathrm{~cm})$ layer of cadmium. Figure $5 . \mathrm{b}$ shows the transfer assembly in the moderating sphere as built. The small "+" in the two figures is the location of the center of the sphere. The "+" is difficult to see in Figure 5.b but is located near the top of the lower plug of the primary encapsulation. The central void region of the primary encapsulation ranges from 0.364 inches $(0.92456 \mathrm{~cm})$ above the center of the 


\section{CALCULATION SHEET}

\begin{tabular}{|c|c|c|c|}
\hline PROJECT No.: & $\begin{array}{l}\text { CALC NO.: } \\
\text { PNNL-19273 }\end{array}$ & REVISION NO.: $\underline{0}$ & SHEET NO.: $\underline{11 / 246}$ \\
\hline \multicolumn{4}{|c|}{ E IN 318LOW SCATTER ROOM } \\
\hline PREPARED BY: R.J. TRAUB & DATE: $\underline{02 / 8 / 10}$ & REVIEWED BY: R.J.MCCONN & DATE: $\underline{03 / 21 / 10}$ \\
\hline
\end{tabular}

sphere to 1.104 inches $(2.80416 \mathrm{~cm})$ above the center of the sphere. The implications of the ${ }^{252} \mathrm{Cf}$ activity being above the center of the $\mathrm{D}_{2} \mathrm{O}$ moderating sphere are discussed in the main body of this calculation package.
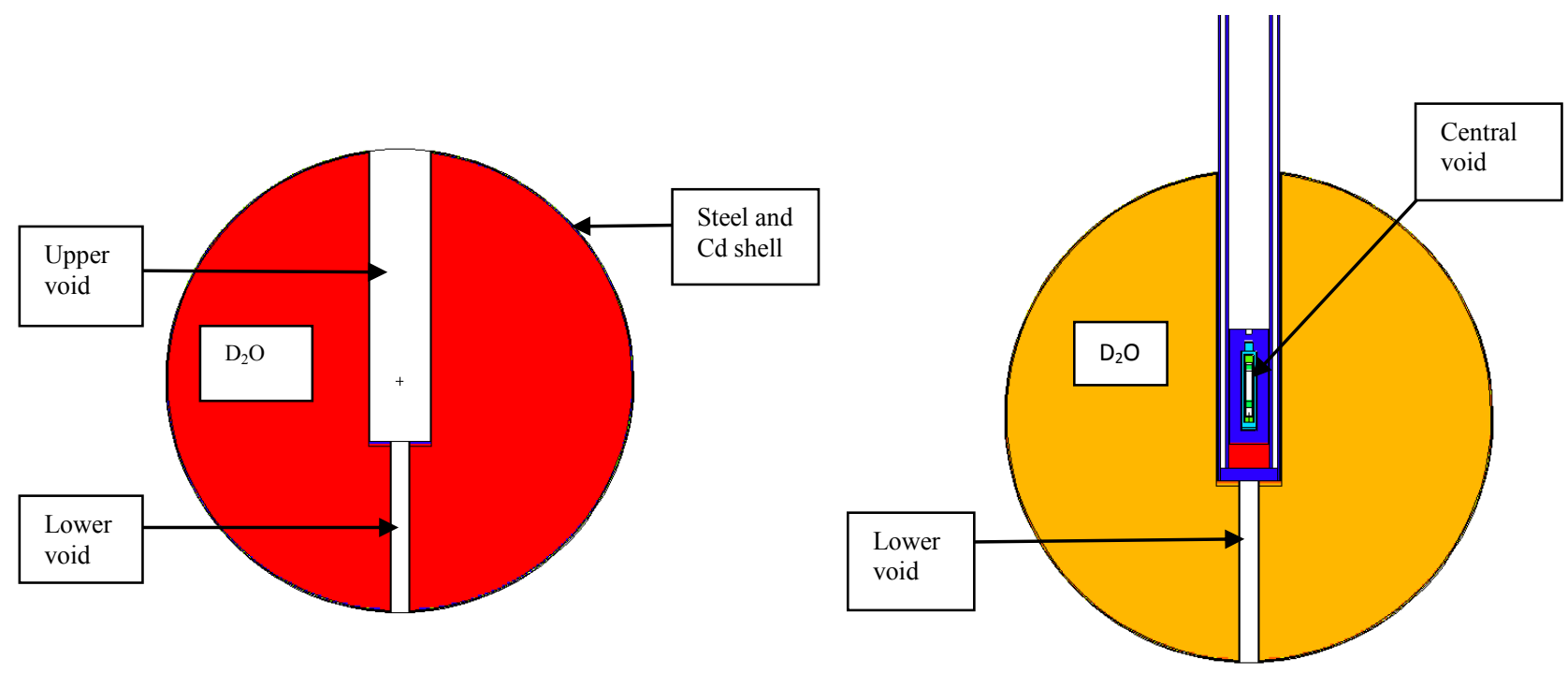

Figure 5.a. $\mathrm{D}_{2} \mathrm{O}$ moderating sphere LSR_D2O_Sphere.mcnp.inp

Figure 5.b. Cf-Sr-3000 moderating sphere assembly. SR3000_D2OSphere.mcnp.inp

The upper void region shown in Figure 5.a was designed to allow the insertion of the transfer tube assembly, as shown in Figure 5.b. The lower void region of the moderating sphere, called out in both figures, had been used to contain a radiation detector that would notify the operator that a radiation source occupied the irradiation position. At the time this report was written nothing except air was located in the lower void region.

\subsection{Radiation Source Term}

The source contains $12,237 \mu \mathrm{g}{ }^{252} \mathrm{Cf}$ as of $7 / 12 / 1996$. The source material is $\mathrm{Cf}_{2} \mathrm{O}_{3}$ and the mass fraction of ${ }^{252} \mathrm{Cf}$ in the oxide was 0.913 . If the density of $\mathrm{Cf}_{2} \mathrm{O}_{3}$ is $11.46 \mathrm{~g} \mathrm{~cm}^{-3}$ (the density of plutonium oxide) and assuming a packing fraction of 1.0 , the volume of $\mathrm{Cf}_{2} \mathrm{O}_{3}$ was approximately

$$
\mathrm{Vol}=\left(0.012237 \mathrm{~g}^{252} \mathrm{Cf}\right) /\left(11.46 \mathrm{~g} \mathrm{~cm}^{-3} * 0.913\right)=1.169 \mathrm{E}-3 \mathrm{~cm}^{3} .
$$




\section{CALCULATION SHEET}

\begin{tabular}{|c|c|c|c|}
\hline \multicolumn{4}{|c|}{$\underline{\text { PNNL-19273 }}$} \\
\hline CALCULATIONS & $00{ }^{252} \mathrm{CF}$ NEUTRO & E IN 318LOW SCATTER ROOM & \\
\hline PREPARED BY: R.J. TRAUB & DATE: $\underline{02 / 8 / 10}$ & REVIEWED BY: $\underline{\text { R.J.MCCONN }}$ & DATE: $\underline{03 / 21 / 10}$ \\
\hline
\end{tabular}

The source was last calibrated at NIST on 3/3/03 and the neutron emission rate was $4.85 \mathrm{E}+09 \mathrm{n} \mathrm{s}^{-1}$.

Table 1 shows a summary of the calculations for the MCNP material definition of the source material. The calculations are based on a mass assay date of 27-Feb-1995. The chemistry of the progeny radioelement $\mathrm{Cm}$ is not certain, but the assumption was that the product would be $\mathrm{Cm}_{2} \mathrm{O}_{3}$. As seen in the table, the elemental composition underwent a significant change from being mostly californium to being mostly curium.

Calculations were performed with Sources4 (Wilson et al 1999; Shores 2000) to determine if the ( $\alpha, n)$ reactions with oxygen in the source material or the in growth of curium isotopes that undergo spontaneous fission might influence the neutron spectrum. The data shown in Table 1 was used as input to Sources4. The results of the calculations, indicate that the total $(\alpha, n)$ neutron production would be very small compared to the ${ }^{252} \mathrm{Cf}$ neutron production. Indeed, even the neutron production from $\mathrm{Cm}$ is much less than the neutron production from ${ }^{252} \mathrm{Cf}$ even though the mass of $\mathrm{Cm}$ greatly exceeds the mass of ${ }^{252} \mathrm{Cf}$. The greatest influence on the neutron spectrum would be that, due to its relative long half-life, ${ }^{250} \mathrm{Cf}$ has increased in importance and is responsible for about $1 \%$ of the neutrons emitted by the neutron source.

To maintain consistency with previous work (Hertel and McDonald 1990) the SDEF card was set to represent the ${ }^{252} \mathrm{Cf}$ fission spectra as a Maxwellian distribution $(\mathrm{kT}=1.42 \mathrm{MeV})$. 


\section{CALCULATION SHEET}

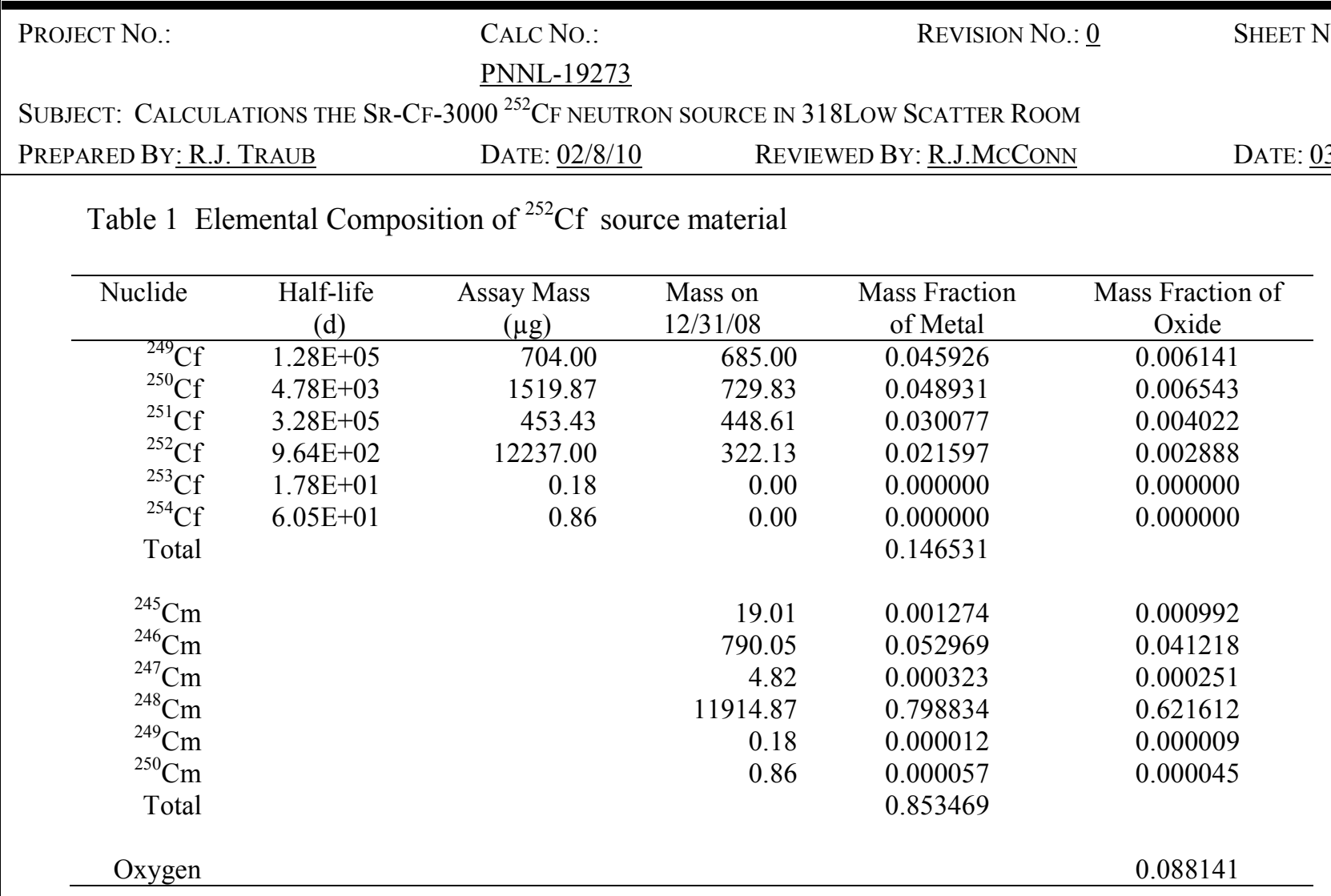

\subsection{Dose Equivalent Calculation}

\section{Dose Factors}

MCNP calculates fluence rates of photons and neutrons in air. Dose conversion factors were used to translate the fluence rates to dose equivalent rates. The dose factors from Appendix 6 of ICRP-21 (ICRP 1973) were used to calculate dose equivalent and the dose factors from Table A.42 of ICRP-74 (ICRP 1996) were used to calculate personal dose equivalent $(\mathrm{Hp}(10))$ and ambient dose equivalent $\left(\mathrm{H}^{*}(10)\right)$.

In a previous report Hertel and McDonald (1991) used the dose factors for ambient dose equivalent provided by Harvey (1987) to perform similar calculations.

\section{Tallies}

MCNP5 Version 1.4 (X-5 Monte Carlo Team, 2003) was used to perform the calculations.

For purposes of describing the tallies, it is useful to consider a reference sphere whose origin is at the same location as the center of the $\mathrm{D}_{2} \mathrm{O}$ moderating sphere. The equator of the reference sphere passes through the origin and is perpendicular to the cylindrical axis of the source encapsulation. The latitude $\delta$ is related to the polar angle ( $\varphi$ in spherical coordinates) by $\delta=90^{\circ}-\varphi$. The equator therefore has latitude $0^{\circ}$, and the top and bottom of the reference sphere have latitude $\pm 90^{\circ}$. 


\begin{tabular}{|c|c|c|c|}
\hline \multicolumn{4}{|c|}{$\begin{array}{l}\text { CALC NO.: } \\
\text { PNNL-19273 } \\
\end{array}$} \\
\hline CT: CALCULATIONS T & $00{ }^{252} \mathrm{CF}$ NeUtro & E IN 318LOW SCATTER ROOM & \\
\hline PREPARED BY: R.J. TRAUB & DATE: $\underline{02 / 8 / 10}$ & REVIEWED BY: R.J.MCCONN & DATE: $\underline{03 / 21 / 10}$ \\
\hline
\end{tabular}

The majority of the tallies were ring detectors (f5 tallies) located on the surface of a reference sphere of either $50-\mathrm{cm}$ radius or $100-\mathrm{cm}$ radius. The tally rings were located on the equator of the reference sphere and at $10^{\circ}$ increments of latitude. The polar tallies (latitude $\pm 90^{\circ}$ ) were collected with point detectors (f5 tallies) also located on the surface of the reference spheres.

Point detector tallies were used to estimate the neutron fluence and dose equivalent rates at the face of a phantom, as would be used during irradiations of dosimeters. For these calculations, the plane is perpendicular to a horizontal line that passes through the center of the dose plane $(Z=X=0.0)$ and the center of the reference sphere. The dose locations are at the center of the dose plane and $5-$ and $10-\mathrm{cm}$. above and below the center point ( $\mathrm{Z}$ direction) and 5 and $10 \mathrm{~cm}$ to the right and left of the center point $(X$ direction). Due to axial symmetry, only the X-positive data are shown.

\subsection{RESULTS}

\section{Fluence Calculations}

Calculations of the neutron emission rate from the transfer capsule assembly (see Figure 3.b) indicate that there is a small amount of neutron multiplication due mainly to (n, xn) type reactions. In the present calculations, the multiplication was only about 1.0003 , which is negligible. The calculations also indicate that virtually all neutrons that are emitted in the assembly will escape from the assembly. The neutron source was calibrated at NIST in the assembly. The figures of this section will show the average neutron fluence from the transfer capsule assembly which is $1.544 \times 10^{+5} \mathrm{n} \cdot \mathrm{cm}^{-2} \cdot \mathrm{s}^{-1}$ at $50 \mathrm{~cm}$ from the center of the reference sphere and $3.858 \times 10^{+4} \mathrm{n} \cdot \mathrm{cm}^{-2} \cdot \mathrm{s}^{-1}$ at $100 \mathrm{~cm}$

Figure 7 shows the moderated and unmoderated neutron fluence at the surface of the 50 -cm radius reference sphere for the situation when the ${ }^{252} \mathrm{Cf}$ activity is located on the surface of the upper frit. The plot on the left side is of the moderated fluence (ASIS) and the unmoderated fluence (TUBE) is shown on the right. The center of the plot represents the center of the reference sphere and the top of the plot represents the north pole (or where the polar angle with respect to the center of axis " $z$ " axis is zero). The large spikes at the top and bottom of the moderated plot are due to the voids in the moderating sphere, as shown in Figure 5.

Figure 7 shows that, when comparing the neutron fluence above and below the equator, the neutron fluence curve of the TUBE geometry is more symmetrical than the ASIS geometry. This difference is because the asymmetry of the TUBE geometry is mainly due to asymmetric distances from the center of the source activity whereas the asymmetry of the ASIS geometry is affected by the moderating sphere and the distance that the neutrons must traverse through the $\mathrm{D}_{2} \mathrm{O}$ moderator.

Figures 8 and 9 are similar to Figure 7 except that in Figure 8 the ${ }^{252} \mathrm{Cf}$ activity was assumed to be in the void region between the two frits and for Figure 9 the ${ }^{252} \mathrm{Cf}$ activity was assumed to be located on the lower frit. Put together, these figures show that the symmetry of the neutron fluence is affected by the 


\section{CALCULATION SHEET}

\begin{tabular}{|c|c|c|c|}
\hline \multicolumn{4}{|c|}{$\begin{array}{l}\text { CALC NO.: } \\
\text { PNNL-19273 }\end{array}$} \\
\hline SUBJECT: CALCULATIONS T & $00{ }^{252} \mathrm{CF}$ NEUTRO & E IN 318LOW SCATTER ROOM & \\
\hline PREPARED BY: R.J. TRAUB & DATE: $\underline{02 / 8 / 10}$ & REVIEWED BY: R.J.MCCONN & DATE: $\underline{03 / 21 / 10}$ \\
\hline
\end{tabular}

location of the ${ }^{252} \mathrm{Cf}$ activity in the source capsule. Furthermore, the presence of the $\mathrm{D}_{2} \mathrm{O}$ moderating sphere enhances the asymmetry that is seen.

Because the center of the ${ }^{252} \mathrm{Cf}$ source is not collocated with the center of the $\mathrm{D}_{2} \mathrm{O}$ moderating sphere when the source is at the irradiation location in the upright position, the neutrons measured above the equator of the reference sphere have been moderated to a different extent than those neutrons that are measured below the equator. Therefore, the neutron spectrum was calculated to determine the extent to which the spectra differ. Lethergy plots of the neutron spectra on the equator of the reference sphere compared to the spectra $10^{\circ}$ above the equator and $10^{\circ}$ below the equator of the reference sphere are shown as Figures 10,11 and 12. The three figures show the plots for the three source locations; upper frit, void between the frits, and lower frit. 


\section{CALCULATION SHEET}

\begin{tabular}{|c|c|c|c|}
\hline PROJECT No.: & $\begin{array}{l}\text { CALC NO.: } \\
\text { PNNL-19273 } \\
\end{array}$ & REVISION NO.: $\underline{0}$ & SHEET NO.: $\underline{16 / 246}$ \\
\hline SuBJeCt: CALCULATIONS & $00{ }^{252}$ CF NEUTRC & E IN 318LOW SCATTER ROOM & \\
\hline PREPARED BY: R.J. TRAUB & DATE: $\underline{02 / 8 / 10}$ & REVIEWED BY: R.J.MCCONN & DATE: $\underline{03 / 21 / 10}$ \\
\hline
\end{tabular}

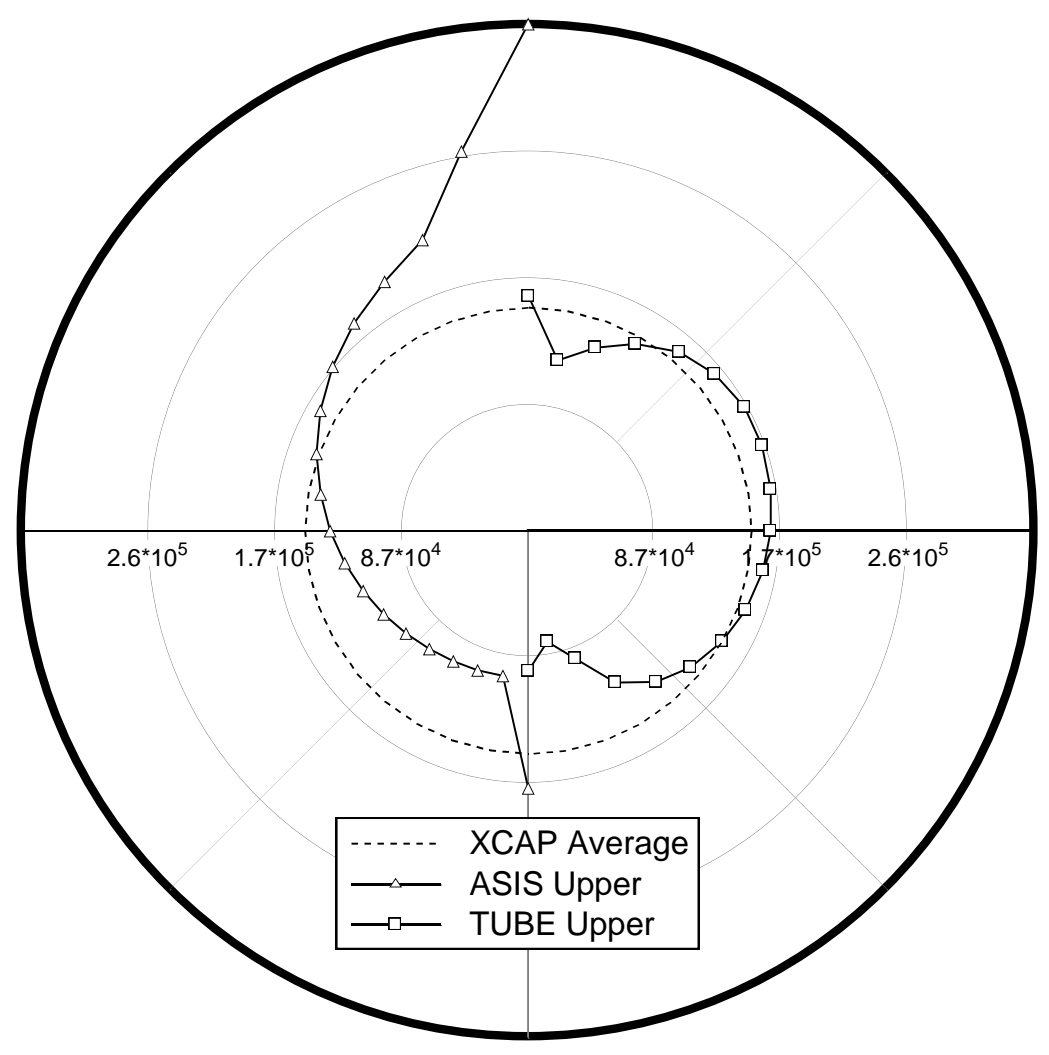

Figure 6. Neutron fluence $\left(\mathrm{n} . \mathrm{cm}^{-2} \cdot \mathrm{s}^{-1}\right)$ at $50 \mathrm{~cm}$ from the ${ }^{252} \mathrm{Cf}$ source. The horizontal line represents the equator of the reference sphere. The dashed line, labeled XCAP average represents the average neutron fluence at $50 \mathrm{~cm}$ from the transfer capsule assembly. The plot on the left side represents the neutron fluence at $50 \mathrm{~cm}$ when the source encapsulation is in the $\mathrm{D}_{2} \mathrm{O}$ moderating sphere. The plot on the right side represents the neutron fluence at $50 \mathrm{~cm}$ when the source encapsulation is in the transfer tube assembly. The ${ }^{252} \mathrm{Cf}$ activity is assumed to be in the upper frit of the source encapsulation. 


\section{CALCULATION SHEET}

\begin{tabular}{|c|c|c|c|}
\hline ProjeCt No.: & $\begin{array}{l}\text { CALC NO.: } \\
\text { PNNL-19273 }\end{array}$ & REVISION NO.: $\underline{0}$ & SHEET NO.: $\underline{17 / 246}$ \\
\hline \multicolumn{4}{|c|}{ E IN 318LOW SCATTER ROOM } \\
\hline PREPARED BY: R.J. TRAUB & DATE: $\underline{02 / 8 / 10}$ & REVIEWED BY: R.J.MCCONN & DATE: $03 / 21 / 10$ \\
\hline
\end{tabular}

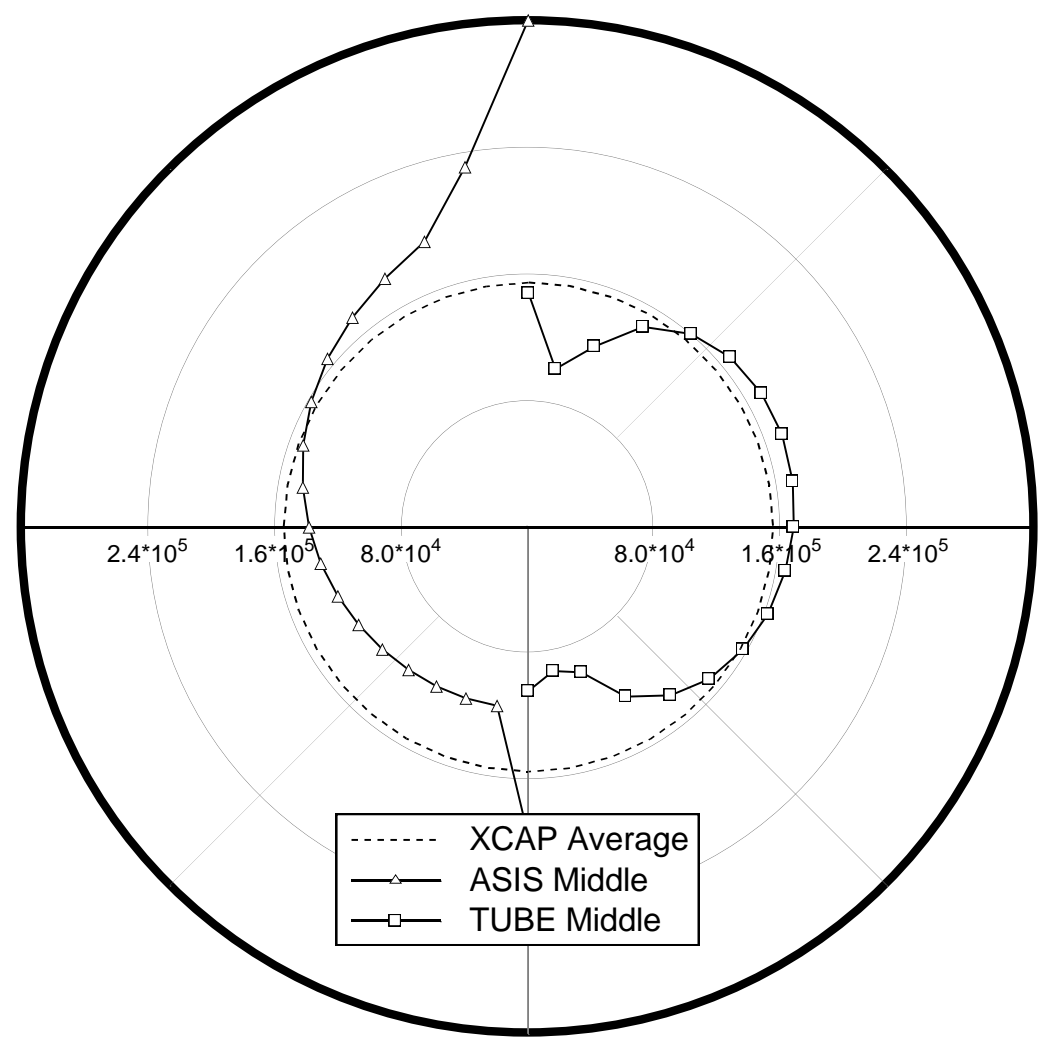

Figure 7. Neutron fluence $\left(\mathrm{n} . \mathrm{cm}^{-2} \cdot \mathrm{s}^{-1}\right)$ at $50 \mathrm{~cm}$ from the ${ }^{252} \mathrm{Cf}$ source. The horizontal line represents the equator of the reference sphere. The dashed line, labeled XCAP average, represents the average neutron fluence at $50 \mathrm{~cm}$ from the transfer capsule assembly. The plot on the left side represents the neutron fluence at $50 \mathrm{~cm}$ when the source encapsulation is in the $\mathrm{D}_{2} \mathrm{O}$ moderating sphere. The plot on the right side represents the neutron fluence at $50 \mathrm{~cm}$ when the source encapsulation is in the transfer tube assembly. The ${ }^{252} \mathrm{Cf}$ activity is assumed to be in the void between the frits of the source encapsulation. 


\section{CALCULATION SHEET}

\begin{tabular}{|c|c|c|c|}
\hline ProjeCt No.: & $\begin{array}{l}\text { CALC NO.: } \\
\text { PNNL-19273 }\end{array}$ & REVISION NO.: $\underline{0}$ & SHEET NO.: $\underline{18 / 246}$ \\
\hline \multicolumn{4}{|c|}{ E IN 318LOW SCATTER ROOM } \\
\hline PREPARED BY: R.J. TRAUB & DATE: $\underline{02 / 8 / 10}$ & REVIEWED BY: R.J.MCCONN & DATE: $03 / 21 / 10$ \\
\hline
\end{tabular}

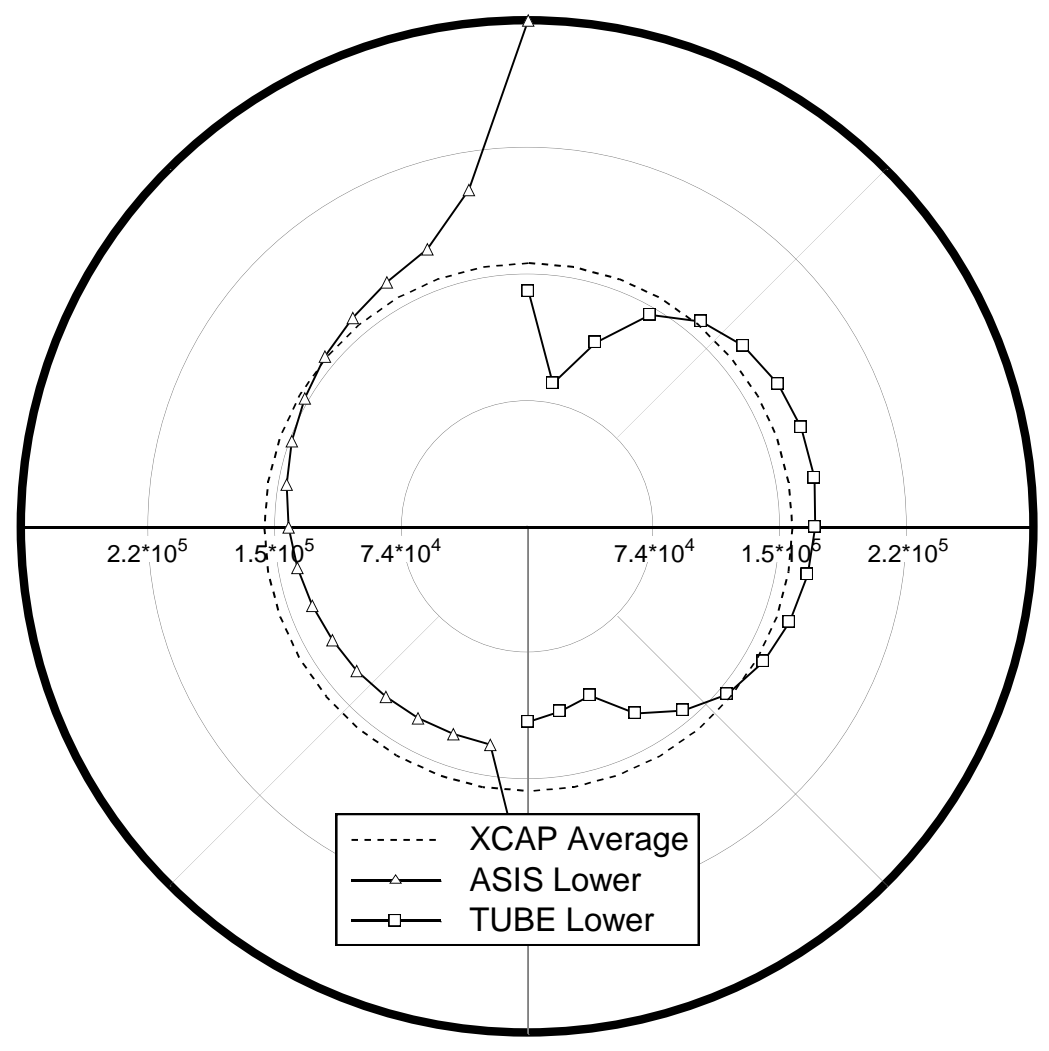

Figure 8 . Neutron fluence $\left(n \cdot \mathrm{cm}^{-2} \cdot \mathrm{s}^{-1}\right)$ at $50 \mathrm{~cm}$ from the ${ }^{252} \mathrm{Cf}$ source. The horizontal line represents the equator of the reference sphere. The dashed line, labeled XCAP average, represents the average neutron fluence at $50 \mathrm{~cm}$ from the transfer capsule assembly. The plot on the left side represents the neutron fluence at $50 \mathrm{~cm}$ when the source encapsulation is in the $\mathrm{D}_{2} \mathrm{O}$ moderating sphere. The plot on the right side represents the neutron fluence at $50 \mathrm{~cm}$ when the source encapsulation is in the transfer tube assembly. The ${ }^{252} \mathrm{Cf}$ activity is assumed to be in the lower frit of the source encapsulation. 


\section{CALCULATION SHEET}

\begin{tabular}{|c|c|c|c|}
\hline \multicolumn{4}{|c|}{$\begin{array}{l}\text { CALC NO.: } \\
\text { PNNL-19273 }\end{array}$} \\
\hline SUBJECT: CALCULATIONS T & $00{ }^{252} \mathrm{CF}$ NEUTRO & E IN 318LOW SCATTER ROOM & \\
\hline Prepared By: R.J. TRAuB & DATE: $02 / 8 / 10$ & REVIEWED BY: R.J.MCCONN & DATE: $03 / 21 / 10$ \\
\hline
\end{tabular}

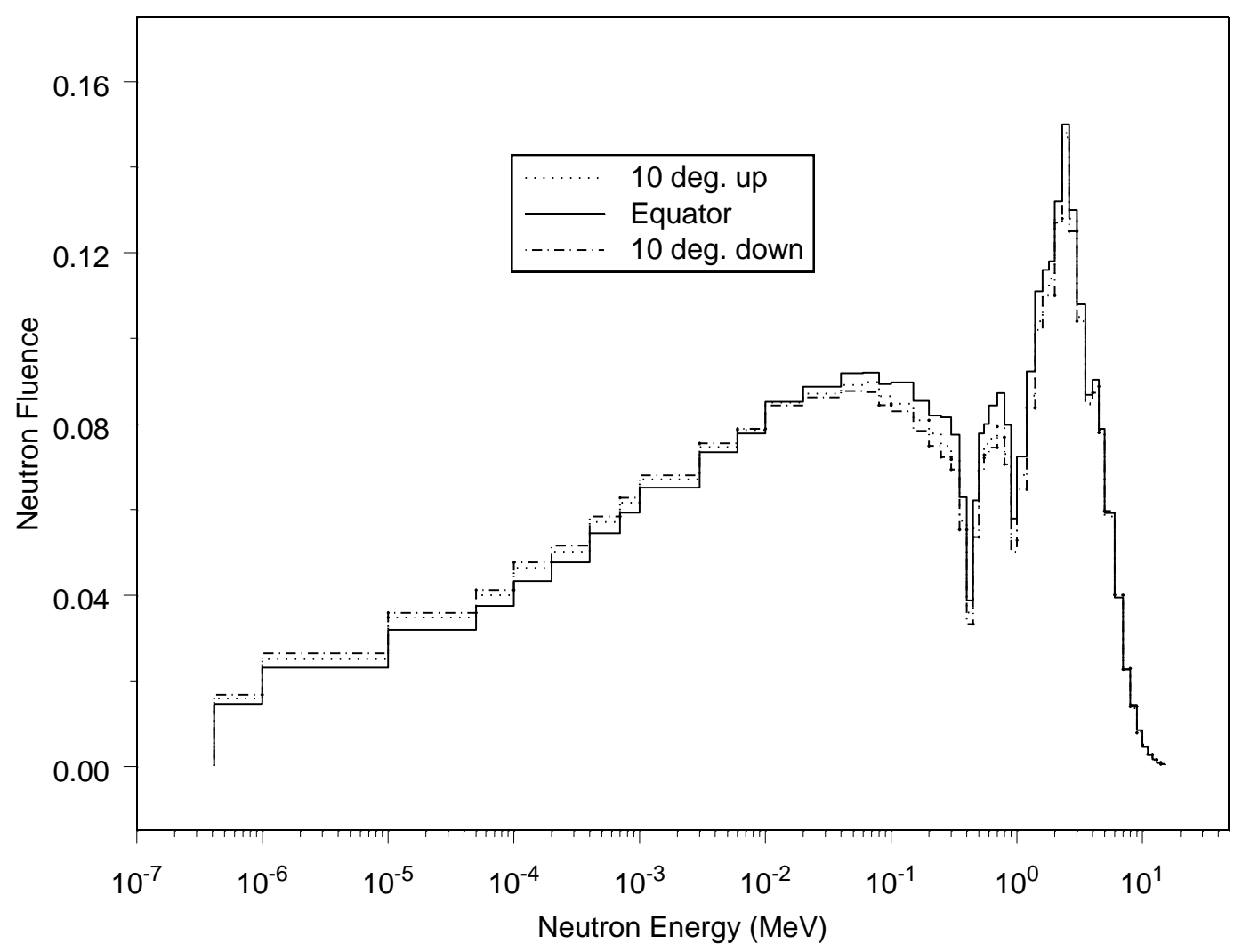

Figure 9. Neutron spectrum (lethargy plots) at $50 \mathrm{~cm}$ from the center of the $\mathrm{D}_{2} \mathrm{O}$ moderating sphere. The three spectra were taken on the equator of the moderating sphere and $10^{\circ}$ above the equator and $10^{\circ}$ below the equator. The ${ }^{252} \mathrm{Cf}$ activity is assumed to be in the upper frit of the source encapsulation. 


\section{CALCULATION SHEET}

\begin{tabular}{|c|c|c|c|}
\hline \multicolumn{4}{|c|}{$\begin{array}{l}\text { CALC NO.: } \\
\text { PNNL-19273 }\end{array}$} \\
\hline SUBJECT: CALCULATIONS T & $00{ }^{252} \mathrm{CF}$ NEUTRO & E IN 318LOW SCATTER ROOM & \\
\hline Prepared By: R.J. TRAuB & DATE: $02 / 8 / 10$ & REVIEWED BY: R.J.MCCONN & DATE: $03 / 21 / 10$ \\
\hline
\end{tabular}

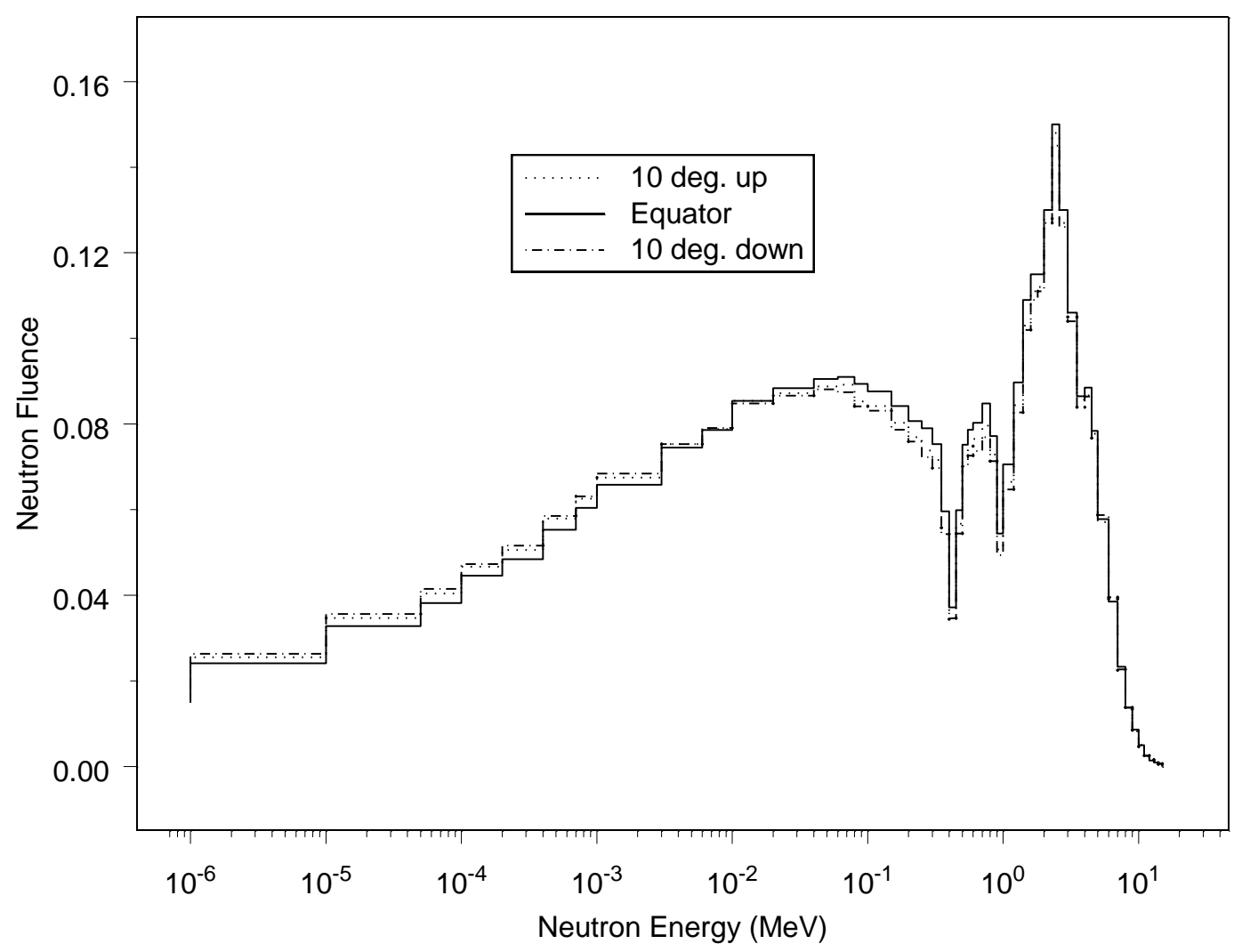

Figure 10. Neutron spectrum (lethargy plots) at $50 \mathrm{~cm}$ from the center of the $\mathrm{D}_{2} \mathrm{O}$ moderating sphere. The three spectra were taken on the equator of the moderating sphere and $10^{\circ}$ above the equator and $10^{\circ}$ below the equator. The ${ }^{252} \mathrm{Cf}$ activity is assumed to be in the void between the frits of the source encapsulation. 


\section{CALCULATION SHEET}

\begin{tabular}{|c|c|c|c|}
\hline PROJECT No.: & $\begin{array}{l}\text { CALC NO.: } \\
\text { PNNL-19273 }\end{array}$ & REVISION NO.: $\underline{0}$ & SHEET NO.: $\underline{21 / 246}$ \\
\hline \multicolumn{4}{|c|}{ PNNL-19273 } \\
\hline PREPARED BY: R.J. TRAUB & DATE: $\underline{02 / 8 / 10}$ & REVIEWED BY: $\underline{\text { R.J.MCCONN }}$ & DATE: $\underline{03 / 21 / 10}$ \\
\hline
\end{tabular}

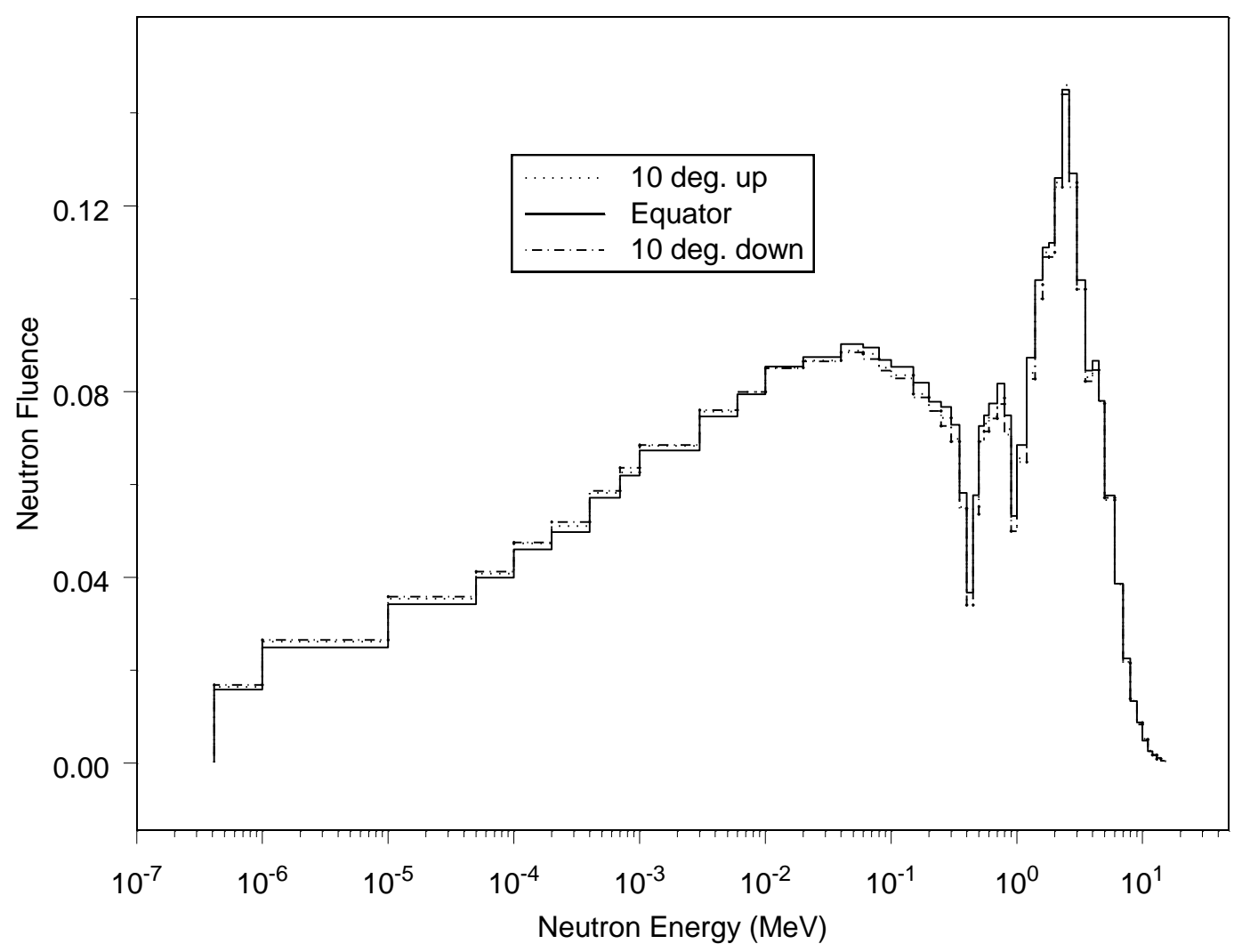

Figure 11. Neutron spectrum (lethargy plots) at $50 \mathrm{~cm}$ from the center of the $\mathrm{D}_{2} \mathrm{O}$ moderating sphere. The three spectra were taken on the equator of the moderating sphere and $10^{\circ}$ above the equator and $10^{\circ}$ below the equator. The ${ }^{252} \mathrm{Cf}$ activity is assumed to be in the lower frit of the source encapsulation. 


\begin{tabular}{|c|c|c|c|}
\hline \multicolumn{4}{|c|}{$\begin{array}{l}\text { CALC NO.: } \\
\text { PNNL-19273 }\end{array}$} \\
\hline SuBJECT: CALCULATIONS T & $00{ }^{252} \mathrm{CF}$ NeUtro & E IN 318LOW SCATTER ROOM & \\
\hline PREPARED BY: R.J. TRAUB & DATE: $\underline{02 / 8 / 10}$ & REVIEWED BY: R.J.MCCONN & DATE: $\underline{03 / 21 / 10}$ \\
\hline
\end{tabular}

\section{Dose Calculations}

Neutron fluence rates, dose rates and dose factors on the surface of the $50-\mathrm{cm}$ and $100-\mathrm{cm}$ reference spheres at $10^{\circ}$ increments above and below the equator of the reference spheres are listed in Tables 2 through Table 13. The tabulated data are for the moderated and unmoderated neutron geometries. Separate tables are provided for the three different source locations; upper frit, middle void region, and lower frit.

Neutron fluence rates on the surface of the flat dosimeter irradiation plane are listed in Tables 14 through Table 16. The tabulated data are for the moderated and unmoderated neutron geometries. Separate tables are provided for the three different source locations; upper frit, middle void region, and lower frit.

Neutron dose factors on the face of the flat phantom plane are listed in Tables 17 through Table 19 for the moderated geometry. Separate tables are provided for the three different source locations; upper frit, middle void region, and lower frit.

Neutron dose factors on the face of the flat phantom plane are listed in Tables 20 through Table 22 for the unmoderated geometry. Separate tables are provided for the three different source locations; upper frit, middle void region, and lower frit.

Neutron dose rates on the face of the flat phantom plane are listed in Tables 23 through Table 25 for the moderated geometry. Separate tables are provided for the three different source locations; upper frit, middle void region, and lower frit.

Neutron dose rates on the face of the flat phantom plane are listed in Tables 26 through Table 28 for the unmoderated geometry. Separate tables are provided for the three different source locations; upper frit, middle void region, and lower frit.

Neutron fluence rates, dose rates, and dose factors on the face of the flat phantom plane when the equator of the measurement sphere is $1 \mathrm{~cm}$ above the center of the reference sphere are listed in Tables 29 through Table 37. These tables are applicable to the situation where calibrations are made with the source encapsulation with an elevated measurement to account for the elevated source location. These tables are for only the unmoderated geometry.

Neutron fluence rates, dose rates, and dose factors on the face of the flat phantom plane when the equator of the measurement sphere is $1.864 \mathrm{~cm}$ above the center of the reference sphere are listed in Tables 38 through Table 46. These tables are applicable when the equator of the reference sphere is elevated to pass through the center of the center void region of the primary source encapsulation. These tables are for only the unmoderated geometry. 


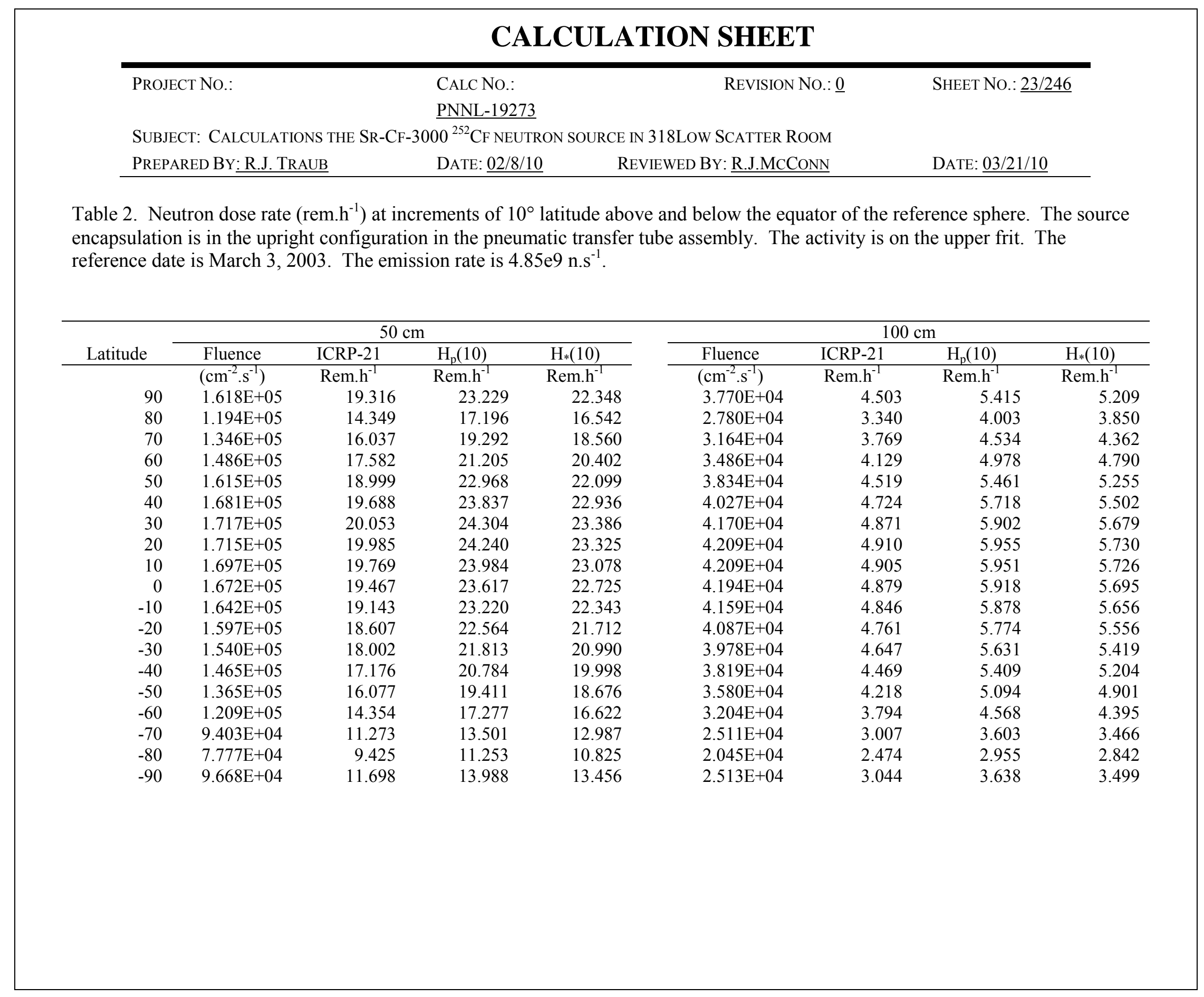




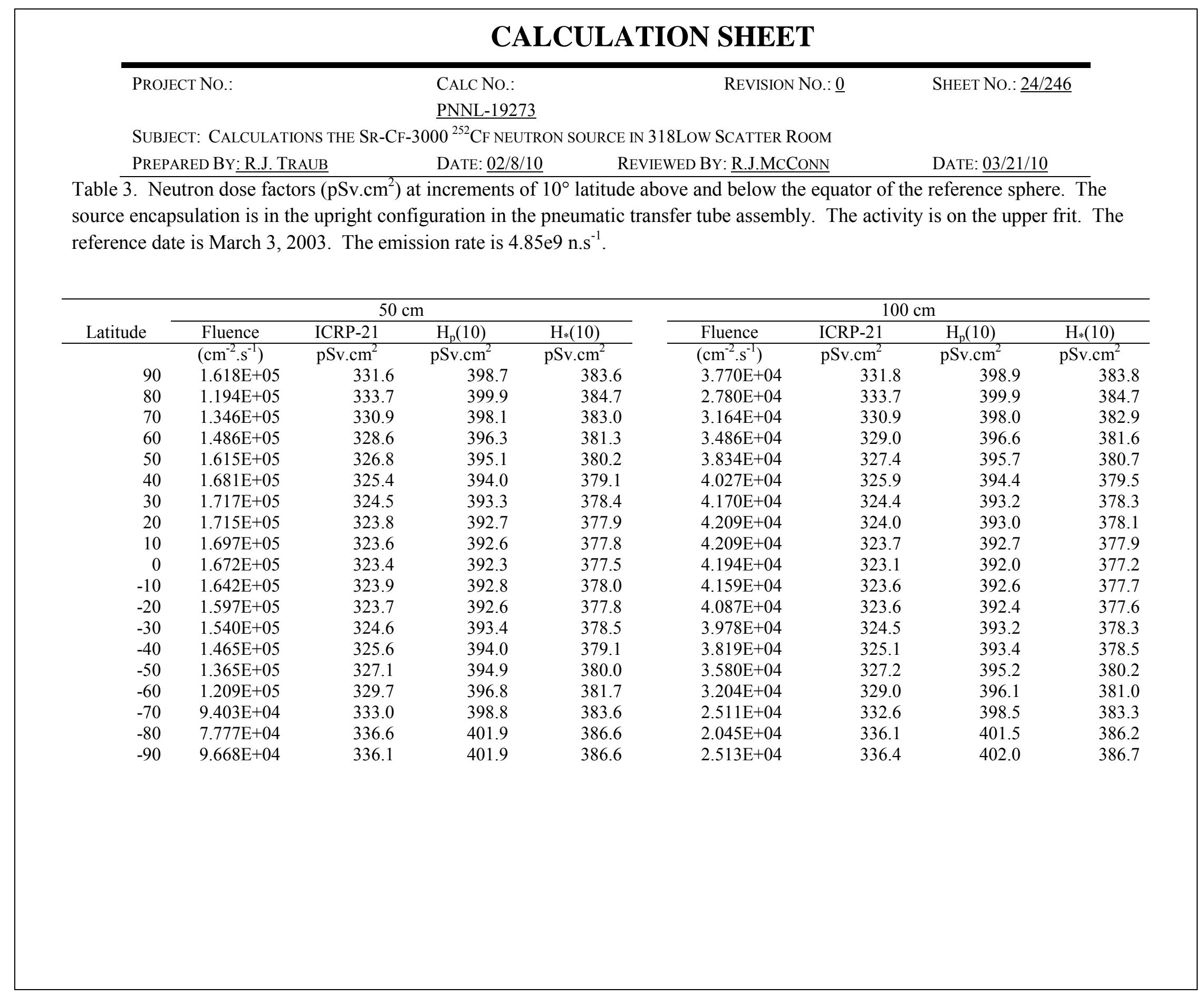




\begin{tabular}{|c|c|c|c|c|c|c|c|c|}
\hline \multicolumn{9}{|c|}{ CALCULATION SHEET } \\
\hline \multirow{2}{*}{\multicolumn{2}{|c|}{ PROJECT No.: }} & & \multicolumn{2}{|c|}{ REVISION NO.: $\underline{0}$} & \multicolumn{2}{|c|}{ SHEET NO.: $\underline{25 / 246}$} \\
\hline & & \multicolumn{6}{|c|}{ 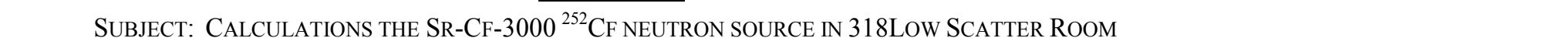 } & \\
\hline \multicolumn{3}{|c|}{ PREPARED BY: R.J. TRAUB } & DATE: $\underline{02 / 8}$ & \multicolumn{3}{|c|}{ REVIEWED BY: R.J.MCCONN } & \multicolumn{2}{|c|}{ DATE: $\underline{03 / 21 / 10}$} \\
\hline \multirow[b]{2}{*}{ Latitude } & \multicolumn{4}{|c|}{$50 \mathrm{~cm}$} & \multicolumn{4}{|c|}{$100 \mathrm{~cm}$} \\
\hline & Fluence & ICRP-21 & $\mathrm{H}_{\mathrm{p}}(10)$ & $\mathrm{H} *(10)$ & Fluence & ICRP-21 & $\mathrm{H}_{\mathrm{p}}(10)$ & $\mathrm{H}_{*}(10)$ \\
\hline & $\left(\mathrm{cm}^{-2} \cdot \mathrm{s}^{-1}\right)$ & Rem.h $\mathrm{h}^{-1}$ & Rem.h ${ }^{-1}$ & Rem.h ${ }^{-1}$ & $\left(\mathrm{~cm}^{-2} \cdot \mathrm{s}^{-1}\right)$ & Rem.h. ${ }^{-1}$ & Rem.h $\mathrm{h}^{-1}$ & Rem.h ${ }^{-1}$ \\
\hline 90 & $1.471 \mathrm{E}+05$ & 17.652 & 21.175 & 20.371 & $3.489 \mathrm{E}+04$ & 4.192 & 5.027 & 4.836 \\
\hline 80 & $1.010 \mathrm{E}+05$ & 12.192 & 14.573 & 14.017 & $2.397 \mathrm{E}+04$ & 2.898 & 3.464 & 3.332 \\
\hline 70 & $1.211 \mathrm{E}+05$ & 14.472 & 17.368 & 16.708 & $2.885 \mathrm{E}+04$ & 3.452 & 4.142 & 3.984 \\
\hline 60 & $1.453 \mathrm{E}+05$ & 17.229 & 20.753 & 19.967 & $3.477 \mathrm{E}+04$ & 4.129 & 4.972 & 4.783 \\
\hline 50 & $1.591 \mathrm{E}+05$ & 18.764 & 22.665 & 21.807 & $3.842 \mathrm{E}+04$ & 4.530 & 5.471 & 5.264 \\
\hline 40 & $1.661 \mathrm{E}+05$ & 19.502 & 23.598 & 22.705 & $4.039 \mathrm{E}+04$ & 4.747 & 5.742 & 5.525 \\
\hline 30 & $1.693 \mathrm{E}+05$ & 19.819 & 24.006 & 23.099 & $4.145 \mathrm{E}+04$ & 4.860 & 5.885 & 5.663 \\
\hline 20 & $1.700 \mathrm{E}+05$ & 19.855 & 24.068 & 23.159 & $4.197 \mathrm{E}+04$ & 4.905 & 5.945 & 5.721 \\
\hline 10 & $1.689 \mathrm{E}+05$ & 19.727 & 23.920 & 23.017 & $4.206 \mathrm{E}+04$ & 4.907 & 5.950 & 5.725 \\
\hline 0 & $1.671 \mathrm{E}+05$ & 19.490 & 23.639 & 22.746 & $4.188 \mathrm{E}+04$ & 4.885 & 5.924 & 5.700 \\
\hline-10 & $1.641 \mathrm{E}+05$ & 19.167 & 23.244 & 22.366 & $4.148 \mathrm{E}+04$ & 4.838 & 5.867 & 5.645 \\
\hline-20 & $1.604 \mathrm{E}+05$ & 18.760 & 22.736 & 21.878 & $4.076 \mathrm{E}+04$ & 4.762 & 5.772 & 5.554 \\
\hline-30 & $1.557 \mathrm{E}+05$ & 18.221 & 22.069 & 21.235 & $3.976 \mathrm{E}+04$ & 4.656 & 5.639 & 5.426 \\
\hline-40 & $1.489 \mathrm{E}+05$ & 17.488 & 21.154 & 20.354 & $3.826 \mathrm{E}+04$ & 4.492 & 5.434 & 5.228 \\
\hline-50 & $1.389 \mathrm{E}+05$ & 16.388 & 19.782 & 19.033 & $3.591 \mathrm{E}+04$ & 4.232 & 5.109 & 4.916 \\
\hline-60 & $1.233 \mathrm{E}+05$ & 14.644 & 17.623 & 16.954 & $3.200 \mathrm{E}+04$ & 3.802 & 4.576 & 4.402 \\
\hline-70 & $9.755 E+04$ & 11.679 & 14.000 & 13.467 & $2.546 \mathrm{E}+04$ & 3.049 & 3.655 & 3.516 \\
\hline-80 & $9.216 \mathrm{E}+04$ & 11.106 & 13.305 & 12.799 & $2.380 \mathrm{E}+04$ & 2.868 & 3.436 & 3.306 \\
\hline-90 & $1.035 \mathrm{E}+05$ & 12.485 & 14.949 & 14.381 & $2.649 \mathrm{E}+04$ & 3.201 & 3.830 & 3.684 \\
\hline & & & & & & & & \\
\hline & & & & & & & & \\
\hline & & & & & & & & \\
\hline & & & & & & & & \\
\hline & & & & & & & & \\
\hline
\end{tabular}




\begin{tabular}{|c|c|c|c|c|c|c|c|c|}
\hline \multicolumn{9}{|c|}{ CALCULATION SHEET } \\
\hline \multicolumn{2}{|c|}{ PROJECT NO.: } & \multicolumn{3}{|c|}{ CALC No.: } & \multicolumn{2}{|c|}{ REVISION NO.: $\underline{0}$} & \multicolumn{2}{|c|}{ SHEET NO.: $\underline{26 / 246}$} \\
\hline \multicolumn{9}{|c|}{ 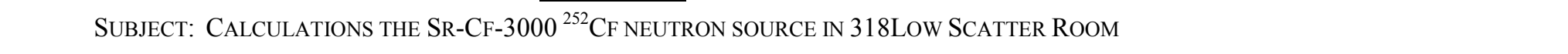 } \\
\hline \multicolumn{3}{|c|}{ PREPARED BY: R.J. TRAUB } & DATE: $\underline{02 / 8}$ & & D BY: R.J.MCC & & \multicolumn{2}{|c|}{ DATE: $\underline{03 / 21 / 10}$} \\
\hline \multirow[b]{2}{*}{ Latitude } & \multicolumn{4}{|c|}{$50 \mathrm{~cm}$} & \multicolumn{4}{|c|}{$100 \mathrm{~cm}$} \\
\hline & Fluence & ICRP-21 & $\mathrm{H}_{\mathrm{p}}(10)$ & $\mathrm{H}_{*}(10)$ & Fluence & ICRP-21 & $\mathrm{H}_{\mathrm{p}}(10)$ & $\mathrm{H}_{*}(10)$ \\
\hline & $\left(\mathrm{cm}^{-2} \cdot \mathrm{s}^{-1}\right)$ & pSv.cm ${ }^{2}$ & pSv.cm ${ }^{2}$ & pSv.cm ${ }^{2}$ & $\left(\mathrm{~cm}^{-2} \cdot \mathrm{s}^{-1}\right)$ & pSv.cm ${ }^{2}$ & pSv.cm ${ }^{2}$ & pSv.cm ${ }^{2}$ \\
\hline 90 & $1.471 \mathrm{E}+05$ & 333.4 & 400.0 & 384.8 & $3.489 \mathrm{E}+04$ & 333.8 & 400.2 & 385.0 \\
\hline 80 & $1.010 \mathrm{E}+05$ & 335.3 & 400.8 & 385.5 & $2.397 \mathrm{E}+04$ & 335.8 & 401.4 & 386.1 \\
\hline 70 & $1.211 \mathrm{E}+05$ & 332.0 & 398.5 & 383.3 & $2.885 \mathrm{E}+04$ & 332.3 & 398.7 & 383.6 \\
\hline 60 & $1.453 \mathrm{E}+05$ & 329.4 & 396.8 & 381.8 & $3.477 \mathrm{E}+04$ & 329.9 & 397.2 & 382.2 \\
\hline 50 & $1.591 \mathrm{E}+05$ & 327.5 & 395.6 & 380.6 & $3.842 \mathrm{E}+04$ & 327.5 & 395.5 & 380.6 \\
\hline 40 & $1.661 \mathrm{E}+05$ & 326.1 & 394.6 & 379.7 & $4.039 \mathrm{E}+04$ & 326.4 & 394.9 & 379.9 \\
\hline 30 & $1.693 \mathrm{E}+05$ & 325.2 & 393.9 & 379.0 & $4.145 \mathrm{E}+04$ & 325.7 & 394.4 & 379.5 \\
\hline 20 & $1.700 \mathrm{E}+05$ & 324.3 & 393.2 & 378.3 & $4.197 \mathrm{E}+04$ & 324.6 & 393.5 & 378.6 \\
\hline 10 & $1.689 \mathrm{E}+05$ & 324.3 & 393.3 & 378.4 & $4.206 \mathrm{E}+04$ & 324.0 & 392.9 & 378.1 \\
\hline 0 & $1.671 \mathrm{E}+05$ & 324.0 & 392.9 & 378.1 & $4.188 \mathrm{E}+04$ & 323.9 & 392.9 & 378.0 \\
\hline-10 & $1.641 \mathrm{E}+05$ & 324.4 & 393.4 & 378.5 & $4.148 \mathrm{E}+04$ & 324.0 & 392.9 & 378.0 \\
\hline-20 & $1.604 \mathrm{E}+05$ & 324.8 & 393.7 & 378.8 & $4.076 \mathrm{E}+04$ & 324.6 & 393.4 & 378.5 \\
\hline-30 & $1.557 \mathrm{E}+05$ & 325.1 & 393.8 & 378.9 & $3.976 \mathrm{E}+04$ & 325.3 & 394.0 & 379.1 \\
\hline-40 & $1.489 \mathrm{E}+05$ & 326.2 & 394.6 & 379.6 & $3.826 \mathrm{E}+04$ & 326.1 & 394.5 & 379.6 \\
\hline-50 & $1.389 \mathrm{E}+05$ & 327.8 & 395.7 & 380.7 & $3.591 \mathrm{E}+04$ & 327.4 & 395.3 & 380.3 \\
\hline-60 & $1.233 \mathrm{E}+05$ & 329.9 & 397.0 & 381.9 & $3.200 \mathrm{E}+04$ & 330.0 & 397.2 & 382.1 \\
\hline-70 & $9.755 \mathrm{E}+04$ & 332.6 & 398.7 & 383.5 & $2.546 \mathrm{E}+04$ & 332.6 & 398.8 & 383.6 \\
\hline-80 & $9.216 \mathrm{E}+04$ & 334.7 & 401.0 & 385.8 & $2.380 \mathrm{E}+04$ & 334.7 & 401.0 & 385.8 \\
\hline-90 & $1.035 \mathrm{E}+05$ & 335.1 & 401.3 & 386.0 & $2.649 \mathrm{E}+04$ & 335.6 & 401.6 & 386.3 \\
\hline
\end{tabular}




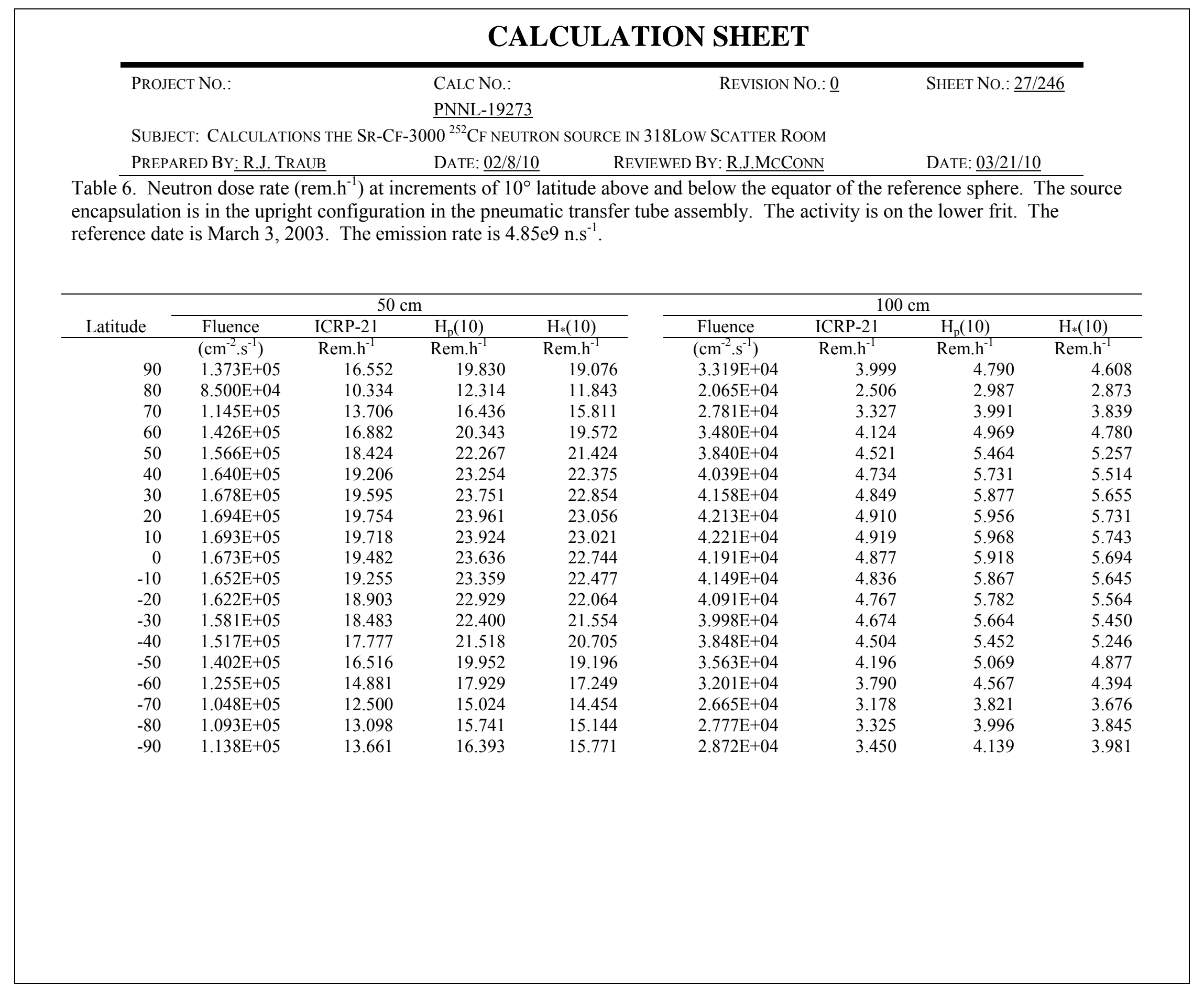




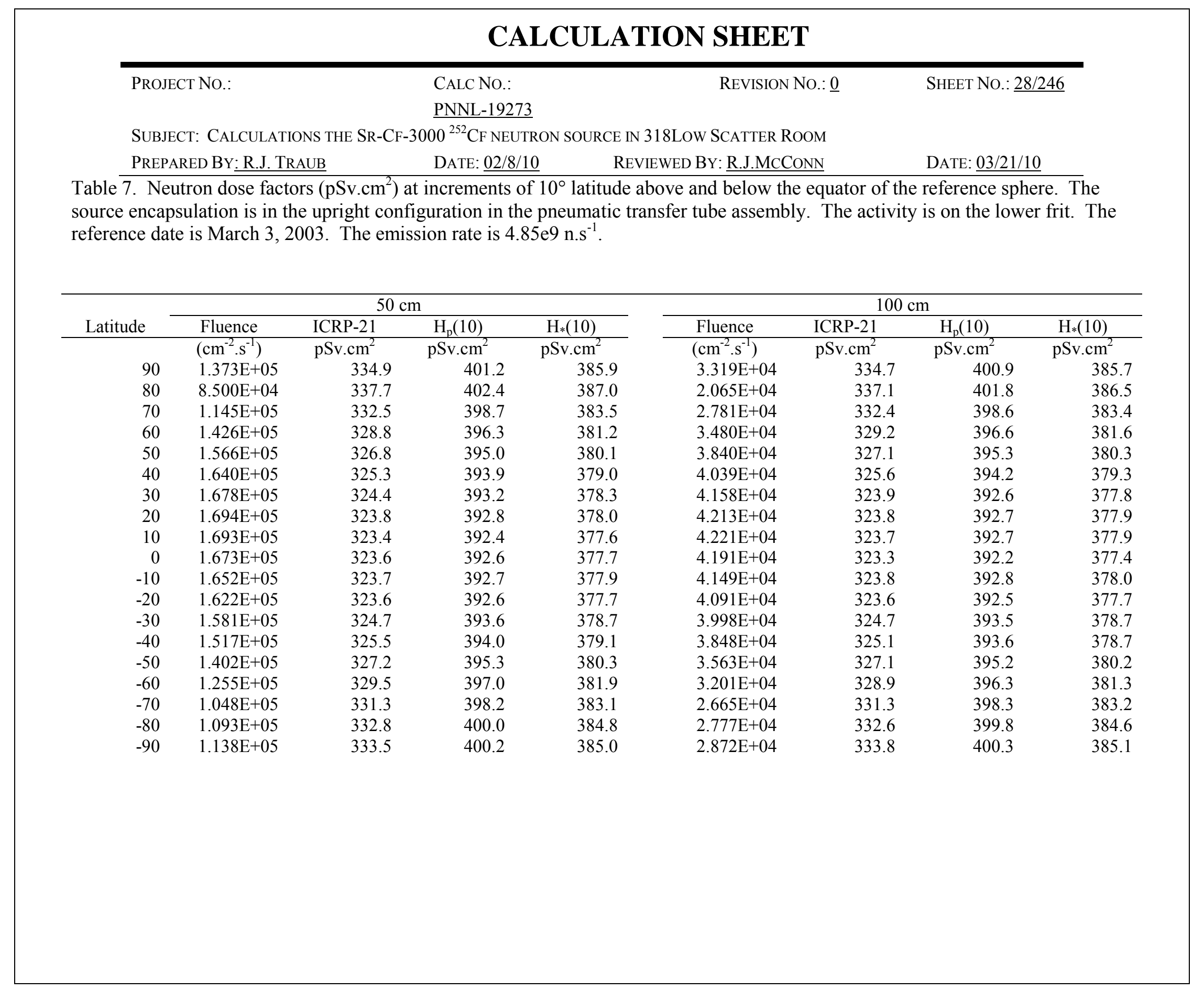




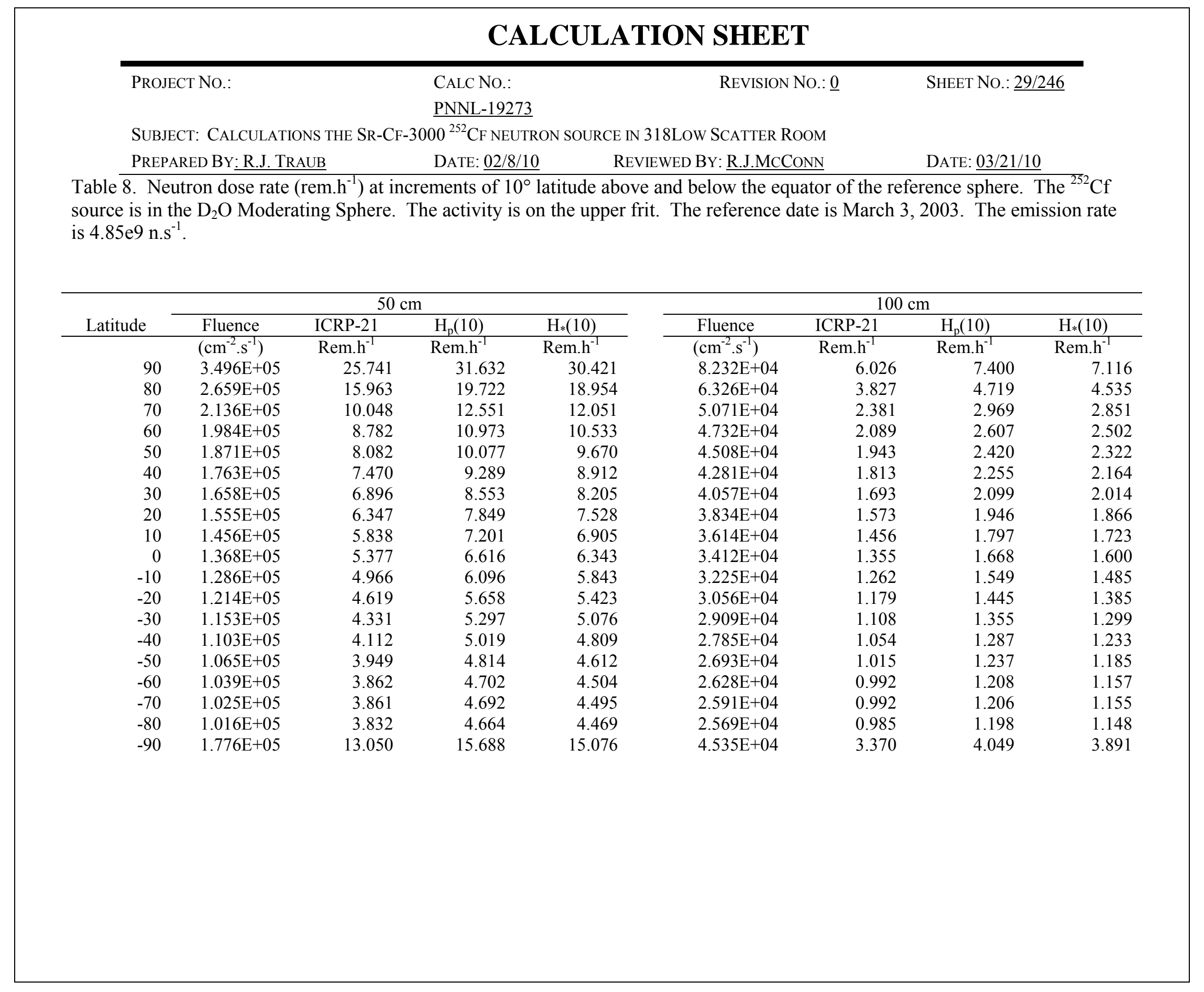




\begin{tabular}{|c|c|c|c|c|c|c|c|c|}
\hline \multicolumn{9}{|c|}{ CALCULATION SHEET } \\
\hline \multirow{2}{*}{\multicolumn{2}{|c|}{ PROJECT No.: }} & & \multicolumn{2}{|c|}{ REVISION NO.: $\underline{0}$} & \\
\hline & & \multicolumn{5}{|c|}{ SUBJECT: CALCULATIONS THE SR-CF-3000 ${ }^{252}$ CF NEUTRON SOURCE IN 318LOW SCATTER ROOM } & SHEET NO.: $\underline{30 / 246}$ & \\
\hline \multicolumn{3}{|c|}{ PREPARED BY: R.J. TRAUB } & DATE: $\underline{02 / 8}$ & & D BY: R.J.MCC & & \multicolumn{2}{|c|}{ DATE: $\underline{03 / 21 / 10}$} \\
\hline \multirow{3}{*}{ Latitude } & \multicolumn{4}{|c|}{$50 \mathrm{~cm}$} & \multicolumn{4}{|c|}{$100 \mathrm{~cm}$} \\
\hline & Fluence & ICRP-21 & $\mathrm{H}_{\mathrm{p}}(10)$ & $\mathrm{H}_{*}(10)$ & Fluence & ICRP-21 & $\mathrm{H}_{\mathrm{p}}(10)$ & $\mathrm{H} *(10)$ \\
\hline & $\left(\mathrm{cm}^{-2} \cdot \mathrm{s}^{-1}\right)$ & pSv.cm ${ }^{2}$ & pSv.cm ${ }^{2}$ & pSv.cm ${ }^{2}$ & $\left(\mathrm{~cm}^{-2} \cdot \mathrm{s}^{-1}\right)$ & pSv.cm ${ }^{2}$ & pSv.cm ${ }^{2}$ & pSv.cm ${ }^{2}$ \\
\hline 90 & $3.496 \mathrm{E}+05$ & 204.5 & 251.3 & 241.7 & $8.232 \mathrm{E}+04$ & 203.3 & 249.7 & 240.1 \\
\hline 80 & $2.659 \mathrm{E}+05$ & 166.8 & 206.1 & 198.0 & $6.326 \mathrm{E}+04$ & 168.0 & 207.2 & 199.1 \\
\hline 70 & $2.136 \mathrm{E}+05$ & 130.7 & 163.2 & 156.7 & $5.071 \mathrm{E}+04$ & 130.4 & 162.7 & 156.2 \\
\hline 60 & $1.984 \mathrm{E}+05$ & 123.0 & 153.6 & 147.5 & $4.732 \mathrm{E}+04$ & 122.6 & 153.0 & 146.9 \\
\hline 50 & $1.871 \mathrm{E}+05$ & 120.0 & 149.6 & 143.6 & $4.508 \mathrm{E}+04$ & 119.7 & 149.1 & 143.1 \\
\hline 40 & $1.763 \mathrm{E}+05$ & 117.7 & 146.4 & 140.4 & $4.281 \mathrm{E}+04$ & 117.7 & 146.3 & 140.4 \\
\hline 30 & $1.658 \mathrm{E}+05$ & 115.5 & 143.3 & 137.5 & $4.057 \mathrm{E}+04$ & 115.9 & 143.7 & 137.9 \\
\hline 20 & $1.555 \mathrm{E}+05$ & 113.4 & 140.2 & 134.5 & $3.834 \mathrm{E}+04$ & 113.9 & 141.0 & 135.2 \\
\hline 10 & $1.456 \mathrm{E}+05$ & 111.4 & 137.4 & 131.7 & $3.614 \mathrm{E}+04$ & 111.9 & 138.1 & 132.5 \\
\hline 0 & $1.368 \mathrm{E}+05$ & 109.2 & 134.4 & 128.8 & $3.412 \mathrm{E}+04$ & 110.3 & 135.8 & 130.2 \\
\hline-10 & $1.286 \mathrm{E}+05$ & 107.3 & 131.7 & 126.2 & $3.225 \mathrm{E}+04$ & 108.7 & 133.5 & 127.9 \\
\hline-20 & $1.214 \mathrm{E}+05$ & 105.7 & 129.5 & 124.1 & $3.056 \mathrm{E}+04$ & 107.2 & 131.3 & 125.9 \\
\hline-30 & $1.153 \mathrm{E}+05$ & 104.3 & 127.6 & 122.3 & $2.909 \mathrm{E}+04$ & 105.8 & 129.4 & 124.0 \\
\hline-40 & $1.103 \mathrm{E}+05$ & 103.6 & 126.4 & 121.1 & $2.785 \mathrm{E}+04$ & 105.1 & 128.4 & 123.0 \\
\hline-50 & $1.065 \mathrm{E}+05$ & 103.0 & 125.6 & 120.3 & $2.693 E+04$ & 104.7 & 127.6 & 122.3 \\
\hline-60 & $1.039 \mathrm{E}+05$ & 103.3 & 125.7 & 120.5 & $2.628 \mathrm{E}+04$ & 104.9 & 127.7 & 122.3 \\
\hline-70 & $1.025 \mathrm{E}+05$ & 104.7 & 127.2 & 121.9 & $2.591 \mathrm{E}+04$ & 106.4 & 129.2 & 123.8 \\
\hline-80 & $1.016 \mathrm{E}+05$ & 104.8 & 127.6 & 122.2 & $2.569 \mathrm{E}+04$ & 106.5 & 129.5 & 124.1 \\
\hline-90 & $1.776 \mathrm{E}+05$ & 204.1 & 245.3 & 235.8 & $4.535 \mathrm{E}+04$ & 206.4 & 248.0 & 238.3 \\
\hline
\end{tabular}




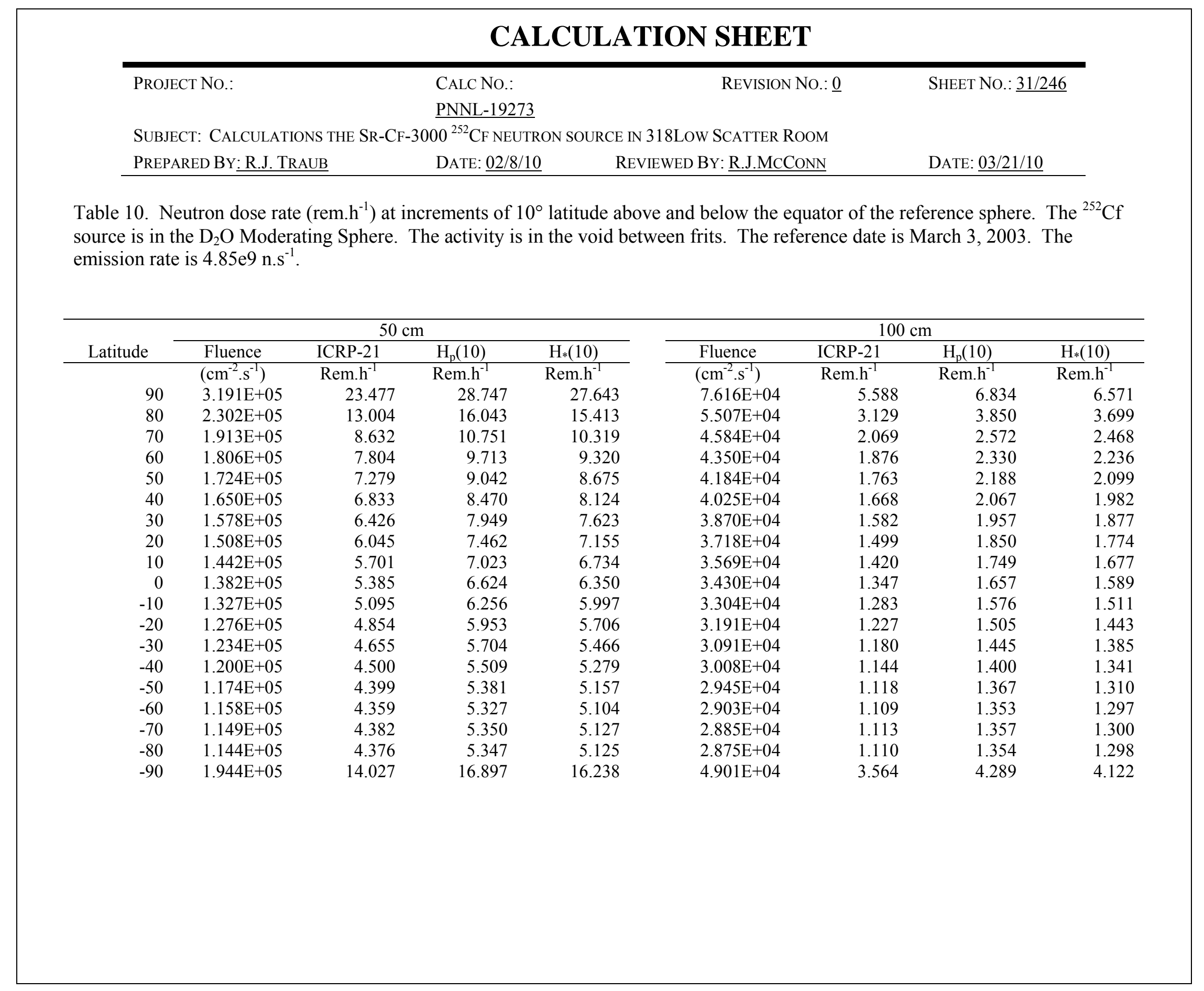




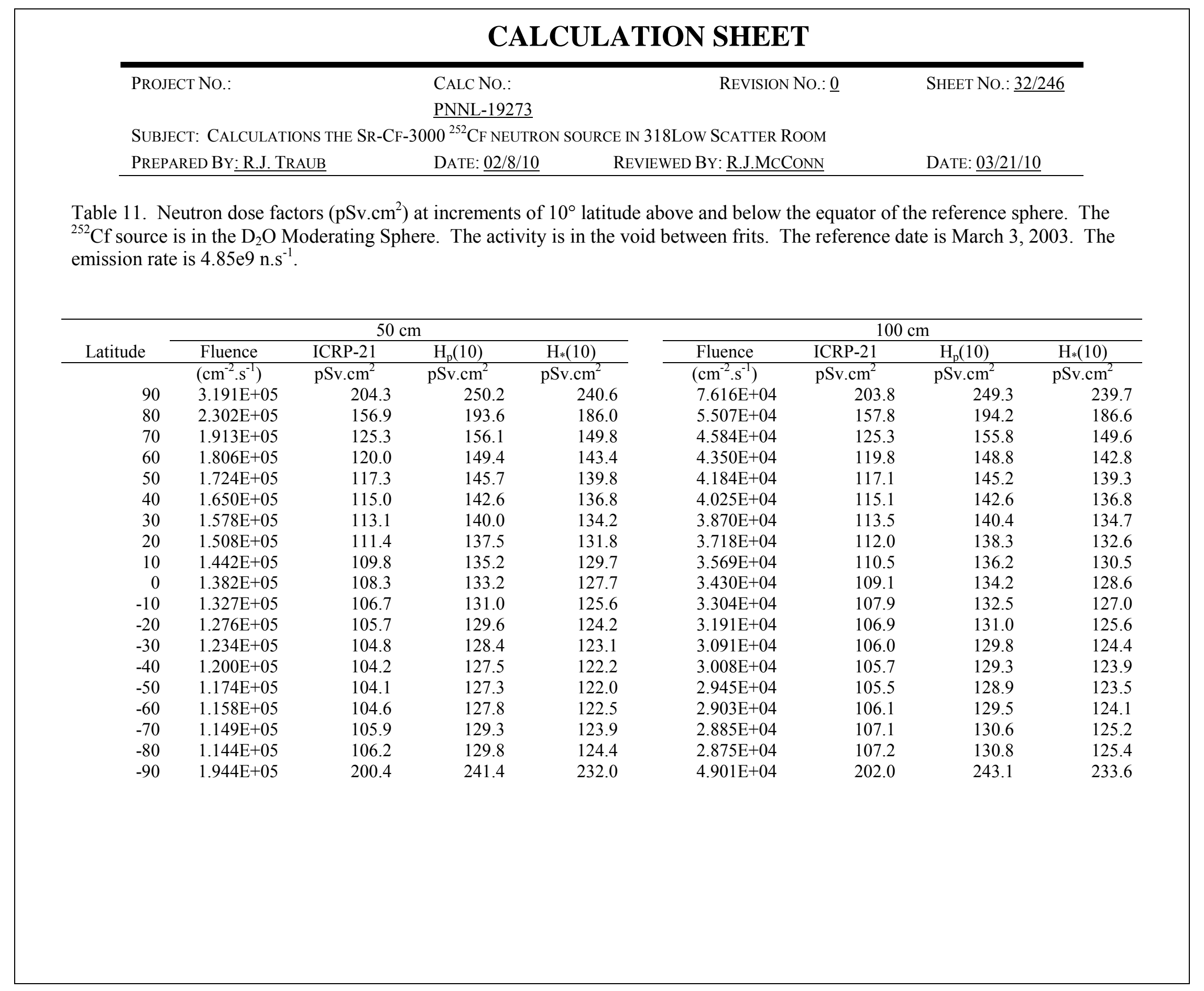




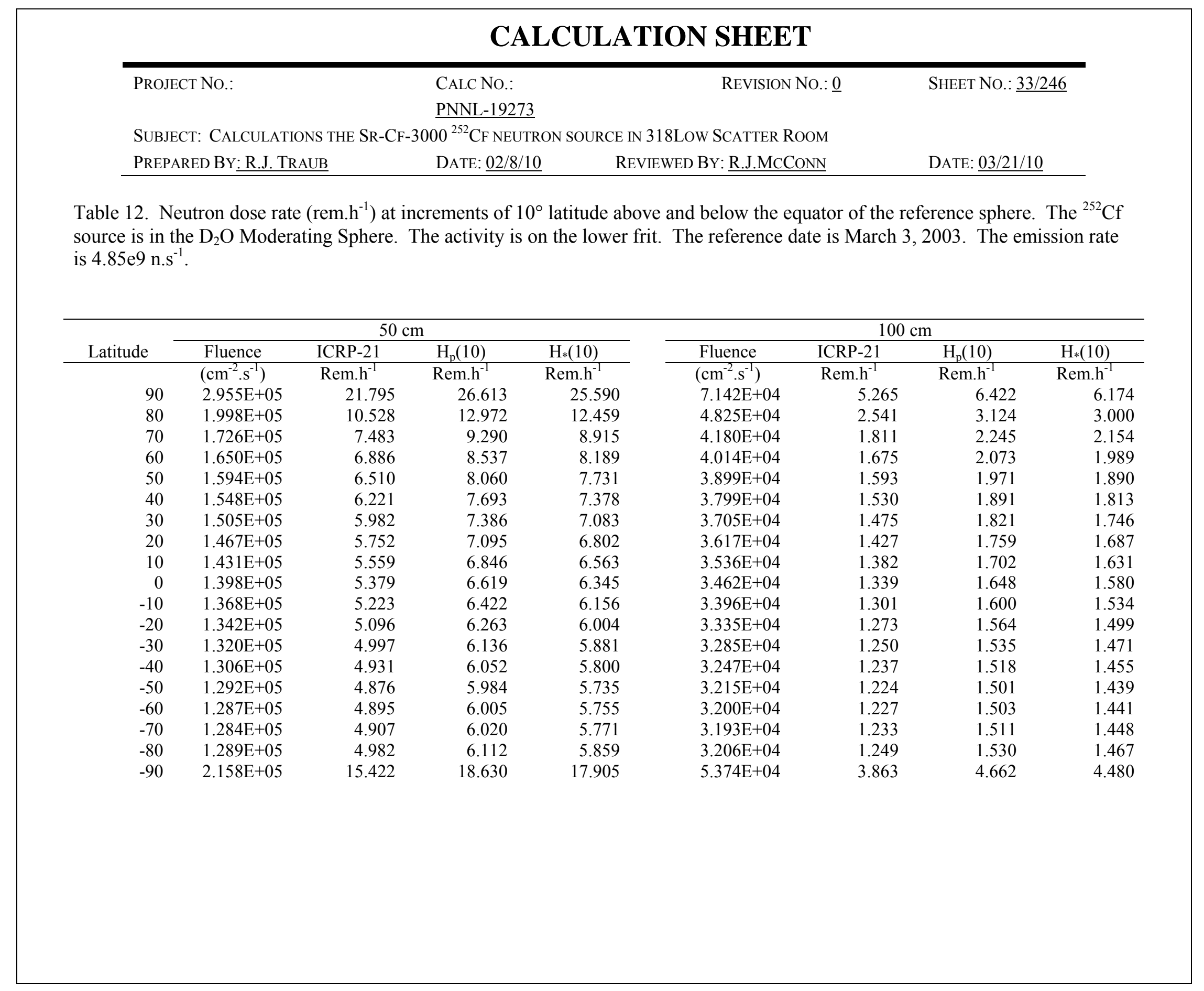




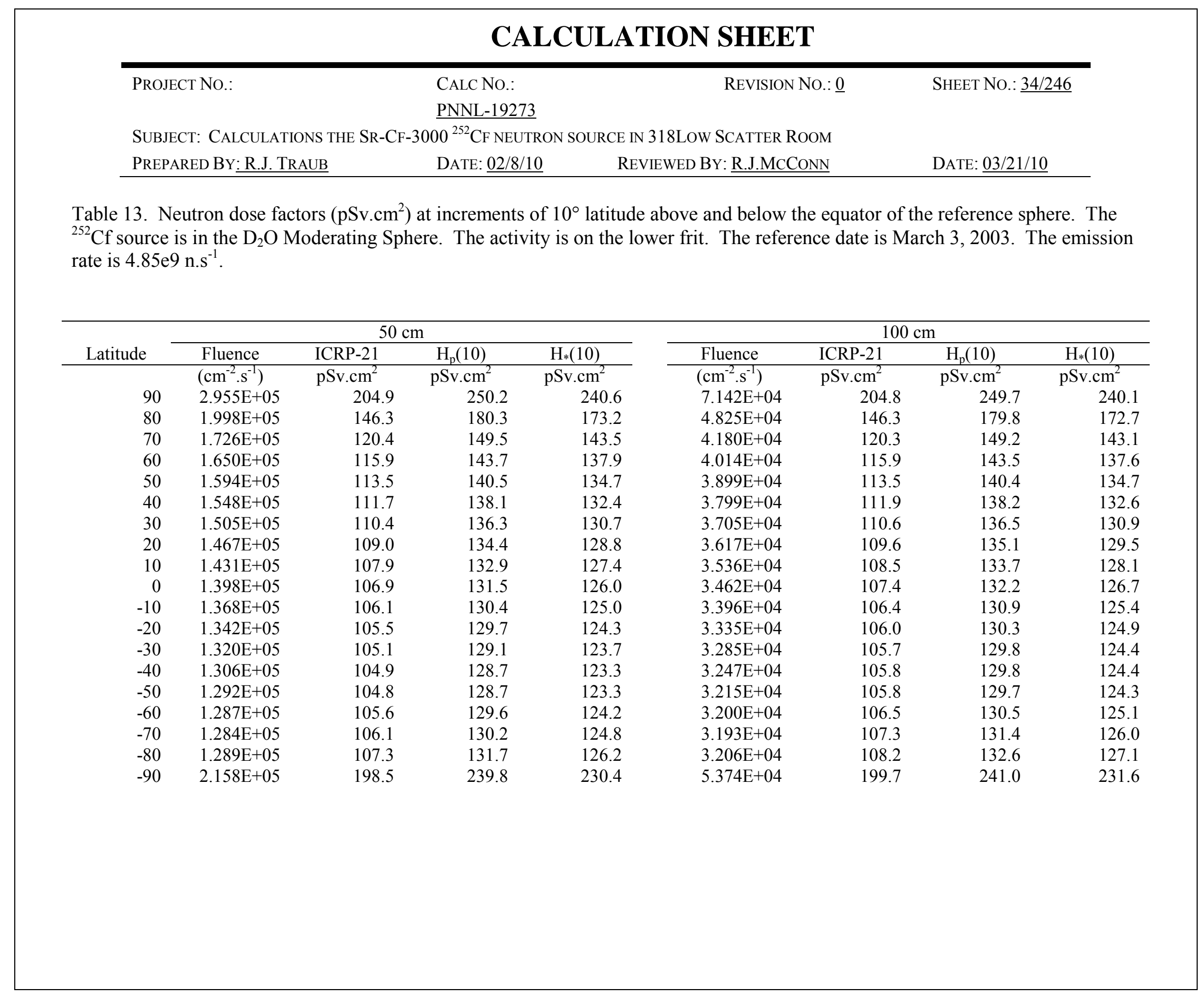




\section{CALCULATION SHEET}

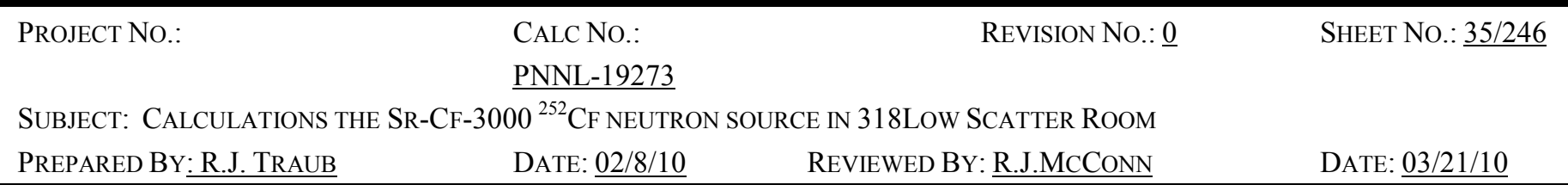

Table 14. Neutron fluence at the surface of a $10-\mathrm{cm} \times 10-\mathrm{cm}$ rectangle $50 \mathrm{~cm}$ and $100 \mathrm{~cm}$ from the center of the reference sphere. The activity is on the upper frit. The reference date is March 3, 2003. The emission rate is $4.85 \mathrm{e} 9 \mathrm{n} \cdot \mathrm{s}^{-1}$.

\begin{tabular}{|c|c|c|c|c|c|c|}
\hline \multirow[b]{2}{*}{$\begin{array}{l}Z \text { position } \\
(\mathrm{cm})\end{array}$} & \multicolumn{3}{|c|}{$50 \mathrm{~cm}$ from center of reference sphere } & \multicolumn{3}{|c|}{$\begin{array}{c}100 \mathrm{~cm} \text { from center of reference } \\
\text { sphere } \\
\text { horizontal Position }(\mathrm{cm})\end{array}$} \\
\hline & 0 & 5 & 10 & 0 & 5 & 10 \\
\hline & \multicolumn{6}{|c|}{$\begin{array}{l}\mathrm{D}_{2} \mathrm{O} \text { Moderating Sphere (Moderated Spectra) } \\
\qquad\left(\mathrm{cm}^{-2} \cdot \mathrm{s}^{-1}\right)\end{array}$} \\
\hline 10 & $1.41 \mathrm{E}+05$ & $1.40 \mathrm{E}+05$ & $1.36 \mathrm{E}+05$ & $3.48 \mathrm{E}+04$ & $3.47 \mathrm{E}+04$ & $3.45 \mathrm{E}+04$ \\
\hline 5 & $1.40 \mathrm{E}+05$ & $1.39 \mathrm{E}+05$ & $1.35 \mathrm{E}+05$ & $3.45 \mathrm{E}+04$ & $3.44 \mathrm{E}+04$ & $3.42 \mathrm{E}+04$ \\
\hline 0 & $1.36 \mathrm{E}+05$ & $1.35 \mathrm{E}+05$ & $1.31 \mathrm{E}+05$ & $3.40 \mathrm{E}+04$ & $3.40 \mathrm{E}+04$ & $3.37 \mathrm{E}+04$ \\
\hline-5 & $1.30 \mathrm{E}+05$ & $1.29 \mathrm{E}+05$ & $1.26 \mathrm{E}+05$ & $3.34 \mathrm{E}+04$ & $3.33 \mathrm{E}+04$ & $3.31 \mathrm{E}+04$ \\
\hline-10 & $1.22 \mathrm{E}+05$ & $1.21 \mathrm{E}+05$ & $1.18 \mathrm{E}+05$ & $3.26 \mathrm{E}+04$ & $3.25 \mathrm{E}+04$ & $3.23 \mathrm{E}+04$ \\
\hline & \multicolumn{6}{|c|}{$\begin{array}{l}\text { Pneumatic Transfer Tube Assembly (Unmoderated Spectra) } \\
\qquad\left(\mathrm{cm}^{-2} \cdot \mathrm{s}^{-1}\right)\end{array}$} \\
\hline 10 & $1.63 \mathrm{E}+05$ & $1.62 \mathrm{E}+05$ & $1.57 \mathrm{E}+05$ & $4.16 \mathrm{E}+04$ & $4.15 \mathrm{E}+04$ & $4.12 \mathrm{E}+04$ \\
\hline 5 & $1.67 \mathrm{E}+05$ & $1.65 \mathrm{E}+05$ & $1.60 \mathrm{E}+05$ & $4.18 \mathrm{E}+04$ & $4.17 \mathrm{E}+04$ & $4.14 \mathrm{E}+04$ \\
\hline 0 & $1.67 \mathrm{E}+05$ & $1.65 \mathrm{E}+05$ & $1.61 \mathrm{E}+05$ & $4.19 \mathrm{E}+04$ & $4.18 \mathrm{E}+04$ & $4.15 \mathrm{E}+04$ \\
\hline-5 & $1.64 \mathrm{E}+05$ & $1.62 \mathrm{E}+05$ & $1.58 \mathrm{E}+05$ & $4.17 \mathrm{E}+04$ & $4.16 \mathrm{E}+04$ & $4.13 E+04$ \\
\hline-10 & $1.57 \mathrm{E}+05$ & $1.56 \mathrm{E}+05$ & $1.52 \mathrm{E}+05$ & 4.13E+04 & $4.12 \mathrm{E}+04$ & $4.09 \mathrm{E}+04$ \\
\hline Range & Maximum & Minimum & Ratio & Maximum & Minimum & Ratio \\
\hline & \multicolumn{6}{|c|}{$\mathrm{D}_{2} \mathrm{O}$ Moderating Sphere (Moderated Spectra) } \\
\hline 10 & $1.41 \mathrm{E}+05$ & $1.18 \mathrm{E}+05$ & $1.19 \mathrm{E}+00$ & $3.48 \mathrm{E}+04$ & $3.23 \mathrm{E}+04$ & $1.08 \mathrm{E}+00$ \\
\hline 5 & $1.40 \mathrm{E}+05$ & $1.29 \mathrm{E}+05$ & $1.08 \mathrm{E}+00$ & $3.45 \mathrm{E}+04$ & $3.33 \mathrm{E}+04$ & $1.04 \mathrm{E}+00$ \\
\hline
\end{tabular}

Pneumatic Transfer Tube Assembly (Unmoderated Spectra)

$\begin{array}{rrrrrrr}10 & 1.67 \mathrm{E}+05 & 1.52 \mathrm{E}+05 & 1.10 \mathrm{E}+00 & 4.19 \mathrm{E}+04 & 4.09 \mathrm{E}+04 & 1.02 \mathrm{E}+00 \\ 5 & 1.67 \mathrm{E}+05 & 1.62 \mathrm{E}+05 & 1.03 \mathrm{E}+00 & 4.19 \mathrm{E}+04 & 4.16 \mathrm{E}+04 & 1.01 \mathrm{E}+00\end{array}$




\section{CALCULATION SHEET}

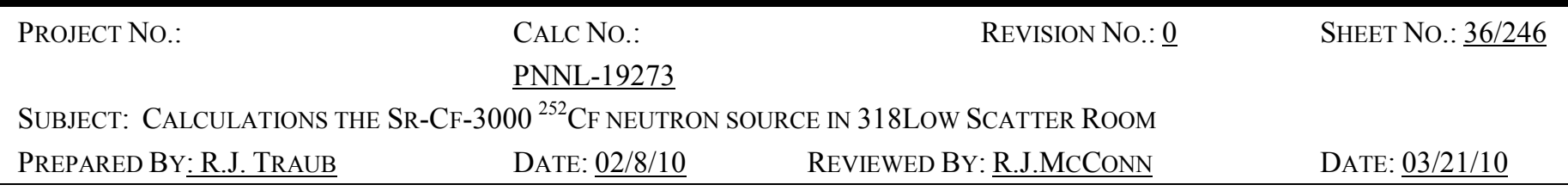

Table 15. Neutron fluence at the surface of a $10-\mathrm{cm} \times 10-\mathrm{cm}$ rectangle $50 \mathrm{~cm}$ and $100 \mathrm{~cm}$ from the center of the reference sphere. The activity is in the void between the frits. The reference date is March 3, 2003. The emission rate is $4.85 \mathrm{e} 9 \mathrm{n} \cdot \mathrm{s}^{-1}$.

\begin{tabular}{|c|c|c|c|c|c|c|}
\hline \multirow{3}{*}{$\begin{array}{l}\text { Z position } \\
(\mathrm{cm})\end{array}$} & \multicolumn{3}{|c|}{$50 \mathrm{~cm}$ from center of reference sphere } & \multicolumn{3}{|c|}{$\begin{array}{c}100 \mathrm{~cm} \text { from center of reference } \\
\text { sphere } \\
\text { horizontal position }(\mathrm{cm})\end{array}$} \\
\hline & \multicolumn{3}{|c|}{ horizontal position $(\mathrm{cm})$} & \multicolumn{3}{|c|}{ horizontal position $(\mathrm{cm})$} \\
\hline & & & & & & \\
\hline & \multicolumn{6}{|c|}{$\begin{array}{c}\mathrm{D}_{2} \mathrm{O} \text { Moderating Sphere (Moderated Spectra) } \\
\qquad\left(\mathrm{cm}^{-2} \cdot \mathrm{s}^{-1}\right)\end{array}$} \\
\hline 10 & $1.40 \mathrm{E}+05$ & $1.38 \mathrm{E}+05$ & $1.34 \mathrm{E}+05$ & $3.48 \mathrm{E}+04$ & $3.47 \mathrm{E}+04$ & $3.45 \mathrm{E}+04$ \\
\hline 5 & $1.41 \mathrm{E}+05$ & $1.39 \mathrm{E}+05$ & $1.35 \mathrm{E}+05$ & $3.47 \mathrm{E}+04$ & $3.46 \mathrm{E}+04$ & $3.44 \mathrm{E}+04$ \\
\hline 0 & $1.39 \mathrm{E}+05$ & $1.37 \mathrm{E}+05$ & $1.33 \mathrm{E}+05$ & $3.44 \mathrm{E}+04$ & $3.43 \mathrm{E}+04$ & $3.41 \mathrm{E}+04$ \\
\hline-5 & $1.34 \mathrm{E}+05$ & $1.33 \mathrm{E}+05$ & $1.29 \mathrm{E}+05$ & $3.40 \mathrm{E}+04$ & $3.39 \mathrm{E}+04$ & $3.36 \mathrm{E}+04$ \\
\hline \multirow[t]{2}{*}{-10} & $1.27 \mathrm{E}+05$ & $1.26 \mathrm{E}+05$ & $1.23 \mathrm{E}+05$ & $3.33 \mathrm{E}+04$ & $3.33 \mathrm{E}+04$ & $3.30 \mathrm{E}+04$ \\
\hline & \multicolumn{6}{|c|}{$\begin{array}{l}\text { Pneumatic Transfer Tube Assembly (Unmoderated Spectra) } \\
\qquad\left(\mathrm{cm}^{-2} \cdot \mathrm{s}^{-1}\right)\end{array}$} \\
\hline 10 & $1.63 \mathrm{E}+05$ & $1.62 \mathrm{E}+05$ & $1.57 \mathrm{E}+05$ & $4.17 \mathrm{E}+04$ & $4.16 \mathrm{E}+04$ & $4.13 \mathrm{E}+04$ \\
\hline 5 & $1.67 \mathrm{E}+05$ & $1.66 \mathrm{E}+05$ & $1.61 \mathrm{E}+05$ & $4.20 \mathrm{E}+04$ & $4.19 \mathrm{E}+04$ & $4.16 \mathrm{E}+04$ \\
\hline 0 & $1.68 \mathrm{E}+05$ & $1.66 \mathrm{E}+05$ & $1.61 \mathrm{E}+05$ & $4.20 \mathrm{E}+04$ & $4.19 \mathrm{E}+04$ & $4.16 \mathrm{E}+04$ \\
\hline-5 & $1.64 \mathrm{E}+05$ & $1.63 \mathrm{E}+05$ & $1.58 \mathrm{E}+05$ & $4.18 \mathrm{E}+04$ & $4.17 \mathrm{E}+04$ & $4.14 \mathrm{E}+04$ \\
\hline-10 & $1.58 \mathrm{E}+05$ & $1.57 \mathrm{E}+05$ & $1.52 \mathrm{E}+05$ & 4.14E+04 & $4.13 \mathrm{E}+04$ & $4.10 \mathrm{E}+04$ \\
\hline \multirow[t]{2}{*}{ Range } & Maximum & Minimum & Ratio & Maximum & Minimum & Ratio \\
\hline & \multicolumn{6}{|c|}{$\mathrm{D}_{2} \mathrm{O}$ Moderating Sphere (Moderated Spectra) } \\
\hline 10 & $1.41 \mathrm{E}+05$ & $1.23 \mathrm{E}+05$ & $1.15 \mathrm{E}+00$ & $3.48 \mathrm{E}+04$ & $3.30 \mathrm{E}+04$ & $1.06 \mathrm{E}+00$ \\
\hline 5 & $1.41 \mathrm{E}+05$ & $1.33 \mathrm{E}+05$ & $1.06 \mathrm{E}+00$ & $3.47 \mathrm{E}+04$ & $3.39 \mathrm{E}+04$ & $1.02 \mathrm{E}+00$ \\
\hline & \multicolumn{6}{|c|}{ Pneumatic Transfer Tube Assembly (Unmoderated Spectra) } \\
\hline 10 & $1.68 \mathrm{E}+05$ & $1.52 \mathrm{E}+05$ & $1.10 \mathrm{E}+00$ & $4.20 \mathrm{E}+04$ & $4.10 \mathrm{E}+04$ & $1.03 \mathrm{E}+00$ \\
\hline 5 & $1.68 \mathrm{E}+05$ & $1.63 \mathrm{E}+05$ & $1.03 \mathrm{E}+00$ & $4.20 \mathrm{E}+04$ & $4.17 \mathrm{E}+04$ & $1.01 \mathrm{E}+00$ \\
\hline
\end{tabular}




\section{CALCULATION SHEET}

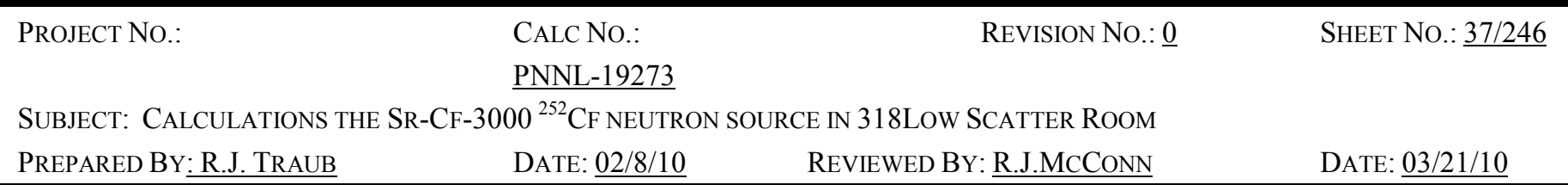

Table 16. Neutron fluence at the surface of a $10-\mathrm{cm} \times 10-\mathrm{cm}$ rectangle $50 \mathrm{~cm}$ and $100 \mathrm{~cm}$ from the center of the reference sphere. The activity is on the lower frit. The reference date is March 3, 2003. The emission rate is $4.85 \mathrm{e} 9 \mathrm{n} \cdot \mathrm{s}^{-1}$.

\begin{tabular}{|c|c|c|c|c|c|c|}
\hline \multirow{2}{*}{$\begin{array}{l}\text { Z position } \\
(\mathrm{cm})\end{array}$} & \multicolumn{3}{|c|}{$50 \mathrm{~cm}$ from center of reference sphere } & \multicolumn{3}{|c|}{$\begin{array}{c}100 \mathrm{~cm} \text { from center of reference } \\
\text { sphere } \\
\text { horizontal position }(\mathrm{cm})\end{array}$} \\
\hline & 0 & 5 & 10 & 0 & 5 & 10 \\
\hline & \multicolumn{6}{|c|}{$\begin{array}{l}\mathrm{D}_{2} \mathrm{O} \text { Moderating Sphere (Moderated Spectra) } \\
\qquad\left(\mathrm{cm}^{-2} \cdot \mathrm{s}^{-1}\right)\end{array}$} \\
\hline 10 & $1.38 \mathrm{E}+05$ & $1.36 \mathrm{E}+05$ & $1.33 \mathrm{E}+05$ & $3.46 \mathrm{E}+04$ & $3.46 \mathrm{E}+04$ & $3.43 \mathrm{E}+04$ \\
\hline 5 & $1.40 \mathrm{E}+05$ & $1.39 \mathrm{E}+05$ & $1.35 \mathrm{E}+05$ & $3.47 \mathrm{E}+04$ & $3.46 \mathrm{E}+04$ & $3.44 \mathrm{E}+04$ \\
\hline 0 & $1.40 \mathrm{E}+05$ & $1.38 \mathrm{E}+05$ & $1.34 \mathrm{E}+05$ & $3.46 \mathrm{E}+04$ & $3.45 \mathrm{E}+04$ & $3.42 \mathrm{E}+04$ \\
\hline-5 & $1.36 \mathrm{E}+05$ & $1.35 E+05$ & $1.31 \mathrm{E}+05$ & $3.43 E+04$ & $3.42 \mathrm{E}+04$ & $3.39 E+04$ \\
\hline \multirow[t]{2}{*}{-10} & $1.31 \mathrm{E}+05$ & $1.30 \mathrm{E}+05$ & $1.26 \mathrm{E}+05$ & $3.38 \mathrm{E}+04$ & $3.38 \mathrm{E}+04$ & $3.35 \mathrm{E}+04$ \\
\hline & \multicolumn{6}{|c|}{$\begin{array}{l}\text { Pneumatic Transfer Tube Assembly (Unmoderated Spectra) } \\
\qquad\left(\mathrm{cm}^{-2} \cdot \mathrm{s}^{-1}\right)\end{array}$} \\
\hline 10 & $1.63 \mathrm{E}+05$ & $1.61 \mathrm{E}+05$ & $1.57 \mathrm{E}+05$ & $4.17 \mathrm{E}+04$ & $4.16 \mathrm{E}+04$ & $4.13 E+04$ \\
\hline 5 & $1.67 \mathrm{E}+05$ & $1.65 \mathrm{E}+05$ & $1.60 \mathrm{E}+05$ & $4.19 \mathrm{E}+04$ & $4.18 \mathrm{E}+04$ & $4.15 \mathrm{E}+04$ \\
\hline 0 & $1.67 \mathrm{E}+05$ & $1.66 \mathrm{E}+05$ & $1.61 \mathrm{E}+05$ & $4.19 \mathrm{E}+04$ & $4.18 \mathrm{E}+04$ & $4.15 \mathrm{E}+04$ \\
\hline-5 & $1.65 \mathrm{E}+05$ & $1.63 \mathrm{E}+05$ & $1.58 \mathrm{E}+05$ & $4.17 \mathrm{E}+04$ & $4.16 \mathrm{E}+04$ & $4.13 \mathrm{E}+04$ \\
\hline-10 & $1.59 \mathrm{E}+05$ & $1.57 \mathrm{E}+05$ & $1.53 \mathrm{E}+05$ & $4.13 \mathrm{E}+04$ & $4.12 \mathrm{E}+04$ & $4.09 \mathrm{E}+04$ \\
\hline Range & Maximum & Minimum & Ratio & Maximum & Minimum & Ratio \\
\hline & \multicolumn{6}{|c|}{$\mathrm{D}_{2} \mathrm{O}$ Moderating Sphere (Moderated Spectra) } \\
\hline 10 & $1.40 \mathrm{E}+05$ & $1.26 \mathrm{E}+05$ & $1.11 \mathrm{E}+00$ & $3.47 \mathrm{E}+04$ & $3.35 \mathrm{E}+04$ & $1.04 \mathrm{E}+00$ \\
\hline 5 & $1.40 \mathrm{E}+05$ & $1.35 \mathrm{E}+05$ & $1.04 \mathrm{E}+00$ & $3.47 \mathrm{E}+04$ & $3.42 \mathrm{E}+04$ & $1.01 \mathrm{E}+00$ \\
\hline & \multicolumn{6}{|c|}{ Pneumatic Transfer Tube Assembly (Unmoderated Spectra) } \\
\hline 10 & $1.67 \mathrm{E}+05$ & $1.53 \mathrm{E}+05$ & 1.095 & 4.19E+04 & $4.09 \mathrm{E}+04$ & 1.025 \\
\hline 5 & $1.67 \mathrm{E}+05$ & $1.63 \mathrm{E}+05$ & 1.027 & $4.19 \mathrm{E}+04$ & $4.16 \mathrm{E}+04$ & 1.007 \\
\hline
\end{tabular}




\section{CALCULATION SHEET}

\begin{tabular}{|c|c|c|c|}
\hline PROJECT No.: & $\begin{array}{l}\text { CALC NO.: } \\
\text { PNNL-19273 }\end{array}$ & REVISION NO.: $\underline{0}$ & SHEET NO.: $\underline{38 / 246}$ \\
\hline \multicolumn{4}{|c|}{$\frac{\text { PNNL-19273 }}{252}$} \\
\hline PREPARED BY: R.J. TRAUB & DATE: $\underline{02 / 8 / 10}$ & REVIEWED BY: $\underline{\text { R.J.MCCONN }}$ & DATE: $\underline{03 / 21 / 10}$ \\
\hline
\end{tabular}

Table 17. Neutron Dose Factors at the surface of a $10-\mathrm{cm} \times 10-\mathrm{cm}$ rectangle $50 \mathrm{~cm}$ and $100 \mathrm{~cm}$ from the center of the reference sphere. The ${ }^{252} \mathrm{Cf}$ source is in the $\mathrm{D}_{2} \mathrm{O}$ moderating sphere. The activity is on the upper frit. The reference date is March 3, 2003. The emission rate is $4.85 \mathrm{e} 9 \mathrm{n} \cdot \mathrm{s}^{-1}$.

\begin{tabular}{|c|c|c|c|c|c|c|}
\hline \multirow{3}{*}{$\begin{array}{l}\text { Z position } \\
(\mathrm{cm})\end{array}$} & \multicolumn{3}{|c|}{$50 \mathrm{~cm}$ from center of reference sphere } & \multicolumn{3}{|c|}{$\begin{array}{l}100 \mathrm{~cm} \text { from center of reference } \\
\text { sphere }\end{array}$} \\
\hline & 0 & $\frac{\text { tal position }}{5}$ & horizontal position $(\mathrm{cm})$ & 0 & 5 & (cm) \\
\hline & & & & & & \\
\hline \multicolumn{7}{|c|}{$\begin{array}{l}\text { ICRP-21 Appendix } 6 \text { Factors } \\
\left(\text { pSv. } \mathrm{cm}^{2}\right)\end{array}$} \\
\hline 10 & 111.6 & 111.6 & 111.6 & 111.4 & 111.4 & 111.4 \\
\hline 5 & 110.4 & 110.3 & 110.4 & 110.9 & 110.8 & 110.8 \\
\hline 0 & 109.2 & 109.1 & 109.2 & 110.3 & 110.3 & 110.3 \\
\hline-5 & 108.0 & 108.0 & 108.0 & 109.8 & 109.8 & 109.8 \\
\hline-10 & 107.0 & 107.0 & 106.9 & 109.3 & 109.3 & 109.3 \\
\hline & \multicolumn{6}{|c|}{$\begin{array}{l}\text { Personal Dose Equivalent } \mathrm{Hp}(10) \\
\left.\text { (pSv. } \mathrm{cm}^{2}\right)\end{array}$} \\
\hline 10 & 137.7 & 137.7 & 137.7 & 137.3 & 137.3 & 137.3 \\
\hline 5 & 136.0 & 135.9 & 135.9 & 136.5 & 136.5 & 136.5 \\
\hline 0 & 134.3 & 134.3 & 134.3 & 135.7 & 135.7 & 135.7 \\
\hline-5 & 132.7 & 132.7 & 132.6 & 135.0 & 135.0 & 135.0 \\
\hline-10 & 131.3 & 131.2 & 131.2 & 134.3 & 134.3 & 134.3 \\
\hline & \multicolumn{6}{|c|}{$\begin{array}{c}\text { Ambient Dose Equivalent } \mathrm{H}^{*}(10) \\
\left(\mathrm{pSv} \cdot \mathrm{cm}^{2}\right)\end{array}$} \\
\hline 10 & 132.1 & 132.0 & 132.0 & 131.6 & 131.6 & 131.6 \\
\hline 5 & 130.3 & 130.3 & 130.3 & 130.9 & 130.9 & 130.9 \\
\hline 0 & 128.7 & 128.7 & 128.7 & 130.1 & 130.1 & 130.1 \\
\hline-5 & 127.2 & 127.2 & 127.1 & 129.5 & 129.4 & 129.4 \\
\hline-10 & 125.9 & 125.8 & 125.8 & 128.8 & 128.8 & 128.7 \\
\hline Dose Factor & Maximum & Minimum & Ratio & Maximum & Minimum & Ratio \\
\hline & \multicolumn{6}{|c|}{ 10-cm Range } \\
\hline ICRP-21 & 111.6 & 106.9 & 1.044 & 111.4 & 109.3 & 1.019 \\
\hline $\mathrm{Hp}(10)$ & 137.7 & 131.2 & 1.050 & 137.3 & 134.3 & 1.022 \\
\hline $\mathrm{H}^{*}(10)$ & 132.1 & 125.8 & 1.050 & 131.6 & 128.7 & 1.022 \\
\hline \multicolumn{7}{|c|}{ 5-cm Range } \\
\hline ICRP-21 & 110.4 & 108.0 & 1.022 & 110.9 & 109.8 & 1.010 \\
\hline$H p(10)$ & 136.0 & 132.7 & 1.025 & 136.5 & 135.0 & 1.011 \\
\hline $\mathrm{H}^{*}(10)$ & 130.3 & 127.2 & 1.025 & 130.9 & 129.4 & 1.011 \\
\hline
\end{tabular}




\section{CALCULATION SHEET}

\begin{tabular}{|c|c|c|c|}
\hline ProjeCt No.: & $\begin{array}{l}\text { CALC NO.: } \\
\text { PNNL-19273 }\end{array}$ & REVISION NO.: $\underline{0}$ & SHEET NO.: $\underline{39 / 246}$ \\
\hline \multicolumn{4}{|c|}{ E IN 318LOW SCATTER ROOM } \\
\hline PREPARED BY: R.J. TRAUB & DATE: $\underline{02 / 8 / 10}$ & REVIEWED BY: R.J.MCCONN & DATE: $03 / 21 / 10$ \\
\hline
\end{tabular}

Table 18. Neutron Dose Factors at the surface of a $10-\mathrm{cm} \times 10-\mathrm{cm}$ rectangle $50 \mathrm{~cm}$ and $100 \mathrm{~cm}$ from the center of the reference sphere. The ${ }^{252} \mathrm{Cf}$ source is in the $\mathrm{D}_{2} \mathrm{O}$ moderating sphere. The activity is in the void between the frits. The reference date is March 3, 2003. The emission rate is $4.85 \mathrm{e} 9 \mathrm{n} \cdot \mathrm{s}^{-1}$.

\begin{tabular}{|c|c|c|c|c|c|c|}
\hline \multirow[b]{3}{*}{$\begin{array}{l}\text { Z position } \\
(\mathrm{cm})\end{array}$} & \multicolumn{3}{|c|}{$50 \mathrm{~cm}$ from center of reference sphere } & \multirow{2}{*}{\multicolumn{3}{|c|}{$\begin{array}{c}100 \mathrm{~cm} \text { from center of reference } \\
\text { sphere } \\
\text { horizontal position }(\mathrm{cm})\end{array}$}} \\
\hline & \multicolumn{3}{|c|}{ horizontal position $(\mathrm{cm})$} & & & \\
\hline & 0 & 5 & 10 & 0 & 5 & 10 \\
\hline \multicolumn{7}{|c|}{$\begin{array}{c}\text { ICRP-21 Appendix } 6 \text { Factors } \\
\left(\mathrm{pSv} \cdot \mathrm{cm}^{2}\right)\end{array}$} \\
\hline 10 & 109.7 & 109.7 & 109.8 & 109.6 & 109.6 & 109.6 \\
\hline 5 & 108.8 & 108.8 & 108.9 & 109.2 & 109.2 & 109.2 \\
\hline 0 & 107.9 & 107.9 & 108.0 & 108.8 & 108.8 & 108.8 \\
\hline-5 & 107.1 & 107.1 & 107.2 & 108.4 & 108.4 & 108.4 \\
\hline-10 & 106.5 & 106.5 & 106.6 & 108.1 & 108.1 & 108.1 \\
\hline & \multicolumn{6}{|c|}{$\begin{array}{l}\text { Personal Dose Equivalent } \mathrm{Hp}(10) \\
\left(\mathrm{pSv} . \mathrm{cm}^{2}\right)\end{array}$} \\
\hline 10 & 135.2 & 135.2 & 135.3 & 135.0 & 134.9 & 134.9 \\
\hline 5 & 133.9 & 133.9 & 134.0 & 134.4 & 134.4 & 134.4 \\
\hline 0 & 132.7 & 132.7 & 132.8 & 133.8 & 133.8 & 133.9 \\
\hline-5 & 131.6 & 131.7 & 131.8 & 133.3 & 133.3 & 133.3 \\
\hline-10 & 130.8 & 130.8 & 130.9 & 132.9 & 132.9 & 132.9 \\
\hline & \multicolumn{6}{|c|}{$\begin{array}{c}\text { Ambient Dose Equivalent } \mathrm{H}^{*}(10) \\
\left(\mathrm{pSv} . \mathrm{cm}^{2}\right)\end{array}$} \\
\hline 10 & 129.6 & 129.7 & 129.7 & 129.4 & 129.4 & 129.4 \\
\hline 5 & 128.4 & 128.4 & 128.5 & 128.8 & 128.8 & 128.8 \\
\hline 0 & 127.2 & 127.3 & 127.4 & 128.3 & 128.3 & 128.3 \\
\hline-5 & 126.2 & 126.2 & 126.3 & 127.8 & 127.8 & 127.8 \\
\hline-10 & 125.4 & 125.3 & 125.5 & 127.4 & 127.4 & 127.4 \\
\hline Dose Factor & Maximum & Minimum & Ratio & Maximum & Minimum & Ratio \\
\hline & \multicolumn{6}{|c|}{ 10-cm Range } \\
\hline ICRP-21 & 109.8 & 106.5 & 1.031 & 109.6 & 108.1 & 1.014 \\
\hline $\mathrm{Hp}(10)$ & 135.3 & 130.8 & 1.035 & 135.0 & 132.9 & 1.016 \\
\hline $\mathrm{H}^{*}(10)$ & 129.7 & 125.3 & 1.035 & 129.4 & 127.4 & 1.016 \\
\hline \multicolumn{7}{|c|}{ 5-cm Range } \\
\hline ICRP-21 & 108.8 & 107.1 & 1.016 & 109.2 & 108.4 & 1.007 \\
\hline $\mathrm{Hp}(10)$ & 133.9 & 131.6 & 1.017 & 134.4 & 133.3 & 1.008 \\
\hline $\mathrm{H}^{*}(10)$ & 128.4 & 126.2 & 1.018 & 128.8 & 127.8 & 1.008 \\
\hline
\end{tabular}




\section{CALCULATION SHEET}

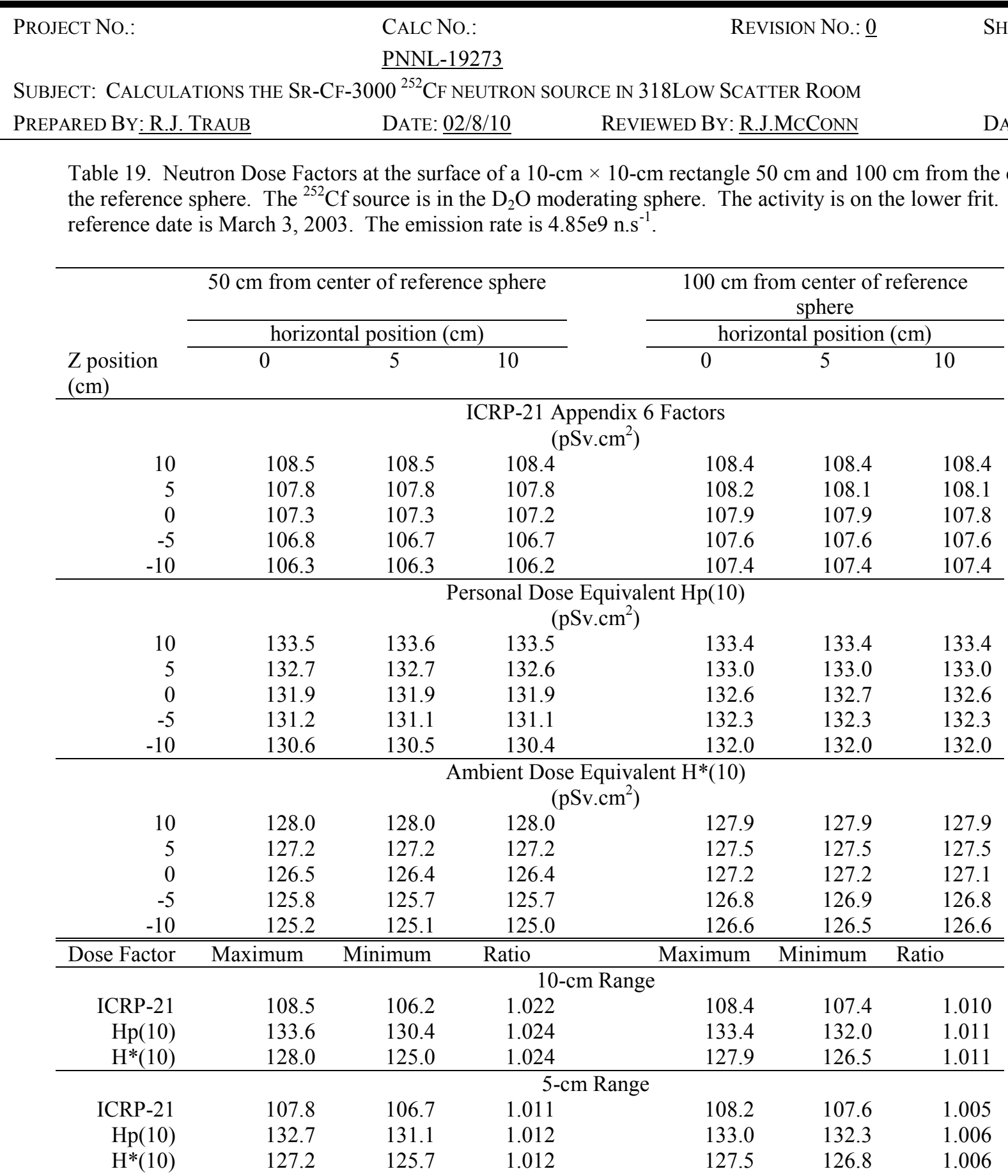




\section{CALCULATION SHEET}

\begin{tabular}{|c|c|c|c|}
\hline PROJECT No.: & $\begin{array}{l}\text { CALC NO.: } \\
\text { PNNL-19273 }\end{array}$ & REVISION NO.: $\underline{0}$ & SHEET NO.: $\underline{41 / 246}$ \\
\hline \multicolumn{4}{|c|}{ PNNL-19273 } \\
\hline PREPARED BY: R.J. TRAUB & DATE: $\underline{02 / 8 / 10}$ & REVIEWED BY: $\underline{\text { R.J.MCCONN }}$ & DATE: $\underline{03 / 21 / 10}$ \\
\hline
\end{tabular}

Table 20. Neutron Dose Factors at the surface of a $10-\mathrm{cm} \times 10-\mathrm{cm}$ rectangle $50 \mathrm{~cm}$ and $100 \mathrm{~cm}$ from the center of the reference sphere. The ${ }^{252} \mathrm{Cf}$ source is in the pneumatic transfer assembly. The activity is on the upper frit. The reference date is March 3, 2003. The emission rate is $4.85 \mathrm{e}^{2} \mathrm{n} \cdot \mathrm{s}^{-1}$.

\begin{tabular}{|c|c|c|c|c|c|c|}
\hline \multirow[b]{2}{*}{$\begin{array}{l}\text { Z position } \\
(\mathrm{cm})\end{array}$} & \multicolumn{3}{|c|}{$\begin{array}{c}50 \mathrm{~cm} \text { from center of reference sphere } \\
\text { horizontal position }(\mathrm{cm})\end{array}$} & \multicolumn{3}{|c|}{$\begin{array}{c}100 \mathrm{~cm} \text { from center of reference } \\
\text { sphere } \\
\text { horizontal Position }(\mathrm{cm})\end{array}$} \\
\hline & 0 & 5 & 10 & 0 & 5 & 10 \\
\hline & \multicolumn{6}{|c|}{$\begin{array}{l}\text { ICRP-21 Appendix } 6 \text { Factors } \\
\left(\mathrm{pSv} . \mathrm{cm}^{2}\right)\end{array}$} \\
\hline 10 & 323.5 & 323.5 & 323.5 & 323.5 & 323.5 & 323.5 \\
\hline 5 & 323.5 & 323.5 & 323.5 & 323.5 & 323.5 & 323.5 \\
\hline 0 & 323.4 & 323.4 & 323.4 & 323.5 & 323.5 & 323.5 \\
\hline-5 & 323.4 & 323.4 & 323.4 & 323.4 & 323.4 & 323.4 \\
\hline-10 & 323.5 & 323.5 & 323.5 & 323.4 & 323.4 & 323.4 \\
\hline & \multicolumn{6}{|c|}{$\begin{array}{c}\text { Personal Dose Equivalent Hp(10) } \\
\left(\mathrm{pSv} . \mathrm{cm}^{2}\right)\end{array}$} \\
\hline 10 & 392.4 & 392.4 & 392.4 & 392.4 & 392.4 & 392.4 \\
\hline 5 & 392.4 & 392.4 & 392.4 & 392.4 & 392.4 & 392.4 \\
\hline 0 & 392.4 & 392.4 & 392.4 & 392.4 & 392.4 & 392.4 \\
\hline-5 & 392.4 & 392.4 & 392.4 & 392.4 & 392.4 & 392.4 \\
\hline-10 & 392.5 & 392.5 & 392.5 & 392.4 & 392.4 & 392.4 \\
\hline & \multicolumn{6}{|c|}{$\begin{array}{c}\text { Ambient Dose Equivalent } \mathrm{H}^{*}(10) \\
\left(\mathrm{pSv} . \mathrm{cm}^{2}\right)\end{array}$} \\
\hline 10 & 377.6 & 377.6 & 377.6 & 377.6 & 377.6 & 377.6 \\
\hline 5 & 377.6 & 377.6 & 377.6 & 377.6 & 377.6 & 377.6 \\
\hline 0 & 377.6 & 377.6 & 377.6 & 377.6 & 377.6 & 377.6 \\
\hline-5 & 377.6 & 377.6 & 377.6 & 377.6 & 377.6 & 377.6 \\
\hline-10 & 377.7 & 377.7 & 377.6 & 377.6 & 377.6 & 377.6 \\
\hline Dose Factor & Maximum & Minimum & Ratio & Maximum & Minimum & Ratio \\
\hline & \multicolumn{6}{|c|}{ 10-cm Range } \\
\hline ICRP-21 & 323.5 & 323.4 & 1.000 & 323.5 & 323.4 & 1.000 \\
\hline $\mathrm{Hp}(10)$ & 392.5 & 392.4 & 1.000 & 392.4 & 392.4 & 1.000 \\
\hline $\mathrm{H}^{*}(10)$ & 377.7 & 377.6 & 1.000 & 377.6 & 377.6 & 1.000 \\
\hline \multicolumn{7}{|c|}{ 5-cm Range } \\
\hline ICRP-21 & 323.5 & 323.4 & 1.000 & 323.5 & 323.4 & 1.000 \\
\hline$H p(10)$ & 392.4 & 392.4 & 1.000 & 392.4 & 392.4 & 1.000 \\
\hline $\mathrm{H}^{*}(10)$ & 377.6 & 377.6 & 1.000 & 377.6 & 377.6 & 1.000 \\
\hline
\end{tabular}




\section{CALCULATION SHEET}

\begin{tabular}{|c|c|c|c|}
\hline PROJECT NO.: & $\begin{array}{l}\text { CALC NO.: } \\
\text { PNNL-19273 }\end{array}$ & REVISION NO.: $\underline{\underline{0}}$ & SHEET NO.: $\underline{42 / 246}$ \\
\hline \multicolumn{4}{|c|}{$\underline{\text { PNNL-19273 }}$} \\
\hline PREPARED BY: R.J. TRAUB & DATE: $\underline{02 / 8 / 10}$ & REVIEWED BY: R.J.MCCONN & DATE: $\underline{03 / 21 / 10}$ \\
\hline
\end{tabular}

Table 21. Neutron Dose Factors at the surface of a $10-\mathrm{cm} \times 10-\mathrm{cm}$ rectangle $50 \mathrm{~cm}$ and $100 \mathrm{~cm}$ from the center of the reference sphere. The ${ }^{252} \mathrm{Cf}$ source is in the pneumatic transfer assembly. The activity is in the void between the frits. The reference date is March 3, 2003. The emission rate is $4.85 \mathrm{e} 9 \mathrm{n} \cdot \mathrm{s}^{-1}$.

\begin{tabular}{|c|c|c|c|c|c|c|}
\hline \multirow[b]{2}{*}{$\begin{array}{l}\text { Z position } \\
(\mathrm{cm})\end{array}$} & \multicolumn{3}{|c|}{$\begin{array}{c}50 \mathrm{~cm} \text { from center of reference sphere } \\
\text { horizontal position }(\mathrm{cm})\end{array}$} & \multicolumn{3}{|c|}{$\begin{array}{c}100 \mathrm{~cm} \text { from center of reference } \\
\text { sphere } \\
\text { horizontal Position }(\mathrm{cm})\end{array}$} \\
\hline & 0 & 5 & 10 & 0 & 5 & 10 \\
\hline & \multicolumn{6}{|c|}{$\begin{array}{l}\text { ICRP-21 Appendix } 6 \text { Factors } \\
\left(\mathrm{pSv} . \mathrm{cm}^{2}\right)\end{array}$} \\
\hline 10 & 323.8 & 323.8 & 323.8 & 323.8 & 323.8 & 323.8 \\
\hline 5 & 323.7 & 323.7 & 323.8 & 323.7 & 323.8 & 323.8 \\
\hline 0 & 323.7 & 323.7 & 323.7 & 323.7 & 323.7 & 323.8 \\
\hline-5 & 323.8 & 323.8 & 323.8 & 323.7 & 323.8 & 323.8 \\
\hline-10 & 323.9 & 323.9 & 323.9 & 323.8 & 323.8 & 323.8 \\
\hline & \multicolumn{6}{|c|}{$\begin{array}{c}\text { Personal Dose Equivalent Hp(10) } \\
\left(\mathrm{pSv} . \mathrm{cm}^{2}\right)\end{array}$} \\
\hline 10 & 392.6 & 392.6 & 392.6 & 392.6 & 392.6 & 392.6 \\
\hline 5 & 392.5 & 392.6 & 392.6 & 392.6 & 392.6 & 392.6 \\
\hline 0 & 392.5 & 392.5 & 392.5 & 392.6 & 392.6 & 392.6 \\
\hline-5 & 392.6 & 392.6 & 392.6 & 392.6 & 392.6 & 392.6 \\
\hline-10 & 392.7 & 392.7 & 392.7 & 392.6 & 392.6 & 392.6 \\
\hline & \multicolumn{6}{|c|}{$\begin{array}{c}\text { Ambient Dose Equivalent } \mathrm{H}^{*}(10) \\
\left(\mathrm{pSv} . \mathrm{cm}^{2}\right)\end{array}$} \\
\hline 10 & 377.8 & 377.8 & 377.8 & 377.7 & 377.8 & 377.8 \\
\hline 5 & 377.7 & 377.7 & 377.7 & 377.7 & 377.7 & 377.7 \\
\hline 0 & 377.7 & 377.7 & 377.7 & 377.7 & 377.7 & 377.7 \\
\hline-5 & 377.7 & 377.8 & 377.8 & 377.7 & 377.7 & 377.7 \\
\hline-10 & 377.8 & 377.8 & 377.8 & 377.8 & 377.8 & 377.8 \\
\hline Dose Factor & Maximum & Minimum & Ratio & Maximum & Minimum & Ratio \\
\hline & \multicolumn{6}{|c|}{ 10-cm Range } \\
\hline ICRP-21 & 323.9 & 323.7 & 1.001 & 323.8 & 323.7 & 1.000 \\
\hline $\mathrm{Hp}(10)$ & 392.7 & 392.5 & 1.000 & 392.6 & 392.6 & 1.000 \\
\hline $\mathrm{H}^{*}(10)$ & 377.8 & 377.7 & 1.000 & 377.8 & 377.7 & 1.000 \\
\hline \multicolumn{7}{|c|}{ 5-cm Range } \\
\hline ICRP-21 & 323.8 & 323.7 & 1.000 & 323.8 & 323.7 & 1.000 \\
\hline$H p(10)$ & 392.6 & 392.5 & 1.000 & 392.6 & 392.6 & 1.000 \\
\hline $\mathrm{H}^{*}(10)$ & 377.8 & 377.7 & 1.000 & 377.7 & 377.7 & 1.000 \\
\hline
\end{tabular}




\section{CALCULATION SHEET}

\begin{tabular}{|c|c|c|c|}
\hline PROJECT No.: & $\begin{array}{l}\text { CALC NO.: } \\
\text { PNNL-19273 }\end{array}$ & REVISION NO.: $\underline{0}$ & SHEET NO.: $\underline{43 / 246}$ \\
\hline \multicolumn{4}{|c|}{ PNNL-19273 } \\
\hline PREPARED BY: R.J. TRAUB & DATE: $\underline{02 / 8 / 10}$ & REVIEWED BY: $\underline{\text { R.J.MCCONN }}$ & DATE: $\underline{03 / 21 / 10}$ \\
\hline
\end{tabular}

Table 22. Neutron Dose Factors at the surface of a $10-\mathrm{cm} \times 10-\mathrm{cm}$ rectangle $50 \mathrm{~cm}$ and $100 \mathrm{~cm}$ from the center of the reference sphere. The ${ }^{252} \mathrm{Cf}$ source is in the pneumatic transfer assembly. The activity is on the lower frit. The reference date is March 3, 2003. The emission rate is $4.85 \mathrm{e}^{2} \mathrm{n} \cdot \mathrm{s}^{-1}$.

\begin{tabular}{|c|c|c|c|c|c|c|}
\hline \multirow{3}{*}{$\begin{array}{l}\text { Z position } \\
(\mathrm{cm})\end{array}$} & \multirow{2}{*}{\multicolumn{3}{|c|}{$\begin{array}{c}50 \mathrm{~cm} \text { from center of reference sphere } \\
\text { horizontal position }(\mathrm{cm})\end{array}$}} & \multicolumn{3}{|c|}{$\begin{array}{c}100 \mathrm{~cm} \text { from center of reference } \\
\text { sphere } \\
\text { horizontal Position }(\mathrm{cm})\end{array}$} \\
\hline & 0 & & & horiz & & $\frac{(\mathrm{cm})}{10}$ \\
\hline & & & 10 & & & \\
\hline & \multicolumn{6}{|c|}{$\begin{array}{l}\text { ICRP-21 Appendix 6 Factors } \\
\left(\mathrm{pSv} . \mathrm{cm}^{2}\right)\end{array}$} \\
\hline 10 & 323.4 & 323.4 & 323.4 & 323.4 & 323.4 & 323.4 \\
\hline 5 & 323.4 & 323.4 & 323.4 & 323.4 & 323.4 & 323.4 \\
\hline 0 & 323.4 & 323.4 & 323.4 & 323.4 & 323.4 & 323.4 \\
\hline-5 & 323.4 & 323.4 & 323.4 & 323.4 & 323.4 & 323.4 \\
\hline-10 & 323.5 & 323.5 & 323.5 & 323.4 & 323.4 & 323.4 \\
\hline & \multicolumn{6}{|c|}{$\begin{array}{c}\text { Personal Dose Equivalent } \mathrm{Hp}(10) \\
\left(\mathrm{pSv} \cdot \mathrm{cm}^{2}\right)\end{array}$} \\
\hline 10 & 392.4 & 392.4 & 392.4 & 392.4 & 392.4 & 392.4 \\
\hline 5 & 392.3 & 392.3 & 392.3 & 392.4 & 392.4 & 392.4 \\
\hline 0 & 392.3 & 392.3 & 392.4 & 392.4 & 392.4 & 392.4 \\
\hline-5 & 392.4 & 392.4 & 392.4 & 392.4 & 392.4 & 392.4 \\
\hline-10 & 392.5 & 392.5 & 392.5 & 392.4 & 392.4 & 392.4 \\
\hline & \multicolumn{6}{|c|}{$\begin{array}{c}\text { Ambient Dose Equivalent } \mathrm{H}^{*}(10) \\
\left(\mathrm{pSv} . \mathrm{cm}^{2}\right)\end{array}$} \\
\hline 10 & 377.5 & 377.5 & 377.5 & 377.5 & 377.5 & 377.5 \\
\hline 5 & 377.5 & 377.5 & 377.5 & 377.5 & 377.5 & 377.5 \\
\hline 0 & 377.5 & 377.5 & 377.5 & 377.6 & 377.6 & 377.6 \\
\hline-5 & 377.6 & 377.6 & 377.6 & 377.6 & 377.6 & 377.6 \\
\hline-10 & 377.6 & 377.6 & 377.6 & 377.6 & 377.6 & 377.6 \\
\hline Dose Factor & Maximum & Minimum & Ratio & Maximum & Minimum & Ratio \\
\hline & \multicolumn{6}{|c|}{ 10-cm Range } \\
\hline ICRP-21 & 323.5 & 323.4 & 1.000 & 323.4 & 323.4 & 1.000 \\
\hline $\operatorname{Hp}(10)$ & 392.5 & 392.3 & 1.000 & 392.4 & 392.4 & 1.000 \\
\hline$H^{*}(10)$ & 377.6 & 377.5 & 1.000 & 377.6 & 377.5 & 1.000 \\
\hline \multicolumn{7}{|c|}{ 5-cm Range } \\
\hline ICRP-21 & 323.4 & 323.4 & 1.000 & 323.4 & 323.4 & 1.000 \\
\hline$H p(10)$ & 392.4 & 392.3 & 1.000 & 392.4 & 392.4 & 1.000 \\
\hline $\mathrm{H}^{*}(10)$ & 377.6 & 377.5 & 1.000 & 377.6 & 377.5 & 1.000 \\
\hline
\end{tabular}




\section{CALCULATION SHEET}

\begin{tabular}{|c|c|c|c|}
\hline PROJECT No.: & $\begin{array}{l}\text { CALC NO.: } \\
\text { PNNL-19273 }\end{array}$ & REVISION NO.: $\underline{0}$ & SHEET NO.: $\underline{44 / 246}$ \\
\hline SUBJECT: CALCULATIONS & $00{ }^{252} \mathrm{CF}$ NEUTRC & CE IN 318LOW SCATTER ROOM & \\
\hline PREPARED BY: R.J. TRAUB & DATE: $\underline{02 / 8 / 10}$ & REVIEWED BY: R.J.MCCONN & DATE: $\underline{03 / 21 / 10}$ \\
\hline
\end{tabular}

Table 23. Neutron dose rate at the surface of a $10-\mathrm{cm} \times 10-\mathrm{cm}$ rectangle $50 \mathrm{~cm}$ and $100 \mathrm{~cm}$ from the center of the reference sphere. The ${ }^{252} \mathrm{Cf}$ source is in the $\mathrm{D}_{2} \mathrm{O}$ moderating sphere. The activity is in the upper frit. The reference date is March 3, 2003. The emission rate is $4.85 \mathrm{e} 9 \mathrm{n} \cdot \mathrm{s}^{-1}$.

\begin{tabular}{|c|c|c|c|c|c|c|}
\hline \multirow{3}{*}{$\begin{array}{l}\text { Z position } \\
(\mathrm{cm})\end{array}$} & \multirow{2}{*}{\multicolumn{3}{|c|}{$\begin{array}{c}50 \mathrm{~cm} \text { from center of reference sphere } \\
\text { horizontal position }(\mathrm{cm})\end{array}$}} & \multicolumn{3}{|c|}{$\begin{array}{c}100 \mathrm{~cm} \text { from center of reference } \\
\text { sphere } \\
\text { horizontal position }(\mathrm{cm})\end{array}$} \\
\hline & 0 & 5 & & 0 & 5 & $\frac{(\mathrm{cm})}{10}$ \\
\hline & & & & & & \\
\hline & \multicolumn{6}{|c|}{$\begin{array}{l}\text { ICRP-21 Appendix } 6 \\
\left(\text { rem. } \mathrm{h}^{-2}\right)\end{array}$} \\
\hline 10 & 5.665 & 5.609 & 5.444 & 1.397 & 1.393 & 1.383 \\
\hline 5 & 5.567 & 5.509 & 5.347 & 1.378 & 1.374 & 1.364 \\
\hline 0 & 5.364 & 5.309 & 5.155 & 1.351 & 1.348 & 1.338 \\
\hline-5 & 5.072 & 5.022 & 4.879 & 1.320 & 1.317 & 1.307 \\
\hline-10 & 4.718 & 4.672 & 4.545 & 1.284 & 1.280 & 1.271 \\
\hline & \multicolumn{6}{|c|}{$\begin{array}{c}\text { Personal Dose Equivalent Hp(10) } \\
\left(\text { rem. } h^{-2}\right)\end{array}$} \\
\hline 10 & 6.989 & 6.920 & 6.715 & 1.722 & 1.717 & 1.704 \\
\hline 5 & 6.858 & 6.786 & 6.587 & 1.696 & 1.692 & 1.680 \\
\hline 0 & 6.599 & 6.531 & 6.342 & 1.663 & 1.659 & 1.647 \\
\hline-5 & 6.231 & 6.170 & 5.995 & 1.624 & 1.619 & 1.607 \\
\hline-10 & 5.789 & 5.733 & 5.577 & 1.578 & 1.573 & 1.562 \\
\hline & \multicolumn{6}{|c|}{$\begin{array}{c}\text { Ambient Dose Equivalent } \mathrm{H}^{*}(10) \\
\left(\text { rem. }^{-2}\right)\end{array}$} \\
\hline 10 & 6.702 & 6.635 & 6.439 & 1.651 & 1.646 & 1.634 \\
\hline 5 & 6.575 & 6.506 & 6.315 & 1.626 & 1.622 & 1.610 \\
\hline 0 & 6.326 & 6.261 & 6.080 & 1.594 & 1.590 & 1.579 \\
\hline-5 & 5.973 & 5.914 & 5.746 & 1.557 & 1.552 & 1.541 \\
\hline-10 & 5.549 & 5.495 & 5.345 & 1.512 & 1.508 & 1.497 \\
\hline Dose Factor & Maximum & Minimum & Ratio & Maximum & Minimum & Ratio \\
\hline & \multicolumn{6}{|c|}{ 10-cm Range } \\
\hline ICRP-21 & 5.665 & 4.545 & 1.247 & 1.397 & 1.271 & 1.099 \\
\hline $\mathrm{Hp}(10)$ & 6.989 & 5.577 & 1.253 & 1.722 & 1.562 & 1.102 \\
\hline $\mathrm{H}^{*}(10)$ & 6.702 & 5.345 & 1.254 & 1.651 & 1.497 & 1.102 \\
\hline \multicolumn{7}{|c|}{ 5-cm Range } \\
\hline ICRP-21 & 5.567 & 5.022 & 1.109 & 1.378 & 1.317 & 1.046 \\
\hline$H p(10)$ & 6.858 & 6.170 & 1.111 & 1.696 & 1.619 & 1.048 \\
\hline $\mathrm{H}^{*}(10)$ & 6.575 & 5.914 & 1.112 & 1.626 & 1.552 & 1.048 \\
\hline
\end{tabular}




\section{CALCULATION SHEET}

\begin{tabular}{|c|c|c|c|}
\hline PROJECT NO.: & $\begin{array}{l}\text { CALC NO.: } \\
\text { PNNL-19273 }\end{array}$ & REVISION NO.: $\underline{0}$ & SHEET NO.: $\underline{45 / 246}$ \\
\hline \multicolumn{4}{|c|}{ PNNL-19273 } \\
\hline PREPARED BY: R.J. TRAUB & DATE: $\underline{02 / 8 / 10}$ & REVIEWED BY: R.J.MCCONN & DATE: $\underline{03 / 21 / 10}$ \\
\hline
\end{tabular}

Table 24. Neutron dose rate at the surface of a $10-\mathrm{cm} \times 10-\mathrm{cm}$ rectangle $50 \mathrm{~cm}$ and $100 \mathrm{~cm}$ from the center of the reference sphere. The ${ }^{252} \mathrm{Cf}$ source is in the $\mathrm{D}_{2} \mathrm{O}$ moderating sphere. The activity is in the void between the frits. The reference date is March 3, 2003. The emission rate is 4.85e $9 \mathrm{n}_{\mathrm{s}} \mathrm{s}^{-1}$.

\begin{tabular}{|c|c|c|c|c|c|c|}
\hline \multirow[b]{3}{*}{$\begin{array}{l}Z \text { position } \\
(\mathrm{cm})\end{array}$} & \multicolumn{3}{|c|}{$50 \mathrm{~cm}$ from center of reference sphere } & \multirow{2}{*}{\multicolumn{3}{|c|}{$\begin{array}{c}100 \mathrm{~cm} \text { from center of reference } \\
\text { sphere } \\
\text { horizontal position }(\mathrm{cm})\end{array}$}} \\
\hline & \multicolumn{3}{|c|}{ horizontal position $(\mathrm{cm})$} & & & \\
\hline & 0 & 5 & 10 & 0 & 5 & 10 \\
\hline & \multicolumn{6}{|c|}{$\begin{array}{l}\text { ICRP-21 Appendix } 6 \\
\left(\text { rem. } h^{-2}\right)\end{array}$} \\
\hline 10 & 5.521 & 5.469 & 5.310 & 1.374 & 1.370 & 1.361 \\
\hline 5 & 5.506 & 5.449 & 5.292 & 1.364 & 1.360 & 1.351 \\
\hline 0 & 5.383 & 5.331 & 5.178 & 1.348 & 1.344 & 1.334 \\
\hline-5 & 5.169 & 5.118 & 4.974 & 1.325 & 1.322 & 1.312 \\
\hline-10 & 4.878 & 4.832 & 4.702 & 1.297 & 1.294 & 1.285 \\
\hline & \multicolumn{6}{|c|}{$\begin{array}{c}\text { Personal Dose Equivalent Hp(10) } \\
\left(\mathrm{rem} \cdot \mathrm{h}^{-2}\right)\end{array}$} \\
\hline 10 & 6.804 & 6.740 & 6.544 & 1.692 & 1.687 & 1.676 \\
\hline 5 & 6.779 & 6.709 & 6.515 & 1.679 & 1.674 & 1.662 \\
\hline 0 & 6.622 & 6.558 & 6.370 & 1.658 & 1.653 & 1.641 \\
\hline-5 & 6.353 & 6.290 & 6.114 & 1.630 & 1.626 & 1.614 \\
\hline-10 & 5.991 & 5.935 & 5.774 & 1.594 & 1.591 & 1.579 \\
\hline & \multicolumn{6}{|c|}{$\begin{array}{c}\text { Ambient Dose Equivalent } \mathrm{H}^{*}(10) \\
\left(\mathrm{rem} \cdot \mathrm{h}^{-2}\right)\end{array}$} \\
\hline 10 & 6.524 & 6.462 & 6.274 & 1.622 & 1.618 & 1.606 \\
\hline 5 & 6.499 & 6.432 & 6.246 & 1.610 & 1.605 & 1.594 \\
\hline 0 & 6.348 & 6.287 & 6.106 & 1.590 & 1.585 & 1.574 \\
\hline-5 & 6.090 & 6.030 & 5.860 & 1.562 & 1.558 & 1.547 \\
\hline-10 & 5.743 & 5.688 & 5.535 & 1.528 & 1.525 & 1.514 \\
\hline Dose Factor & Maximum & "Minimum & Ratio & Maximum & Minimum & Ratio \\
\hline & \multicolumn{6}{|c|}{ 10-cm Range } \\
\hline ICRP-21 & 5.521 & 4.702 & 1.174 & 1.374 & 1.285 & 1.070 \\
\hline $\mathrm{Hp}(10)$ & 6.804 & 5.774 & 1.178 & 1.692 & 1.579 & 1.072 \\
\hline $\mathrm{H}^{*}(10)$ & 6.524 & 5.535 & 1.179 & 1.622 & 1.514 & 1.072 \\
\hline \multicolumn{7}{|c|}{ 5-cm Range } \\
\hline ICRP-21 & 5.506 & 5.118 & 1.076 & 1.364 & 1.322 & 1.032 \\
\hline $\mathrm{Hp}(10)$ & 6.779 & 6.290 & 1.078 & 1.679 & 1.626 & 1.033 \\
\hline $\mathrm{H}^{*}(10)$ & 6.499 & 6.030 & 1.078 & 1.610 & 1.558 & 1.033 \\
\hline
\end{tabular}




\section{CALCULATION SHEET}

\begin{tabular}{|c|c|c|c|}
\hline PROJECT No.: & $\begin{array}{l}\text { CALC NO.: } \\
\text { PNNL-19273 }\end{array}$ & REVISION NO.: $\underline{0}$ & SHEET NO.: $\underline{46 / 246}$ \\
\hline SUBJECT: CALCULATIONS & $00{ }^{252} \mathrm{CF}$ NEUTRC & CE IN 318LOW SCATTER ROOM & \\
\hline PREPARED BY: R.J. TRAUB & DATE: $\underline{02 / 8 / 10}$ & REVIEWED BY: R.J.MCCONN & DATE: $\underline{03 / 21 / 10}$ \\
\hline
\end{tabular}

Table 25. Neutron dose rate at the surface of a $10-\mathrm{cm} \times 10-\mathrm{cm}$ rectangle $50 \mathrm{~cm}$ and $100 \mathrm{~cm}$ from the center of the reference sphere. The ${ }^{252} \mathrm{Cf}$ source is in the $\mathrm{D}_{2} \mathrm{O}$ moderating sphere. The activity is in the lower frit. The reference date is March 3, 2003. The emission rate is $4.85 \mathrm{e} 9 \mathrm{n} . \mathrm{s}^{-1}$.

\begin{tabular}{|c|c|c|c|c|c|c|}
\hline \multirow[b]{3}{*}{$\begin{array}{l}Z \text { position } \\
(\mathrm{cm})\end{array}$} & \multirow{2}{*}{\multicolumn{3}{|c|}{$\begin{array}{l}50 \mathrm{~cm} \text { from center of the reference } \\
\text { sphere } \\
\text { horizontal position }(\mathrm{cm})\end{array}$}} & \multirow{2}{*}{\multicolumn{3}{|c|}{$\begin{array}{l}100 \mathrm{~cm} \text { from center of the reference } \\
\text { sphere } \\
\text { horizontal position }(\mathrm{cm})\end{array}$}} \\
\hline & & & & & & \\
\hline & 0 & 5 & 10 & 0 & 5 & 10 \\
\hline & \multicolumn{6}{|c|}{$\begin{array}{l}\text { ICRP-21 Appendix } 6 \\
\left(\text { rem. } h^{-2}\right)\end{array}$} \\
\hline 10 & 5.378 & 5.325 & 5.172 & 1.352 & 1.349 & 1.339 \\
\hline 5 & 5.435 & 5.381 & 5.223 & 1.351 & 1.347 & 1.337 \\
\hline 0 & 5.390 & 5.334 & 5.178 & 1.342 & 1.339 & 1.329 \\
\hline-5 & 5.243 & 5.188 & 5.038 & 1.328 & 1.325 & 1.315 \\
\hline-10 & 5.009 & 4.959 & 4.819 & 1.308 & 1.305 & 1.295 \\
\hline & \multicolumn{6}{|c|}{$\begin{array}{c}\text { Personal Dose Equivalent } \mathrm{Hp}(10) \\
\left(\mathrm{rem}^{\left.-\mathrm{h}^{-2}\right)}\right.\end{array}$} \\
\hline 10 & 6.621 & 6.556 & 6.368 & 1.664 & 1.660 & 1.647 \\
\hline 5 & 6.687 & 6.620 & 6.426 & 1.662 & 1.658 & 1.645 \\
\hline 0 & 6.627 & 6.558 & 6.367 & 1.651 & 1.647 & 1.635 \\
\hline-5 & 6.443 & 6.377 & 6.192 & 1.633 & 1.629 & 1.617 \\
\hline-10 & 6.154 & 6.092 & 5.920 & 1.608 & 1.604 & 1.592 \\
\hline & \multicolumn{6}{|c|}{$\begin{array}{c}\text { Ambient Dose Equivalent } \mathrm{H}^{*}(10) \\
\left(\mathrm{rem} \cdot \mathrm{h}^{-2}\right)\end{array}$} \\
\hline 10 & 6.347 & 6.285 & 6.105 & 1.595 & 1.592 & 1.579 \\
\hline 5 & 6.410 & 6.346 & 6.160 & 1.593 & 1.589 & 1.577 \\
\hline 0 & 6.353 & 6.287 & 6.103 & 1.583 & 1.579 & 1.567 \\
\hline-5 & 6.177 & 6.112 & 5.936 & 1.566 & 1.562 & 1.550 \\
\hline-10 & 5.899 & 5.839 & 5.675 & 1.541 & 1.537 & 1.526 \\
\hline Dose Factor & Maximum & Minimum & Ratio & Maximum & Minimum & Ratio \\
\hline & \multicolumn{6}{|c|}{$10-\mathrm{cm}$ Range } \\
\hline ICRP-21 & 5.435 & 4.819 & 1.128 & 1.352 & 1.295 & 1.044 \\
\hline $\operatorname{Hp}(10)$ & 6.687 & 5.920 & 1.130 & 1.664 & 1.592 & 1.045 \\
\hline $\mathrm{H}^{*}(10)$ & 6.410 & 5.675 & 1.130 & 1.595 & 1.526 & 1.045 \\
\hline \multicolumn{7}{|c|}{ 5-cm Range } \\
\hline ICRP-21 & 5.435 & 5.188 & 1.048 & 1.351 & 1.325 & 1.019 \\
\hline $\mathrm{Hp}(10)$ & 6.687 & 6.377 & 1.049 & 1.662 & 1.629 & 1.020 \\
\hline $\mathrm{H}^{*}(10)$ & 6.410 & 6.112 & 1.049 & 1.593 & 1.562 & 1.020 \\
\hline
\end{tabular}




\section{CALCULATION SHEET}

\begin{tabular}{|c|c|c|c|}
\hline PROJECT No.: & $\begin{array}{l}\text { CALC NO.: } \\
\text { PNNL-19273 }\end{array}$ & REVISION NO.: $\underline{0}$ & SHEET NO.: $\underline{47 / 246}$ \\
\hline SUBJECT: CALCULATIONS T & $00{ }^{252} \mathrm{CF}$ NEUTRC & EE IN 318LOW SCATTER ROOM & \\
\hline PREPARED BY: R.J. TRAUB & DATE: $\underline{02 / 8 / 10}$ & REVIEWED BY: $\underline{\text { R.J.MCCONN }}$ & DATE: $\underline{03 / 21 / 10}$ \\
\hline
\end{tabular}

Table 26. Neutron dose equivalent rate at the surface of a $10-\mathrm{cm} \times 10-\mathrm{cm}$ rectangle $50 \mathrm{~cm}$ and $100 \mathrm{~cm}$ from the center of the reference sphere. The ${ }^{252} \mathrm{Cf}$ source is in the pneumatic transfer tube. The activity is in the upper frit. The reference date is March 3, 2003. The emission rate is $4.85 \mathrm{e} 9 \mathrm{n} \cdot \mathrm{s}^{-1}$.

\begin{tabular}{|c|c|c|c|c|c|c|}
\hline \multirow[b]{3}{*}{$\begin{array}{l}\text { Z position } \\
(\mathrm{cm})\end{array}$} & \multirow{2}{*}{\multicolumn{3}{|c|}{$\begin{array}{l}50 \mathrm{~cm} \text { from center of the reference } \\
\text { sphere } \\
\text { horizontal position }(\mathrm{cm})\end{array}$}} & \multirow{2}{*}{\multicolumn{3}{|c|}{$\begin{array}{l}100 \mathrm{~cm} \text { from center of the reference } \\
\text { sphere } \\
\text { horizontal position }(\mathrm{cm})\end{array}$}} \\
\hline & & & & & & \\
\hline & 0 & 5 & 10 & 0 & 5 & 10 \\
\hline & \multicolumn{6}{|c|}{$\begin{array}{l}\text { ICRP-21 Appendix } 6 \\
\left(\mathrm{rem}^{-2} \mathrm{~h}^{-2}\right)\end{array}$} \\
\hline 10 & 19.010 & 18.827 & 18.298 & 4.842 & 4.830 & 4.795 \\
\hline 5 & 19.424 & 19.233 & 18.682 & 4.870 & 4.858 & 4.822 \\
\hline 0 & 19.455 & 19.263 & 18.712 & 4.875 & 4.863 & 4.827 \\
\hline-5 & 19.095 & 18.911 & 18.380 & 4.858 & 4.846 & 4.810 \\
\hline-10 & 18.333 & 18.164 & 17.675 & 4.814 & 4.802 & 4.767 \\
\hline & \multicolumn{6}{|c|}{$\begin{array}{c}\text { Personal Dose Equivalent } \mathrm{Hp}(10) \\
\left(\mathrm{rem}^{-2} \mathrm{~h}^{-2}\right)\end{array}$} \\
\hline 10 & 23.064 & 22.842 & 22.200 & 5.874 & 5.860 & 5.817 \\
\hline 5 & 23.564 & 23.332 & 22.664 & 5.908 & 5.893 & 5.850 \\
\hline 0 & 23.602 & 23.370 & 22.701 & 5.914 & 5.899 & 5.856 \\
\hline-5 & 23.166 & 22.943 & 22.299 & 5.893 & 5.879 & 5.835 \\
\hline-10 & 22.241 & 22.035 & 21.442 & 5.841 & 5.826 & 5.784 \\
\hline & \multicolumn{6}{|c|}{$\begin{array}{c}\text { Ambient Dose Equivalent } \mathrm{H}^{*}(10) \\
\left(\mathrm{rem} \cdot \mathrm{h}^{-2}\right)\end{array}$} \\
\hline 10 & 22.193 & 21.979 & 21.361 & 5.653 & 5.639 & 5.597 \\
\hline 5 & 22.674 & 22.451 & 21.808 & 5.685 & 5.671 & 5.629 \\
\hline 0 & 22.711 & 22.487 & 21.844 & 5.691 & 5.677 & 5.635 \\
\hline-5 & 22.292 & 22.077 & 21.457 & 5.671 & 5.657 & 5.615 \\
\hline-10 & 21.401 & 21.203 & 20.632 & 5.620 & 5.606 & 5.565 \\
\hline Dose Factor & Maximum & "Minimum & Ratio & Maximum & "Minimum & Ratio \\
\hline & \multicolumn{6}{|c|}{ 10-cm Range } \\
\hline ICRP-21 & 19.455 & 17.675 & 1.101 & 4.875 & 4.767 & 1.023 \\
\hline $\mathrm{Hp}(10)$ & 23.602 & 21.442 & 1.101 & 5.914 & 5.784 & 1.022 \\
\hline $\mathrm{H}^{*}(10)$ & 22.711 & 20.632 & 1.101 & 5.691 & 5.565 & 1.022 \\
\hline \multicolumn{7}{|c|}{ 5-cm Range } \\
\hline ICRP-21 & 19.455 & 18.911 & 1.029 & 4.875 & 4.846 & 1.006 \\
\hline$H p(10)$ & 23.602 & 22.943 & 1.029 & 5.914 & 5.879 & 1.006 \\
\hline $\mathrm{H}^{*}(10)$ & 22.711 & 22.077 & 1.029 & 5.691 & 5.657 & 1.006 \\
\hline
\end{tabular}




\section{CALCULATION SHEET}

\begin{tabular}{|c|c|c|c|}
\hline PROJECT No.: & $\begin{array}{l}\text { CALC NO.: } \\
\text { PNNL-19273 }\end{array}$ & REVISION NO.: $\underline{0}$ & SHEET NO.: $\underline{48 / 246}$ \\
\hline SUBJECT: CALCULATIONS T & $00{ }^{252} \mathrm{CF}$ NEUTRC & EE IN 318LOW SCATTER ROOM & \\
\hline PREPARED BY: R.J. TRAUB & DATE: $\underline{02 / 8 / 10}$ & REVIEWED BY: $\underline{\text { R.J.MCCONN }}$ & DATE: $\underline{03 / 21 / 10}$ \\
\hline
\end{tabular}

Table 27. Neutron dose equivalent rate at the surface of a $10-\mathrm{cm} \times 10-\mathrm{cm}$ rectangle $50 \mathrm{~cm}$ and $100 \mathrm{~cm}$ from the center of the reference sphere. The ${ }^{252} \mathrm{Cf}$ source is in the pneumatic transfer tube. The activity is in the void between the frits. The reference date is March 3, 2003. The emission rate is $4.85 \mathrm{e} 9 \mathrm{n} \cdot \mathrm{s}^{-1}$.

\begin{tabular}{|c|c|c|c|c|c|c|}
\hline \multirow[b]{3}{*}{$\begin{array}{l}\text { Z position } \\
(\mathrm{cm})\end{array}$} & \multirow{2}{*}{\multicolumn{3}{|c|}{$\begin{array}{l}50 \mathrm{~cm} \text { from center of the reference } \\
\text { sphere } \\
\text { horizontal position }(\mathrm{cm})\end{array}$}} & \multirow{2}{*}{\multicolumn{3}{|c|}{$\begin{array}{l}100 \mathrm{~cm} \text { from center of the reference } \\
\text { sphere } \\
\text { horizontal position }(\mathrm{cm})\end{array}$}} \\
\hline & & & & & & \\
\hline & 0 & 5 & 10 & 0 & 5 & 10 \\
\hline & \multicolumn{6}{|c|}{$\begin{array}{l}\text { ICRP-21 Appendix } 6 \\
\left(\text { rem. } \mathrm{h}^{-2}\right)\end{array}$} \\
\hline 10 & 19.022 & 18.842 & 18.315 & 4.862 & 4.850 & 4.815 \\
\hline 5 & 19.481 & 19.293 & 18.741 & 4.891 & 4.879 & 4.843 \\
\hline 0 & 19.530 & 19.341 & 18.787 & 4.893 & 4.882 & 4.846 \\
\hline-5 & 19.162 & 18.981 & 18.447 & 4.869 & 4.858 & 4.823 \\
\hline-10 & 18.423 & 18.255 & 17.762 & 4.820 & 4.809 & 4.774 \\
\hline & \multicolumn{6}{|c|}{$\begin{array}{c}\text { Personal Dose Equivalent Hp(10) } \\
\left(\text { rem. } \mathrm{h}^{-2}\right)\end{array}$} \\
\hline 10 & 23.063 & 22.844 & 22.205 & 5.895 & 5.881 & 5.838 \\
\hline 5 & 23.622 & 23.393 & 22.724 & 5.930 & 5.916 & 5.873 \\
\hline 0 & 23.681 & 23.452 & 22.780 & 5.934 & 5.919 & 5.876 \\
\hline-5 & 23.234 & 23.014 & 22.367 & 5.905 & 5.890 & 5.848 \\
\hline-10 & 22.335 & 22.131 & 21.533 & 5.845 & 5.831 & 5.789 \\
\hline & \multicolumn{6}{|c|}{$\begin{array}{c}\text { Ambient Dose Equivalent } \mathrm{H}^{*}(10) \\
\left(\mathrm{rem} \cdot \mathrm{h}^{-2}\right)\end{array}$} \\
\hline 10 & 22.192 & 21.981 & 21.367 & 5.672 & 5.658 & 5.617 \\
\hline 5 & 22.730 & 22.509 & 21.866 & 5.706 & 5.692 & 5.651 \\
\hline 0 & 22.787 & 22.566 & 21.919 & 5.709 & 5.696 & 5.654 \\
\hline-5 & 22.357 & 22.145 & 21.522 & 5.681 & 5.668 & 5.627 \\
\hline-10 & 21.491 & 21.295 & 20.719 & 5.624 & 5.611 & 5.570 \\
\hline Dose Factor & Maximum & Minimum & Ratio & Maximum & Minimum & Ratio \\
\hline & \multicolumn{6}{|c|}{ 10-cm Range } \\
\hline ICRP-21 & 19.530 & 17.762 & 1.100 & 4.893 & 4.774 & 1.025 \\
\hline $\mathrm{Hp}(10)$ & 23.681 & 21.533 & 1.100 & 5.934 & 5.789 & 1.025 \\
\hline $\mathrm{H}^{*}(10)$ & 22.787 & 20.719 & 1.100 & 5.709 & 5.570 & 1.025 \\
\hline \multicolumn{7}{|c|}{ 5-cm Range } \\
\hline ICRP-21 & 19.530 & 18.981 & 1.029 & 4.893 & 4.858 & 1.007 \\
\hline$H p(10)$ & 23.681 & 23.014 & 1.029 & 5.934 & 5.890 & 1.007 \\
\hline$H^{*}(10)$ & 22.787 & 22.145 & 1.029 & 5.709 & 5.668 & 1.007 \\
\hline
\end{tabular}




\section{CALCULATION SHEET}

\begin{tabular}{|c|c|c|c|}
\hline PROJECT No.: & $\begin{array}{l}\text { CALC NO.: } \\
\text { PNNL-19273 }\end{array}$ & REVISION NO.: $\underline{0}$ & SHEET NO.: $\underline{49 / 246}$ \\
\hline SUBJECT: CALCULATIONS T & $00{ }^{252} \mathrm{CF}$ NEUTRC & EE IN 318LOW SCATTER ROOM & \\
\hline PREPARED BY: R.J. TRAUB & DATE: $\underline{02 / 8 / 10}$ & REVIEWED BY: $\underline{\text { R.J.MCCONN }}$ & DATE: $\underline{03 / 21 / 10}$ \\
\hline
\end{tabular}

Table 28. Neutron dose equivalent rate at the surface of a $10-\mathrm{cm} \times 10-\mathrm{cm}$ rectangle $50 \mathrm{~cm}$ and $100 \mathrm{~cm}$ from the center of the reference sphere. The ${ }^{252} \mathrm{Cf}$ source is in the pneumatic transfer tube. The activity is in the lower frit. The reference date is March 3, 2003. The emission rate is $4.85 \mathrm{e} 9 \mathrm{n} . \mathrm{s}^{-1}$.

\begin{tabular}{|c|c|c|c|c|c|c|}
\hline \multirow[b]{3}{*}{$\begin{array}{l}\text { Z position } \\
(\mathrm{cm})\end{array}$} & \multirow{2}{*}{\multicolumn{3}{|c|}{$\begin{array}{l}50 \mathrm{~cm} \text { from center of the reference } \\
\text { sphere } \\
\text { horizontal position }(\mathrm{cm})\end{array}$}} & \multirow{2}{*}{\multicolumn{3}{|c|}{$\begin{array}{l}100 \mathrm{~cm} \text { from center of the reference } \\
\text { sphere } \\
\text { horizontal position }(\mathrm{cm})\end{array}$}} \\
\hline & & & & & & \\
\hline & 0 & 5 & 10 & 0 & 5 & 10 \\
\hline & \multicolumn{6}{|c|}{$\begin{array}{l}\text { ICRP-21 Appendix } 6 \\
\left(\text { rem. } \mathrm{h}^{-2}\right)\end{array}$} \\
\hline 10 & 18.955 & 18.775 & 18.253 & 4.852 & 4.840 & 4.805 \\
\hline 5 & 19.416 & 19.227 & 18.678 & 4.875 & 4.863 & 4.827 \\
\hline 0 & 19.472 & 19.281 & 18.729 & 4.875 & 4.863 & 4.827 \\
\hline-5 & 19.153 & 18.968 & 18.432 & 4.852 & 4.841 & 4.805 \\
\hline-10 & 18.464 & 18.292 & 17.793 & 4.804 & 4.793 & 4.758 \\
\hline & \multicolumn{6}{|c|}{$\begin{array}{c}\text { Personal Dose Equivalent Hp(10) } \\
\left(\text { rem. } \mathrm{h}^{-2}\right)\end{array}$} \\
\hline 10 & 22.997 & 22.778 & 22.144 & 5.887 & 5.872 & 5.830 \\
\hline 5 & 23.556 & 23.327 & 22.660 & 5.915 & 5.900 & 5.856 \\
\hline 0 & 23.623 & 23.391 & 22.721 & 5.914 & 5.899 & 5.856 \\
\hline-5 & 23.238 & 23.013 & 22.363 & 5.887 & 5.873 & 5.829 \\
\hline-10 & 22.401 & 22.193 & 21.587 & 5.829 & 5.815 & 5.773 \\
\hline & \multicolumn{6}{|c|}{$\begin{array}{c}\text { Ambient Dose Equivalent } \mathrm{H}^{*}(10) \\
\left(\mathrm{rem} \cdot \mathrm{h}^{-2}\right)\end{array}$} \\
\hline 10 & 22.129 & 21.918 & 21.308 & 5.665 & 5.651 & 5.609 \\
\hline 5 & 22.666 & 22.446 & 21.804 & 5.691 & 5.677 & 5.635 \\
\hline 0 & 22.731 & 22.508 & 21.863 & 5.690 & 5.677 & 5.634 \\
\hline-5 & 22.360 & 22.144 & 21.519 & 5.665 & 5.651 & 5.609 \\
\hline-10 & 21.555 & 21.355 & 20.772 & 5.609 & 5.596 & 5.555 \\
\hline Dose Factor & Maximum & Minimum & Ratio & Maximum & Minimum & Ratio \\
\hline & \multicolumn{6}{|c|}{ 10-cm Range } \\
\hline ICRP-21 & 19.472 & 17.793 & 1.094 & 4.875 & 4.758 & 1.025 \\
\hline$H p(10)$ & 23.623 & 21.587 & 1.094 & 5.915 & 5.773 & 1.025 \\
\hline $\mathrm{H}^{*}(10)$ & 22.731 & 20.772 & 1.094 & 5.691 & 5.555 & 1.025 \\
\hline \multicolumn{7}{|c|}{ 5-cm Range } \\
\hline ICRP-21 & 19.472 & 18.968 & 1.027 & 4.875 & 4.841 & 1.007 \\
\hline$H p(10)$ & 23.623 & 23.013 & 1.027 & 5.915 & 5.873 & 1.007 \\
\hline $\mathrm{H}^{*}(10)$ & 22.731 & 22.144 & 1.027 & 5.691 & 5.651 & 1.007 \\
\hline
\end{tabular}




\section{CALCULATION SHEET}

\begin{tabular}{|c|c|c|c|}
\hline \multirow{2}{*}{\multicolumn{4}{|c|}{$\underline{\text { PNNL-19273 }}$}} \\
\hline & & & \\
\hline PREPARED BY: R.J. TRAUB & DATE: $\underline{02 / 8 / 10}$ & REVIEWED BY: R.J.MCCONN & DATE: $\underline{03 / 21 / 10}$ \\
\hline
\end{tabular}

Table 29. Neutron fluence at the surface of a $10-\mathrm{cm} \times 10-\mathrm{cm}$ rectangle $50 \mathrm{~cm}$ and $100 \mathrm{~cm}$ from the center of the reference sphere. The dose rectangle has been elevated $1 \mathrm{~cm}$. relative to the equator of the reference sphere. The activity is on the upper frit. The reference date is March 3, 2003. The emission rate is $4.85 \mathrm{e} 9 \mathrm{n} \cdot \mathrm{s}^{-1}$.

\begin{tabular}{|c|c|c|}
\hline & $50 \mathrm{~cm}$ from center of reference sphere & $\begin{array}{l}100 \mathrm{~cm} \text { from center of reference } \\
\text { sphere }\end{array}$ \\
\hline & horizontal position $(\mathrm{cm})$ & horizontal position $(\mathrm{cm})$ \\
\hline $\begin{array}{l}\text { Z position } \\
(\mathrm{cm})\end{array}$ & $\begin{array}{lll}0 & 5 & 10\end{array}$ & $\begin{array}{lll}0 & 5 & 10\end{array}$ \\
\hline
\end{tabular}
$(\mathrm{cm})$

\begin{tabular}{|c|c|c|c|c|c|c|}
\hline & \multicolumn{6}{|c|}{$\begin{array}{l}\text { Pneumatic Transfer Tube Assembly (Unmoderated Spectra) } \\
\qquad\left(\mathrm{cm}^{-2} \cdot \mathrm{s}^{-1}\right)\end{array}$} \\
\hline 11 & $1.62 \mathrm{E}+05$ & $1.61 \mathrm{E}+05$ & $1.56 \mathrm{E}+05$ & $4.15 \mathrm{E}+04$ & $4.14 \mathrm{E}+04$ & $4.11 \mathrm{E}+04$ \\
\hline 6 & $1.66 \mathrm{E}+05$ & $1.65 \mathrm{E}+05$ & $1.60 \mathrm{E}+05$ & $4.18 \mathrm{E}+04$ & $4.17 \mathrm{E}+04$ & $4.14 \mathrm{E}+04$ \\
\hline 1 & $1.67 \mathrm{E}+05$ & $1.66 \mathrm{E}+05$ & $1.61 \mathrm{E}+05$ & $4.19 \mathrm{E}+04$ & $4.18 \mathrm{E}+04$ & $4.14 \mathrm{E}+04$ \\
\hline-4 & $1.65 \mathrm{E}+05$ & $1.63 \mathrm{E}+05$ & $1.59 \mathrm{E}+05$ & $4.18 \mathrm{E}+04$ & $4.17 \mathrm{E}+04$ & $4.13 \mathrm{E}+04$ \\
\hline-9 & $1.59 \mathrm{E}+05$ & $1.57 \mathrm{E}+05$ & $1.53 \mathrm{E}+05$ & $4.14 \mathrm{E}+04$ & $4.13 \mathrm{E}+04$ & $4.10 \mathrm{E}+04$ \\
\hline Range & Maximum & Minimum & Ratio & Maximum & Minimum & Ratio \\
\hline \multicolumn{7}{|c|}{ Pneumatic Transfer Tube Assembly (Unmoderated Spectra) } \\
\hline 10 & $1.67 \mathrm{E}+05$ & $1.53 \mathrm{E}+05$ & $1.09 \mathrm{E}+00$ & $4.19 \mathrm{E}+04$ & $4.10 \mathrm{E}+04$ & $1.02 \mathrm{E}+00$ \\
\hline 5 & $1.67 \mathrm{E}+05$ & $1.63 \mathrm{E}+05$ & $1.02 \mathrm{E}+00$ & $4.19 \mathrm{E}+04$ & $4.17 \mathrm{E}+04$ & $1.00 \mathrm{E}+00$ \\
\hline
\end{tabular}




\section{CALCULATION SHEET}

\begin{tabular}{|c|c|c|c|}
\hline ProjeCt No.: & $\begin{array}{l}\text { CALC NO.: } \\
\text { PNNL-19273 }\end{array}$ & REVISION NO.: $\underline{0}$ & SHEET NO.: $\underline{51 / 246}$ \\
\hline \multicolumn{4}{|c|}{ E IN 318LOW SCATTER ROOM } \\
\hline PREPARED BY: R.J. TRAUB & DATE: $\underline{02 / 8 / 10}$ & REVIEWED BY: R.J.MCCONN & DATE: $03 / 21 / 10$ \\
\hline
\end{tabular}

Table 30. Neutron dose factors at the surface of a $10-\mathrm{cm} \times 10-\mathrm{cm}$ rectangle $50 \mathrm{~cm}$ and $100 \mathrm{~cm}$ from the center of the reference sphere. The ${ }^{252} \mathrm{Cf}$ source is in the pneumatic transfer tube assembly. The dose rectangle has been elevated $1 \mathrm{~cm}$. relative to the equator of the reference sphere. The activity is on the upper frit. The reference date is March 3, 2003. The emission rate is $4.85 \mathrm{e} 9 \mathrm{n} \cdot \mathrm{s}^{-1}$.

\begin{tabular}{|c|c|c|c|c|c|c|}
\hline \multirow[b]{3}{*}{$\begin{array}{l}Z \text { position } \\
(\mathrm{cm})\end{array}$} & \multirow{2}{*}{\multicolumn{3}{|c|}{$\begin{array}{l}50 \mathrm{~cm} \text { from center of the reference } \\
\text { sphere } \\
\text { horizontal position }(\mathrm{cm})\end{array}$}} & \multirow{2}{*}{\multicolumn{3}{|c|}{$\begin{array}{l}100 \mathrm{~cm} \text { from center of the reference } \\
\text { sphere } \\
\text { horizontal position }(\mathrm{cm})\end{array}$}} \\
\hline & & & & & & \\
\hline & 0 & 5 & 10 & 0 & 5 & 10 \\
\hline & \multicolumn{6}{|c|}{$\begin{array}{l}\text { ICRP-21 Appendix } 6 \\
\left(\mathrm{pSv} . \mathrm{cm}^{2}\right)\end{array}$} \\
\hline 11 & 323.5 & 323.5 & 323.5 & 323.5 & 323.5 & 323.5 \\
\hline 6 & 323.4 & 323.4 & 323.4 & 323.5 & 323.5 & 323.5 \\
\hline 1 & 323.4 & 323.4 & 323.4 & 323.5 & 323.5 & 323.5 \\
\hline-4 & 323.4 & 323.4 & 323.4 & 323.4 & 323.4 & 323.4 \\
\hline-9 & 323.5 & 323.5 & 323.5 & 323.4 & 323.4 & 323.4 \\
\hline & \multicolumn{6}{|c|}{$\begin{array}{c}\text { Personal Dose Equivalent Hp(10) } \\
\left(\mathrm{pSv} . \mathrm{cm}^{2}\right)\end{array}$} \\
\hline 11 & 392.5 & 392.4 & 392.4 & 392.4 & 392.4 & 392.4 \\
\hline 6 & 392.4 & 392.4 & 392.4 & 392.4 & 392.4 & 392.4 \\
\hline 1 & 392.4 & 392.4 & 392.4 & 392.4 & 392.4 & 392.4 \\
\hline-4 & 392.4 & 392.4 & 392.4 & 392.4 & 392.4 & 392.4 \\
\hline-9 & 392.4 & 392.4 & 392.4 & 392.4 & 392.4 & 392.4 \\
\hline & \multicolumn{6}{|c|}{$\begin{array}{c}\text { Ambient Dose Equivalent } \mathrm{H}^{*}(10) \\
\left(\mathrm{pSv} \cdot \mathrm{cm}^{2}\right)\end{array}$} \\
\hline 11 & 377.6 & 377.6 & 377.6 & 377.6 & 377.6 & 377.6 \\
\hline 6 & 377.6 & 377.6 & 377.6 & 377.6 & 377.6 & 377.6 \\
\hline 1 & 377.6 & 377.6 & 377.6 & 377.6 & 377.6 & 377.6 \\
\hline-4 & 377.6 & 377.6 & 377.6 & 377.6 & 377.6 & 377.6 \\
\hline-9 & 377.6 & 377.6 & 377.6 & 377.6 & 377.6 & 377.6 \\
\hline Dose Factor & Maximum & Minimum & Ratio & Maximum & Minimum & Ratio \\
\hline & \multicolumn{6}{|c|}{ 10-cm Range } \\
\hline ICRP-21 & 323.5 & 323.4 & 1.000 & 323.5 & 323.4 & 1.000 \\
\hline $\mathrm{Hp}(10)$ & 392.5 & 392.4 & 1.000 & 392.4 & 392.4 & 1.000 \\
\hline $\mathrm{H}^{*}(10)$ & 377.6 & 377.6 & 1.000 & 377.6 & 377.6 & 1.000 \\
\hline & \multicolumn{6}{|c|}{ 5-cm Range } \\
\hline ICRP-21 & 323.4 & 323.4 & 1.000 & 323.5 & 323.4 & 1.000 \\
\hline Hp(10) & 392.4 & 392.4 & 1.000 & 392.4 & 392.4 & 1.000 \\
\hline $\mathrm{H}^{*}(10)$ & 377.6 & 377.6 & 1.000 & 377.6 & 377.6 & 1.000 \\
\hline
\end{tabular}




\section{CALCULATION SHEET}

\begin{tabular}{|c|c|c|c|}
\hline PROJECT No.: & $\begin{array}{l}\text { CALC NO.: } \\
\text { PNNL-19273 } \\
\end{array}$ & REVISION NO.: $\underline{0}$ & SHEET NO.: $\underline{52 / 246}$ \\
\hline SuBJECT: CALCULATIONS & $00{ }^{252} \mathrm{CF}$ NEUTR & CE IN 318LOW SCATTER ROOM & \\
\hline PREPARED BY: R.J. TRAUB & DATE: $\underline{02 / 8 / 10}$ & REVIEWED BY: R.J.MCCONN & DATE: $\underline{03 / 21 / 10}$ \\
\hline
\end{tabular}

Table 31. Neutron dose rates at the surface of a $10-\mathrm{cm} \times 10-\mathrm{cm}$ rectangle $50 \mathrm{~cm}$ and $100 \mathrm{~cm}$ from the center of the reference sphere. The ${ }^{252} \mathrm{Cf}$ source is in the pneumatic transfer tube assembly. The activity is on the upper frit. The dose rectangle has been elevated $1 \mathrm{~cm}$. relative to the equator of the reference sphere. The reference date is March 3, 2003. The emission rate is $4.85 \mathrm{e} 9 \mathrm{n} . \mathrm{s}^{-1}$.

\begin{tabular}{|c|c|c|c|c|c|c|}
\hline \multirow{3}{*}{$\begin{array}{l}Z \text { position } \\
(\mathrm{cm})\end{array}$} & \multicolumn{3}{|c|}{$\begin{array}{l}50 \mathrm{~cm} \text { from center of the reference } \\
\text { sphere } \\
\text { horizontal position }(\mathrm{cm})\end{array}$} & \multicolumn{3}{|c|}{$\begin{array}{l}100 \mathrm{~cm} \text { from center of the reference } \\
\text { sphere } \\
\text { horizontal position }(\mathrm{cm})\end{array}$} \\
\hline & 0 & 5 & 10 & 0 & 5 & 10 \\
\hline & & & & & & \\
\hline & \multicolumn{6}{|c|}{$\frac{\text { ICRP-21 Appendix } 6}{\left(\text { rem. } \mathrm{h}^{-1}\right)}$} \\
\hline 11 & 18.880 & 18.699 & 18.177 & 4.833 & 4.821 & 4.786 \\
\hline 6 & 19.371 & 19.181 & 18.632 & 4.866 & 4.854 & 4.818 \\
\hline 1 & 19.475 & 19.283 & 18.730 & 4.875 & 4.863 & 4.827 \\
\hline-4 & 19.200 & 19.013 & 18.476 & 4.863 & 4.851 & 4.815 \\
\hline-9 & 18.512 & 18.339 & 17.841 & 4.824 & 4.813 & 4.777 \\
\hline & \multirow{2}{*}{\multicolumn{6}{|c|}{$\underline{\text { Personal Dose Equivalent } \mathrm{Hp}(10)}$}} \\
\hline & & & & & & \\
\hline 11 & 22.907 & 22.687 & 22.053 & 5.863 & 5.849 & 5.806 \\
\hline 6 & 23.500 & 23.271 & 22.605 & 5.903 & 5.888 & 5.845 \\
\hline 1 & 23.626 & 23.393 & 22.722 & 5.914 & 5.899 & 5.855 \\
\hline-4 & 23.293 & 23.067 & 22.416 & 5.899 & 5.885 & 5.841 \\
\hline-9 & 22.457 & 22.248 & 21.643 & 5.853 & 5.839 & 5.796 \\
\hline & \multirow{2}{*}{\multicolumn{6}{|c|}{ Ambient Dose Equivalent $\mathrm{H}^{*}(10)$}} \\
\hline & & & & & & \\
\hline 11 & 22.042 & 21.830 & 21.221 & 5.642 & 5.628 & 5.587 \\
\hline 6 & 22.613 & 22.392 & 21.751 & 5.680 & 5.666 & 5.624 \\
\hline 1 & 22.733 & 22.510 & 21.864 & 5.690 & 5.676 & 5.634 \\
\hline-4 & 22.414 & 22.196 & 21.569 & 5.676 & 5.662 & 5.621 \\
\hline-9 & 21.610 & 21.408 & 20.826 & 5.632 & 5.618 & 5.577 \\
\hline \multirow[t]{2}{*}{ Dose Factor } & Maximum & Minimum & Ratio & Maximum & Minimum & Ratio \\
\hline & \multicolumn{6}{|c|}{$\underline{10-\mathrm{cm} \text { Range }}$} \\
\hline ICRP-21 & 19.475 & 17.841 & $1.0 \overline{2}$ & 4.875 & 4.777 & 1.020 \\
\hline Hp(10) & 23.626 & 21.643 & 1.092 & 5.914 & 5.796 & 1.020 \\
\hline $\mathrm{H}^{*}(10)$ & 22.733 & 20.826 & 1.092 & 5.690 & 5.577 & 1.020 \\
\hline & \multicolumn{6}{|c|}{ 5-cm Range } \\
\hline ICRP-21 & 19.475 & 19.013 & $1.02 \overline{4}$ & 4.875 & 4.851 & 1.005 \\
\hline Hp(10) & 23.626 & 23.067 & 1.024 & 5.914 & 5.885 & 1.005 \\
\hline $\mathrm{H}^{*}(10)$ & 22.733 & 22.196 & 1.024 & 5.690 & 5.662 & 1.005 \\
\hline
\end{tabular}




\section{CALCULATION SHEET}

\begin{tabular}{|c|c|c|c|}
\hline PROJECT No.: & $\begin{array}{l}\text { CALC NO.: } \\
\text { PNNL-19273 } \\
\end{array}$ & REVISION NO.: $\underline{0}$ & SHEET NO.: $\underline{53 / 246}$ \\
\hline \multicolumn{4}{|c|}{$\frac{\text { PNNL-19273 }}{00{ }^{252} \text { CF NEUTRO }}$} \\
\hline PREPARED BY: R.J. TRAUB & DATE: $\underline{02 / 8 / 10}$ & REVIEWED BY: R.J.MCCONN & DATE: $\underline{03 / 21 / 10}$ \\
\hline
\end{tabular}

Table 32. Neutron fluence at the surface of a $10-\mathrm{cm} \times 10-\mathrm{cm}$ rectangle $50 \mathrm{~cm}$ and $100 \mathrm{~cm}$ from the center of the reference sphere. The ${ }^{252} \mathrm{Cf}$ source is in the pneumatic transfer tube assembly. The dose rectangle has been elevated $1 \mathrm{~cm}$. relative to the equator of the reference sphere. The activity is in the void between the frits. The reference date is March 3, 2003. The emission rate is $4.85 \mathrm{e} 9 \mathrm{n} \cdot \mathrm{s}^{-1}$.

\begin{tabular}{rccccccc}
\hline & \multicolumn{3}{c}{$50 \mathrm{~cm}$ from center of reference sphere } & & \multicolumn{3}{c}{$100 \mathrm{~cm}$ from center of reference } \\
sphere
\end{tabular}




\section{CALCULATION SHEET}

\begin{tabular}{|c|c|c|c|}
\hline ProjeCt No.: & $\begin{array}{l}\text { CALC NO.: } \\
\text { PNNL-19273 }\end{array}$ & REVISION NO.: $\underline{0}$ & SHEET NO.: $\underline{54 / 246}$ \\
\hline T: CALCULATIONS T & $00{ }^{252} \mathrm{CF}$ NEUTR & E IN 318LOW SCATTER ROOM & \\
\hline PREPARED BY: R.J. TRAUB & DATE: $\underline{02 / 8 / 10}$ & REVIEWED BY: R.J.MCCONN & DATE: $\underline{03 / 21 / 10}$ \\
\hline
\end{tabular}

Table 33. Neutron dose factors at the surface of a $10-\mathrm{cm} \times 10-\mathrm{cm}$ rectangle $50 \mathrm{~cm}$ and $100 \mathrm{~cm}$ from the center of the reference sphere. The ${ }^{252} \mathrm{Cf}$ source is in the pneumatic transfer tube assembly. The dose rectangle has been elevated $1 \mathrm{~cm}$. relative to the equator of the reference sphere. The activity is in the void between the frits. The reference date is March 3, 2003. The emission rate is $4.85 \mathrm{e} 9 \mathrm{n} . \mathrm{s}^{-1}$.

\begin{tabular}{|c|c|c|c|c|c|c|}
\hline \multirow[b]{3}{*}{$\begin{array}{l}\text { Z position } \\
(\mathrm{cm})\end{array}$} & \multirow{2}{*}{\multicolumn{3}{|c|}{$\begin{array}{l}50 \mathrm{~cm} \text { from center of the reference } \\
\text { sphere } \\
\text { horizontal position }(\mathrm{cm})\end{array}$}} & \multirow{2}{*}{\multicolumn{3}{|c|}{$\begin{array}{l}100 \mathrm{~cm} \text { from center of the reference } \\
\text { sphere } \\
\text { horizontal position }(\mathrm{cm})\end{array}$}} \\
\hline & & & & & & \\
\hline & 0 & 5 & 10 & 0 & 5 & 10 \\
\hline & \multicolumn{6}{|c|}{$\begin{array}{l}\text { ICRP-21 Appendix } 6 \\
(\text { pSv.cm²) }\end{array}$} \\
\hline 11 & 323.8 & 323.8 & 323.9 & 323.8 & 323.8 & 323.8 \\
\hline 6 & 323.7 & 323.8 & 323.8 & 323.7 & 323.8 & 323.8 \\
\hline 1 & 323.7 & 323.7 & 323.7 & 323.7 & 323.7 & 323.8 \\
\hline-4 & 323.8 & 323.8 & 323.8 & 323.7 & 323.8 & 323.8 \\
\hline-9 & 323.9 & 323.9 & 323.9 & 323.8 & 323.8 & 323.8 \\
\hline & \multicolumn{6}{|c|}{$\begin{array}{c}\text { Personal Dose Equivalent Hp(10) } \\
\left(\mathrm{pSv} . \mathrm{cm}^{2}\right)\end{array}$} \\
\hline 11 & 392.6 & 392.6 & 392.6 & 392.6 & 392.6 & 392.6 \\
\hline 6 & 392.5 & 392.6 & 392.6 & 392.6 & 392.6 & 392.6 \\
\hline 1 & 392.5 & 392.5 & 392.5 & 392.6 & 392.6 & 392.6 \\
\hline-4 & 392.6 & 392.6 & 392.6 & 392.6 & 392.6 & 392.6 \\
\hline-9 & 392.6 & 392.7 & 392.7 & 392.6 & 392.6 & 392.6 \\
\hline & \multicolumn{6}{|c|}{$\begin{array}{c}\text { Ambient Dose Equivalent } \mathrm{H}^{*}(10) \\
\left(\mathrm{pSv} \cdot \mathrm{cm}^{2}\right)\end{array}$} \\
\hline 11 & 377.8 & 377.8 & 377.8 & 377.7 & 377.8 & 377.8 \\
\hline 6 & 377.7 & 377.7 & 377.7 & 377.7 & 377.7 & 377.7 \\
\hline 1 & 377.7 & 377.7 & 377.7 & 377.7 & 377.7 & 377.7 \\
\hline-4 & 377.7 & 377.7 & 377.7 & 377.7 & 377.7 & 377.7 \\
\hline-9 & 377.8 & 377.8 & 377.8 & 377.8 & 377.8 & 377.8 \\
\hline Dose Factor & Maximum & Minimum & Ratio & Maximum & Minimum & Ratio \\
\hline & \multicolumn{6}{|c|}{ 10-cm Range } \\
\hline ICRP-21 & 323.9 & 323.7 & 1.001 & 323.8 & 323.7 & 1.000 \\
\hline $\mathrm{Hp}(10)$ & 392.7 & 392.5 & 1.000 & 392.6 & 392.6 & 1.000 \\
\hline $\mathrm{H}^{*}(10)$ & 377.8 & 377.7 & 1.000 & 377.8 & 377.7 & 1.000 \\
\hline & \multicolumn{6}{|c|}{ 5-cm Range } \\
\hline ICRP-21 & 323.8 & 323.7 & 1.000 & 323.8 & 323.7 & 1.000 \\
\hline $\mathrm{Hp}(10)$ & 392.6 & 392.5 & 1.000 & 392.6 & 392.6 & 1.000 \\
\hline $\mathrm{H}^{*}(10)$ & 377.7 & 377.7 & 1.000 & 377.7 & 377.7 & 1.000 \\
\hline
\end{tabular}




\section{CALCULATION SHEET}

\begin{tabular}{|c|c|c|c|}
\hline PROJECT No.: & $\begin{array}{l}\text { CALC NO.: } \\
\text { PNNL-19273 }\end{array}$ & REVISION NO.: $\underline{0}$ & SHEET NO.: $\underline{55 / 246}$ \\
\hline SUBJECT: CALCULATIONS & $00{ }^{252} \mathrm{CF}$ NEUTRC & CE IN 318LOW SCATTER ROOM & \\
\hline PREPARED BY: R.J. TRAUB & DATE: $\underline{02 / 8 / 10}$ & REVIEWED BY: R.J.MCCONN & DATE: $\underline{03 / 21 / 10}$ \\
\hline
\end{tabular}

Table 34. Neutron dose rates at the surface of a $10-\mathrm{cm} \times 10-\mathrm{cm}$ rectangle $50 \mathrm{~cm}$ and $100 \mathrm{~cm}$ from the center of the reference sphere. The ${ }^{252} \mathrm{Cf}$ source is in the pneumatic transfer tube assembly. The activity is in the void between the frits. The dose rectangle has been elevated $1 \mathrm{~cm}$. relative to the equator of the reference sphere. The reference date is March 3, 2003. The emission rate is $4.85 \mathrm{e} 9 \mathrm{n} \cdot \mathrm{s}^{-1}$.

\begin{tabular}{|c|c|c|c|c|c|c|}
\hline \multirow[b]{3}{*}{$\begin{array}{l}\text { Z position } \\
(\mathrm{cm})\end{array}$} & \multirow{2}{*}{\multicolumn{3}{|c|}{$\begin{array}{l}50 \mathrm{~cm} \text { from center of the reference } \\
\text { sphere } \\
\text { horizontal position }(\mathrm{cm})\end{array}$}} & \multirow{2}{*}{\multicolumn{3}{|c|}{$\begin{array}{l}100 \mathrm{~cm} \text { from center of the reference } \\
\text { sphere } \\
\text { horizontal position }(\mathrm{cm})\end{array}$}} \\
\hline & & & & & & \\
\hline & 0 & 5 & 10 & 0 & 5 & 10 \\
\hline & \multicolumn{6}{|c|}{$\begin{array}{l}\text { ICRP-21 Appendix } 6 \\
\left(\text { rem. }^{-1}\right)\end{array}$} \\
\hline 11 & 18.885 & 18.707 & 18.188 & 4.853 & 4.841 & 4.806 \\
\hline 6 & 19.422 & 19.234 & 18.686 & 4.887 & 4.875 & 4.840 \\
\hline 1 & 19.555 & 19.366 & 18.810 & 4.895 & 4.884 & 4.848 \\
\hline-4 & 19.268 & 19.084 & 18.546 & 4.877 & 4.865 & 4.830 \\
\hline-9 & 18.599 & 18.428 & 17.924 & 4.832 & 4.821 & 4.786 \\
\hline & \multicolumn{6}{|c|}{$\begin{array}{c}\text { Personal Dose Equivalent } \mathrm{Hp}(10) \\
\left(\mathrm{rem}^{-1} \mathrm{~h}^{-1}\right)\end{array}$} \\
\hline 11 & 22.896 & 22.680 & 22.051 & 5.884 & 5.870 & 5.827 \\
\hline 6 & 23.550 & 23.322 & 22.656 & 5.926 & 5.912 & 5.868 \\
\hline 1 & 23.712 & 23.482 & 22.808 & 5.936 & 5.922 & 5.878 \\
\hline-4 & 23.363 & 23.140 & 22.486 & 5.913 & 5.899 & 5.856 \\
\hline-9 & 22.549 & 22.341 & 21.731 & 5.859 & 5.846 & 5.803 \\
\hline & \multicolumn{6}{|c|}{$\begin{array}{c}\text { Ambient Dose Equivalent } \mathrm{H}^{*}(10) \\
\left(\mathrm{rem} \cdot \mathrm{h}^{-1}\right)\end{array}$} \\
\hline 11 & 22.031 & 21.823 & 21.218 & 5.662 & 5.648 & 5.607 \\
\hline 6 & 22.660 & 22.441 & 21.801 & 5.702 & 5.688 & 5.647 \\
\hline 1 & 22.817 & 22.595 & 21.947 & 5.712 & 5.698 & 5.656 \\
\hline-4 & 22.481 & 22.266 & 21.637 & 5.690 & 5.676 & 5.635 \\
\hline-9 & 21.697 & 21.497 & 20.910 & 5.638 & 5.625 & 5.584 \\
\hline Dose Factor & Maximum & Minimum & Ratio & Maximum & Minimum & Ratio \\
\hline & \multicolumn{6}{|c|}{ 10-cm Range } \\
\hline ICRP-21 & 19.555 & 17.924 & 1.091 & 4.895 & 4.786 & 1.023 \\
\hline $\mathrm{Hp}(10)$ & 23.712 & 21.731 & 1.091 & 5.936 & 5.803 & 1.023 \\
\hline $\mathrm{H}^{*}(10)$ & 22.817 & 20.910 & 1.091 & 5.712 & 5.584 & 1.023 \\
\hline \multicolumn{7}{|c|}{ 5-cm Range } \\
\hline ICRP-21 & 19.555 & 19.084 & 1.025 & 4.895 & 4.865 & 1.006 \\
\hline Hp(10) & 23.712 & 23.140 & 1.025 & 5.936 & 5.899 & 1.006 \\
\hline $\mathrm{H}^{*}(10)$ & 22.817 & 22.266 & 1.025 & 5.712 & 5.676 & 1.006 \\
\hline
\end{tabular}




\section{CALCULATION SHEET}

\begin{tabular}{|c|c|c|c|}
\hline PROJECT No.: & $\begin{array}{l}\text { CALC NO.: } \\
\text { PNNL-19273 }\end{array}$ & REVISION NO.: $\underline{0}$ & SHEET NO.: $\underline{56 / 246}$ \\
\hline \multicolumn{4}{|c|}{1050} \\
\hline PREPARED BY: R.J. TRAUB & DATE: $\underline{02 / 8 / 10}$ & REVIEWED BY: R.J.MCCONN & DATE: $\underline{03 / 21 / 10}$ \\
\hline
\end{tabular}

Table 35. Neutron fluence at the surface of a $10-\mathrm{cm} \times 10-\mathrm{cm}$ rectangle $50 \mathrm{~cm}$ and $100 \mathrm{~cm}$ from the center of the reference sphere. The ${ }^{252} \mathrm{Cf}$ source is in the pneumatic transfer tube assembly. The dose rectangle has been elevated $1 \mathrm{~cm}$. relative to the equator of the reference sphere. The activity is on the lower frit. The reference date is March 3, 2003. The emission rate is $4.85 \mathrm{e} 9 \mathrm{n} \cdot \mathrm{s}^{-1}$.

\begin{tabular}{rccccccc}
\hline & \multicolumn{3}{c}{$50 \mathrm{~cm}$ from center of reference sphere } & & \multicolumn{3}{c}{$100 \mathrm{~cm}$ from center of reference } \\
sphere
\end{tabular}




\section{CALCULATION SHEET}

\begin{tabular}{|c|c|c|c|}
\hline ProjeCt No.: & $\begin{array}{l}\text { CALC NO.: } \\
\text { PNNL-19273 }\end{array}$ & REVISION NO.: $\underline{0}$ & SHEET NO.: $\underline{57 / 246}$ \\
\hline \multicolumn{4}{|c|}{ E IN 318LOW SCATTER ROOM } \\
\hline PREPARED BY: R.J. TRAUB & DATE: $\underline{02 / 8 / 10}$ & REVIEWED BY: R.J.MCCONN & DATE: $03 / 21 / 10$ \\
\hline
\end{tabular}

Table 36. Neutron dose factors at the surface of a $10-\mathrm{cm} \times 10-\mathrm{cm}$ rectangle $50 \mathrm{~cm}$ and $100 \mathrm{~cm}$ from the center of the reference sphere. The ${ }^{252} \mathrm{Cf}$ source is in the pneumatic transfer tube assembly. The dose rectangle has been elevated $1 \mathrm{~cm}$. relative to the equator of the reference sphere. The activity is on the lower frit. The reference date is March 3, 2003. The emission rate is $4.85 \mathrm{e} 9 \mathrm{n} \cdot \mathrm{s}^{-1}$.

\begin{tabular}{|c|c|c|c|c|c|c|}
\hline \multirow[b]{3}{*}{$\begin{array}{l}\text { Z position } \\
(\mathrm{cm})\end{array}$} & \multirow{2}{*}{\multicolumn{3}{|c|}{$\begin{array}{l}50 \mathrm{~cm} \text { from center of the reference } \\
\text { sphere } \\
\text { horizontal position }(\mathrm{cm})\end{array}$}} & \multirow{2}{*}{\multicolumn{3}{|c|}{$\begin{array}{l}100 \mathrm{~cm} \text { from center of the reference } \\
\text { sphere } \\
\text { horizontal position }(\mathrm{cm})\end{array}$}} \\
\hline & & & & & & \\
\hline & 0 & 5 & 10 & 0 & 5 & 10 \\
\hline & \multicolumn{6}{|c|}{$\begin{array}{c}\text { ICRP-21 Appendix } 6 \\
(\text { pSv.cm²) }\end{array}$} \\
\hline 11 & 323.4 & 323.4 & 323.4 & 323.4 & 323.4 & 323.4 \\
\hline 6 & 323.4 & 323.4 & 323.4 & 323.4 & 323.4 & 323.4 \\
\hline 1 & 323.4 & 323.4 & 323.4 & 323.4 & 323.4 & 323.4 \\
\hline-4 & 323.4 & 323.4 & 323.4 & 323.4 & 323.4 & 323.4 \\
\hline-9 & 323.5 & 323.5 & 323.5 & 323.4 & 323.4 & 323.4 \\
\hline & \multicolumn{6}{|c|}{$\begin{array}{c}\text { Personal Dose Equivalent Hp(10) } \\
\left(\mathrm{pSv} . \mathrm{cm}^{2}\right)\end{array}$} \\
\hline 11 & 392.4 & 392.4 & 392.4 & 392.4 & 392.4 & 392.4 \\
\hline 6 & 392.3 & 392.3 & 392.3 & 392.4 & 392.4 & 392.4 \\
\hline 1 & 392.4 & 392.4 & 392.4 & 392.4 & 392.4 & 392.4 \\
\hline-4 & 392.4 & 392.4 & 392.4 & 392.4 & 392.4 & 392.4 \\
\hline-9 & 392.4 & 392.4 & 392.4 & 392.4 & 392.4 & 392.4 \\
\hline & \multicolumn{6}{|c|}{$\begin{array}{c}\text { Ambient Dose Equivalent } \mathrm{H}^{*}(10) \\
\left(\mathrm{pSv} \cdot \mathrm{cm}^{2}\right)\end{array}$} \\
\hline 11 & 377.6 & 377.6 & 377.6 & 377.5 & 377.5 & 377.6 \\
\hline 6 & 377.5 & 377.5 & 377.5 & 377.5 & 377.5 & 377.6 \\
\hline 1 & 377.5 & 377.5 & 377.5 & 377.6 & 377.6 & 377.6 \\
\hline-4 & 377.6 & 377.6 & 377.6 & 377.6 & 377.6 & 377.6 \\
\hline-9 & 377.6 & 377.6 & 377.6 & 377.6 & 377.6 & 377.6 \\
\hline Dose Factor & Maximum & Minimum & Ratio & Maximum & Minimum & Ratio \\
\hline & \multicolumn{6}{|c|}{ 10-cm Range } \\
\hline ICRP-21 & 323.5 & 323.4 & 1.000 & 323.4 & 323.4 & 1.000 \\
\hline Hp(10) & 392.4 & 392.3 & 1.000 & 392.4 & 392.4 & 1.000 \\
\hline $\mathrm{H}^{*}(10)$ & 377.6 & 377.5 & 1.000 & 377.6 & 377.5 & 1.000 \\
\hline & \multicolumn{6}{|c|}{ 5-cm Range } \\
\hline ICRP-21 & 323.4 & 323.4 & 1.000 & 323.4 & 323.4 & 1.000 \\
\hline Hp(10) & 392.4 & 392.3 & 1.000 & 392.4 & 392.4 & 1.000 \\
\hline $\mathrm{H}^{*}(10)$ & 377.6 & 377.5 & 1.000 & 377.6 & 377.5 & 1.000 \\
\hline
\end{tabular}




\section{CALCULATION SHEET}

\begin{tabular}{|c|c|c|c|}
\hline PROJECT No.: & $\begin{array}{l}\text { CALC NO.: } \\
\text { PNNL-19273 }\end{array}$ & REVISION NO.: $\underline{0}$ & SHEET NO.: $\underline{58 / 246}$ \\
\hline SUBJECT: CALCULATIONS & $00{ }^{252} \mathrm{CF}$ NEUTRC & CE IN 318LOW SCATTER ROOM & \\
\hline PREPARED BY: R.J. TRAUB & DATE: $\underline{02 / 8 / 10}$ & REVIEWED BY: R.J.MCCONN & DATE: $\underline{03 / 21 / 10}$ \\
\hline
\end{tabular}

Table 37. Neutron dose rates at the surface of a $10-\mathrm{cm} \times 10-\mathrm{cm}$ rectangle $50 \mathrm{~cm}$ and $100 \mathrm{~cm}$ from the center of the reference sphere. The ${ }^{252} \mathrm{Cf}$ source is in the pneumatic transfer tube assembly. The activity is on the lower frit. The dose rectangle has been elevated $1 \mathrm{~cm}$. relative to the equator of the reference sphere.

\begin{tabular}{|c|c|c|c|c|c|c|}
\hline \multirow[b]{2}{*}{$\begin{array}{l}\text { Z position } \\
(\mathrm{cm})\end{array}$} & \multicolumn{3}{|c|}{$\begin{array}{l}50 \mathrm{~cm} \text { from center of the reference } \\
\text { sphere } \\
\text { horizontal position }(\mathrm{cm})\end{array}$} & \multicolumn{3}{|c|}{$\begin{array}{l}100 \mathrm{~cm} \text { from center of the reference } \\
\text { sphere } \\
\text { horizontal position }(\mathrm{cm})\end{array}$} \\
\hline & 0 & 5 & 10 & 0 & 5 & 10 \\
\hline & \multicolumn{6}{|c|}{$\begin{array}{l}\text { ICRP-21 Appendix } 6 \\
\left(\mathrm{rem}^{-1} \mathrm{~h}^{-1}\right)\end{array}$} \\
\hline 11 & 18.815 & 18.636 & 18.122 & 4.845 & 4.833 & 4.798 \\
\hline 6 & 19.359 & 19.171 & 18.625 & 4.873 & 4.861 & 4.825 \\
\hline 1 & 19.486 & 19.296 & 18.743 & 4.876 & 4.864 & 4.828 \\
\hline-4 & 19.249 & 19.063 & 18.522 & 4.859 & 4.847 & 4.812 \\
\hline-9 & 18.629 & 18.455 & 17.947 & 4.816 & 4.805 & 4.770 \\
\hline & \multicolumn{6}{|c|}{$\begin{array}{c}\text { Personal Dose Equivalent Hp(10) } \\
\left(\text { rem. } h^{-1}\right)\end{array}$} \\
\hline 11 & 22.826 & 22.610 & 21.986 & 5.878 & 5.863 & 5.821 \\
\hline 6 & 23.487 & 23.258 & 22.596 & 5.912 & 5.897 & 5.854 \\
\hline 1 & 23.640 & 23.408 & 22.737 & 5.915 & 5.901 & 5.857 \\
\hline-4 & 23.354 & 23.128 & 22.472 & 5.895 & 5.881 & 5.838 \\
\hline-9 & 22.602 & 22.390 & 21.774 & 5.843 & 5.829 & 5.787 \\
\hline & \multicolumn{6}{|c|}{$\begin{array}{c}\text { Ambient Dose Equivalent } \mathrm{H}^{*}(10) \\
\left(\mathrm{rem} \cdot \mathrm{h}^{-1}\right)\end{array}$} \\
\hline 11 & 21.964 & 21.756 & 21.156 & 5.656 & 5.642 & 5.601 \\
\hline 6 & 22.600 & 22.380 & 21.743 & 5.689 & 5.674 & 5.633 \\
\hline 1 & 22.747 & 22.524 & 21.878 & 5.692 & 5.678 & 5.636 \\
\hline-4 & 22.473 & 22.255 & 21.623 & 5.673 & 5.659 & 5.617 \\
\hline-9 & 21.749 & 21.545 & 20.952 & 5.623 & 5.609 & 5.568 \\
\hline Dose Factor & Maximum & Minimum & Ratio & Maximum & Minimum & Ratio \\
\hline & \multicolumn{6}{|c|}{ 10-cm Range } \\
\hline ICRP-21 & 19.486 & 17.947 & 1.086 & 4.876 & 4.770 & 1.022 \\
\hline $\mathrm{Hp}(10)$ & 23.640 & 21.774 & 1.086 & 5.915 & 5.787 & 1.022 \\
\hline $\mathrm{H}^{*}(10)$ & 22.747 & 20.952 & 1.086 & 5.692 & 5.568 & 1.022 \\
\hline \multicolumn{7}{|c|}{ 5-cm Range } \\
\hline ICRP-21 & 19.486 & 19.063 & 1.022 & 4.876 & 4.847 & 1.006 \\
\hline$H p(10)$ & 23.640 & 23.128 & 1.022 & 5.915 & 5.881 & 1.006 \\
\hline$H^{*}(10)$ & 22.747 & 22.255 & 1.022 & 5.692 & 5.659 & 1.006 \\
\hline
\end{tabular}




\section{CALCULATION SHEET}

\begin{tabular}{|c|c|c|c|}
\hline PROJECT No.: & $\begin{array}{l}\text { CALC NO.: } \\
\text { PNNL-19273 } \\
\end{array}$ & REVISION NO.: $\underline{0}$ & SHEET NO.: $\underline{59 / 246}$ \\
\hline \multicolumn{4}{|c|}{$\frac{\text { PNNL-19273 }}{00{ }^{252} \text { CF NEUTRO }}$} \\
\hline PREPARED BY: R.J. TRAUB & DATE: $\underline{02 / 8 / 10}$ & REVIEWED BY: R.J.MCCONN & DATE: $\underline{03 / 21 / 10}$ \\
\hline
\end{tabular}

Table 38. Neutron fluence at the surface of a $10-\mathrm{cm} \times 10-\mathrm{cm}$ rectangle $50 \mathrm{~cm}$ and $100 \mathrm{~cm}$ from the center of the reference sphere. The ${ }^{252} \mathrm{Cf}$ source is in the pneumatic transfer tube assembly. The dose rectangle has been elevated $1.864 \mathrm{~cm}$. relative to the equator of the reference sphere. The activity is on the upper frit. The reference date is March 3, 2003. The emission rate is $4.85 \mathrm{e} 9 \mathrm{n} \cdot \mathrm{s}^{-1}$.

\begin{tabular}{rccccccc}
\hline & \multicolumn{3}{c}{$50 \mathrm{~cm}$ from center of reference sphere } & & \multicolumn{3}{c}{$100 \mathrm{~cm}$ from center of reference } \\
sphere
\end{tabular}




\section{CALCULATION SHEET}

\begin{tabular}{|c|c|c|c|}
\hline PROJECT No.: & $\begin{array}{l}\text { CALC NO.: } \\
\text { PNNL-19273 }\end{array}$ & REVISION NO.: $\underline{0}$ & SHEET NO.: $\underline{60 / 246}$ \\
\hline SUBJECT: CALCULATIONS & $00{ }^{252} \mathrm{CF}$ NEUTRC & CE IN 318LOW SCATTER ROOM & \\
\hline PREPARED BY: R.J. TRAUB & DATE: $\underline{02 / 8 / 10}$ & REVIEWED BY: R.J.MCCONN & DATE: $\underline{03 / 21 / 10}$ \\
\hline
\end{tabular}

Table 39. Neutron dose rates at the surface of a $10-\mathrm{cm} \times 10-\mathrm{cm}$ rectangle $50 \mathrm{~cm}$ and $100 \mathrm{~cm}$ from the center of the reference sphere. The ${ }^{252} \mathrm{Cf}$ source is in the pneumatic transfer tube assembly. The activity is on the upper frit. The dose rectangle has been elevated $1.864 \mathrm{~cm}$. relative to the equator of the reference sphere. The reference date is March 3, 2003. The emission rate is $4.85 \mathrm{e} 9 \mathrm{n} \cdot \mathrm{s}^{-1}$.

\begin{tabular}{|c|c|c|c|c|c|c|}
\hline \multirow[b]{3}{*}{$\begin{array}{l}\text { Z position } \\
(\mathrm{cm})\end{array}$} & \multirow{2}{*}{\multicolumn{3}{|c|}{$\begin{array}{l}50 \mathrm{~cm} \text { from center of the reference } \\
\text { sphere } \\
\text { horizontal position }(\mathrm{cm})\end{array}$}} & \multirow{2}{*}{\multicolumn{3}{|c|}{$\begin{array}{l}100 \mathrm{~cm} \text { from center of the reference } \\
\text { sphere } \\
\text { horizontal position }(\mathrm{cm})\end{array}$}} \\
\hline & & & & & & \\
\hline & 0 & 5 & 10 & 0 & 5 & 10 \\
\hline & \multicolumn{6}{|c|}{$\begin{array}{l}\text { ICRP-21 Appendix } 6 \\
\left(\text { rem. }^{-1}\right)\end{array}$} \\
\hline 11.864 & 18.758 & 18.579 & 18.062 & 4.824 & 4.812 & 4.777 \\
\hline 6.864 & 19.312 & 19.124 & 18.578 & 4.862 & 4.850 & 4.814 \\
\hline 1.864 & 19.479 & 19.286 & 18.733 & 4.874 & 4.862 & 4.826 \\
\hline-3.136 & 19.276 & 19.088 & 18.547 & 4.866 & 4.854 & 4.818 \\
\hline \multirow[t]{2}{*}{-8.136} & 18.656 & 18.481 & 17.974 & 4.833 & 4.821 & 4.786 \\
\hline & \multicolumn{6}{|c|}{$\begin{array}{l}\text { Personal Dose Equivalent } \mathrm{Hp}(10) \\
\left(\mathrm{rem} \cdot \mathrm{h}^{-1}\right)\end{array}$} \\
\hline 11.864 & 22.758 & 22.541 & 21.914 & 5.853 & 5.838 & 5.796 \\
\hline 6.864 & 23.430 & 23.201 & 22.539 & 5.898 & 5.883 & 5.840 \\
\hline 1.864 & 23.630 & 23.397 & 22.725 & 5.913 & 5.898 & 5.854 \\
\hline-3.136 & 23.386 & 23.158 & 22.501 & 5.904 & 5.889 & 5.845 \\
\hline \multirow[t]{2}{*}{-8.136} & 22.632 & 22.420 & 21.806 & 5.863 & 5.849 & 5.806 \\
\hline & \multicolumn{6}{|c|}{$\begin{array}{c}\text { Ambient Dose Equivalent } \mathrm{H}^{*}(10) \\
\left(\mathrm{rem} \cdot \mathrm{h}^{-1}\right)\end{array}$} \\
\hline 11.864 & 21.898 & 21.690 & 21.087 & 5.632 & 5.618 & 5.577 \\
\hline 6.864 & 22.545 & 22.325 & 21.688 & 5.675 & 5.661 & 5.619 \\
\hline 1.864 & 22.738 & 22.514 & 21.867 & 5.689 & 5.675 & 5.633 \\
\hline-3.136 & 22.503 & 22.284 & 21.652 & 5.681 & 5.667 & 5.625 \\
\hline-8.136 & 21.778 & 21.573 & 20.983 & 5.642 & 5.628 & 5.587 \\
\hline Dose Factor & Maximum & Minimum & Ratio & Maximum & Minimum & Ratio \\
\hline & \multicolumn{6}{|c|}{ 10-cm Range } \\
\hline ICRP-21 & 19.479 & 17.974 & 1.084 & 4.874 & 4.777 & 1.020 \\
\hline $\mathrm{Hp}(10)$ & 23.630 & 21.806 & 1.084 & 5.913 & 5.796 & 1.020 \\
\hline $\mathrm{H}^{*}(10)$ & 22.738 & 20.983 & 1.084 & 5.689 & 5.577 & 1.020 \\
\hline \multicolumn{7}{|c|}{ 5-cm Range } \\
\hline ICRP-21 & 19.479 & 19.088 & 1.020 & 4.874 & 4.850 & 1.005 \\
\hline Hp(10) & 23.630 & 23.158 & 1.020 & 5.913 & 5.883 & 1.005 \\
\hline $\mathrm{H}^{*}(10)$ & 22.738 & 22.284 & 1.020 & 5.689 & 5.661 & 1.005 \\
\hline
\end{tabular}




\section{CALCULATION SHEET}

\begin{tabular}{|c|c|c|c|}
\hline ProjeCt No.: & $\begin{array}{l}\text { CALC NO.: } \\
\text { PNNL-19273 }\end{array}$ & REVISION NO.: $\underline{0}$ & SHEET NO.: $\underline{61 / 246}$ \\
\hline \multicolumn{4}{|c|}{ E IN 318LOW SCATTER ROOM } \\
\hline PREPARED BY: R.J. TRAUB & DATE: $\underline{02 / 8 / 10}$ & REVIEWED BY: R.J.MCCONN & DATE: $03 / 21 / 10$ \\
\hline
\end{tabular}

Table 40. Neutron dose factors at the surface of a $10-\mathrm{cm} \times 10-\mathrm{cm}$ rectangle $50 \mathrm{~cm}$ and $100 \mathrm{~cm}$ from the center of the reference sphere. The ${ }^{252} \mathrm{Cf}$ source is in the pneumatic transfer tube assembly. The dose rectangle has been elevated $1.864 \mathrm{~cm}$. relative to the equator of the reference sphere. The activity is on the upper frit. The reference date is March 3, 2003. The emission rate is $4.85 \mathrm{e} 9 \mathrm{n} \cdot \mathrm{s}^{-1}$.

\begin{tabular}{|c|c|c|c|c|c|c|}
\hline \multirow[b]{3}{*}{$\begin{array}{l}Z \text { position } \\
(\mathrm{cm})\end{array}$} & \multirow{2}{*}{\multicolumn{3}{|c|}{$\begin{array}{l}50 \mathrm{~cm} \text { from center of the reference } \\
\text { sphere } \\
\text { horizontal position }(\mathrm{cm})\end{array}$}} & \multirow{2}{*}{\multicolumn{3}{|c|}{$\begin{array}{l}100 \mathrm{~cm} \text { from center of the reference } \\
\text { sphere } \\
\text { horizontal position }(\mathrm{cm})\end{array}$}} \\
\hline & & & & & & \\
\hline & 0 & 5 & 10 & 0 & 5 & 10 \\
\hline & \multicolumn{6}{|c|}{$\begin{array}{l}\text { ICRP-21 Appendix } 6 \\
\left(\mathrm{pSv} \cdot \mathrm{cm}^{2}\right)\end{array}$} \\
\hline 11.864 & 323.5 & 323.5 & 323.5 & 323.5 & 323.5 & 323.5 \\
\hline 6.864 & 323.4 & 323.4 & 323.4 & 323.5 & 323.5 & 323.5 \\
\hline 1.864 & 323.4 & 323.4 & 323.4 & 323.5 & 323.5 & 323.5 \\
\hline-3.136 & 323.4 & 323.4 & 323.4 & 323.4 & 323.4 & 323.4 \\
\hline \multirow[t]{2}{*}{-8.136} & 323.5 & 323.5 & 323.5 & 323.4 & 323.4 & 323.4 \\
\hline & \multicolumn{6}{|c|}{$\begin{array}{l}\text { Personal Dose Equivalent Hp(10) } \\
\text { (pSv.cm²) }\end{array}$} \\
\hline 11.864 & 392.5 & 392.5 & 392.5 & 392.4 & 392.4 & 392.4 \\
\hline 6.864 & 392.4 & 392.4 & 392.4 & 392.4 & 392.4 & 392.4 \\
\hline 1.864 & 392.4 & 392.4 & 392.4 & 392.4 & 392.4 & 392.4 \\
\hline-3.136 & 392.4 & 392.4 & 392.4 & 392.4 & 392.4 & 392.4 \\
\hline \multirow[t]{2}{*}{-8.136} & 392.4 & 392.4 & 392.4 & 392.4 & 392.4 & 392.4 \\
\hline & \multicolumn{6}{|c|}{$\begin{array}{c}\text { Ambient Dose Equivalent } \mathrm{H}^{*}(10) \\
\left(\mathrm{pSv} \cdot \mathrm{cm}^{2}\right)\end{array}$} \\
\hline 11.864 & 377.6 & 377.6 & 377.6 & 377.6 & 377.6 & 377.6 \\
\hline 6.864 & 377.6 & 377.6 & 377.6 & 377.6 & 377.6 & 377.6 \\
\hline 1.864 & 377.6 & 377.6 & 377.6 & 377.6 & 377.6 & 377.6 \\
\hline-3.136 & 377.6 & 377.6 & 377.5 & 377.6 & 377.6 & 377.6 \\
\hline-8.136 & 377.6 & 377.6 & 377.6 & 377.6 & 377.6 & 377.6 \\
\hline \multirow[t]{2}{*}{ Dose Factor } & Maximum & Minimum & Ratio & Maximum & Minimum & Ratio \\
\hline & \multicolumn{6}{|c|}{ 10-cm Range } \\
\hline ICRP-21 & 323.5 & 323.4 & 1.000 & 323.5 & 323.4 & 1.000 \\
\hline $\mathrm{Hp}(10)$ & 392.5 & 392.4 & 1.000 & 392.4 & 392.4 & 1.000 \\
\hline \multirow[t]{2}{*}{$\mathrm{H}^{*}(10)$} & 377.6 & 377.5 & 1.000 & 377.6 & 377.6 & 1.000 \\
\hline & \multicolumn{6}{|c|}{ 5-cm Range } \\
\hline ICRP-21 & 323.4 & 323.4 & 1.000 & 323.5 & 323.4 & 1.000 \\
\hline Hp(10) & 392.4 & 392.4 & 1.000 & 392.4 & 392.4 & 1.000 \\
\hline $\mathrm{H}^{*}(10)$ & 377.6 & 377.6 & 1.000 & 377.6 & 377.6 & 1.000 \\
\hline
\end{tabular}




\section{CALCULATION SHEET}

\begin{tabular}{|c|c|c|c|}
\hline PROJECT No.: & $\begin{array}{l}\text { CALC NO.: } \\
\text { PNNL-19273 }\end{array}$ & REVISION NO.: $\underline{0}$ & SHEET NO.: $\underline{62 / 246}$ \\
\hline \multicolumn{4}{|c|}{1050} \\
\hline PREPARED BY: R.J. TRAUB & DATE: $\underline{02 / 8 / 10}$ & REVIEWED BY: R.J.MCCONN & DATE: $\underline{03 / 21 / 10}$ \\
\hline
\end{tabular}

Table 41. Neutron fluence at the surface of a $10-\mathrm{cm} \times 10-\mathrm{cm}$ rectangle $50 \mathrm{~cm}$ and $100 \mathrm{~cm}$ from the center of the reference sphere. The ${ }^{252} \mathrm{Cf}$ source is in the pneumatic transfer tube assembly. The dose rectangle has been elevated $1.864 \mathrm{~cm}$. relative to the equator of the reference sphere. The activity is in the void between the frits. The reference date is March 3, 2003. The emission rate is $4.85 \mathrm{e} 9 \mathrm{n} \cdot \mathrm{s}^{-1}$.

\begin{tabular}{|c|c|c|c|c|c|c|}
\hline \multirow[b]{2}{*}{$\begin{array}{l}Z \text { position } \\
(\mathrm{cm})\end{array}$} & \multicolumn{3}{|c|}{$50 \mathrm{~cm}$ from center of reference sphere } & \multicolumn{3}{|c|}{$\begin{array}{l}100 \mathrm{~cm} \text { from center of reference } \\
\text { sphere }\end{array}$} \\
\hline & 0 & 5 & 10 & 0 & 5 & 10 \\
\hline & \multicolumn{6}{|c|}{$\begin{array}{l}\text { Pneumatic Transfer Tube Assembly (Unmoderated Spectra) } \\
\qquad\left(\mathrm{cm}^{-2} \cdot \mathrm{s}^{-1}\right)\end{array}$} \\
\hline 11.864 & $1.61 \mathrm{E}+05$ & $1.59 \mathrm{E}+05$ & $1.55 \mathrm{E}+05$ & $4.16 \mathrm{E}+04$ & $4.15 E+04$ & $4.12 E+04$ \\
\hline 6.864 & $1.66 \mathrm{E}+05$ & $1.64 \mathrm{E}+05$ & $1.60 \mathrm{E}+05$ & $4.19 \mathrm{E}+04$ & $4.18 \mathrm{E}+04$ & $4.15 \mathrm{E}+04$ \\
\hline 1.864 & $1.68 \mathrm{E}+05$ & $1.66 \mathrm{E}+05$ & $1.61 \mathrm{E}+05$ & $4.20 \mathrm{E}+04$ & $4.19 \mathrm{E}+04$ & $4.16 \mathrm{E}+04$ \\
\hline-3.136 & $1.66 \mathrm{E}+05$ & $1.64 \mathrm{E}+05$ & $1.60 \mathrm{E}+05$ & 4.19E+04 & $4.18 \mathrm{E}+04$ & $4.15 \mathrm{E}+04$ \\
\hline-8.136 & $1.61 \mathrm{E}+05$ & $1.59 \mathrm{E}+05$ & $1.55 \mathrm{E}+05$ & $4.15 \mathrm{E}+04$ & $4.14 \mathrm{E}+04$ & $4.11 \mathrm{E}+04$ \\
\hline Range & Maximum & Minimum & Ratio & Maximum & Minimum & Ratio \\
\hline & \multicolumn{6}{|c|}{ Pneumatic Transfer Tube Assembly (Unmoderated Spectra) } \\
\hline 10 & $1.68 \mathrm{E}+05$ & $1.55 \mathrm{E}+05$ & 1.084 & $4.20 \mathrm{E}+04$ & $4.11 \mathrm{E}+04$ & 1.021 \\
\hline 5 & $1.68 \mathrm{E}+05$ & $1.64 \mathrm{E}+05$ & 1.021 & $4.20 \mathrm{E}+04$ & $4.18 \mathrm{E}+04$ & 1.005 \\
\hline
\end{tabular}




\section{CALCULATION SHEET}

\begin{tabular}{|c|c|c|c|}
\hline PROJECT No.: & $\begin{array}{l}\text { CALC NO.: } \\
\text { PNNL-19273 }\end{array}$ & REVISION NO.: $\underline{0}$ & SHEET NO.: $\underline{63 / 246}$ \\
\hline \multicolumn{4}{|c|}{ PNNL-19273 } \\
\hline PREPARED BY: R.J. TRAUB & DATE: $\underline{02 / 8 / 10}$ & REVIEWED BY: $\underline{\text { R.J.MCCONN }}$ & DATE: $\underline{03 / 21 / 10}$ \\
\hline
\end{tabular}

Table 42. Calculated dose rates at the surface of a $10-\mathrm{cm} \times 10-\mathrm{cm}$ rectangle $50 \mathrm{~cm}$ and $100 \mathrm{~cm}$ from the center of the reference sphere. The ${ }^{252} \mathrm{Cf}$ source is in the pneumatic transfer tube assembly. The activity is in the void between the frits. The dose rectangle has been elevated $1.864 \mathrm{~cm}$. relative to the equator of the reference sphere. The reference date is March 3, 2003. The emission rate is $4.85 \mathrm{e} 9 \mathrm{n} . \mathrm{s}^{-1}$.

\begin{tabular}{|c|c|c|c|c|c|c|}
\hline \multirow[b]{3}{*}{$\begin{array}{l}\text { Z position } \\
(\mathrm{cm})\end{array}$} & \multirow{2}{*}{\multicolumn{3}{|c|}{$\begin{array}{l}50 \mathrm{~cm} \text { from center of the reference } \\
\text { sphere } \\
\text { horizontal position }(\mathrm{cm})\end{array}$}} & \multirow{2}{*}{\multicolumn{3}{|c|}{$\begin{array}{l}100 \mathrm{~cm} \text { from center of the reference } \\
\text { sphere } \\
\text { horizontal position }(\mathrm{cm})\end{array}$}} \\
\hline & & & & & & \\
\hline & 0 & 5 & 10 & 0 & 5 & 10 \\
\hline & \multicolumn{6}{|c|}{$\begin{array}{l}\text { ICRP-21 Appendix } 6 \\
\left(\text { rem. }^{-1}\right)\end{array}$} \\
\hline 11.864 & 18.756 & 18.580 & 18.067 & 4.844 & 4.833 & 4.798 \\
\hline 6.864 & 19.357 & 19.170 & 18.626 & 4.883 & 4.871 & 4.836 \\
\hline 1.864 & 19.562 & 19.373 & 18.817 & 4.896 & 4.884 & 4.848 \\
\hline-3.136 & 19.346 & 19.161 & 18.617 & 4.882 & 4.870 & 4.834 \\
\hline \multirow[t]{2}{*}{-8.136} & 18.738 & 18.564 & 18.054 & 4.841 & 4.830 & 4.795 \\
\hline & \multicolumn{6}{|c|}{$\begin{array}{l}\text { Personal Dose Equivalent } \mathrm{Hp}(10) \\
\left(\mathrm{rem} \cdot \mathrm{h}^{-1}\right)\end{array}$} \\
\hline 11.864 & 22.738 & 22.525 & 21.904 & 5.874 & 5.860 & 5.817 \\
\hline 6.864 & 23.470 & 23.244 & 22.583 & 5.921 & 5.907 & 5.863 \\
\hline 1.864 & 23.720 & 23.491 & 22.816 & 5.936 & 5.922 & 5.879 \\
\hline-3.136 & 23.458 & 23.233 & 22.573 & 5.919 & 5.905 & 5.862 \\
\hline \multirow[t]{2}{*}{-8.136} & 22.719 & 22.507 & 21.889 & 5.871 & 5.857 & 5.814 \\
\hline & \multicolumn{6}{|c|}{$\begin{array}{c}\text { Ambient Dose Equivalent } \mathrm{H}^{*}(10) \\
\left(\mathrm{rem} \cdot \mathrm{h}^{-1}\right)\end{array}$} \\
\hline 11.864 & 21.879 & 21.674 & 21.076 & 5.652 & 5.638 & 5.597 \\
\hline 6.864 & 22.583 & 22.366 & 21.730 & 5.697 & 5.684 & 5.642 \\
\hline 1.864 & 22.824 & 22.603 & 21.954 & 5.712 & 5.698 & 5.657 \\
\hline-3.136 & 22.572 & 22.355 & 21.721 & 5.696 & 5.682 & 5.640 \\
\hline-8.136 & 21.861 & 21.657 & 21.062 & 5.649 & 5.636 & 5.595 \\
\hline \multirow[t]{2}{*}{ Dose Factor } & Maximum & Minimum & Ratio & Maximum & Minimum & Ratio \\
\hline & \multicolumn{6}{|c|}{ 10-cm Range } \\
\hline ICRP-21 & 19.562 & 18.054 & 1.084 & 4.896 & 4.795 & 1.021 \\
\hline $\mathrm{Hp}(10)$ & 23.720 & 21.889 & 1.084 & 5.936 & 5.814 & 1.021 \\
\hline $\mathrm{H}^{*}(10)$ & 22.824 & 21.062 & 1.084 & 5.712 & 5.595 & 1.021 \\
\hline \multicolumn{7}{|c|}{ 5-cm Range } \\
\hline ICRP-21 & 19.562 & 19.161 & 1.021 & 4.896 & 4.870 & 1.005 \\
\hline Hp(10) & 23.720 & 23.233 & 1.021 & 5.936 & 5.905 & 1.005 \\
\hline $\mathrm{H}^{*}(10)$ & 22.824 & 22.355 & 1.021 & 5.712 & 5.682 & 1.005 \\
\hline
\end{tabular}




\section{CALCULATION SHEET}

\begin{tabular}{|c|c|c|c|}
\hline PROJECT No.: & $\begin{array}{l}\text { CALC NO.: } \\
\text { PNNL-19273 }\end{array}$ & REVISION NO.: $\underline{0}$ & SHEET NO.: $\underline{64 / 246}$ \\
\hline \multicolumn{4}{|c|}{ SUBJECT: CALCULATIONS THE SR-CF-3000 ${ }^{252}$ CF NEUTRON SOURCE IN 318LOW SCATTER ROOM } \\
\hline PREPARED BY: R.J. TRAUB & DATE: $\underline{02 / 8 / 10}$ & REVIEWED BY: R.J.MCCONN & DATE: $\underline{03 / 21 / 10}$ \\
\hline
\end{tabular}

Table 43. Neutron dose factors at the surface of a $10-\mathrm{cm} \times 10-\mathrm{cm}$ rectangle $50 \mathrm{~cm}$ and $100 \mathrm{~cm}$ from the center of the reference sphere. The ${ }^{252} \mathrm{Cf}$ source is in the pneumatic transfer tube assembly. The dose rectangle has been elevated $1.864 \mathrm{~cm}$. relative to the equator of the reference sphere. The activity is in the void between the frits. The reference date is March 3, 2003. The emission rate is $4.85 \mathrm{e} 9 \mathrm{n} . \mathrm{s}^{-1}$.

\begin{tabular}{|c|c|c|c|c|c|c|}
\hline \multirow[b]{3}{*}{$\begin{array}{l}Z \text { position } \\
(\mathrm{cm})\end{array}$} & \multirow{2}{*}{\multicolumn{3}{|c|}{$\begin{array}{l}50 \mathrm{~cm} \text { from center of the reference } \\
\text { sphere } \\
\text { horizontal position }(\mathrm{cm})\end{array}$}} & \multirow{2}{*}{\multicolumn{3}{|c|}{$\begin{array}{l}100 \mathrm{~cm} \text { from center of the reference } \\
\text { sphere } \\
\text { horizontal position }(\mathrm{cm})\end{array}$}} \\
\hline & & & & & & \\
\hline & 0 & 5 & 10 & 0 & 5 & 10 \\
\hline & \multicolumn{6}{|c|}{$\begin{array}{l}\text { ICRP-21 Appendix } 6 \\
\left(\mathrm{pSv} . \mathrm{cm}^{2}\right)\end{array}$} \\
\hline 11.864 & 323.9 & 323.9 & 323.9 & 323.8 & 323.8 & 323.8 \\
\hline 6.864 & 323.8 & 323.8 & 323.8 & 323.8 & 323.8 & 323.8 \\
\hline 1.864 & 323.7 & 323.7 & 323.7 & 323.7 & 323.7 & 323.8 \\
\hline-3.136 & 323.7 & 323.8 & 323.8 & 323.7 & 323.8 & 323.8 \\
\hline \multirow[t]{2}{*}{-8.136} & 323.8 & 323.8 & 323.9 & 323.8 & 323.8 & 323.8 \\
\hline & \multicolumn{6}{|c|}{$\begin{array}{l}\text { Personal Dose Equivalent Hp(10) } \\
\text { (pSv.cm²) }\end{array}$} \\
\hline 11.864 & 392.6 & 392.6 & 392.6 & 392.6 & 392.6 & 392.6 \\
\hline 6.864 & 392.6 & 392.6 & 392.6 & 392.6 & 392.6 & 392.6 \\
\hline 1.864 & 392.5 & 392.5 & 392.5 & 392.6 & 392.6 & 392.6 \\
\hline-3.136 & 392.6 & 392.6 & 392.6 & 392.6 & 392.6 & 392.6 \\
\hline \multirow[t]{2}{*}{-8.136} & 392.6 & 392.6 & 392.6 & 392.6 & 392.6 & 392.6 \\
\hline & \multicolumn{6}{|c|}{$\begin{array}{c}\text { Ambient Dose Equivalent } \mathrm{H}^{*}(10) \\
\left(\mathrm{pSv} \cdot \mathrm{cm}^{2}\right)\end{array}$} \\
\hline 11.864 & 377.8 & 377.8 & 377.8 & 377.8 & 377.8 & 377.8 \\
\hline 6.864 & 377.7 & 377.7 & 377.7 & 377.7 & 377.7 & 377.7 \\
\hline 1.864 & 377.7 & 377.7 & 377.7 & 377.7 & 377.7 & 377.7 \\
\hline-3.136 & 377.7 & 377.7 & 377.7 & 377.7 & 377.7 & 377.7 \\
\hline-8.136 & 377.8 & 377.8 & 377.8 & 377.7 & 377.8 & 377.8 \\
\hline \multirow[t]{2}{*}{ Dose Factor } & Maximum & Minimum & Ratio & Maximum & Minimum & Ratio \\
\hline & \multicolumn{6}{|c|}{ 10-cm Range } \\
\hline ICRP-21 & 323.9 & 323.7 & 1.000 & 323.8 & 323.7 & 1.000 \\
\hline $\mathrm{Hp}(10)$ & 392.6 & 392.5 & 1.000 & 392.6 & 392.6 & 1.000 \\
\hline $\mathrm{H}^{*}(10)$ & 377.8 & 377.7 & 1.000 & 377.8 & 377.7 & 1.000 \\
\hline & \multicolumn{6}{|c|}{ 5-cm Range } \\
\hline ICRP-21 & 323.8 & 323.7 & 1.000 & 323.8 & 323.7 & 1.000 \\
\hline Hp(10) & 392.6 & 392.5 & 1.000 & 392.6 & 392.6 & 1.000 \\
\hline $\mathrm{H}^{*}(10)$ & 377.7 & 377.7 & 1.000 & 377.7 & 377.7 & 1.000 \\
\hline
\end{tabular}




\section{CALCULATION SHEET}

\begin{tabular}{|c|c|c|c|}
\hline Project No.: & $\begin{array}{l}\text { CALC NO.: } \\
\text { PNNL-19273 } \\
\end{array}$ & REVISION NO.: $\underline{0}$ & SHEET NO.: $\underline{65 / 246}$ \\
\hline SUBJECT: CALCULATIONS & $00{ }^{252} \mathrm{CF}$ NEUTRC & EE IN 318LOW SCATTER ROOM & \\
\hline PREPARED BY: R.J. TRAUB & DATE: $\underline{02 / 8 / 10}$ & REVIEWED BY: R.J.MCCONN & DATE: $\underline{03 / 21 / 10}$ \\
\hline
\end{tabular}

Table 44. Neutron fluence at the surface of a $10-\mathrm{cm} \times 10-\mathrm{cm}$ rectangle $50 \mathrm{~cm}$ and $100 \mathrm{~cm}$ from the center of the reference sphere. The ${ }^{252} \mathrm{Cf}$ source is in the pneumatic transfer tube assembly. The dose rectangle has been elevated $1.864 \mathrm{~cm}$. relative to the equator of the reference sphere. The activity is on the lower frit. The reference date is March 3, 2003. The emission rate is $4.85 \mathrm{e} 9 \mathrm{n} \cdot \mathrm{s}^{-1}$.

\begin{tabular}{rccccccc}
\hline & \multicolumn{3}{c}{$50 \mathrm{~cm}$ from center of reference sphere } & & \multicolumn{3}{c}{$100 \mathrm{~cm}$ from center of reference } \\
sphere
\end{tabular}




\section{CALCULATION SHEET}

\begin{tabular}{|c|c|c|c|}
\hline PROJECT No.: & $\begin{array}{l}\text { CALC NO.: } \\
\text { PNNL-19273 }\end{array}$ & REVISION NO.: $\underline{0}$ & SHEET NO.: $\underline{66 / 246}$ \\
\hline \multicolumn{4}{|c|}{ PNNL-19273 } \\
\hline PREPARED BY: R.J. TRAUB & DATE: $\underline{02 / 8 / 10}$ & REVIEWED BY: $\underline{\text { R.J.MCCONN }}$ & DATE: $\underline{03 / 21 / 10}$ \\
\hline
\end{tabular}

Table 45. Calculated dose rates at the surface of a $10-\mathrm{cm} \times 10-\mathrm{cm}$ rectangle $50 \mathrm{~cm}$ and $100 \mathrm{~cm}$ from the center of the reference sphere. The ${ }^{252} \mathrm{Cf}$ source is in the pneumatic transfer tube assembly. The activity is on the lower frit. The dose rectangle has been elevated $1.864 \mathrm{~cm}$. relative to the equator of the reference sphere. The reference date is March 3, 2003. The emission rate is $4.85 \mathrm{e} 9 \mathrm{n} \cdot \mathrm{s}^{-1}$.

\begin{tabular}{|c|c|c|c|c|c|c|}
\hline \multirow[b]{3}{*}{$\begin{array}{l}\text { Z position } \\
(\mathrm{cm})\end{array}$} & \multirow{2}{*}{\multicolumn{3}{|c|}{$\begin{array}{l}50 \mathrm{~cm} \text { from center of the reference } \\
\text { sphere } \\
\text { horizontal position }(\mathrm{cm})\end{array}$}} & \multirow{2}{*}{\multicolumn{3}{|c|}{$\begin{array}{l}100 \mathrm{~cm} \text { from center of the reference } \\
\text { sphere } \\
\text { horizontal position }(\mathrm{cm})\end{array}$}} \\
\hline & & & & & & \\
\hline & 0 & 5 & 10 & 0 & 5 & 10 \\
\hline & \multicolumn{6}{|c|}{$\begin{array}{l}\text { ICRP-21 Appendix } 6 \\
\left(\text { rem. }^{-1}\right)\end{array}$} \\
\hline 11.864 & 18.681 & 18.506 & 17.999 & 4.837 & 4.826 & 4.791 \\
\hline 6.864 & 19.296 & 19.109 & 18.568 & 4.870 & 4.858 & 4.822 \\
\hline 1.864 & 19.492 & 19.301 & 18.748 & 4.877 & 4.865 & 4.829 \\
\hline-3.136 & 19.321 & 19.133 & 18.589 & 4.864 & 4.852 & 4.817 \\
\hline \multirow[t]{2}{*}{-8.136} & 18.762 & 18.585 & 18.070 & 4.826 & 4.814 & 4.779 \\
\hline & \multicolumn{6}{|c|}{$\begin{array}{l}\text { Personal Dose Equivalent } \mathrm{Hp}(10) \\
\left(\mathrm{rem} \cdot \mathrm{h}^{-1}\right)\end{array}$} \\
\hline 11.864 & 22.664 & 22.451 & 21.836 & 5.869 & 5.855 & 5.812 \\
\hline 6.864 & 23.411 & 23.184 & 22.527 & 5.908 & 5.894 & 5.850 \\
\hline 1.864 & 23.646 & 23.415 & 22.743 & 5.916 & 5.902 & 5.858 \\
\hline-3.136 & 23.441 & 23.212 & 22.552 & 5.901 & 5.887 & 5.843 \\
\hline \multirow[t]{2}{*}{-8.136} & 22.763 & 22.548 & 21.923 & 5.855 & 5.841 & 5.798 \\
\hline & \multicolumn{6}{|c|}{$\begin{array}{c}\text { Ambient Dose Equivalent } \mathrm{H}^{*}(10) \\
\left(\mathrm{rem} \cdot \mathrm{h}^{-1}\right)\end{array}$} \\
\hline 11.864 & 21.808 & 21.603 & 21.011 & 5.647 & 5.634 & 5.592 \\
\hline 6.864 & 22.527 & 22.308 & 21.676 & 5.685 & 5.671 & 5.630 \\
\hline 1.864 & 22.753 & 22.531 & 21.884 & 5.693 & 5.679 & 5.637 \\
\hline-3.136 & 22.556 & 22.336 & 21.700 & 5.678 & 5.665 & 5.623 \\
\hline-8.136 & 21.904 & 21.697 & 21.096 & 5.634 & 5.620 & 5.579 \\
\hline \multirow[t]{2}{*}{ Dose Factor } & Maximum & Minimum & Ratio & Maximum & Minimum & Ratio \\
\hline & \multicolumn{6}{|c|}{ 10-cm Range } \\
\hline ICRP-21 & 19.492 & 17.999 & 1.083 & 4.877 & 4.779 & 1.020 \\
\hline $\mathrm{Hp}(10)$ & 23.646 & 21.836 & 1.083 & 5.916 & 5.798 & 1.020 \\
\hline $\mathrm{H}^{*}(10)$ & 22.753 & 21.011 & 1.083 & 5.693 & 5.579 & 1.020 \\
\hline \multicolumn{7}{|c|}{ 5-cm Range } \\
\hline ICRP-21 & 19.492 & 19.109 & 1.020 & 4.877 & 4.852 & 1.005 \\
\hline Hp(10) & 23.646 & 23.184 & 1.020 & 5.916 & 5.887 & 1.005 \\
\hline $\mathrm{H}^{*}(10)$ & 22.753 & 22.308 & 1.020 & 5.693 & 5.665 & 1.005 \\
\hline
\end{tabular}




\section{CALCULATION SHEET}

\begin{tabular}{|c|c|c|c|}
\hline ProjeCt No.: & $\begin{array}{l}\text { CALC NO.: } \\
\text { PNNL-19273 } \\
\end{array}$ & REVISION NO.: $\underline{0}$ & SHEET NO.: $\underline{67 / 246}$ \\
\hline \multicolumn{4}{|c|}{$\frac{\text { PNNL-19273 }}{000^{252} \text { CF NEUTR }}$} \\
\hline PREPARED BY: R.J. TRAUB & DATE: $\underline{02 / 8 / 10}$ & REVIEWED BY: R.J.MCCONN & DATE: $03 / 21 / 10$ \\
\hline
\end{tabular}

Table 46. Neutron dose factors at the surface of a $10-\mathrm{cm} \times 10-\mathrm{cm}$ rectangle $50 \mathrm{~cm}$ and $100 \mathrm{~cm}$ from the center of the reference sphere. The ${ }^{252} \mathrm{Cf}$ source is in the pneumatic transfer tube assembly. The dose rectangle has been elevated $1.864 \mathrm{~cm}$. relative to the equator of the reference sphere. The activity is on the lower frit. The reference date is March 3, 2003. The emission rate is $4.85 \mathrm{e} 9 \mathrm{n} \cdot \mathrm{s}^{-1}$.

\begin{tabular}{|c|c|c|c|c|c|c|}
\hline \multirow[b]{3}{*}{$\begin{array}{l}Z \text { position } \\
(\mathrm{cm})\end{array}$} & \multirow{2}{*}{\multicolumn{3}{|c|}{$\begin{array}{l}50 \mathrm{~cm} \text { from center of the reference } \\
\text { sphere } \\
\text { horizontal position }(\mathrm{cm})\end{array}$}} & \multirow{2}{*}{\multicolumn{3}{|c|}{$\begin{array}{l}100 \mathrm{~cm} \text { from center of the reference } \\
\text { sphere } \\
\text { horizontal position }(\mathrm{cm})\end{array}$}} \\
\hline & & & & & & \\
\hline & 0 & 5 & 10 & 0 & 5 & 10 \\
\hline & \multicolumn{6}{|c|}{$\begin{array}{l}\text { ICRP-21 Appendix } 6 \\
\left(\mathrm{pSv} . \mathrm{cm}^{2}\right)\end{array}$} \\
\hline 11.864 & 323.4 & 323.4 & 323.4 & 323.4 & 323.4 & 323.4 \\
\hline 6.864 & 323.4 & 323.4 & 323.4 & 323.4 & 323.4 & 323.4 \\
\hline 1.864 & 323.4 & 323.4 & 323.4 & 323.4 & 323.4 & 323.4 \\
\hline-3.136 & 323.4 & 323.4 & 323.4 & 323.4 & 323.4 & 323.4 \\
\hline \multirow[t]{2}{*}{-8.136} & 323.4 & 323.5 & 323.5 & 323.4 & 323.4 & 323.4 \\
\hline & \multicolumn{6}{|c|}{$\begin{array}{l}\text { Personal Dose Equivalent Hp(10) } \\
\text { (pSv.cm²) }\end{array}$} \\
\hline 11.864 & 392.4 & 392.4 & 392.4 & 392.4 & 392.4 & 392.4 \\
\hline 6.864 & 392.3 & 392.3 & 392.3 & 392.4 & 392.4 & 392.4 \\
\hline 1.864 & 392.3 & 392.3 & 392.4 & 392.4 & 392.4 & 392.4 \\
\hline-3.136 & 392.4 & 392.4 & 392.4 & 392.4 & 392.4 & 392.4 \\
\hline \multirow[t]{2}{*}{-8.136} & 392.4 & 392.4 & 392.4 & 392.4 & 392.4 & 392.4 \\
\hline & \multicolumn{6}{|c|}{$\begin{array}{c}\text { Ambient Dose Equivalent } \mathrm{H}^{*}(10) \\
\left(\mathrm{pSv} \cdot \mathrm{cm}^{2}\right)\end{array}$} \\
\hline 11.864 & 377.6 & 377.6 & 377.6 & 377.5 & 377.5 & 377.5 \\
\hline 6.864 & 377.5 & 377.5 & 377.5 & 377.5 & 377.5 & 377.5 \\
\hline 1.864 & 377.5 & 377.5 & 377.5 & 377.6 & 377.6 & 377.6 \\
\hline-3.136 & 377.5 & 377.5 & 377.6 & 377.6 & 377.6 & 377.6 \\
\hline-8.136 & 377.6 & 377.6 & 377.6 & 377.6 & 377.6 & 377.6 \\
\hline \multirow[t]{2}{*}{ Dose Factor } & Maximum & Minimum & Ratio & Maximum & Minimum & Ratio \\
\hline & \multicolumn{6}{|c|}{ 10-cm Range } \\
\hline ICRP-21 & 323.5 & 323.4 & 1.000 & 323.4 & 323.4 & 1.000 \\
\hline Hp(10) & 392.4 & 392.3 & 1.000 & 392.4 & 392.4 & 1.000 \\
\hline \multirow[t]{2}{*}{$\mathrm{H}^{*}(10)$} & 377.6 & 377.5 & 1.000 & 377.6 & 377.5 & 1.000 \\
\hline & \multicolumn{6}{|c|}{ 5-cm Range } \\
\hline ICRP-21 & 323.4 & 323.4 & 1.000 & 323.4 & 323.4 & 1.000 \\
\hline Hp(10) & 392.4 & 392.3 & 1.000 & 392.4 & 392.4 & 1.000 \\
\hline $\mathrm{H}^{*}(10)$ & 377.5 & 377.5 & 1.000 & 377.6 & 377.5 & 1.000 \\
\hline
\end{tabular}




\section{CALCULATION SHEET}

PROJECT NO:

CALC NO:

REVISION NO.: $\underline{0}$

SHEET NO.: $\underline{68 / 246}$

PNNL-19273

SubJeCt: CAlCUlations the SR-CF-3000 ${ }^{252}$ CF NeUtron SOURCE IN 318Low SCATTER RoOM

PREPARED BY: R.J. TRAUB DATE: 02/8/10 REVIEWED BY: R.J.MCCONN

DATE: $\underline{03 / 21 / 10}$

The data show that when the $\mathrm{D}_{2} \mathrm{O}$ moderating sphere is present the dose rates are highly asymmetric across the face of the dose plane. The highest dose rate is at the upper center and the lowest dose rate is at the lower edges. At $50 \mathrm{~cm}$ from the center of the $\mathrm{D}_{2} \mathrm{O}$ moderating sphere, the ratio of the maximum to minimum dose rate is 1.18 and at $100 \mathrm{~cm} 1.08$.

The neutron fluence-to-dose-equivalent conversion factors calculated for this report are summarized and compared to published values in Table 47. The calculated coefficients for the unmoderated ${ }^{252} \mathrm{Cf}$ spectra agree very well with published reference values, within about $2 \%$. The calculated coefficients for the $\mathrm{D}_{2} \mathrm{O}$ moderated ${ }^{252} \mathrm{Cf}$ spectra, however, differ from published reference values by about $22 \%$. The PNNL coefficients are higher than the published values which could be interpreted to mean that the PNNL $\mathrm{D}_{2} \mathrm{O}$ moderated spectrum is harder, i.e. higher average energy, than the spectrum published by the ISO (2000), which formed the basis for the published coefficients. Figure 13 shows the ISO and PNNL spectra for $\mathrm{D}_{2} \mathrm{O}$ moderated ${ }^{252} \mathrm{Cf}$ neutrons. This figure clearly shows that the moderated portion of the ISO spectra tends to lower energy than does the PNNL spectra.

The cause of the discrepancy in the $\mathrm{D}_{2} \mathrm{O}$ moderated spectra is likely due to enhanced void region that surrounds the ${ }^{252} \mathrm{Cf}$ source in the PNNL $\mathrm{D}_{2} \mathrm{O}$ moderating sphere (see Figures 5.a and 5.b). The description of the source and $\mathrm{D}_{2} \mathrm{O}$ moderating sphere, described in ISO 8529-1:2000(E), had a small void region. Because neutrons are not moderated in a void or metals, the PNNL $\mathrm{D}_{2} \mathrm{O}$ moderating geometry would be expected to result in higher energy neutrons than would the ISO geometry.

Table 47. Comparison of neutron fluence-to-dose-equivalent conversion factors published by international agencies and those prepared for this report.

\begin{tabular}{|c|c|c|c|c|}
\hline \multirow{3}{*}{ Quantity } & \multicolumn{4}{|c|}{ Calibration Field } \\
\hline & \multicolumn{2}{|c|}{ ISO spectra } & \multicolumn{2}{|c|}{ PNNL spectra } \\
\hline & Bare & $\mathrm{D}_{2} \mathrm{O}$ Moderated & Bare & $\mathrm{D}_{2} \mathrm{O}$ Moderated \\
\hline \multirow{2}{*}{$\begin{array}{l}\text { Ambient dose equivalent, } \mathrm{H}^{*}(10) \\
\text { Personal dose equivalent, } \mathrm{H}_{\mathrm{p}}(10)\end{array}$} & $385^{\mathrm{a}}$ & $105^{\mathrm{a}}$ & $378^{\mathrm{c}}$ & $128^{\mathrm{c}}$ \\
\hline & $400^{\mathrm{b}}$ & $110^{\mathrm{b}}$ & $392^{\mathrm{c}}$ & $133^{\mathrm{c}}$ \\
\hline \multicolumn{5}{|l|}{ a. ISO 8529-1:2000(E), IAEA (2001) } \\
\hline \multicolumn{5}{|l|}{ b. IAEA (2001) } \\
\hline \multicolumn{5}{|c|}{ c. Average of Upper Frit, Middle Void, and Lower Frit source locations } \\
\hline
\end{tabular}




\section{CALCULATION SHEET}

\begin{tabular}{|c|c|c|c|}
\hline PROJECT No.: & $\begin{array}{l}\text { CALC NO.: } \\
\text { PNNL-19273 }\end{array}$ & REVISION NO.: $\underline{0}$ & SHEET NO.: $\underline{69 / 246}$ \\
\hline SUBJECT: CALCULATIONS & $00{ }^{252}$ CF NEUTRO & CE IN 318LOW SCATTER ROOM & \\
\hline PREPARED BY: R.J. TRAUB & DATE: $\underline{02 / 8 / 10}$ & REVIEWED BY: R.J.MCCONN & DATE: $\underline{03 / 21 / 10}$ \\
\hline
\end{tabular}

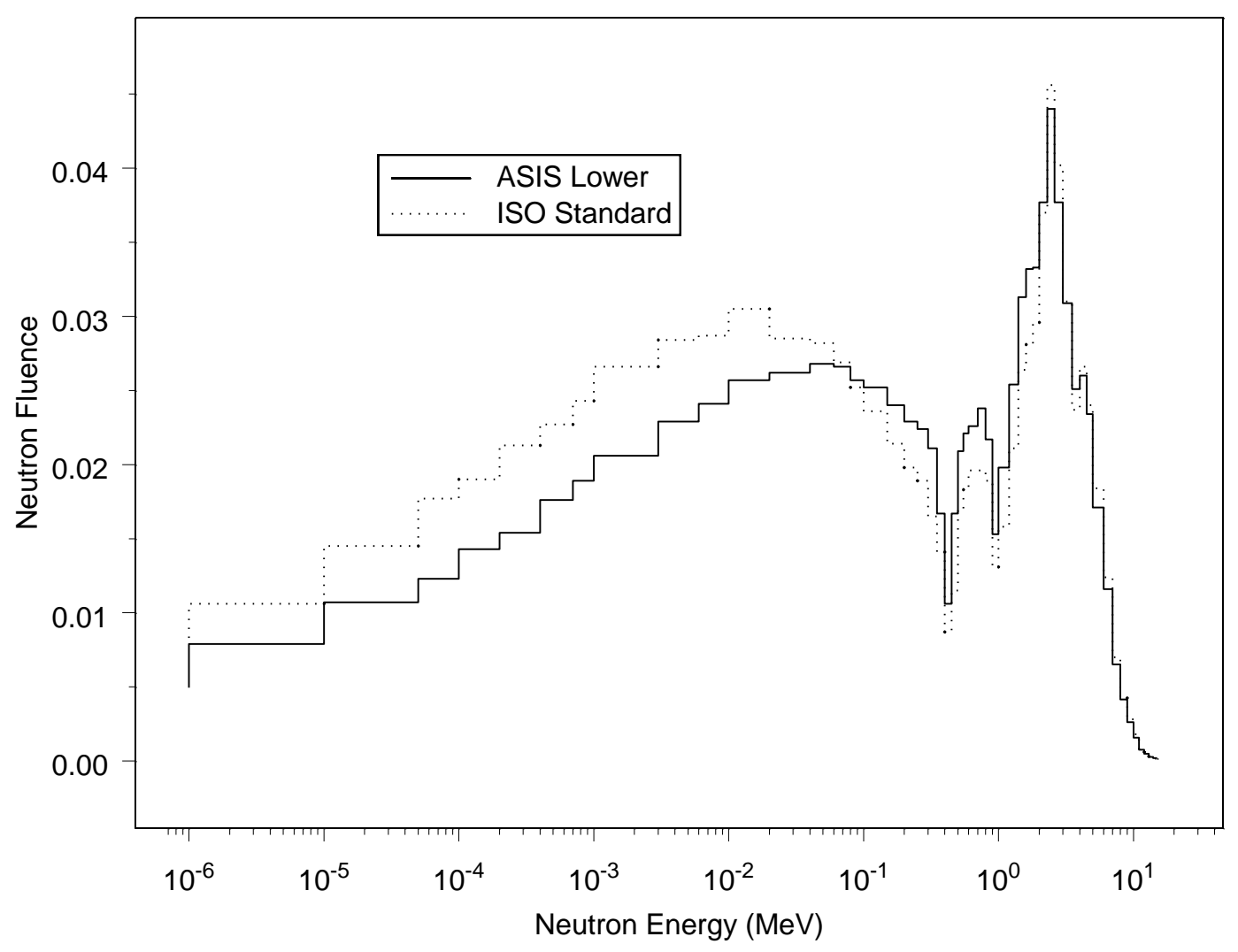

Figure 12. Neutron spectrum (lethargy plots) at $50 \mathrm{~cm}$ from the center of the $\mathrm{D}_{2} \mathrm{O}$ moderating sphere. The ASIS spectrum represents the spectrum on the equator of the $\mathrm{D}_{2} \mathrm{O}$ moderating sphere. The ${ }^{252} \mathrm{Cf}$ activity is assumed to be in the lower frit of the source encapsulation. The ISO standard spectrum was obtained from ISO 8529-1:2000(E) 


\section{CALCULATION SHEET}

\section{PROJECT NO.: \\ CALC NO.: \\ REVISION NO.: $\underline{0}$ \\ SHEET NO.: $70 / 246$ \\ PNNL-19273 \\ SubJeCt: CALCUlations THE SR-CF-3000 ${ }^{252}$ CF NEUTRON SOURCE IN 318 LOW SCATTER ROOM \\ PREPARED BY: R.J. TRAUB \\ DATE: $\underline{02 / 8 / 10}$ \\ REVIEWED BY: R.J.MCCONN \\ DATE: $\underline{03 / 21 / 10}$ \\ 6.0 Conclusion / Discussion}

The most important conclusion from these calculations is that the location of the ${ }^{252} \mathrm{Cf}$ activity in the SRCf-3000 source is not where it had been thought to be. The postulated source location was at the approximate center of the $\mathrm{D}_{2} \mathrm{O}$ moderating sphere. This was found not to be the case. The actual source location is approximately $2 \mathrm{~cm}$ above the equator of the moderating sphere. The $2-\mathrm{cm}$ offset is large when compared to the $15-\mathrm{cm}$ radius of the sphere.

The location of the ${ }^{252} \mathrm{Cf}$ source material with respect to the center of the $\mathrm{D}_{2} \mathrm{O}$ moderating sphere should be known to the users. The results of the calculations presented here show how the 2-cm offset can result in large variations between the calculated and postulated doses administered to dosimeters.

The calculations also indicate that the error associated with the incorrect positioning of the source activity is less important when the neutrons are unmoderated than when the neutrons are moderated. The difference is that, for the unmoderated case, the error is caused mainly by the error in estimated distance and is relatively small. In the case of the moderated neutrons, the error is caused by the distance traveled through the moderating medium and the geometry of the sphere. 


\section{CALCULATION SHEET}

\begin{tabular}{|c|c|c|c|}
\hline PROJECT No.: & $\begin{array}{l}\text { CALC NO.: } \\
\text { PNNL-19273 }\end{array}$ & REVISION NO.: $\underline{0}$ & SHEET NO.: $\underline{71 / 246}$ \\
\hline \multicolumn{4}{|c|}{$\frac{\text { PNNL-19273 }}{3000^{252} \mathrm{CF} \text { NEUTRO }}$} \\
\hline PREPARED BY: R.J. TRAUB & DATE: $\underline{02 / 8 / 10}$ & REVIEWED BY: R.J.MCCONN & DATE: $\underline{03 / 21 / 10}$ \\
\hline
\end{tabular}

1. X-5 Monte Carlo Team. 2003. MCNP - A General Monte Carlo N-Particle Transport Code, Version 5. Volume II: User's Guide. LA-CP-03-0245. Los Alamos National Laboratory. Los Alamos, NM. MCNP is available from the Radiation Safety Information Computational Center at www-rsicc.ornl.gov.

2. Cleveland WS and SJ Devlin, (1988) Locally-weighted Regression: An Approach to Regression Analysis by Local Fitting. J. Am. Statist. Assoc., Vol. 83, pp 596-610.

3. Harvey JR 1987. The Individual Monitoring Quantity for Neutrons and its relationship with fluence. Radiation Protection Dosiometry 20(1/2):19-24.

4. Hertel, NE and JC McDonald. 1990. Calculations of Aniostrophy Factors and Dose Equivalents for Unmoderated ${ }^{252}$ Cf Sources. Radiation Protection Dosimetry 32(2):77-82.

5. Hertel, NE and JC McDonald. 1991. Calculated Dosimetric Quantities for $\mathrm{D}_{2} \mathrm{O}$ moderated ${ }^{252} \mathrm{Cf}$ Sources. Radiation Protection Dosimetry. 35(1):23-30.

6. International Atomic Energy Agency (IAEA). 2001. Compendium of Neutron Spectra and Detector Responses for Radiation Protection Purposes. Supplement to Technical Reports Series No. 318. Technical Series Reports No. 403. International Atomic Energy Agency, Vienna, Austria.

7. International Commission on Radiological Protection (ICRP). 1973. Data for Protection against Ionizing Radiation Protection from External Sources, ICRP Publication 21. Pergamon Press, Oxford.

8. International Commission on Radiological Protection (ICRP). 1996. Conversion Coefficients for use in Radiological Protection against External Radiation, ICRP Publication 74. Annals of the ICRP 26(3/4):1-204.

9. International Organization for Standardization (ISO). 2000. Reference neutron radiations - Part 1: Characteristics and methods of production. ISO 8529-1:2000(E). ISO, Geneva, Switzerland.

10. Shores EF. 2000. "Data Updates for the SOURCES-4A Computer Code," LA-UR-00-5016.

11. Weisstein, E.W. "Latitude.” From MathWorld - A Wolfram Web Reseouce. http://mathworld.wolfram.com/Latitude.html. (referenced: 27-July-2009).

12. Weisstein, E.W. "Spherical Coordinates.” From MathWorld - A Wolfram Web Resource. http://mathworld.wolfram.com/SphericalCoordinates.html. (referenced: 27-July-2009). 


\section{CALCULATION SHEET}

PROJECT NO.:

CALC NO.:

REVISION NO.: $\underline{0}$

SHEET NO.: $72 / 246$

$\underline{\text { PNNL-19273 }}$

SubJECT: CAlCUlations THE SR-CF-3000 ${ }^{252}$ CF NEUTRON SOURCE IN 318LOW SCATTER RoOM

PREPARED BY: R.J. TRAUB DATE: $\underline{02 / 8 / 10}$ REVIEWED BY: R.J.MCCONN

DATE: $\underline{03 / 21 / 10}$

13. Wilson WB, RT Perry, WS Charlton,TA Parish,GP Estes, TH Brown, ED Arthur, M Bozoian, TR England, DG Madland and JE Stewart. 1999. SOURCES 4A: A Code for Calculating (a,n), Spontaneous Fission, and Delayed Neutron Sources and Spectra. LA-13639-MS. Los Alamos National Laboratory, Los Alamos, NM 87545. Code available from RSICC (http://wwwrsicc.ornl.gov) as code package CCC-661, Sources-4C. 


\section{CALCULATION SHEET}

PROJECT NO.:

CALC NO:

REVISION NO.: $\underline{0}$

SHEET NO.: $\underline{73 / 246}$

PNNL-19273

SubJeCt: CALCULATIONS THE SR-CF-3000 ${ }^{252}$ CF NEUTRON SOURCE IN 318 LOW SCATTER RoOM

PREPARED BY: R.J. TRAUB $\quad$ DATE: $\underline{02 / 8 / 10}$ REVIEWED BY: R.J.MCCONN

DATE: $03 / 21 / 10$

\section{Appendix A}

\section{MCNP Development Files}

This Appendix contains the files that were used to develop the MCNP input files. These geometry development files contain the cell and surface definitions and show the evolution of the MCNP geometry from the primary encapsulation to the full geometry that includes the pneumatic transfer tubing and the $\mathrm{D}_{2} \mathrm{O}$ moderating sphere. These MCNP input files contain only enough information so that Vised won't throw an error when it reads them.

Table A.1 identifies the files and links the files to the geometry figures shown in the main text of the report.

\begin{tabular}{|c|c|c|}
\hline $\begin{array}{c}\text { File Name } \\
\text { (append with monp.inp) }\end{array}$ & $\begin{array}{l}\text { Main Text } \\
\text { Figure \# }\end{array}$ & What's There \\
\hline Sr3000_Primary & 1.a & $\begin{array}{l}\text { MCNP input file. Surface values should exactly match the } \\
\text { values in DWG M-12541-CP-338E. }\end{array}$ \\
\hline Sr3000_Primary_flip & $1 . \mathrm{b}$ & $\begin{array}{l}\text { Inverted version of CR3000_Primary. Changed sign of PZ } \\
\text { surface cards. Changed sign of PZ surfaces in the cell } \\
\text { definitions. }\end{array}$ \\
\hline Sr3000_Secondary & 2.a & $\begin{array}{l}\text { The (empty) secondary encapsulation for the source } \\
\text { encapsulation }\end{array}$ \\
\hline Sr3000 SourceAssembly & $2 . b$ & The primary and secondary encapsulations together. \\
\hline Sr3000_TransferCapsule & $3 . \mathrm{a}$ & $\begin{array}{l}\text { The transfer capsule. Surface values match those in } \\
\text { "figure 2" }\end{array}$ \\
\hline Sr3000_TransferCapsuleAssembly & 3.b & The Sr-Cf-3000 source is in the transfer capsule. \\
\hline LSR_TransferTubeAssembly & 4.a & $\begin{array}{l}\text { The pneumatic transfer tube in the Low Scatter Room } \\
\text { (LSR). The surfaces match the surface heights in the LSR. }\end{array}$ \\
\hline Sr3000_TransferTubeAssembly & 4.b & $\begin{array}{l}\text { The pneumatic transfer tube in the Low Scatter Room } \\
\text { (LSR) with the transfer capsule assembly (Fig 3.b) } \\
\text { inserted. }\end{array}$ \\
\hline LSR_D2O_Sphere & 5.a & $\begin{array}{l}\text { The } \mathrm{D}_{2} \mathrm{O} \text { moderating sphere. This sphere has a cadmium } \\
\text { cover. The surfaces place the sphere in the actual location } \\
\text { of the sphere in the LSR. }\end{array}$ \\
\hline SR3000_D2OSphere & $5 . b$ & $\begin{array}{l}\text { The } \mathrm{D}_{2} \mathrm{O} \text { moderating sphere with the transfer tube } \\
\text { assembly and transfer capsule assembly. }\end{array}$ \\
\hline
\end{tabular}




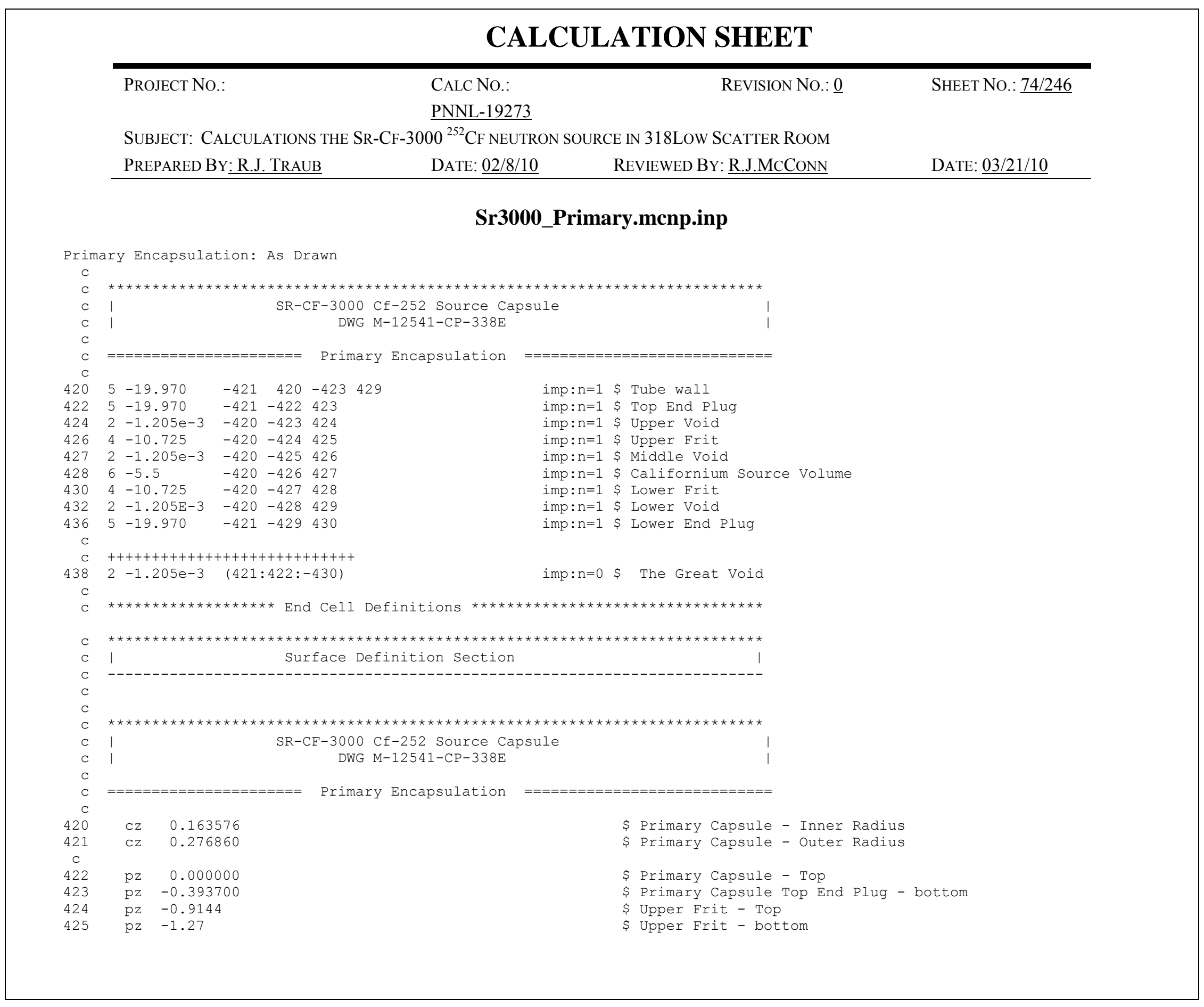




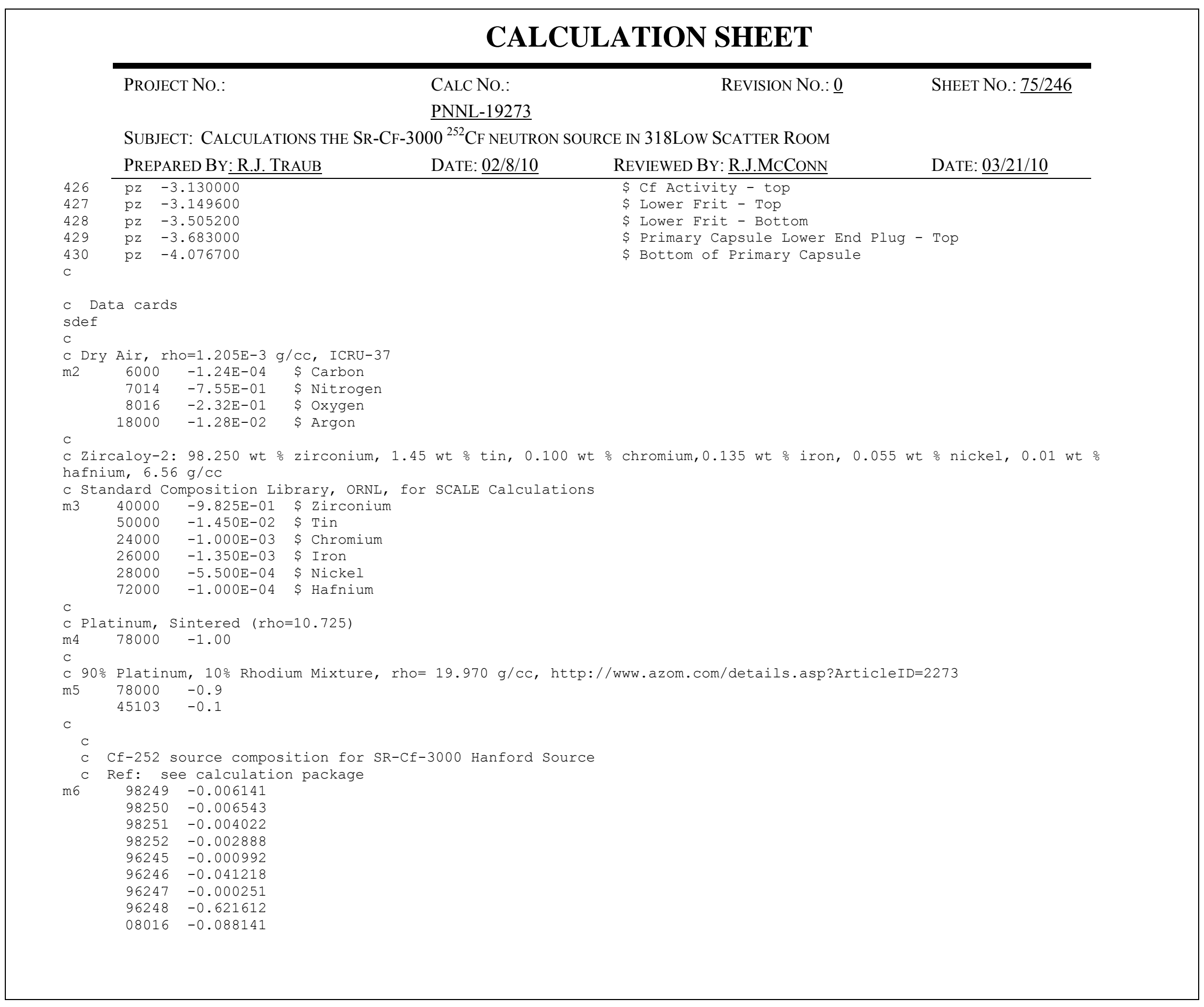




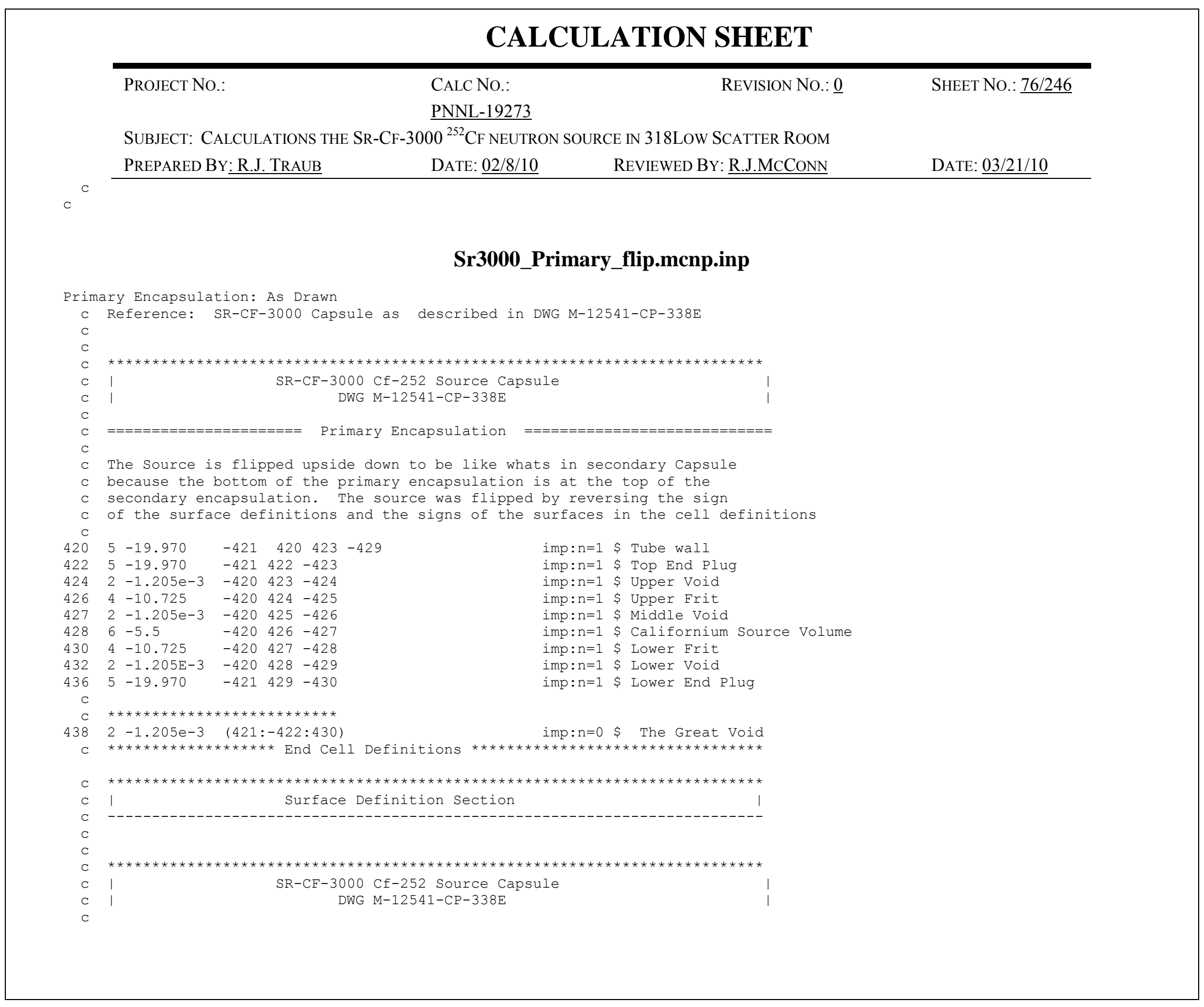




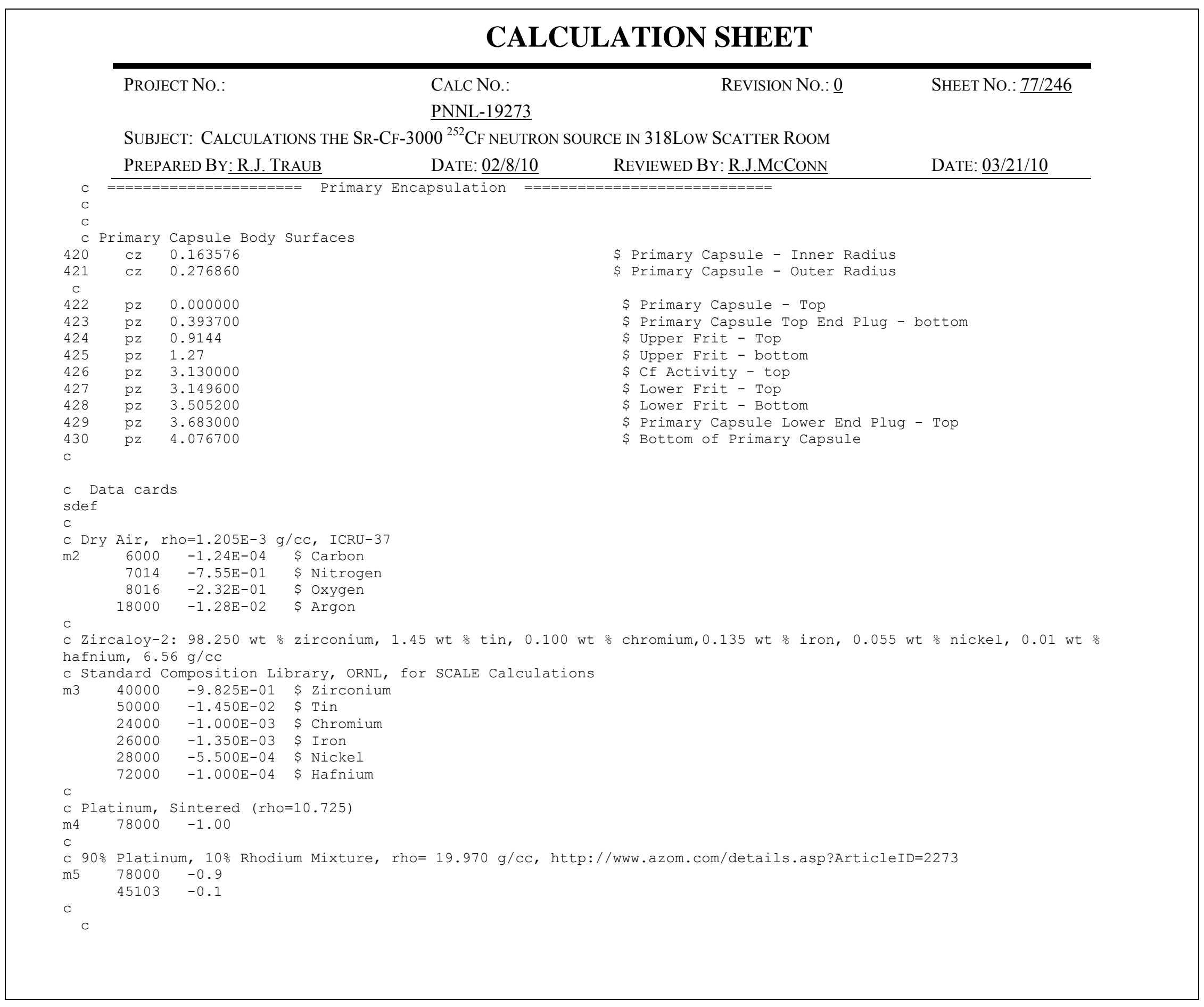




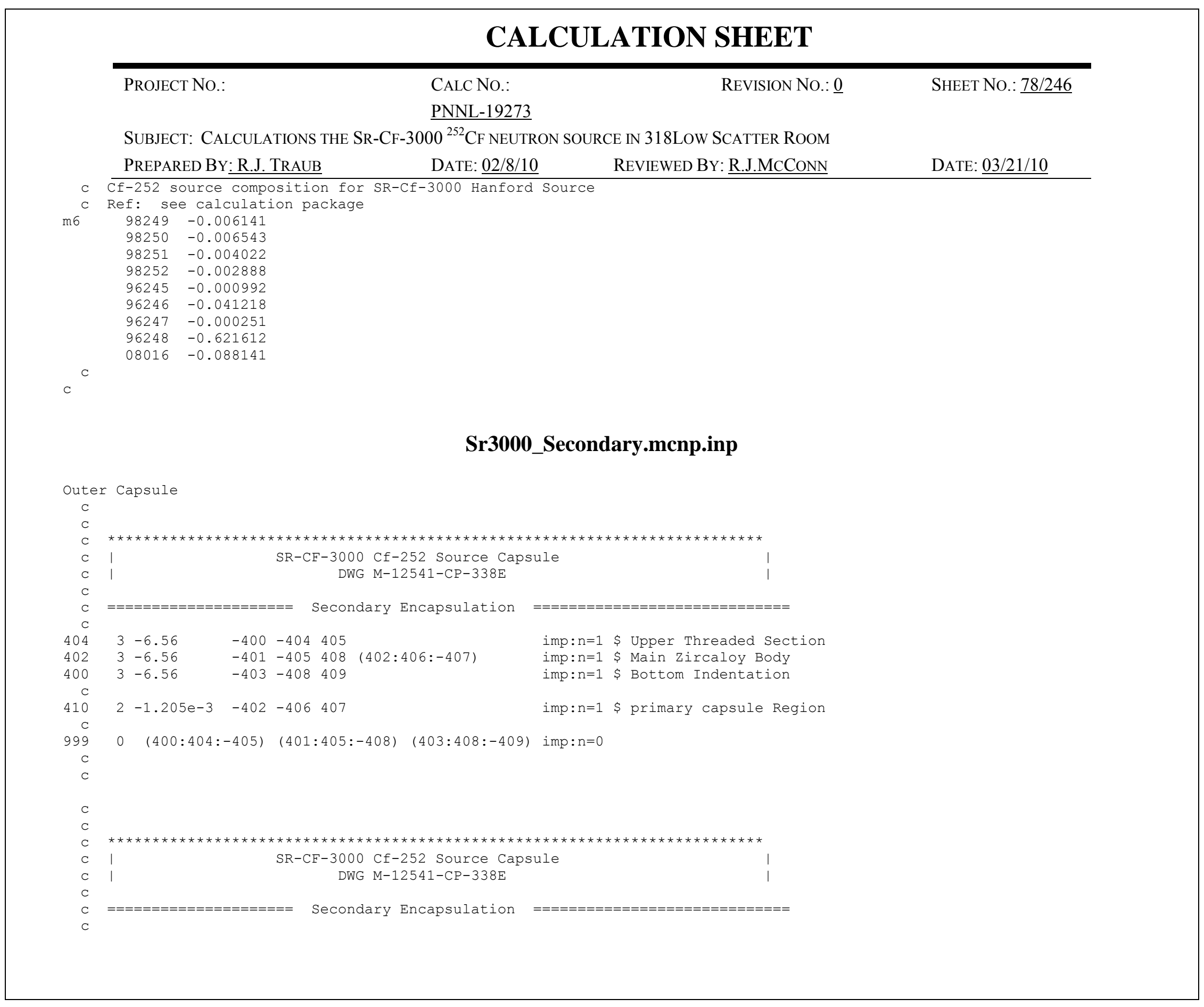




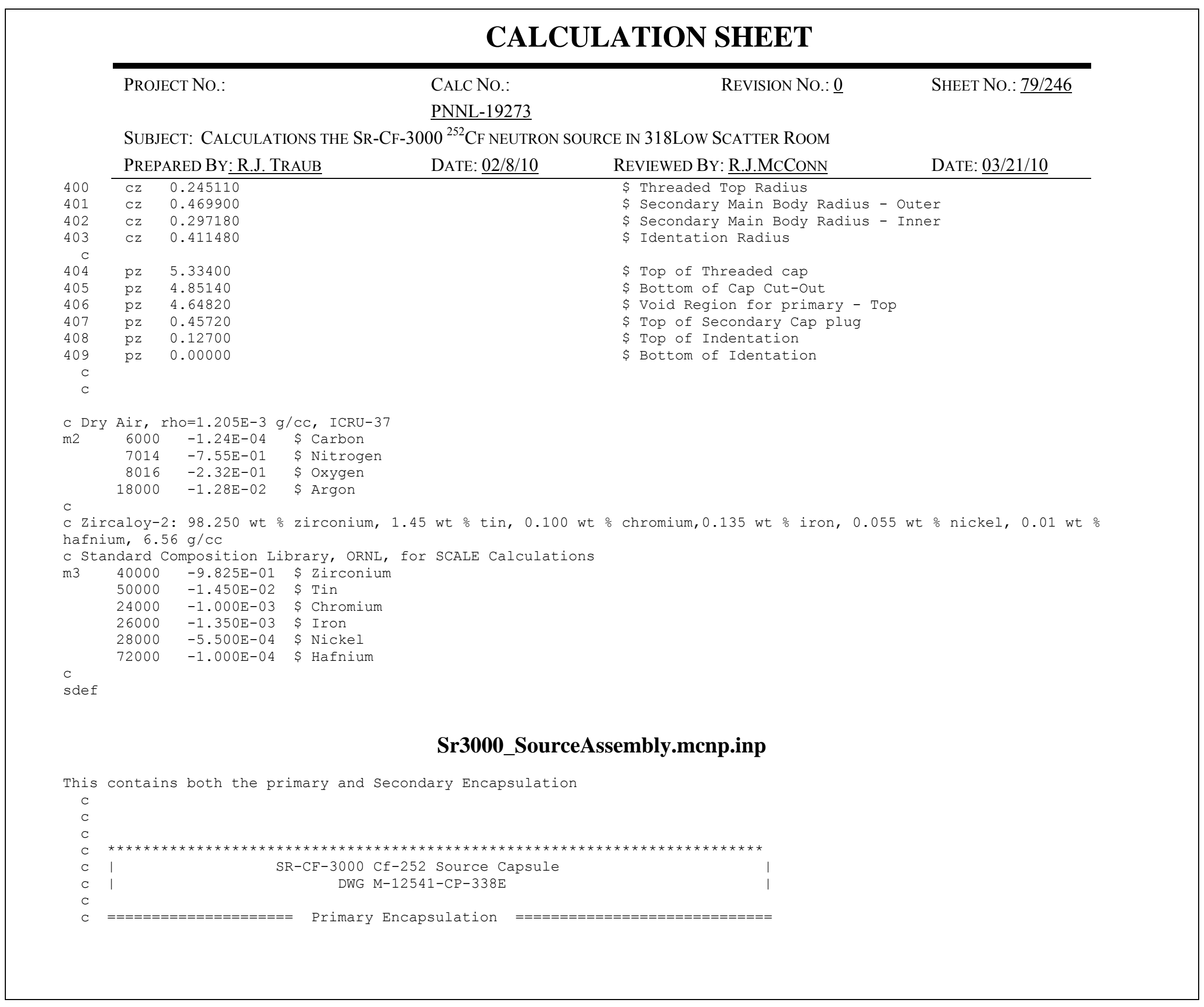




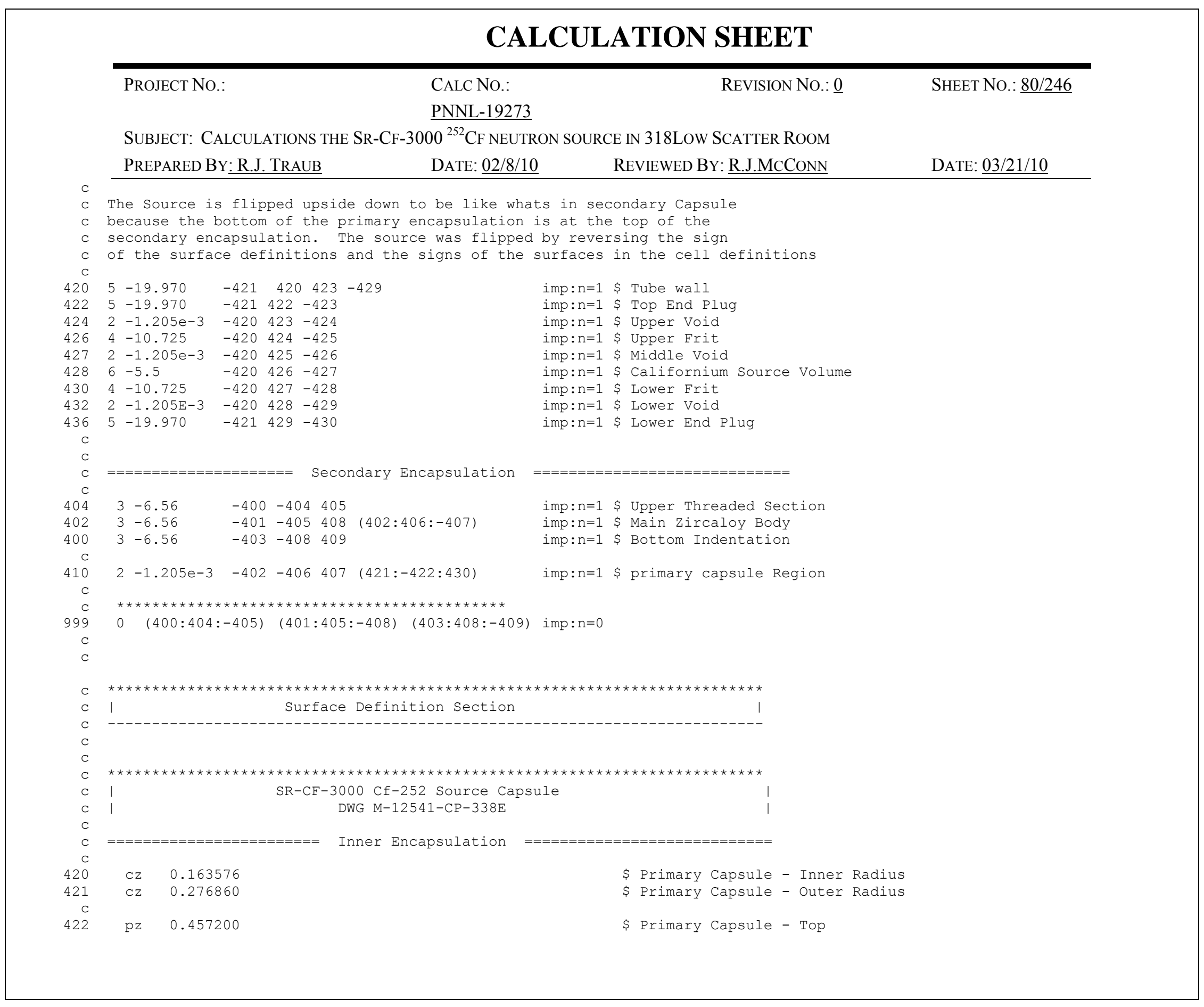




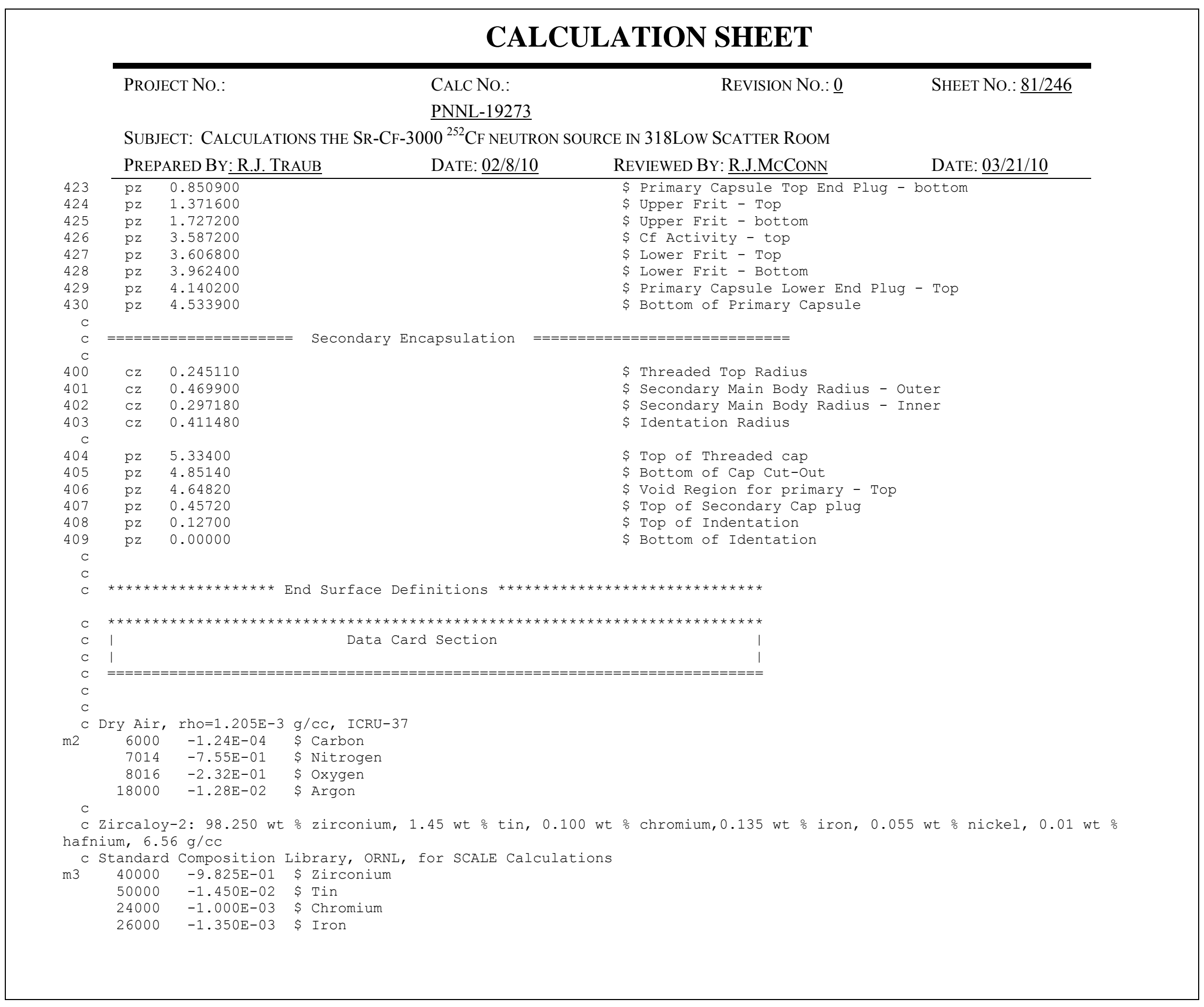




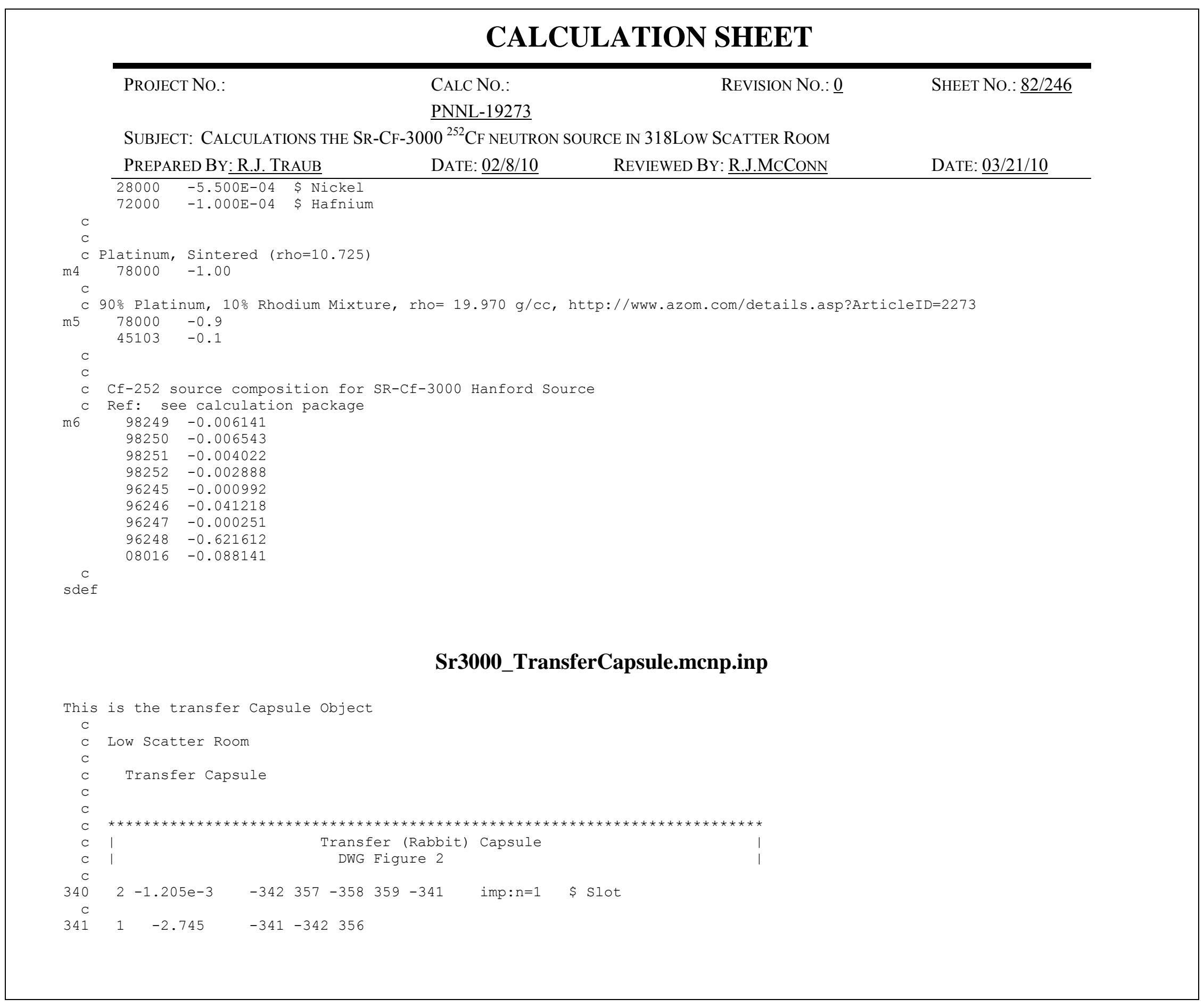




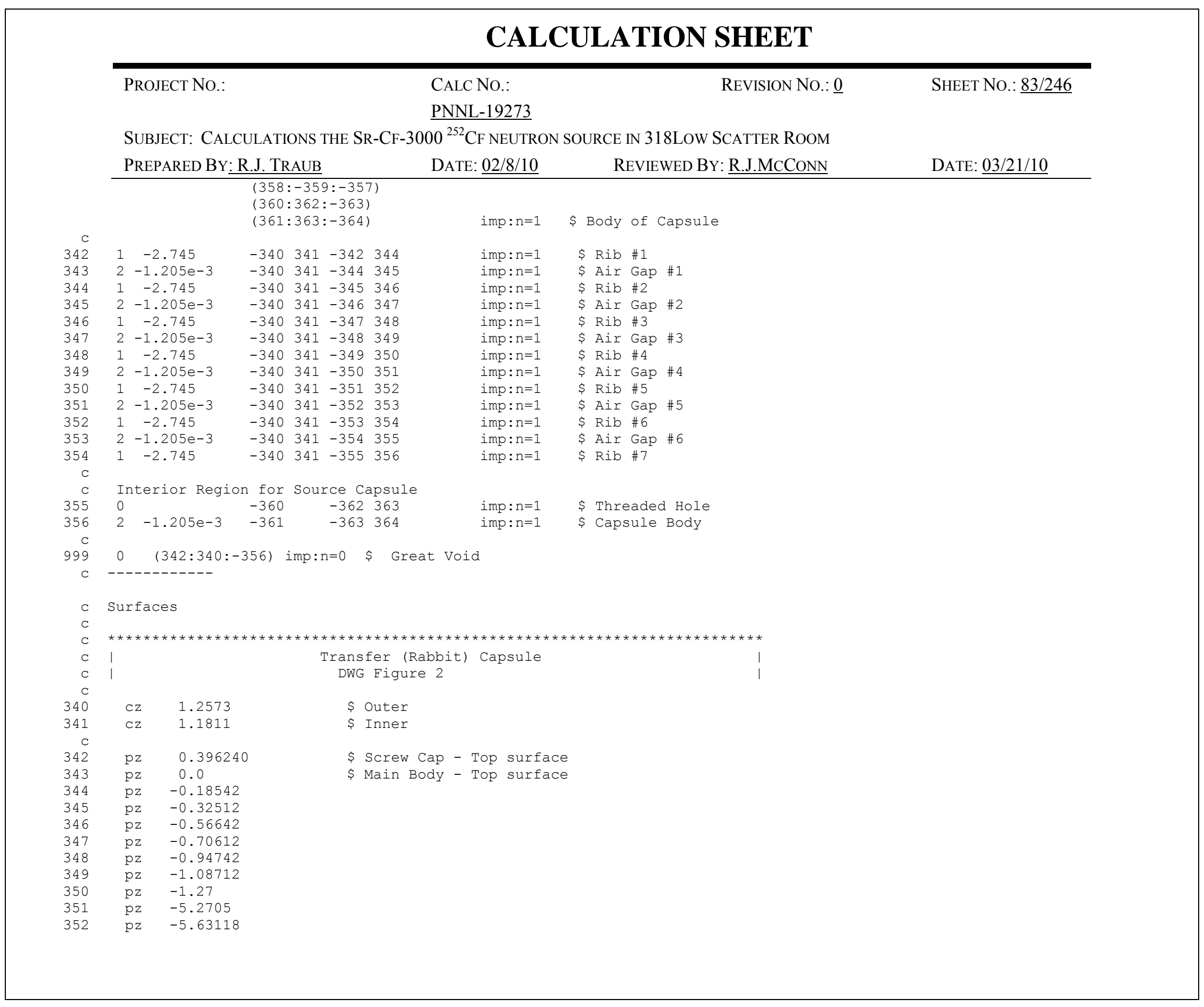




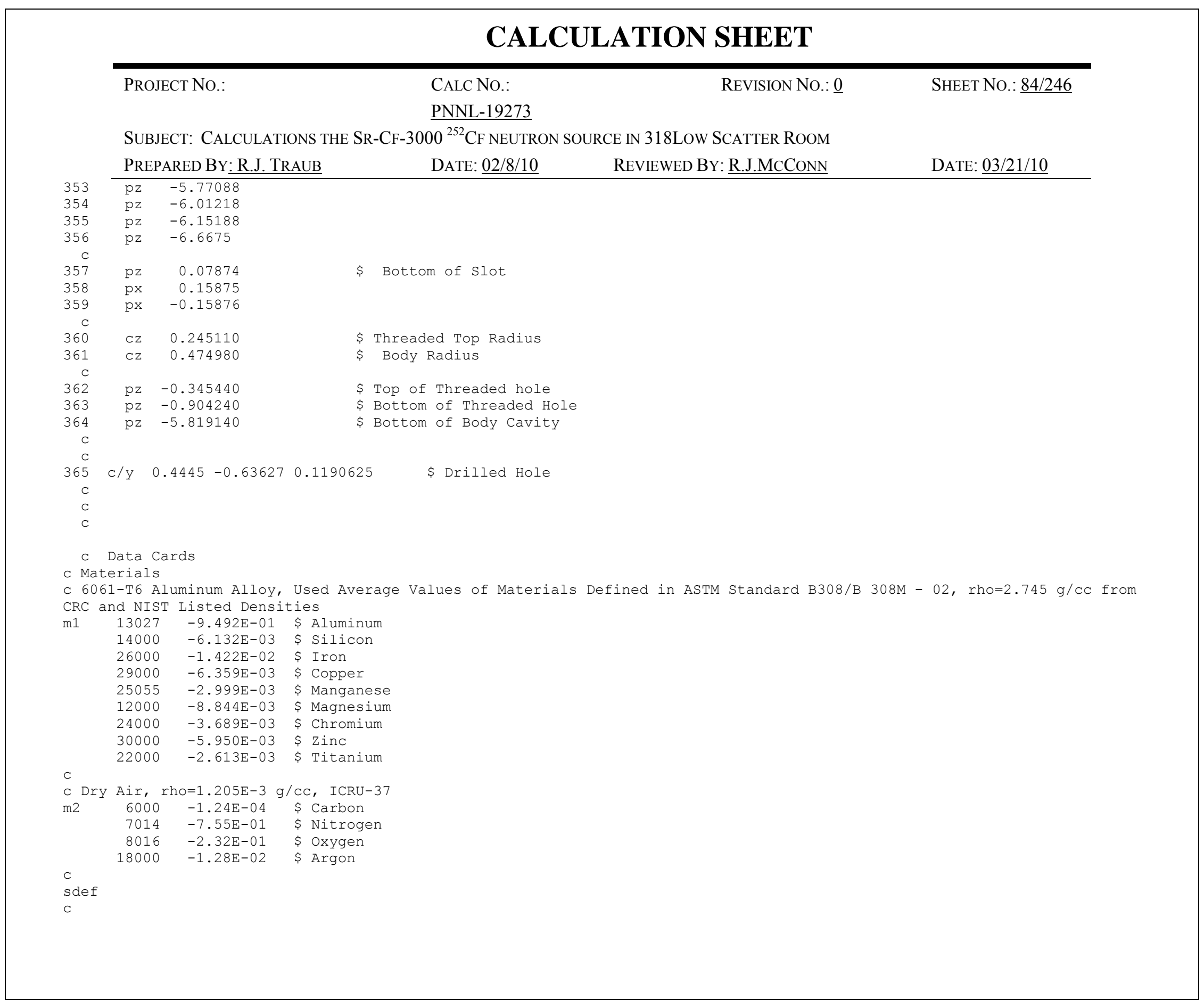




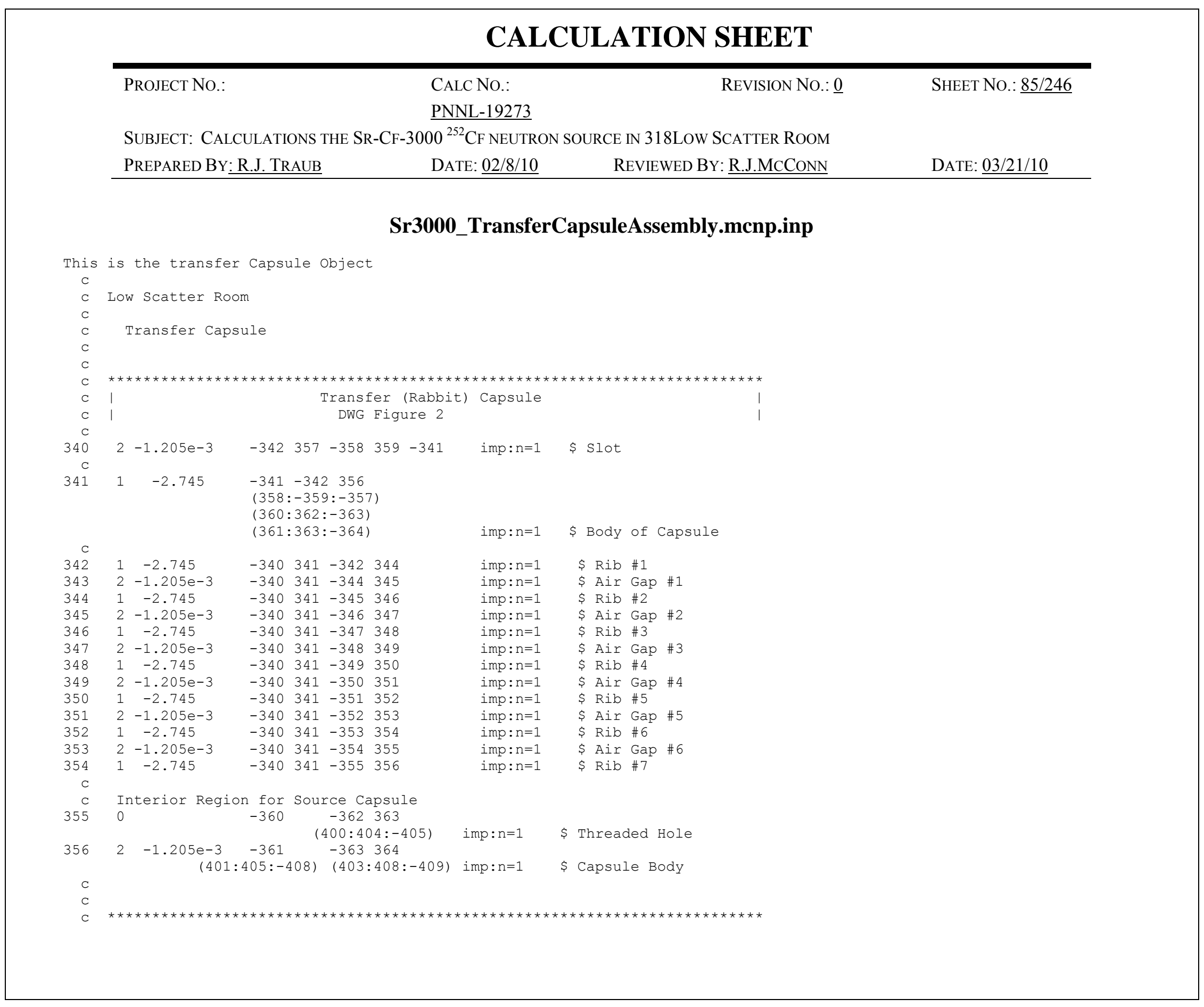




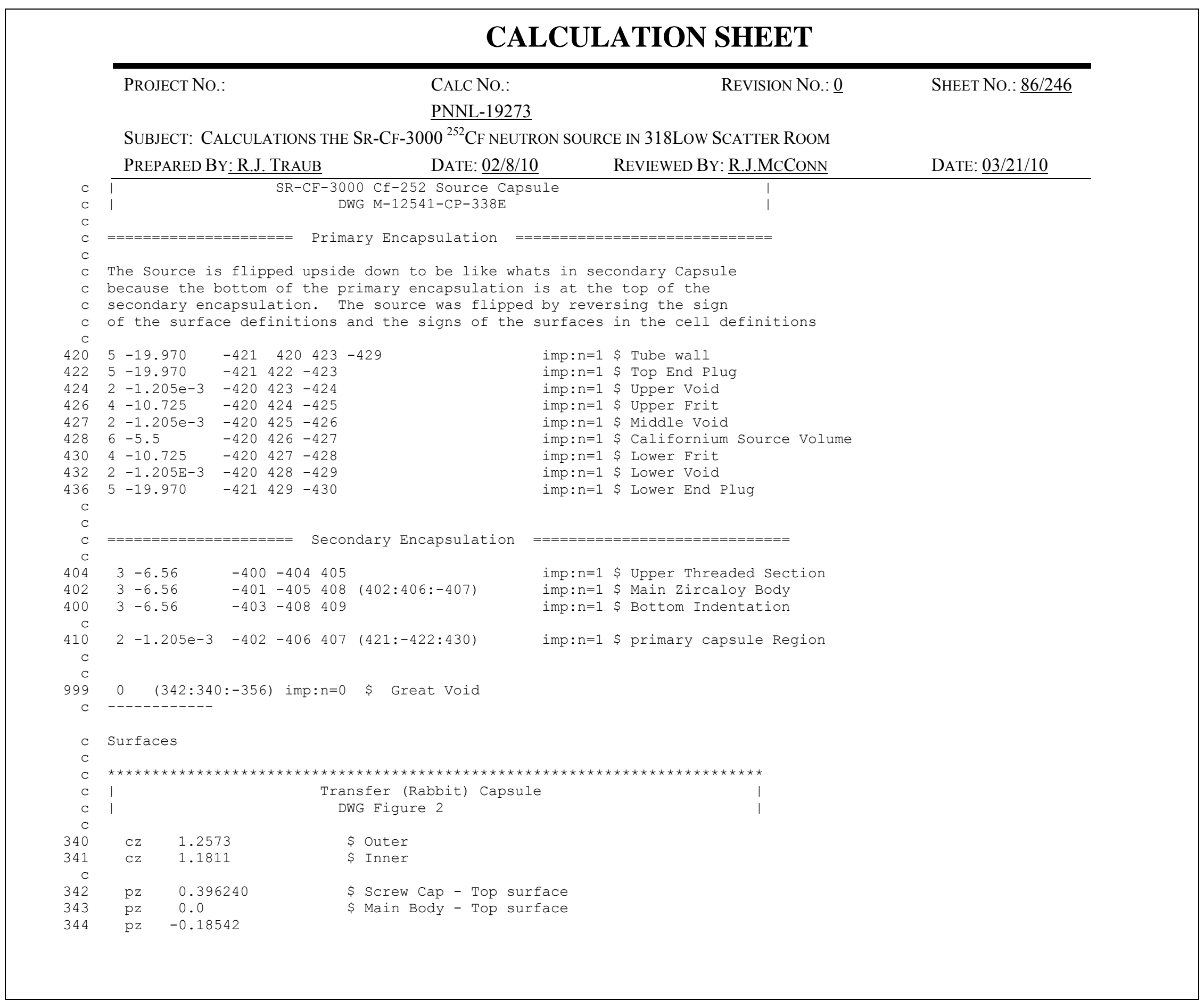




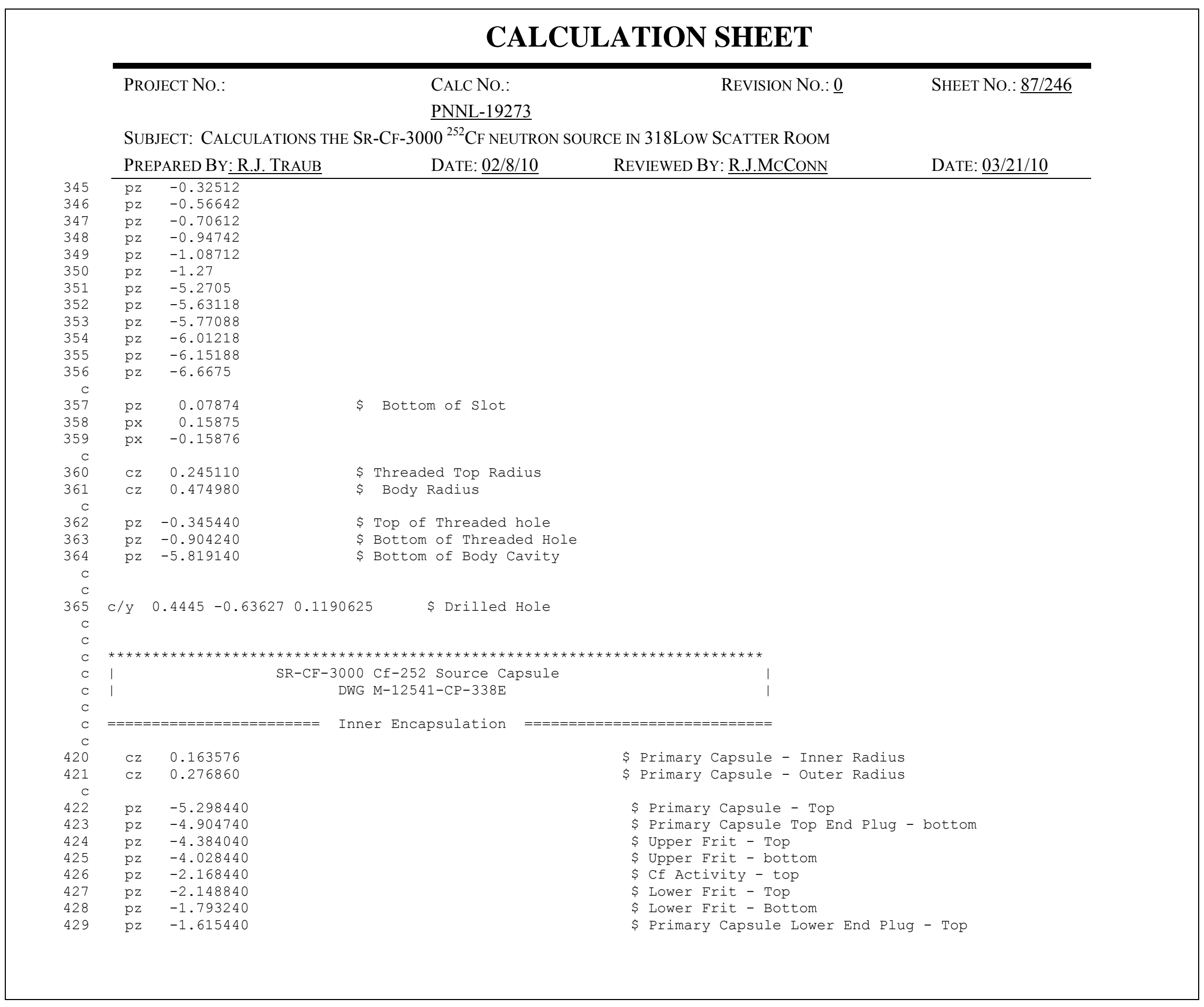




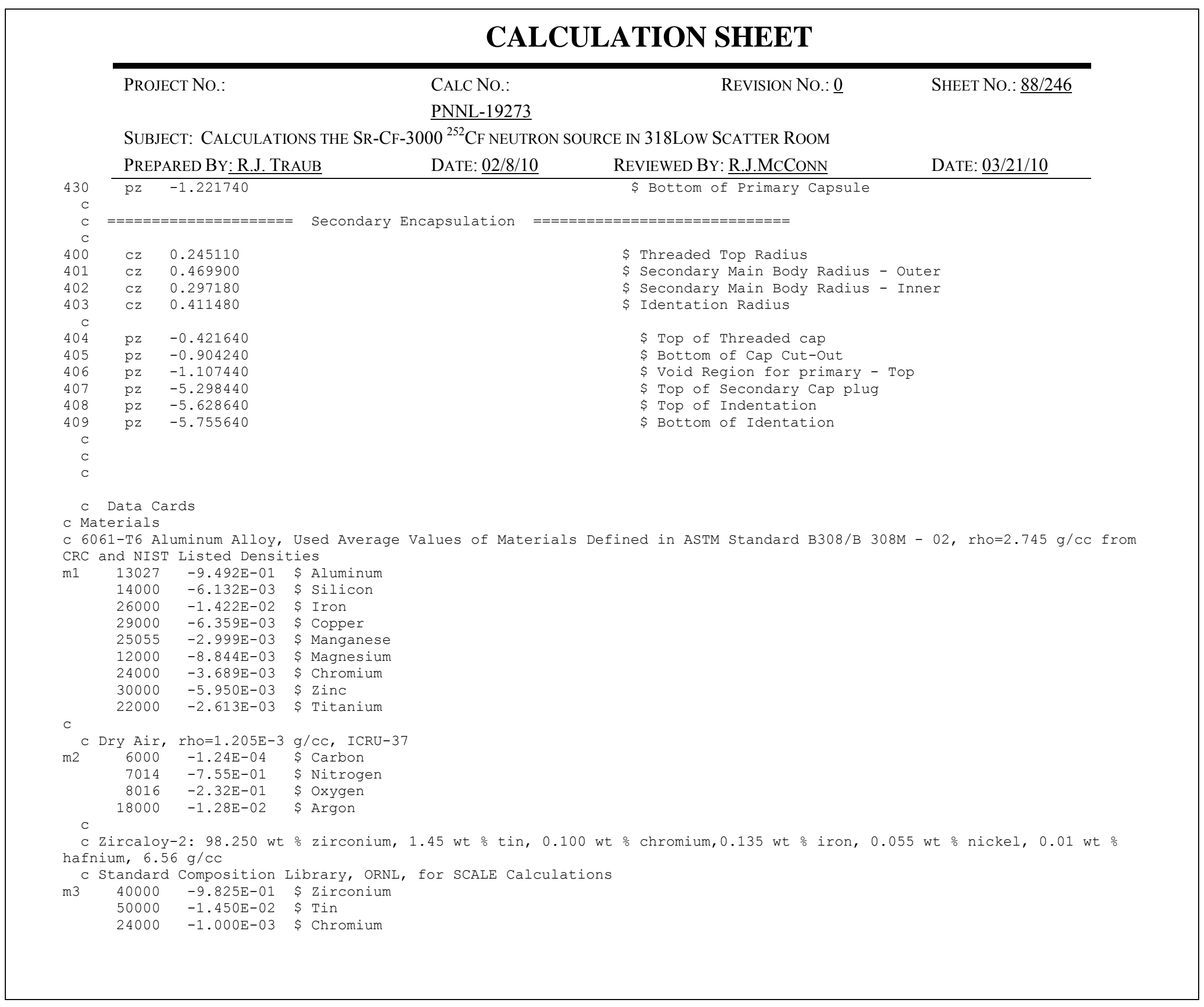




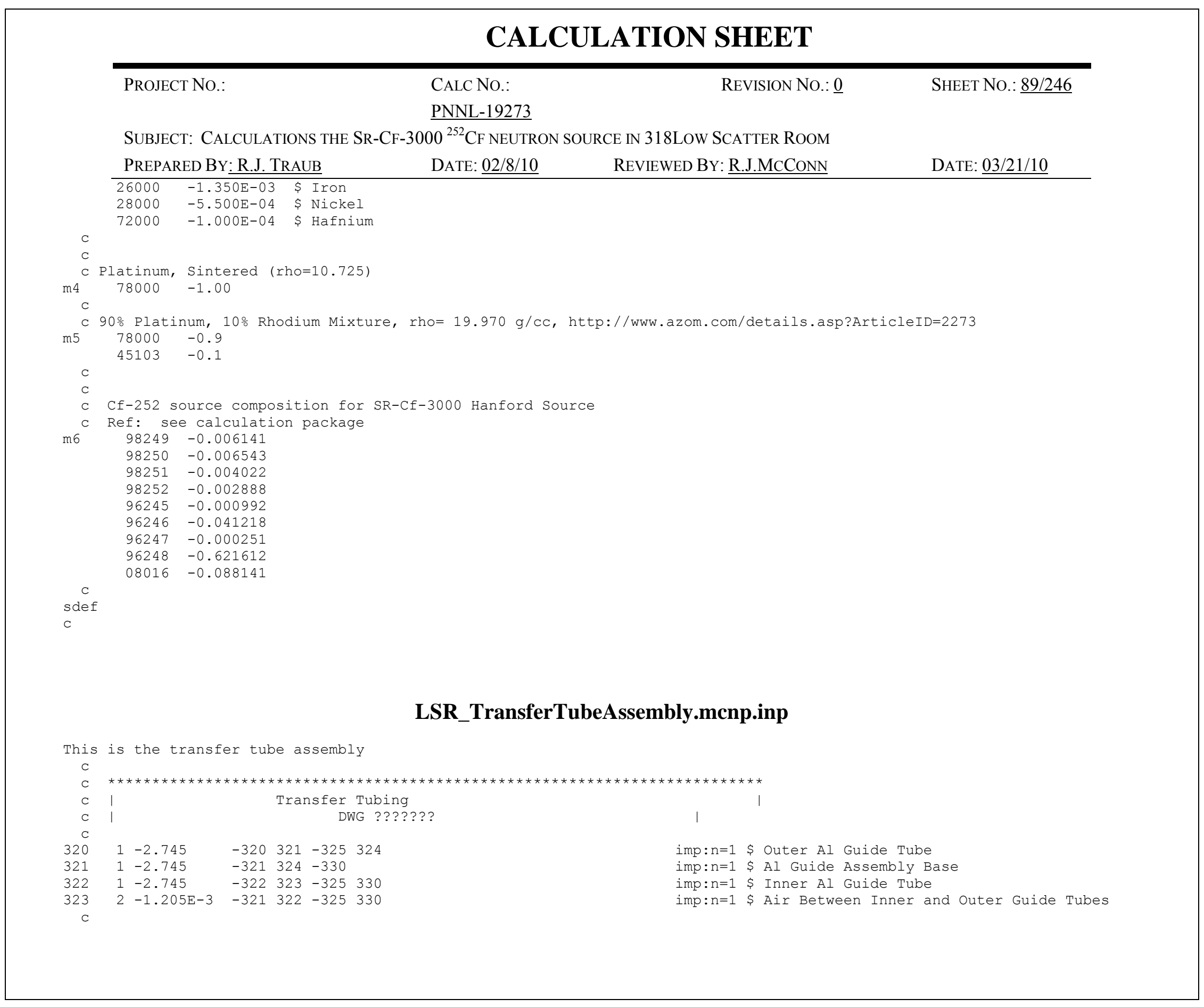




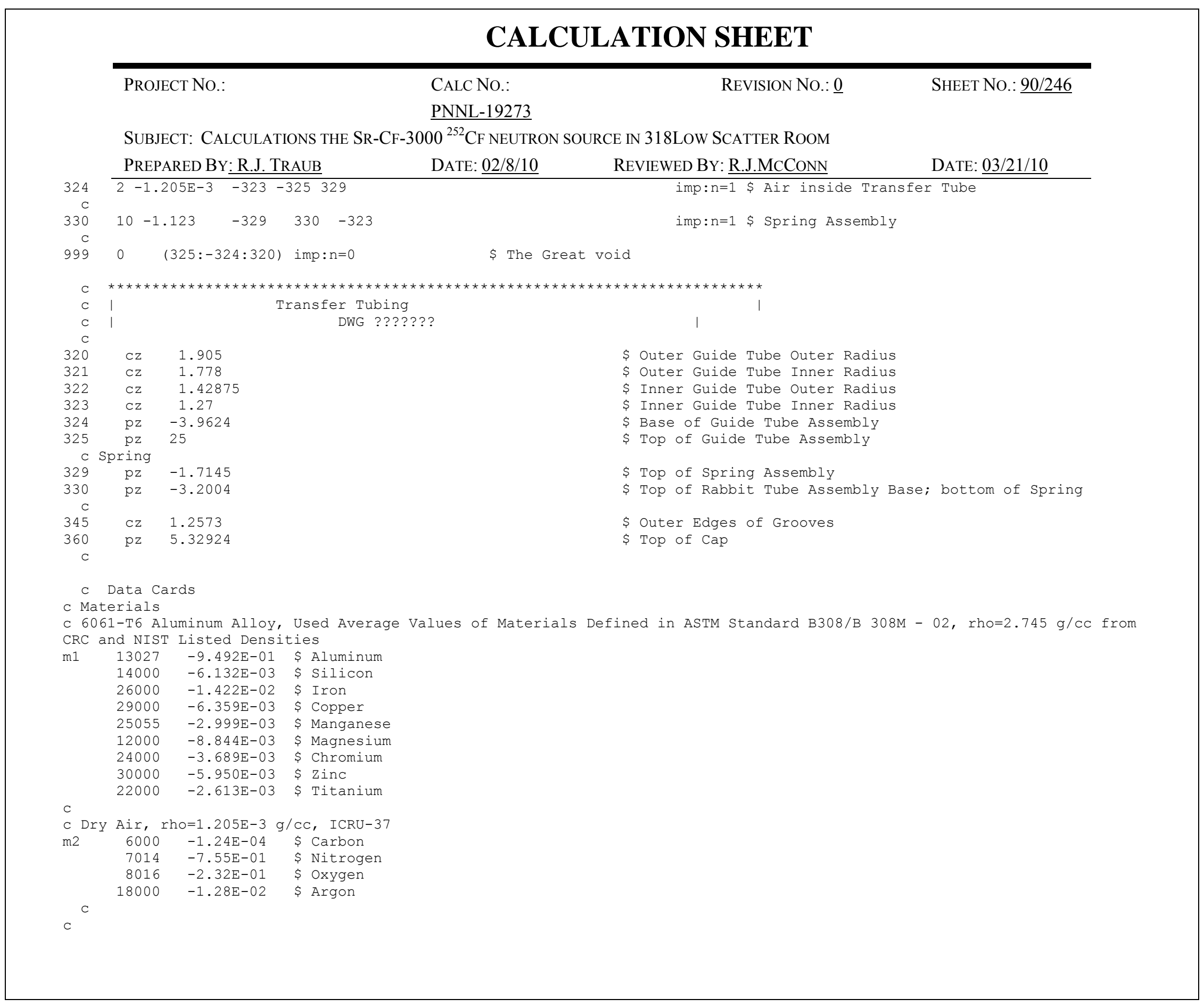




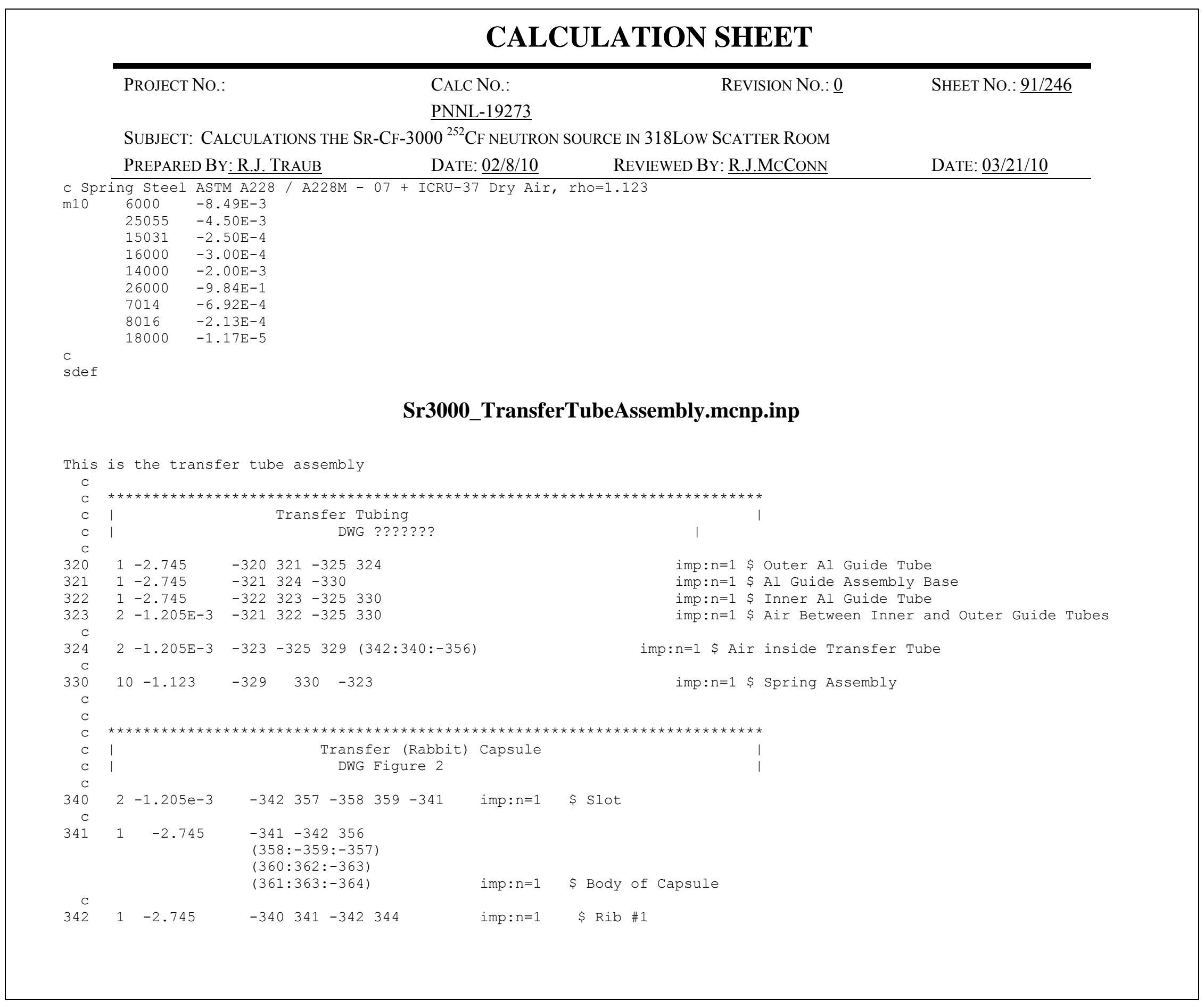




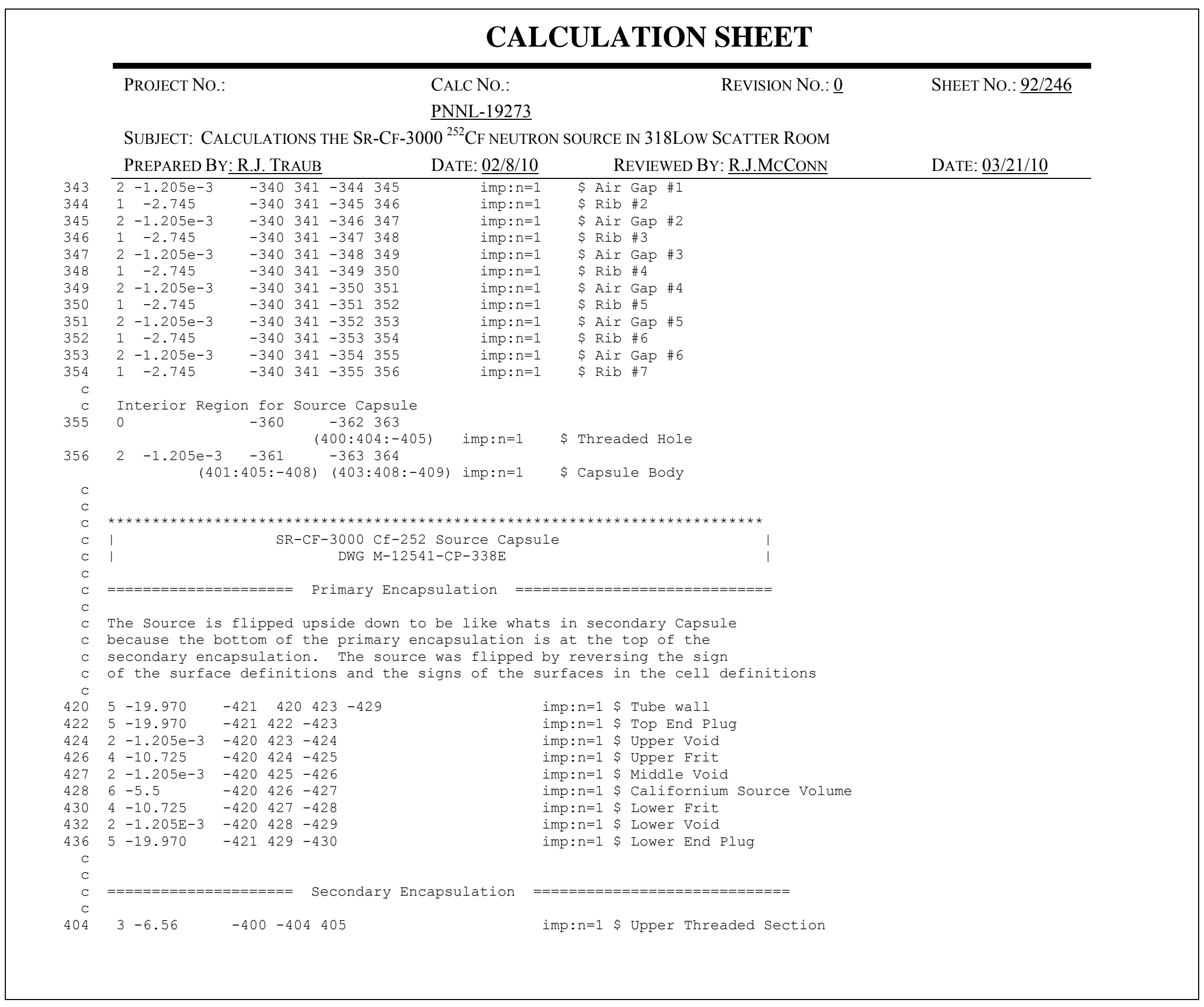




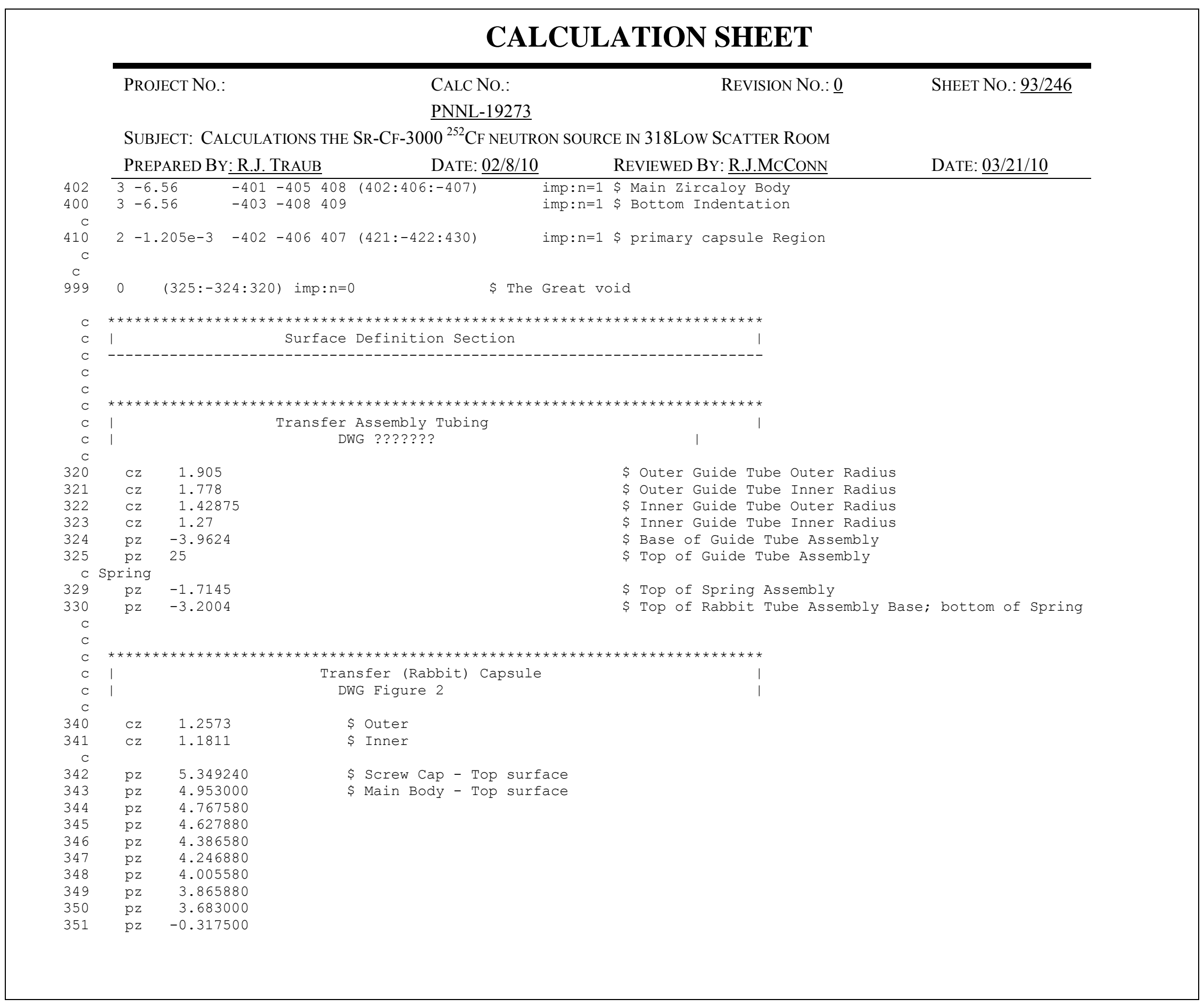




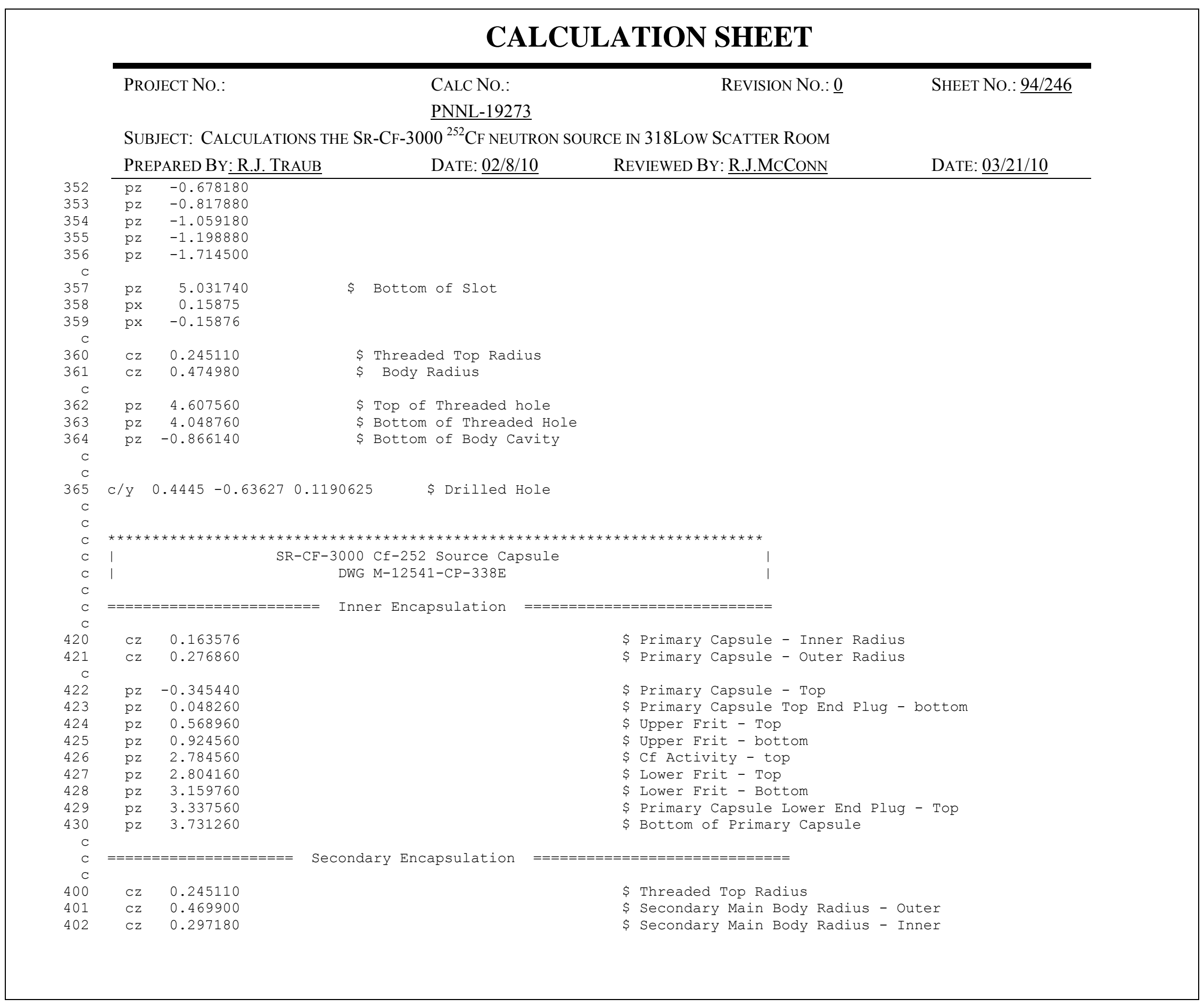




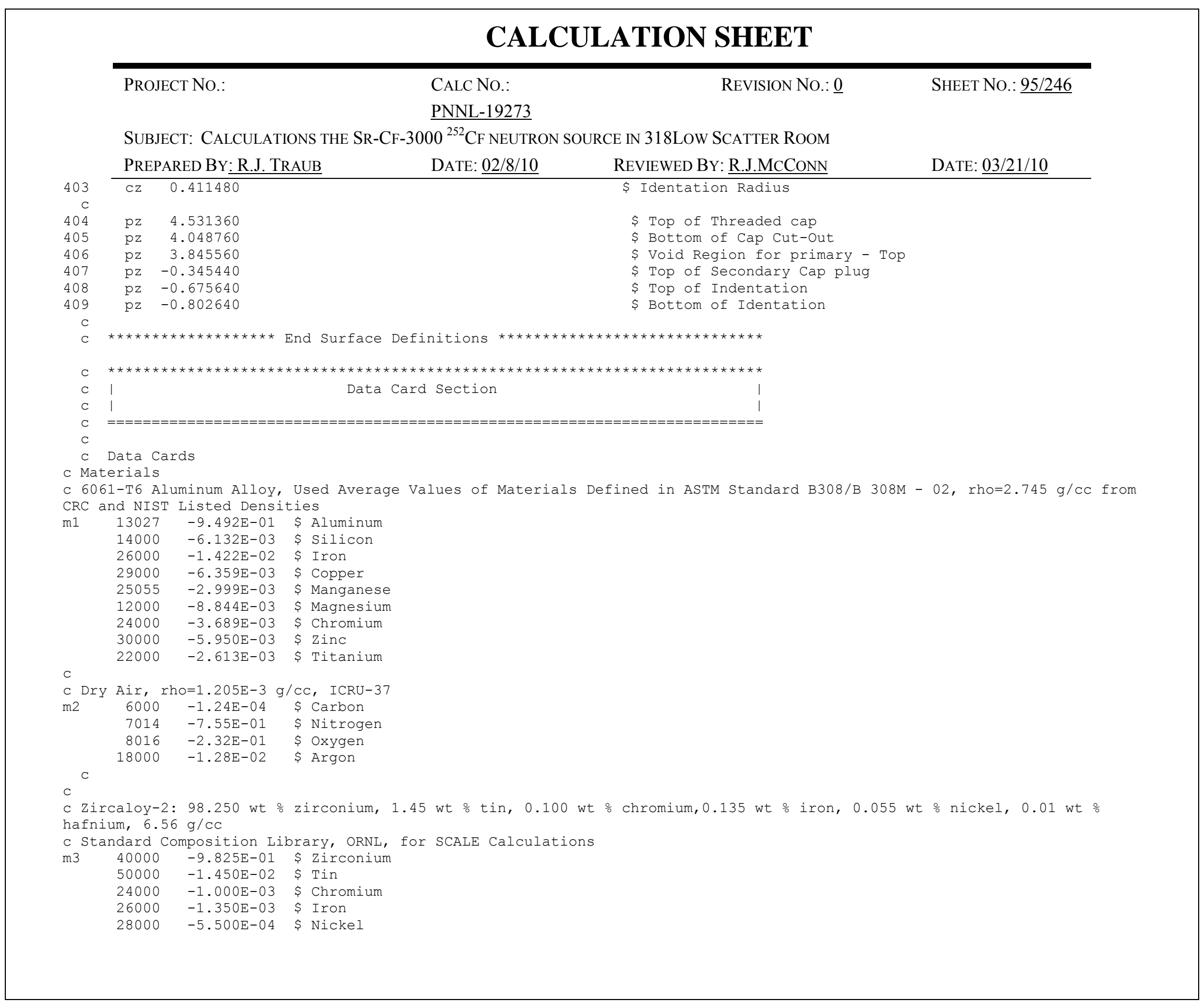




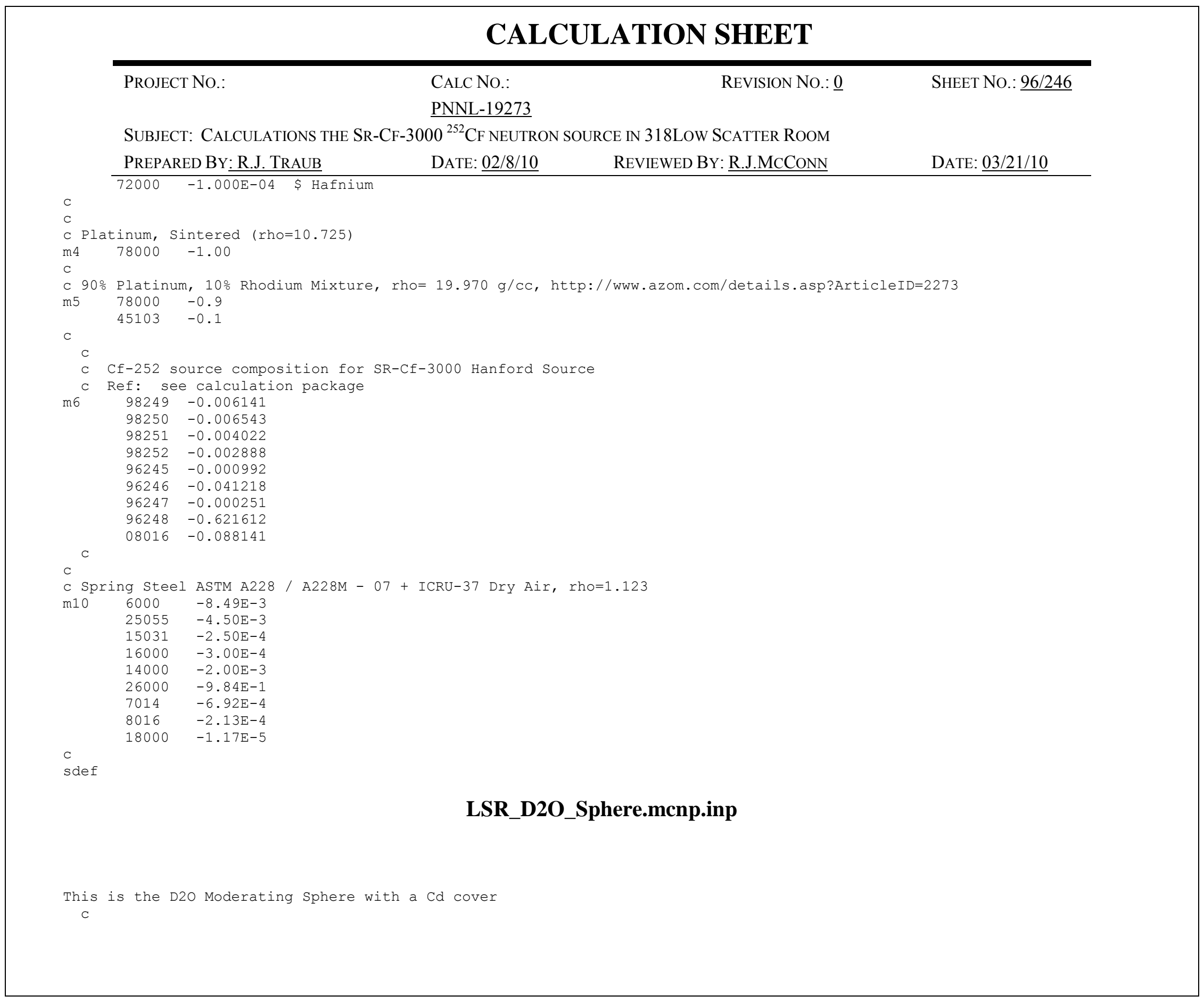




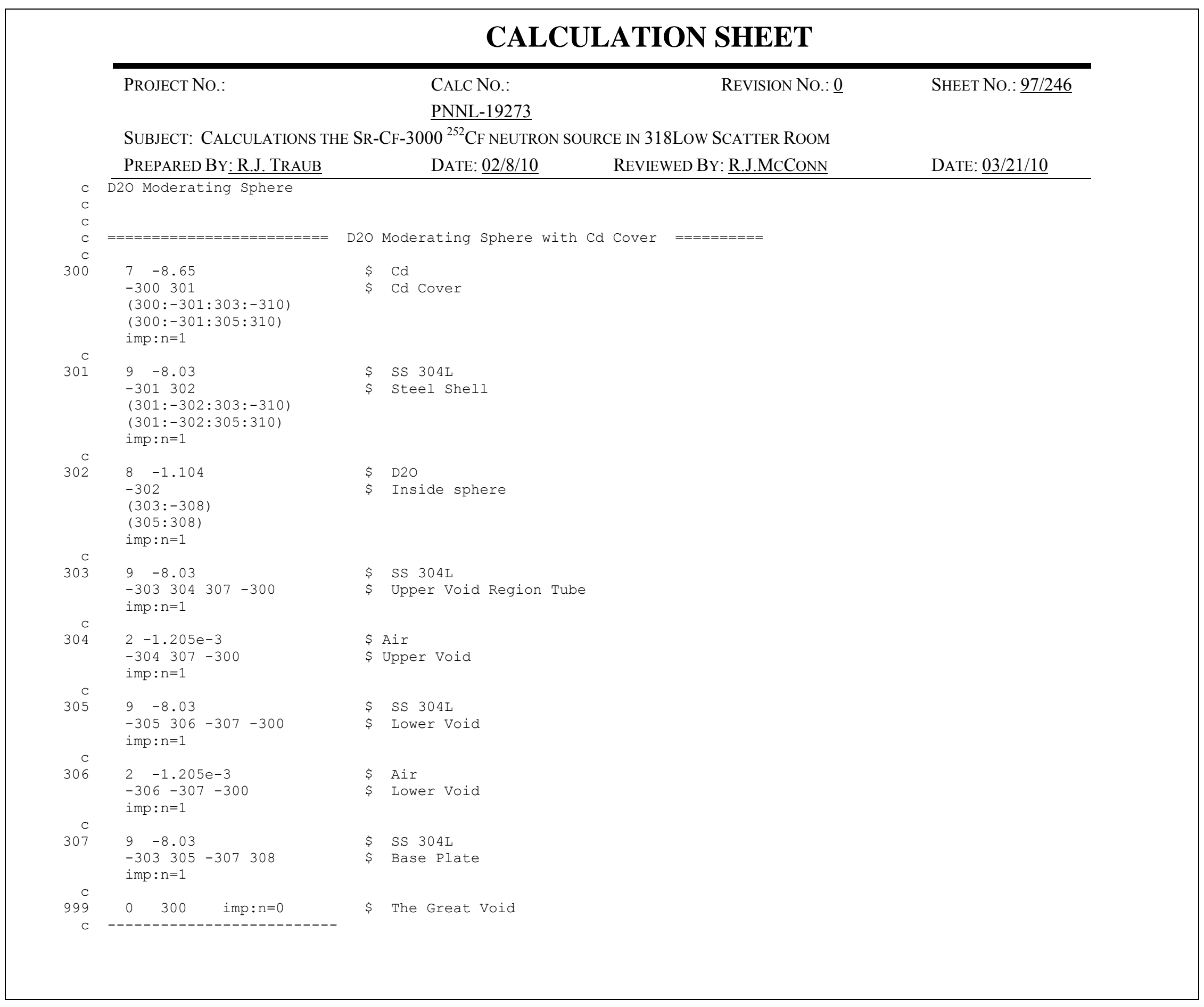




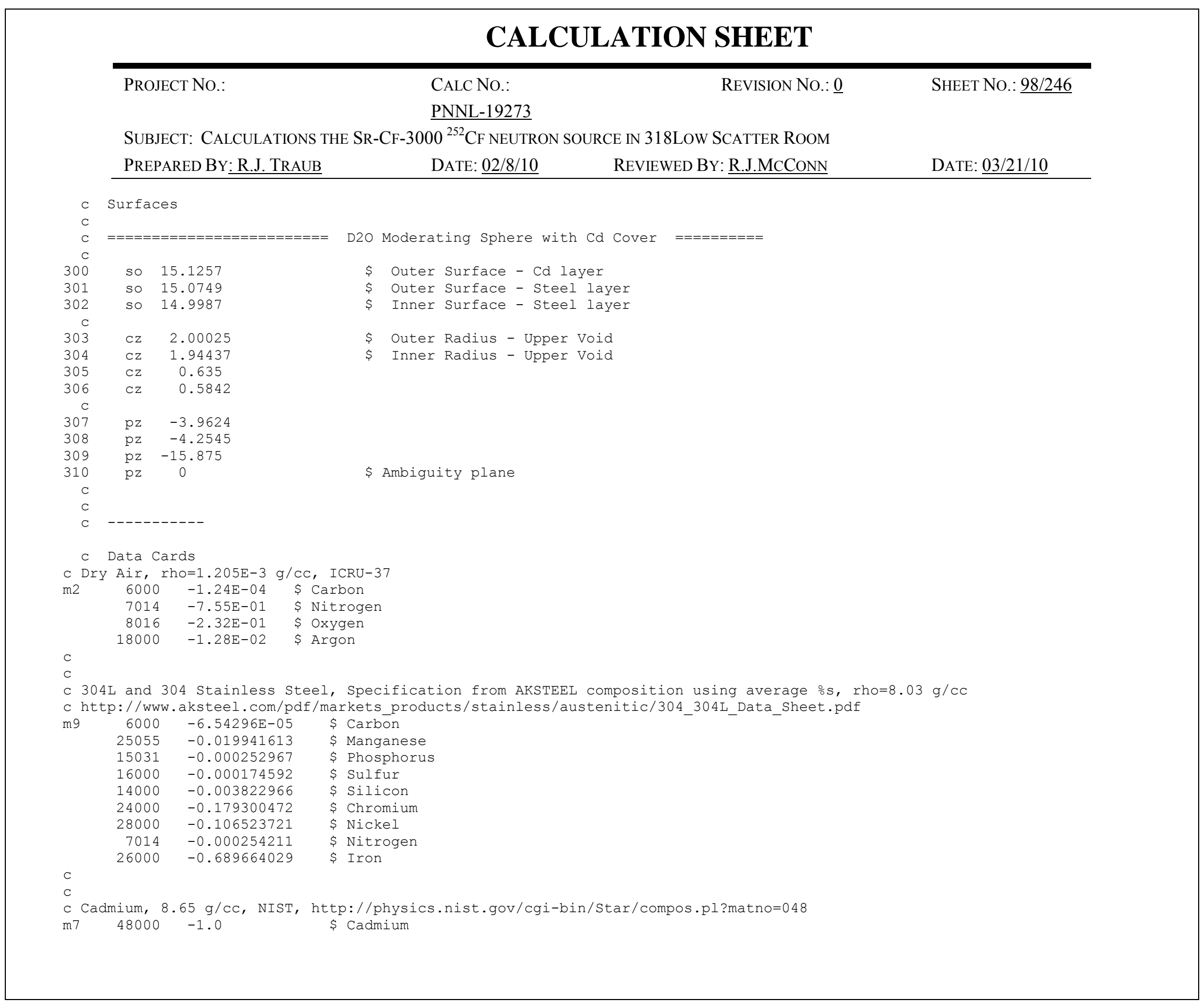




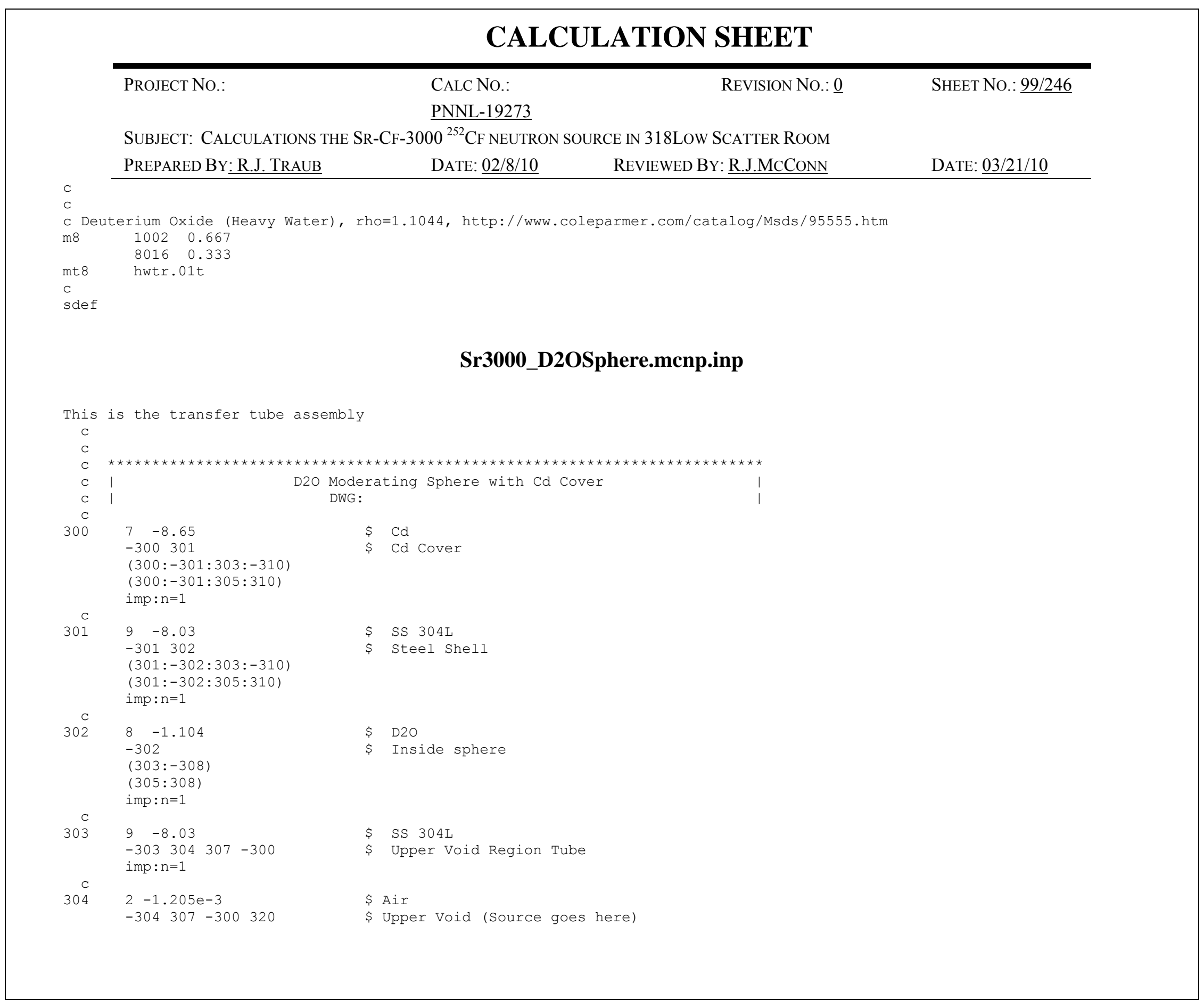




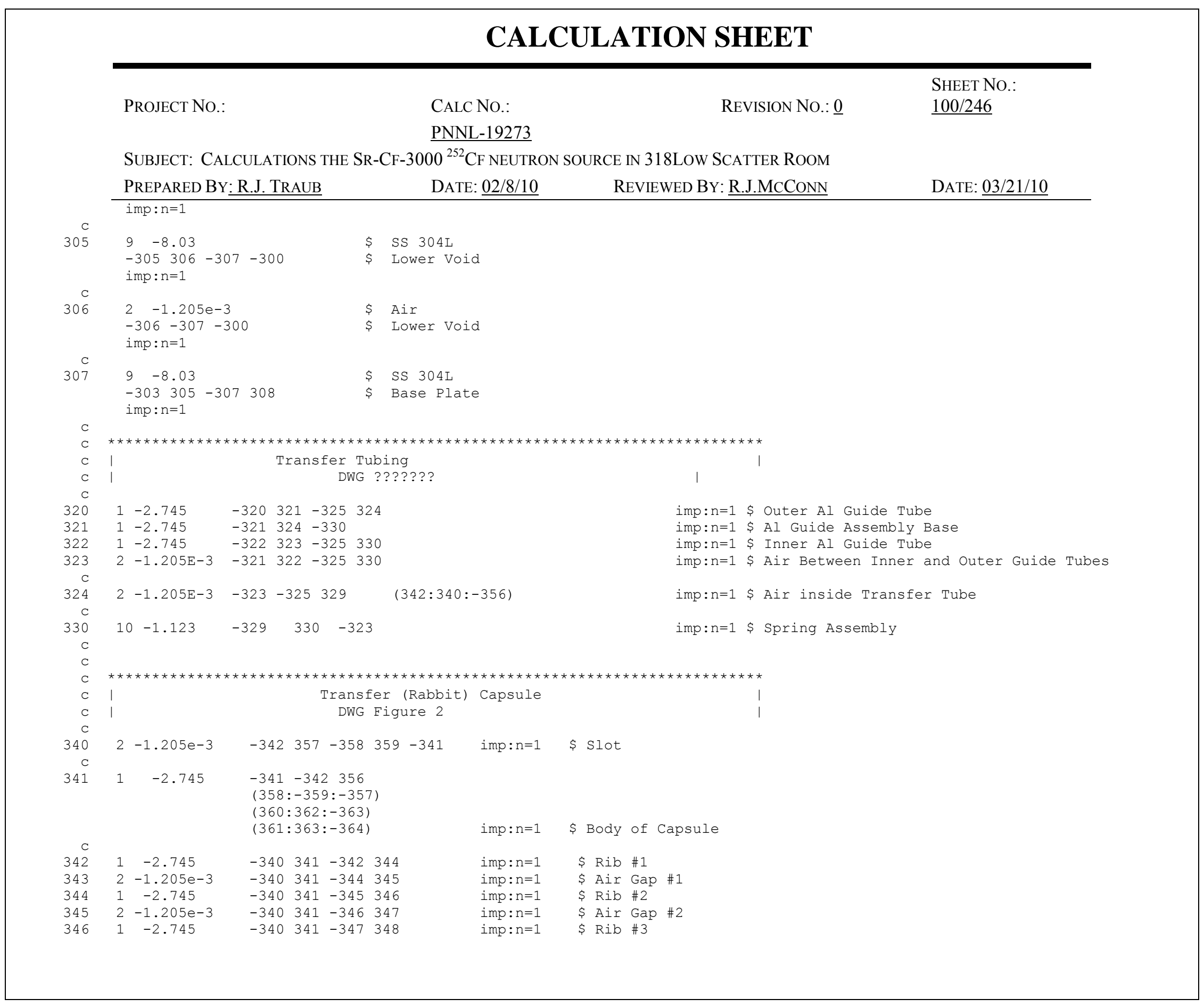




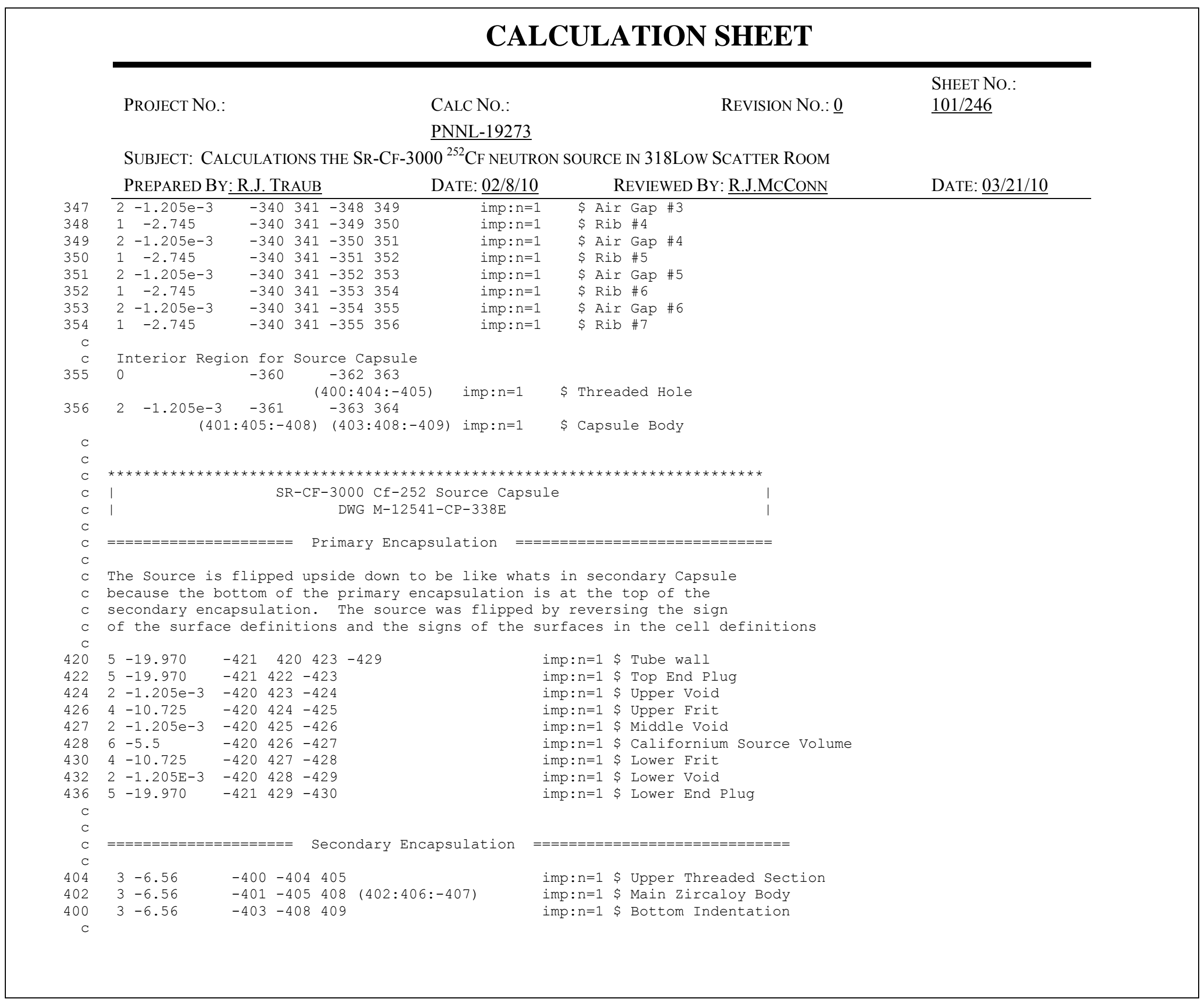




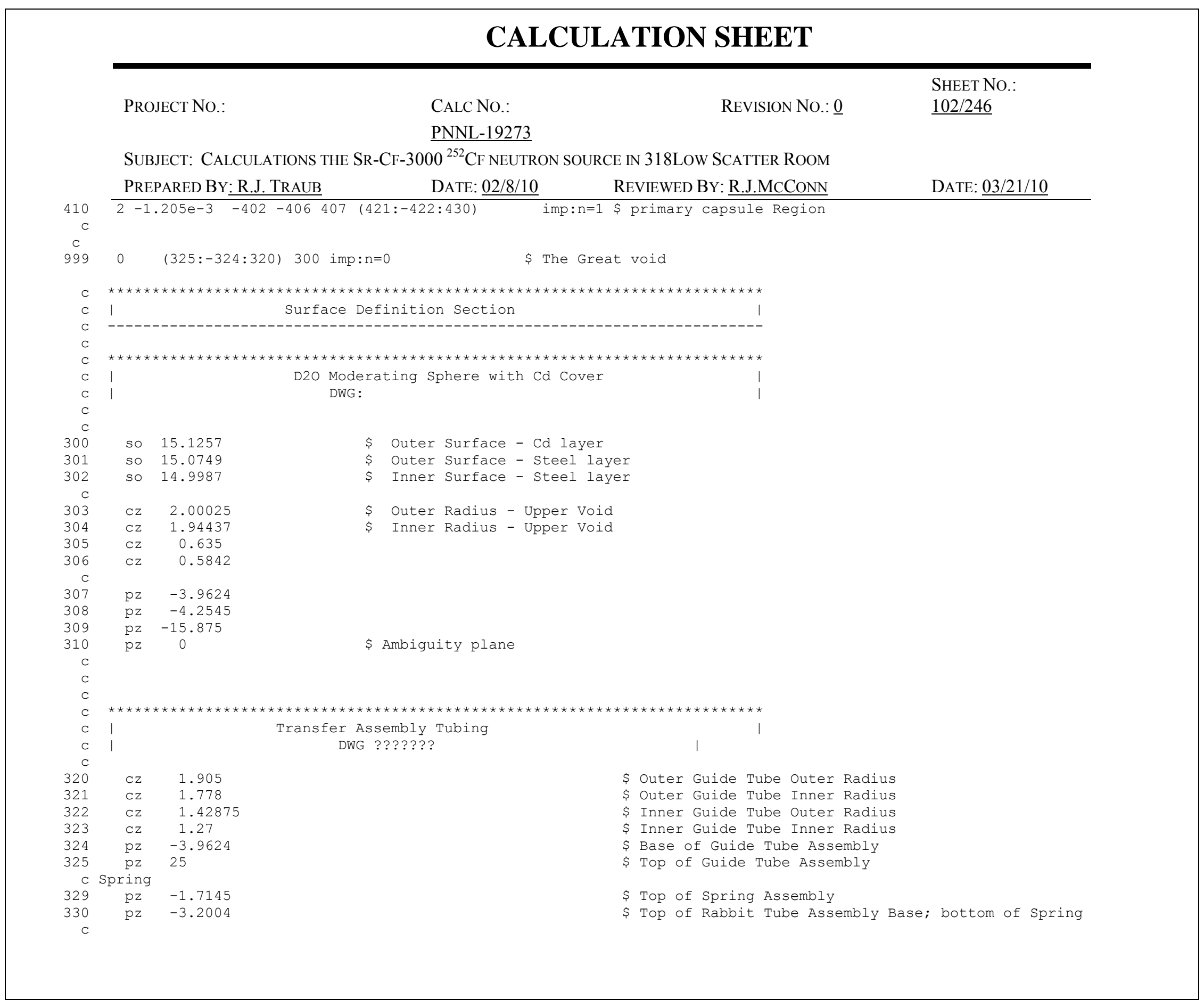




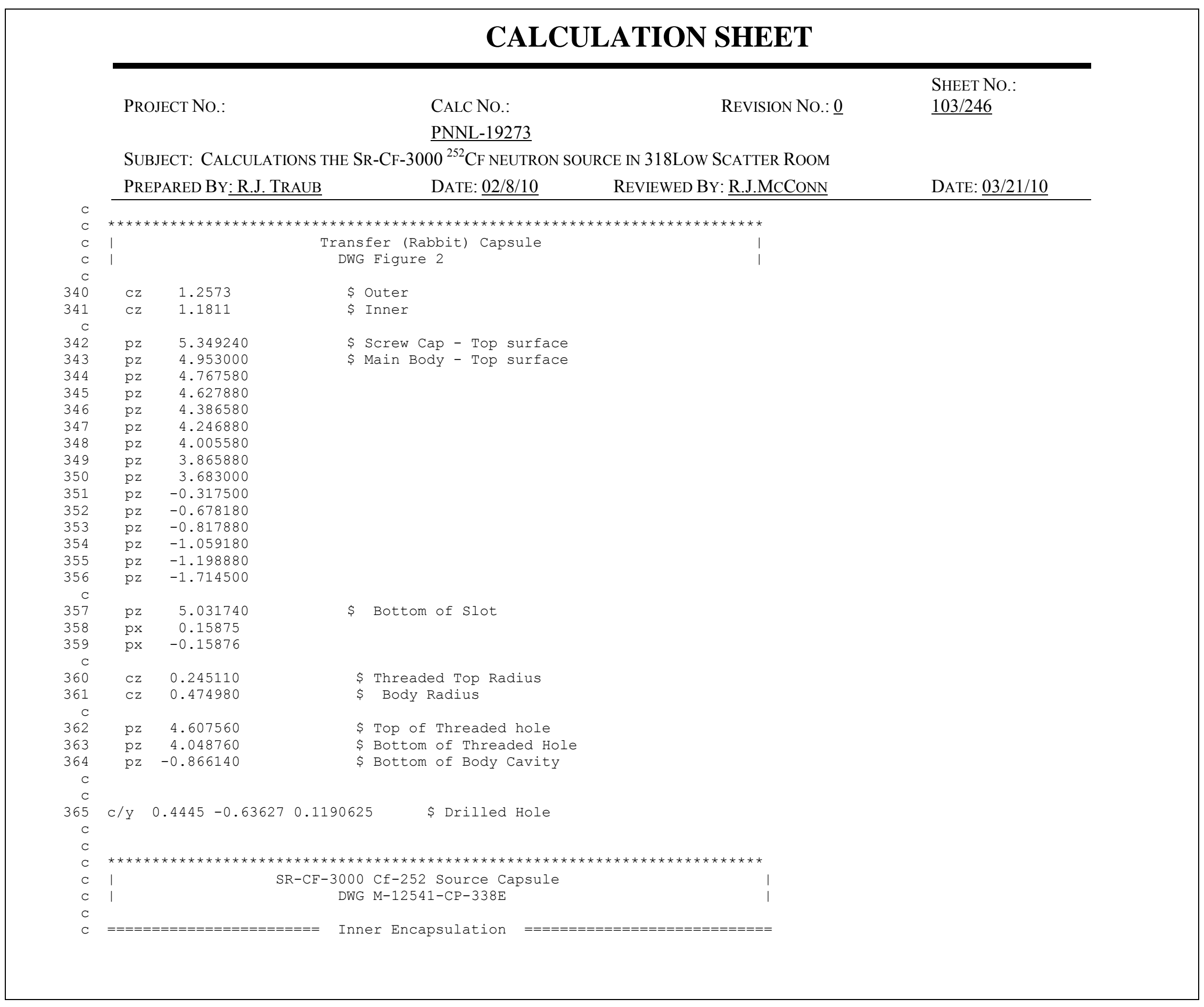




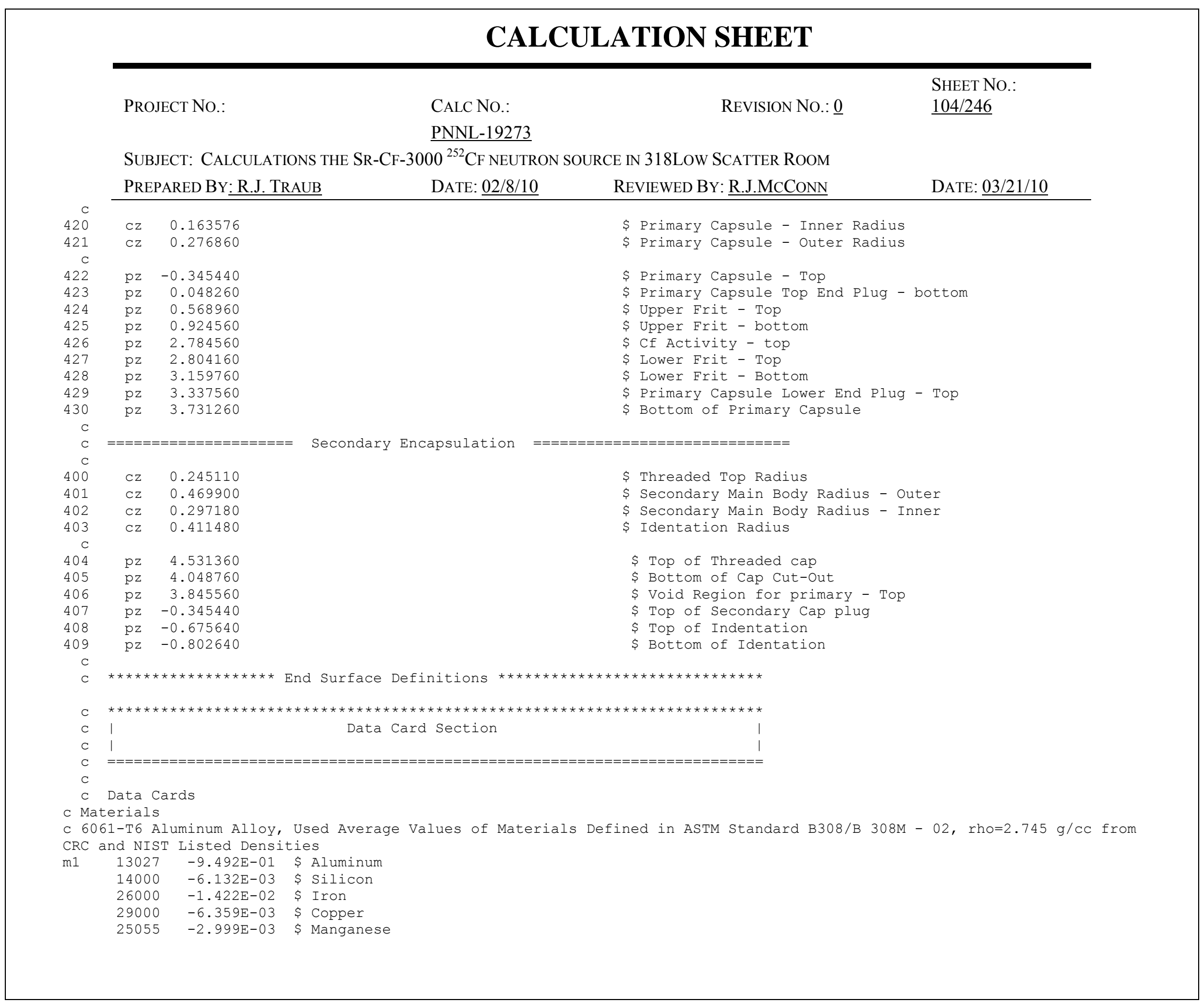




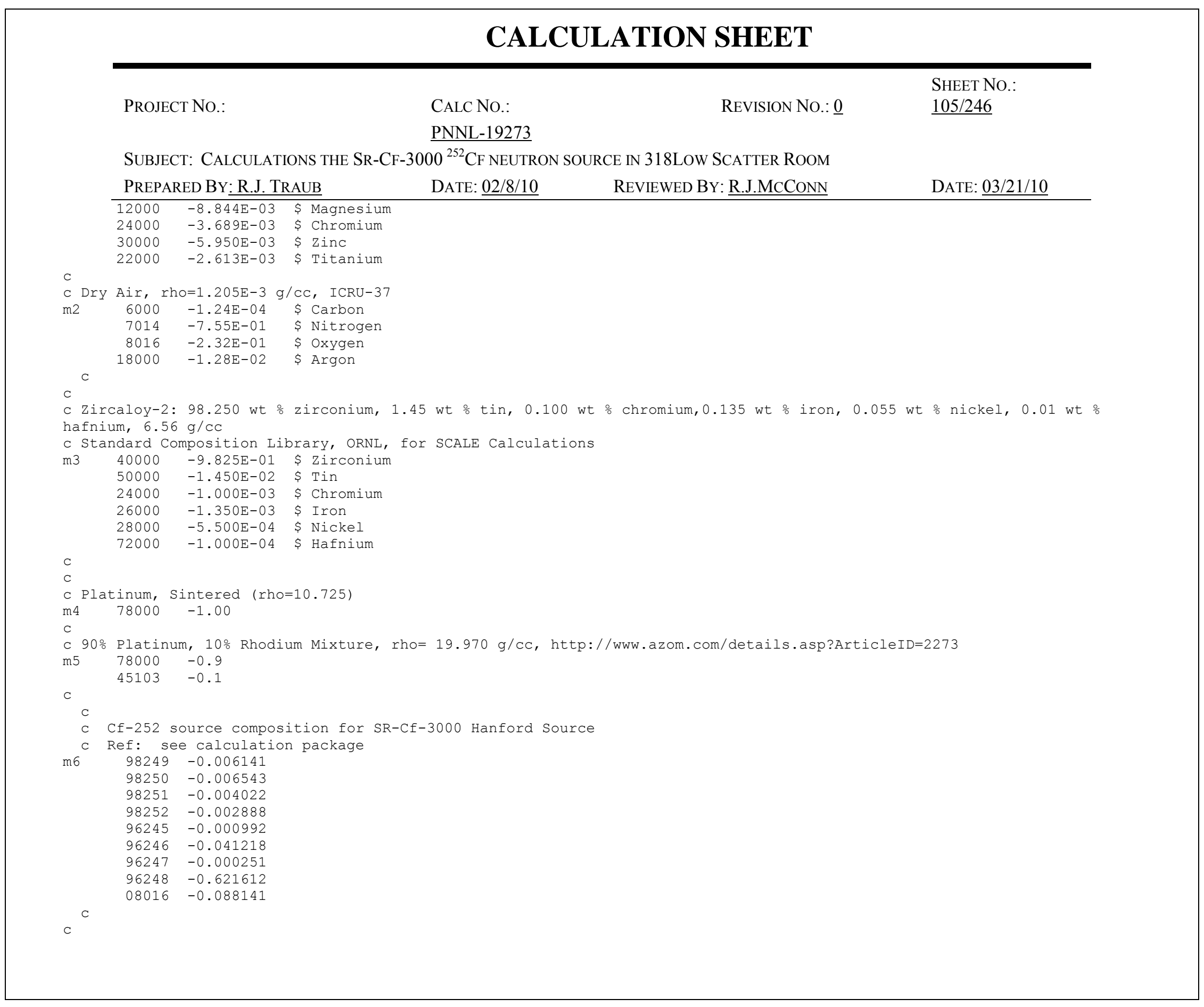




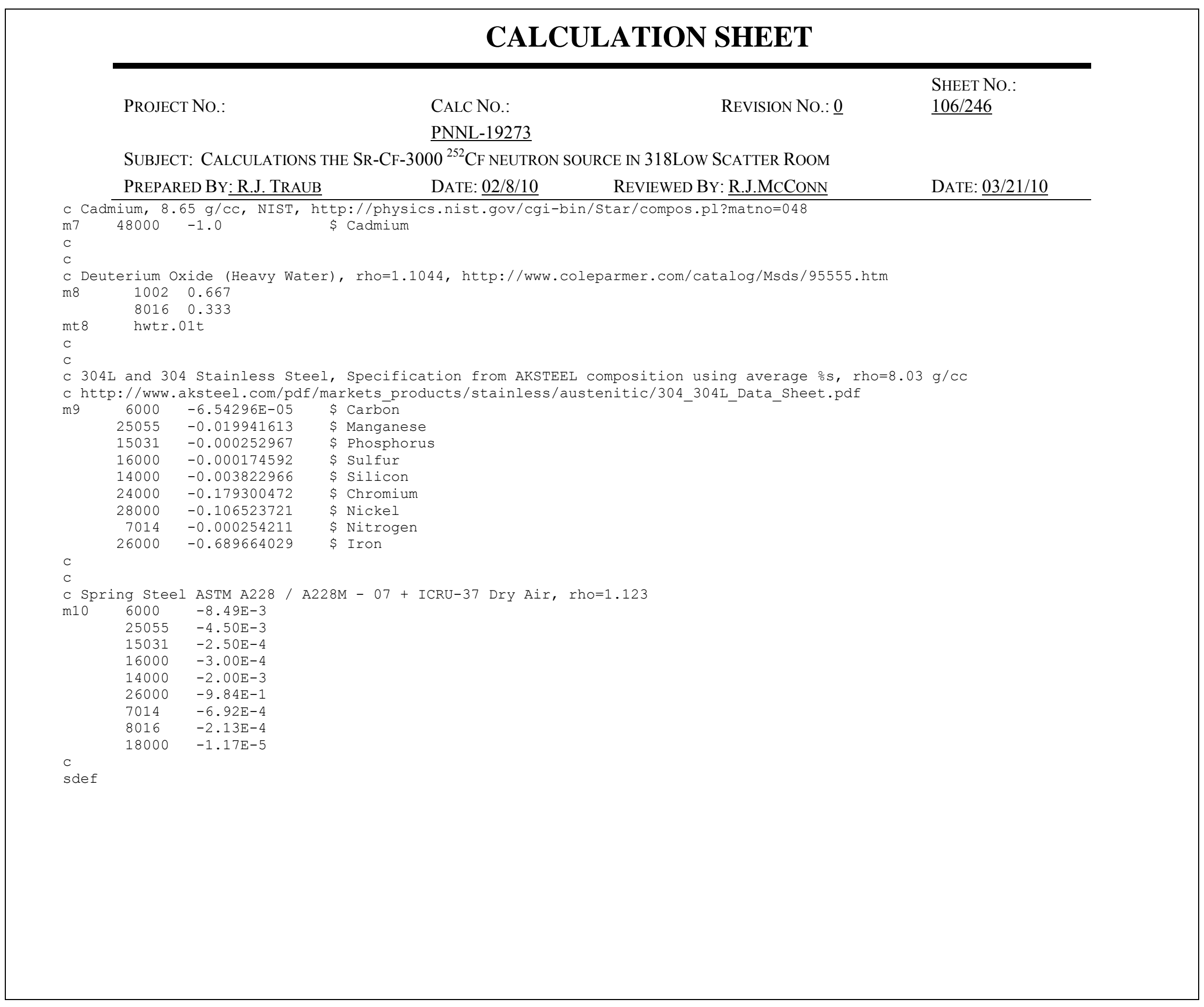




\section{CALCULATION SHEET}

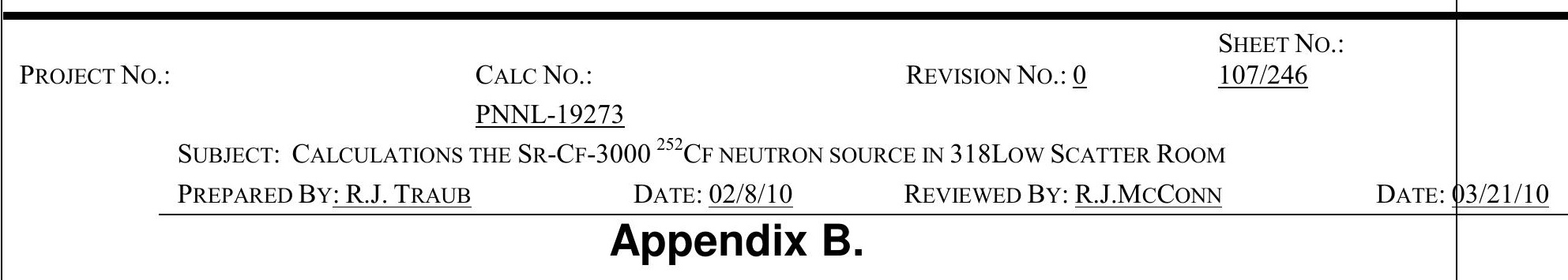

\section{Engineering Drawings}




\section{CALCULATION SHEET}

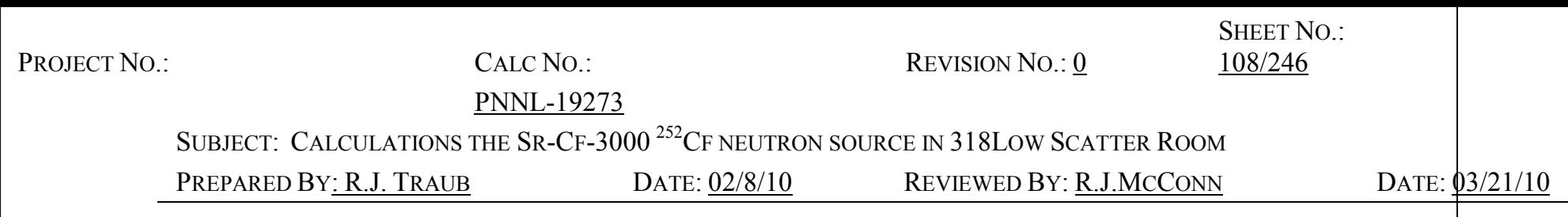

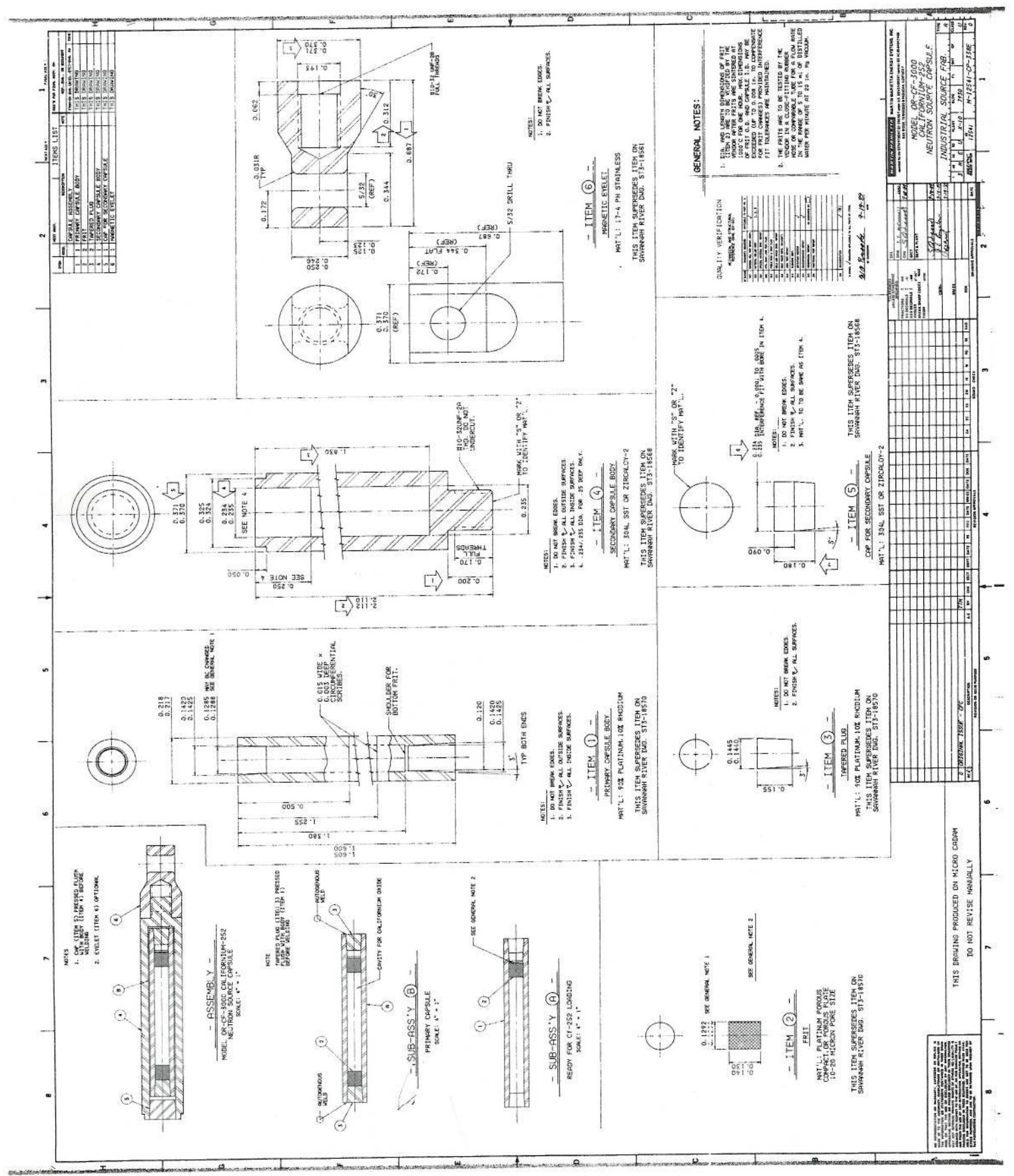




\section{CALCULATION SHEET}

\begin{tabular}{|c|c|c|c|}
\hline PROJECT No.: & $\begin{array}{l}\text { CALC NO.: } \\
\text { PNNL-19273 }\end{array}$ & REVISION NO.: $\underline{0}$ & $\begin{array}{l}\text { SHEET NO. } \\
\underline{109 / 246}\end{array}$ \\
\hline
\end{tabular}
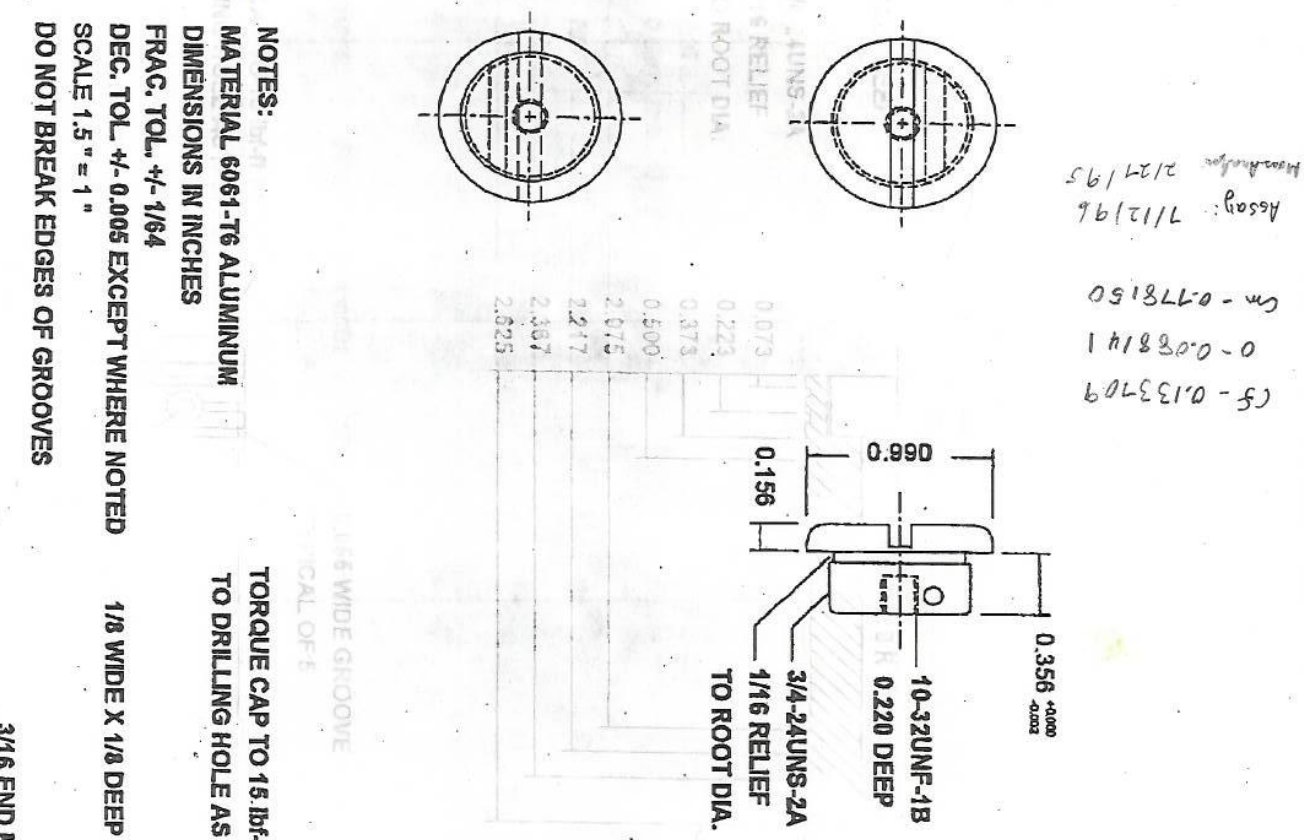

b०L $\{\varepsilon / 0-f)$
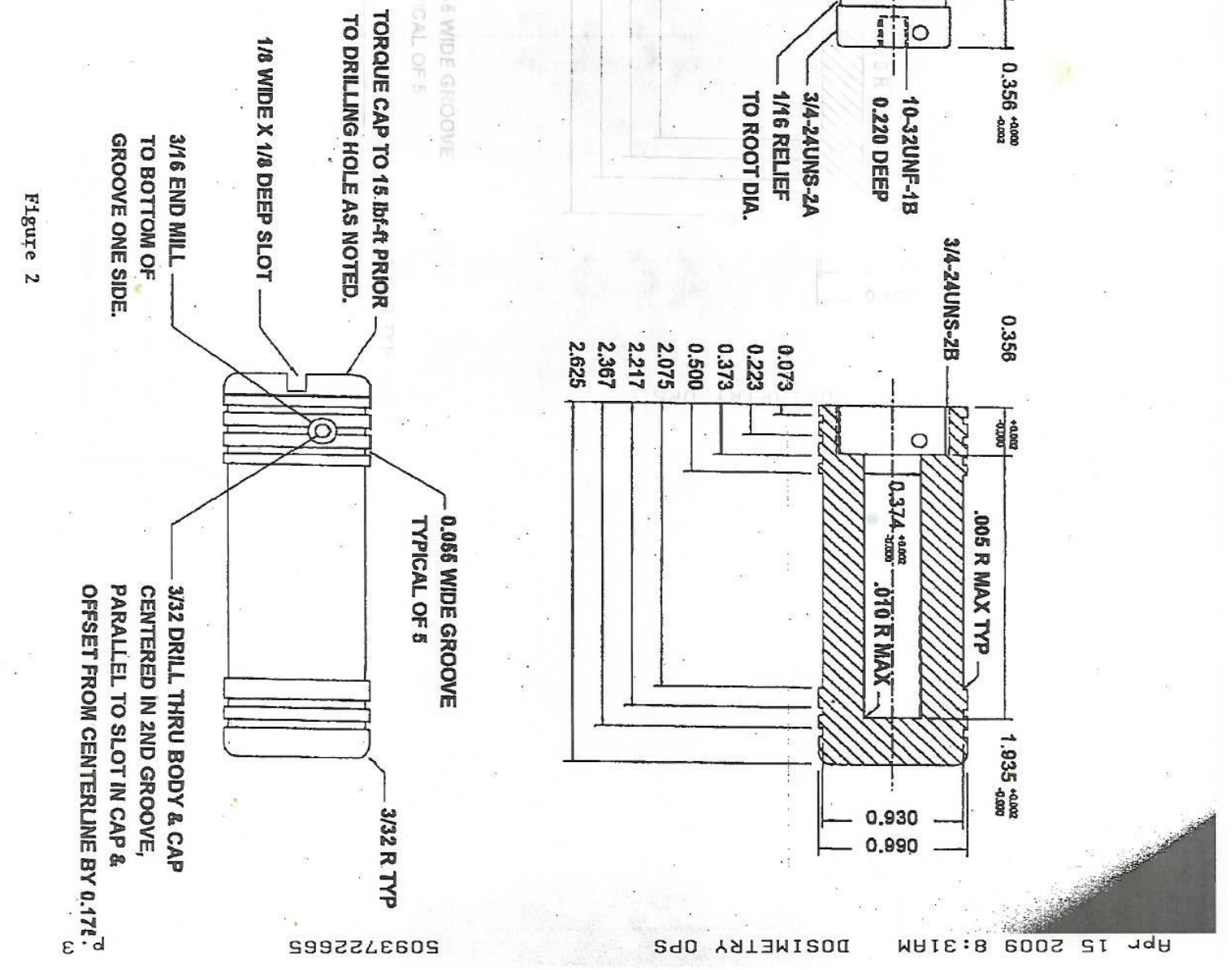


\section{CALCULATION SHEET}

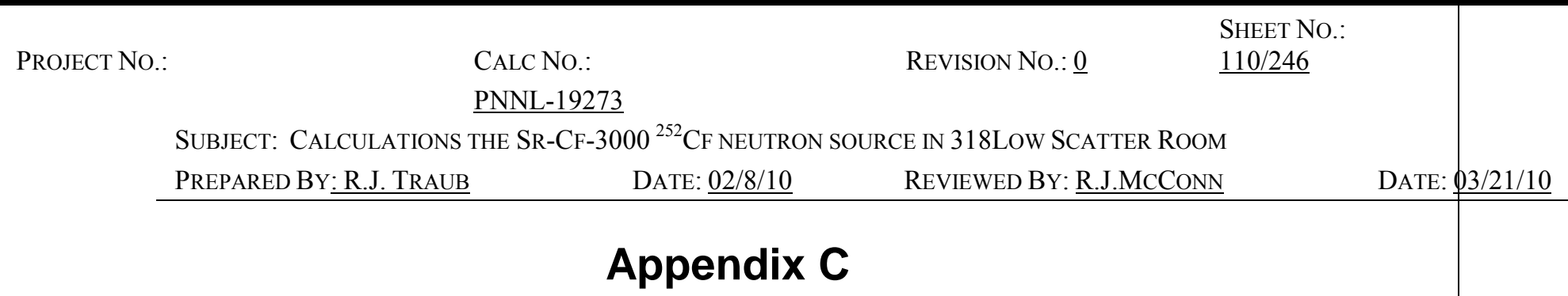

\section{MCNP Input and Output File Example}




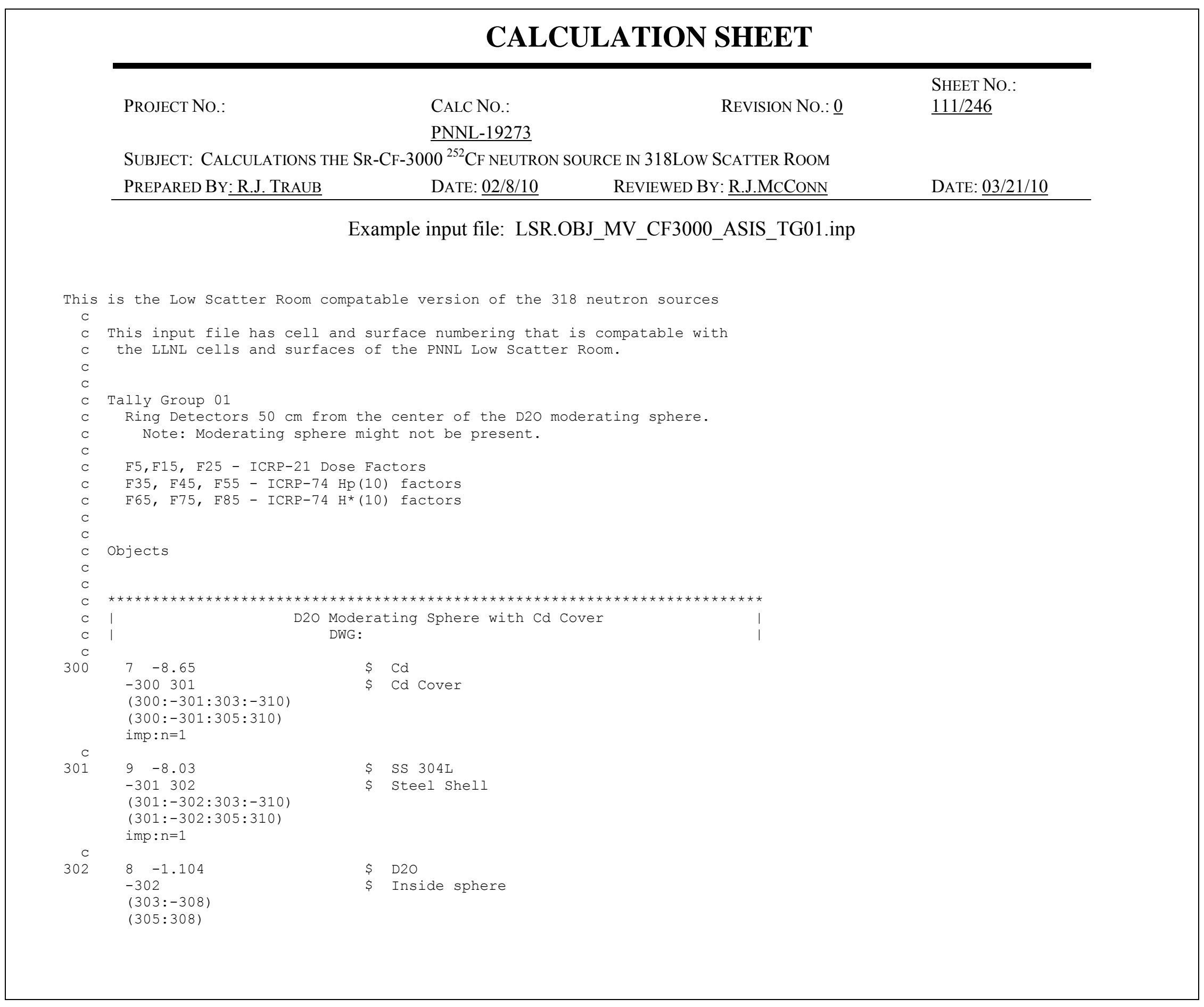




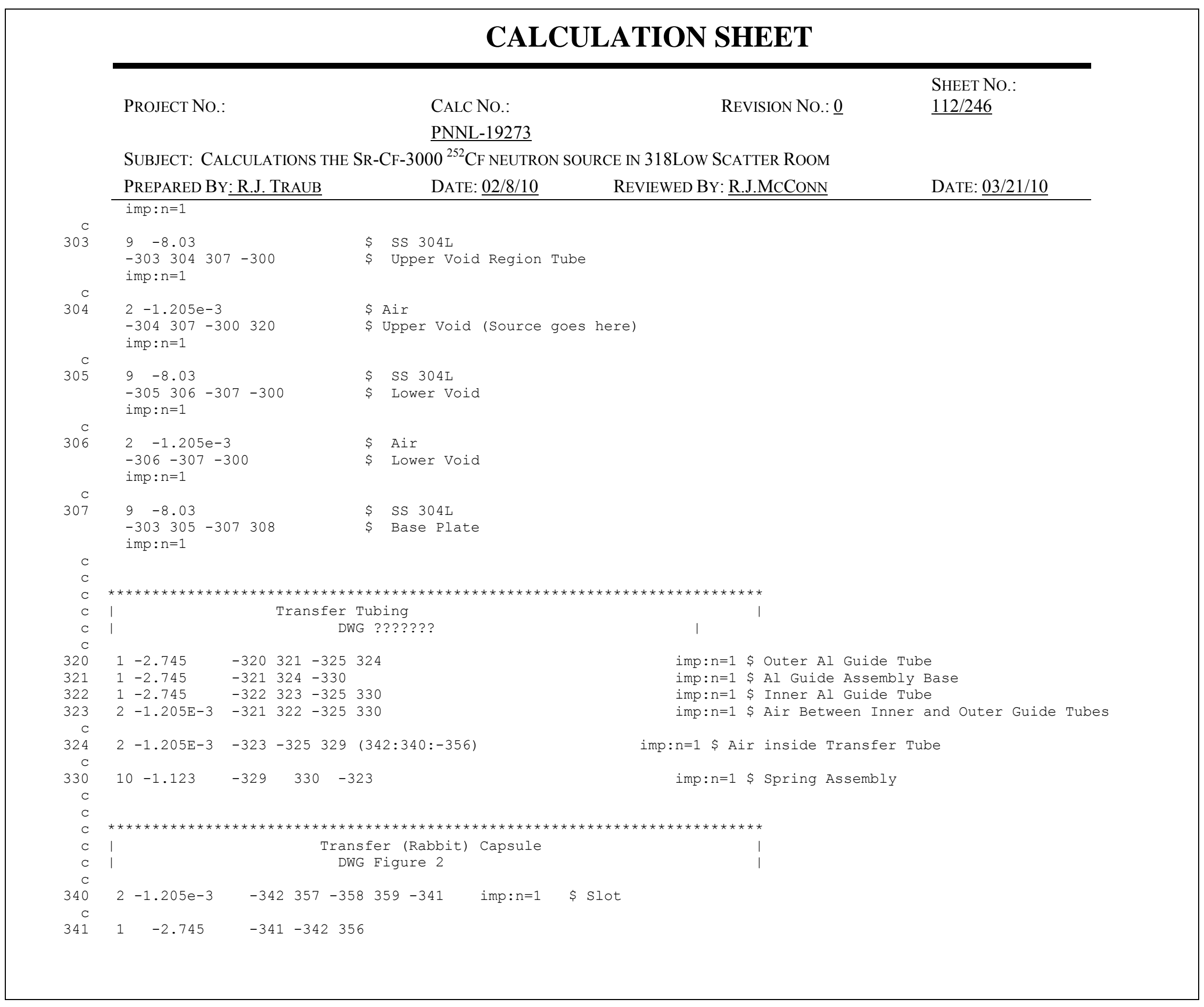




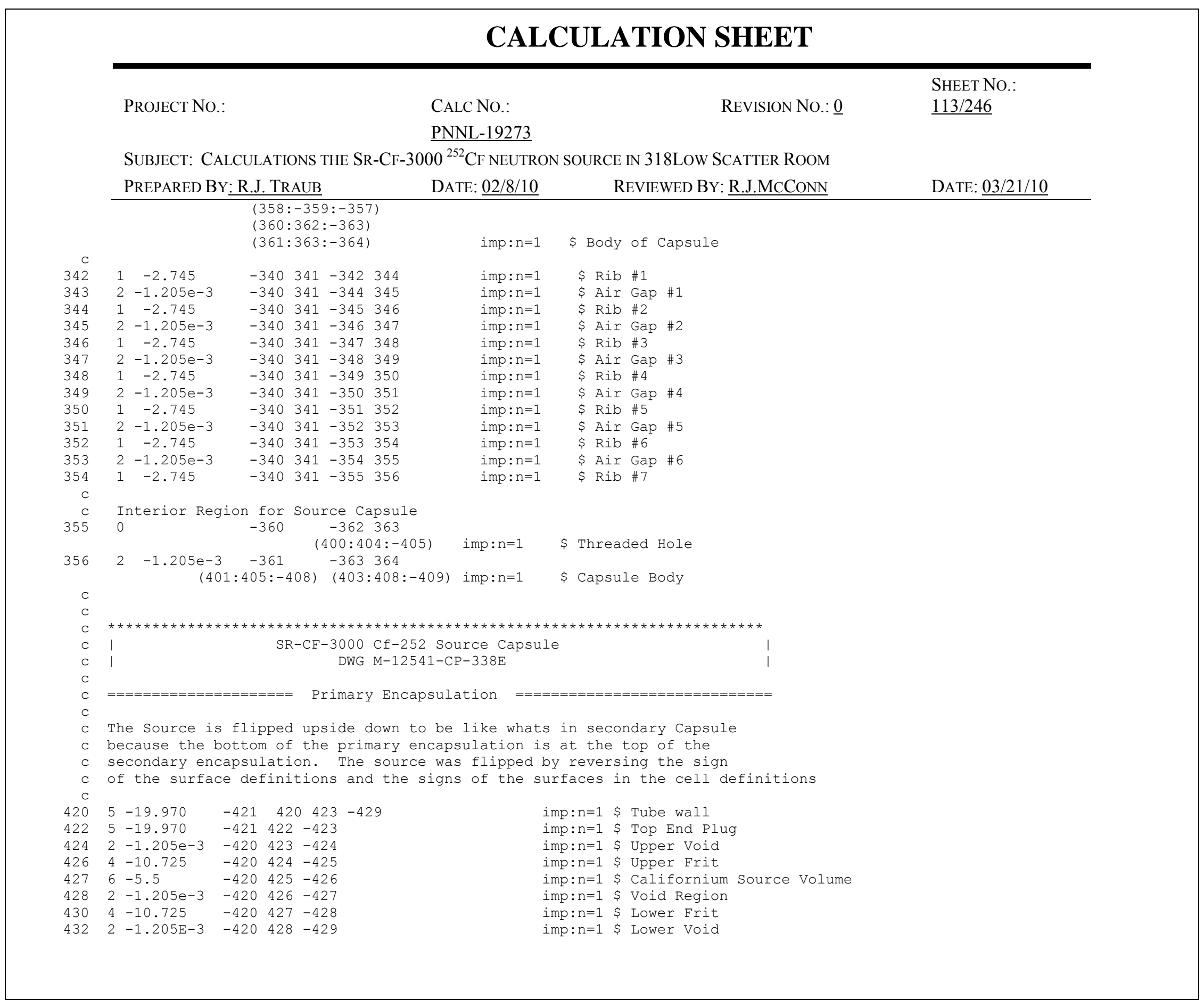




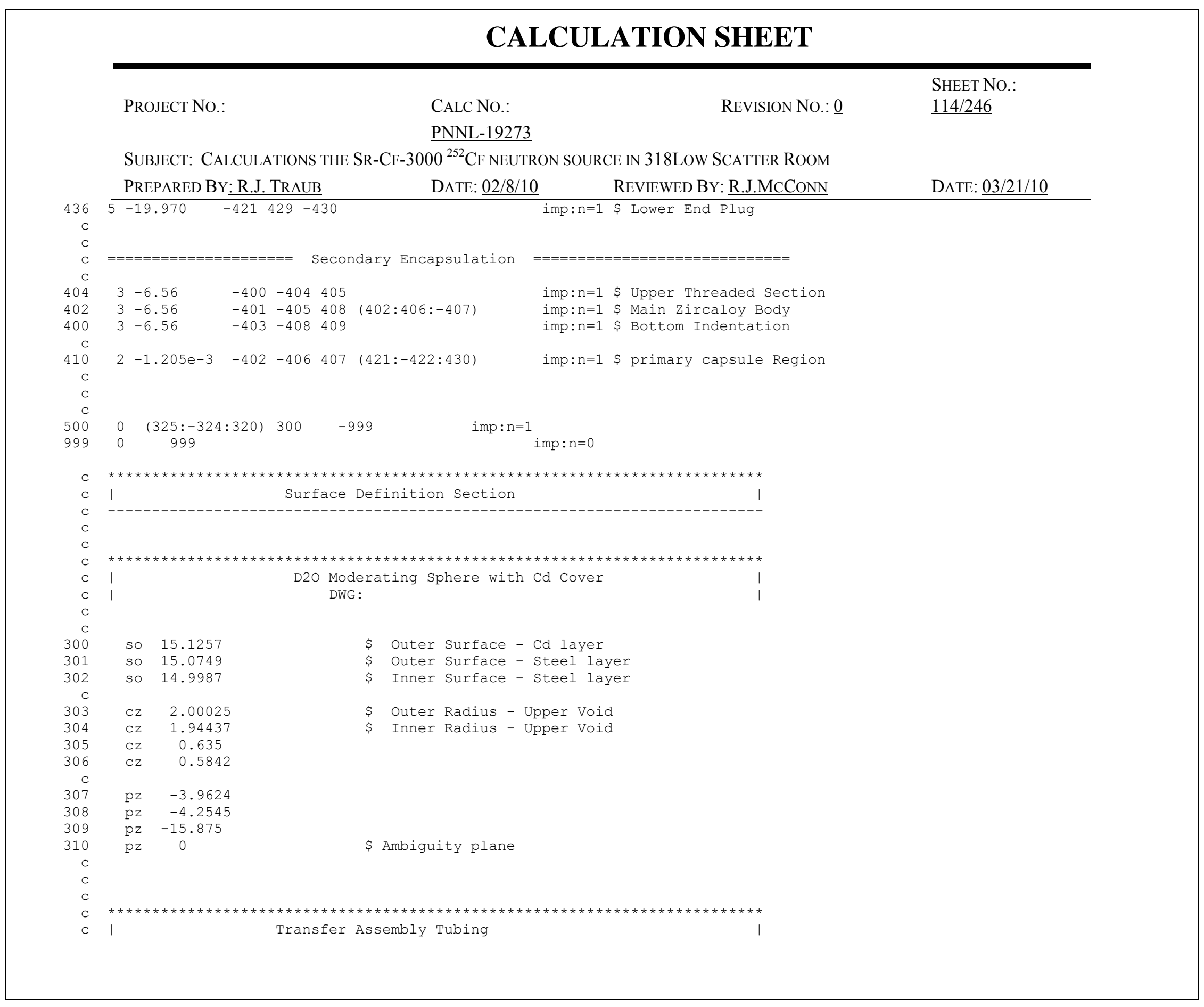




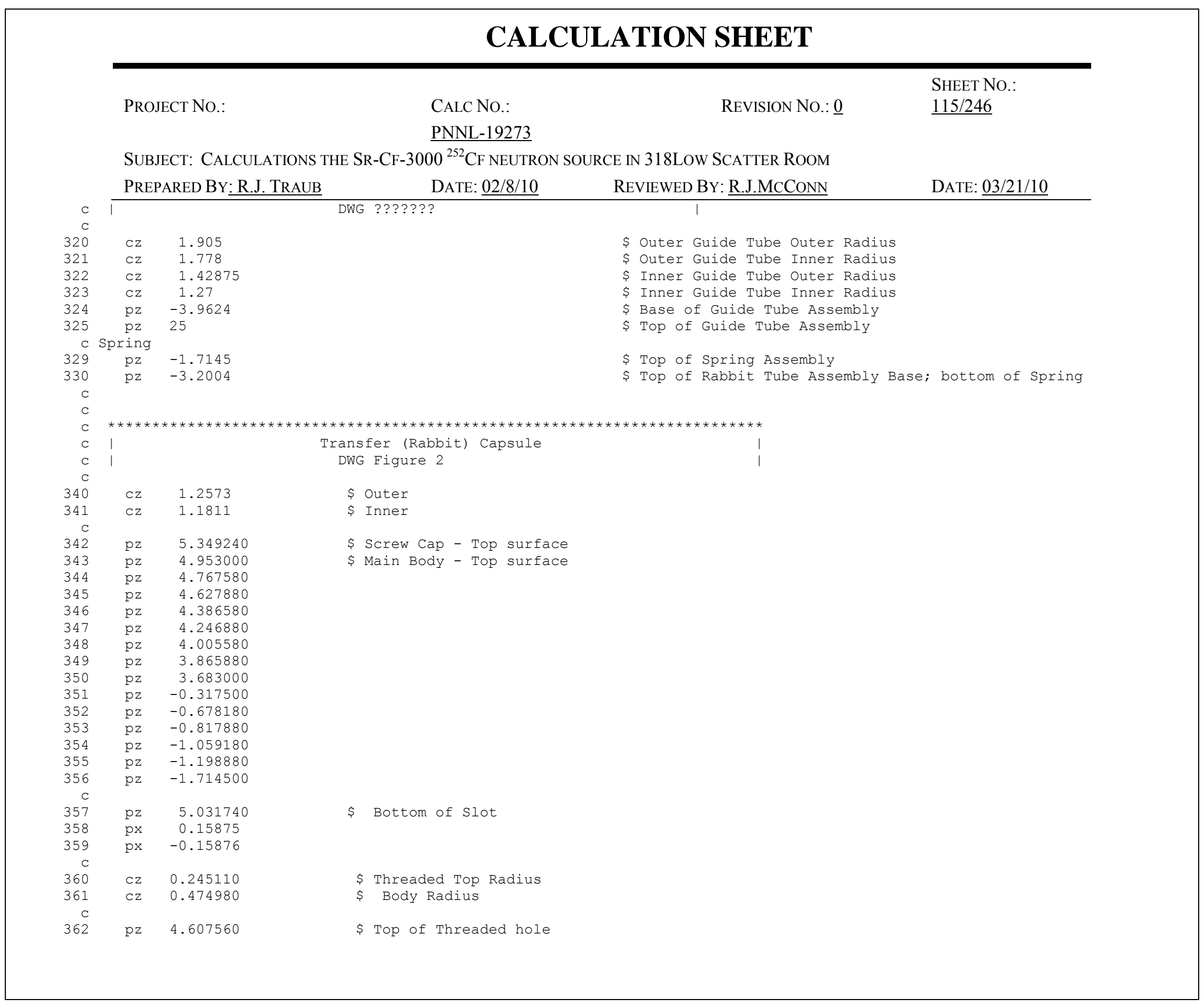




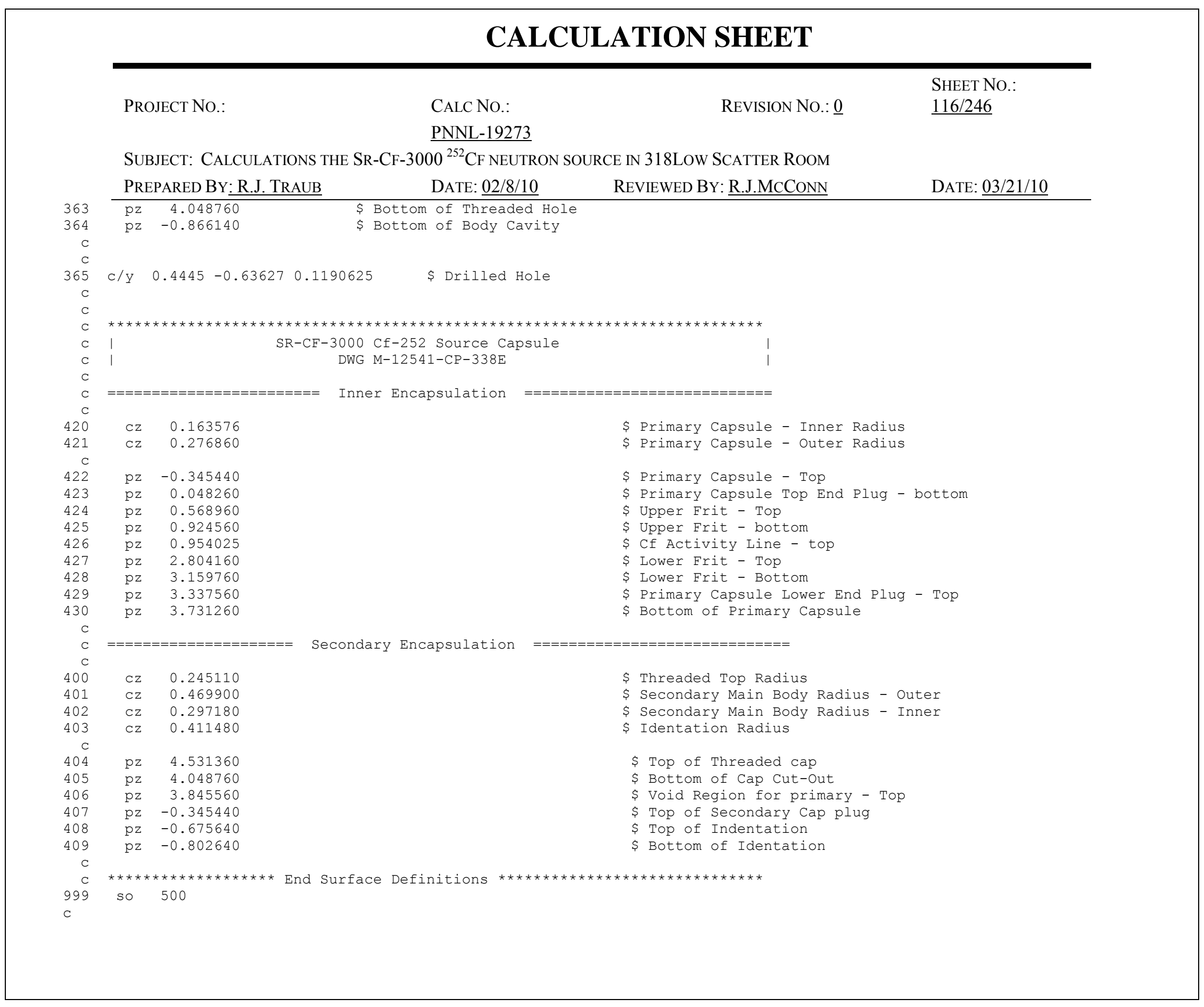




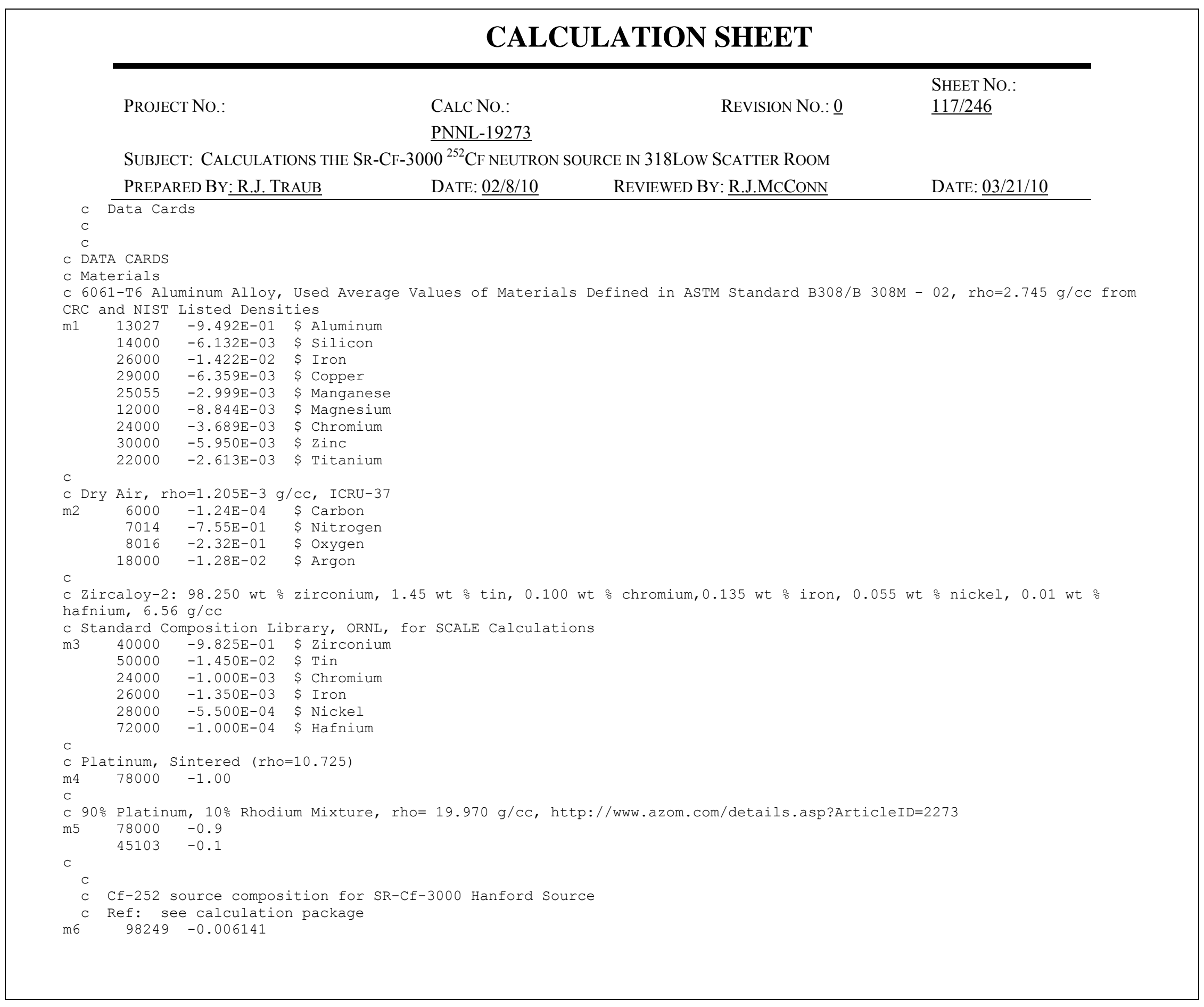




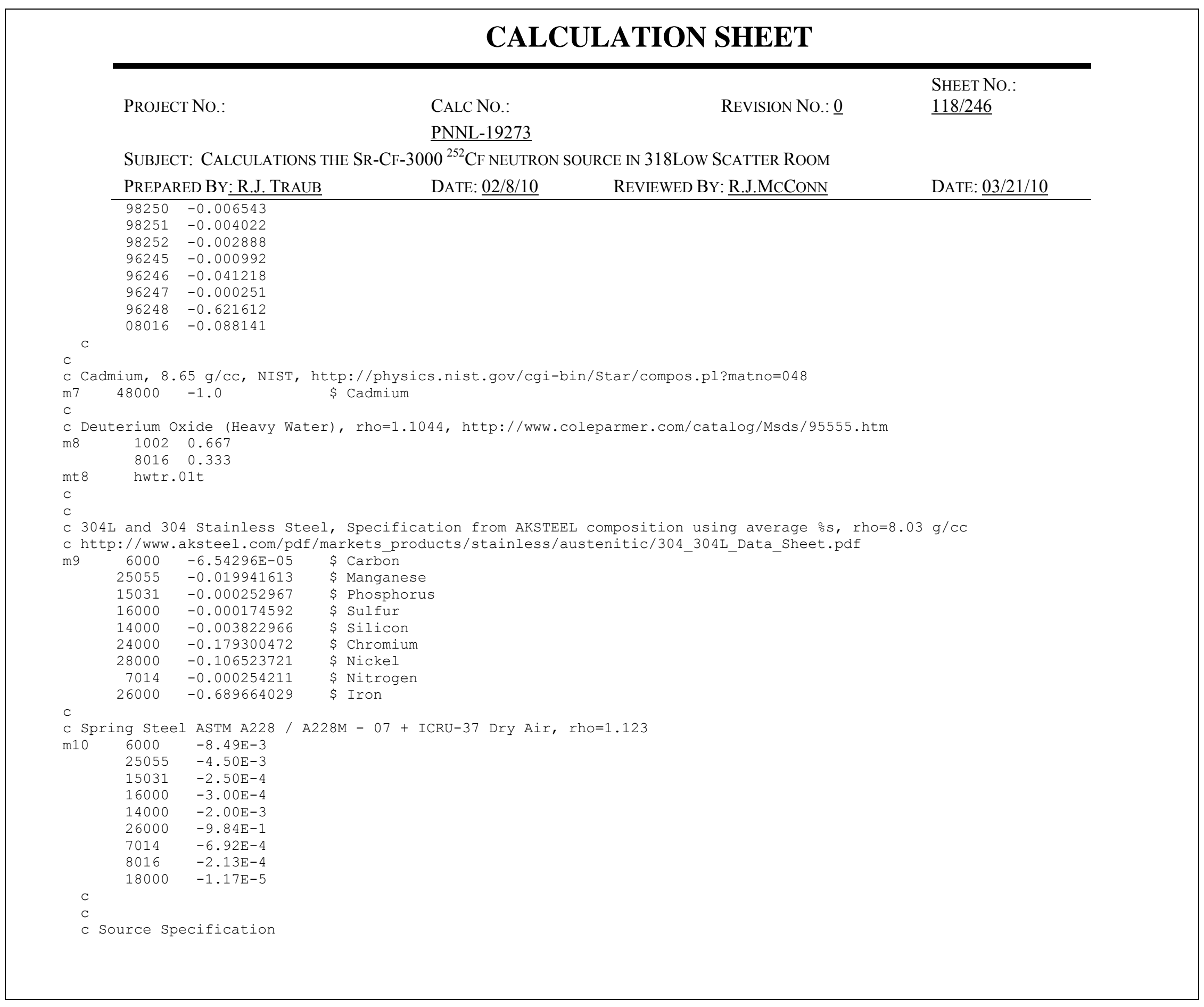




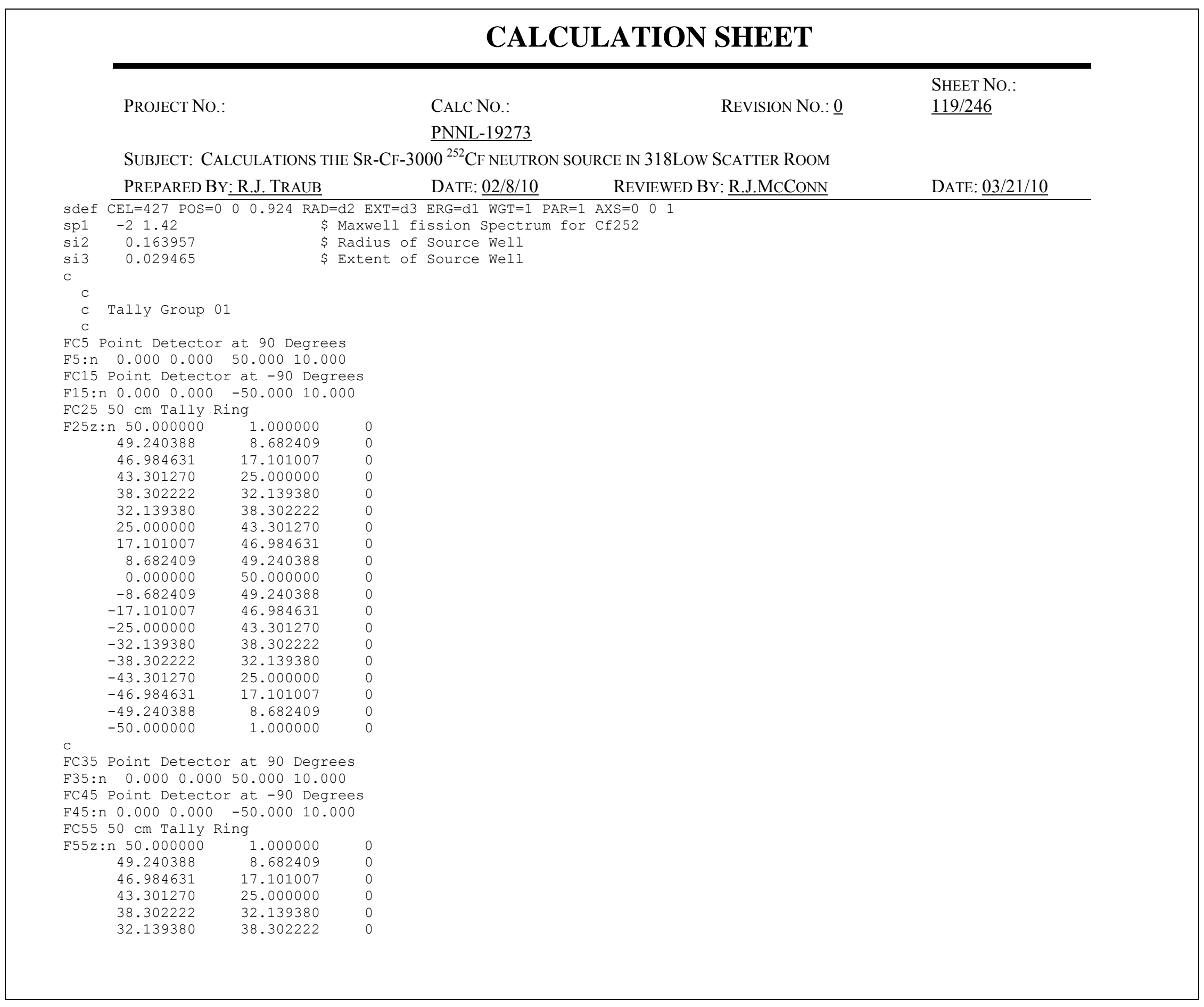




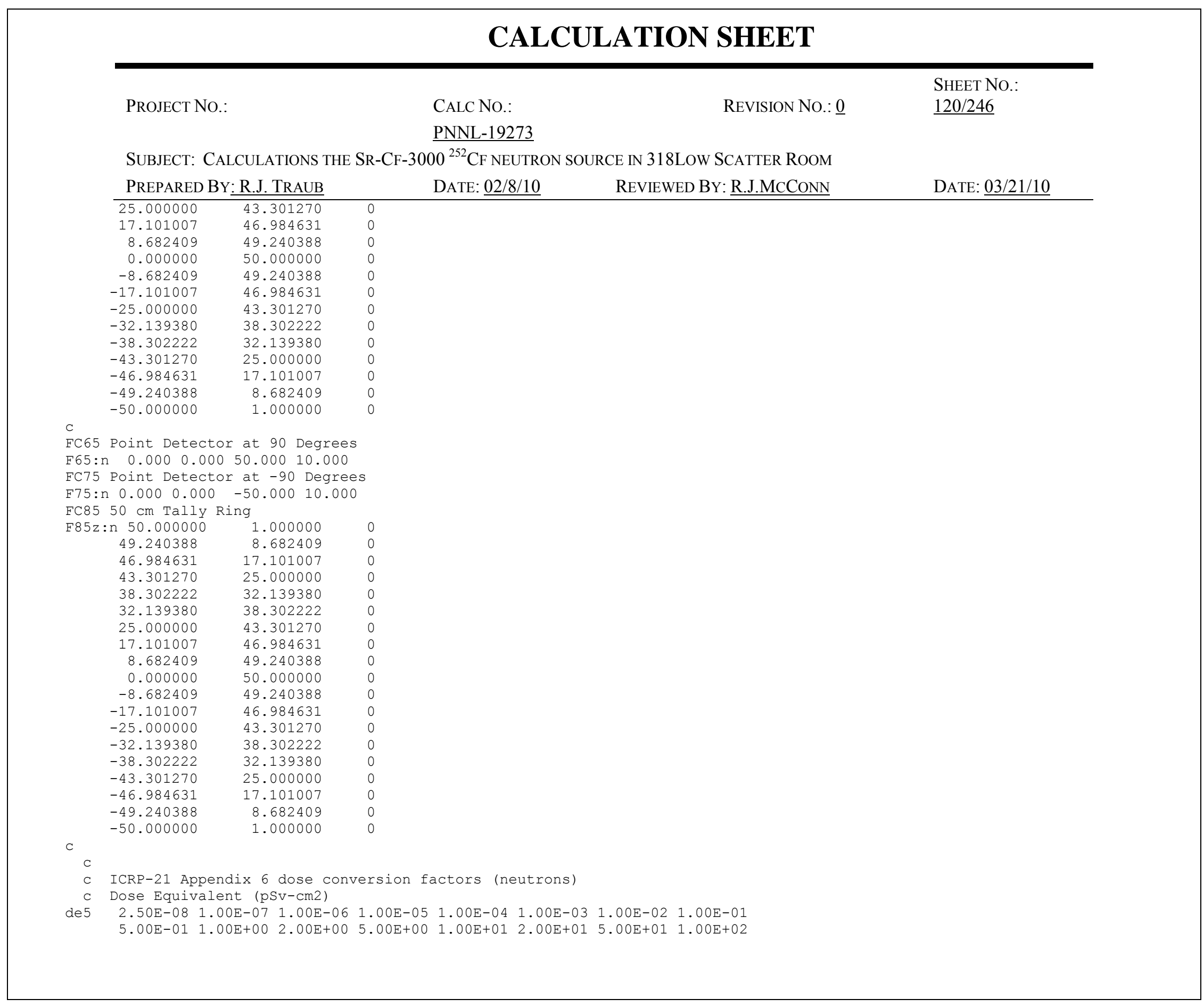




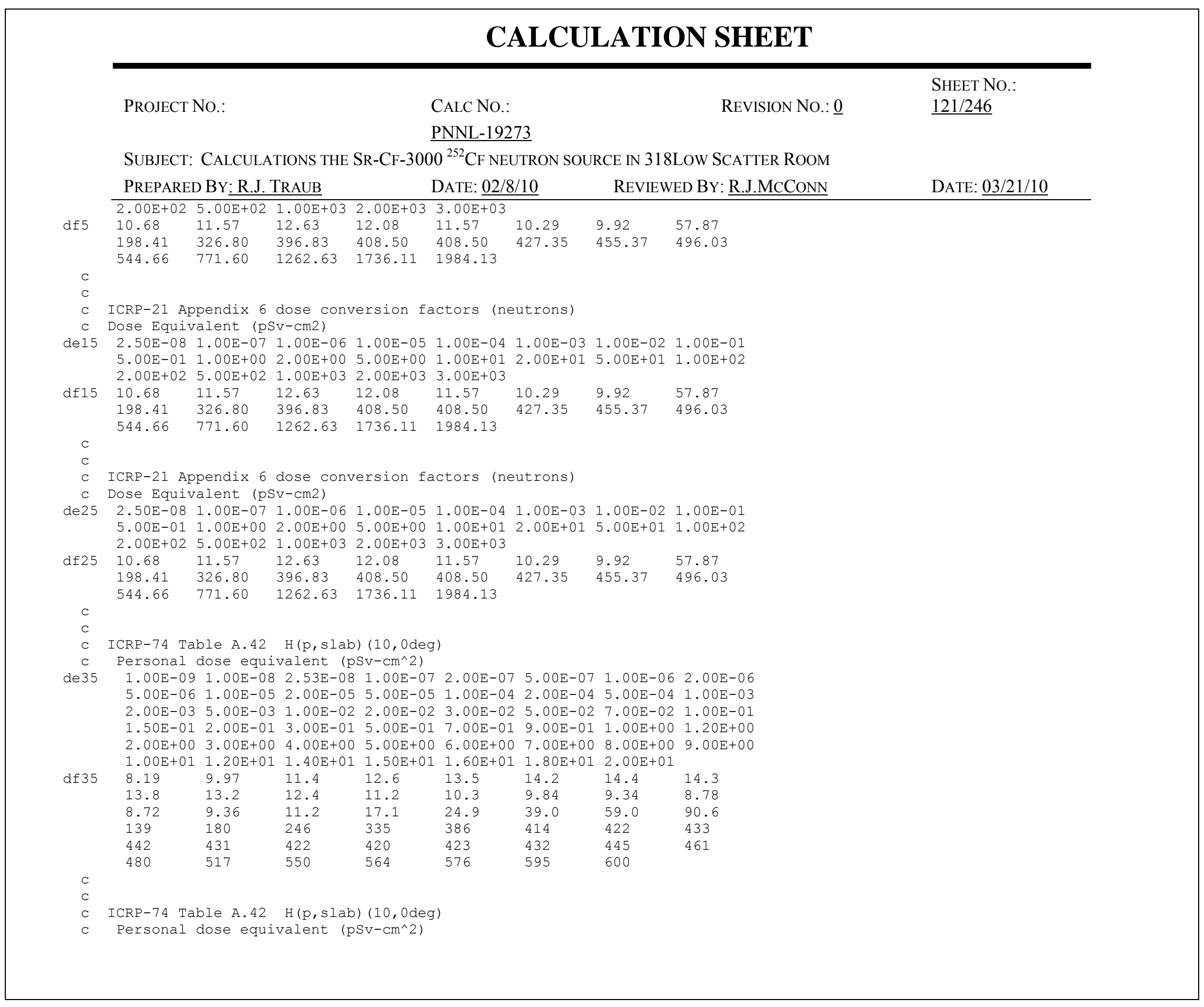




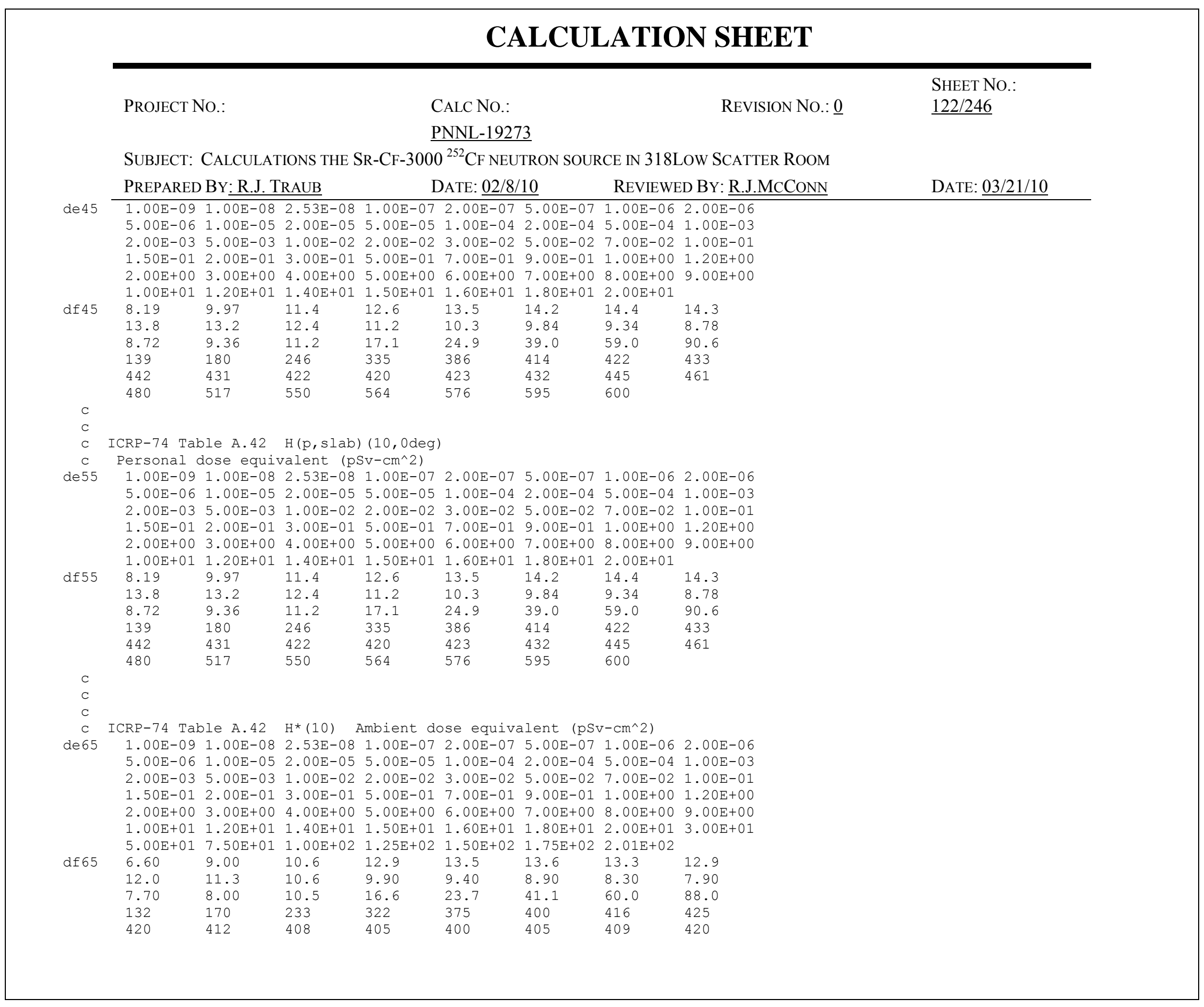




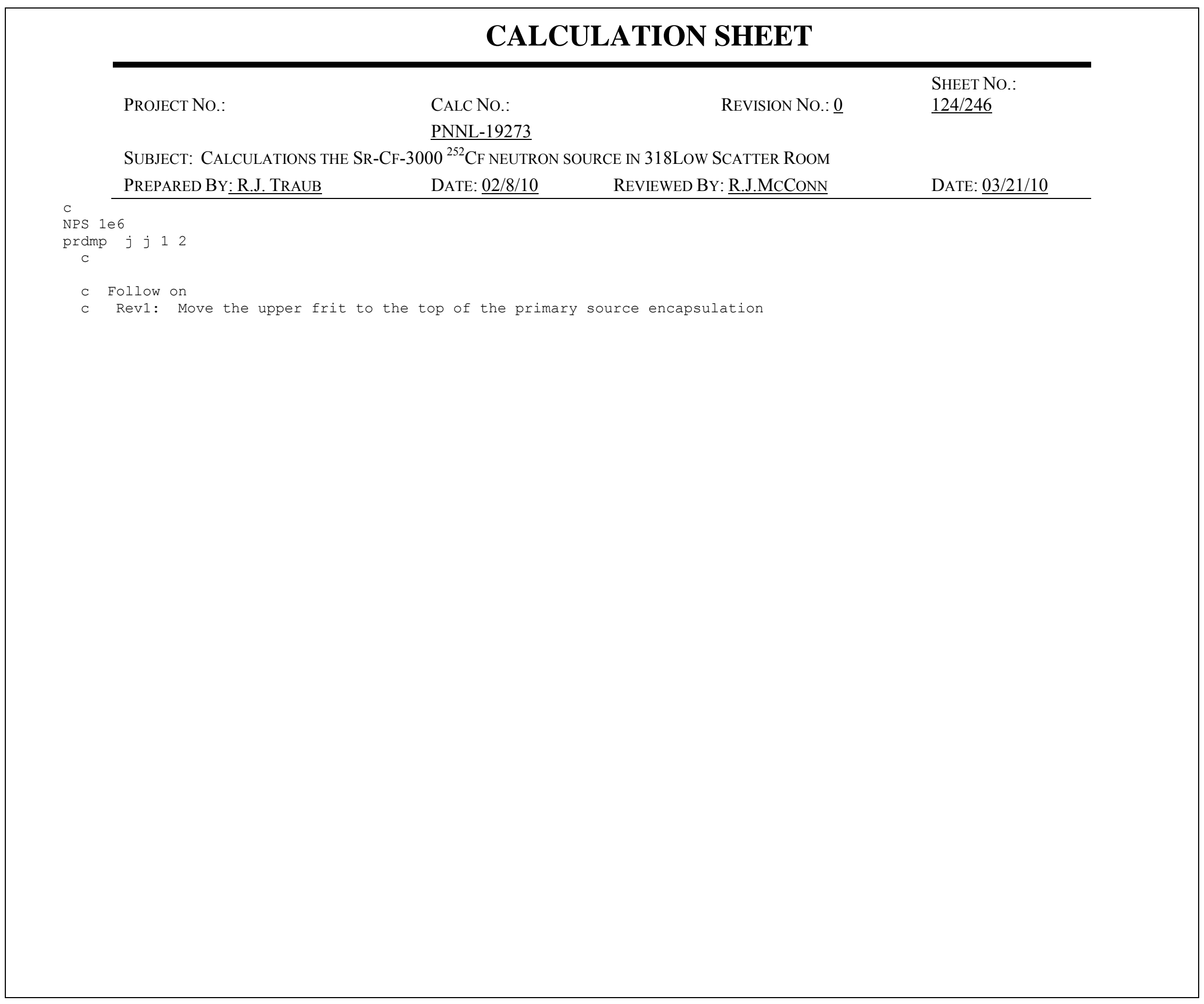




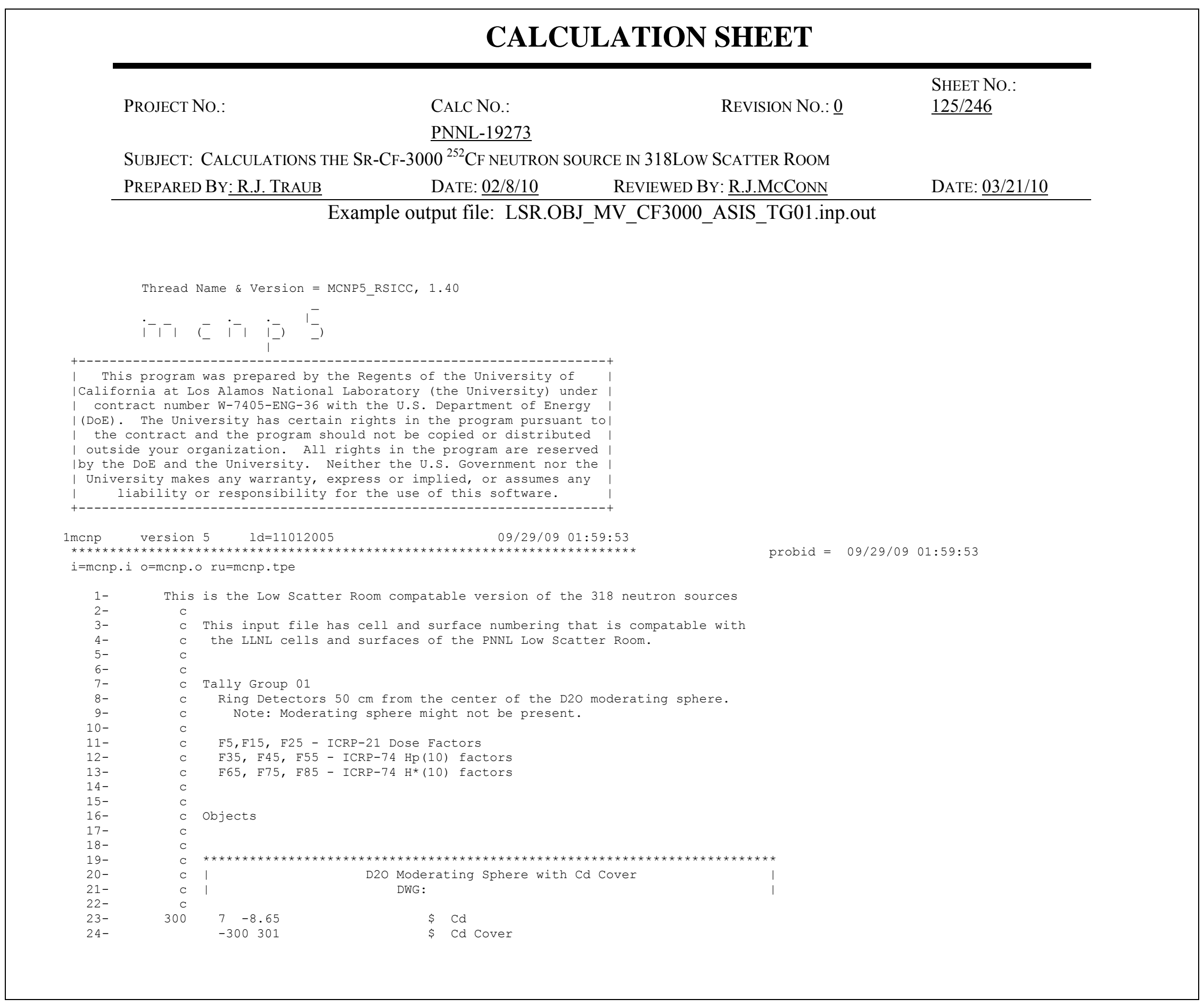




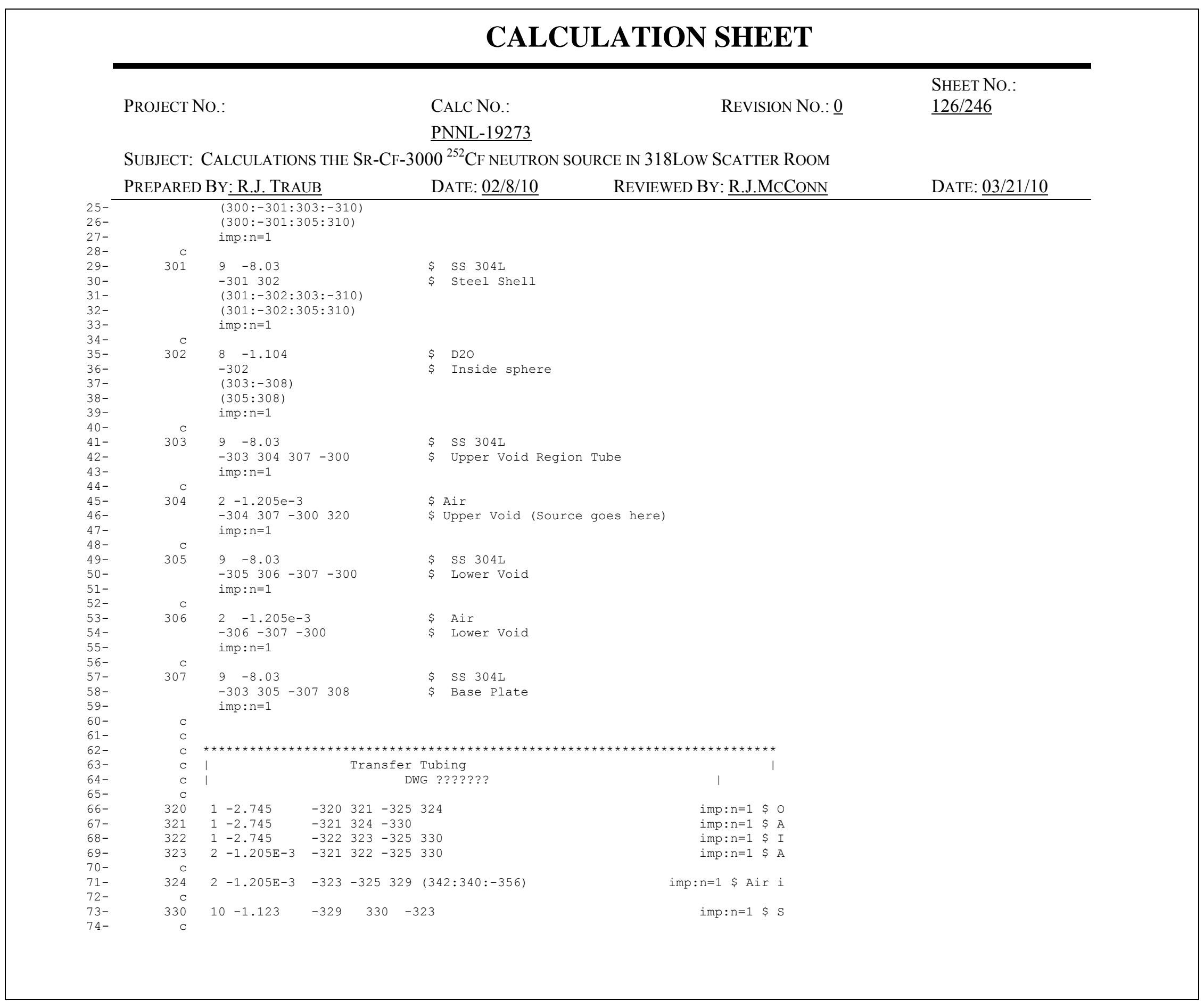




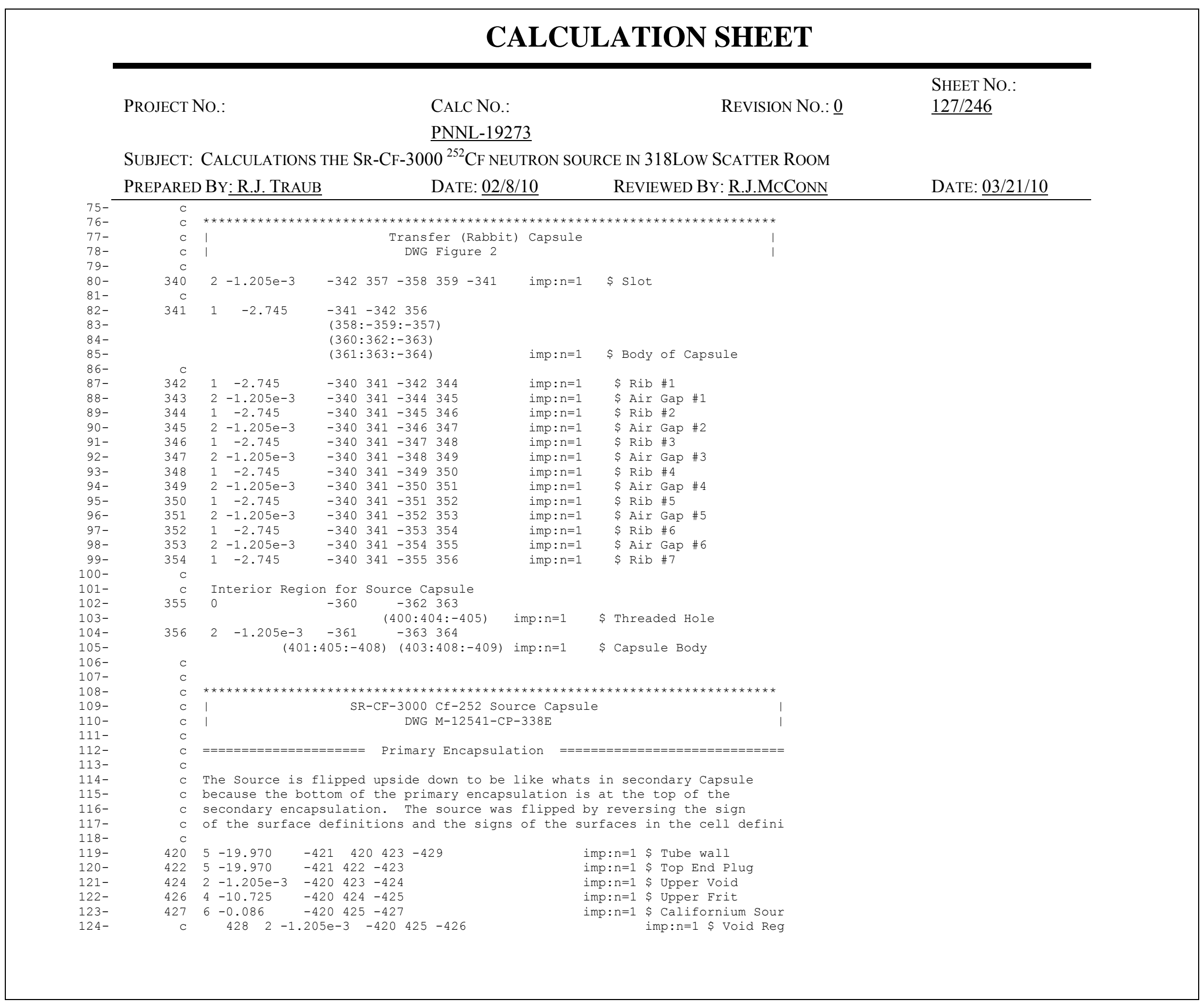




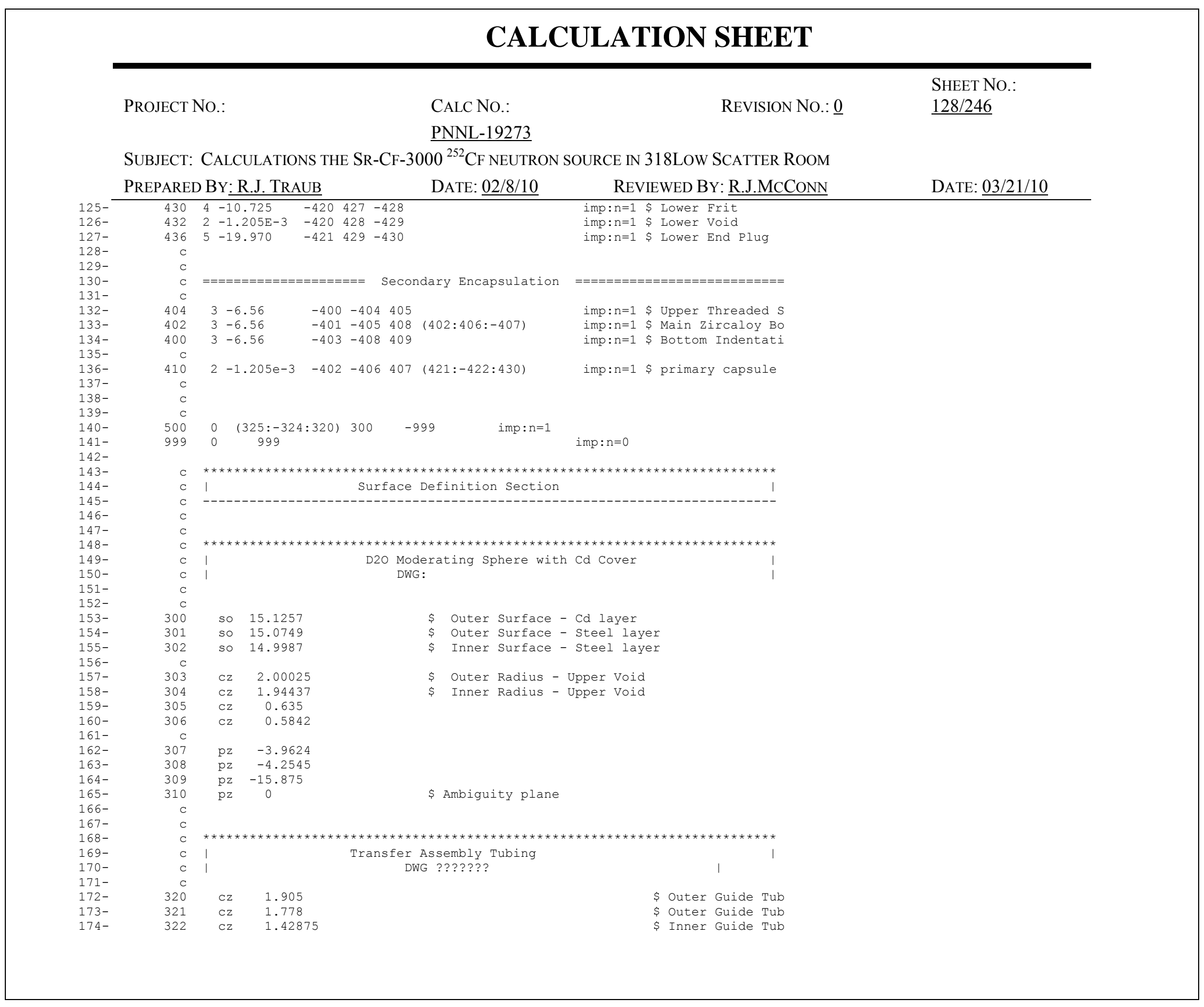




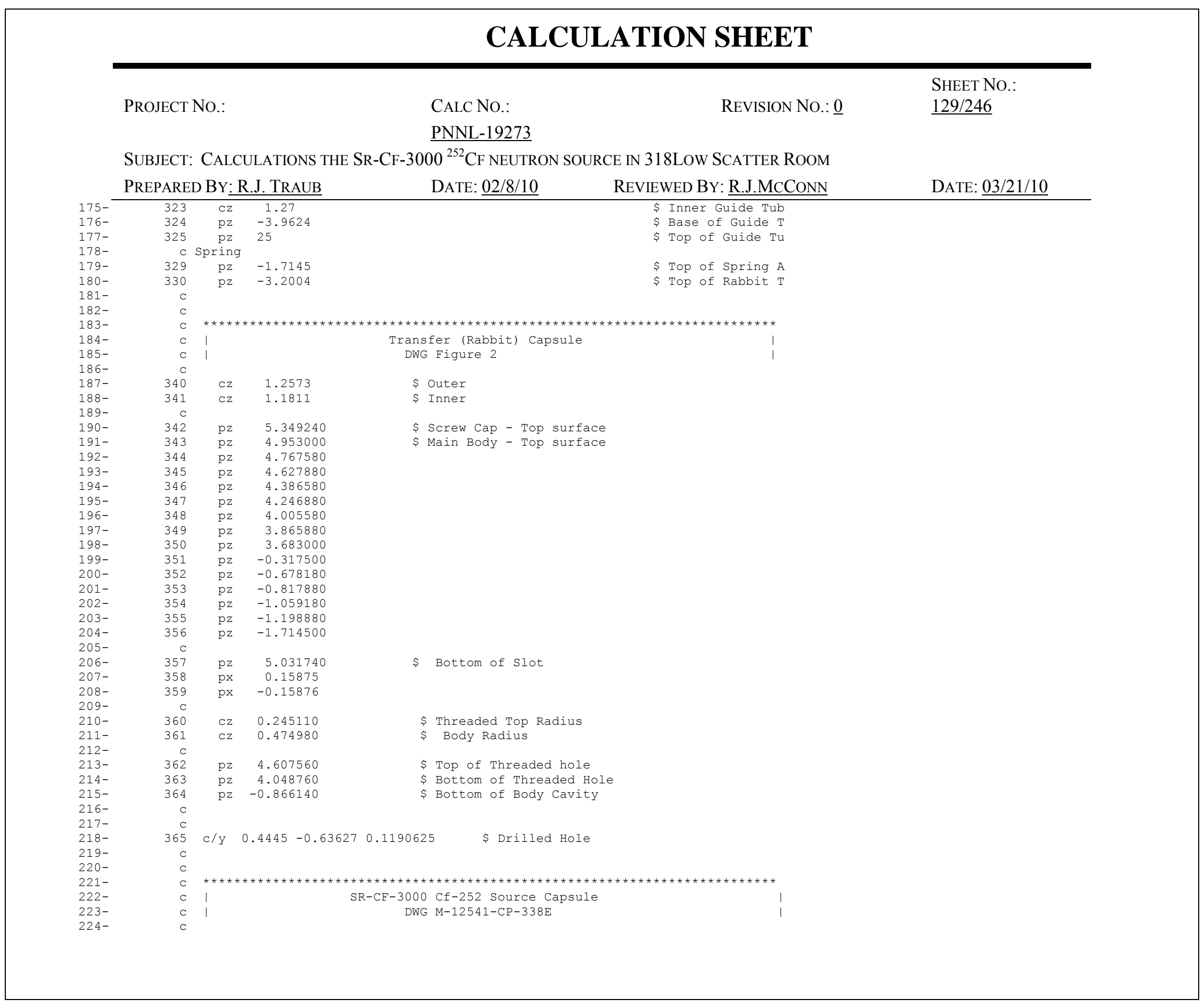




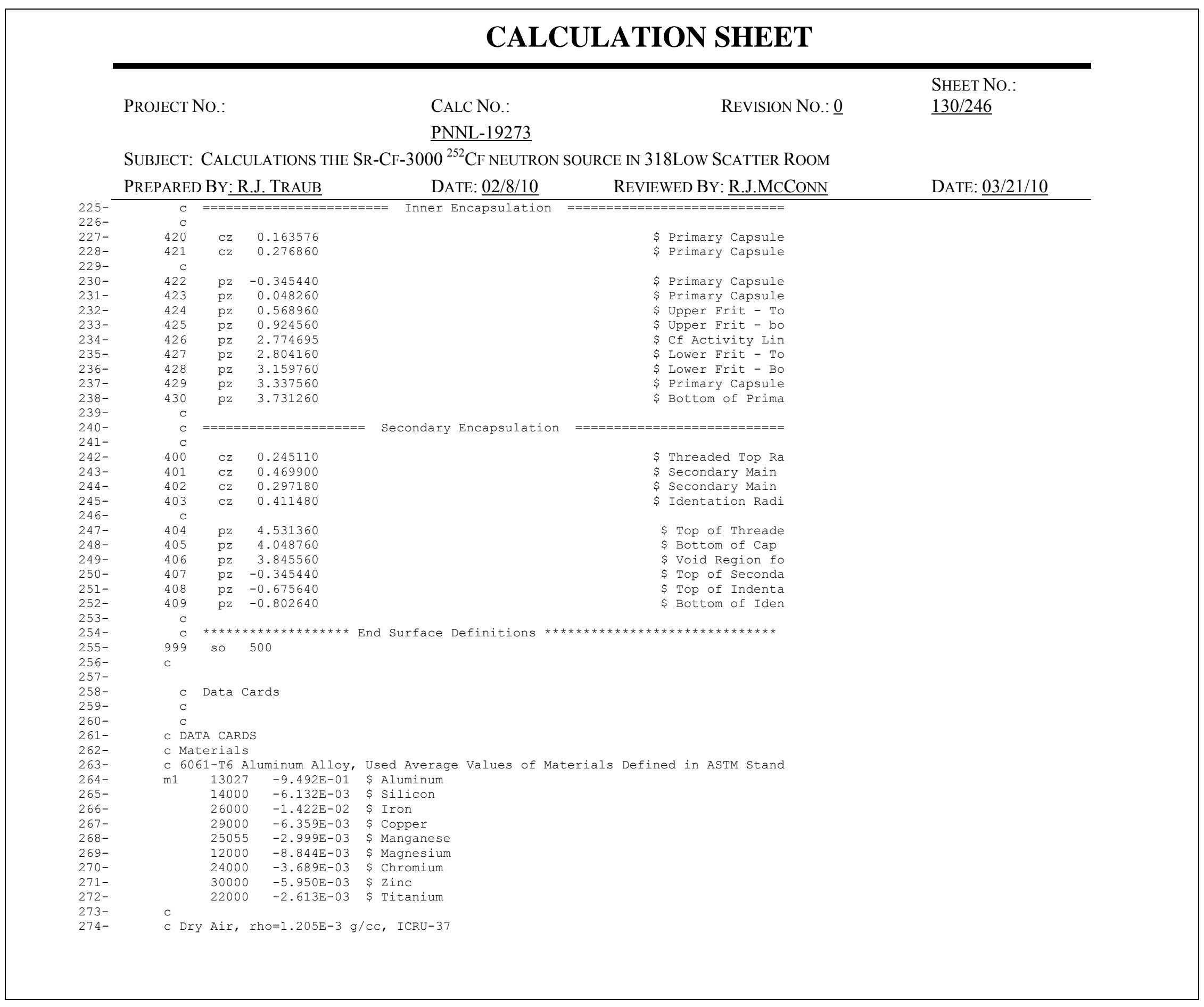




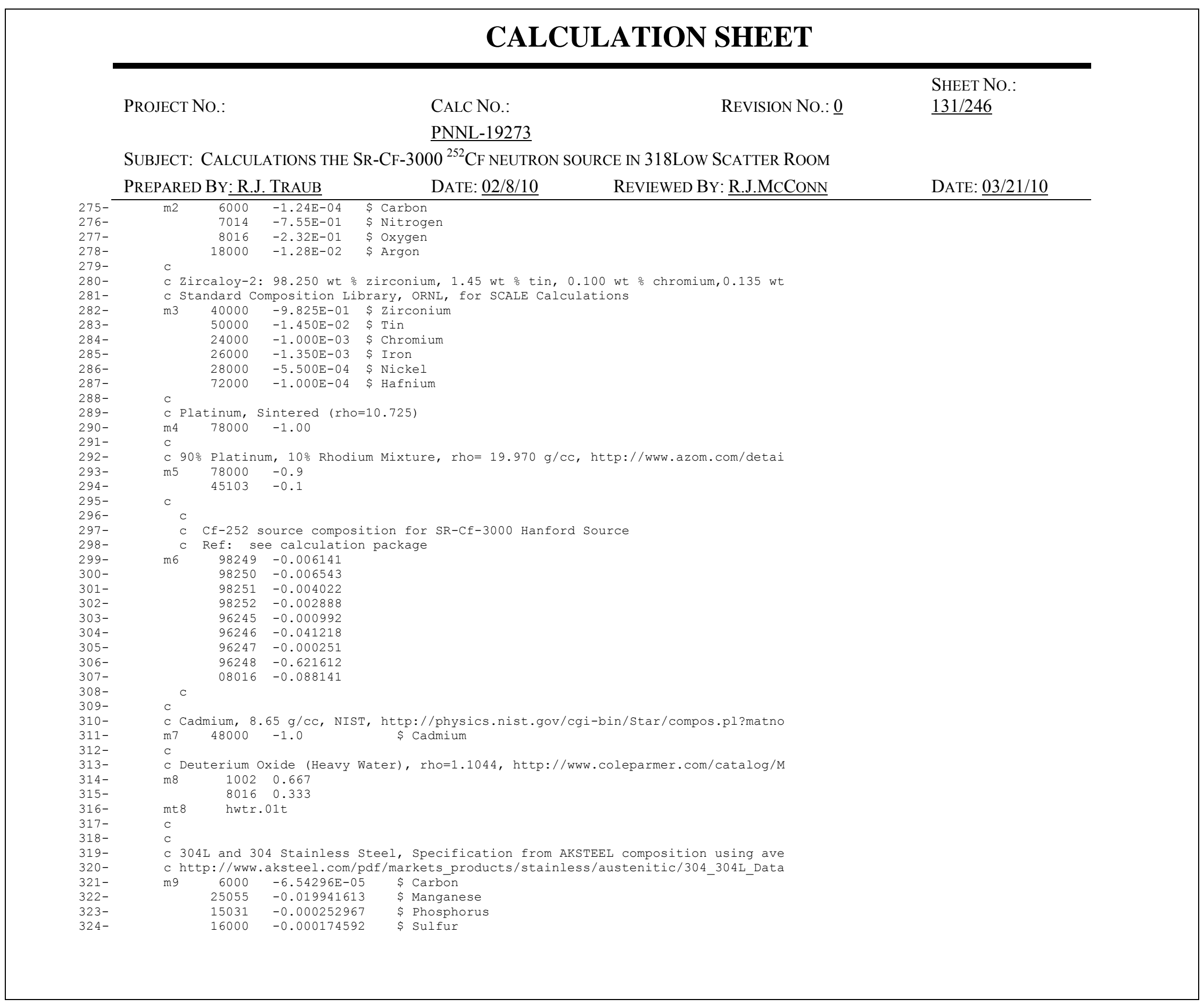




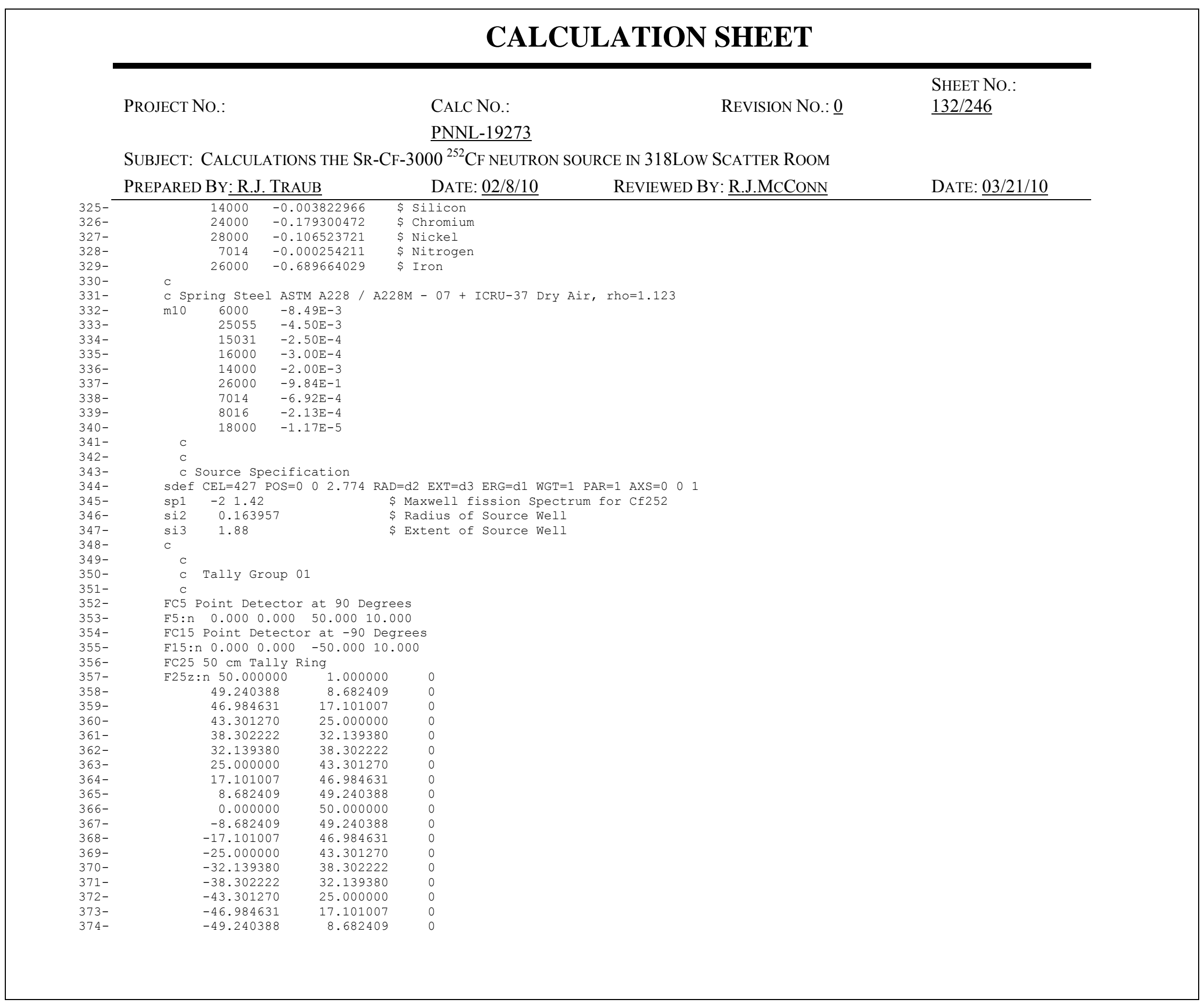




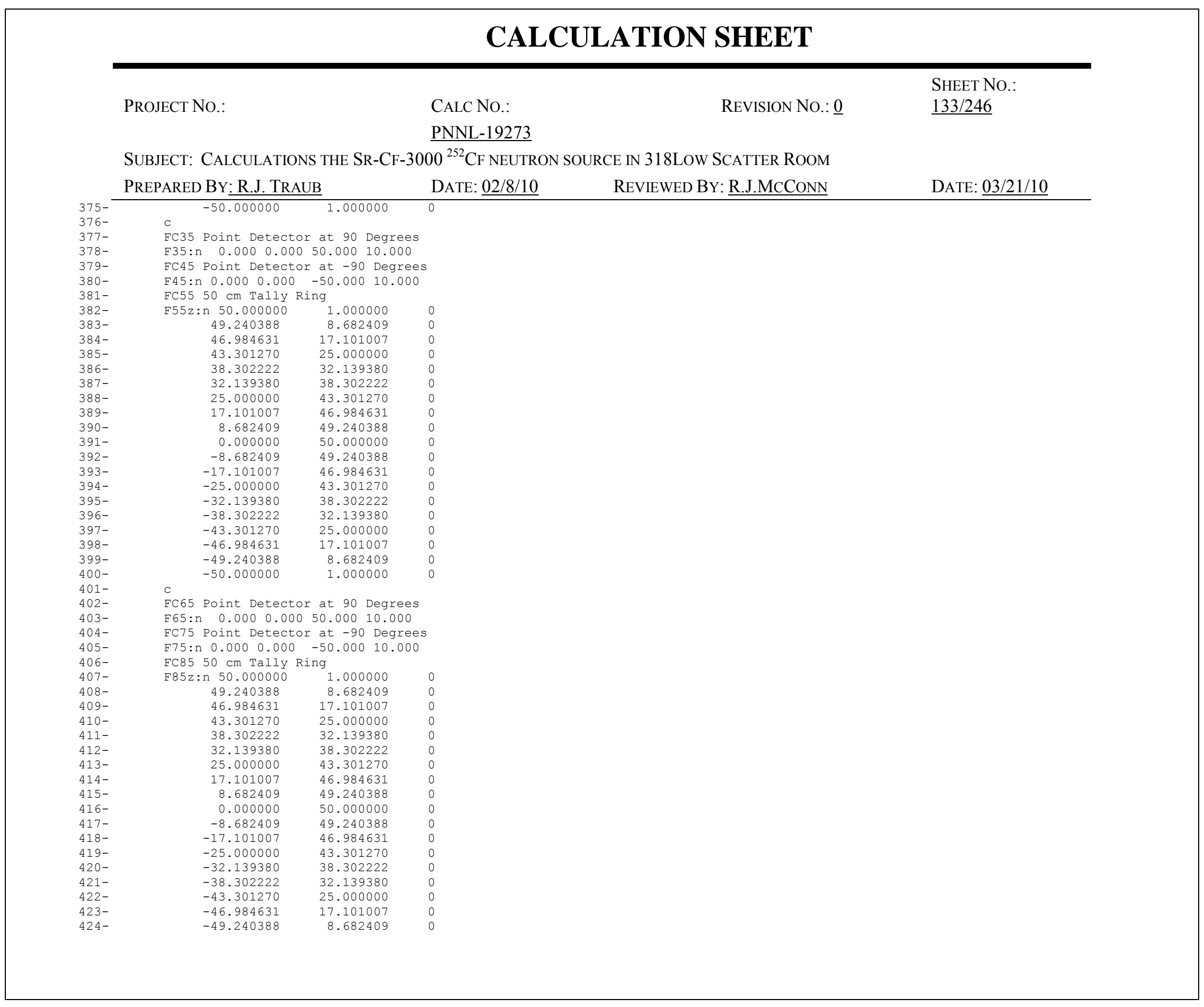




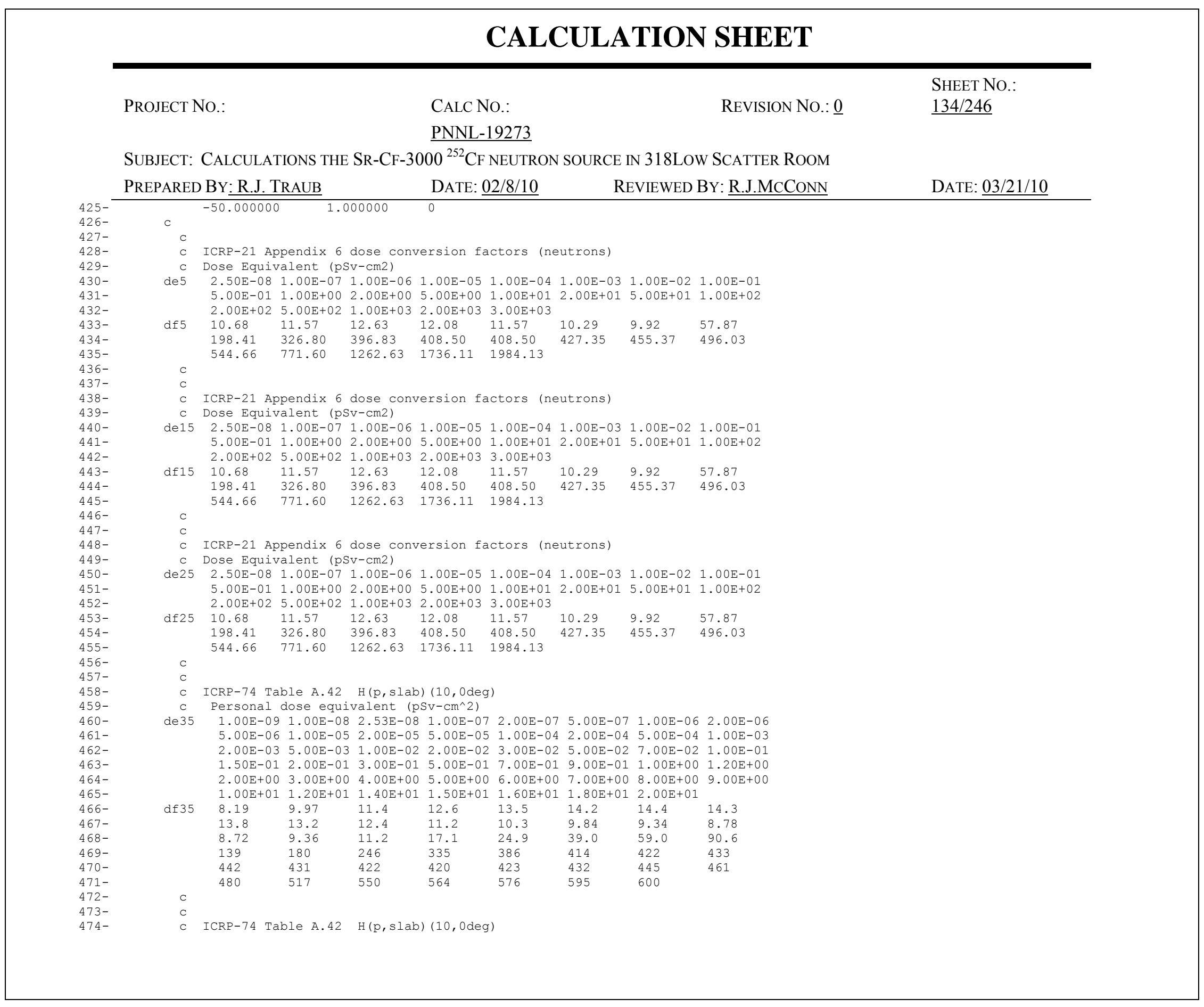




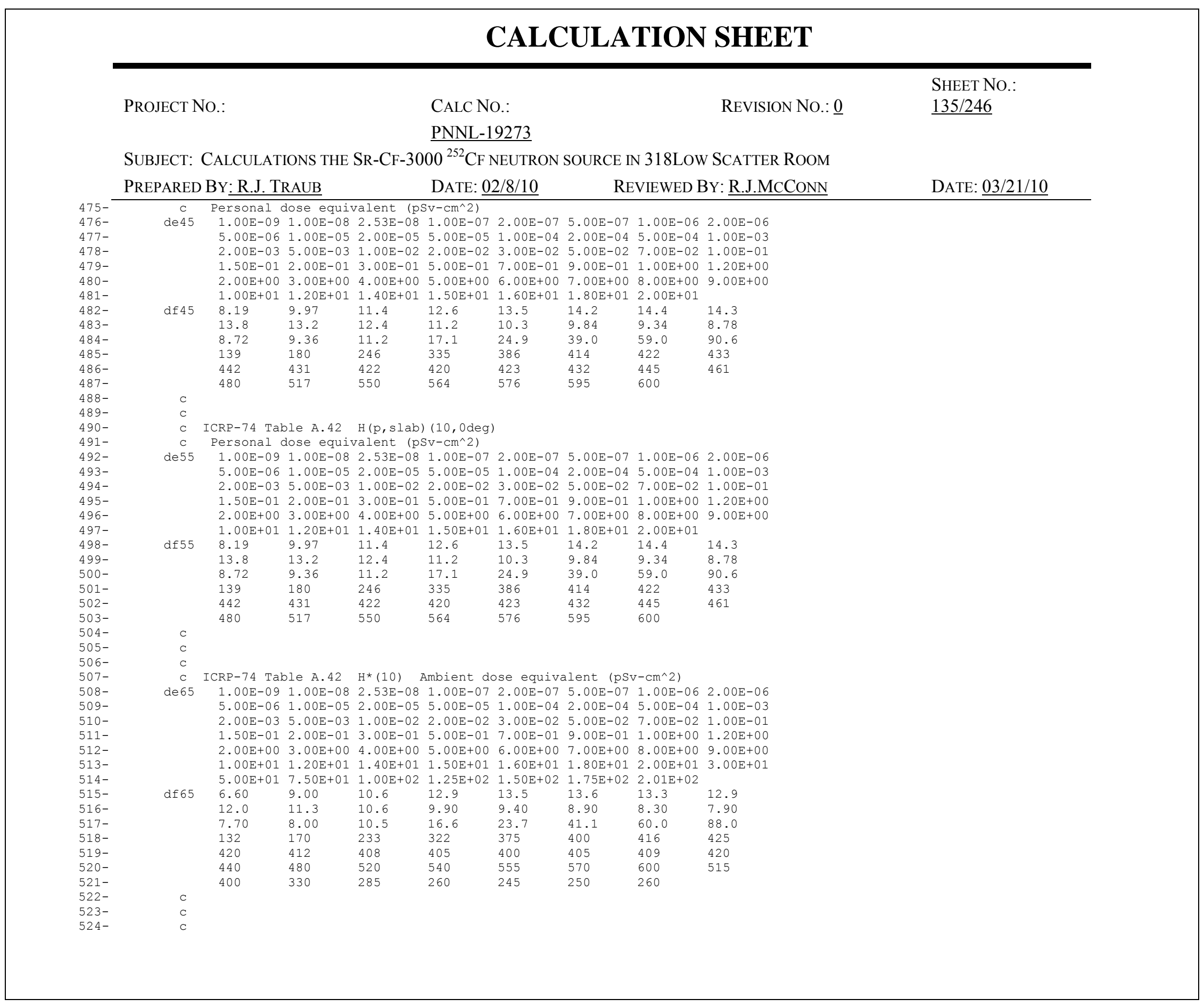




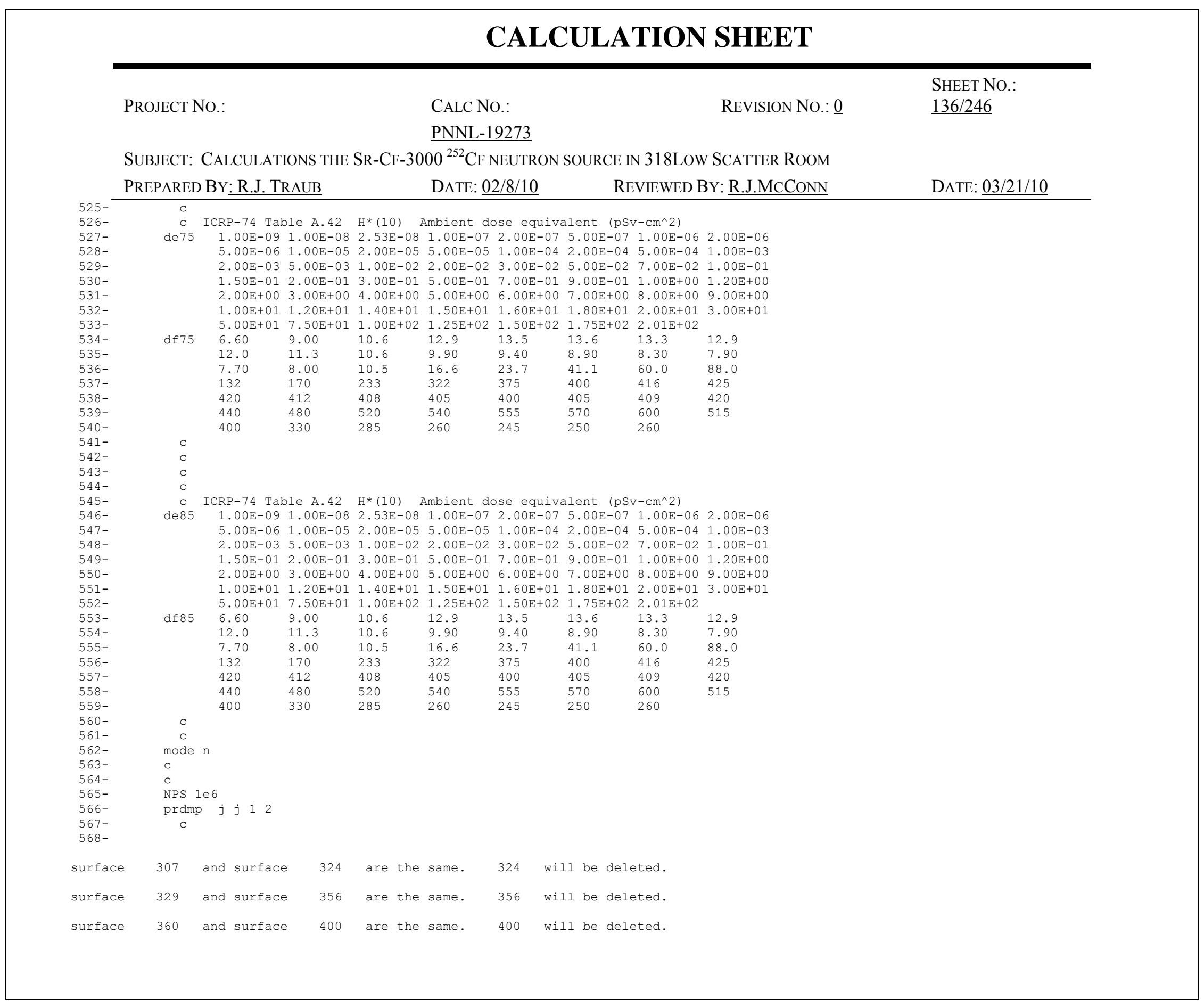




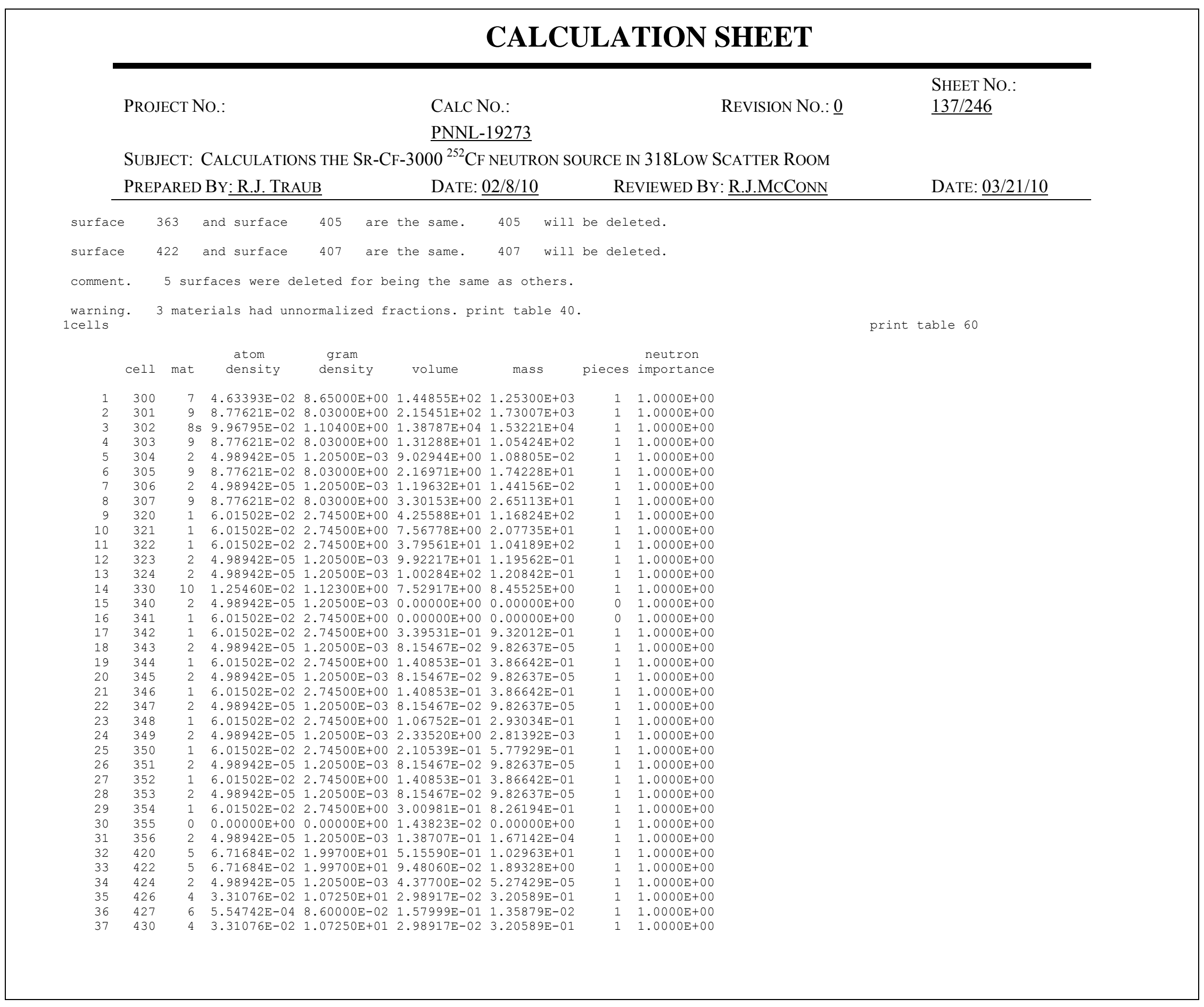




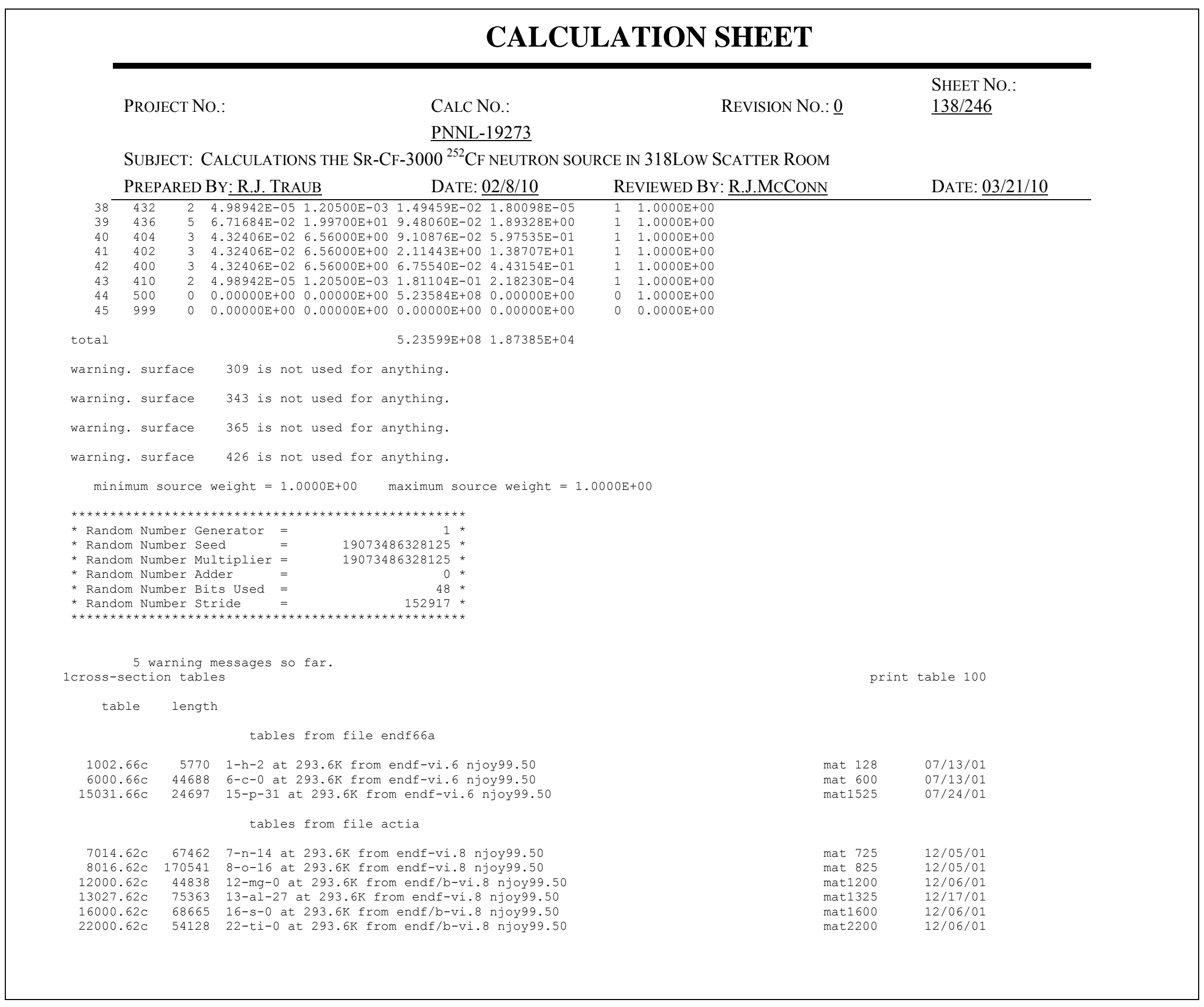




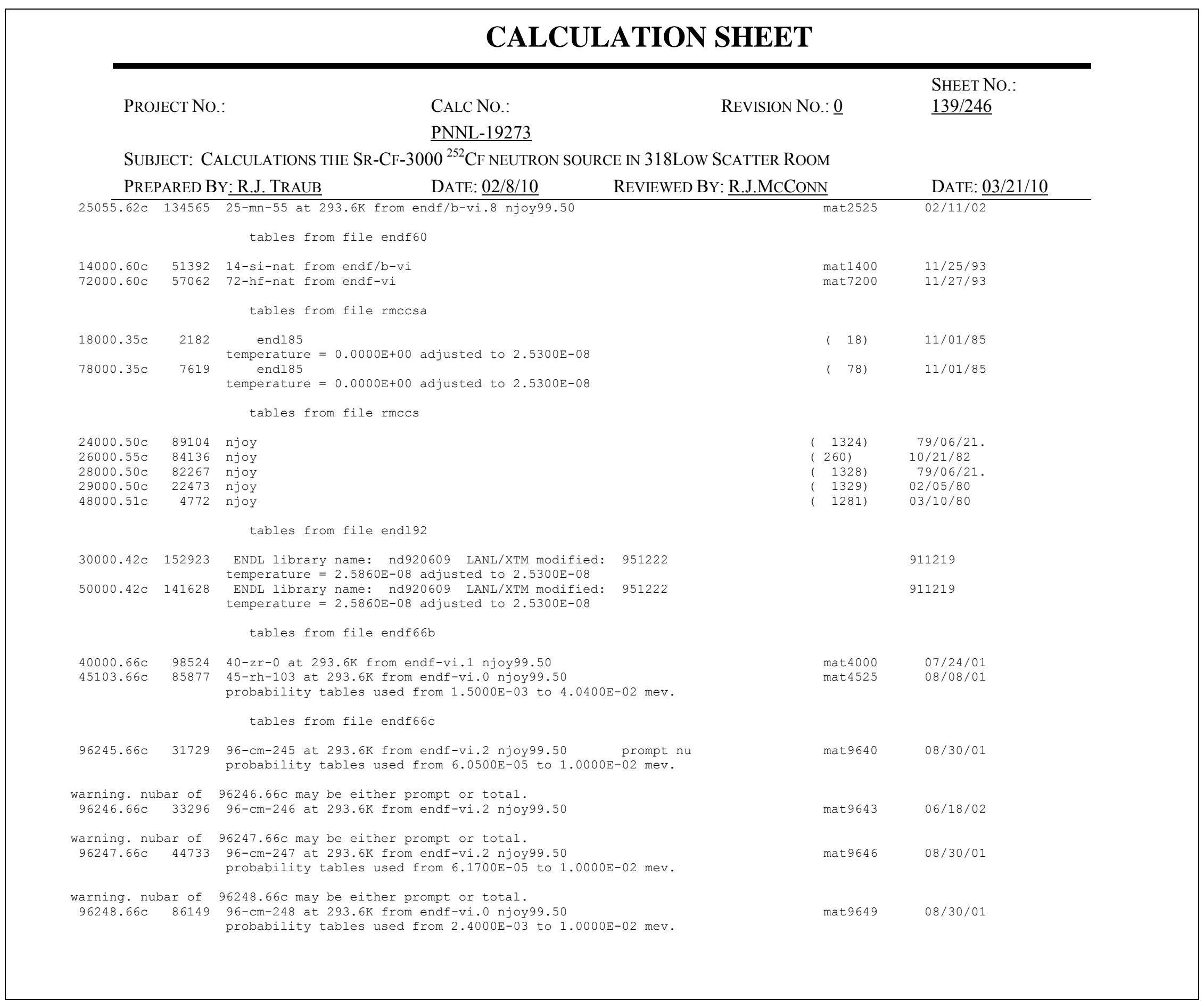




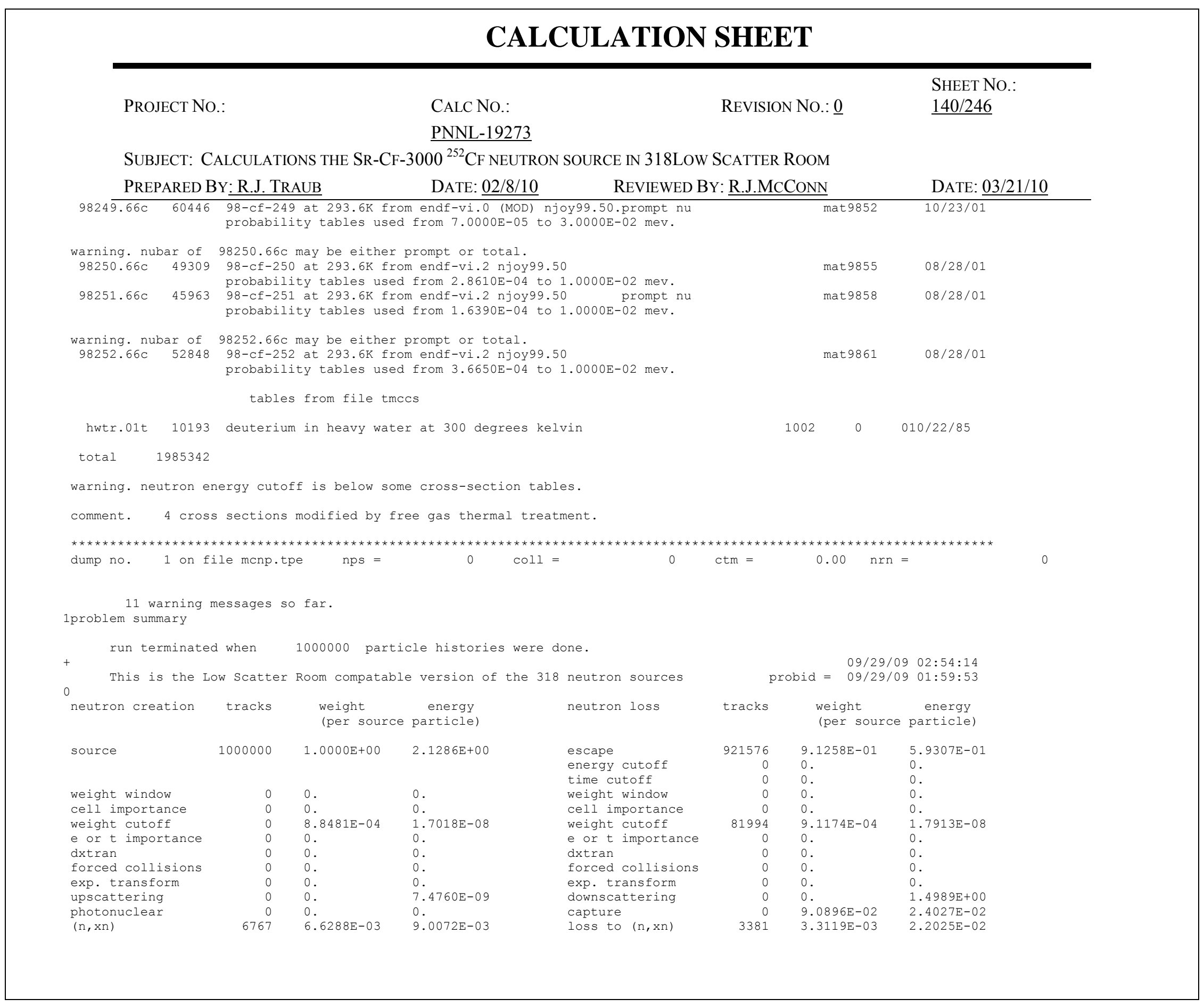




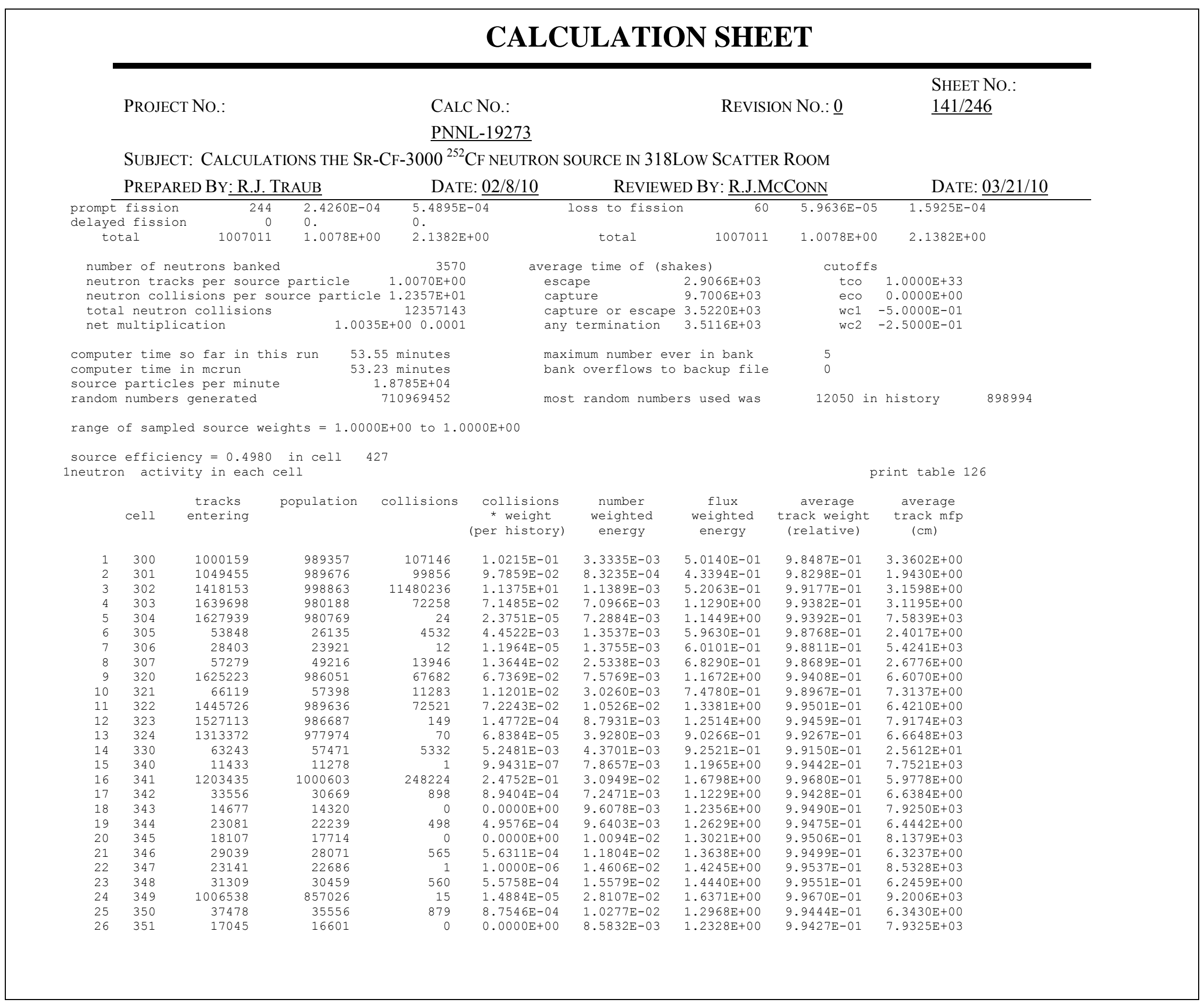




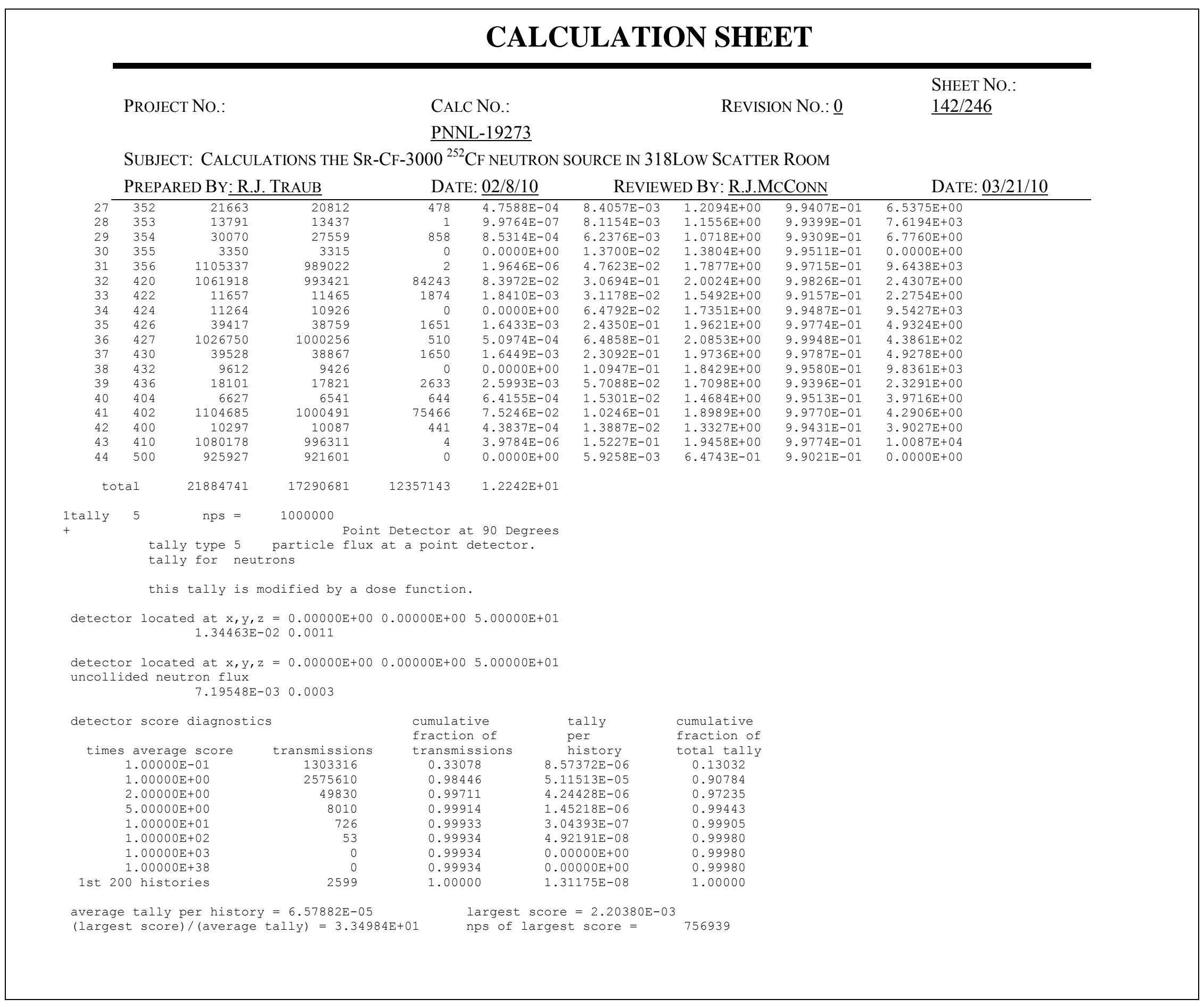




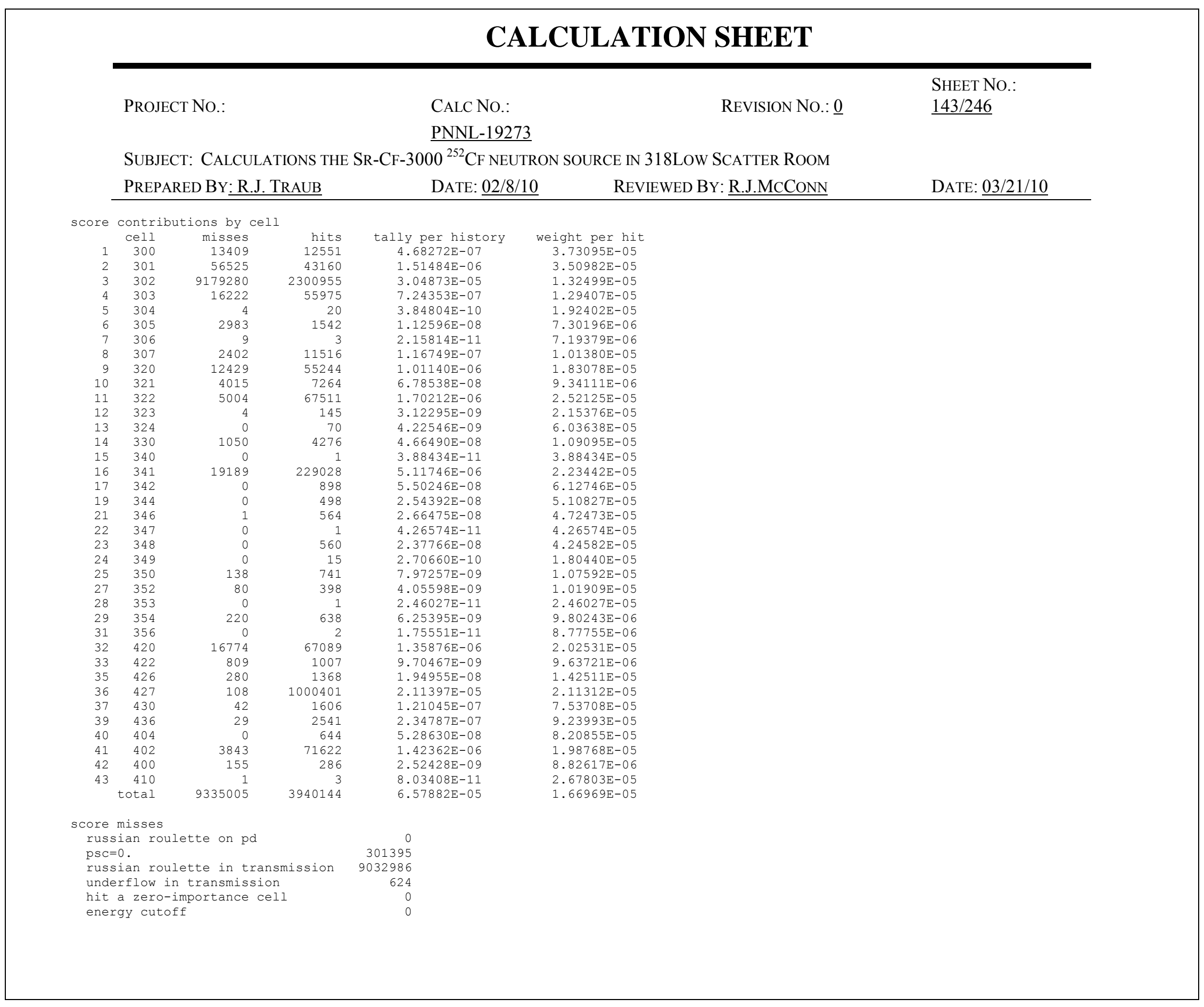




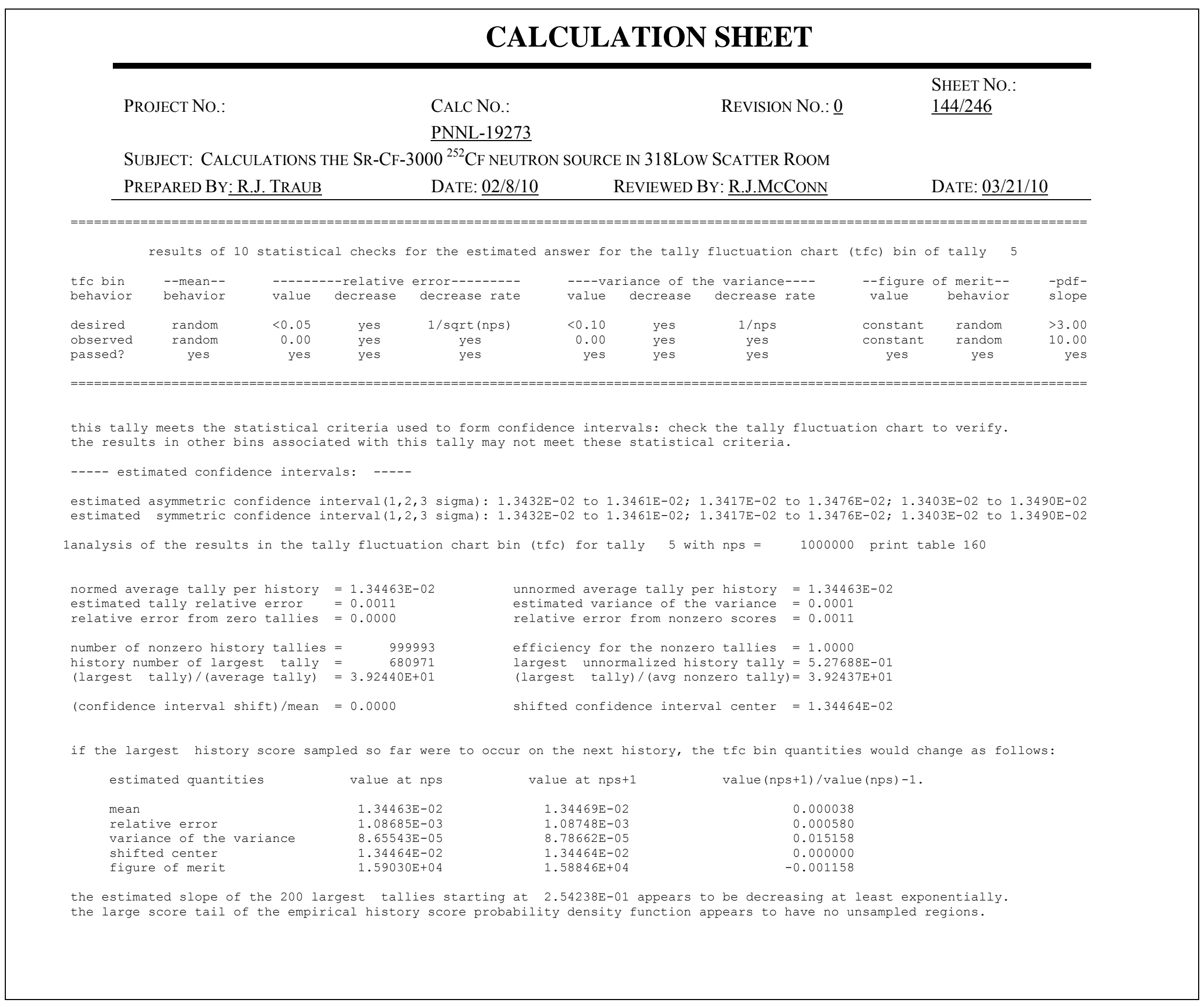




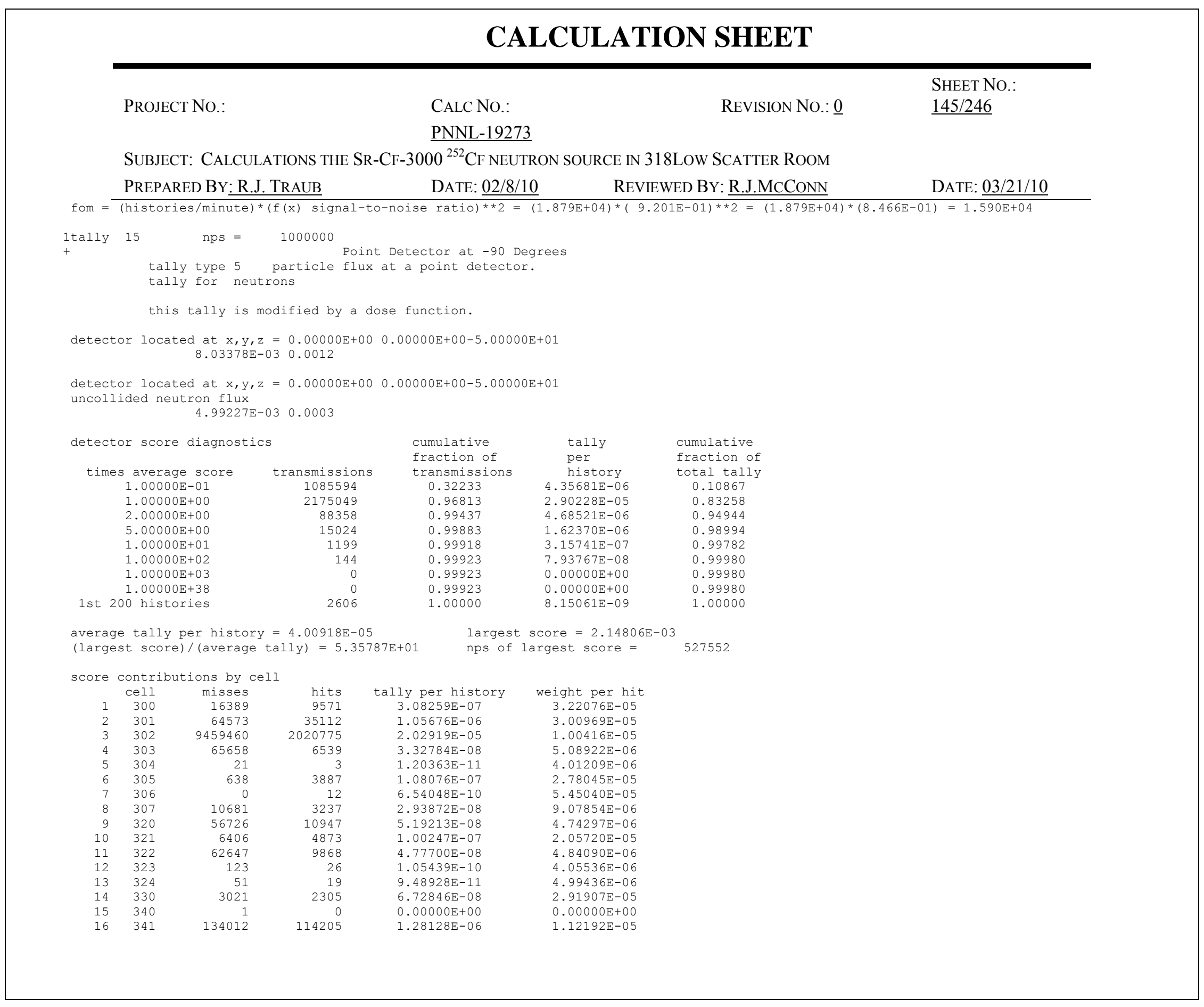




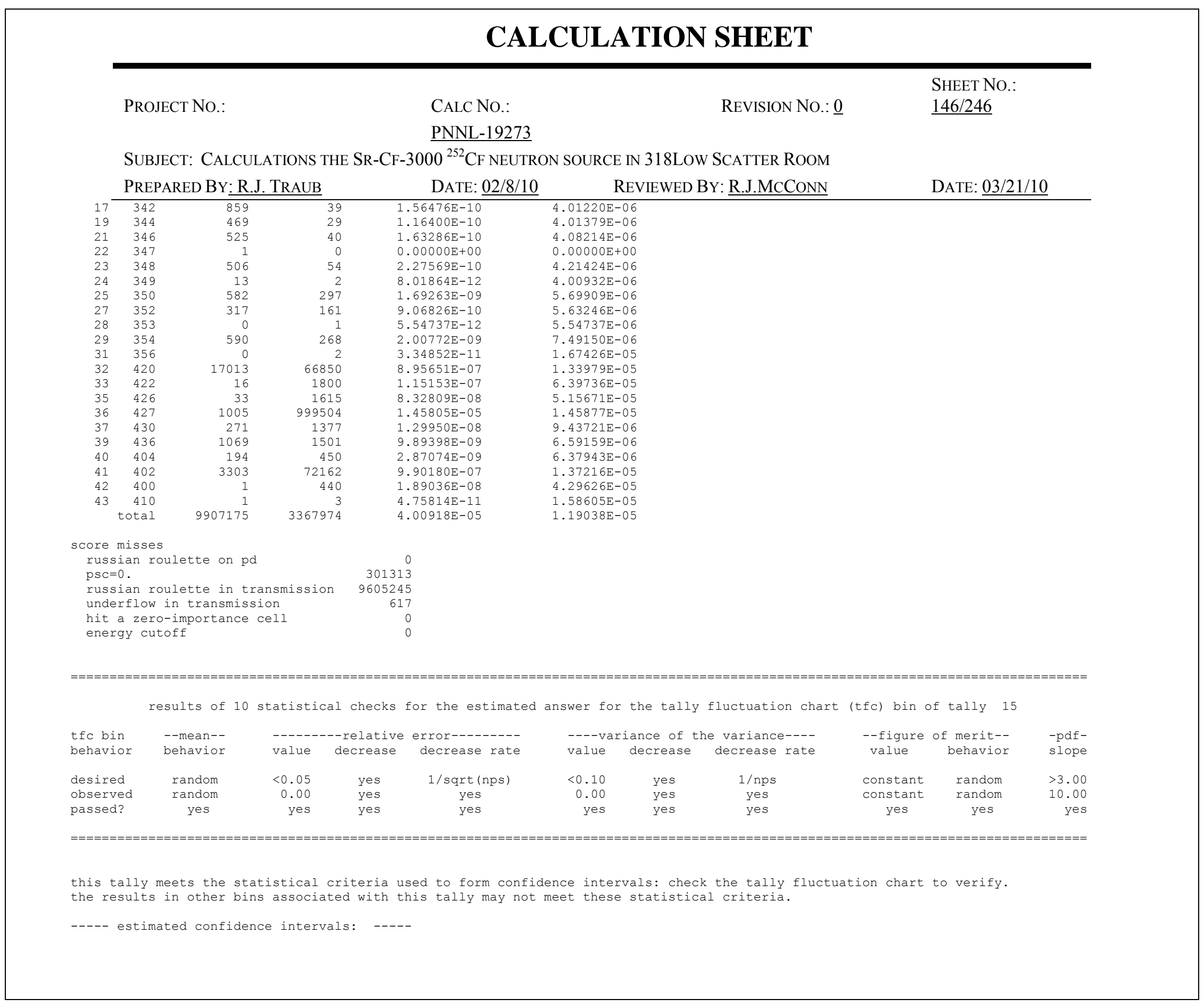




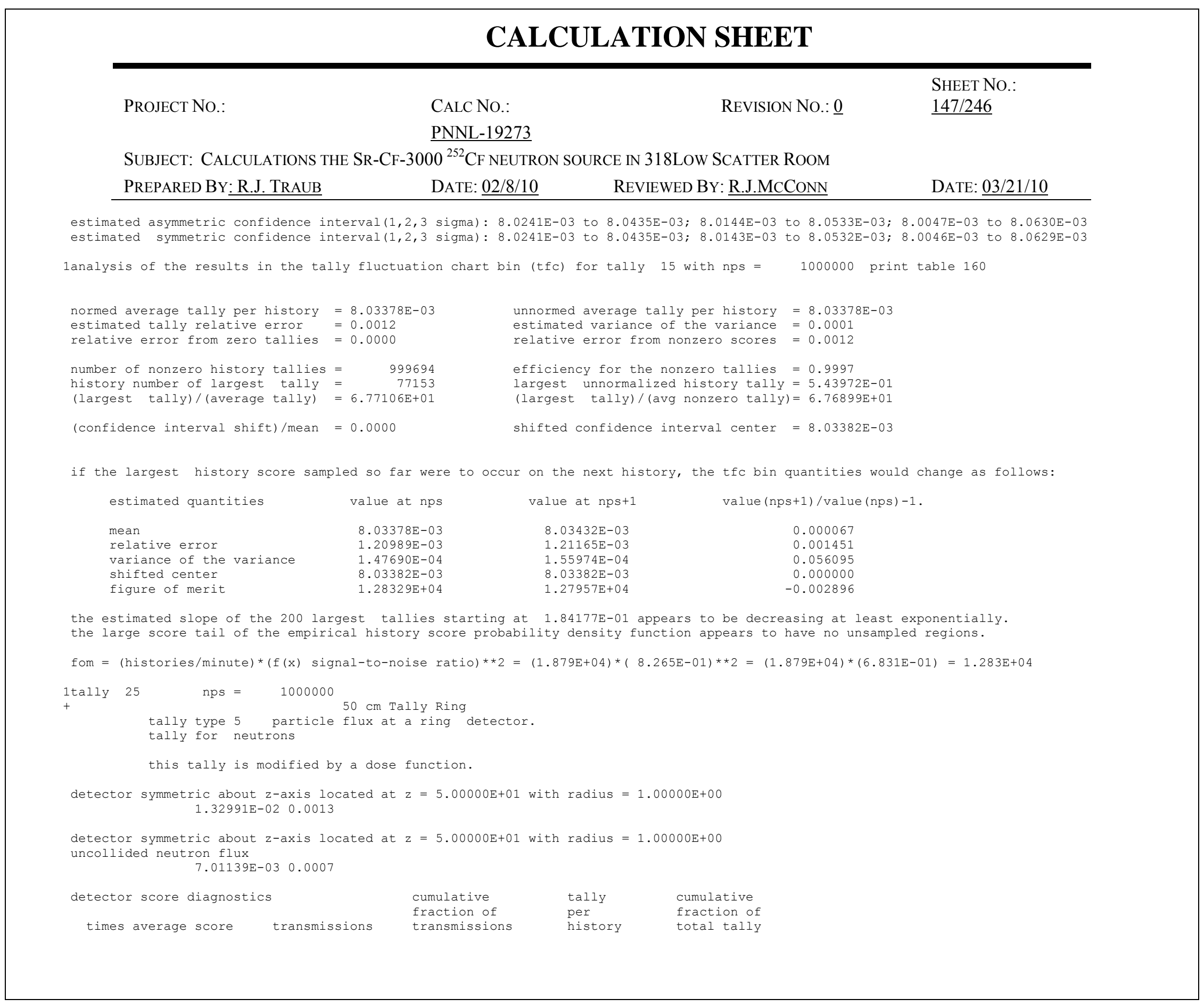




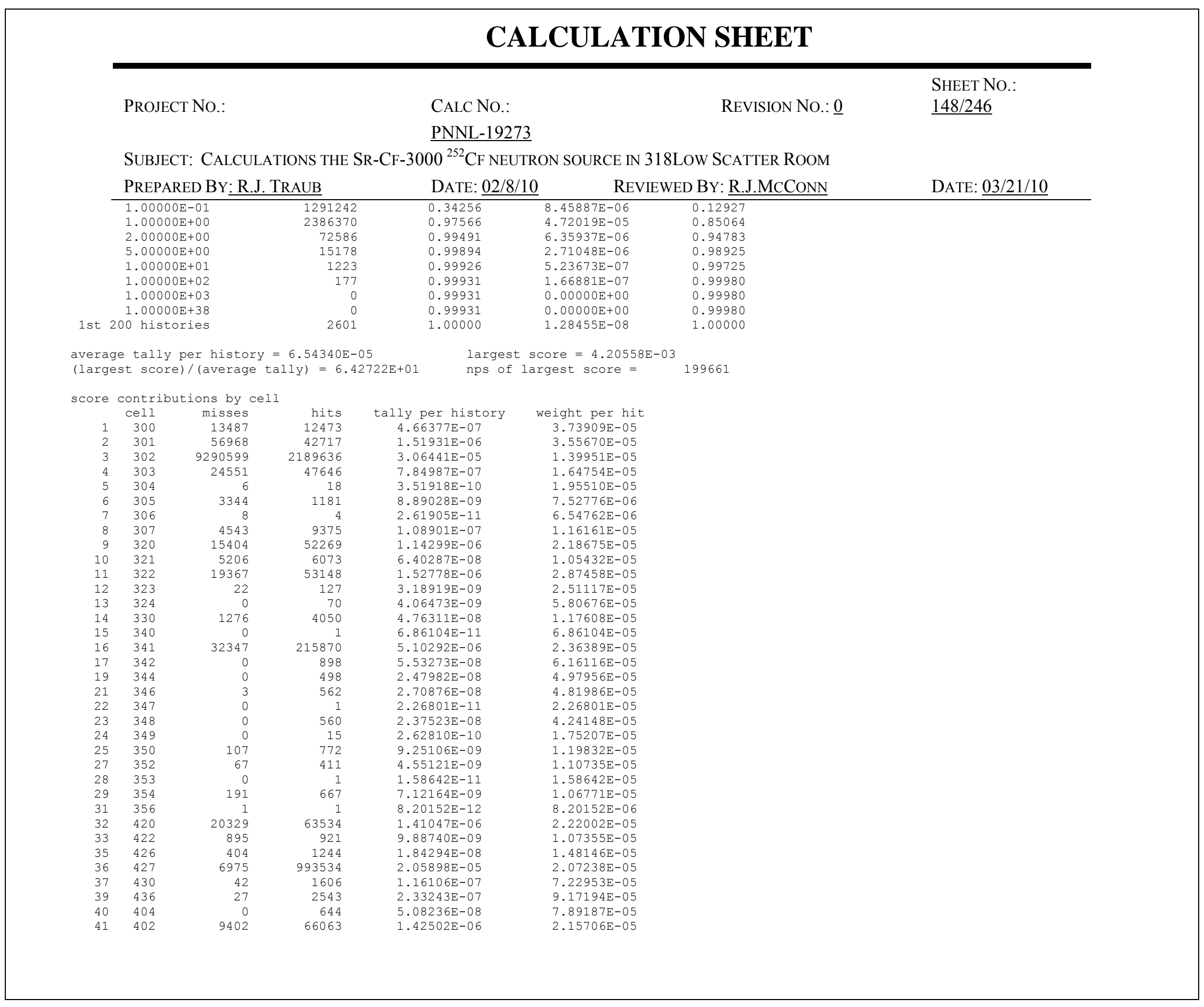




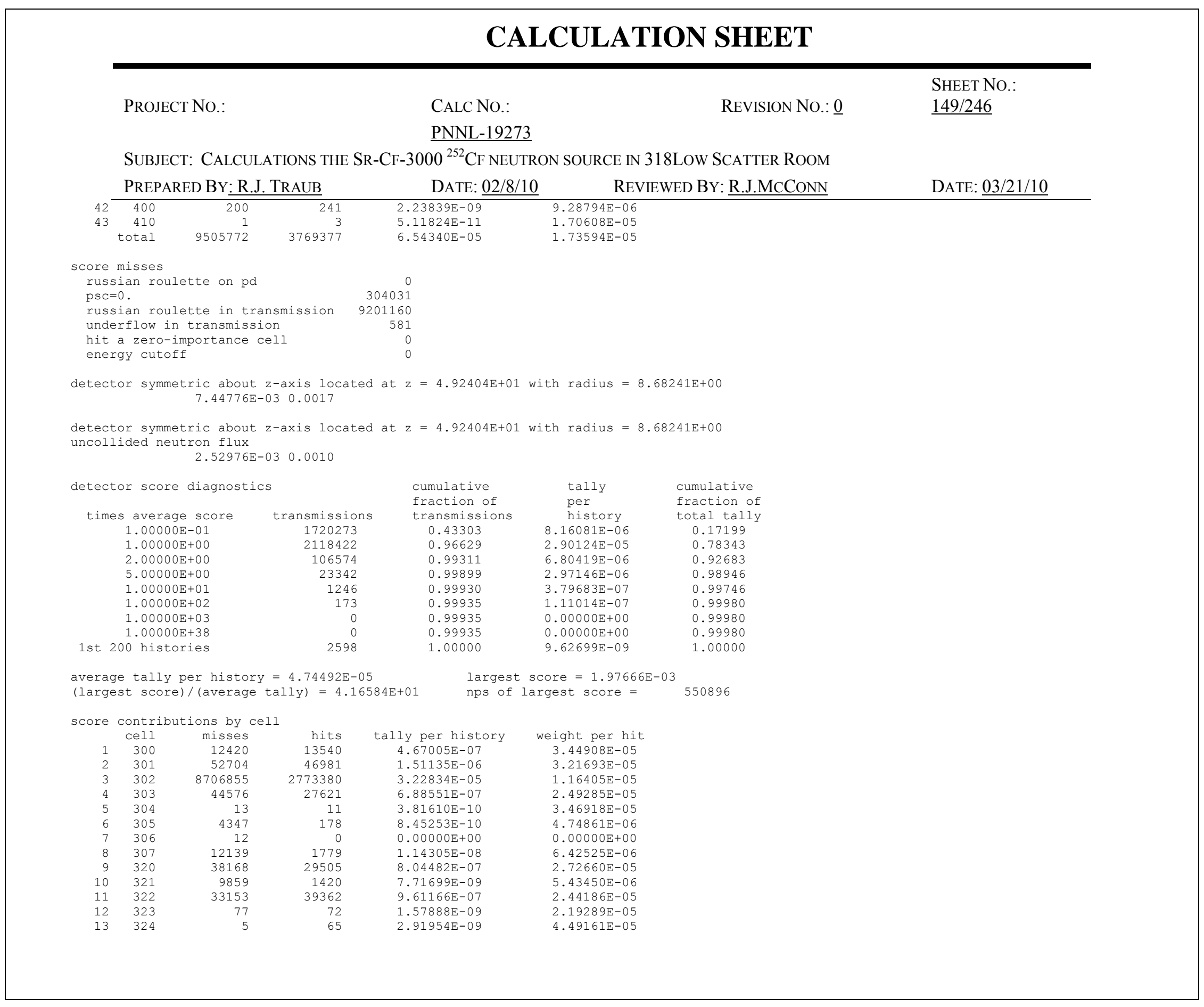




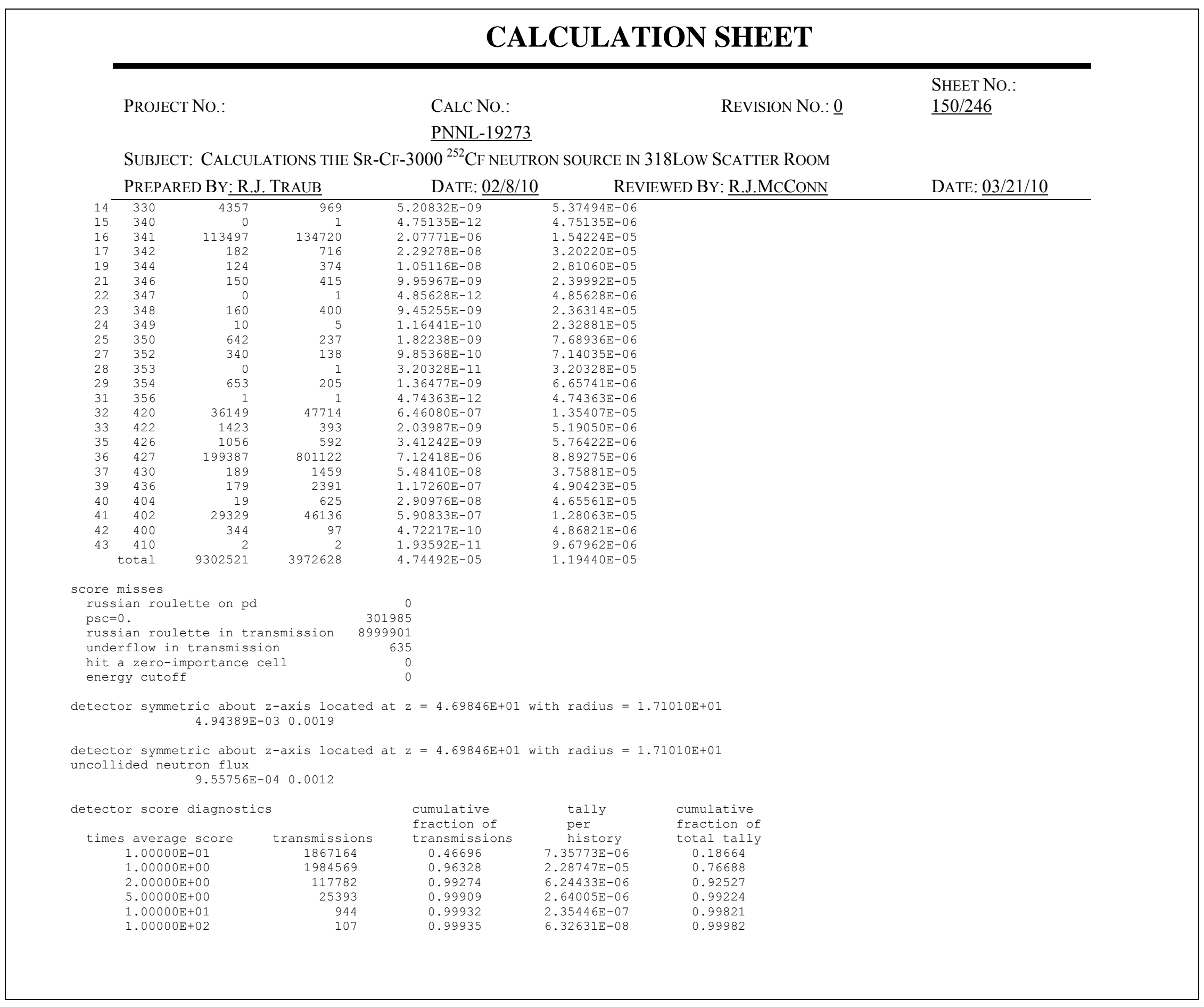




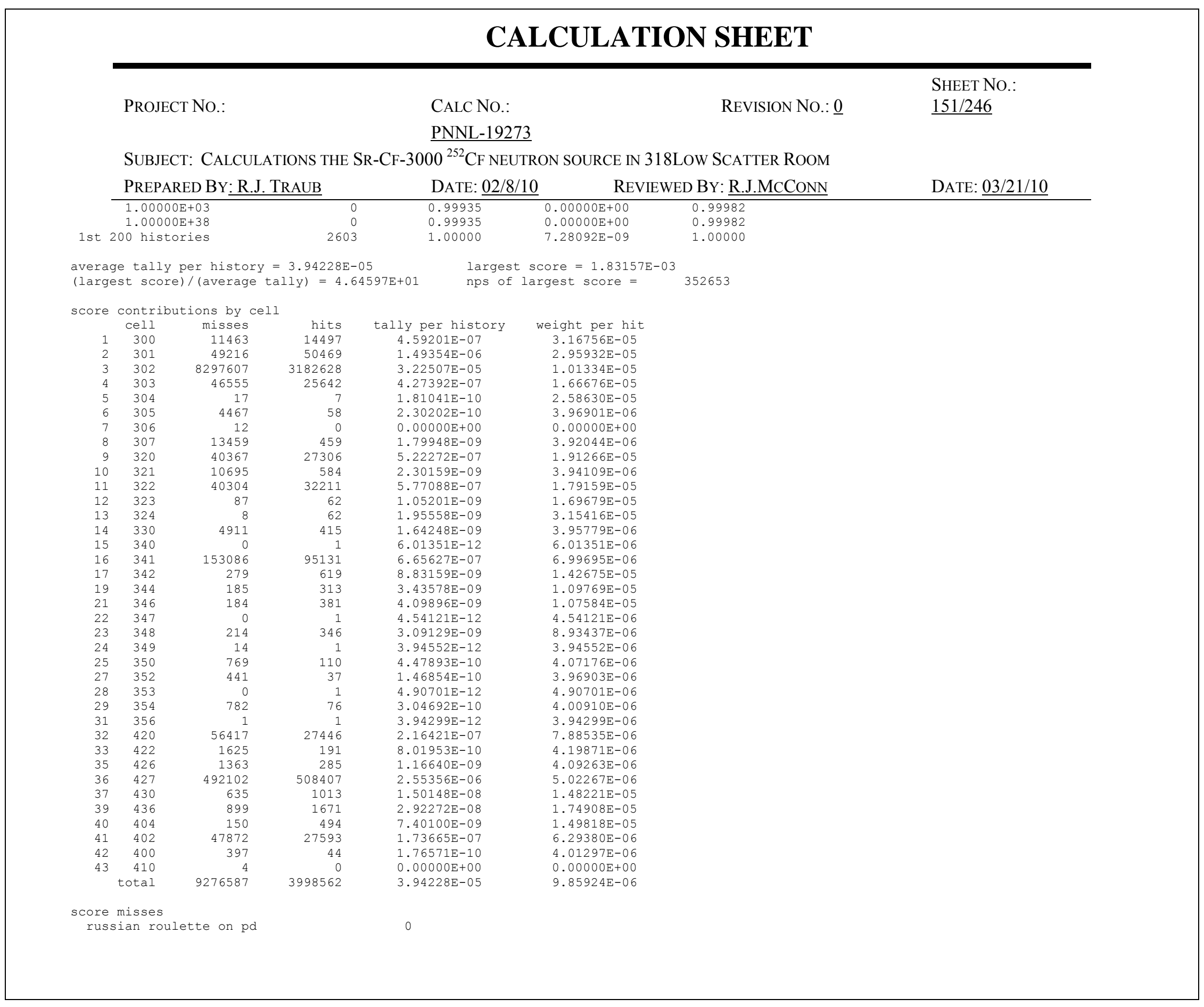




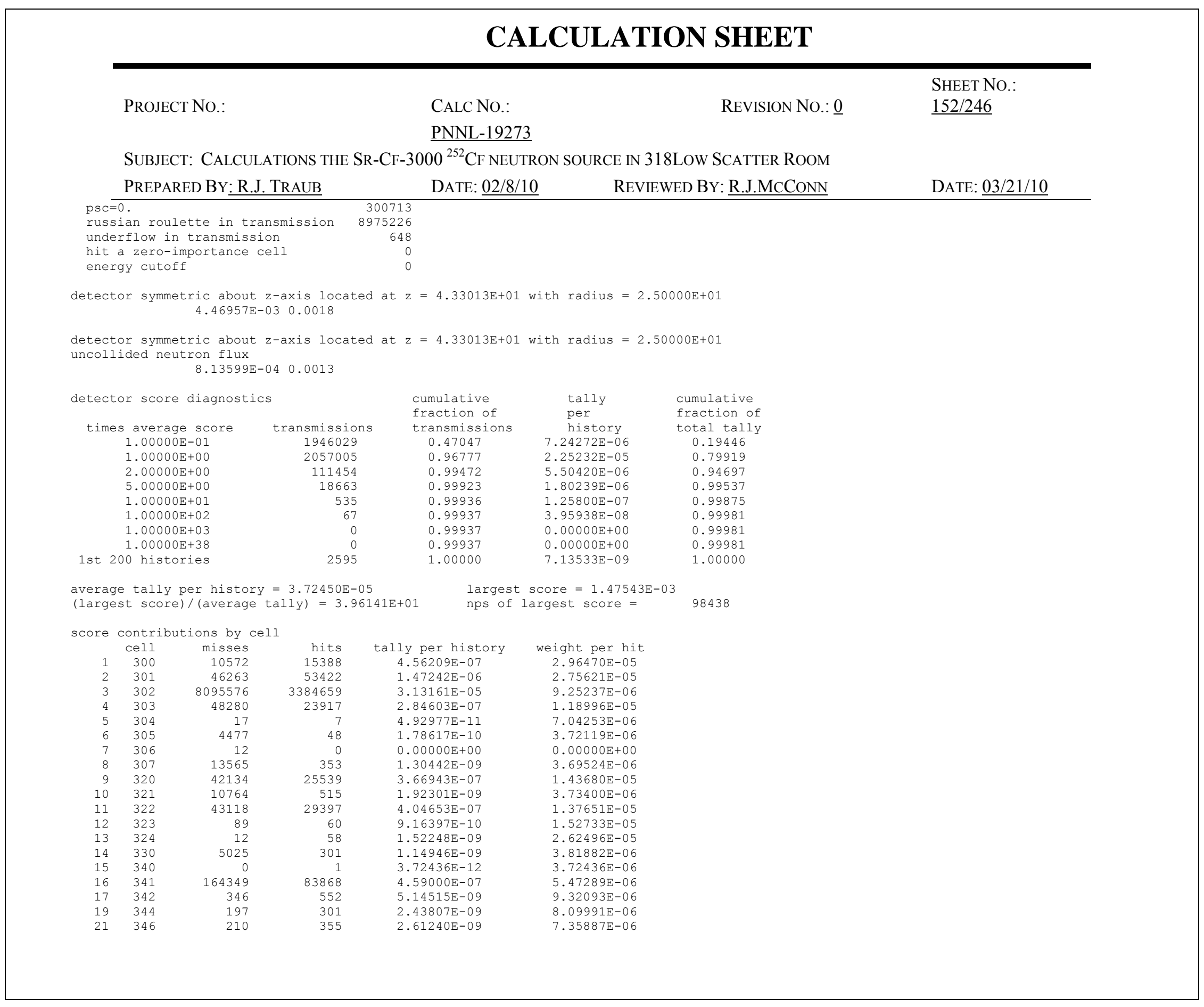




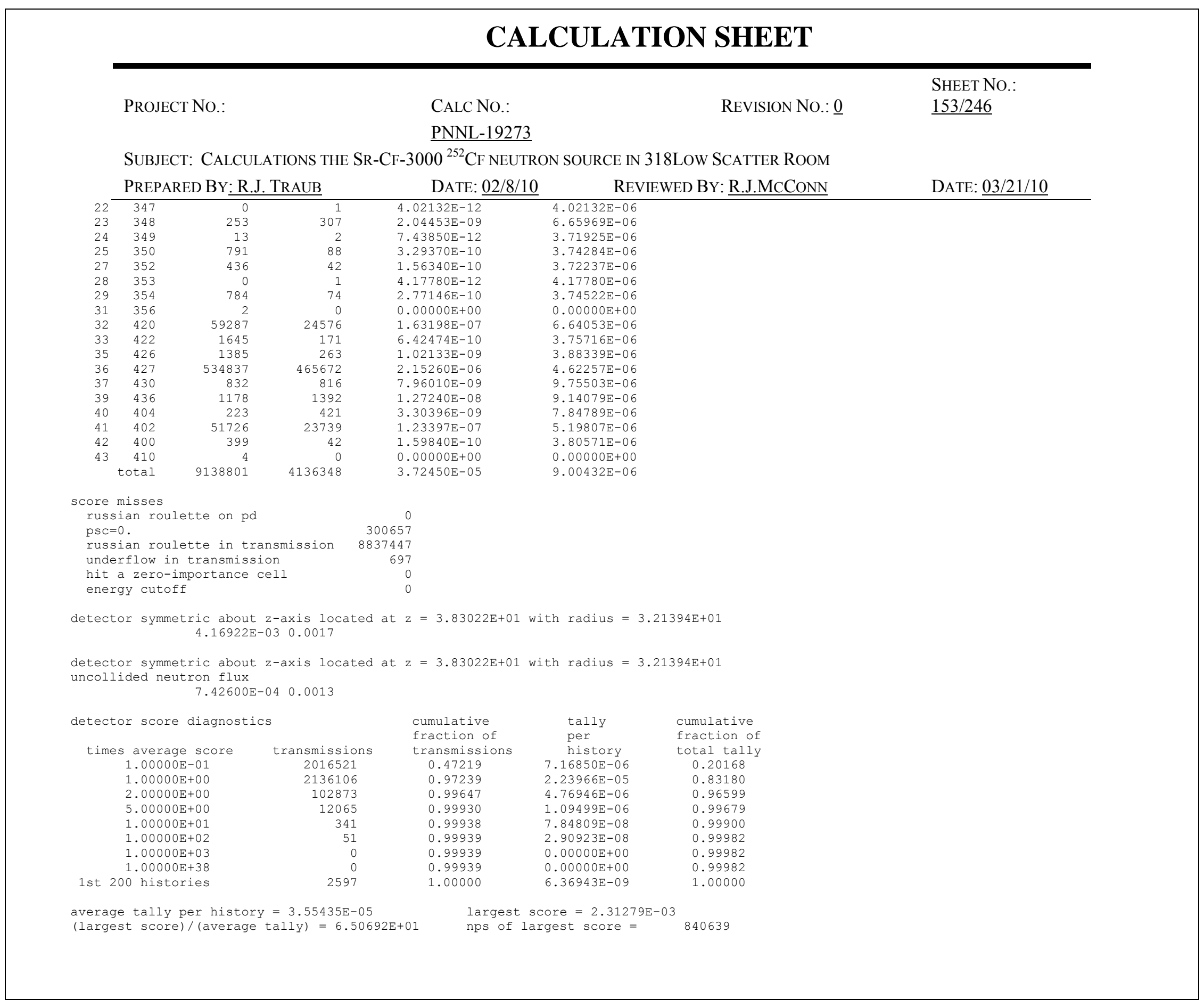




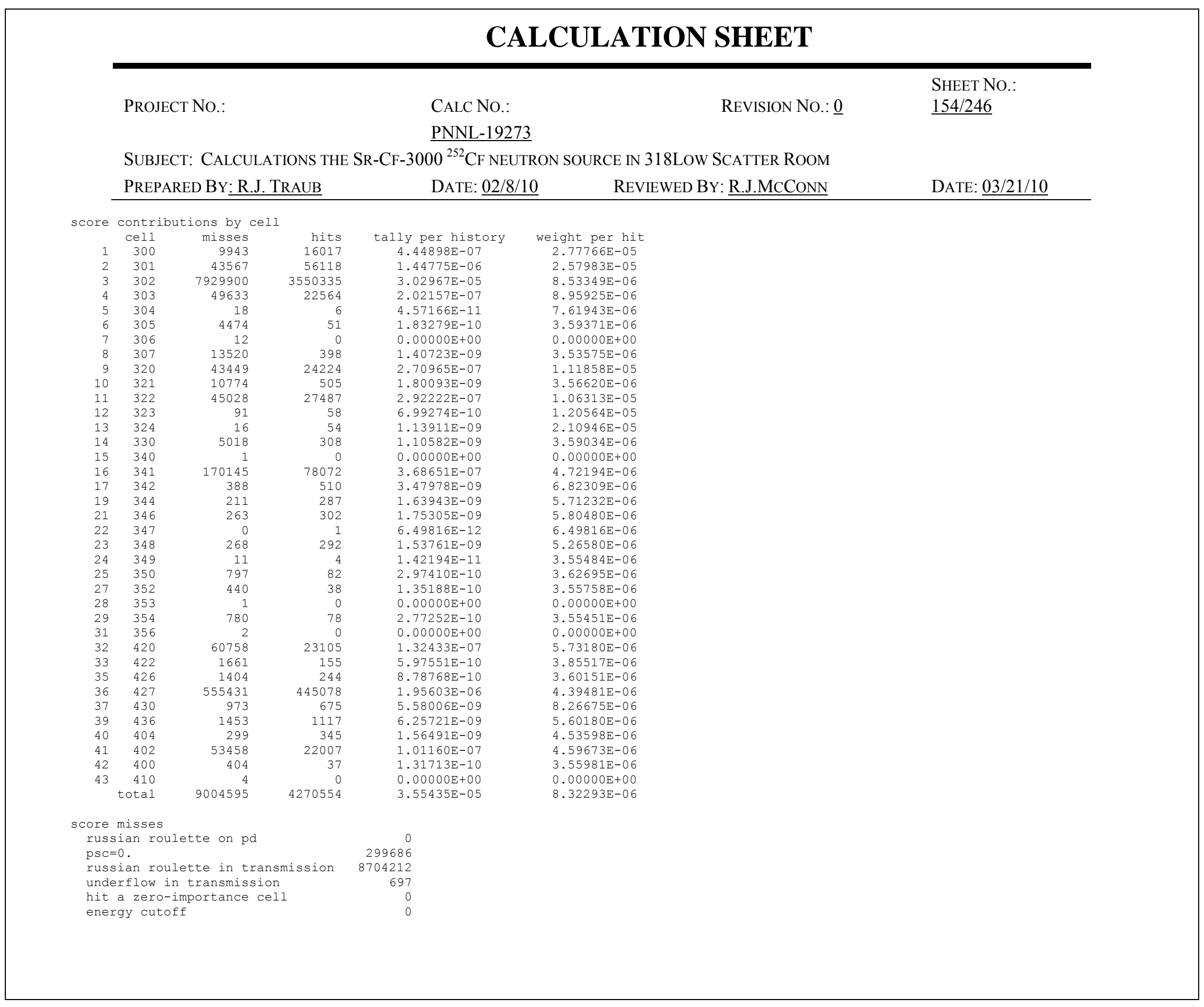




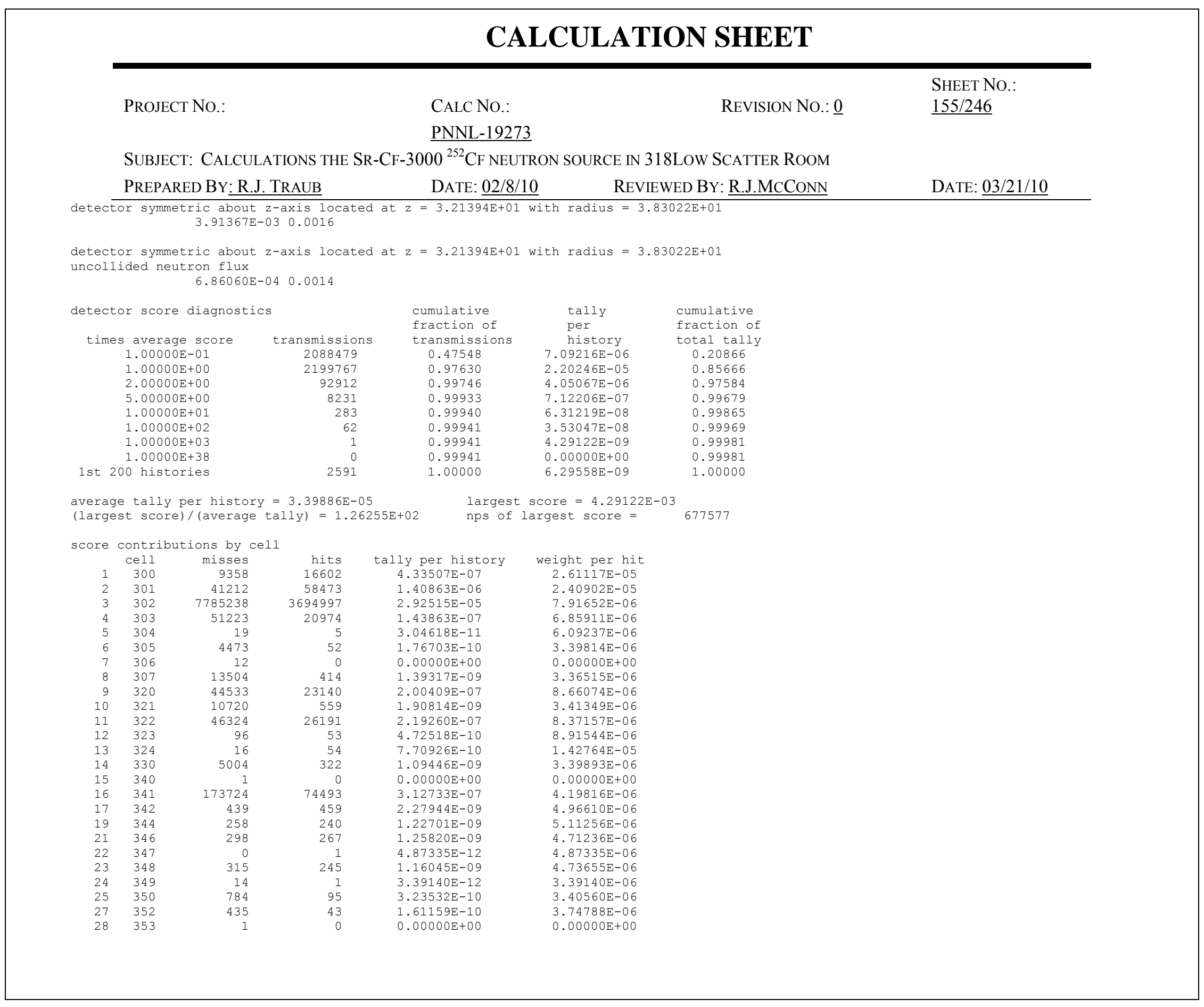




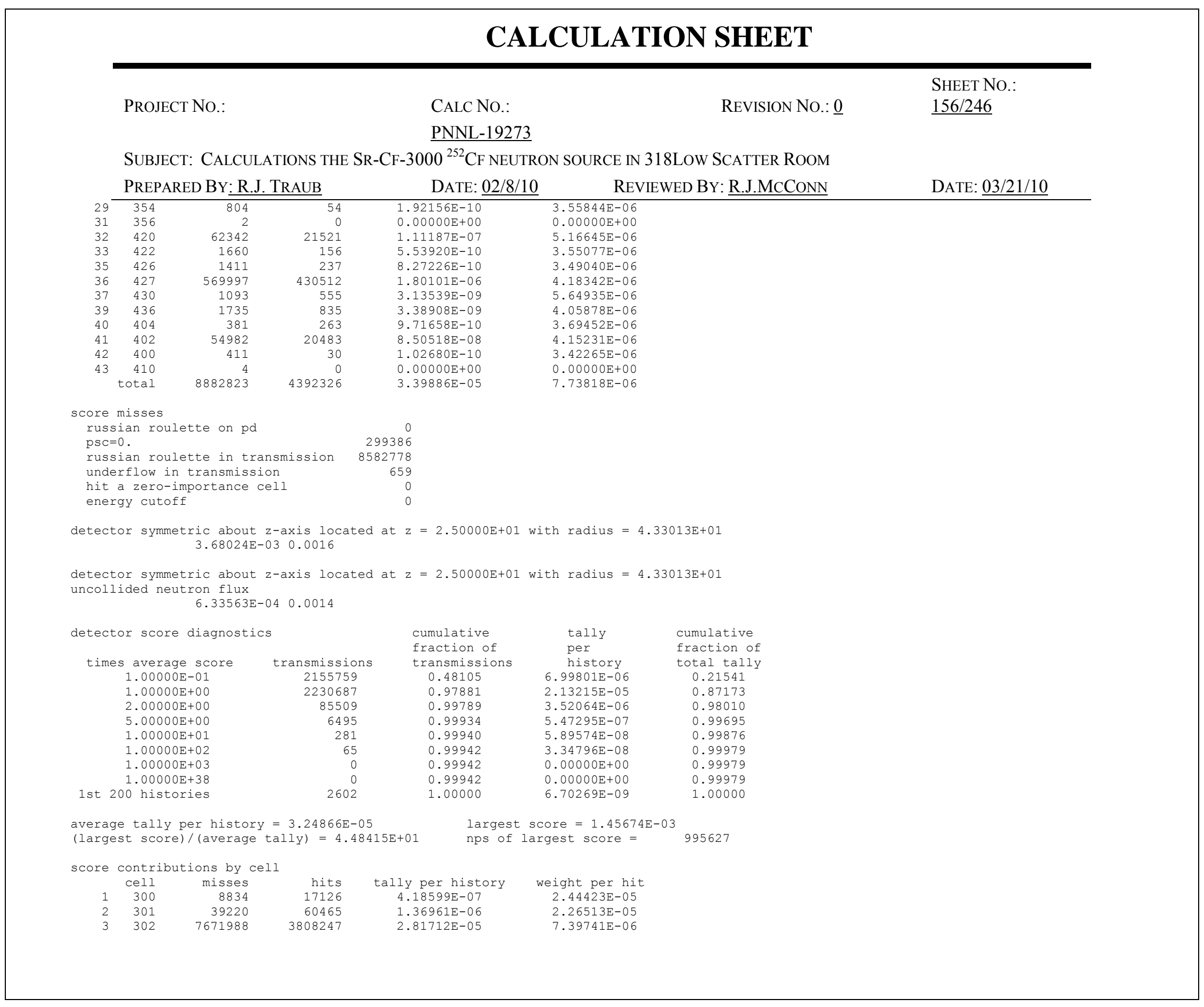




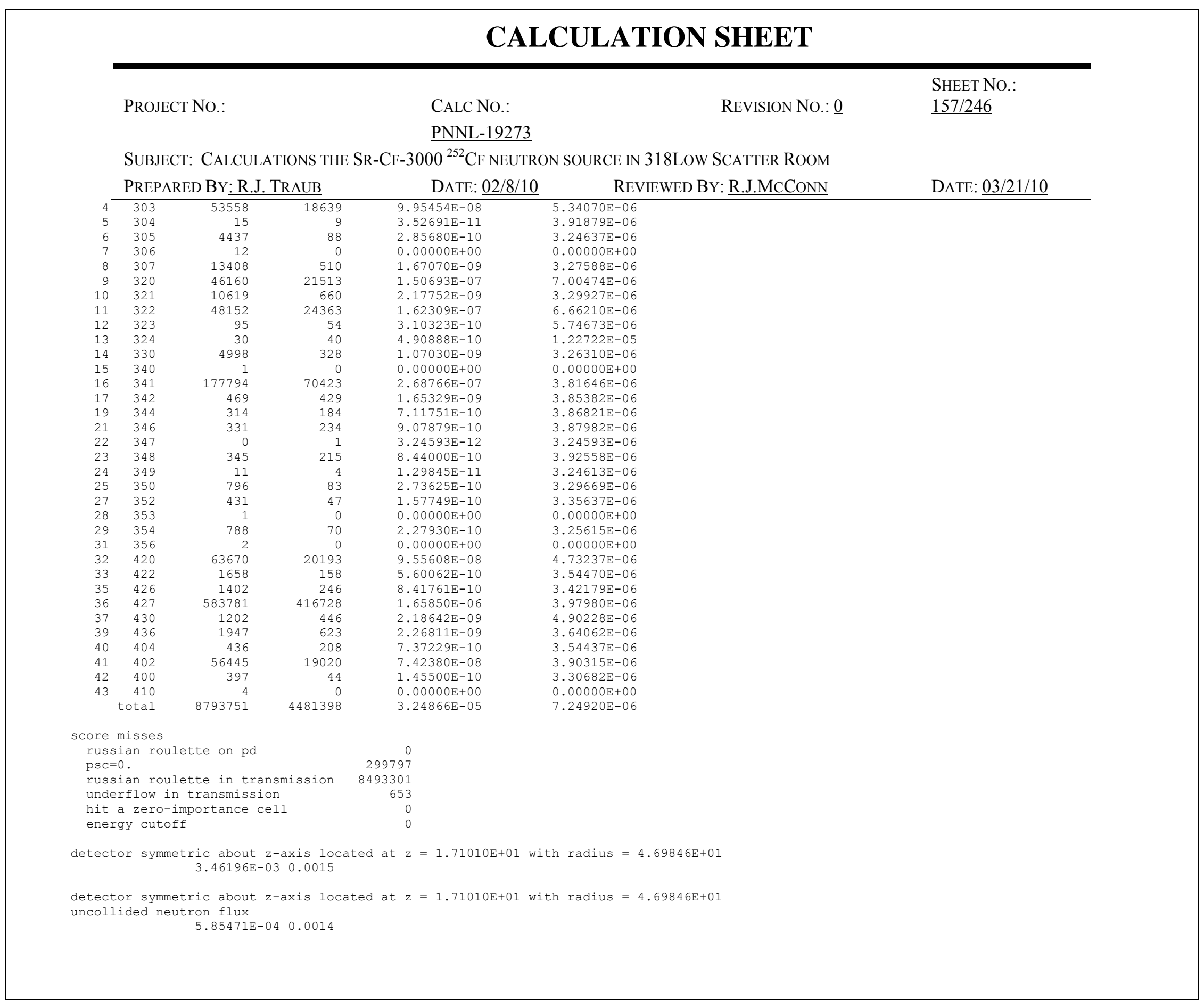




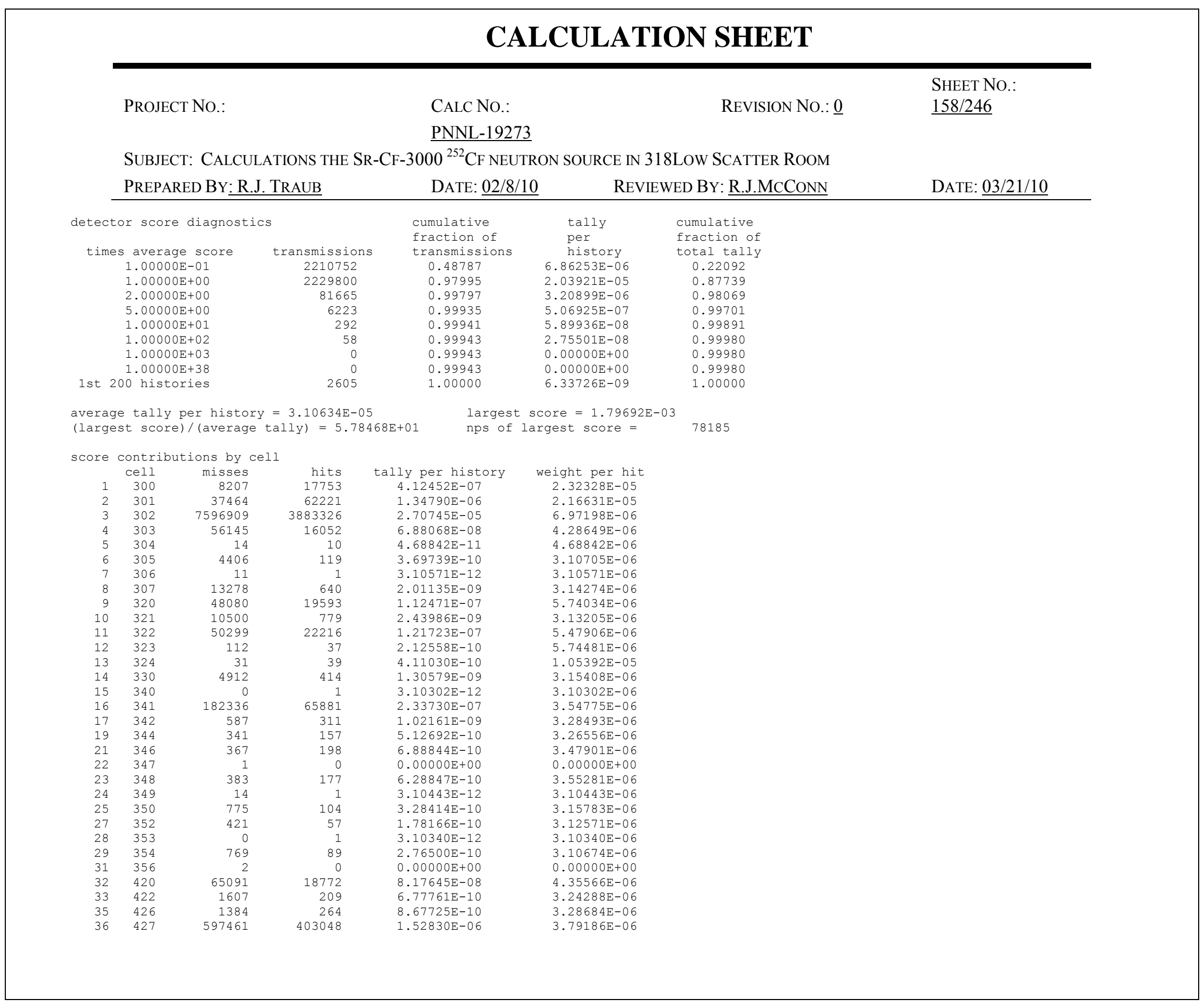




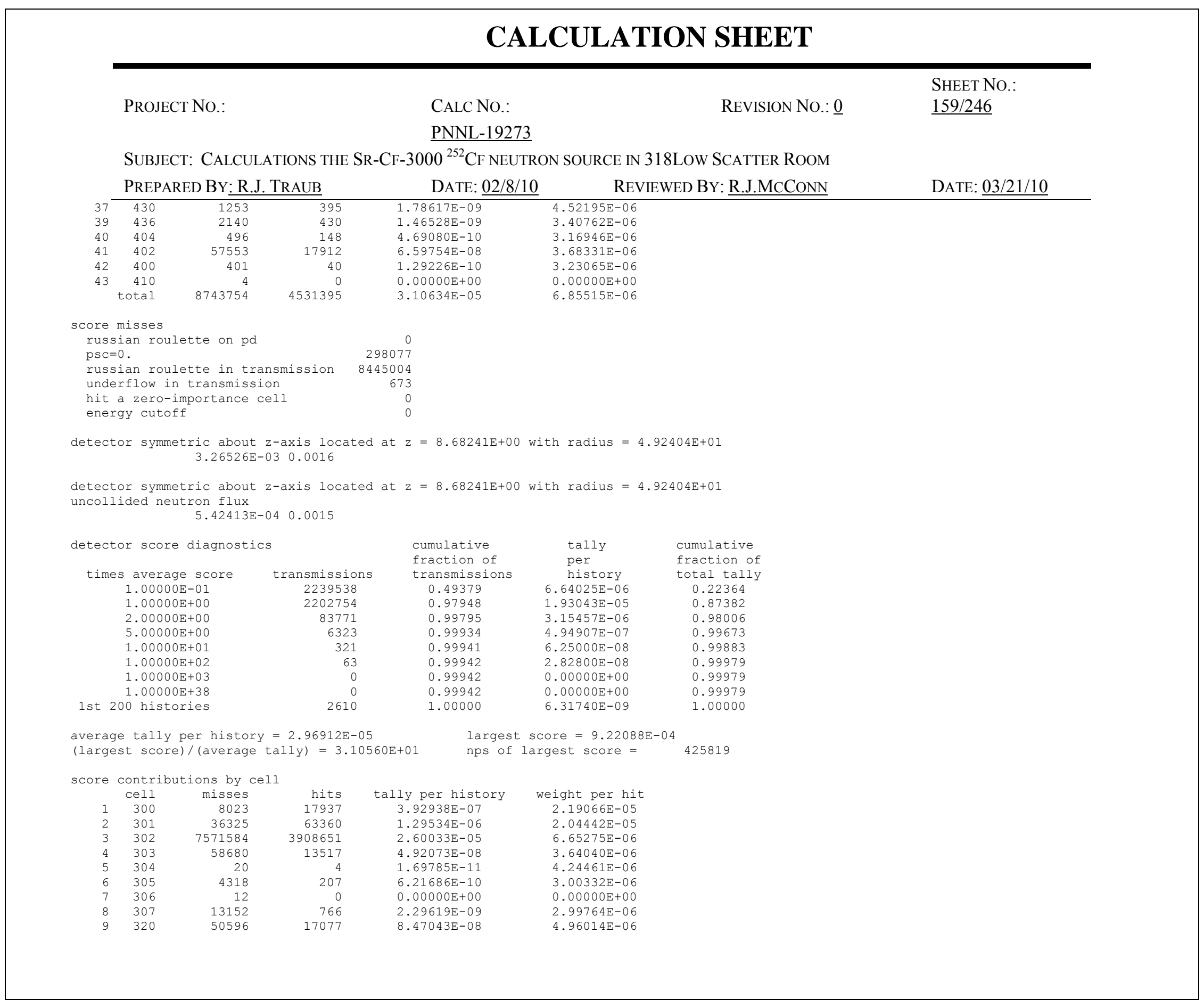




\begin{tabular}{|c|c|c|c|c|c|c|c|}
\hline \multicolumn{8}{|c|}{ CALCULATION SHEET } \\
\hline & PROJE & No.: & & $\begin{array}{l}\text { CALC NO.: } \\
\text { PNNL-19273 }\end{array}$ & & REVISION NO.: $\underline{0}$ & $\begin{array}{l}\text { SHEET NO.: } \\
\underline{160 / 246}\end{array}$ \\
\hline & \multicolumn{7}{|c|}{ 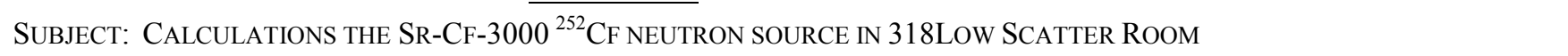 } \\
\hline & \multicolumn{3}{|c|}{ PREPARED BY: R.J. TRAUB } & DATE: $\underline{02 / 8 / 10}$ & \multicolumn{2}{|c|}{ REVIEWED BY: R.J.MCCONN } & \multirow[t]{2}{*}{ DATE: $\underline{03 / 21 / 10}$} \\
\hline $10^{\circ}$ & 321 & 1023 & 1047 & $3.13188 \mathrm{E}-09$ & $2.99129 \mathrm{E}-06$ & & \\
\hline 11 & 322 & 5267 & 19844 & $9.45121 \mathrm{E}-08$ & $4.76275 \mathrm{E}-06$ & & \\
\hline 12 & 323 & 11 & 30 & $1.62043 \mathrm{E}-10$ & $5.40143 \mathrm{E}-06$ & & \\
\hline 13 & 324 & 3 & 31 & $3.73458 \mathrm{E}-10$ & $1.20470 \mathrm{E}-05$ & & \\
\hline 14 & 330 & 485 & 472 & 1.41878E-09 & $3.00590 \mathrm{E}-06$ & & \\
\hline 15 & $\begin{array}{l}340 \\
347\end{array}$ & $18534 \mathrm{rat}$ & $\begin{array}{r}0 \\
62875\end{array}$ & $0.00000 \mathrm{E}+00$ & $\begin{array}{l}0.00000 \mathrm{E}+00 \\
3.3476 \mathrm{E}-06\end{array}$ & & \\
\hline $\begin{array}{l}16 \\
17\end{array}$ & $\begin{array}{l}341 \\
342\end{array}$ & $\begin{array}{r}18534 \\
70\end{array}$ & $\begin{array}{r}62875 \\
198\end{array}$ & $\begin{array}{l}2.10239 \mathrm{E}-07 \\
5.98558 \mathrm{E}-10\end{array}$ & $\begin{array}{l}3.34376 \mathrm{E}-06 \\
3.02302 \mathrm{E}-06\end{array}$ & & \\
\hline 19 & 344 & 39 & $\begin{array}{l}198 \\
108\end{array}$ & $\begin{array}{l}5.98558 \mathrm{E}-10 \\
3.36268 \mathrm{E}-10\end{array}$ & $\begin{array}{l}3.02302 \mathrm{E}-06 \\
3.11359 \mathrm{E}-06\end{array}$ & & \\
\hline 21 & 346 & 42 & 142 & $4.29997 \mathrm{E}-10$ & $3.02815 \mathrm{E}-06$ & & \\
\hline 22 & 347 & te & 0 & $0.00000 \mathrm{E}+00$ & $0.00000 \mathrm{E}+00$ & & \\
\hline 23 & 348 & 42 & 137 & $4.20978 \mathrm{E}-10$ & $3.07283 \mathrm{E}-06$ & & \\
\hline 24 & 349 & 1 & 3 & $8.89624 \mathrm{E}-12$ & $2.96541 \mathrm{E}-06$ & & \\
\hline 25 & 350 & 75 & 121 & $3.66911 \mathrm{E}-10$ & $3.03232 \mathrm{E}-06$ & & \\
\hline 27 & 352 & 43 & 48 & 1.43195E-10 & $2.98324 \mathrm{E}-06$ & & \\
\hline 28 & 353 & 75 & 0 & $0.00000 E+00$ & $0.00000 \mathrm{E}+00$ & & \\
\hline 29 & 354 & 75 & 105 & $3.11984 \mathrm{E}-10$ & $2.97128 \mathrm{E}-06$ & & \\
\hline $\begin{array}{l}31 \\
32\end{array}$ & $\begin{array}{l}356 \\
420\end{array}$ & 6606 & $\begin{aligned} 0 \\
17800\end{aligned}$ & $0.00000 \mathrm{E}+00$ & $0.00000 \mathrm{E}+00$ & & \\
\hline $\begin{array}{l}32 \\
33 \\
3\end{array}$ & $\begin{array}{l}420 \\
422\end{array}$ & $\begin{array}{r}6606 \\
160\end{array}$ & $\begin{array}{r}17800 \\
215\end{array}$ & $\begin{array}{l}7.31333 \mathrm{E}-08 \\
6\end{array}$ & $\begin{array}{l}4.10861 \mathrm{E}-06 \\
3.08442 E-06\end{array}$ & & \\
\hline 35 & $\begin{array}{l}422 \\
426\end{array}$ & 136 & 279 & $\begin{array}{l}6.55411 \mathrm{E}-10 \\
8.91169 \mathrm{E}-10\end{array}$ & $\begin{array}{l}3.4842 \mathrm{E}-06 \\
3.19415 \mathrm{E}-06\end{array}$ & & \\
\hline 36 & 427 & 60792 & 392584 & $1.41368 \mathrm{E}-06$ & $3.60096 \mathrm{E}-06$ & & \\
\hline 37 & 430 & 133 & 310 & $1.14618 \mathrm{E}-09$ & $3.69735 \mathrm{E}-06$ & & \\
\hline 39 & 436 & 215 & 416 & $1.27769 \mathrm{E}-09$ & $3.07136 \mathrm{E}-06$ & & \\
\hline 40 & 404 & 50 & 136 & $4.11709 \mathrm{E}-10$ & $3.02727 \mathrm{E}-06$ & & \\
\hline 41 & 402 & 5854 & 16924 & $5.90243 \mathrm{E}-08$ & $3.48761 \mathrm{E}-06$ & & \\
\hline 42 & & 40 & 36 & $1.06757 E-10$ & $2.96546 \mathrm{E}-06$ & & \\
\hline 43 & $\begin{array}{r}410 \\
\text { total }\end{array}$ & 873976 & 4535380 & $\begin{array}{l}0.00000 \mathrm{E}+00 \\
2.96912 \mathrm{E}-05\end{array}$ & $\begin{array}{l}0.00000 \mathrm{E}+00 \\
6.54657 \mathrm{E}-06\end{array}$ & & \\
\hline \multirow{2}{*}{\multicolumn{8}{|c|}{$\begin{array}{l}\text { score misses } \\
\text { russian roulette on pd }\end{array}$}} \\
\hline & & & & & & & \\
\hline \multirow{2}{*}{\multicolumn{8}{|c|}{$\begin{array}{lr} & 298348 \\
\text { psc }=0 . & \end{array}$}} \\
\hline \\
\hline & & & & & & & \\
\hline \multicolumn{8}{|c|}{ energy cutoff } \\
\hline \multicolumn{8}{|c|}{$\begin{array}{c}\text { detector symmetric about } z \text {-axis located at } z=0.00000 \mathrm{E}+00 \text { with radius }=5.00000 \mathrm{E}+01 \\
3.08406 \mathrm{E}-030.0016\end{array}$} \\
\hline \multirow{2}{*}{\multicolumn{8}{|c|}{$\begin{array}{l}\text { detector symmetric about } z \text {-axis located at } z=0.00000 \mathrm{E}+00 \text { with radius }=5.00000 \mathrm{E}+01 \\
\text { uncollided neutron flux } \\
5.01801 \mathrm{E}-04 \quad 0.0015\end{array}$}} \\
\hline & & & & & & & \\
\hline \multicolumn{4}{|c|}{ detector score diagnostics } & cumulative & tally & cumulative & \\
\hline tim & os avera & score & transmissions & $\begin{array}{l}\text { fraction of } \\
\text { transmissions }\end{array}$ & $\begin{array}{l}\text { per } \\
\text { history }\end{array}$ & fraction of & \\
\hline & 1.0000 & -01 & 2228068 & 0.49660 & $6.33970 \mathrm{E}-06$ & 0.22272 & \\
\hline & 1.0000 & & 2157685 & 0.97752 & $1.82190 \mathrm{E}-05$ & 0.86277 & \\
\hline
\end{tabular}




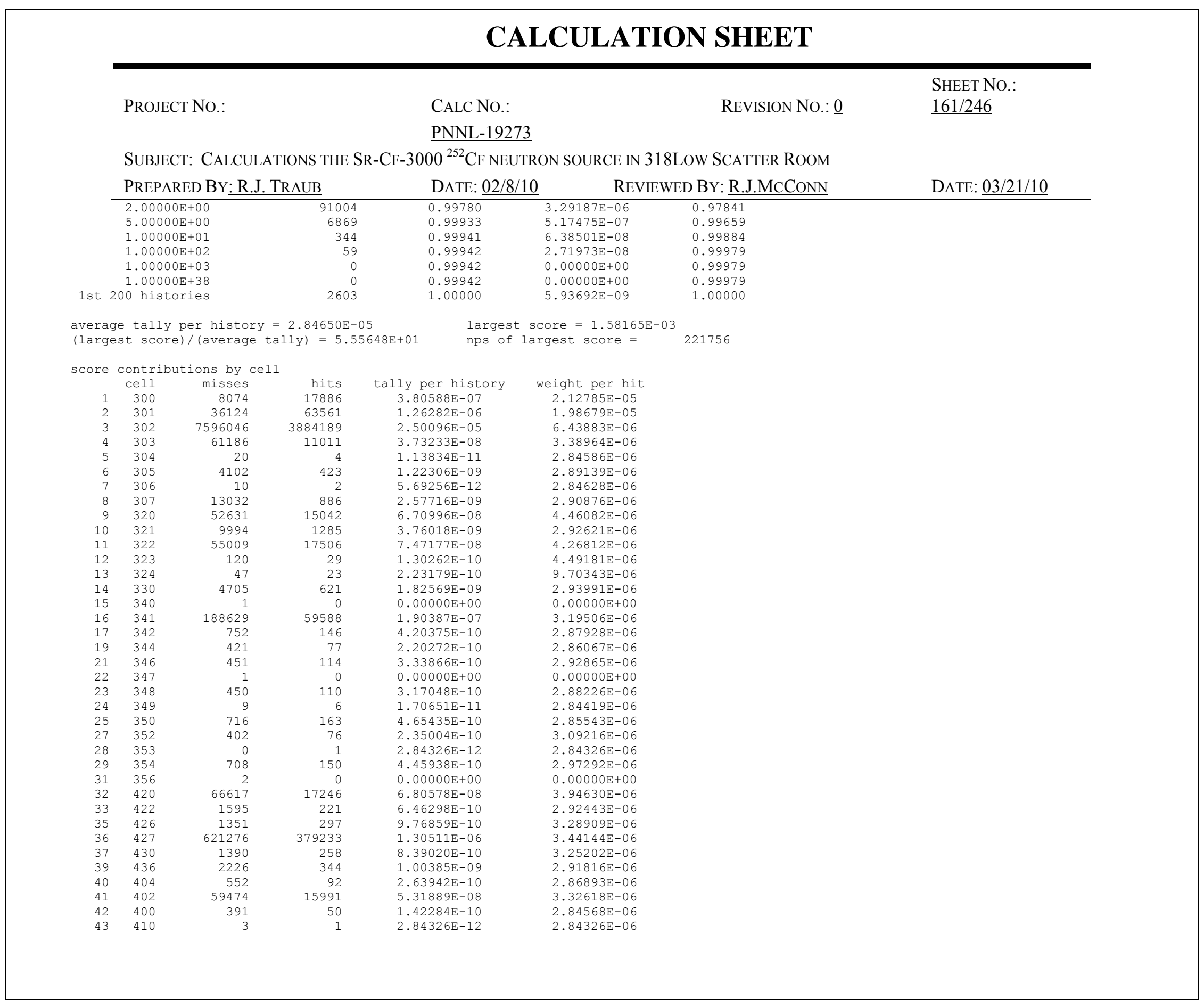




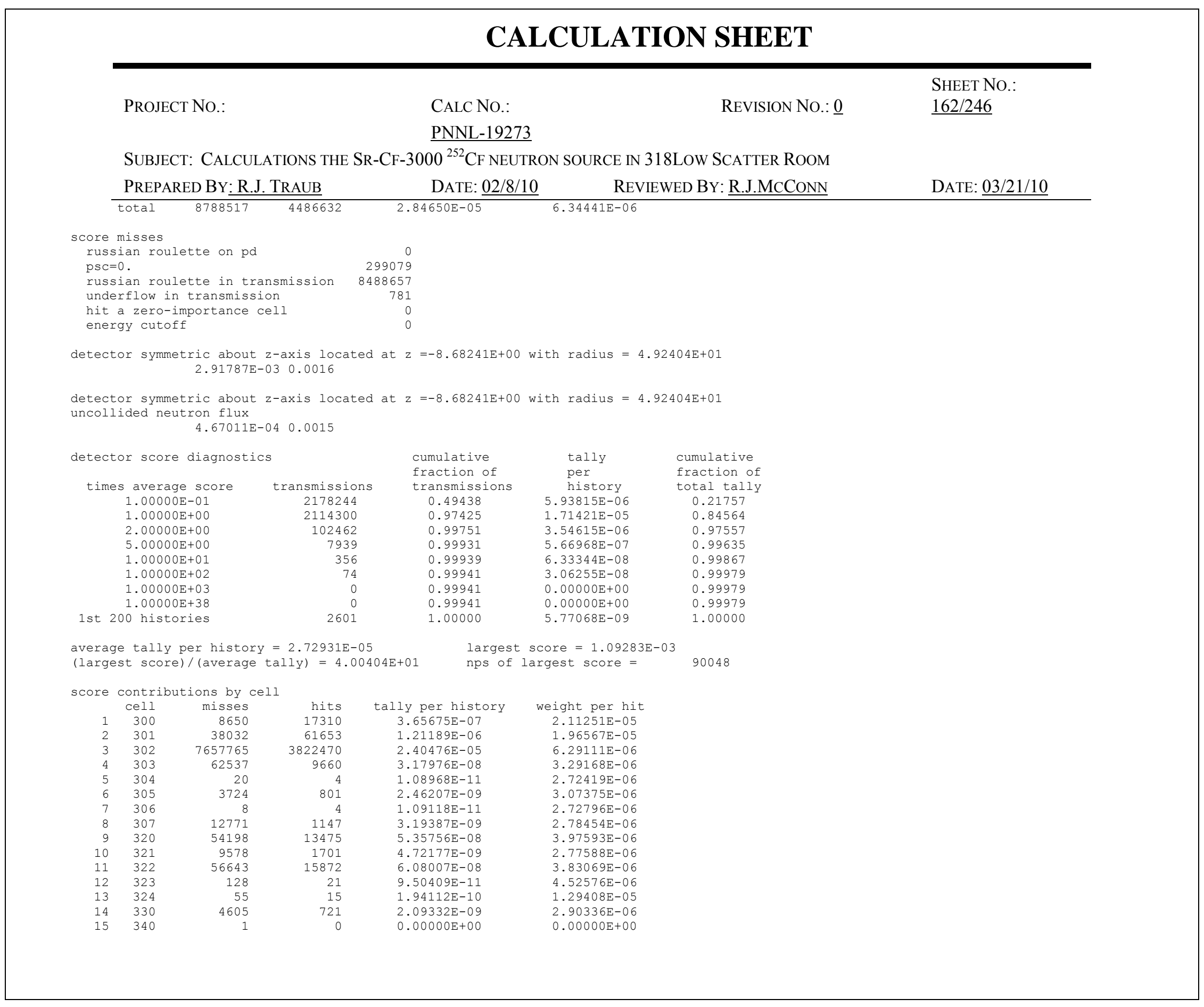




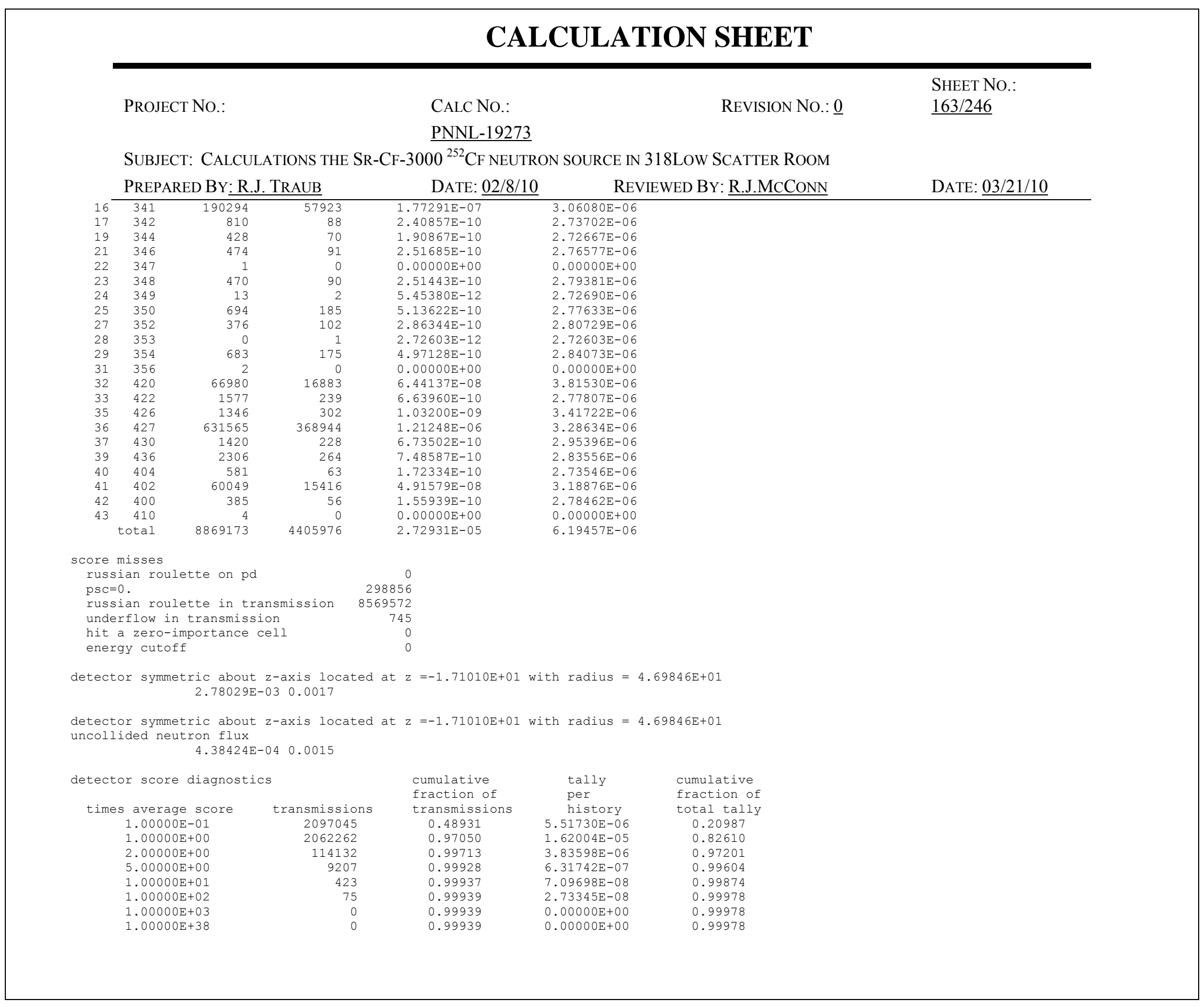




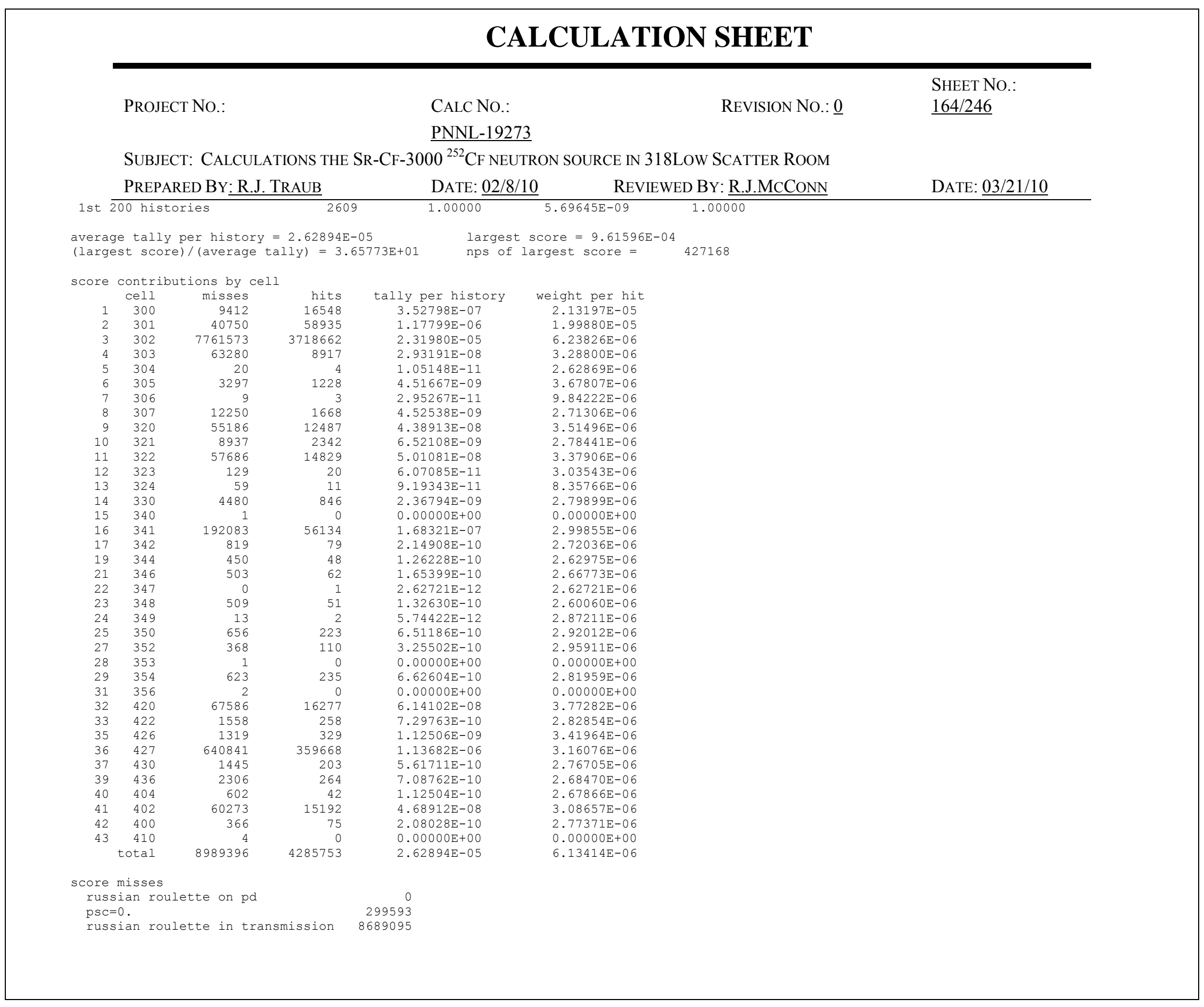




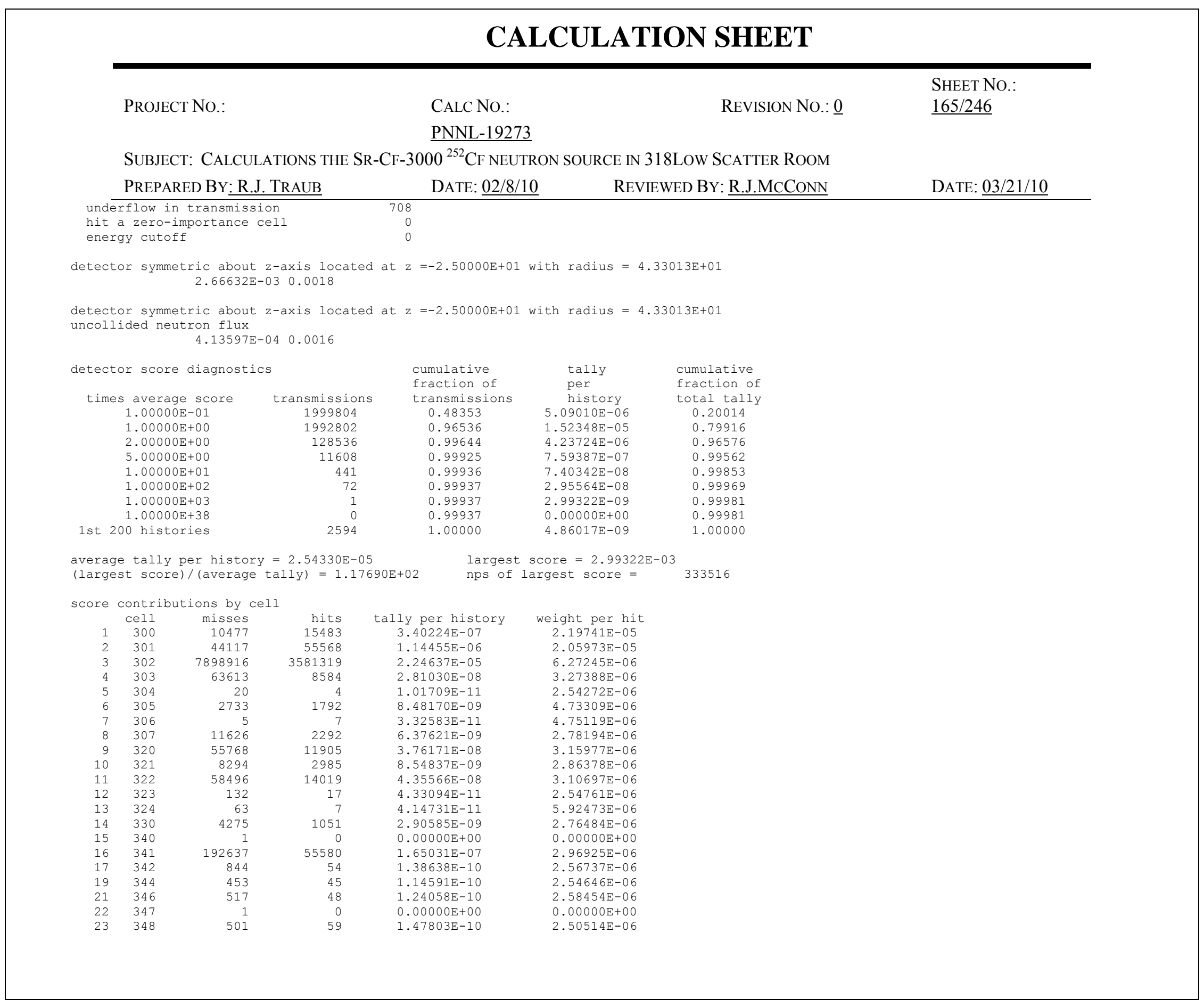




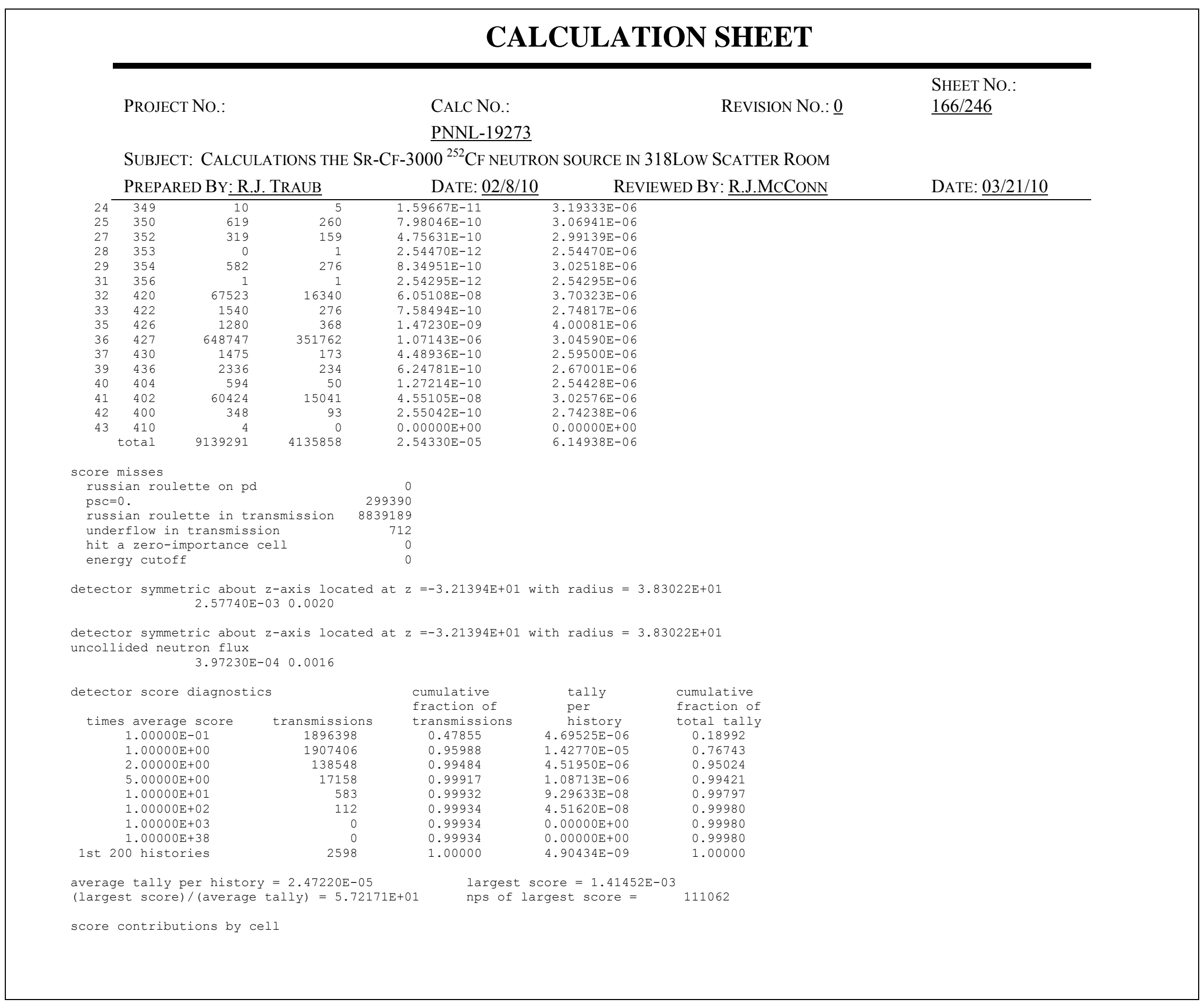




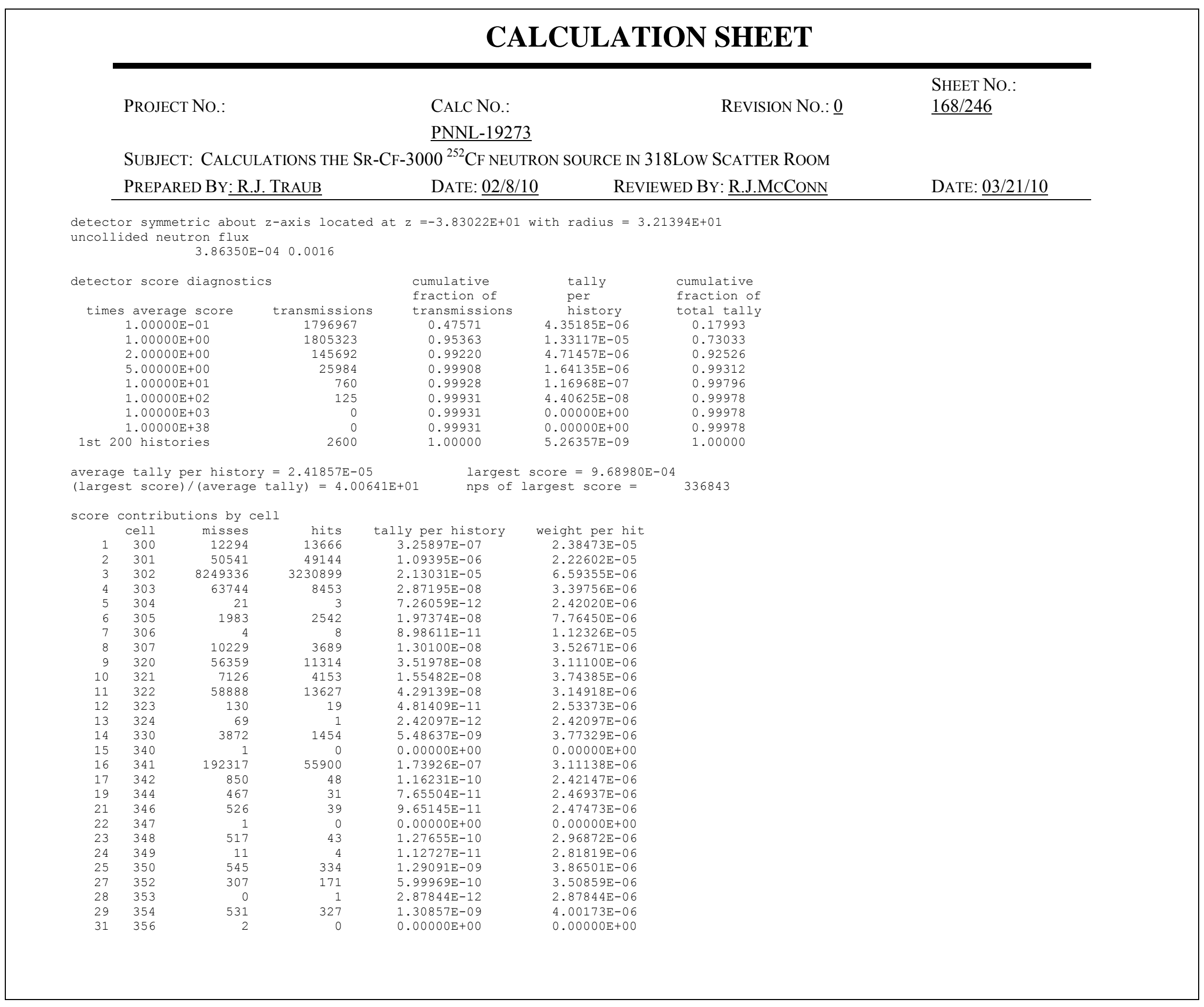




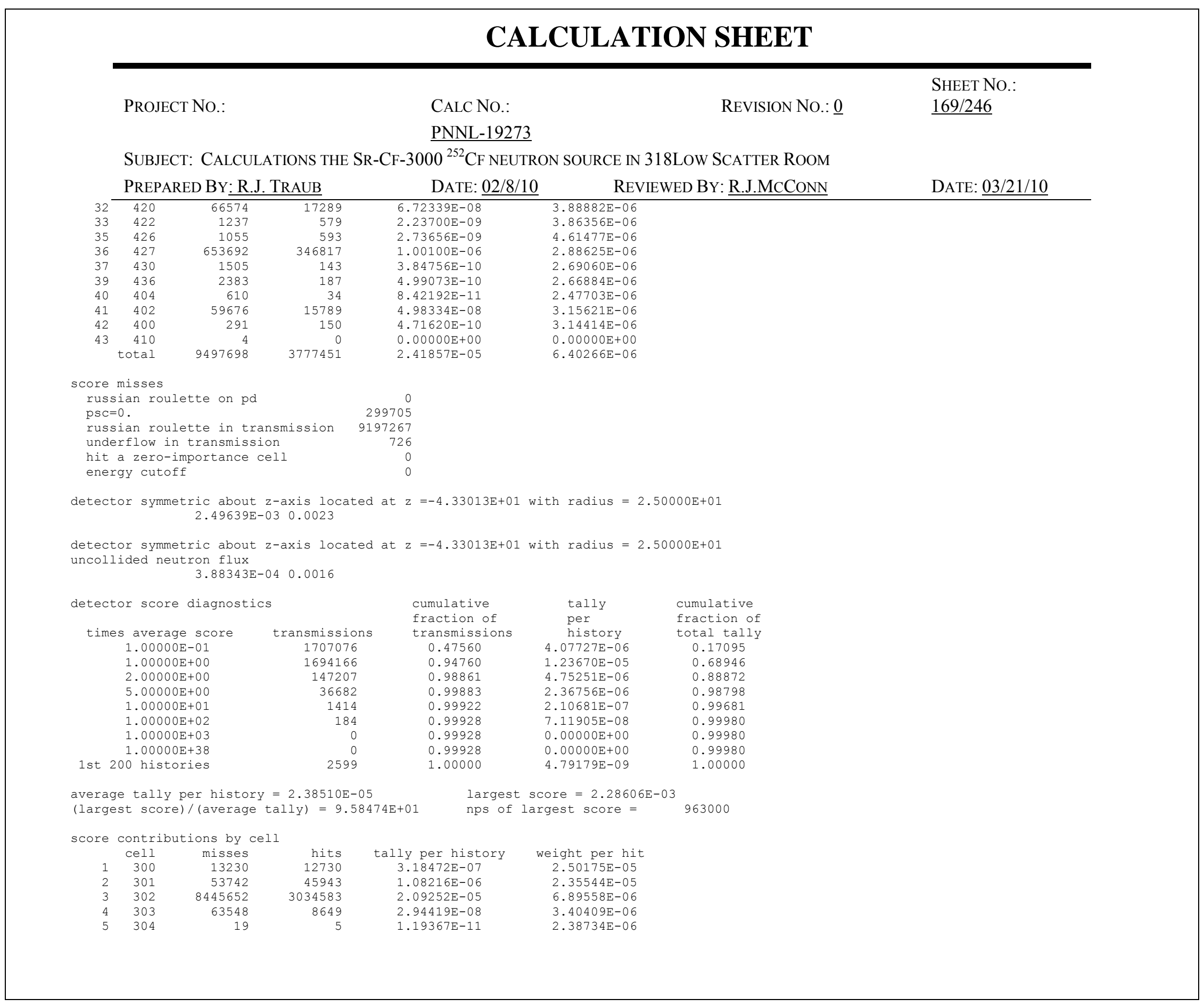




\begin{tabular}{|c|c|c|c|c|c|c|}
\hline \multicolumn{7}{|c|}{ CALCULATION SHEET } \\
\hline & PROJE & No.: & & $\begin{array}{l}\text { CALC NO.: } \\
\text { PNNL-19273 }\end{array}$ & REVISION NO.: $\underline{0}$ & $\begin{array}{l}\text { SHEET NO.: } \\
\underline{170 / 246}\end{array}$ \\
\hline & \multicolumn{6}{|c|}{ 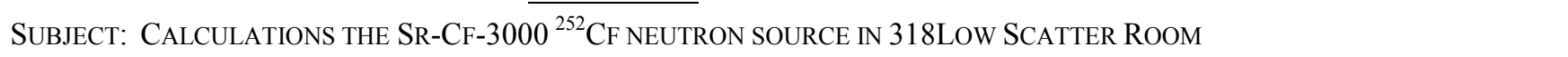 } \\
\hline & \multicolumn{3}{|c|}{ PREPARED BY: R.J. TRAUB } & DATE: $\underline{02 / 8 / 10}$ & REVIEWED BY: R.J.MCCONN & \multirow[t]{2}{*}{ DATE: $\underline{03 / 21 / 10}$} \\
\hline 6 & 305 & 1741 & 2784 & $2.74531 \mathrm{E}-08$ & $9.86104 \mathrm{E}-06$ & \\
\hline 7 & 306 & 4 & 8 & $1.00338 \mathrm{E}-10$ & $1.25422 \mathrm{E}-05$ & \\
\hline 8 & 307 & 9671 & 4247 & 1.80257E-08 & $4.24433 \mathrm{E}-06$ & \\
\hline 9 & 320 & 56251 & 11422 & $3.67824 \mathrm{E}-08$ & $3.22031 \mathrm{E}-06$ & \\
\hline 10 & 321 & 6594 & 4685 & $2.02778 \mathrm{E}-08$ & $4.32825 \mathrm{E}-06$ & \\
\hline 11 & $\begin{array}{l}322 \\
323\end{array}$ & $\begin{array}{r}58480 \\
121\end{array}$ & $\begin{array}{r}14035 \\
28\end{array}$ & $\begin{array}{l}4.58173 \mathrm{E}-08 \\
7.15686 \mathrm{E}-11\end{array}$ & $\begin{array}{l}3.26450 \mathrm{E}-06 \\
2.5602 \mathrm{E}-06\end{array}$ & \\
\hline $\begin{array}{l}12 \\
13\end{array}$ & $\begin{array}{l}323 \\
324\end{array}$ & $\begin{array}{r}121 \\
68\end{array}$ & $\begin{array}{r}28 \\
2\end{array}$ & $\begin{array}{l}7.15686 \mathrm{E}-11 \\
4.76974 \mathrm{E}-12\end{array}$ & $\begin{array}{l}2.5502 \mathrm{E}-06 \\
2.38487 \mathrm{E}-06\end{array}$ & \\
\hline 14 & 330 & 3749 & 1577 & $\begin{array}{l}4.699 / 4 \mathrm{E}-12 \\
7.88631 \mathrm{E}-09\end{array}$ & $\begin{array}{l}2.3848 / \mathrm{E}-06 \\
5.00083 \mathrm{E}-06\end{array}$ & \\
\hline 15 & 340 & 1 & 0 & $0.00000 \mathrm{E}+00$ & $0.00000 \mathrm{E}+00$ & \\
\hline 16 & 341 & 190839 & 57378 & $1.86302 \mathrm{E}-07$ & $3.24692 \mathrm{E}-06$ & \\
\hline 17 & 342 & 852 & 46 & $1.10235 \mathrm{E}-10$ & $2.39642 \mathrm{E}-06$ & \\
\hline 19 & 344 & 471 & 27 & $6.81865 \mathrm{E}-11$ & $2.52543 \mathrm{E}-06$ & \\
\hline 21 & 346 & 520 & 45 & $1.07796 \mathrm{E}-10$ & $2.39546 \mathrm{E}-06$ & \\
\hline 22 & 347 & 1 & 0 & $0.00000 E+00$ & $0.00000 E+00$ & \\
\hline 23 & 348 & 516 & 44 & $1.08098 \mathrm{E}-10$ & $2.45678 \mathrm{E}-06$ & \\
\hline 24 & 349 & 10 & 5 & $1.31794 \mathrm{E}-11$ & $2.63589 \mathrm{E}-06$ & \\
\hline $\begin{array}{l}25 \\
27\end{array}$ & $\begin{array}{r}350 \\
352\end{array}$ & $\begin{array}{l}518 \\
283\end{array}$ & $\begin{array}{l}361 \\
195\end{array}$ & $\begin{array}{l}1.47345 \mathrm{E}-09 \\
73658 \mathrm{E}-10\end{array}$ & $4.08158 \mathrm{E}-06$ & \\
\hline $\begin{array}{l}27 \\
28\end{array}$ & $\begin{array}{l}352 \\
353\end{array}$ & $\begin{array}{r}283 \\
0\end{array}$ & $\begin{array}{r}195 \\
1\end{array}$ & $\begin{array}{l}7.31658 \mathrm{E}-10 \\
3.12971 \mathrm{E}-12\end{array}$ & $\begin{array}{l}3.75209 \mathrm{E}-06 \\
3.12971 \mathrm{E}-06\end{array}$ & \\
\hline 29 & 354 & 513 & 345 & $1.64283 \mathrm{E}-09$ & $4.76182 \mathrm{E}-06$ & \\
\hline 31 & 356 & 2 & 0 & $0.00000 \mathrm{E}+00$ & $0.00000 \mathrm{E}+00$ & \\
\hline 32 & 420 & 66240 & 17623 & 7.56763E-08 & $4.29418 \mathrm{E}-06$ & \\
\hline 33 & 422 & 1107 & 709 & $4.44689 \mathrm{E}-09$ & $6.27205 \mathrm{E}-06$ & \\
\hline 35 & 426 & 1009 & 639 & $4.39124 \mathrm{E}-09$ & $6.87205 \mathrm{E}-06$ & \\
\hline 36 & 427 & 646301 & 354208 & $1.00793 \mathrm{E}-06$ & $2.84558 \mathrm{E}-06$ & \\
\hline $\begin{array}{l}37 \\
39\end{array}$ & 430 & 1519 & 129 & $3.45975 \mathrm{E}-10$ & $2.68198 \mathrm{E}-06$ & \\
\hline $\begin{array}{l}39 \\
40\end{array}$ & $\begin{array}{l}436 \\
404\end{array}$ & $\begin{array}{r}2392 \\
587\end{array}$ & $\begin{array}{r}178 \\
57\end{array}$ & 4.35373E-10 & $2.44591 \mathrm{E}-06$ & \\
\hline 40 & 404 & $\begin{array}{r}587 \\
58986\end{array}$ & $\begin{array}{r}57 \\
16479\end{array}$ & $\begin{array}{l}1.36747 \mathrm{E}-10 \\
5.4765 \mathrm{~F}-08\end{array}$ & $2.39907 \mathrm{E}-06$ & \\
\hline $\begin{array}{l}41 \\
42\end{array}$ & $\begin{array}{l}402 \\
400\end{array}$ & $\begin{array}{r}58986 \\
281\end{array}$ & $\begin{array}{r}16479 \\
160\end{array}$ & $\begin{array}{l}5.4655 \mathrm{E}-08 \\
6.25365 \mathrm{E}-10\end{array}$ & $\begin{array}{l}3.32335 \mathrm{E}-06 \\
3.90853 \mathrm{E}-06\end{array}$ & \\
\hline 43 & & & & $2.38736 \mathrm{E}-12$ & $2.38736 \mathrm{E}-06$ & \\
\hline & total & 9685821 & 3589328 & $2.38510 \mathrm{E}-05$ & $6.64498 \mathrm{E}-06$ & \\
\hline \multicolumn{7}{|c|}{ score misses } \\
\hline \multicolumn{4}{|c|}{ 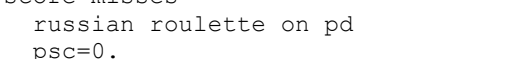 } & 0 & & \\
\hline \multicolumn{7}{|c|}{$\begin{array}{l}\text { psce=0. } \\
\text { russian roulette in transmission } \quad 9384720\end{array}$} \\
\hline \multirow{2}{*}{\multicolumn{7}{|c|}{$\begin{array}{l}\text { underflow in transmission } \\
\text { hit a zero-importance cell }\end{array}$}} \\
\hline \multicolumn{5}{|c|}{$\begin{array}{l}\text { hit a zero-mimortance cell } \\
\text { energy cutoff }\end{array}$} & & \\
\hline \multicolumn{7}{|c|}{$\begin{array}{l}\text { detector symmetric about } z \text {-axis located at } z=-4.69846 \mathrm{E}+01 \text { with radius }=1.71010 \mathrm{E}+01 \\
2.50994 \mathrm{E}-030.0026\end{array}$} \\
\hline \multirow{2}{*}{\multicolumn{7}{|c|}{$\begin{array}{l}\text { detector symmetric about } z \text {-axis located at } z=-4.69846 \mathrm{E}+01 \text { with radius }=1.71010 \mathrm{E}+01 \\
\text { uncollided neutron flux } \\
\qquad \begin{array}{l}4.02016 \mathrm{E}-04 \quad 0.0015\end{array}\end{array}$}} \\
\hline & & & & & & \\
\hline \multicolumn{4}{|c|}{ detector score diagnostics } & cumulative & cumulative & \\
\hline
\end{tabular}




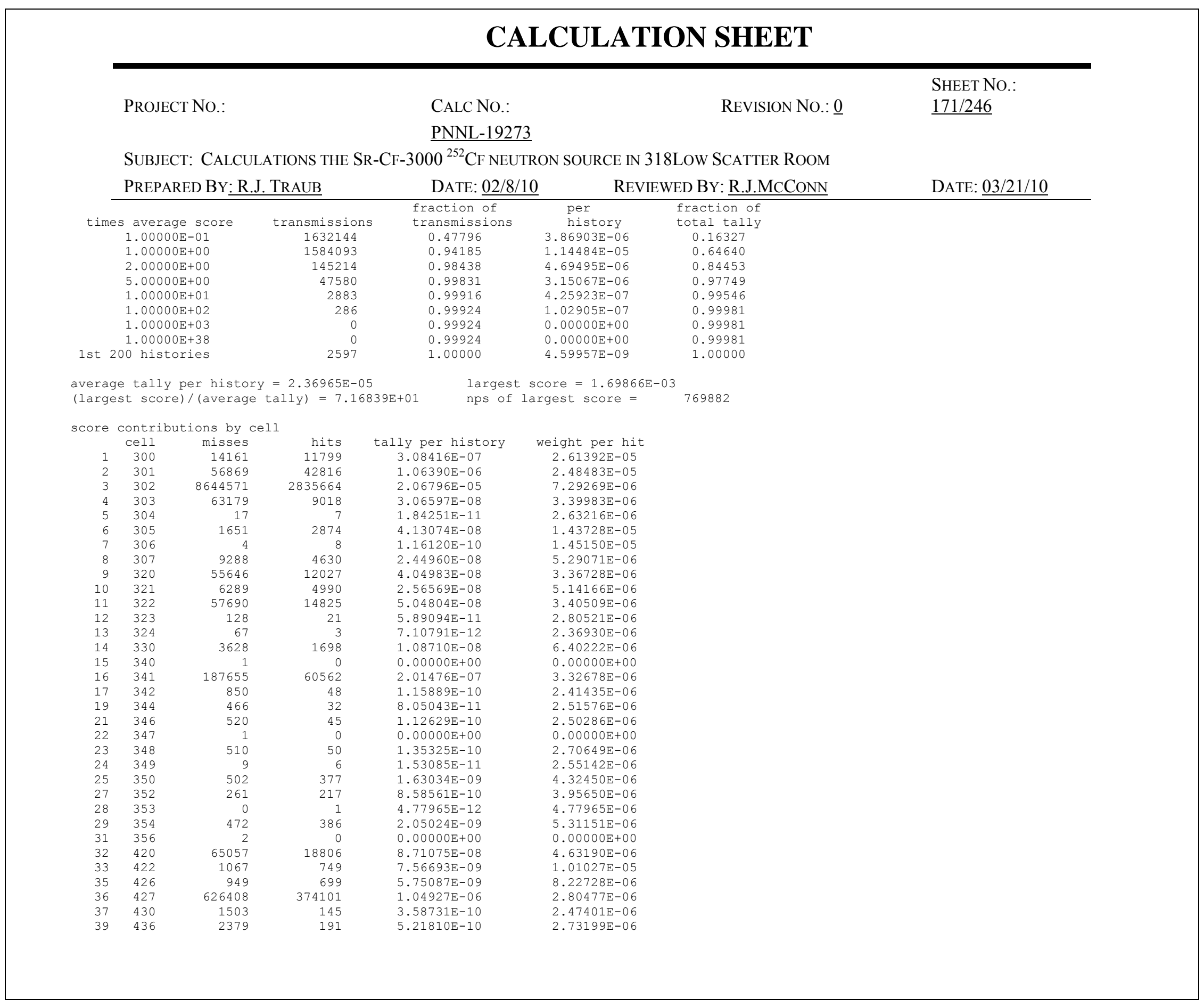




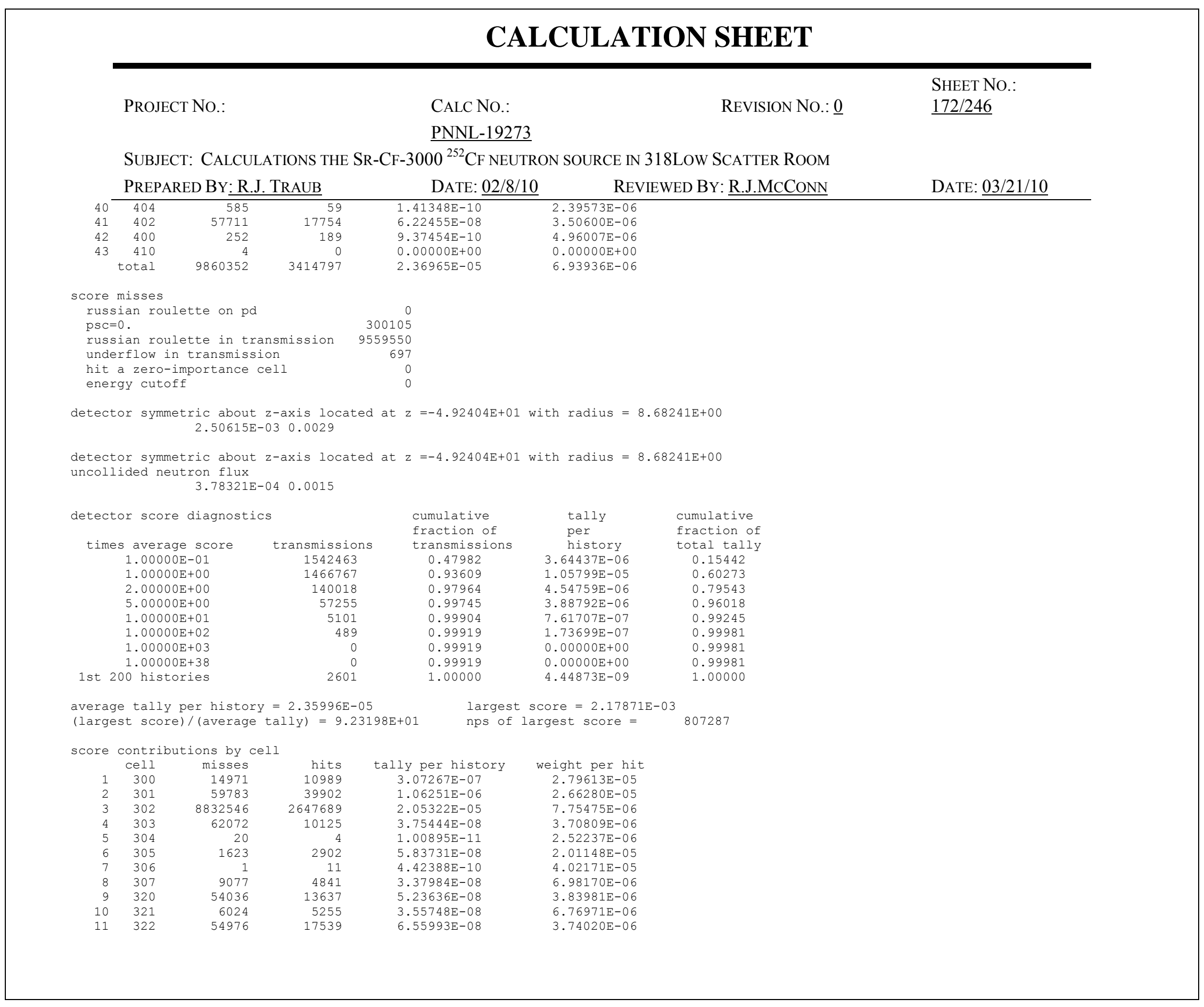




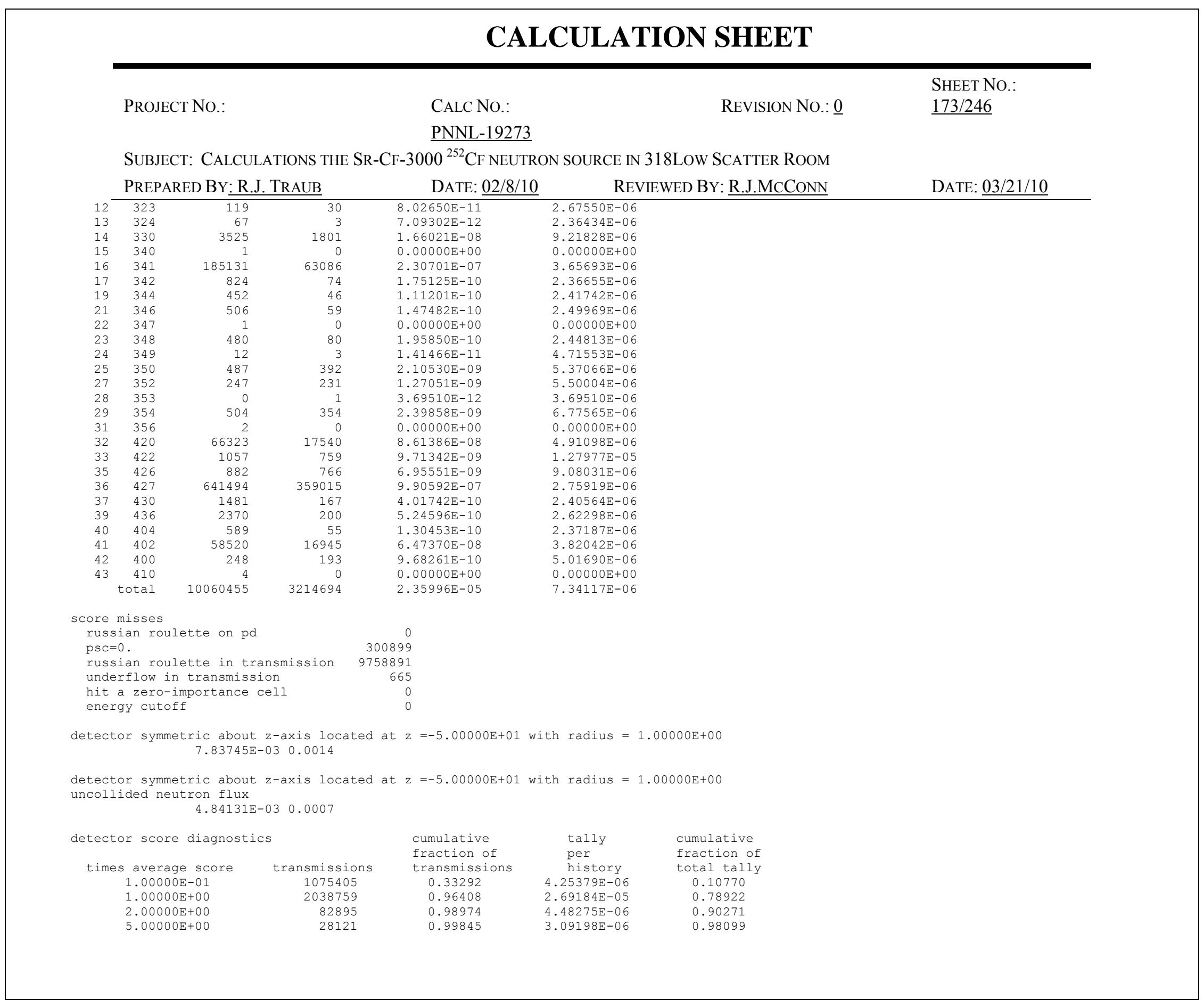




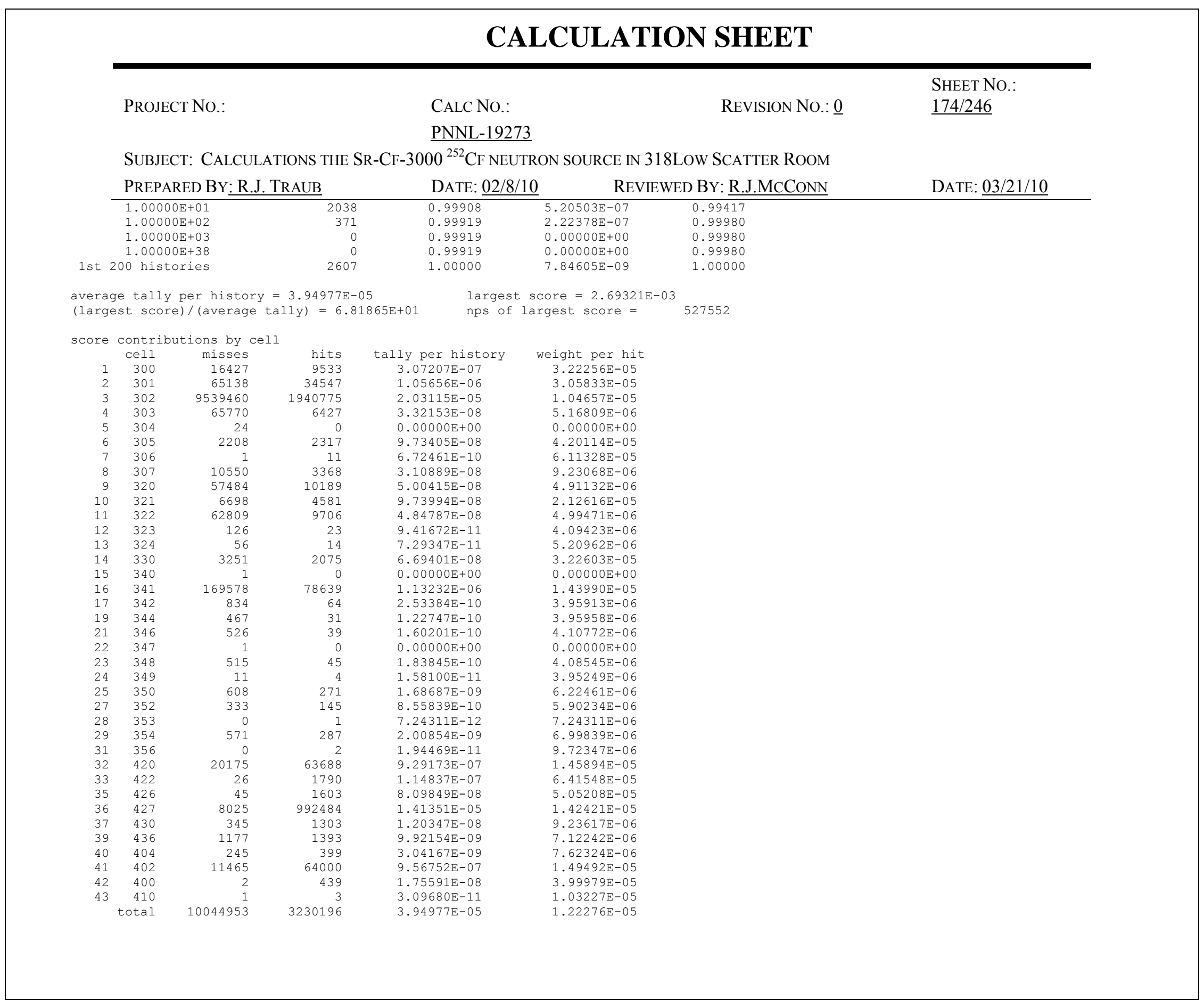




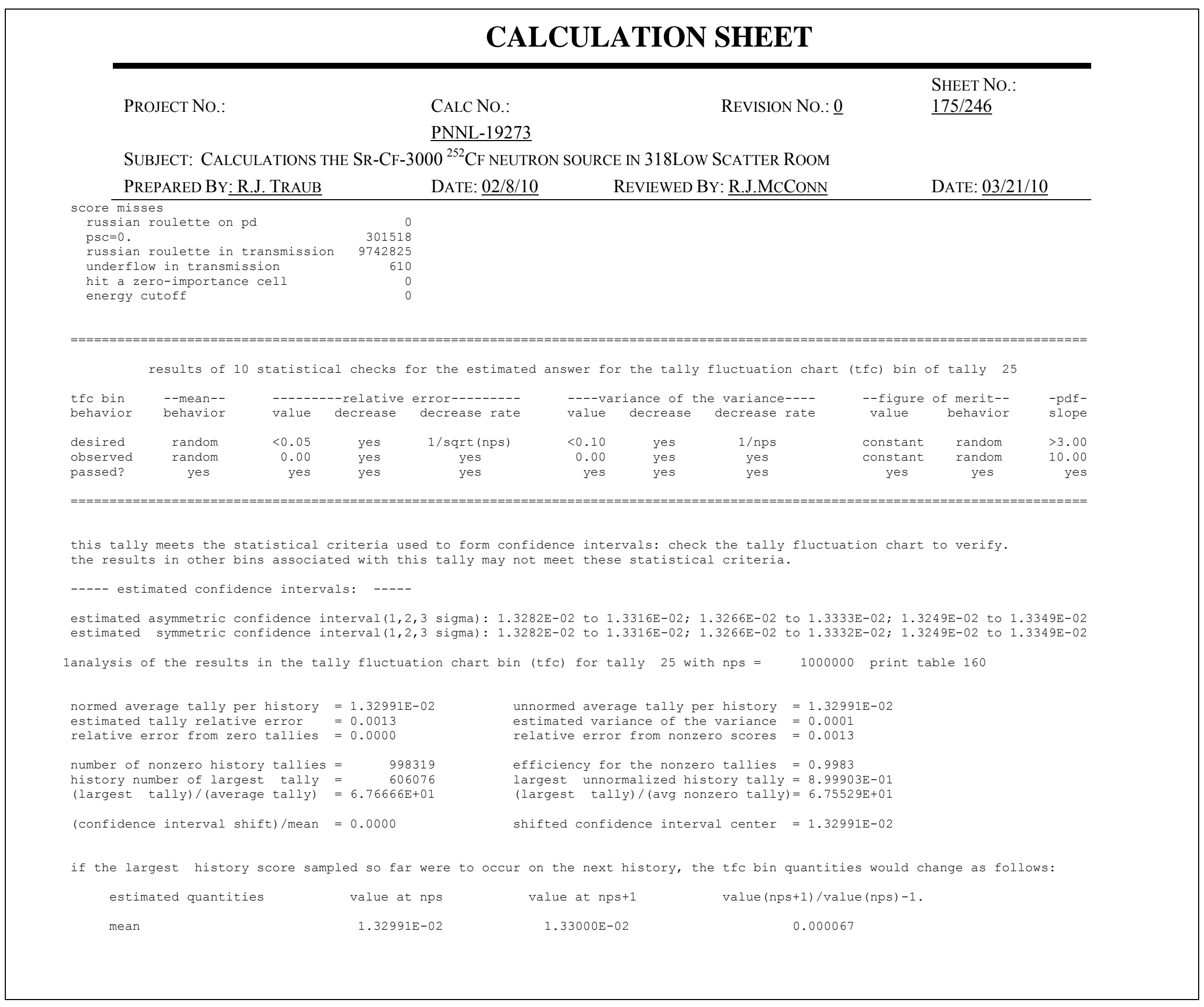




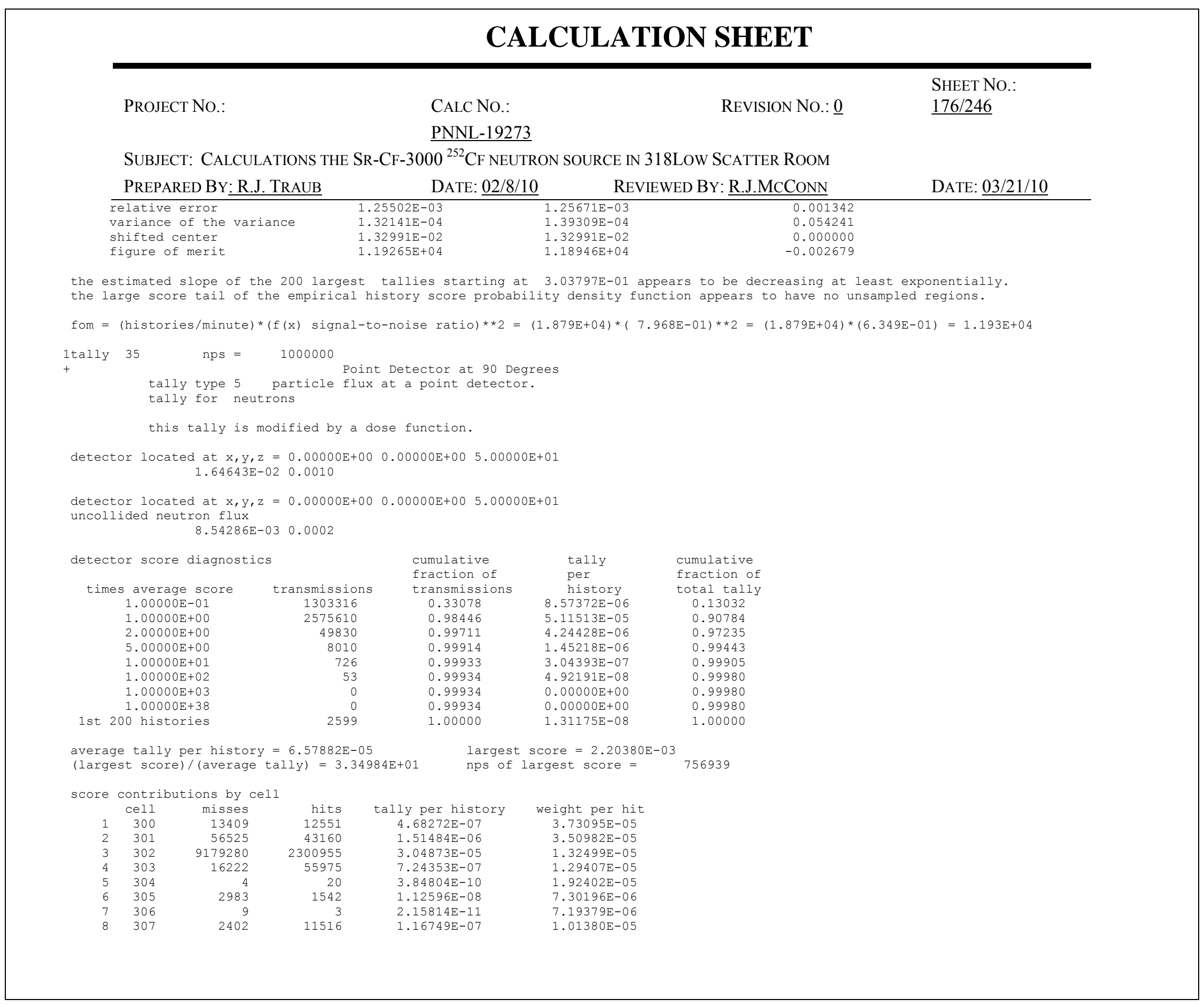




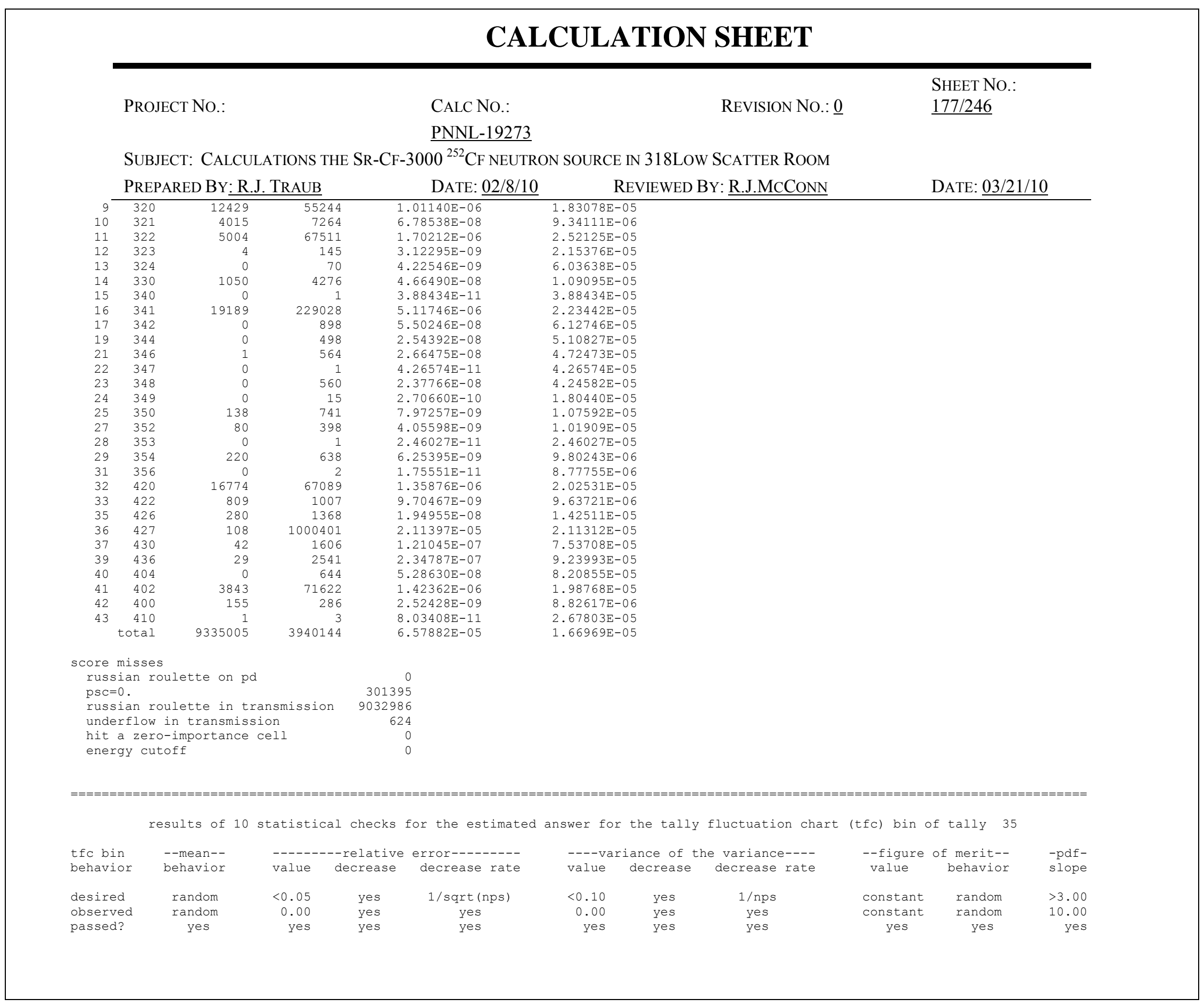




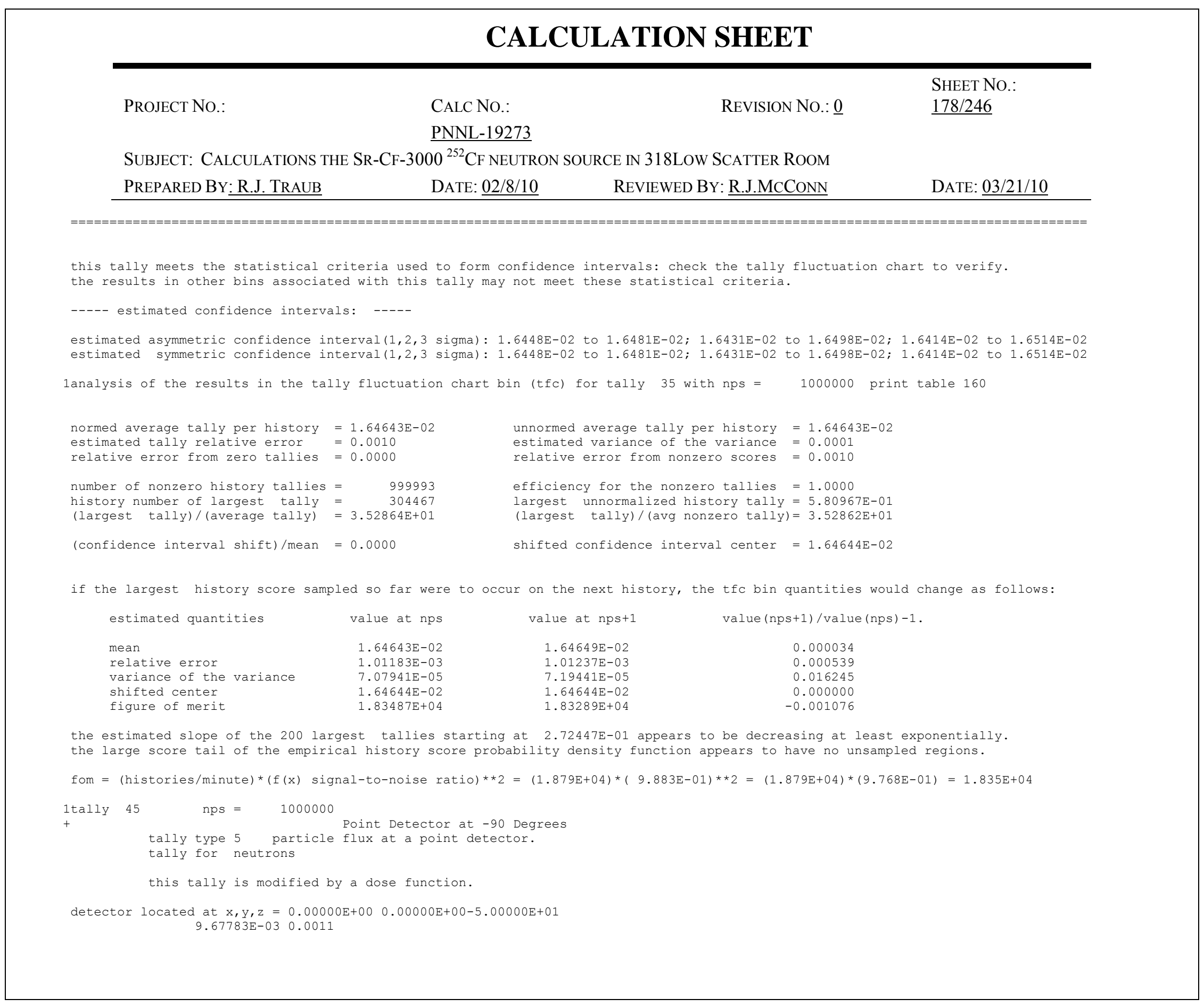




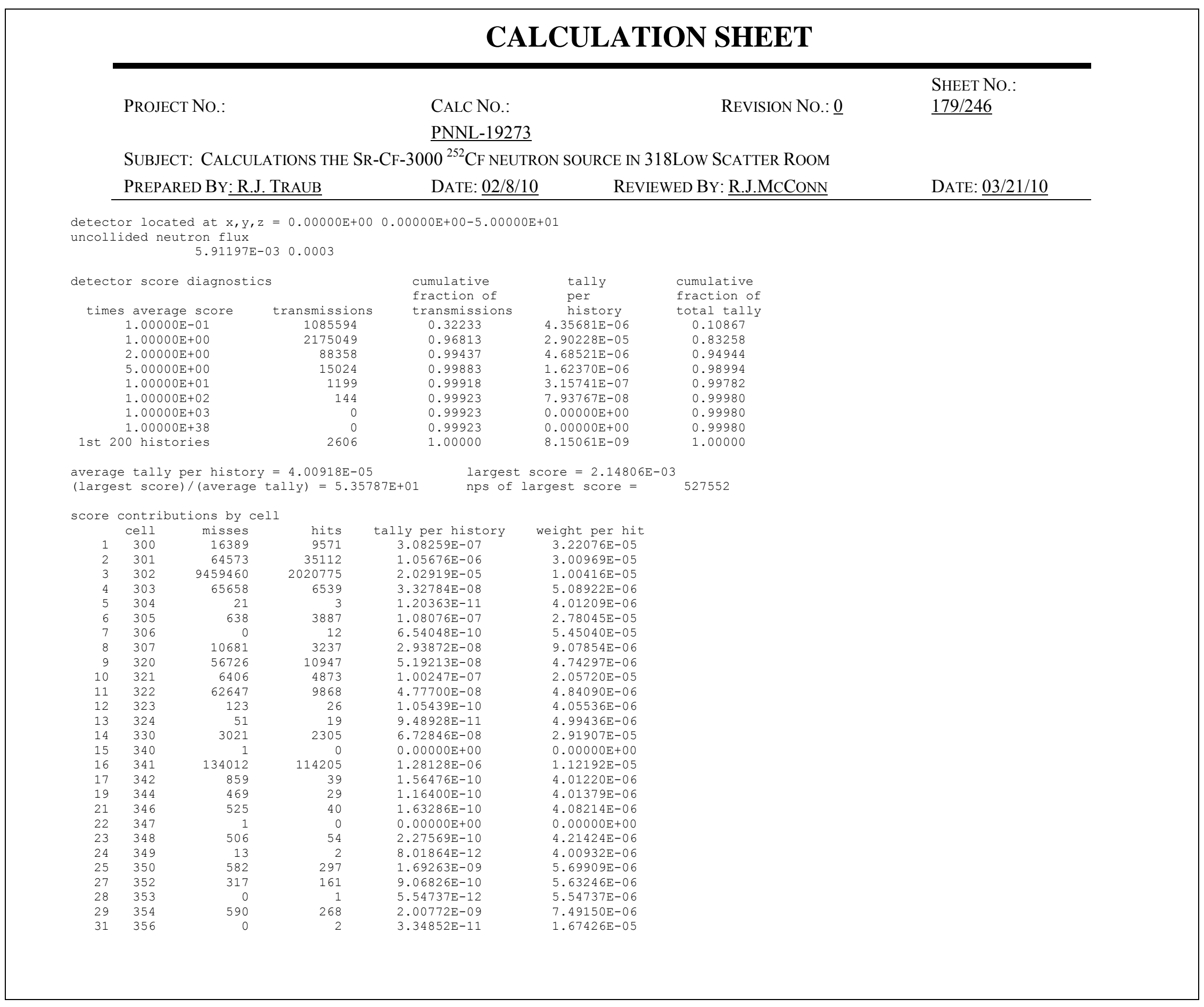




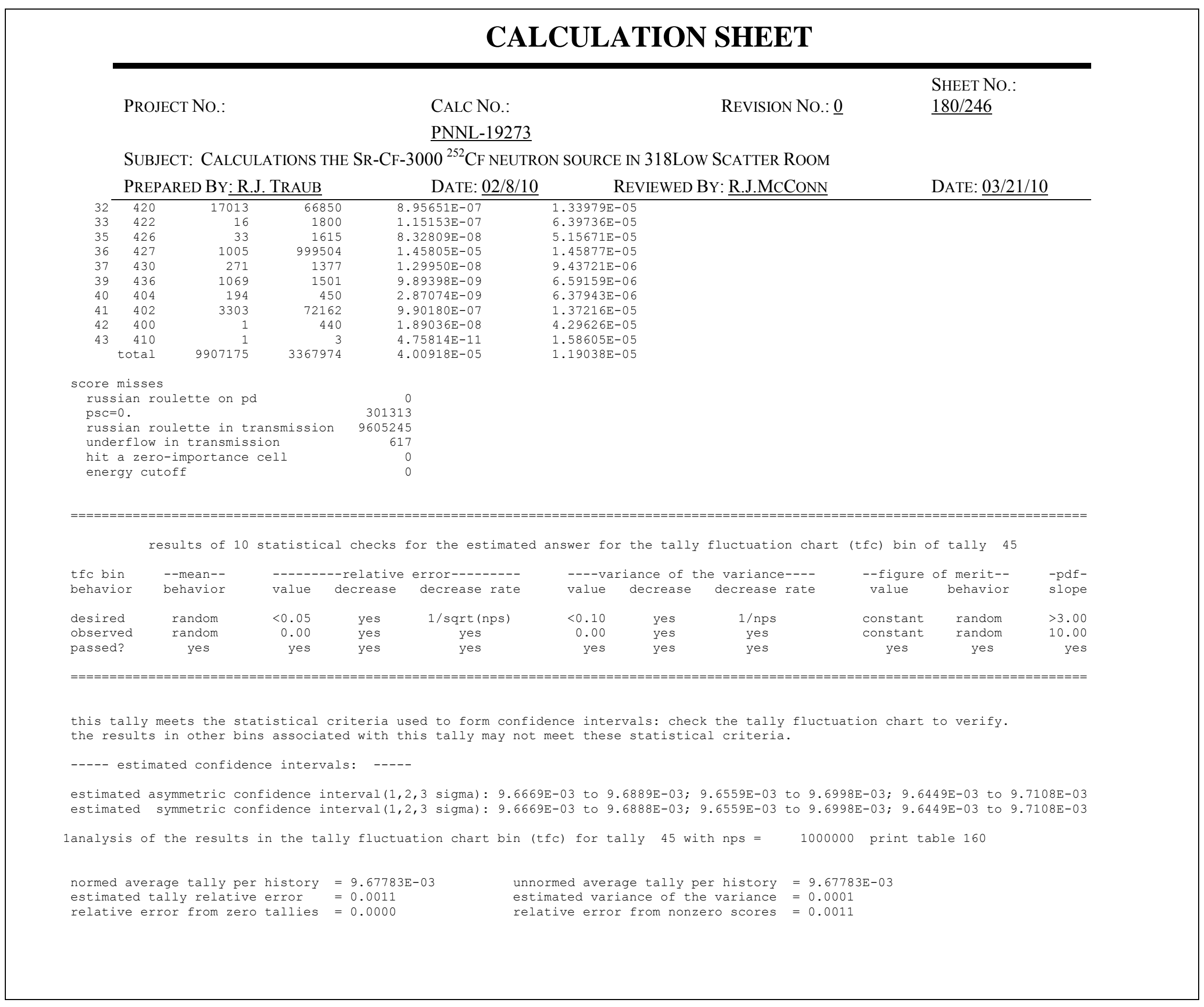




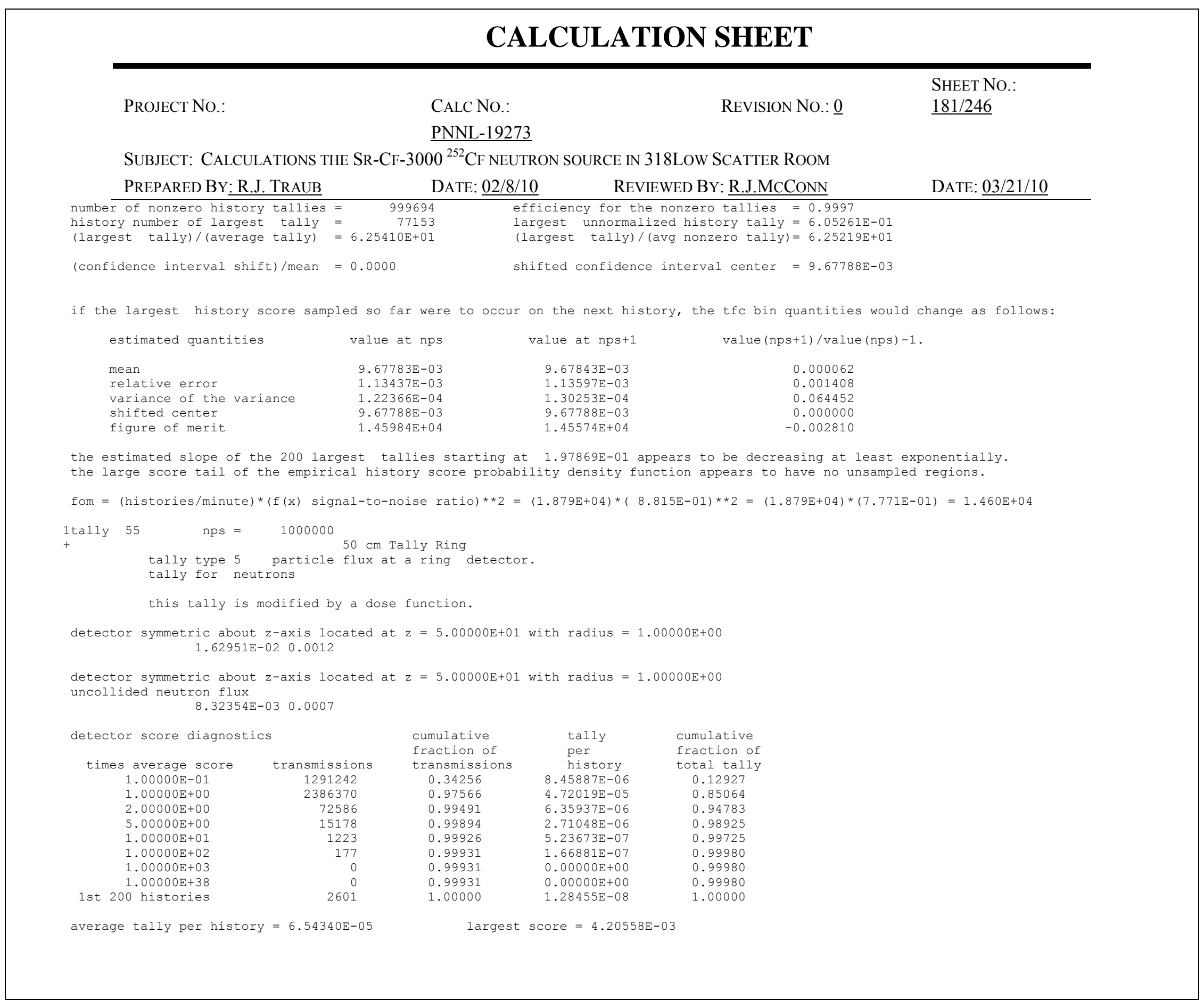




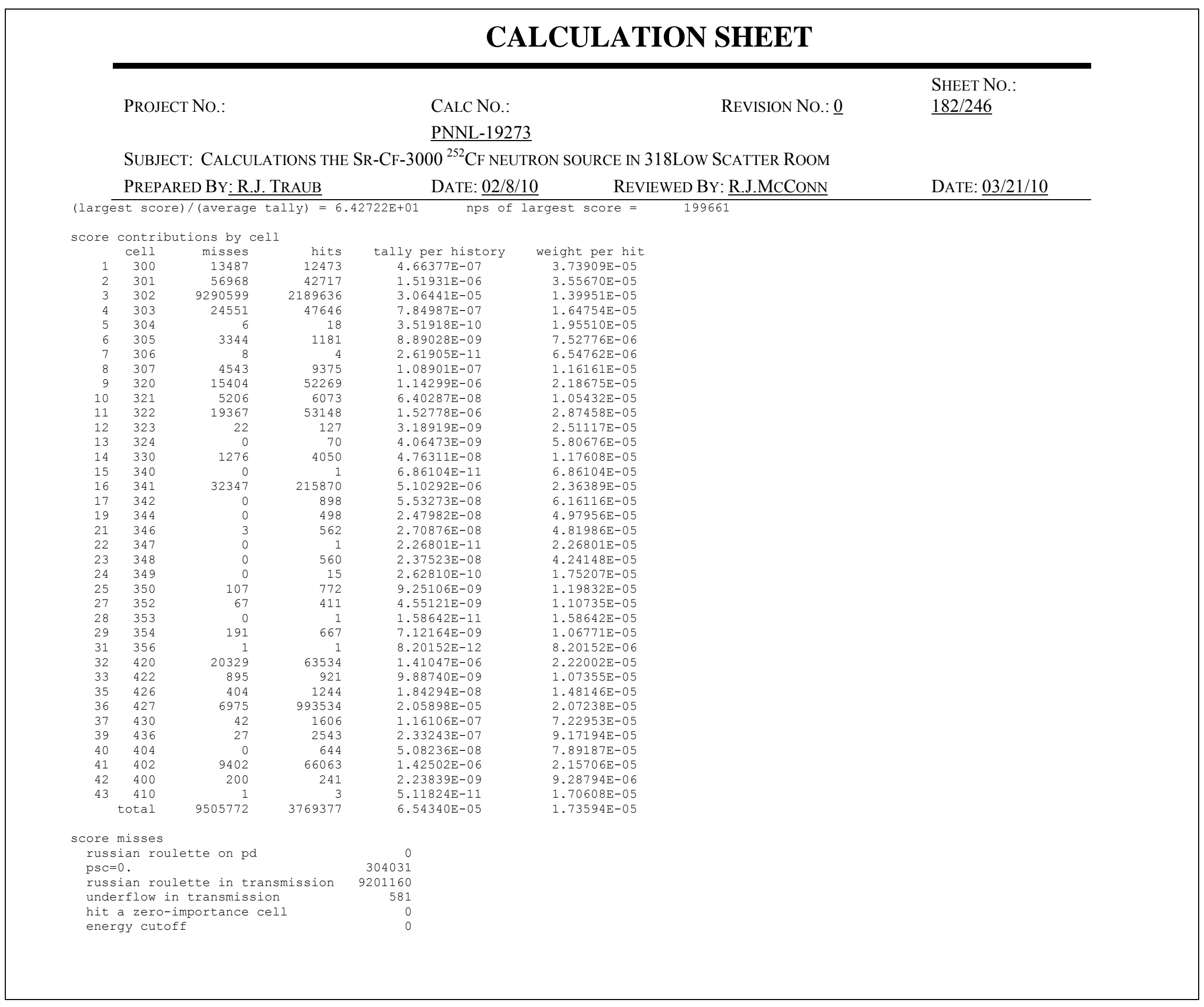




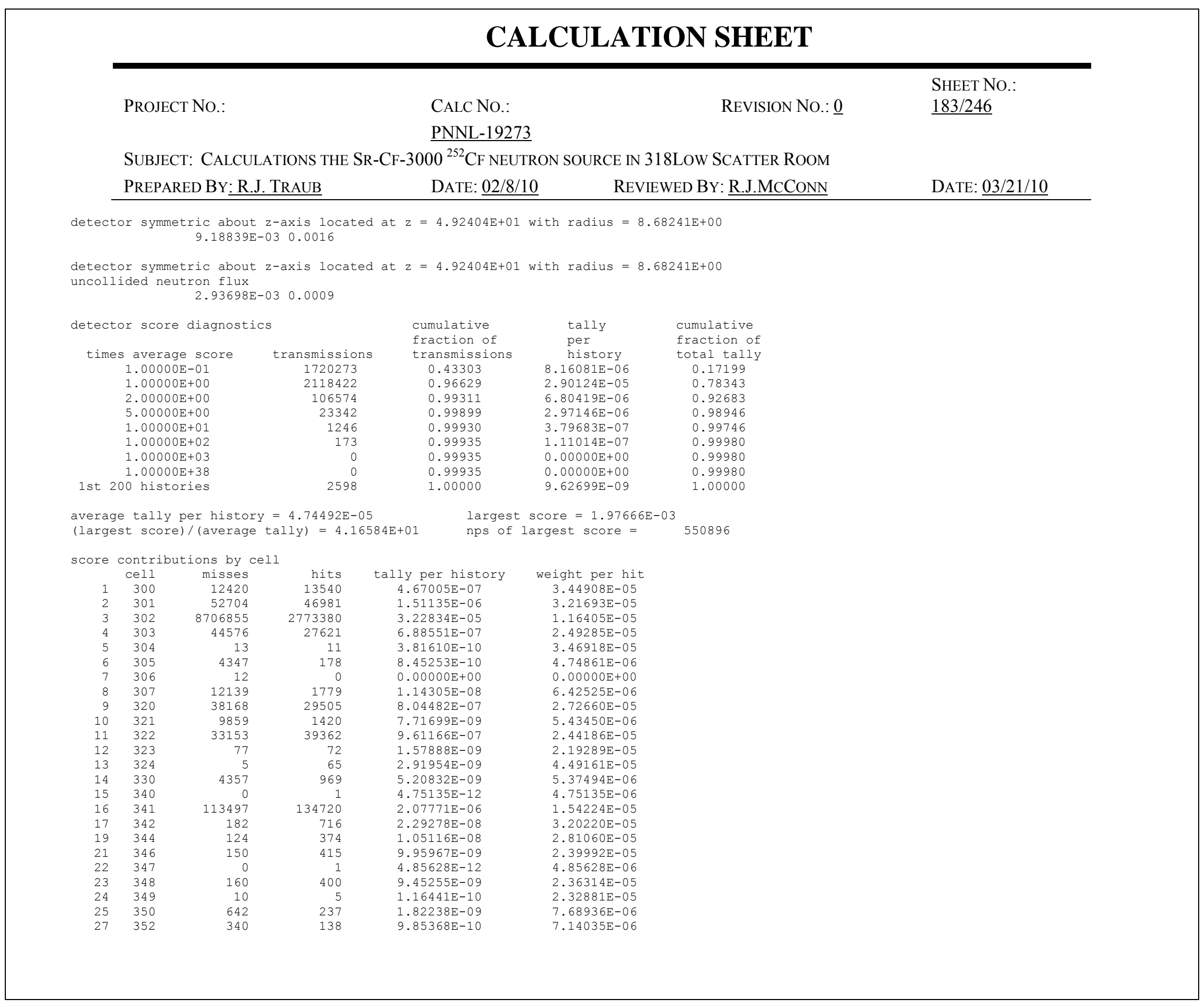




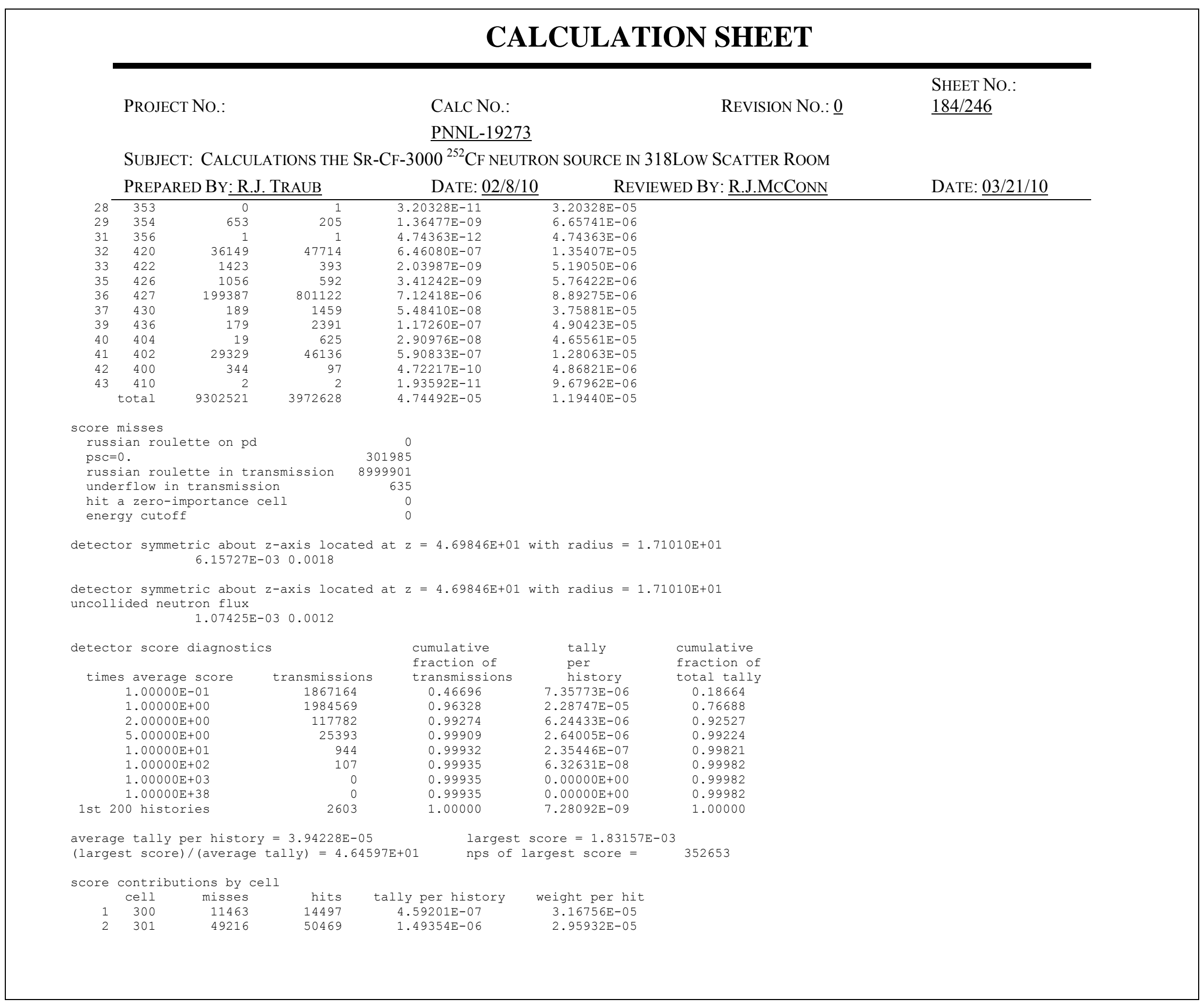




\begin{tabular}{|c|c|c|c|c|c|c|}
\hline \multicolumn{7}{|c|}{ CALCULATION SHEET } \\
\hline & PROJE & r No.: & & $\begin{array}{l}\text { CALC NO.: } \\
\text { PNNL-19273 }\end{array}$ & REVISION NO.: $\underline{0}$ & $\begin{array}{l}\text { SHEET NO.: } \\
\underline{185 / 246}\end{array}$ \\
\hline & \multicolumn{6}{|c|}{ 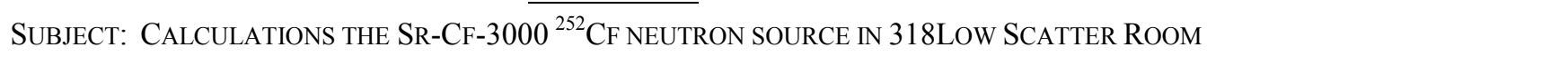 } \\
\hline & \multicolumn{3}{|c|}{ PREPARED BY: R.J. TRAUB } & DATE: $\underline{02 / 8 / 10}$ & \multirow{2}{*}{ REVIEWED BY: R.J.MCCONN } & \multirow[t]{2}{*}{ DATE: $\underline{03 / 21 / 10}$} \\
\hline 3 & 302 & 8297607 & 3182628 & $3.22507 \mathrm{E}-05$ & & \\
\hline 4 & 303 & 46555 & 25642 & $4.27392 \mathrm{E}-07$ & $1.66676 \mathrm{E}-05$ & \\
\hline 5 & 304 & 17 & $\begin{array}{r}7 \\
-5\end{array}$ & $1.81041 \mathrm{E}-10$ & $2.58630 \mathrm{E}-05$ & \\
\hline 6 & 305 & 4467 & 58 & $2.30202 E-10$ & $3.96901 \mathrm{E}-06$ & \\
\hline 7 & 306 & 12 & ${ }^{0}$ & $0.00000 \mathrm{E}+00$ & $\begin{array}{l}0.00000 \mathrm{E}+00 \\
3.92044 \mathrm{E}-06\end{array}$ & \\
\hline 8 & 307 & 13459 & 459 & $1.79948 \mathrm{E}-09$ & $3.92044 \mathrm{E}-06$ & \\
\hline 9 & 320 & 40367 & 27306 & $5.22272 \mathrm{E}-07$ & $1.91266 \mathrm{E}-05$ & \\
\hline 10 & $\begin{array}{l}321 \\
322\end{array}$ & $\begin{array}{l}10695 \\
40304\end{array}$ & $\begin{array}{r}584 \\
32211\end{array}$ & $\begin{array}{l}2.30159 \mathrm{E}-09 \\
5\end{array}$ & $3.94109 \mathrm{E}-06$ & \\
\hline $\begin{array}{l}11 \\
12\end{array}$ & $\begin{array}{l}322 \\
323\end{array}$ & $\begin{array}{r}40304 \\
87\end{array}$ & $\begin{array}{r}32211 \\
62\end{array}$ & $\begin{array}{l}5.77088 \mathrm{E}-07 \\
1.05201 \mathrm{E}-09\end{array}$ & $\begin{array}{l}1.79159 \mathrm{E}-05 \\
1.6679 \mathrm{E}-05\end{array}$ & \\
\hline 13 & 324 & 8 & 62 & $\begin{array}{l}1.05201 \mathrm{E}-09 \\
1.95558 \mathrm{E}-09\end{array}$ & $\begin{array}{l}1.6679 \mathrm{E}-05 \\
3.15416 \mathrm{E}-05\end{array}$ & \\
\hline 14 & 330 & 4911 & 415 & $1.64248 \mathrm{E}-09$ & $3.95779 \mathrm{E}-06$ & \\
\hline 15 & 340 & 0 & 1 & $6.01351 \mathrm{E}-12$ & $6.01351 \mathrm{E}-06$ & \\
\hline 16 & 341 & 153086 & 95131 & $6.65627 \mathrm{E}-07$ & $6.99695 \mathrm{E}-06$ & \\
\hline 17 & 342 & 279 & 619 & $8.83159 \mathrm{E}-09$ & $1.42675 \mathrm{E}-05$ & \\
\hline 19 & 344 & 185 & 313 & $3.43578 E-09$ & $1.09769 \mathrm{E}-05$ & \\
\hline 21 & 346 & 184 & 381 & $4.09896 \mathrm{E}-09$ & $1.07584 \mathrm{E}-05$ & \\
\hline $\begin{array}{l}22 \\
23\end{array}$ & $\begin{array}{l}347 \\
348\end{array}$ & $\begin{array}{r}0 \\
214\end{array}$ & 346 & 4.54121E-12 & $\begin{array}{l}4.54121 \mathrm{E}-06 \\
8\end{array}$ & \\
\hline $\begin{array}{l}23 \\
24\end{array}$ & $\begin{array}{l}348 \\
349\end{array}$ & $\begin{array}{r}214 \\
14\end{array}$ & $\begin{array}{r}346 \\
1\end{array}$ & $\begin{array}{l}3.09129 \mathrm{E}-09 \\
3.94552 \mathrm{E}-12\end{array}$ & $\begin{array}{l}8.93437 E-06 \\
3\end{array}$ & \\
\hline $\begin{array}{l}24 \\
25\end{array}$ & $\begin{array}{l}3499 \\
350\end{array}$ & $\begin{array}{r}14 \\
769\end{array}$ & 110 & $\begin{array}{l}3.94552 \mathrm{E}-12 \\
4.47893 \mathrm{E}-10\end{array}$ & $\begin{array}{l}3.94552 \mathrm{E}-06 \\
4.07176 \mathrm{E}-06\end{array}$ & \\
\hline 27 & 352 & 441 & 37 & $1.46854 \mathrm{E}-10$ & $3.96903 \mathrm{E}-06$ & \\
\hline 28 & 353 & 0 & 1 & $4.90701 \mathrm{E}-12$ & $4.90701 \mathrm{E}-06$ & \\
\hline 29 & 354 & 782 & 76 & $3.04692 \mathrm{E}-10$ & $4.00910 \mathrm{E}-06$ & \\
\hline 31 & 356 & 1 & 1 & $3.94299 \mathrm{E}-12$ & $3.94299 \mathrm{E}-06$ & \\
\hline 32 & 420 & 56417 & 27446 & $2.16421 \mathrm{E}-07$ & $7.88535 \mathrm{E}-06$ & \\
\hline 33 & 422 & $\begin{array}{l}1625 \\
1363\end{array}$ & 191 & 8.01953E-10 & 4.19871E-06 & \\
\hline $\begin{array}{l}35 \\
36\end{array}$ & 426 & $\begin{array}{r}1363 \\
492102\end{array}$ & 285 & $\begin{array}{l}1.16640 \mathrm{E}-09 \\
\end{array}$ & $4.09263 \mathrm{E}-06$ & \\
\hline $\begin{array}{l}36 \\
37\end{array}$ & $\begin{array}{l}427 \\
430\end{array}$ & $\begin{array}{r}492102 \\
635\end{array}$ & $\begin{array}{r}508407 \\
1013\end{array}$ & $\begin{array}{l}2.55356 \mathrm{E}-06 \\
1.50148 \mathrm{E}-08\end{array}$ & $5.02267 \mathrm{E}-06$ & \\
\hline 39 & $\begin{array}{l}350 \\
436\end{array}$ & $\begin{array}{l}635 \\
899\end{array}$ & $\begin{array}{l}1013 \\
1671\end{array}$ & $\begin{array}{l}1.20148 \mathrm{E}-08 \\
2.92272 \mathrm{E}-08\end{array}$ & $\begin{array}{l}1.48221 \mathrm{E}-05 \\
1.74908 \mathrm{E}-05\end{array}$ & \\
\hline 40 & 404 & 150 & 494 & $7.40100 \mathrm{E}-09$ & $1.49818 \mathrm{E}-05$ & \\
\hline 41 & 402 & 47872 & 27593 & $1.73665 \mathrm{E}-07$ & $6.29380 \mathrm{E}-06$ & \\
\hline 42 & 400 & 397 & 44 & $1.76571 \mathrm{E}-10$ & $4.01297 \mathrm{E}-06$ & \\
\hline 43 & & & & $0.00000 \mathrm{E}+00$ & $0.00000 \mathrm{E}+00$ & \\
\hline & total & 9276587 & 3998562 & $3.94228 \mathrm{E}-05$ & $9.85924 \mathrm{E}-06$ & \\
\hline \\
\hline \multirow{2}{*}{\multicolumn{7}{|c|}{$\begin{array}{lr}\text { russian roulette on pd } & 0 \\
\text { psco. } & 300713 \\
\text { russian roulette in transmission } & 8975226\end{array}$}} \\
\hline & & & & & & \\
\hline \\
\hline \multirow{2}{*}{\multicolumn{7}{|c|}{$\begin{array}{ll}\text { hit a zero-importance cell } & 0 \\
\text { energy cutoff } & 0\end{array}$}} \\
\hline \multirow{2}{*}{\multicolumn{7}{|c|}{$\begin{array}{l}\text { detector symmetric about } z \text {-axis located at } z=4.33013 \mathrm{E}+01 \text { with radius }=2.50000 \mathrm{E}+01 \\
5.56290 \mathrm{E}-030.0017\end{array}$}} \\
\hline & & & & & & \\
\hline \multicolumn{7}{|c|}{$\begin{array}{l}\text { detector symmetric about } z \text {-axis located at } z=4.33013 \mathrm{E}+01 \text { with radius }=2.50000 \mathrm{E}+01 \\
\text { uncollided neutron flux }\end{array}$} \\
\hline
\end{tabular}




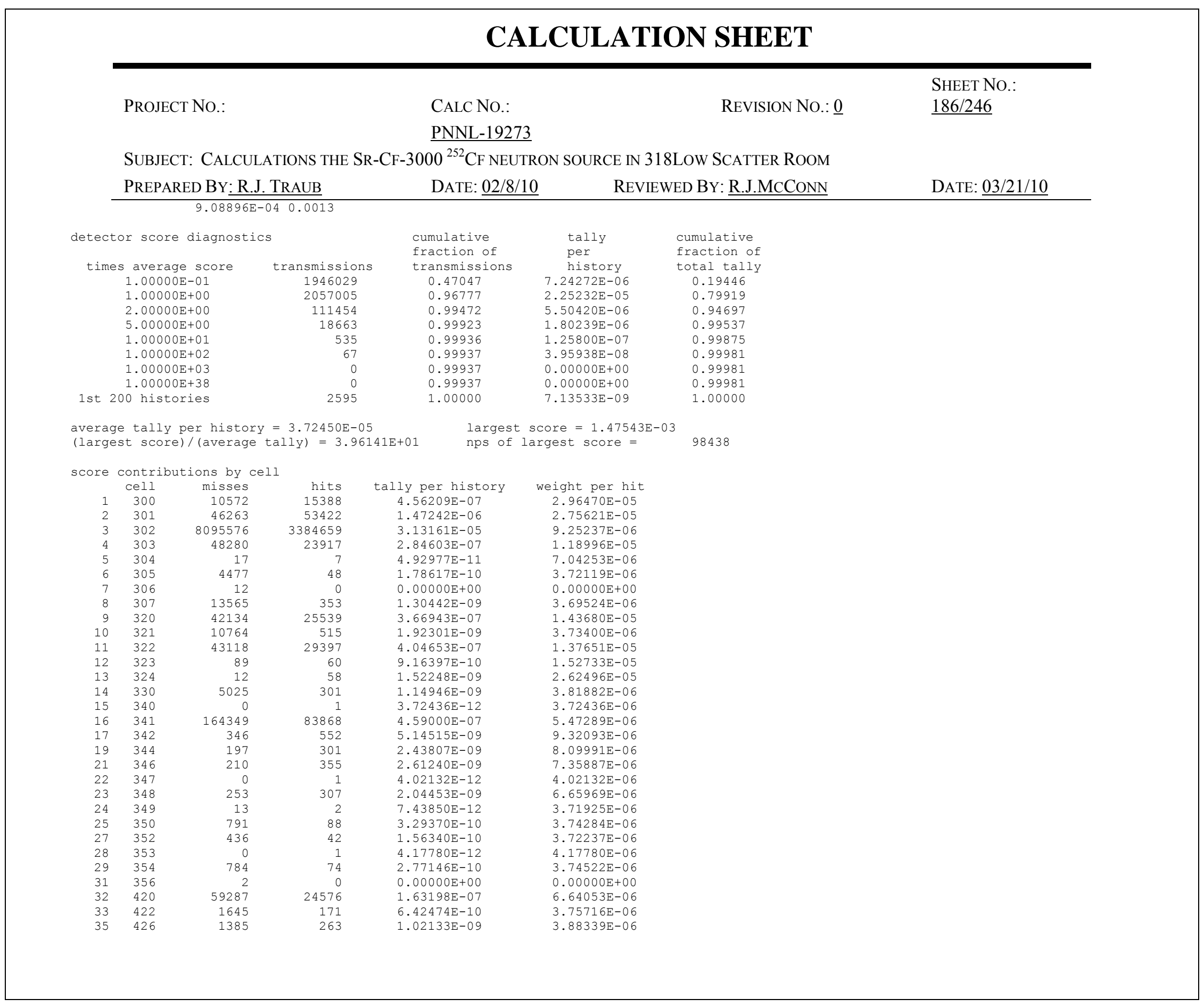




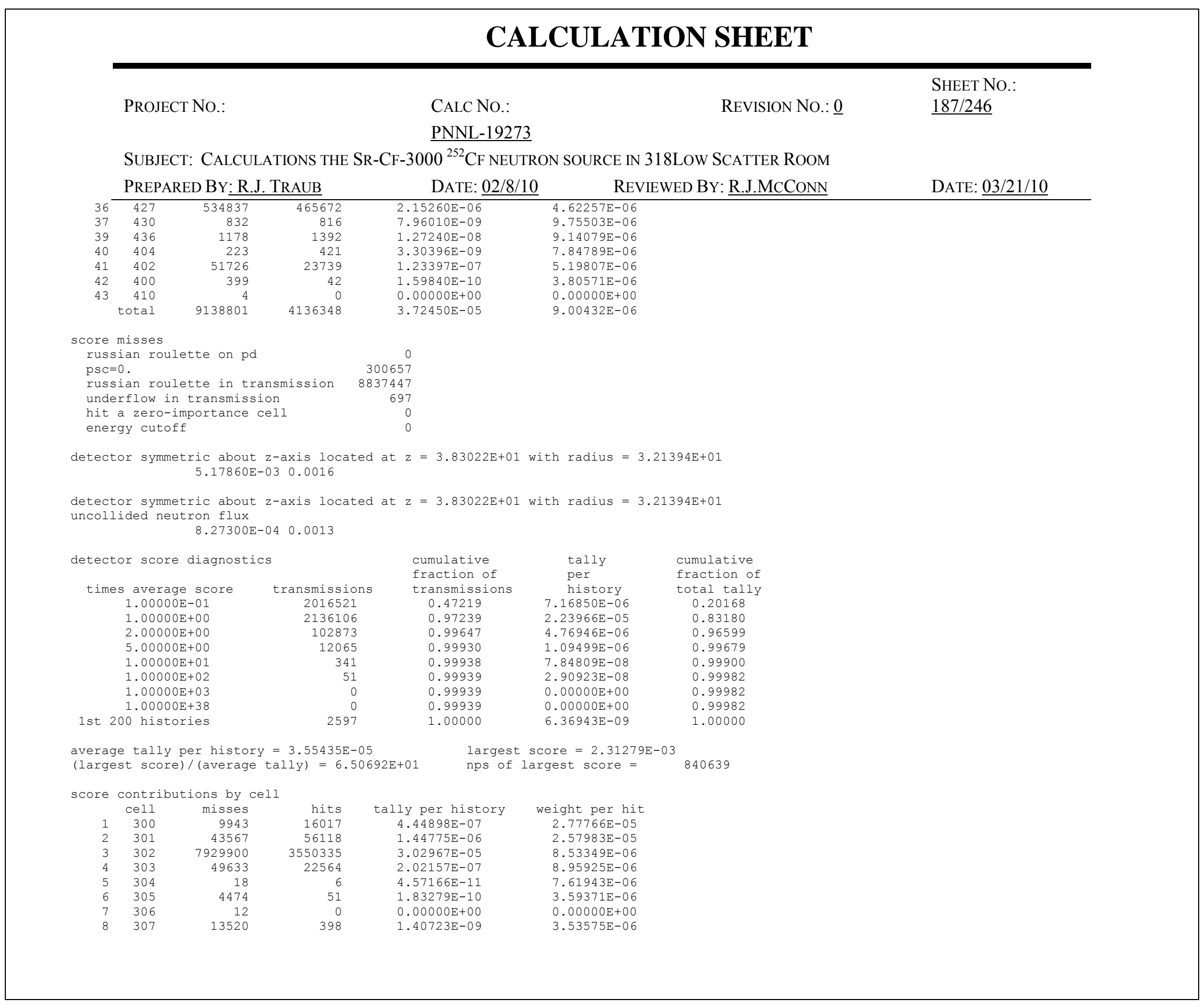




\begin{tabular}{|c|c|c|c|c|c|c|c|}
\hline \multicolumn{8}{|c|}{ CALCULATION SHEET } \\
\hline & PROJE & No.: & & $\begin{array}{l}\text { CALC NO.: } \\
\text { PNNL-19273 }\end{array}$ & & REVISION NO.: $\underline{0}$ & $\begin{array}{l}\text { SHEET NO.: } \\
\underline{188 / 246}\end{array}$ \\
\hline & \multicolumn{7}{|c|}{ 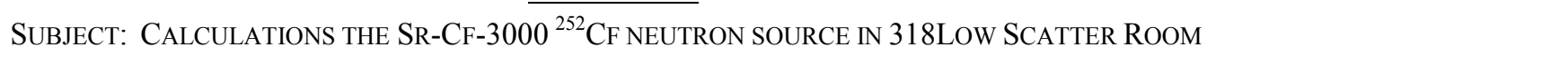 } \\
\hline & \multicolumn{3}{|c|}{ PREPARED BY: R.J. TRAUB } & DATE: $\underline{02 / 8 / 10}$ & \multicolumn{2}{|c|}{ REVIEWED BY: R.J.MCCONN } & \multirow[t]{2}{*}{ DATE: $\underline{03 / 21 / 10}$} \\
\hline 9 & 320 & 4344 & 24224 & $2.70965 \mathrm{E}-07$ & $1.11858 \mathrm{E}-05$ & & \\
\hline 10 & 321 & 1077 & 505 & $1.80093 E-09$ & $3.56620 \mathrm{E}-06$ & & \\
\hline 11 & 322 & 4502 & 27487 & $2.92222 \mathrm{E}-07$ & $1.06313 \mathrm{E}-05$ & & \\
\hline 12 & 323 & 9 & 58 & $6.99274 \mathrm{E}-10$ & $1.20564 \mathrm{E}-05$ & & \\
\hline 13 & 324 & 1 & 54 & 1.13911E-09 & $2.10946 \mathrm{E}-05$ & & \\
\hline 14 & 330 & 501 & 308 & 1.10582E-09 & $\begin{array}{l}3.59034 \mathrm{E}-06 \\
0.0000 \mathrm{E}+00\end{array}$ & & \\
\hline $\begin{array}{l}15 \\
16\end{array}$ & $\begin{array}{l}340 \\
341\end{array}$ & 17014 & $\begin{array}{r}0 \\
78072\end{array}$ & $\begin{array}{l}0.00000 \mathrm{E}+00 \\
3.68651 \mathrm{E}-07\end{array}$ & $\begin{array}{l}0.00000 \mathrm{E}+00 \\
4.72194 \mathrm{E}-06\end{array}$ & & \\
\hline 17 & 342 & $\begin{array}{r}1704 \\
38\end{array}$ & $\begin{array}{r}8012 \\
510\end{array}$ & $\begin{array}{l}3.68651 \mathrm{E}-01 \\
3.47978 \mathrm{E}-09\end{array}$ & $\begin{array}{l}4.2194 \mathrm{E}-06 \\
6.82309 \mathrm{E}-06\end{array}$ & & \\
\hline 19 & 344 & 21 & 287 & $1.63943 \mathrm{E}-09$ & $5.71232 \mathrm{E}-06$ & & \\
\hline 21 & 346 & 26 & 302 & $1.75305 \mathrm{E}-09$ & $5.80480 \mathrm{E}-06$ & & \\
\hline 22 & 347 & 皮 & 1 & $6.49816 \mathrm{E}-12$ & $6.49816 \mathrm{E}-06$ & & \\
\hline 23 & 348 & 26 & 292 & $1.53761 \mathrm{E}-09$ & $5.26580 \mathrm{E}-06$ & & \\
\hline 24 & 349 & 1 & 4 & $1.42194 \mathrm{E}-11$ & $3.55484 \mathrm{E}-06$ & & \\
\hline 25 & 350 & 79 & 82 & $2.97410 \mathrm{E}-10$ & $3.62695 \mathrm{E}-06$ & & \\
\hline 27 & 352 & 44 & 38 & $1.35188 \mathrm{E}-10$ & $3.55758 \mathrm{E}-06$ & & \\
\hline 28 & 353 & 78 & 0 & $0.00000 \mathrm{E}+00$ & $0.00000 \mathrm{E}+00$ & & \\
\hline $\begin{array}{l}29 \\
31\end{array}$ & $\begin{array}{l}354 \\
356\end{array}$ & 78 & $\begin{array}{r}78 \\
0\end{array}$ & $2.77252 \mathrm{E}-10$ & $3.55451 \mathrm{E}-06$ & & \\
\hline $\begin{array}{l}31 \\
32\end{array}$ & $\begin{array}{l}356 \\
420\end{array}$ & 6075 & $\begin{array}{r}0 \\
23105\end{array}$ & $\begin{array}{l}0.00000 \mathrm{E}+00 \\
1.32433 \mathrm{E}-07\end{array}$ & $\begin{array}{l}0.00000 \mathrm{E}+00 \\
5.73180 \mathrm{E}-06\end{array}$ & & \\
\hline 33 & 422 & 166 & 155 & $5.97551 \mathrm{E}-10$ & $3.85517 \mathrm{E}-06$ & & \\
\hline 35 & 426 & 140 & 244 & $8.78768 \mathrm{E}-10$ & $3.60151 \mathrm{E}-06$ & & \\
\hline 36 & 427 & 55543 & 445078 & 1.95603E-06 & $4.39481 \mathrm{E}-06$ & & \\
\hline 37 & 430 & 97 & 675 & $5.58006 \mathrm{E}-09$ & $8.26675 \mathrm{E}-06$ & & \\
\hline 39 & 436 & 145 & 1117 & $6.25721 \mathrm{E}-09$ & $5.60180 \mathrm{E}-06$ & & \\
\hline 40 & 404 & 29 & 345 & $1.56491 \mathrm{E}-09$ & $4.53598 \mathrm{E}-06$ & & \\
\hline 41 & 402 & 5345 & 22007 & $1.01160 \mathrm{E}-07$ & 4.59673E-06 & & \\
\hline \multirow{2}{*}{$\begin{array}{l}42 \\
43\end{array}$} & 400 & 40 & 37 & $1.31713 \mathrm{E}-10$ & $3.55981 \mathrm{E}-06$ & & \\
\hline & $\begin{array}{r}410 \\
\text { total }\end{array}$ & 900459 & $\begin{array}{r}0 \\
4270554\end{array}$ & $\begin{array}{l}0.00000 \mathrm{E}+00 \\
3.55435 \mathrm{E}-05\end{array}$ & $\begin{array}{l}0.00000 \mathrm{E}+00 \\
8.32293 \mathrm{E}-06\end{array}$ & & \\
\hline \\
\hline & & & & & & & \\
\hline \multicolumn{8}{|c|}{$\begin{array}{l}\text { psc }=0 . \\
\text { russian roulette in transmission } \begin{array}{r}299686 \\
8704212\end{array}\end{array}$} \\
\hline \multirow{2}{*}{\multicolumn{8}{|c|}{$\begin{array}{lr}\text { underflow in transmission } & 697 \\
\text { hit a zero-importance cell } & 0\end{array}$}} \\
\hline \multicolumn{7}{|c|}{$\begin{array}{ll}\text { hit a zero-importance cell } & 0 \\
\text { energy cutoff } & 0\end{array}$} & \\
\hline \multicolumn{8}{|c|}{$\begin{aligned} \text { detector symmetric about } z \text {-axis located at } z=3.21394 \mathrm{E}+01 \text { with radius } & =3.83022 \mathrm{E}+01 \\
& 4.85091 \mathrm{E}-03 \quad 0.0015\end{aligned}$} \\
\hline \multirow{2}{*}{\multicolumn{8}{|c|}{$\begin{array}{l}\text { detector symmetric about } z \text {-axis located at } z=3.21394 \mathrm{E}+01 \text { with radius }=3.83022 \mathrm{E}+01 \\
\text { uncollided neutron flux } \\
7.62716 \mathrm{E}-04 \quad 0.0013\end{array}$}} \\
\hline & & & & & & & \\
\hline \multirow{2}{*}{\multicolumn{4}{|c|}{ detector score diagnostics }} & cumulative & tally & cumulative & \\
\hline tim & & & & $\begin{array}{l}\text { fraction of } \\
\text { transmissions }\end{array}$ & $\begin{array}{l}\text { per } \\
\text { history }\end{array}$ & $\begin{array}{l}\text { fraction of } \\
\text { total tally }\end{array}$ & \\
\hline & 1.0000 & -01 & 2088479 & 0.47548 & $7.09216 \mathrm{E}-06$ & 0.20866 & \\
\hline
\end{tabular}




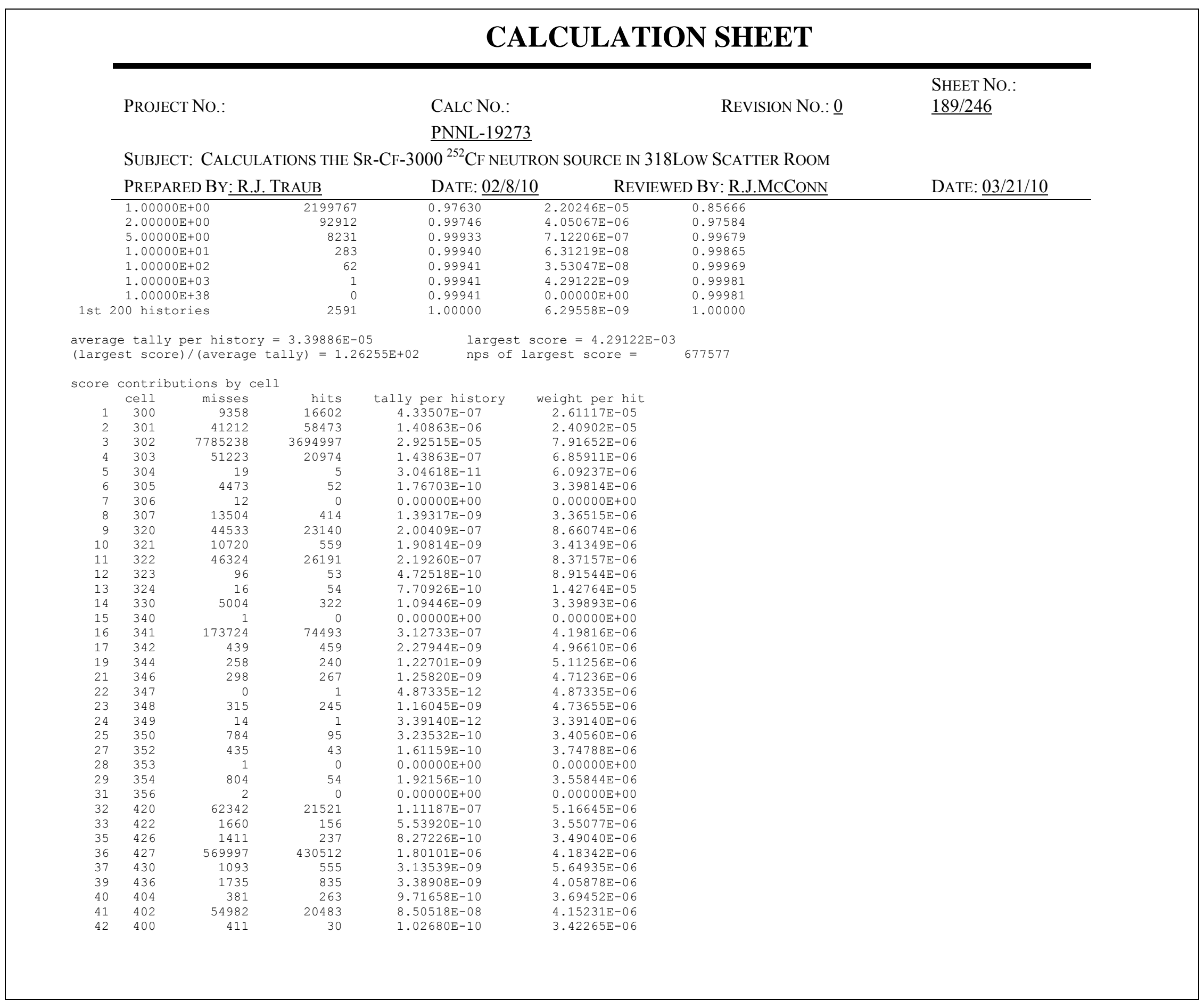




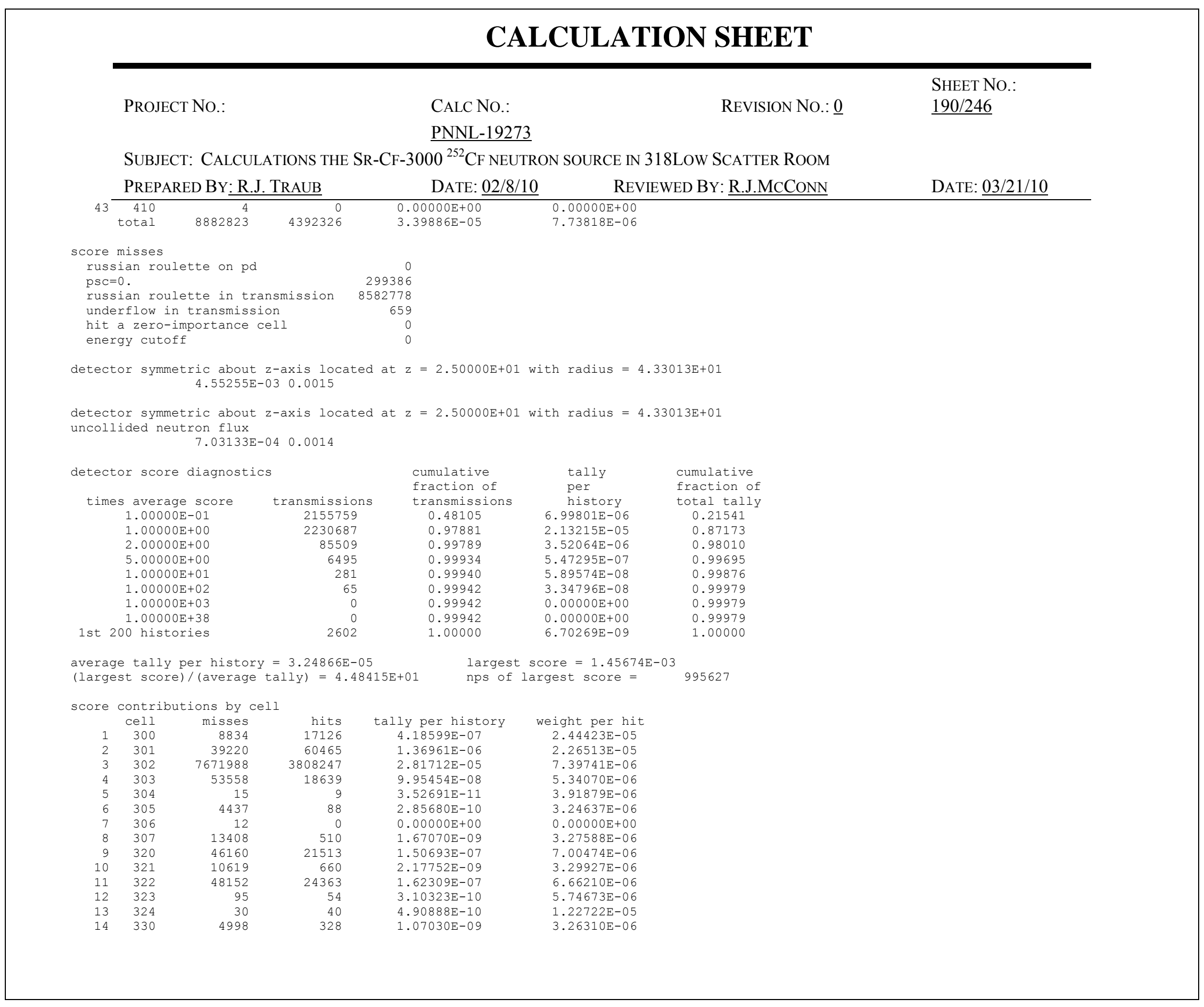




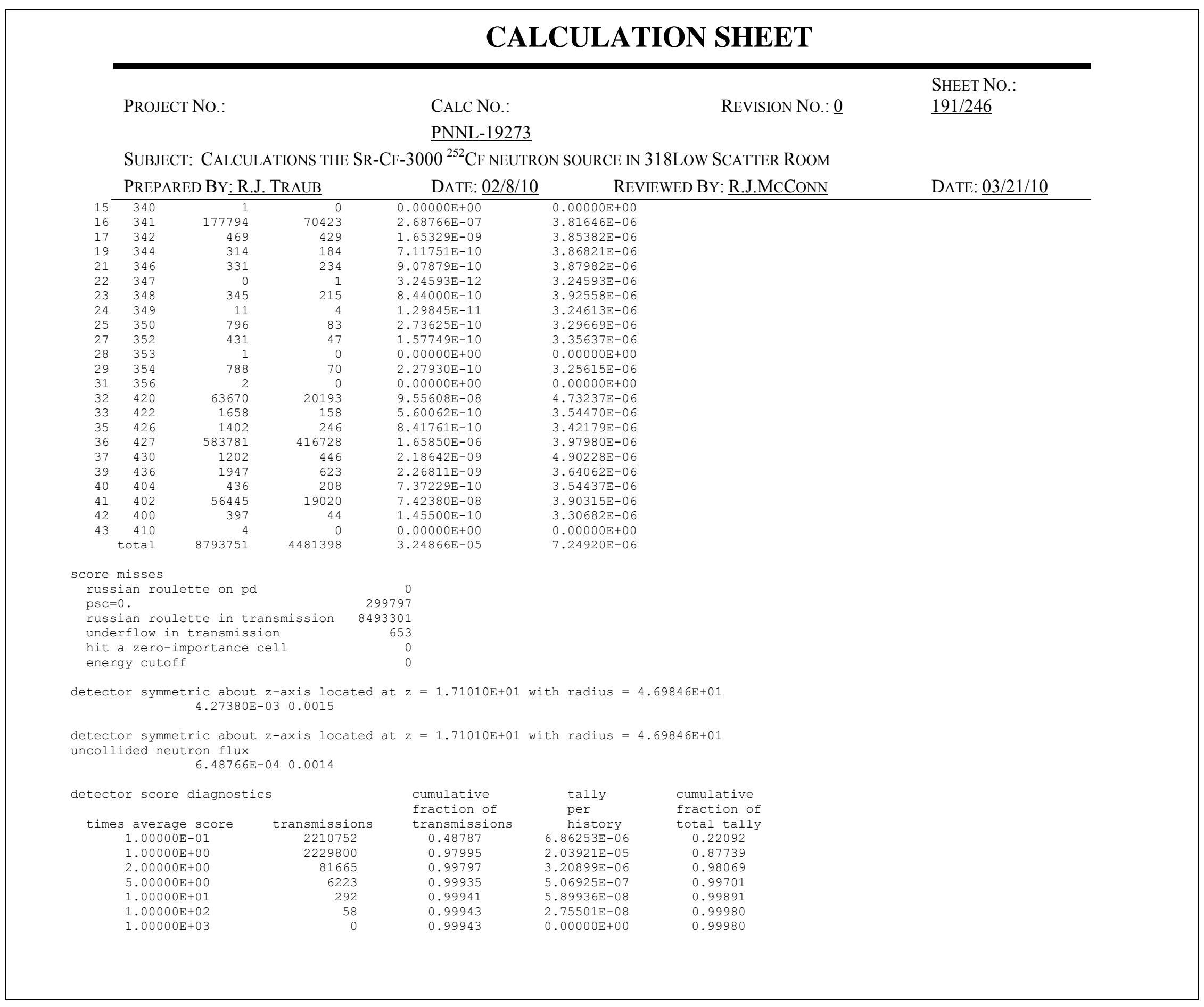




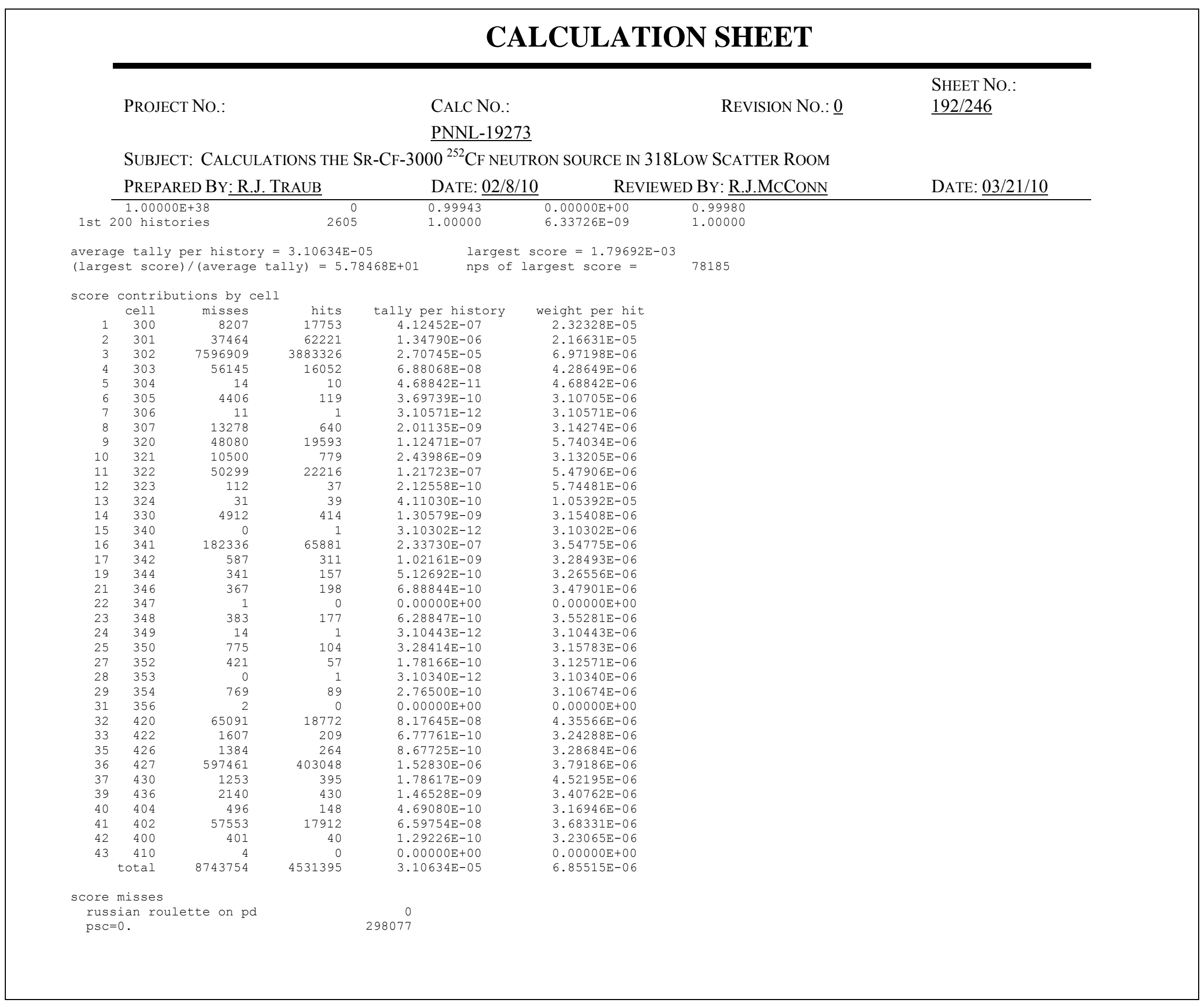




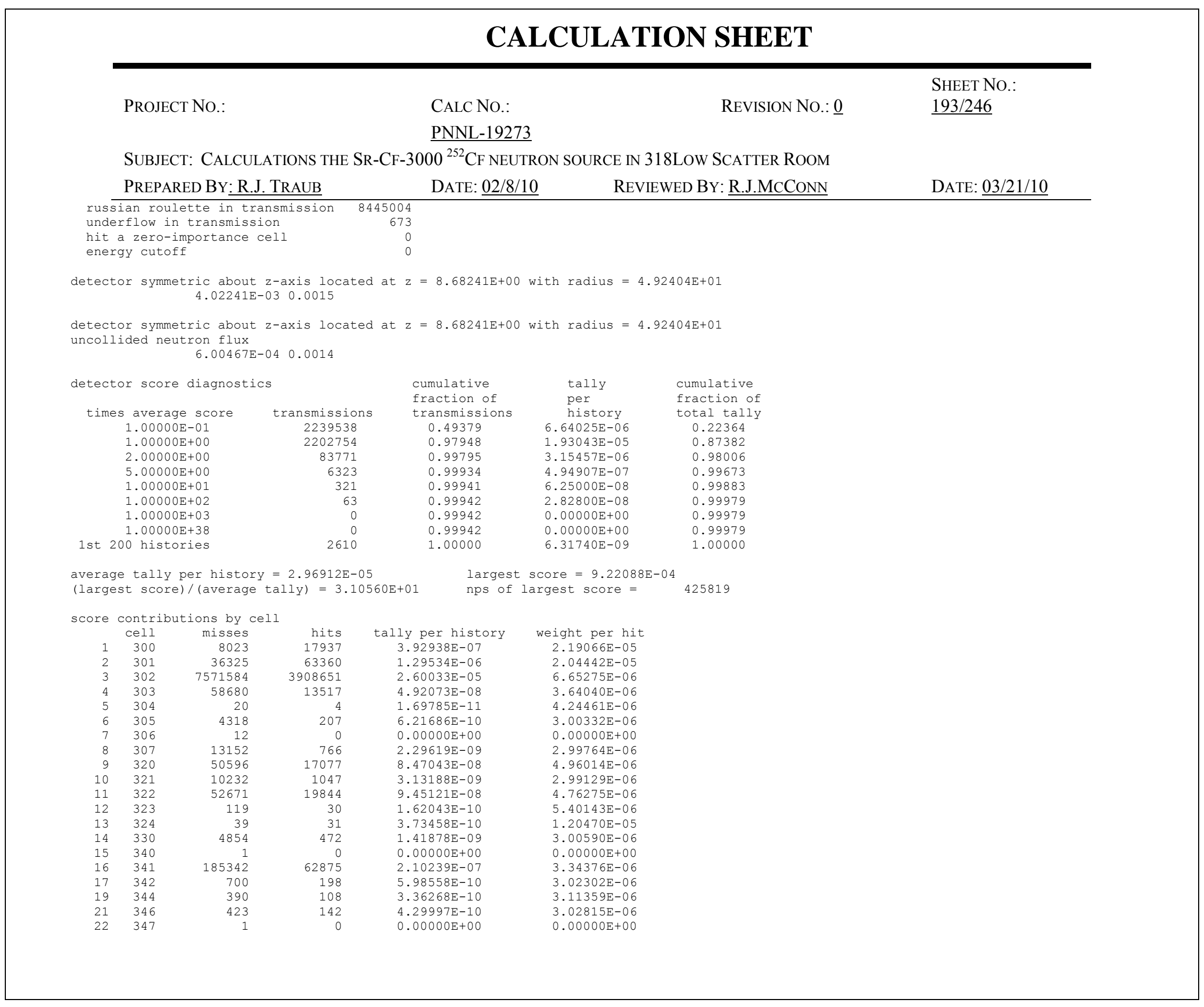




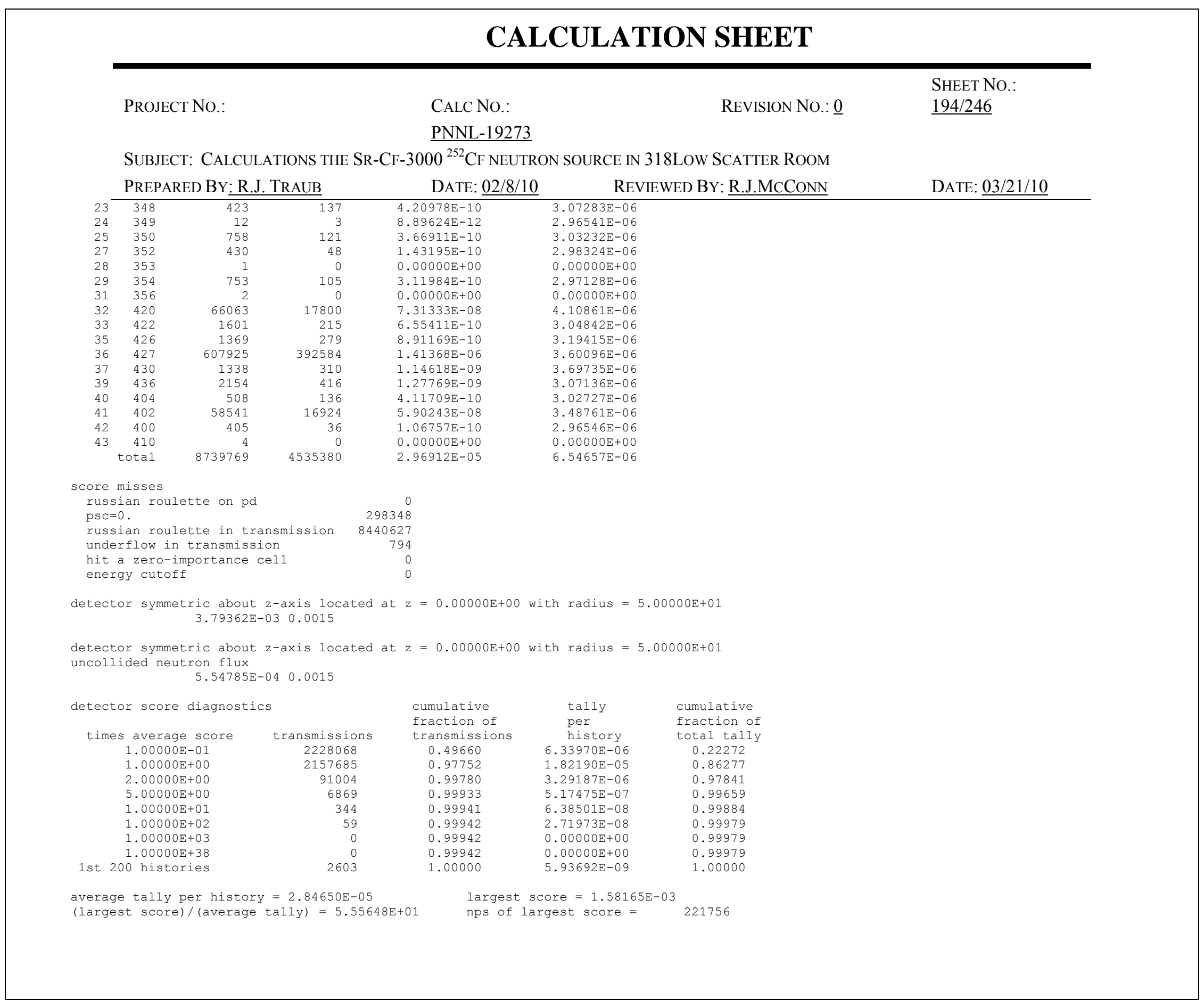




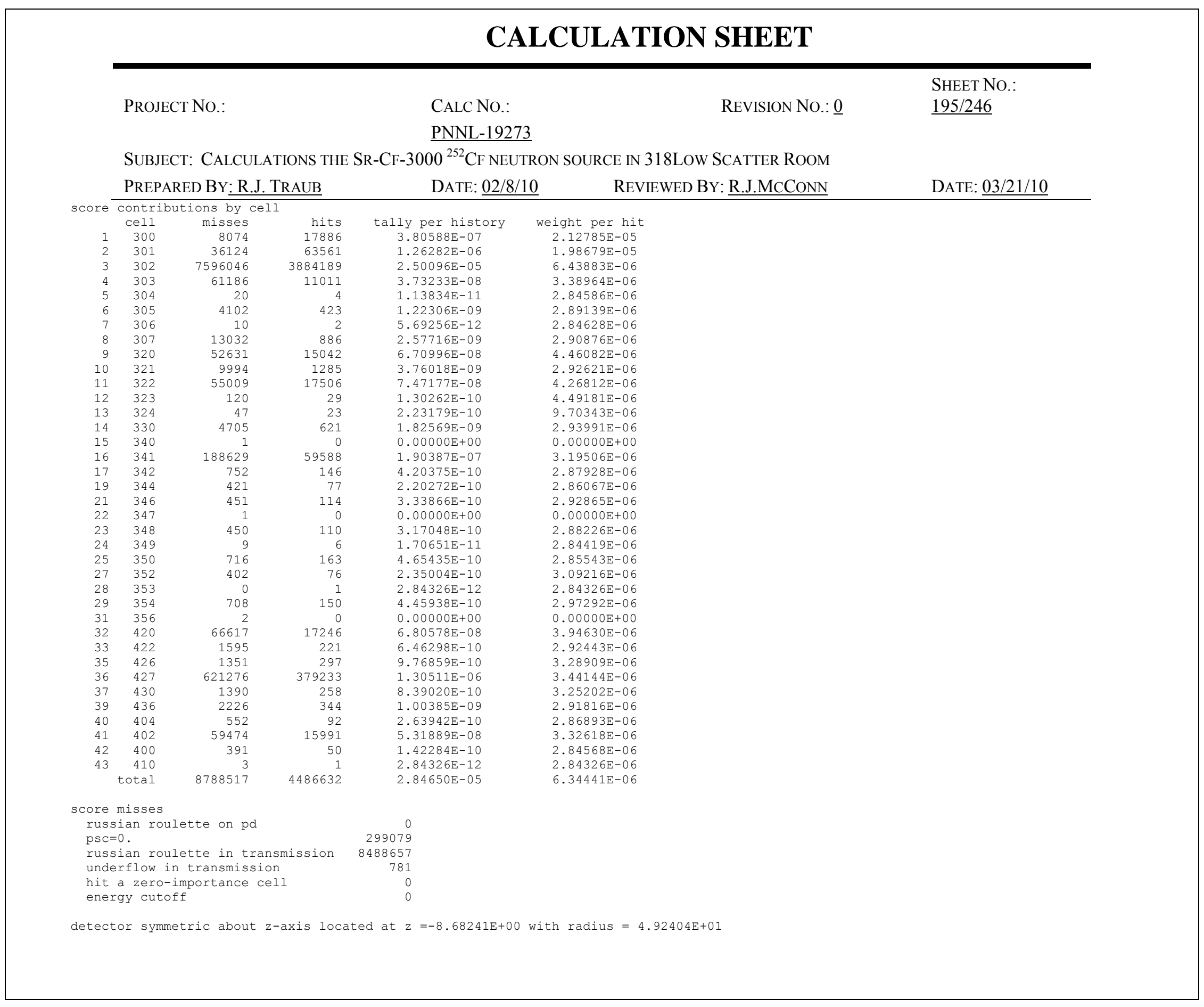




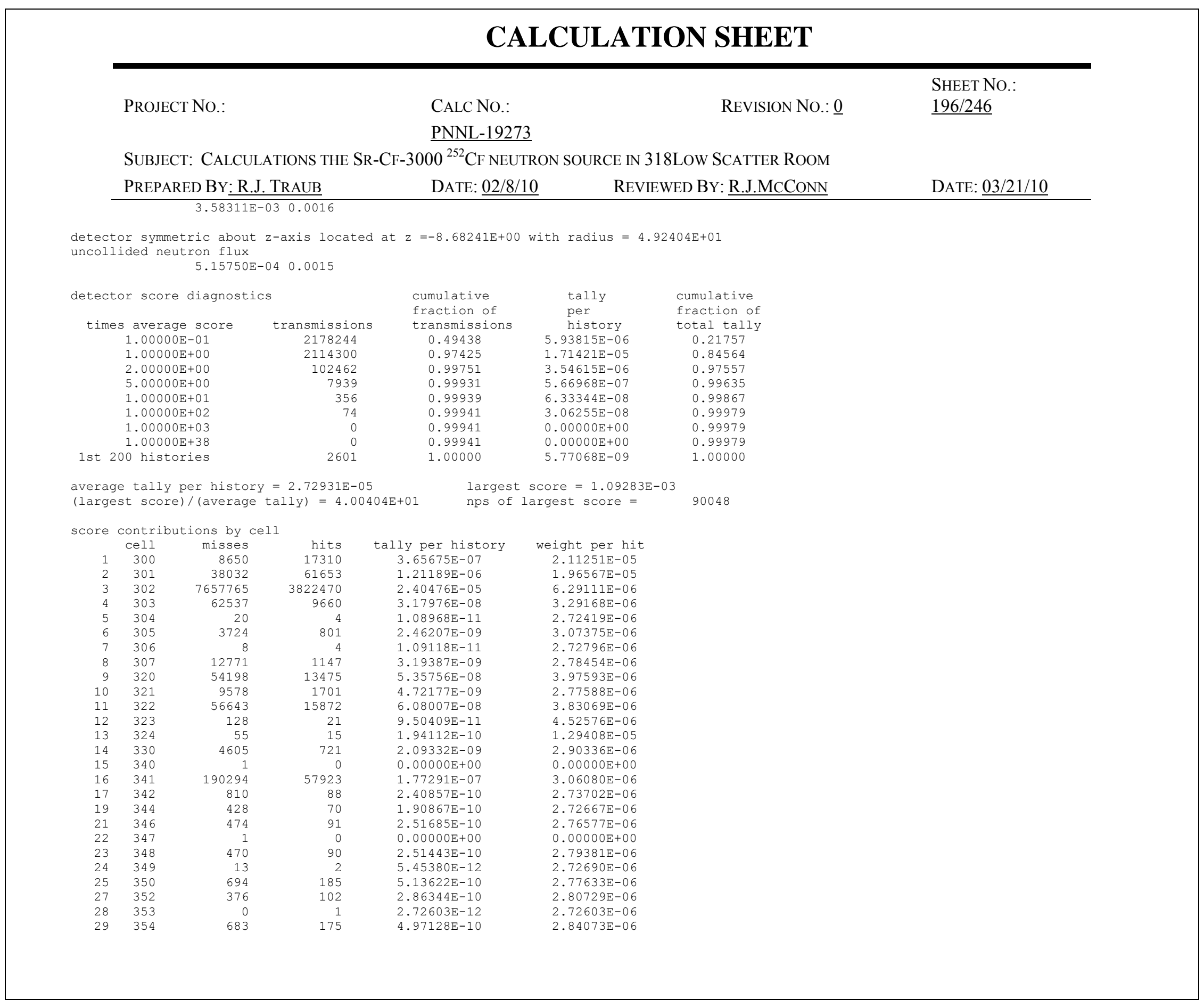




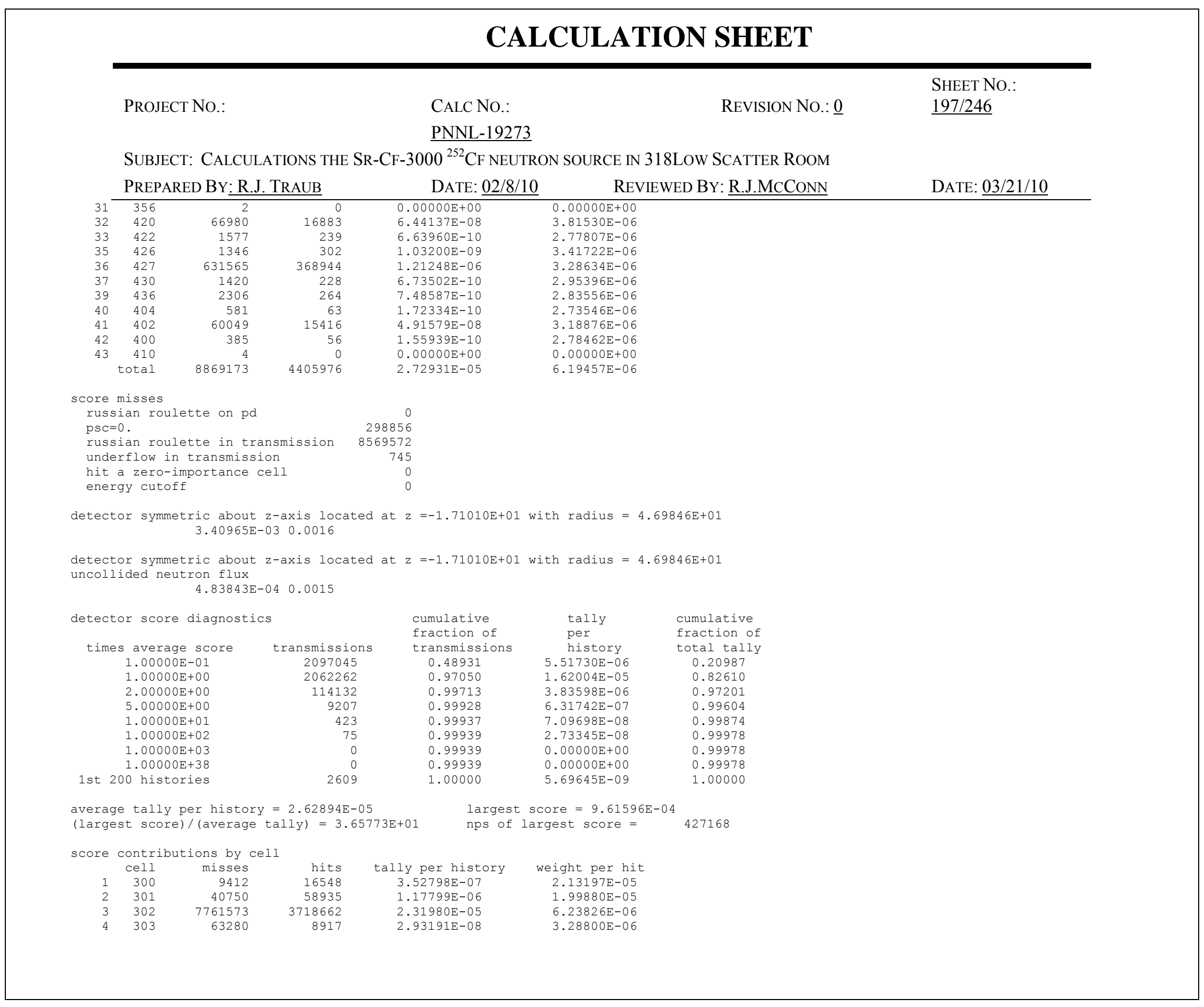




\begin{tabular}{|c|c|c|c|c|c|c|c|}
\hline \multicolumn{8}{|c|}{ CALCULATION SHEET } \\
\hline \multicolumn{4}{|c|}{ Project No.: } & \multicolumn{2}{|l|}{$\begin{array}{l}\text { CALC NO.: } \\
\text { PNNL-19273 }\end{array}$} & REVISION No.: $\underline{0}$ & $\begin{array}{l}\text { SHEET NO.: } \\
\underline{199 / 246}\end{array}$ \\
\hline \multicolumn{8}{|c|}{ 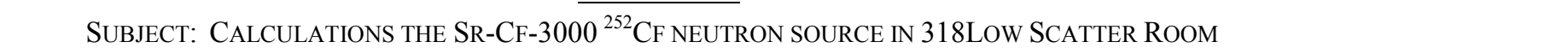 } \\
\hline \multicolumn{4}{|c|}{ PREPARED BY: R.J. TRAUB } & \multicolumn{2}{|c|}{ DATE: $\underline{02 / 8 / 10} \quad$ REVI } & WED BY: R.J.MCCONN & \multirow[t]{2}{*}{ DATE: $\underline{03 / 21 / 10}$} \\
\hline \multicolumn{4}{|c|}{ detector score diagnostics } & $\begin{array}{l}\text { cumulative } \\
\text { fraction of }\end{array}$ & $\begin{array}{l}\text { tally } \\
\text { per }\end{array}$ & $\begin{array}{l}\text { cumulative } \\
\text { fraction of }\end{array}$ & \\
\hline \multirow{2}{*}{\multicolumn{3}{|c|}{$\begin{array}{c}\text { times average score } \\
1.00000 \mathrm{E}-01\end{array}$}} & transmissions & transmissions & history & total tally & \\
\hline & & & $\begin{array}{l}1999804 \\
1992802\end{array}$ & $\begin{array}{l}0.48353 \\
0.96536\end{array}$ & $\begin{array}{l}5.09010 \mathrm{E}-06 \\
1.52348 \mathrm{E}-05\end{array}$ & $\begin{array}{l}0.20014 \\
0.7916\end{array}$ & \\
\hline \multicolumn{3}{|c|}{$\begin{array}{l}1.00000 \mathrm{E}+00 \\
2.00000 \mathrm{E}+00\end{array}$} & 128536 & 0.99644 & $4.23724 \mathrm{E}-06$ & 0.96576 & \\
\hline \multirow{2}{*}{\multicolumn{3}{|c|}{$5.00000 \mathrm{E}+00$}} & 11608 & 0.99925 & $7.59387 \mathrm{E}-07$ & 0.99562 & \\
\hline \multirow{2}{*}{\multicolumn{3}{|c|}{$\begin{array}{l}1.00000 \mathrm{E}+01 \\
1.0000 \mathrm{E}+02\end{array}$}} & 441 & 0.99936 & $7.40342 \mathrm{E}-08$ & 0.99853 & \\
\hline & & & $\begin{array}{r}72 \\
1\end{array}$ & 0.99937 & $2.95564 \mathrm{E}-08$ & 0.99969 & \\
\hline \multicolumn{3}{|c|}{$\begin{array}{l}1.00000 \mathrm{E}+03 \\
1.00000 \mathrm{E}+38\end{array}$} & $\begin{array}{l}1 \\
0\end{array}$ & $\begin{array}{l}0.99937 \\
0.99937\end{array}$ & $\begin{array}{l}2.99322 \mathrm{E}-09 \\
0.00000 \mathrm{E}+00\end{array}$ & $\begin{array}{l}0.99981 \\
0.99981\end{array}$ & \\
\hline \multicolumn{3}{|c|}{ 1st 200 histories } & 2594 & 1.00000 & $\begin{array}{l}0.00000 \mathrm{E}+00 \\
4.86017 \mathrm{E}-09\end{array}$ & $\begin{array}{l}0.99981 \\
1.00000\end{array}$ & \\
\hline \multicolumn{5}{|c|}{$\begin{array}{l}\text { average tally per history }=2.54330 \mathrm{E}-05 \\
(\text { largest score }) /(\text { average tally })=1.17690 \mathrm{E}+02\end{array}$} & $\begin{array}{l}\text { score }=2.99322 \mathrm{E} \\
\text { argest score }=\end{array}$ & 03333516 & \\
\hline \multicolumn{8}{|c|}{ score contributions by cell } \\
\hline & cell & misses & hits & & weight per hit & & \\
\hline 1 & & 10477 & 15483 & $3.40224 \mathrm{E}-07$ & $2.19741 \mathrm{E}-05$ & & \\
\hline $\begin{array}{l}2 \\
3\end{array}$ & $\begin{array}{l}301 \\
302\end{array}$ & $\begin{array}{r}44117 \\
789816\end{array}$ & $\begin{array}{r}55568 \\
3581319\end{array}$ & $1.14455 \mathrm{E}-06$ & 2.05973E-05 & & \\
\hline 4 & 302 & $\begin{array}{r}836916 \\
63613\end{array}$ & $\begin{array}{r}8581319 \\
8584\end{array}$ & $\begin{array}{l}2.243 / \mathrm{E}-05 \\
2.81030 \mathrm{E}-08\end{array}$ & $\begin{array}{l}6.2745 \mathrm{E}-06 \\
3.27388 \mathrm{E}-06\end{array}$ & & \\
\hline 5 & 304 & 20 & 4 & $1.01709 \mathrm{E}-11$ & $2.54272 \mathrm{E}-06$ & & \\
\hline 6 & 305 & 2733 & 1792 & $8.48170 \mathrm{E}-09$ & $4.73309 \mathrm{E}-06$ & & \\
\hline 7 & 306 & 5 & 7 & 3.32583E-11 & $4.75119 \mathrm{E}-06$ & & \\
\hline 8 & 307 & $\begin{array}{l}11626 \\
5576\end{array}$ & 2292 & $6.37621 \mathrm{E}-09$ & $2.78194 \mathrm{E}-06$ & & \\
\hline 9 & $\begin{array}{l}320 \\
321\end{array}$ & $\begin{array}{r}55768 \\
894\end{array}$ & $\begin{array}{r}11905 \\
2985\end{array}$ & $3.76171 \mathrm{E}-08$ & $3.15977 \mathrm{E}-06$ & & \\
\hline $\begin{array}{l}10 \\
11\end{array}$ & $\begin{array}{l}321 \\
322\end{array}$ & $\begin{array}{r}8294 \\
5896\end{array}$ & $\begin{array}{r}2985 \\
14019\end{array}$ & $\begin{array}{l}8.54837 \mathrm{E}-09 \\
4.3566 \mathrm{E}-08\end{array}$ & $\begin{array}{l}2.86378 \mathrm{E}-06 \\
3.10697 \mathrm{E}-06\end{array}$ & & \\
\hline $\begin{array}{l}11 \\
12\end{array}$ & 323 & $\begin{array}{r}5496 \\
132\end{array}$ & $\begin{array}{r}14019 \\
17\end{array}$ & $\begin{array}{l}4.33566 \mathrm{E}-08 \\
4.33094 \mathrm{E}-11\end{array}$ & $\begin{array}{l}3.10697 \mathrm{E}-06 \\
2.54761 \mathrm{E}-06\end{array}$ & & \\
\hline 13 & 324 & 63 & 7 & $4.14731 \mathrm{E}-11$ & $5.92473 \mathrm{E}-06$ & & \\
\hline 14 & 330 & 4275 & 1051 & $2.90585 \mathrm{E}-09$ & $2.76484 \mathrm{E}-06$ & & \\
\hline 15 & 340 & 1 & 0 & $0.00000 \mathrm{E}+00$ & $0.00000 \mathrm{E}+00$ & & \\
\hline 16 & 341 & 192637 & 55580 & $1.65031 \mathrm{E}-07$ & $2.96925 \mathrm{E}-06$ & & \\
\hline 17 & 342 & 844 & 54 & 1.38638E-10 & $2.56737 \mathrm{E}-06$ & & \\
\hline 19 & 344 & 453 & 45 & 1.14591E-10 & $2.54646 \mathrm{E}-06$ & & \\
\hline 21 & $\begin{array}{l}346 \\
347\end{array}$ & 517 & 48 & $\begin{array}{l}1.24058 \mathrm{E}-10 \\
0\end{array}$ & $2.58454 \mathrm{E}-06$ & & \\
\hline $\begin{array}{l}22 \\
23\end{array}$ & $\begin{array}{l}347 \\
348\end{array}$ & $\begin{array}{r}1 \\
501\end{array}$ & $\begin{array}{r}0 \\
59\end{array}$ & $\begin{array}{l}0.00000 \mathrm{E}+00 \\
1.47803 E-10\end{array}$ & $0.00000 \mathrm{E}+00$ & & \\
\hline 24 & $\begin{array}{l}348 \\
349\end{array}$ & $\begin{array}{r}501 \\
10\end{array}$ & $\begin{array}{r}59 \\
5\end{array}$ & $\begin{array}{l}1.47803 \mathrm{E}-10 \\
1.59667 \mathrm{E}-11\end{array}$ & $\begin{array}{l}2.20514 \mathrm{E}-06 \\
3.19333 \mathrm{E}-06\end{array}$ & & \\
\hline 25 & 350 & 619 & 260 & $7.98046 \mathrm{E}-10$ & $3.06941 \mathrm{E}-06$ & & \\
\hline 27 & 352 & 319 & 159 & $4.75631 \mathrm{E}-10$ & $2.99139 \mathrm{E}-06$ & & \\
\hline 28 & 353 & 0 & 1 & $2.54470 \mathrm{E}-12$ & $2.54470 \mathrm{E}-06$ & & \\
\hline 29 & 354 & 582 & 276 & 8.34951E-10 & $3.02518 \mathrm{E}-06$ & & \\
\hline 31 & 356 & 1 & 1 & $2.54295 E-12$ & $2.54295 \mathrm{E}-06$ & & \\
\hline $\begin{array}{l}32 \\
33\end{array}$ & $\begin{array}{l}420 \\
422\end{array}$ & $\begin{array}{r}67523 \\
1540\end{array}$ & $\begin{array}{l}16340 \\
276\end{array}$ & $6.05108 \mathrm{E}-08$ & $3.70323 \mathrm{E}-06$ & & \\
\hline $\begin{array}{l}33 \\
35\end{array}$ & 422 & 1540 & $\begin{array}{l}276 \\
368\end{array}$ & 7.58494E-10 & $2.74817 \mathrm{E}-06$ & & \\
\hline $\begin{array}{l}35 \\
36\end{array}$ & 426 & $\begin{array}{r}1280 \\
648747\end{array}$ & $\begin{array}{r}368 \\
351762\end{array}$ & $\begin{array}{l}1.47230 \mathrm{E}-09 \\
\end{array}$ & $4.00081 \mathrm{E}-06$ & & \\
\hline $\begin{array}{l}36 \\
37\end{array}$ & $\begin{array}{l}427 \\
430\end{array}$ & $\begin{array}{r}648747 \\
1475\end{array}$ & $\begin{array}{r}351762 \\
173\end{array}$ & $\begin{array}{l}1.07143 \mathrm{E}-06 \\
4.48936 \mathrm{E}-10\end{array}$ & $\begin{array}{l}3.04590 \mathrm{E}-06 \\
2.59500 \mathrm{E}-06\end{array}$ & & \\
\hline
\end{tabular}




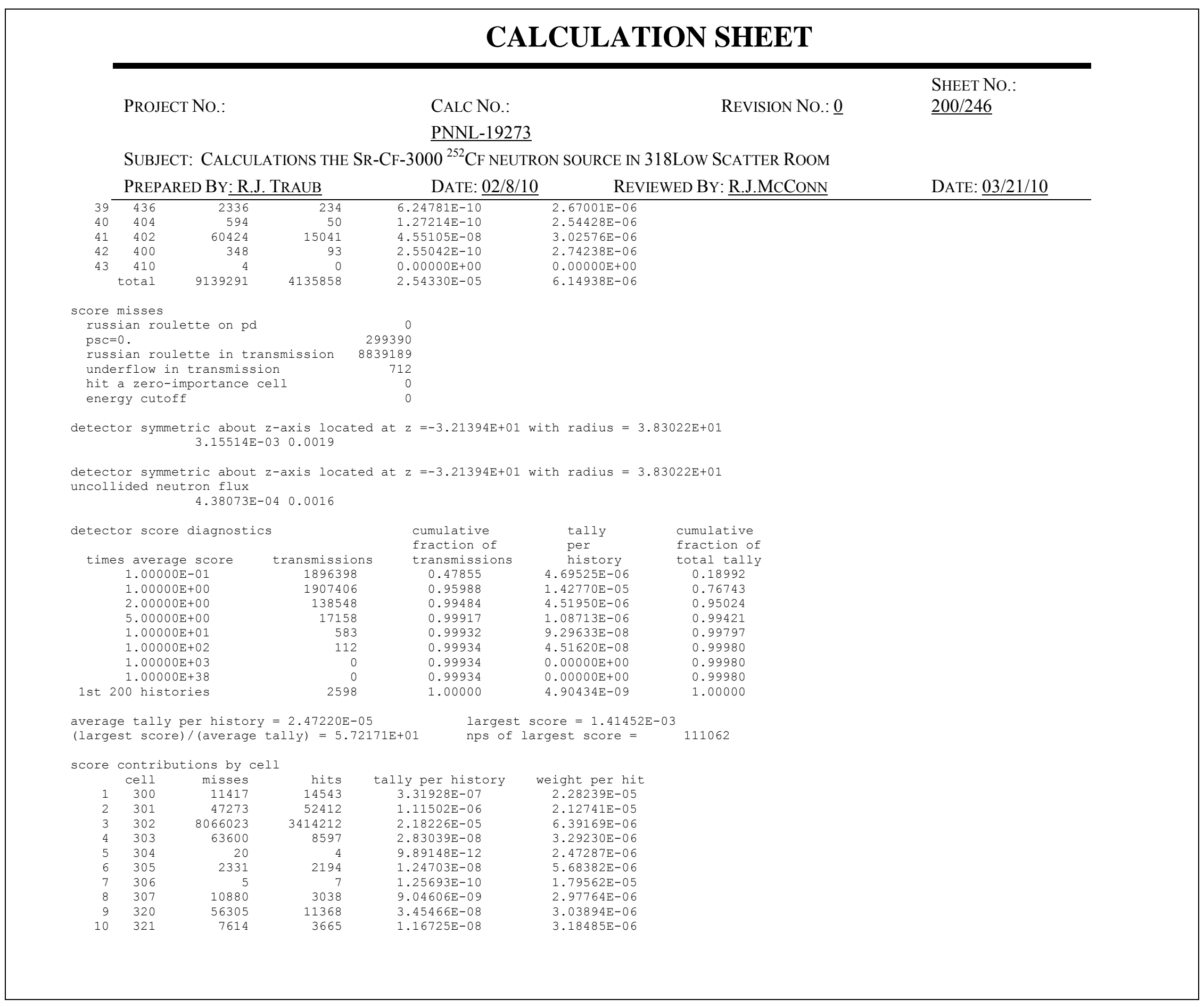




\begin{tabular}{|c|c|c|c|c|c|c|c|}
\hline \multicolumn{8}{|c|}{ CALCULATION SHEET } \\
\hline & PROJE & No.: & & $\begin{array}{l}\text { CALC NO.: } \\
\text { PNNL-19273 }\end{array}$ & & REVISION NO.: $\underline{0}$ & $\begin{array}{l}\text { SHEET NO.: } \\
\underline{201 / 246}\end{array}$ \\
\hline & \multicolumn{7}{|c|}{ SubJECT: CAlCULATIONS THE SR-CF- $3000{ }^{252}$ CF NEUTRON SOURCE IN 318LOW SCATTER ROOM } \\
\hline & \multicolumn{3}{|c|}{ PREPARED BY: R.J. TRAUB } & DATE: $\underline{02 / 8 / 10}$ & \multicolumn{2}{|c|}{ REVIEWED BY: R.J.MCCONN } & \multirow[t]{2}{*}{ DATE: $\underline{03 / 21 / 10}$} \\
\hline 11 & 322 & 588 & 13638 & $4.13373 \mathrm{E}-08$ & $3.03104 \mathrm{E}-06$ & & \\
\hline 12 & 323 & 12 & 23 & $5.75330 \mathrm{E}-11$ & $2.50144 \mathrm{E}-06$ & & \\
\hline 13 & 324 & & 1 & $2.47697 \mathrm{E}-12$ & $2.47697 \mathrm{E}-06$ & & \\
\hline 14 & 330 & 405 & 1269 & 3.85183E-09 & $3.03532 \mathrm{E}-06$ & & \\
\hline 15 & 340 & 1929 & 0 & $0.00000 \mathrm{E}+00$ & $0.00000 \mathrm{E}+00$ & & \\
\hline 16 & 341 & 1929 & 55286 & $\begin{array}{l}1.66169 \mathrm{E}-07 \\
0\end{array}$ & $\begin{array}{l}3.00563 \mathrm{E}-06 \\
2.4756 \mathrm{E}-06\end{array}$ & & \\
\hline $\begin{array}{l}17 \\
19\end{array}$ & $\begin{array}{l}342 \\
344\end{array}$ & $\begin{array}{l}85 \\
4\end{array}$ & $\begin{array}{l}40 \\
28\end{array}$ & 9.90254E-11 & $\begin{array}{l}2.47564 \mathrm{E}-06 \\
2.55802 \mathrm{E}-06\end{array}$ & & \\
\hline 21 & 346 & 52 & 44 & $1.08912 \mathrm{E}-10$ & $2.47527 \mathrm{E}-06$ & & \\
\hline 22 & 347 & 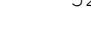 & $\begin{array}{r}44 \\
0\end{array}$ & $0.00000 \mathrm{E}+00$ & $0.00000 \mathrm{E}+00$ & & \\
\hline 23 & 348 & 50 & 52 & $1.29364 \mathrm{E}-10$ & $2.48777 \mathrm{E}-06$ & & \\
\hline 24 & 349 & & 5 & $1.26782 \mathrm{E}-11$ & $2.53564 \mathrm{E}-06$ & & \\
\hline 25 & 350 & 5 & 302 & $1.00374 \mathrm{E}-09$ & $3.32364 \mathrm{E}-06$ & & \\
\hline 27 & 352 & 32 & 157 & $5.00168 \mathrm{E}-10$ & $3.18578 \mathrm{E}-06$ & & \\
\hline 28 & 353 & 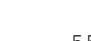 & 0 & $0.00000 E+00$ & $0.00000 \mathrm{E}+00$ & & \\
\hline 29 & 354 & 55 & 307 & 1.08415E-09 & $3.53142 \mathrm{E}-06$ & & \\
\hline 31 & 356 & c7o & 0 & $0.00000 \mathrm{E}+00$ & $0.00000 \mathrm{E}+00$ & & \\
\hline $\begin{array}{l}32 \\
33\end{array}$ & $\begin{array}{l}420 \\
422\end{array}$ & $\begin{array}{l}670 \\
140\end{array}$ & $\begin{array}{r}16800 \\
410\end{array}$ & $6.23909 \mathrm{E}-08$ & $3.71375 \mathrm{E}-06$ & & \\
\hline $\begin{array}{l}33 \\
35\end{array}$ & $\begin{array}{l}422 \\
426\end{array}$ & $\begin{array}{l}14 \mathrm{C} \\
11 \mathrm{C}\end{array}$ & $\begin{array}{l}410 \\
486\end{array}$ & $\begin{array}{l}1.18756 \mathrm{E}-09 \\
1.97261 \mathrm{E}-09\end{array}$ & $\begin{array}{l}2.88186 \mathrm{E}-06 \\
4.0586 \mathrm{E}-06\end{array}$ & & \\
\hline 36 & 427 & 6524 & 348102 & $1.02870 \mathrm{E}-06$ & $2.95517 \mathrm{E}-06$ & & \\
\hline 37 & 430 & 148 & 160 & $4.03906 \mathrm{E}-10$ & $2.52441 \mathrm{E}-06$ & & \\
\hline 39 & 436 & 23 & 201 & $5.27534 \mathrm{E}-10$ & $2.62455 \mathrm{E}-06$ & & \\
\hline 40 & 404 & 5 & 54 & 1.33645E-10 & $2.47490 \mathrm{E}-06$ & & \\
\hline 41 & 402 & 6018 & 15282 & $4.62179 \mathrm{E}-08$ & $3.02433 E-06$ & & \\
\hline & & 32 & 116 & $2.98554 \mathrm{E}-10$ & $2.57374 \mathrm{E}-06$ & & \\
\hline 43 & $\begin{array}{r}410 \\
\text { total }\end{array}$ & 93123 & $\begin{array}{r}0 \\
3962803\end{array}$ & $\begin{array}{l}0.00000 \mathrm{E}+00 \\
2.47220 \mathrm{E}-05\end{array}$ & $\begin{array}{l}0.00000 \mathrm{E}+00 \\
6.23850 \mathrm{E}-06\end{array}$ & & \\
\hline \multirow{2}{*}{\multicolumn{8}{|c|}{$\begin{array}{l}\text { score misses } \\
\text { russian roulette on pd }\end{array}$}} \\
\hline & & & & & & & \\
\hline \multirow{2}{*}{\multicolumn{8}{|c|}{$\begin{array}{lr} & 299667 \\
\text { psc }=0 . & \text {. } \\
\text { russian roulette in transmission } & 9011987\end{array}$}} \\
\hline \multirow{2}{*}{\multicolumn{8}{|c|}{$\begin{array}{l}\text { russian roulette in transmission } 901987 \\
\text { underflow in transmission }\end{array}$}} \\
\hline & & & & & & & \\
\hline \multicolumn{8}{|c|}{$\begin{array}{ll}\text { hit a zero-importance cell } & 0 \\
\text { energy cutoff } & 0\end{array}$} \\
\hline \multicolumn{8}{|c|}{$\begin{array}{c}\text { detector symmetric about } z \text {-axis located at } z=-3.83022 \mathrm{E}+01 \text { with radius }=3.21394 \mathrm{E}+01 \\
3.08214 \mathrm{E}-03 \quad 0.0021\end{array}$} \\
\hline \multirow{2}{*}{\multicolumn{8}{|c|}{$\begin{array}{l}\text { detector symmetric about } z \text {-axis located at } z=-3.83022 \mathrm{E}+01 \text { with radius }=3.21394 \mathrm{E}+01 \\
\text { uncollided neutron flux } \\
4.26178 \mathrm{E}-040.0016\end{array}$}} \\
\hline & & & & & & & \\
\hline \multirow{2}{*}{\multicolumn{4}{|c|}{ detector score diagnostics }} & cumulative & tally & cumulative & \\
\hline & & & & fraction of & & fraction of & \\
\hline time & es avera & score & transmissions & $\begin{array}{l}\text { transmissions } \\
0.47571\end{array}$ & $\begin{array}{c}\text { history } \\
435185 \mathrm{~F}-06\end{array}$ & total tally & \\
\hline & 1.0000 & & 1805323 & 0.95363 & $\begin{array}{l}1.33117 \mathrm{E}-05 \\
1.03\end{array}$ & 0.73033 & \\
\hline & 2.0000 & & 145692 & 0.99220 & $4.71457 \mathrm{E}-06$ & 0.92526 & \\
\hline
\end{tabular}




\begin{tabular}{|c|c|c|c|c|c|c|c|}
\hline \multicolumn{8}{|c|}{ CALCULATION SHEET } \\
\hline \multicolumn{3}{|c|}{ PROJECT No.: } & & & & REVISION NO.: $\underline{0}$ & $\begin{array}{l}\text { SHEET NO.: } \\
\underline{202 / 246}\end{array}$ \\
\hline \multicolumn{8}{|c|}{ SubJeCt: CAlCUlations THE SR-CF-3000 $\frac{\text { PNNL-19273 }}{{ }^{252} \text { CF NEUTRC }}$} \\
\hline \multicolumn{4}{|c|}{ PREPARED BY: R.J. TRAUB } & \multicolumn{2}{|c|}{ DATE: $\underline{02 / 8 / 10} \quad \mathrm{RE}$} & REVIEWED BY: R.J.MCCONN & \multirow[t]{6}{*}{ DATE: $\underline{03 / 21 / 10}$} \\
\hline & 5.000 & +00 & 25984 & 0.99908 & $1.64135 \mathrm{E}-06$ & 0.99312 & \\
\hline & 1.000 & & 760 & 0.99928 & $1.16968 \mathrm{E}-07$ & 0.99796 & \\
\hline & 1.000 & & 125 & 0.99931 & $4.40625 \mathrm{E}-08$ & 0.99978 & \\
\hline & $\begin{array}{l}1.000 \\
1.000\end{array}$ & $\begin{array}{l}8+03 \\
+38\end{array}$ & $\begin{array}{l}0 \\
0 \\
0\end{array}-x-a r$ & $\begin{array}{l}0.99931 \\
0.99931\end{array}$ & $\begin{array}{l}0.00000 \mathrm{E}+00 \\
0.00000 \mathrm{E}+00\end{array}$ & 0.99978 & \\
\hline 1 st 2 & 00 his & ies & 2600 & $\begin{array}{l}0.99931 \\
1.00000\end{array}$ & $\begin{array}{l}0.00000 E+00 \\
5.26357 E-09\end{array}$ & $\begin{array}{l}0.99978 \\
1.00000\end{array}$ & \\
\hline \multicolumn{7}{|c|}{$\begin{array}{ll}\text { average tally per history }=2.41857 \mathrm{E}-05 & \text { lar } \\
(\text { largest score }) /(\text { average tally })=4.00641 \mathrm{E}+01 & \text { nps }\end{array}$} & \\
\hline \multicolumn{7}{|c|}{ score contributions by cell } & \\
\hline & cell & misses & hits & tally per history & weight per hit & & \\
\hline 1 & 300 & 12294 & 13666 & $3.25897 \mathrm{E}-07$ & $2.38473 \mathrm{E}-05$ & & \\
\hline 2 & & 50541 & 49144 & $1.09395 \mathrm{E}-06$ & $2.22602 \mathrm{E}-05$ & & \\
\hline 3 & $\begin{array}{l}302 \\
303\end{array}$ & 8249336 & $\begin{array}{r}3230899 \\
8453\end{array}$ & $\begin{array}{l}2.13031 \mathrm{E}-05 \\
2.8795 \mathrm{E}-08\end{array}$ & $\begin{array}{l}6.59355 \mathrm{E}-06 \\
3.3976 \mathrm{E}-06\end{array}$ & & \\
\hline $\begin{array}{l}4 \\
5\end{array}$ & 304 & $\begin{array}{r}63744 \\
21\end{array}$ & $\begin{array}{r}8453 \\
3\end{array}$ & $\begin{array}{l}2.87195 \mathrm{E}-08 \\
7.2605 \mathrm{E}-12\end{array}$ & $\begin{array}{l}3.39756 \mathrm{E}-06 \\
2.42020 \mathrm{E}-06\end{array}$ & & \\
\hline 6 & 305 & 1983 & 2542 & $1.97374 \mathrm{E}-08$ & $7.76450 \mathrm{E}-06$ & & \\
\hline 7 & 306 & $\begin{array}{r}1983 \\
4\end{array}$ & $\begin{array}{r}2542 \\
8\end{array}$ & $8.98611 \mathrm{E}-11$ & $1.12326 \mathrm{E}-05$ & & \\
\hline 8 & 307 & 10229 & 3689 & $1.30100 \mathrm{E}-08$ & $3.52671 \mathrm{E}-06$ & & \\
\hline 9 & 320 & 56359 & 11314 & $3.51978 \mathrm{E}-08$ & $3.11100 \mathrm{E}-06$ & & \\
\hline 10 & 321 & 7126 & 4153 & $1.55482 E-08$ & $3.74385 E-06$ & & \\
\hline 11 & 322 & 58888 & 13627 & $4.29139 \mathrm{E}-08$ & $3.14918 \mathrm{E}-06$ & & \\
\hline 12 & 323 & 130 & 19 & $4.81409 \mathrm{E}-11$ & $2.53373 \mathrm{E}-06$ & & \\
\hline 13 & 324 & 69 & 1 & $2.42097 \mathrm{E}-12$ & $2.42097 \mathrm{E}-06$ & & \\
\hline 14 & 330 & 3872 & 1454 & 5.48637E-09 & $3.77329 \mathrm{E}-06$ & & \\
\hline 15 & $\begin{array}{l}340 \\
341\end{array}$ & 192317 & 5500 & $0.00000 \mathrm{E}+00$ & $0.00000 \mathrm{E}+00$ & & \\
\hline $\begin{array}{l}16 \\
17\end{array}$ & $\begin{array}{l}341 \\
342\end{array}$ & $\begin{array}{r}192317 \\
850\end{array}$ & $\begin{array}{r}55900 \\
48\end{array}$ & $\begin{array}{l}1.73926 \mathrm{E}-07 \\
1.16231 \mathrm{E}-10\end{array}$ & $\begin{array}{l}3.11138 \mathrm{E}-06 \\
2.4214 \mathrm{E}-06\end{array}$ & & \\
\hline 19 & 344 & 467 & 31 & $\begin{array}{l}1.16231 \mathrm{E}-10 \\
7.65504 \mathrm{E}-11\end{array}$ & $\begin{array}{l}2.42147 \mathrm{E}-06 \\
2.46937 \mathrm{E}-06\end{array}$ & & \\
\hline 21 & 346 & 526 & 39 & $9.65145 \mathrm{E}-11$ & $2.47473 \mathrm{E}-06$ & & \\
\hline 22 & 347 & 1 & 0 & $0.00000 \mathrm{E}+00$ & $0.00000 \mathrm{E}+00$ & & \\
\hline 23 & 348 & 517 & 43 & $1.27655 \mathrm{E}-10$ & $2.96872 \mathrm{E}-06$ & & \\
\hline 24 & 349 & 11 & 4 & $1.12727 \mathrm{E}-11$ & $2.81819 \mathrm{E}-06$ & & \\
\hline 25 & 350 & 545 & 334 & $1.29091 \mathrm{E}-09$ & $3.86501 \mathrm{E}-06$ & & \\
\hline 27 & 352 & 307 & 171 & $5.99969 \mathrm{E}-10$ & $3.50859 \mathrm{E}-06$ & & \\
\hline 28 & $\begin{array}{l}353 \\
354\end{array}$ & $\begin{array}{r}0 \\
531\end{array}$ & $\begin{array}{r}1 \\
327\end{array}$ & $2.87844 \mathrm{E}-12$ & $2.87844 \mathrm{E}-06$ & & \\
\hline $\begin{array}{r}29 \\
31\end{array}$ & $\begin{array}{l}354 \\
356\end{array}$ & 531 & 327 & $\begin{array}{l}1.30857 \mathrm{E}-09 \\
0.0000 \mathrm{E}+00\end{array}$ & 4.00173E-06 & & \\
\hline $\begin{array}{l}31 \\
32\end{array}$ & $\begin{array}{l}356 \\
420\end{array}$ & $\begin{array}{r}2 \\
66574\end{array}$ & $\begin{array}{r}0 \\
17289\end{array}$ & $\begin{array}{l}0.00000 \mathrm{E}+00 \\
6.72339 \mathrm{E}-08\end{array}$ & $\begin{array}{l}0.00000 E+00 \\
3.88882 E-06\end{array}$ & & \\
\hline 33 & 422 & 1237 & 579 & $\begin{array}{l}6.2339 \mathrm{E}-08 \\
2.23700 \mathrm{E}-09\end{array}$ & $\begin{array}{l}3.88828 \mathrm{E}-06 \\
3.86356 \mathrm{E}-06\end{array}$ & & \\
\hline 35 & 426 & 1055 & 593 & $2.73656 \mathrm{E}-09$ & $4.61477 \mathrm{E}-06$ & & \\
\hline 36 & 427 & 653692 & 346817 & $1.00100 \mathrm{E}-06$ & $2.88625 \mathrm{E}-06$ & & \\
\hline 37 & 430 & 1505 & 143 & $3.84756 \mathrm{E}-10$ & $2.69060 \mathrm{E}-06$ & & \\
\hline 39 & 436 & 2383 & 187 & $4.99073 E-10$ & $2.66884 \mathrm{E}-06$ & & \\
\hline 40 & 404 & 610 & 34 & $8.42192 \mathrm{E}-11$ & $2.47703 \mathrm{E}-06$ & & \\
\hline 41 & 402 & 59676 & 15789 & $\begin{array}{l}4.98334 \mathrm{E}-08 \\
4\end{array}$ & $3.15621 \mathrm{E}-06$ & & \\
\hline \multirow{2}{*}{$\begin{array}{l}42 \\
43\end{array}$} & $\begin{array}{l}400 \\
410\end{array}$ & $\begin{array}{r}291 \\
4\end{array}$ & $\begin{array}{r}150 \\
0\end{array}$ & $\begin{array}{l}4.71620 \mathrm{E}-10 \\
\end{array}$ & $3.14414 \mathrm{E}-06$ & & \\
\hline & $\begin{array}{r}410 \\
\text { total }\end{array}$ & $\begin{array}{r}4 \\
9497698\end{array}$ & $\begin{array}{r}0 \\
3777451\end{array}$ & $\begin{array}{l}0.00000 \mathrm{E}+00 \\
2.41857 \mathrm{E}-05\end{array}$ & $\begin{array}{l}0.00000 \mathrm{E}+00 \\
6.40266 \mathrm{E}-06\end{array}$ & & \\
\hline
\end{tabular}




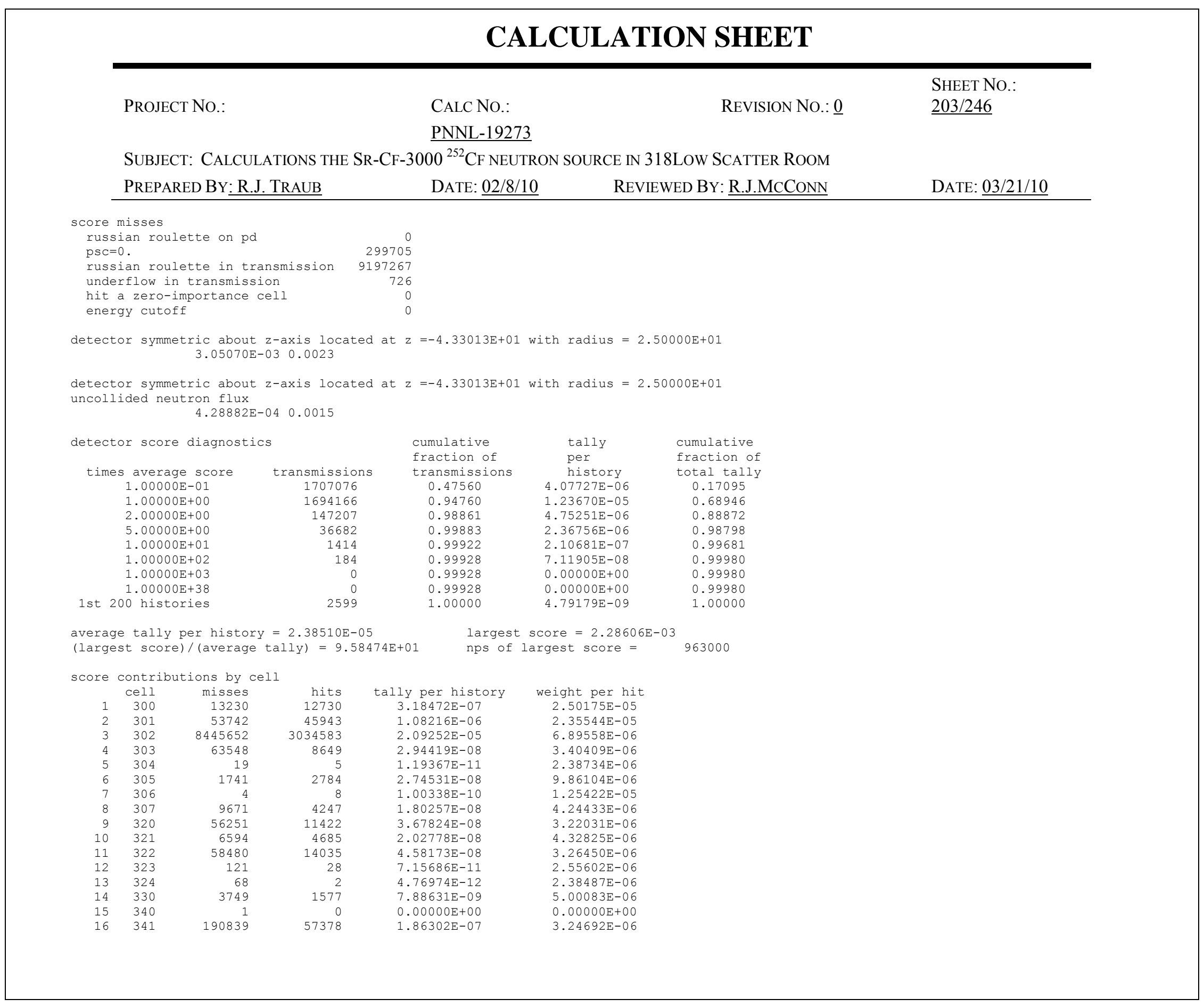




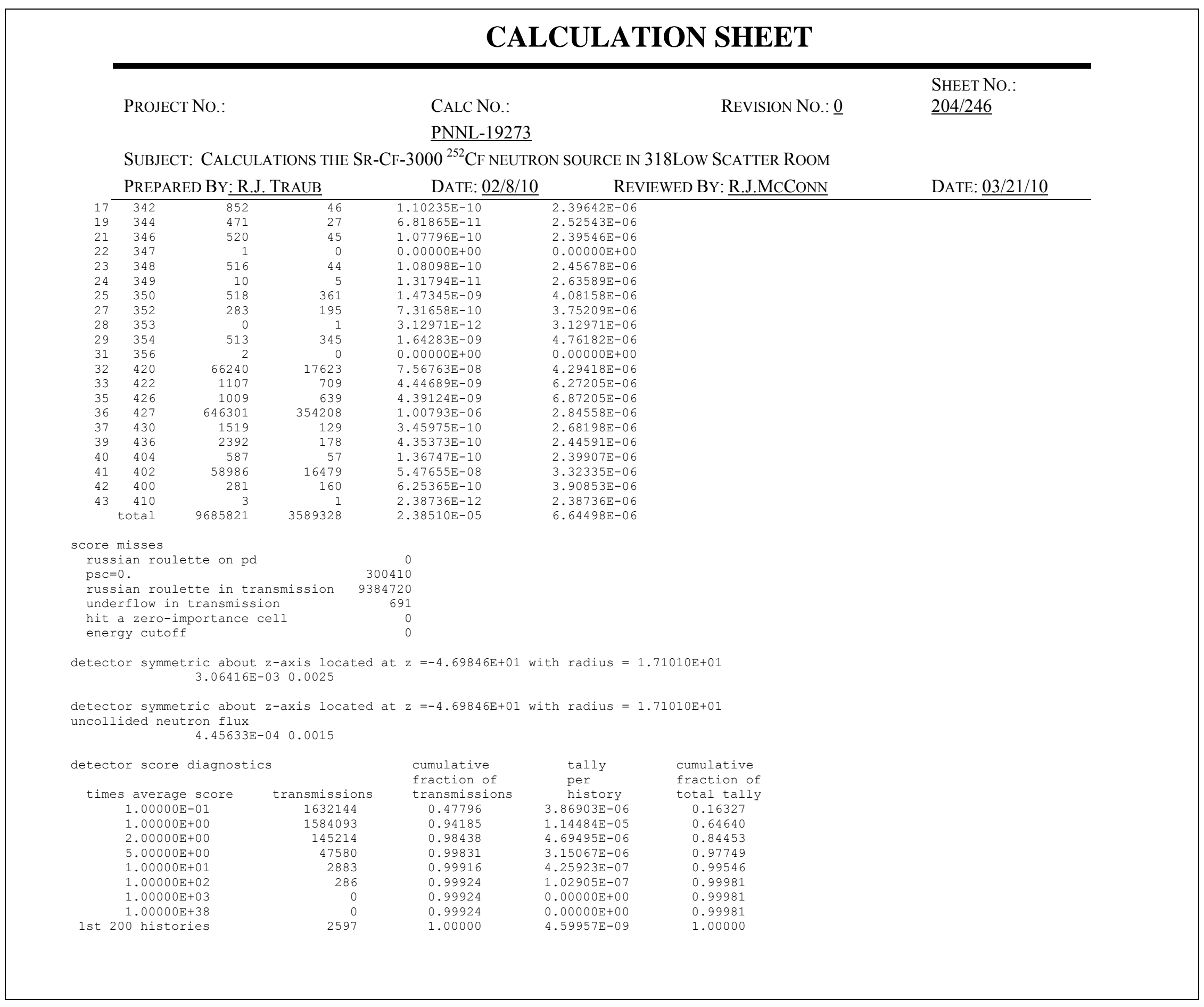




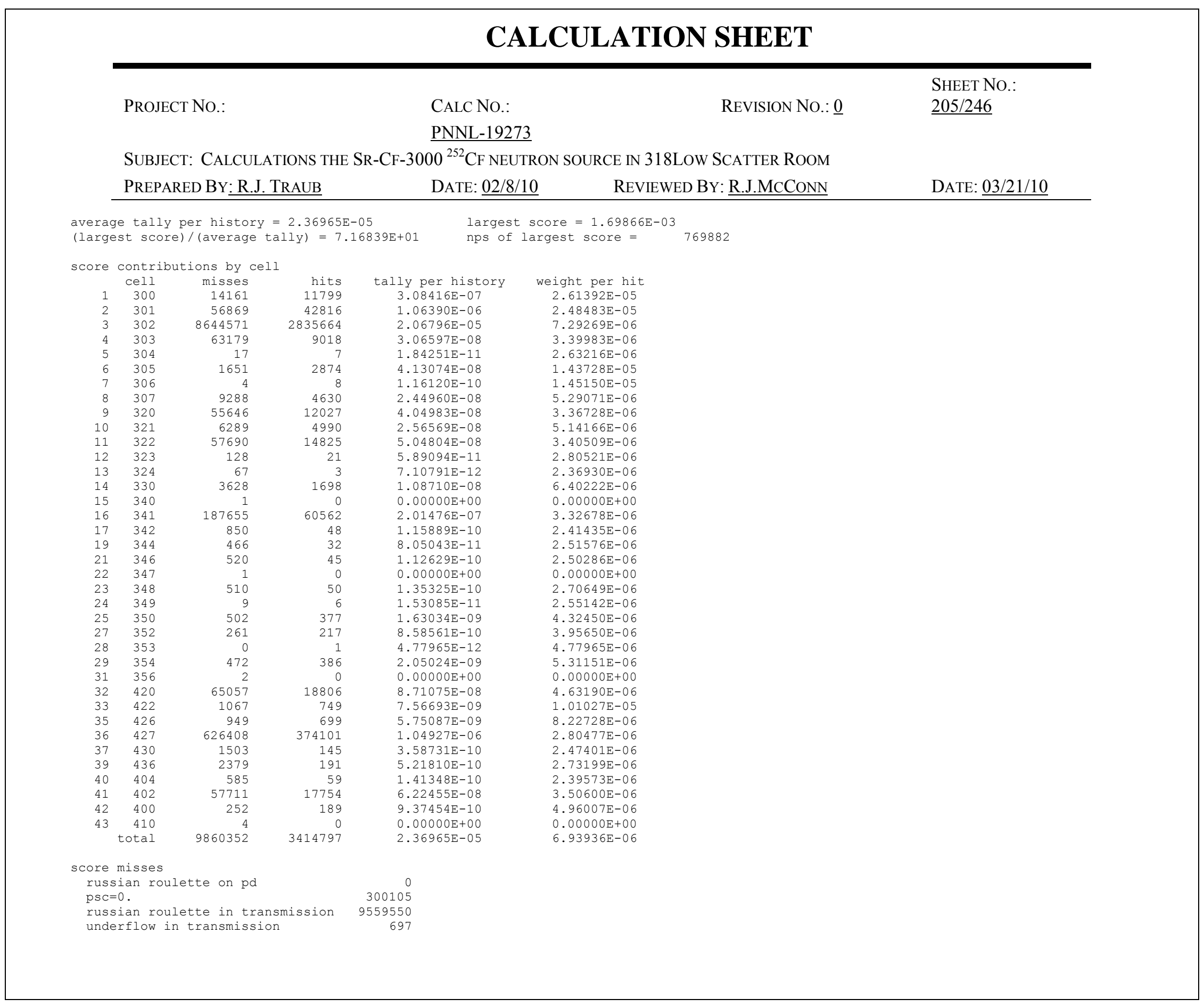




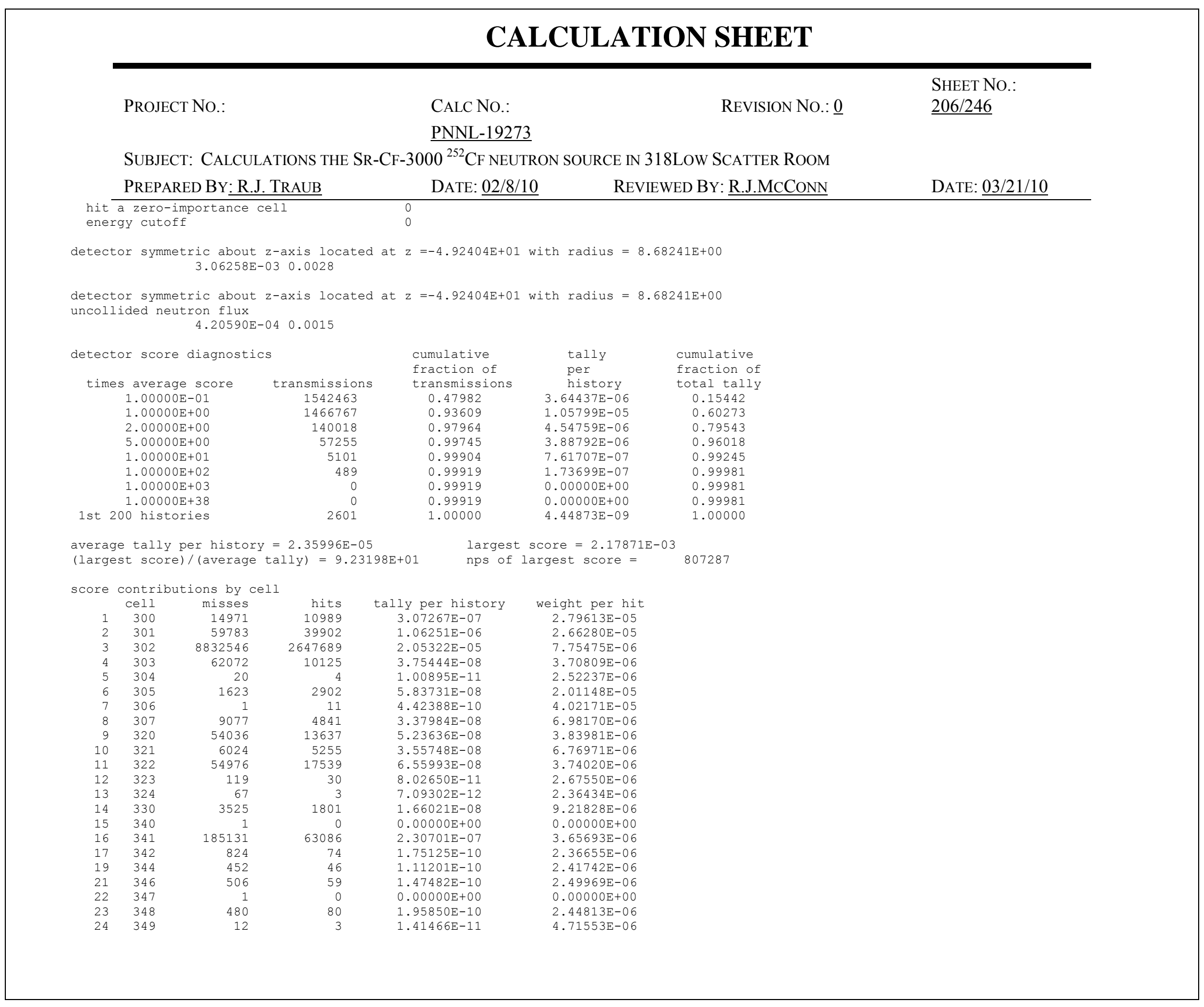




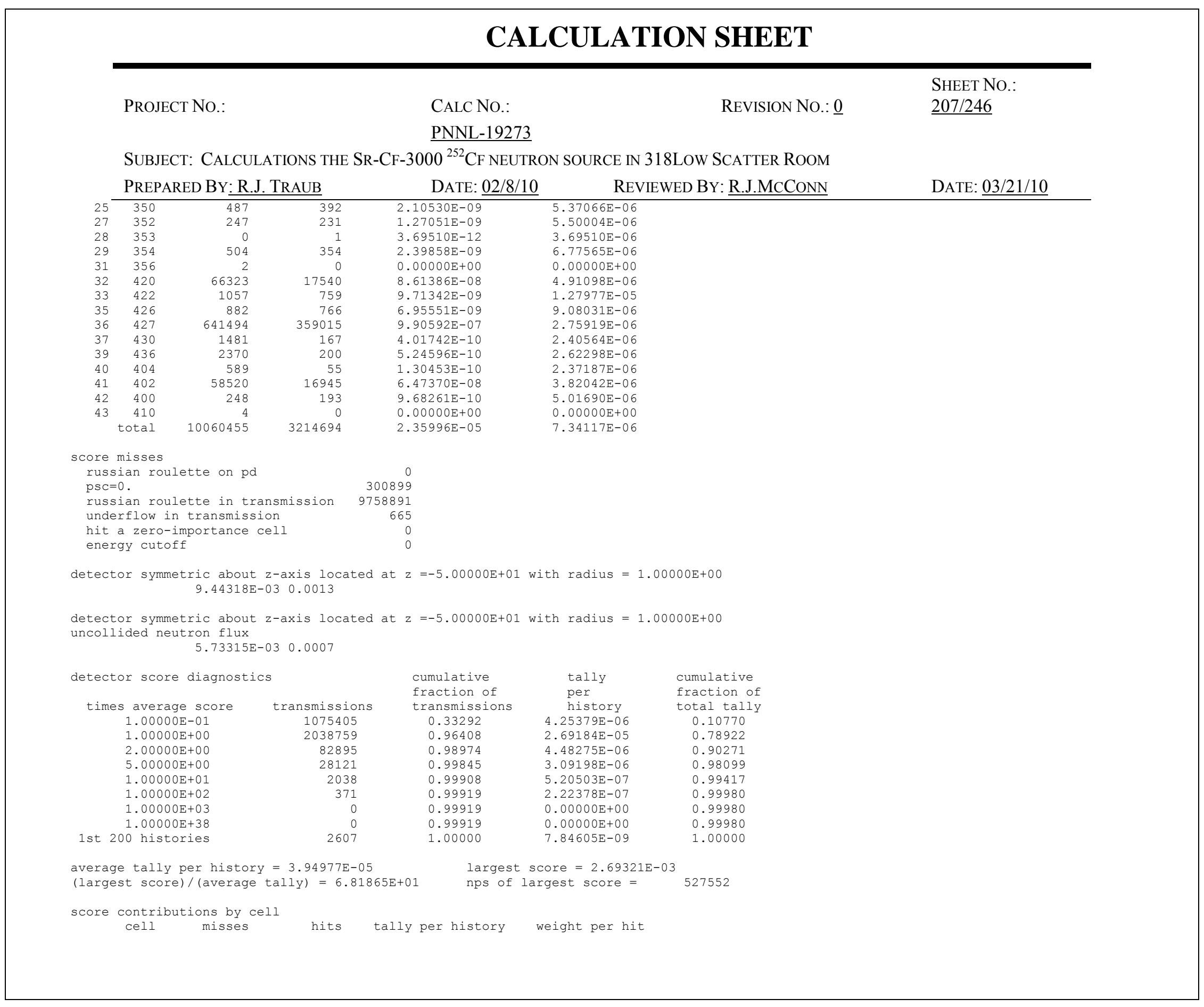




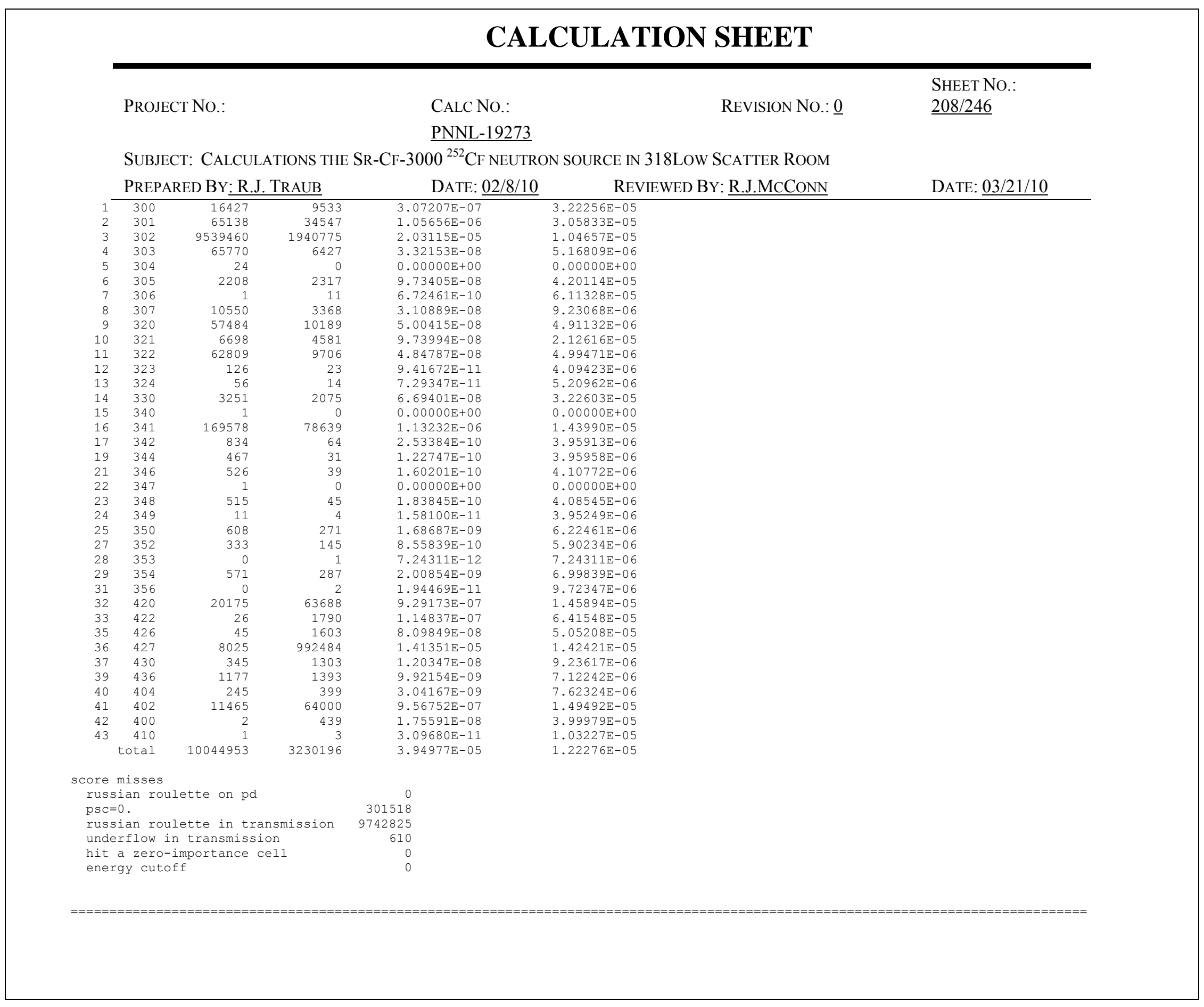




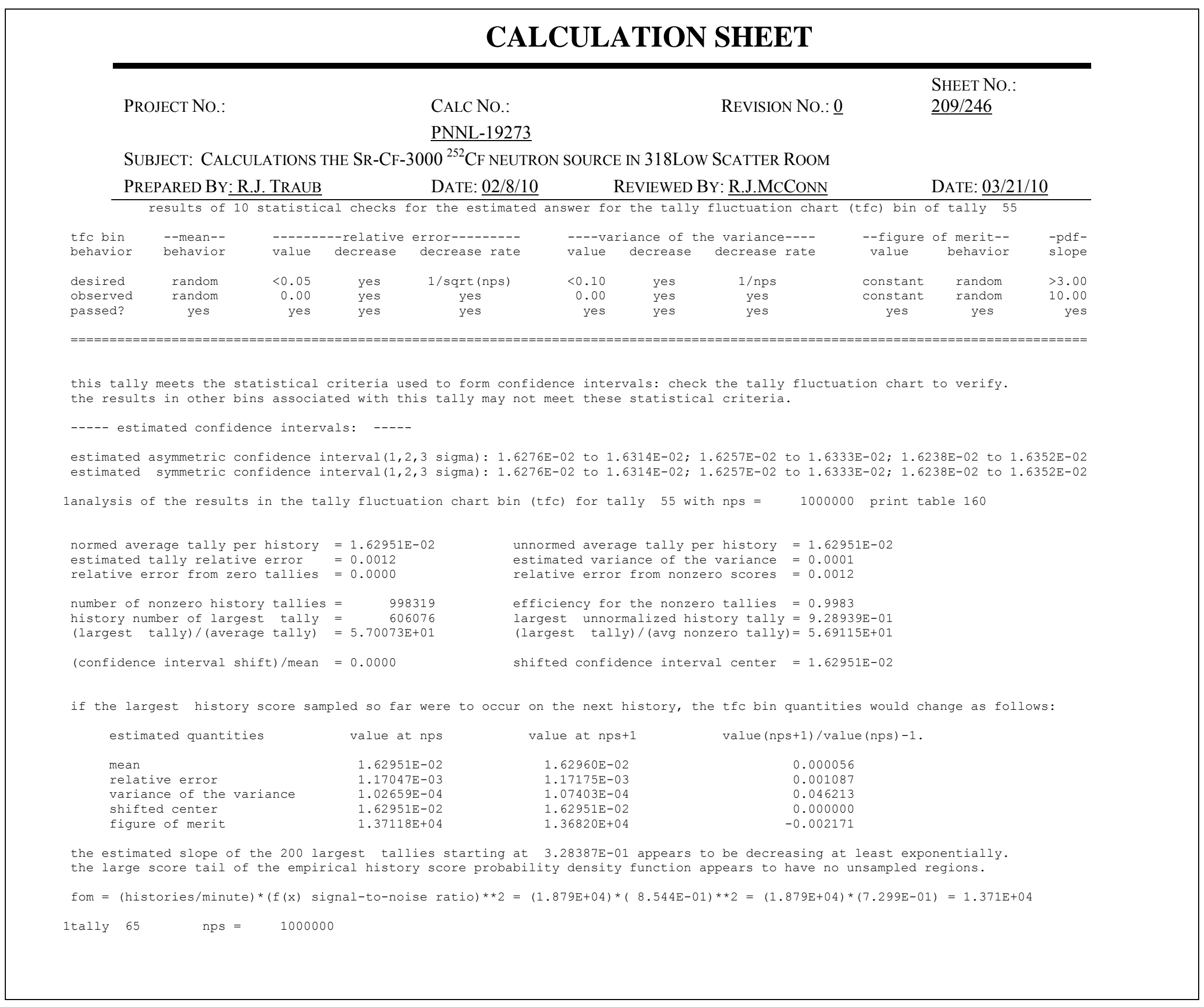




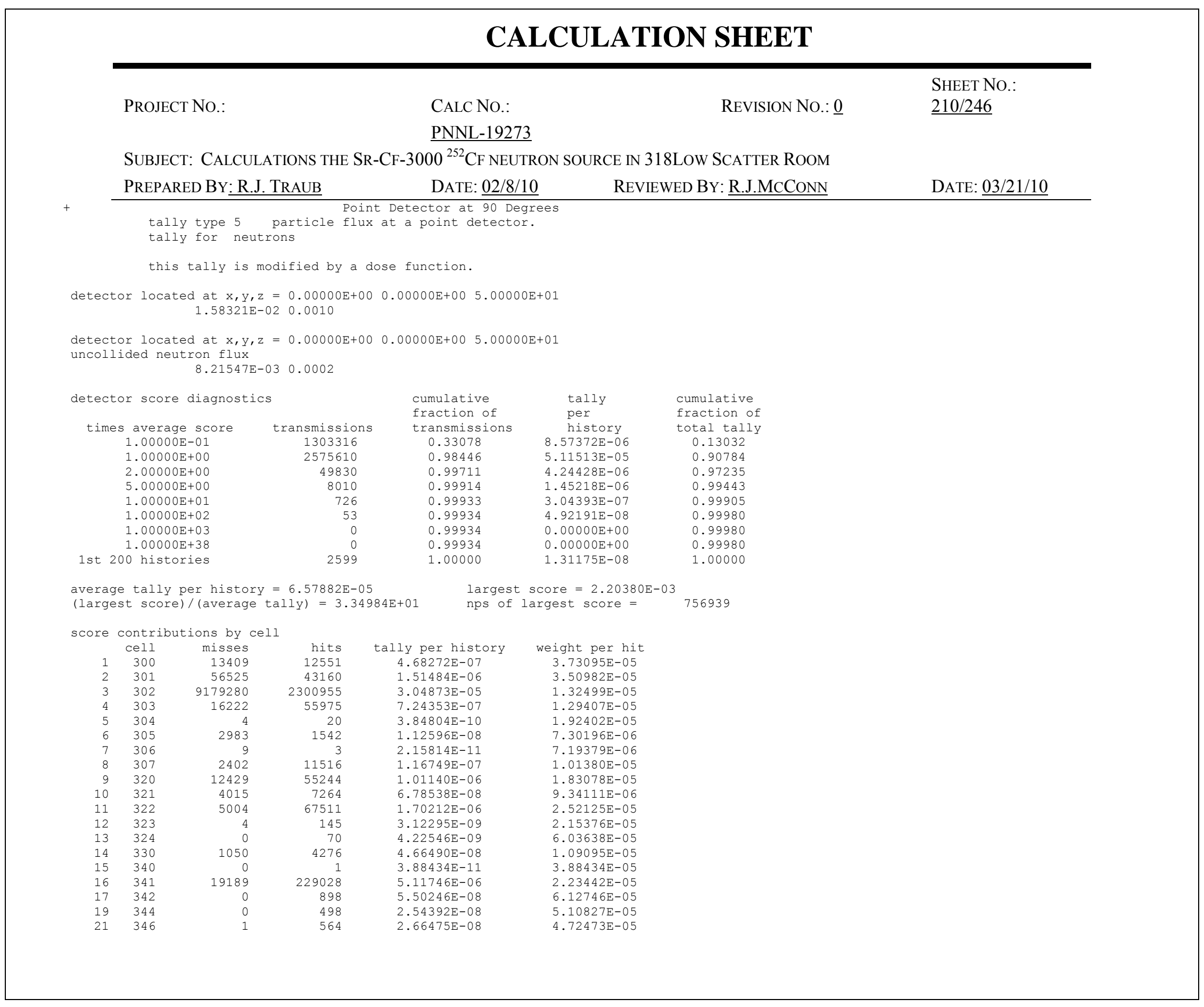




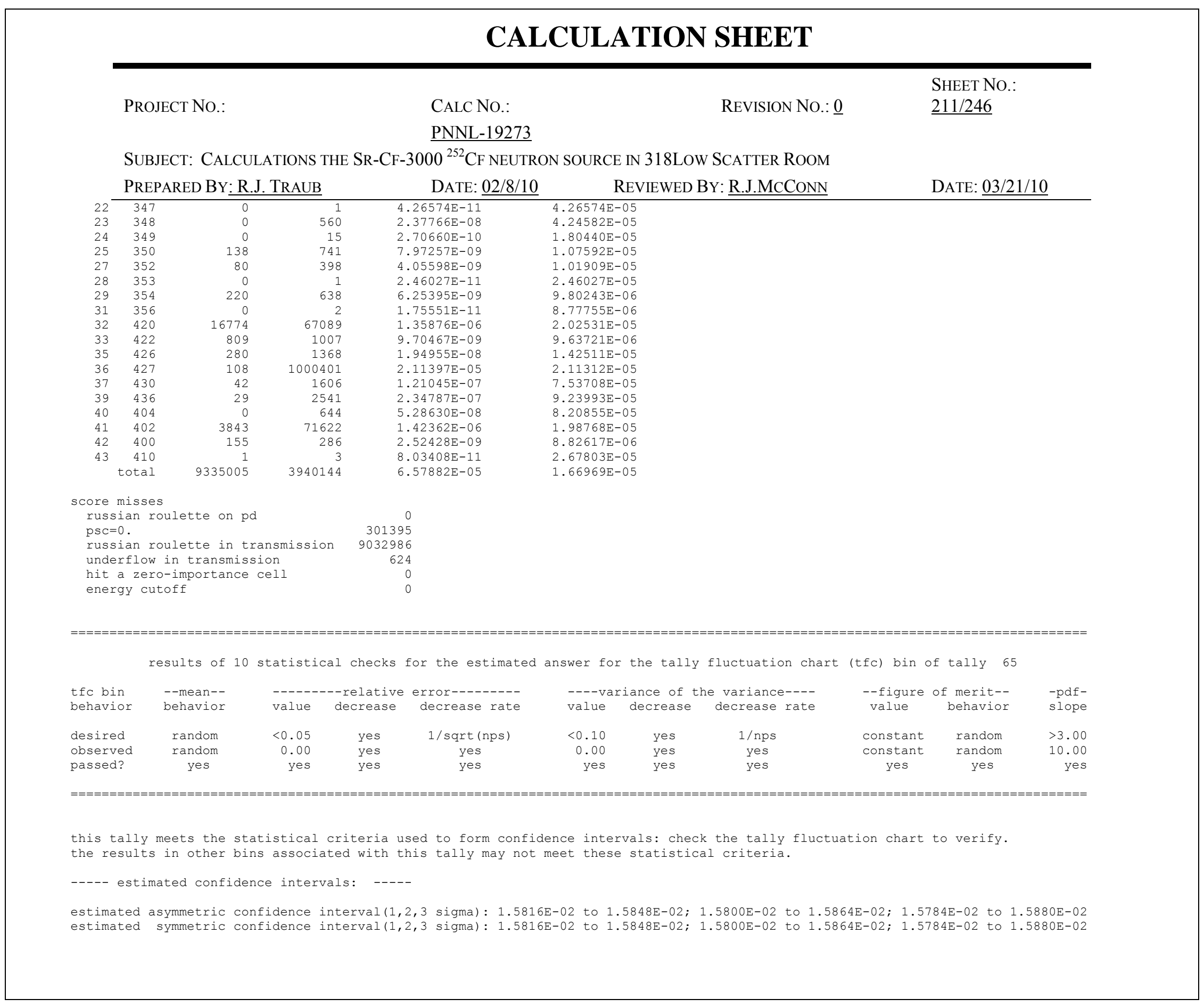




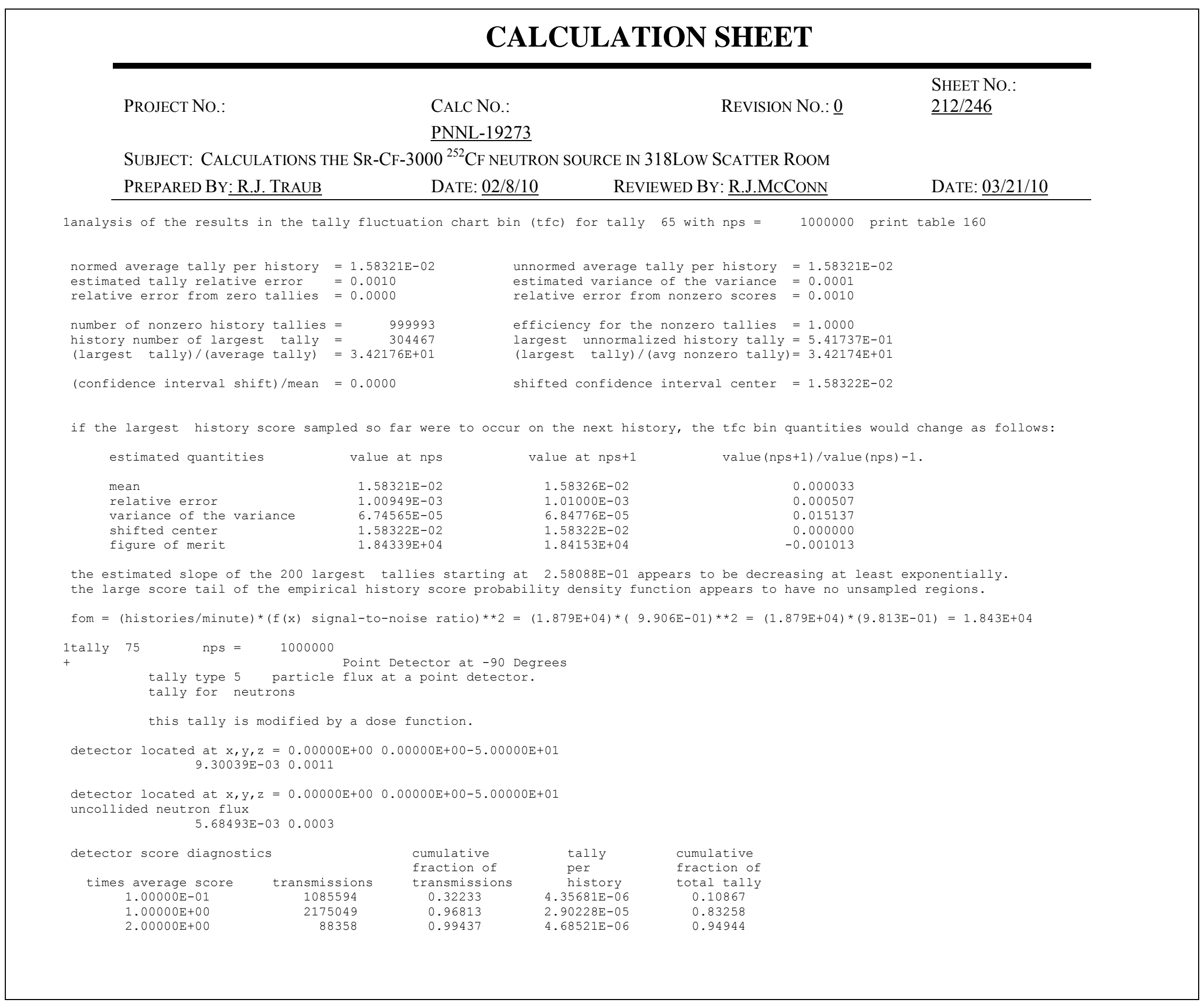




\begin{tabular}{|c|c|c|c|c|c|c|c|}
\hline \multicolumn{8}{|c|}{ CALCULATION SHEET } \\
\hline \multicolumn{3}{|c|}{ PROJECT No.: } & & & & REVISION NO.: $\underline{0}$ & $\begin{array}{l}\text { SHEET NO.: } \\
\underline{213 / 246}\end{array}$ \\
\hline \multicolumn{8}{|c|}{ SuBJeCt: CALCULATIONS THE SR-CF-3000 $\frac{\text { PNNL-19273 }}{{ }^{252} \text { CF NEUTRC }}$} \\
\hline \multicolumn{4}{|c|}{ PREPARED BY: R.J. TRAUB } & \multicolumn{2}{|c|}{ DATE: $\underline{02 / 8 / 10} \quad \mathrm{RI}$} & REVIEWED BY: R.J.MCCONN & \multirow[t]{6}{*}{ DATE: $\underline{03 / 21 / 10}$} \\
\hline & 5.000 & +00 & 15024 & 0.99883 & $1.62370 \mathrm{E}-06$ & 0.98994 & \\
\hline & 1.000 & & 1199 & 0.99918 & $3.15741 \mathrm{E}-07$ & 0.99782 & \\
\hline & 1.000 & & 144 & 0.99923 & 7.93767E-08 & 0.99980 & \\
\hline & $\begin{array}{l}1.000 \\
1.000\end{array}$ & $\begin{array}{ll}+03 \\
+38\end{array}$ & $\begin{array}{l}0 \\
0 \\
0\end{array}-x-a r$ & $\begin{array}{l}0.99923 \\
0.99923\end{array}$ & $\begin{array}{l}0.00000 \mathrm{E}+00 \\
0.00000 \mathrm{E}+00\end{array}$ & 0.99980 & \\
\hline 1 st 2 & 00 his & ties & 2606 & $\begin{array}{l}0.99923 \\
1.00000\end{array}$ & $\begin{array}{l}0.00000 \mathrm{E}+00 \\
8.15061 \mathrm{E}-09\end{array}$ & & \\
\hline \multicolumn{7}{|c|}{$\begin{array}{ll}\text { average tally per history }=4.00918 \mathrm{E}-05 & \text { lar } \\
(\text { largest score }) /(\text { average tally })=5.35787 \mathrm{E}+01 & \text { nps }\end{array}$} & \\
\hline \multicolumn{7}{|c|}{ score contributions by cell } & \\
\hline & cell & misses & hits & tally per history & weight per hit & & \\
\hline 1 & 300 & 16389 & 9571 & $3.08259 \mathrm{E}-07$ & $3.22076 \mathrm{E}-05$ & & \\
\hline 2 & & 64573 & 35112 & $1.05676 \mathrm{E}-06$ & $3.00969 \mathrm{E}-05$ & & \\
\hline 3 & $\begin{array}{l}302 \\
303\end{array}$ & $\begin{array}{r}9459460 \\
6558\end{array}$ & $\begin{array}{r}2020775 \\
6539\end{array}$ & $\begin{array}{l}2.02919 \mathrm{E}-05 \\
3.32784 \mathrm{E}-08\end{array}$ & $\begin{array}{l}1.00416 \mathrm{E}-05 \\
5.0892 \mathrm{E}-06\end{array}$ & & \\
\hline $\begin{array}{l}4 \\
5\end{array}$ & 304 & $\begin{array}{r}65658 \\
21\end{array}$ & $\begin{array}{r}6539 \\
3\end{array}$ & $\begin{array}{l}3.32784 \mathrm{E}-08 \\
1.20363 \mathrm{E}-11\end{array}$ & $\begin{array}{l}5.08922 \mathrm{E}-06 \\
4.01209 \mathrm{E}-06\end{array}$ & & \\
\hline 6 & 305 & 638 & 3887 & $1.08076 \mathrm{E}-07$ & $2.78045 \mathrm{E}-05$ & & \\
\hline 7 & 306 & & 12 & $6.54048 \mathrm{E}-10$ & $5.45040 \mathrm{E}-05$ & & \\
\hline 8 & 307 & 10681 & 3237 & $2.93872 \mathrm{E}-08$ & $9.07854 \mathrm{E}-06$ & & \\
\hline 9 & 320 & 56726 & 10947 & $5.19213 E-08$ & $4.74297 \mathrm{E}-06$ & & \\
\hline 10 & 321 & 6406 & 4873 & $1.00247 E-07$ & $2.05720 \mathrm{E}-05$ & & \\
\hline 11 & 322 & 62647 & 9868 & $4.77700 \mathrm{E}-08$ & $4.84090 \mathrm{E}-06$ & & \\
\hline 12 & 323 & 123 & 26 & $1.05439 \mathrm{E}-10$ & $4.05536 \mathrm{E}-06$ & & \\
\hline 13 & 324 & 51 & 19 & $9.48928 \mathrm{E}-11$ & $4.99436 \mathrm{E}-06$ & & \\
\hline 14 & 330 & 3021 & 2305 & $6.72846 \mathrm{E}-08$ & $2.91907 E-05$ & & \\
\hline 15 & $\begin{array}{l}340 \\
341\end{array}$ & $\begin{array}{r}1 \\
134012\end{array}$ & $\begin{array}{r}0 \\
114205\end{array}$ & $0.00000 \mathrm{E}+00$ & $0.00000 \mathrm{E}+00$ & & \\
\hline $\begin{array}{l}16 \\
17\end{array}$ & $\begin{array}{l}341 \\
342\end{array}$ & $\begin{array}{r}134012 \\
859\end{array}$ & $\begin{array}{r}114205 \\
39\end{array}$ & $\begin{array}{l}1.28128 \mathrm{E}-06 \\
1.5676 \mathrm{E}-10\end{array}$ & $\begin{array}{l}1.12192 \mathrm{E}-05 \\
4.0120 \mathrm{E}-06\end{array}$ & & \\
\hline 19 & 344 & 469 & $\begin{array}{l}39 \\
29\end{array}$ & $\begin{array}{l}1.64676 \mathrm{E}-10 \\
1.16400 \mathrm{E}-10\end{array}$ & $\begin{array}{l}4.0120 \mathrm{E}-06 \\
4.01379 \mathrm{E}-06\end{array}$ & & \\
\hline 21 & 346 & 525 & 40 & $1.63286 \mathrm{E}-10$ & $4.08214 \mathrm{E}-06$ & & \\
\hline 22 & 347 & 1 & 0 & $0.00000 \mathrm{E}+00$ & $0.00000 \mathrm{E}+00$ & & \\
\hline 23 & 348 & 506 & 54 & $2.27569 \mathrm{E}-10$ & $4.21424 \mathrm{E}-06$ & & \\
\hline 24 & 349 & 13 & 2 & $8.01864 \mathrm{E}-12$ & $4.00932 \mathrm{E}-06$ & & \\
\hline 25 & 350 & 582 & 297 & $1.69263 E-09$ & $5.69909 \mathrm{E}-06$ & & \\
\hline 27 & 352 & 317 & 161 & $9.06826 \mathrm{E}-10$ & $5.63246 \mathrm{E}-06$ & & \\
\hline 28 & $\begin{array}{l}353 \\
354\end{array}$ & $\begin{array}{r}0 \\
590\end{array}$ & $\begin{array}{r}1 \\
268\end{array}$ & $5.54737 \mathrm{E}-12$ & $5.54737 \mathrm{E}-06$ & & \\
\hline $\begin{array}{r}29 \\
31\end{array}$ & $\begin{array}{l}354 \\
356\end{array}$ & $\begin{array}{r}590 \\
0\end{array}$ & $\begin{array}{r}268 \\
2\end{array}$ & $\begin{array}{l}2.00772 \mathrm{E}-09 \\
3.34852 \mathrm{E}-11\end{array}$ & $\begin{array}{l}7.49150 \mathrm{E}-06 \\
1\end{array}$ & & \\
\hline $\begin{array}{l}31 \\
32\end{array}$ & $\begin{array}{l}\begin{array}{l}356 \\
420\end{array} \\
\end{array}$ & 17013 & $\begin{array}{r}2 \\
66850\end{array}$ & $\begin{array}{l}3.34852 \mathrm{E}-11 \\
8.95651 \mathrm{E}-07\end{array}$ & $\begin{array}{l}1.67426 \mathrm{E}-05 \\
1.33979 \mathrm{E}-05\end{array}$ & & \\
\hline 33 & 422 & 16 & 1800 & $\begin{array}{l}8.95651 \mathrm{E}-01 \\
1.15153 \mathrm{E}-07\end{array}$ & $\begin{array}{l}1.39736 \mathrm{E}-05 \\
6.397\end{array}$ & & \\
\hline 35 & 426 & 33 & 1615 & $8.32809 \mathrm{E}-08$ & $5.15671 \mathrm{E}-05$ & & \\
\hline 36 & 427 & 1005 & 999504 & $1.45805 \mathrm{E}-05$ & $1.45877 \mathrm{E}-05$ & & \\
\hline 37 & 430 & 271 & 1377 & $1.29950 \mathrm{E}-08$ & $9.43721 \mathrm{E}-06$ & & \\
\hline 39 & 436 & 1069 & 1501 & 9.89398E-09 & $6.59159 \mathrm{E}-06$ & & \\
\hline 40 & 404 & 194 & $\begin{array}{r}450 \\
\end{array}$ & $2.87074 \mathrm{E}-09$ & $6.37943 \mathrm{E}-06$ & & \\
\hline 41 & 402 & 3303 & 72162 & 9.90180E-07 & $1.37216 \mathrm{E}-05$ & & \\
\hline \multirow{2}{*}{$\begin{array}{l}42 \\
43\end{array}$} & $\begin{array}{l}400 \\
410\end{array}$ & 1 & $\begin{array}{r}440 \\
3\end{array}$ & $\begin{array}{l}1.89036 \mathrm{E}-08 \\
4.75814 \mathrm{E}-1\end{array}$ & $4.29626 \mathrm{E}-05$ & & \\
\hline & $\begin{array}{r}410 \\
\text { total }\end{array}$ & $\begin{array}{r}1 \\
9907175\end{array}$ & 3367974 & $\begin{array}{l}4.75814 \mathrm{E}-11 \\
4.00918 \mathrm{E}-05\end{array}$ & $\begin{array}{l}1.58605 \mathrm{E}-05 \\
1.19038 \mathrm{E}-05\end{array}$ & & \\
\hline
\end{tabular}




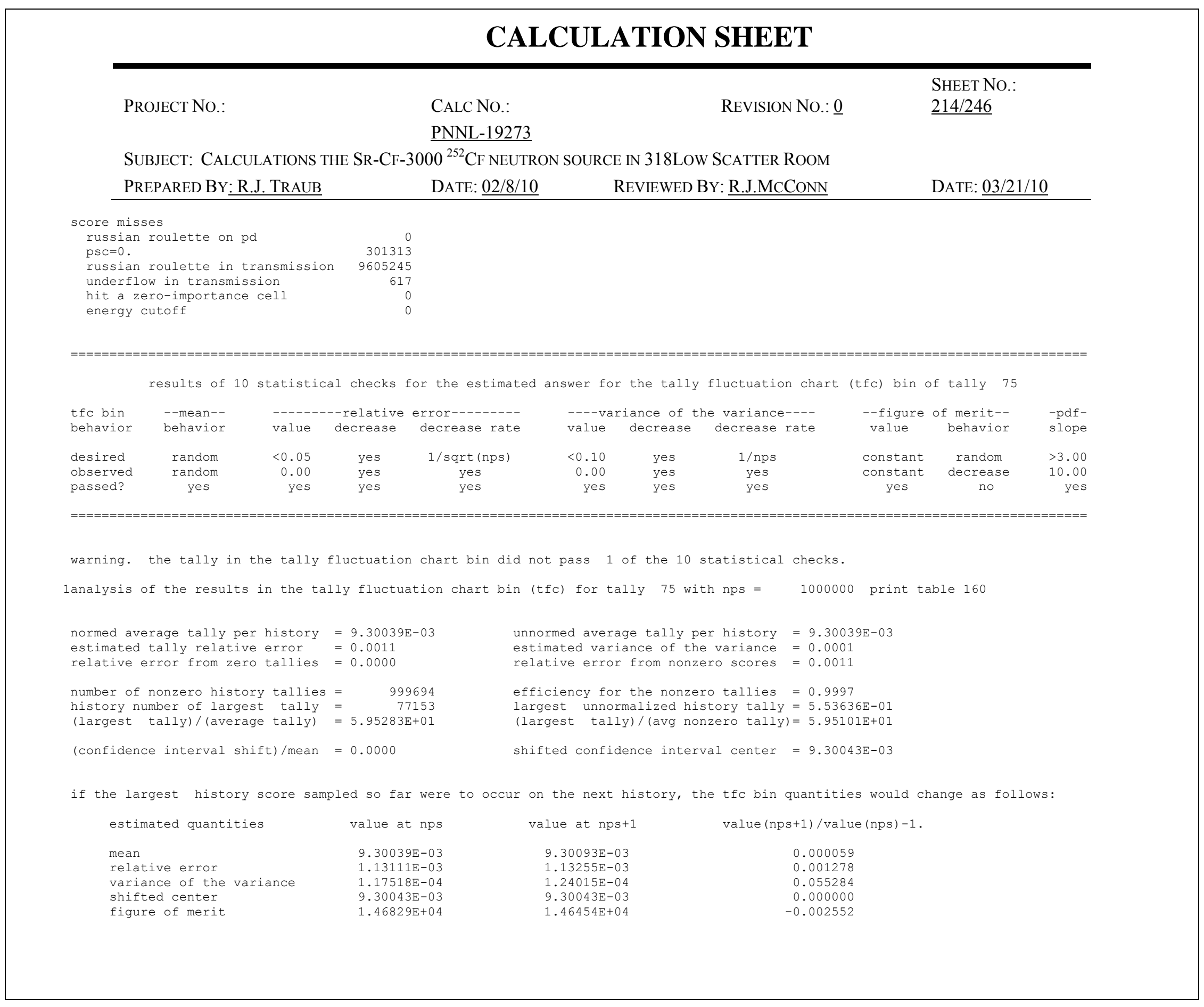




\section{CALCULATION SHEET}

\begin{tabular}{|c|c|c|c|}
\hline PROJECT No.: & $\begin{array}{l}\text { CALC NO.: } \\
\text { PNNL-19273 }\end{array}$ & REVISION NO.: $\underline{0}$ & $\begin{array}{l}\text { SHEET NO.: } \\
\underline{215 / 246}\end{array}$ \\
\hline \multicolumn{4}{|c|}{ SUBJECT: CALCULATIONS THE SR-CF-3000 ${ }^{252}$ CF NEUTRON SOURCE IN 318LOW SCATTER ROOM } \\
\hline PREPARED BY: R.J. TRAUB & DATE: $02 / 8 / 10$ & REVIEWED BY: R.J.MCCONN & DATE: $03 / 21 / 10$ \\
\hline
\end{tabular}

the estimated slope of the 200 largest tallies starting at $1.88931 \mathrm{E}-01$ appears to be decreasing at least exponentially.
the large score tail of the empirical history score probability density function appears to have no unsampled regions.

fom $=($ histories $/$ minute $) *(\mathrm{f}(\mathrm{x})$ signal-to-noise ratio $) * * 2=(1.879 \mathrm{E}+04) *(8.841 \mathrm{E}-01) * * 2=(1.879 \mathrm{E}+04) *(7.816 \mathrm{E}-01)=1.468 \mathrm{E}+04$

lunnormed tally density for tally $75 \quad$ nonzero tally mean $(\mathrm{m})=9.303 \mathrm{E}-03 \mathrm{nps}=1000000$ print table 161

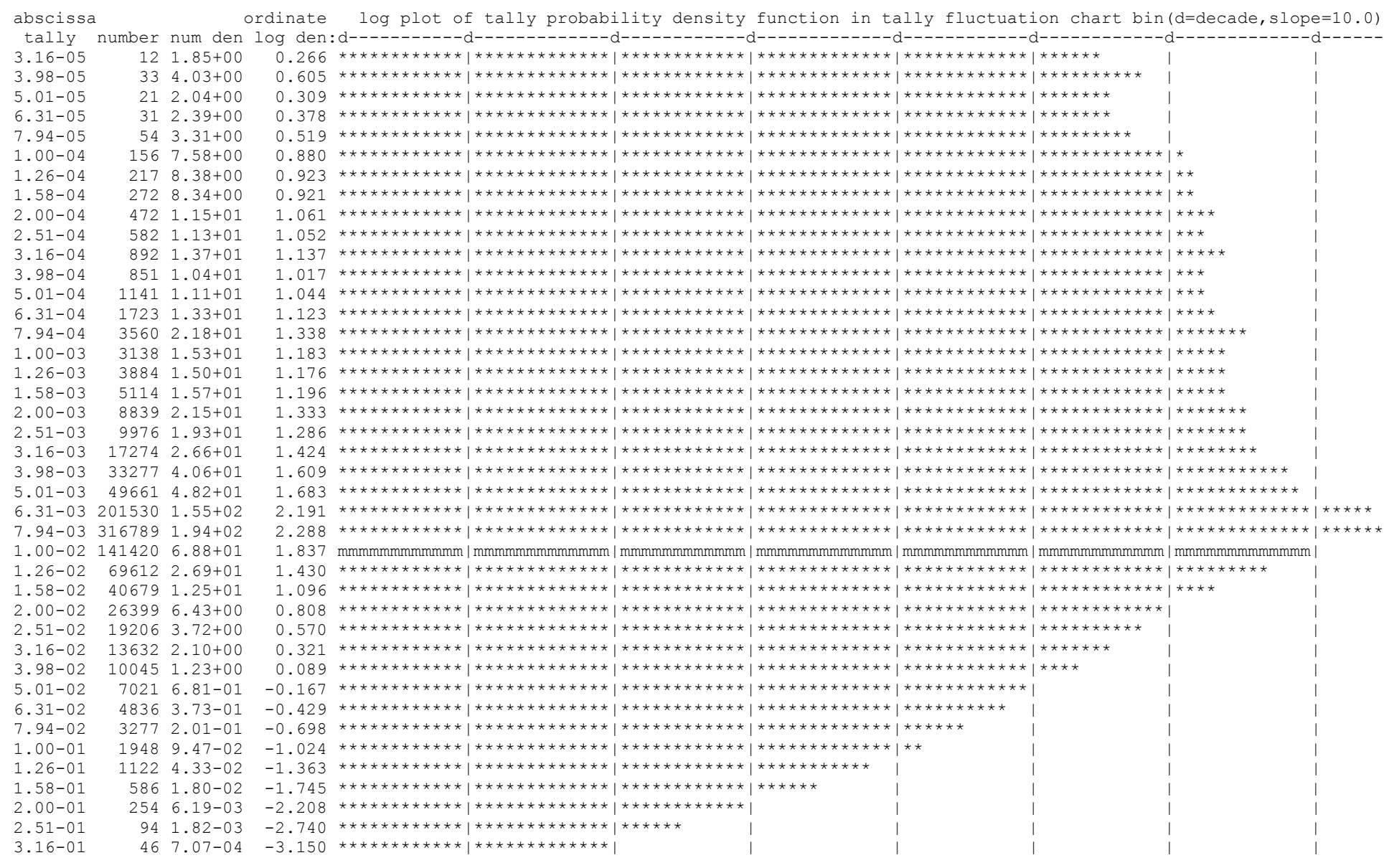




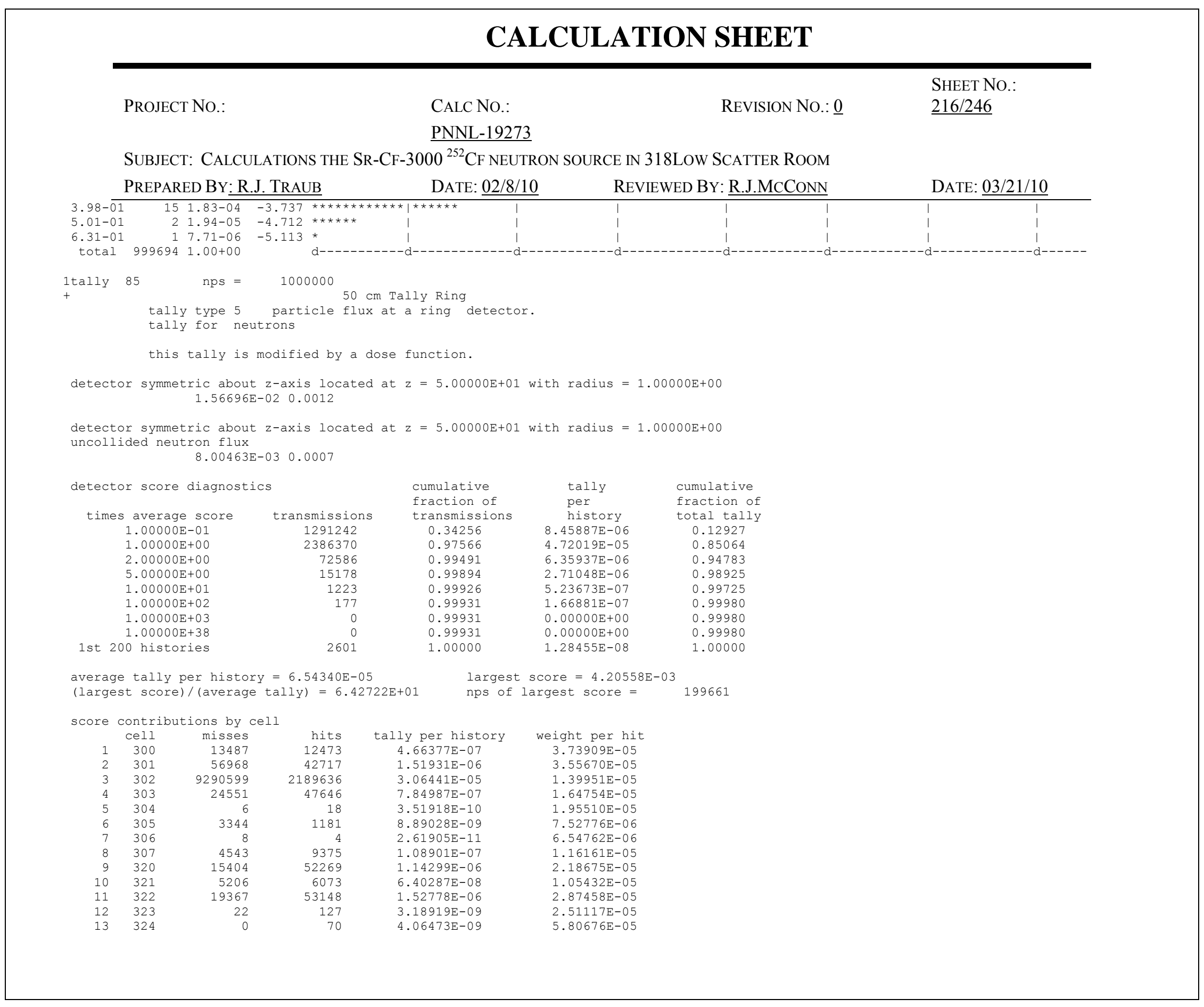




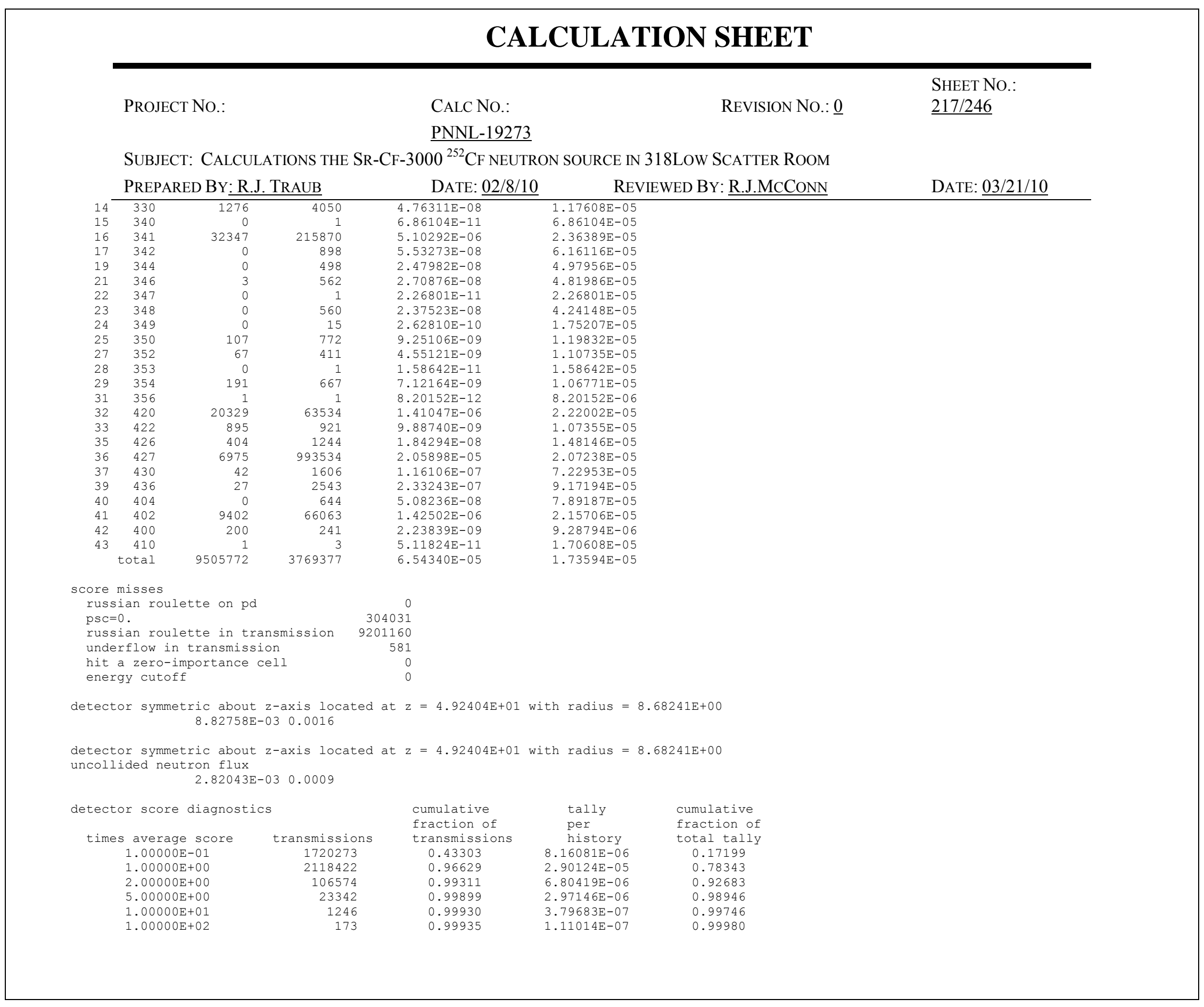




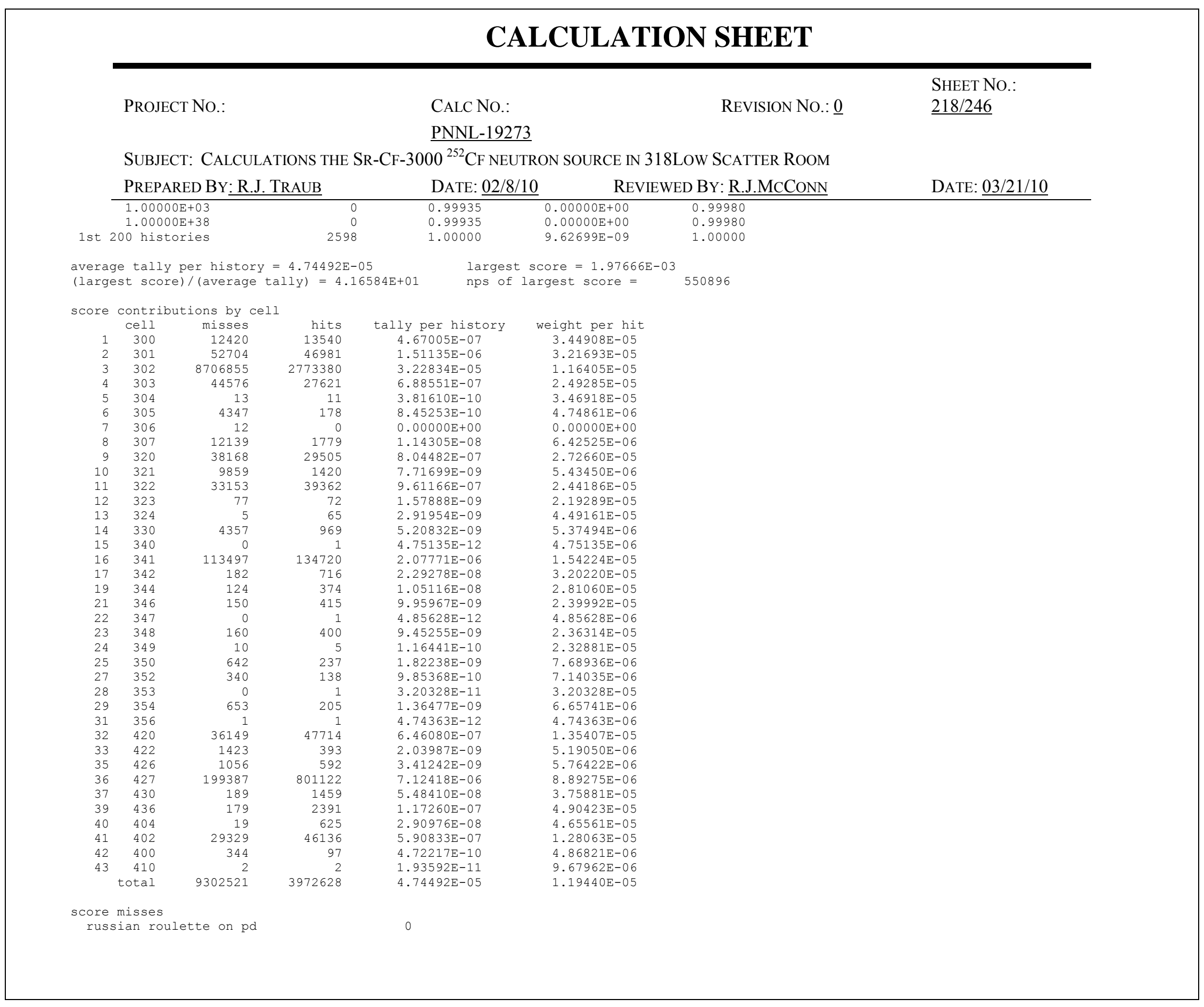




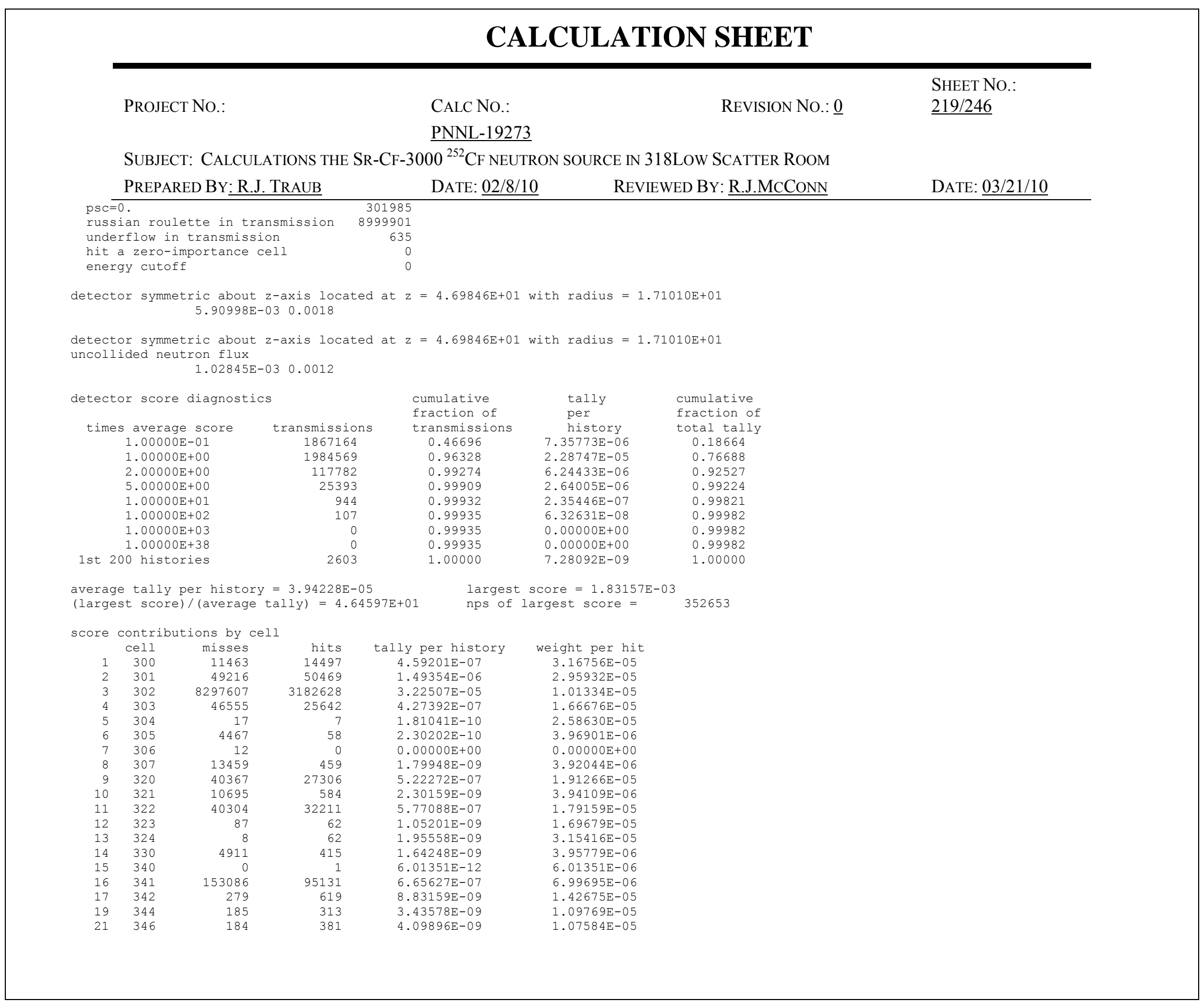




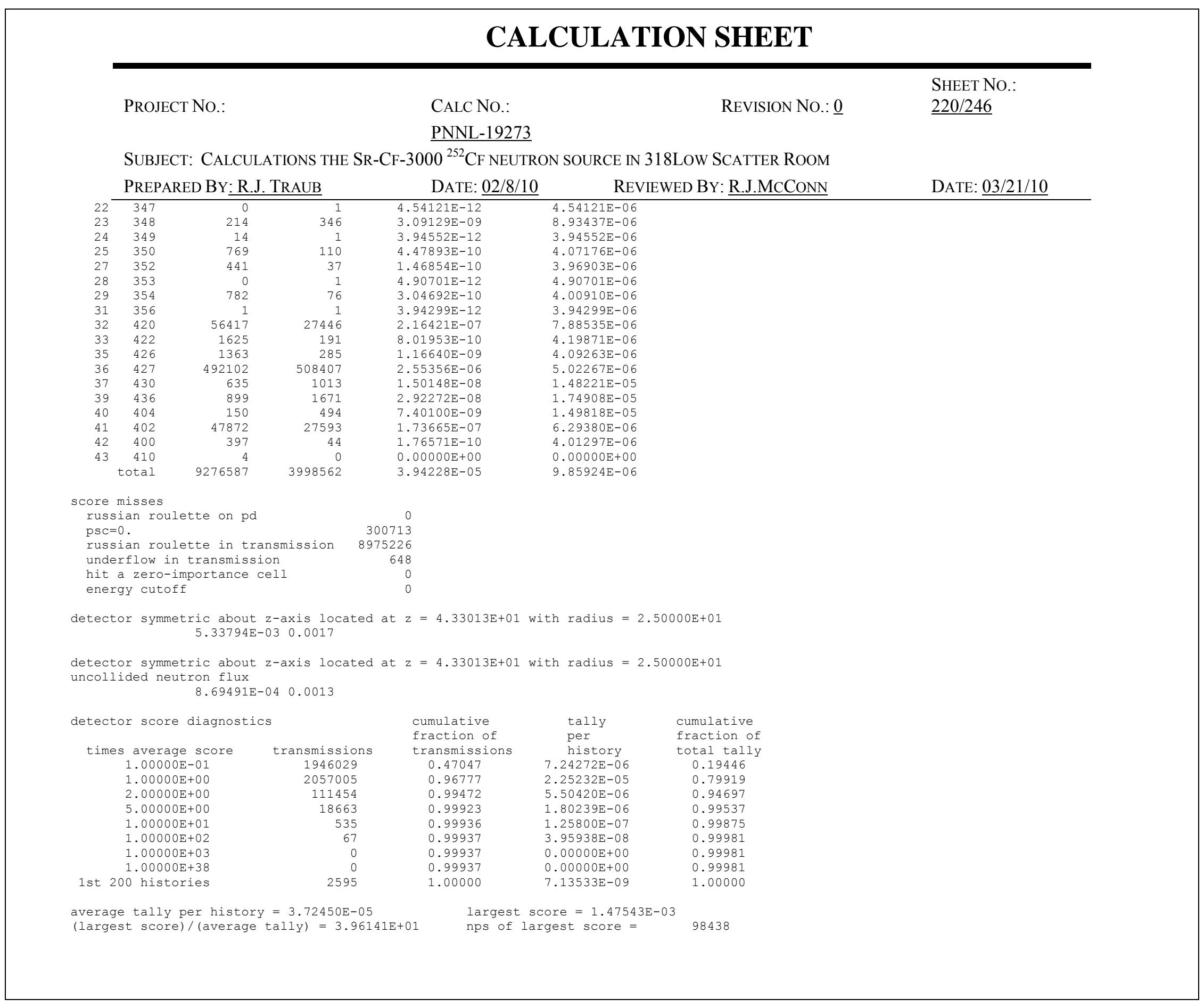




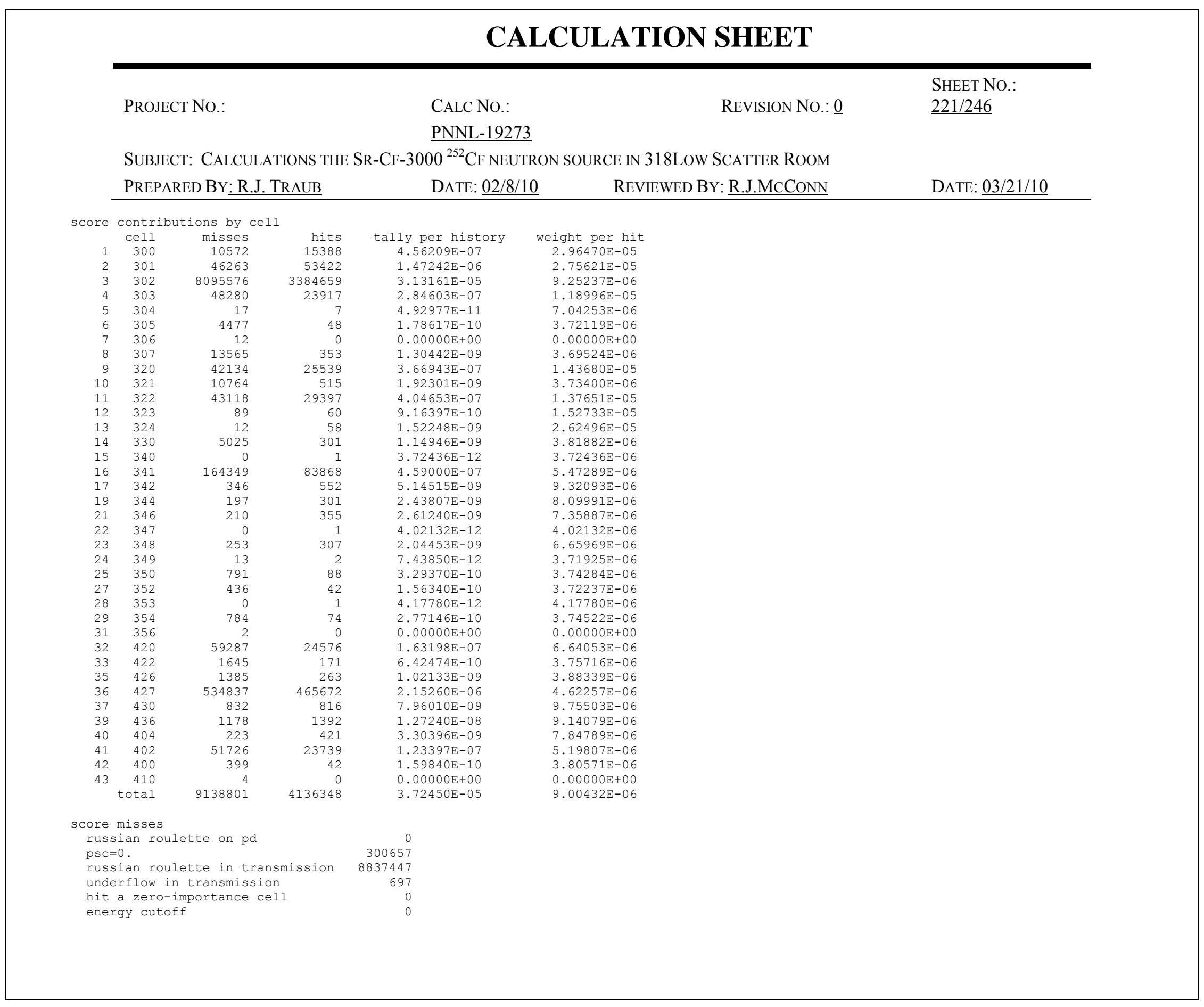




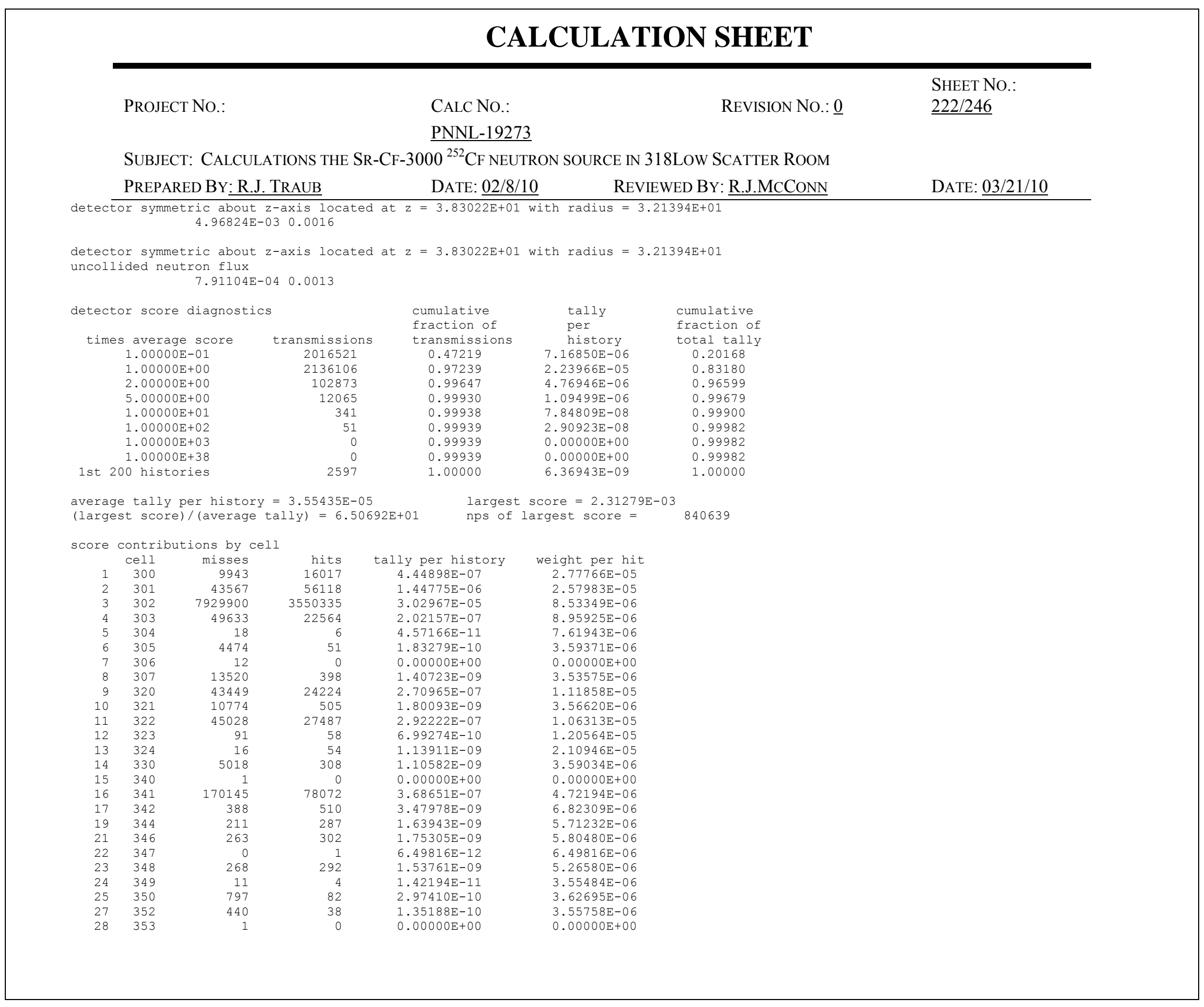




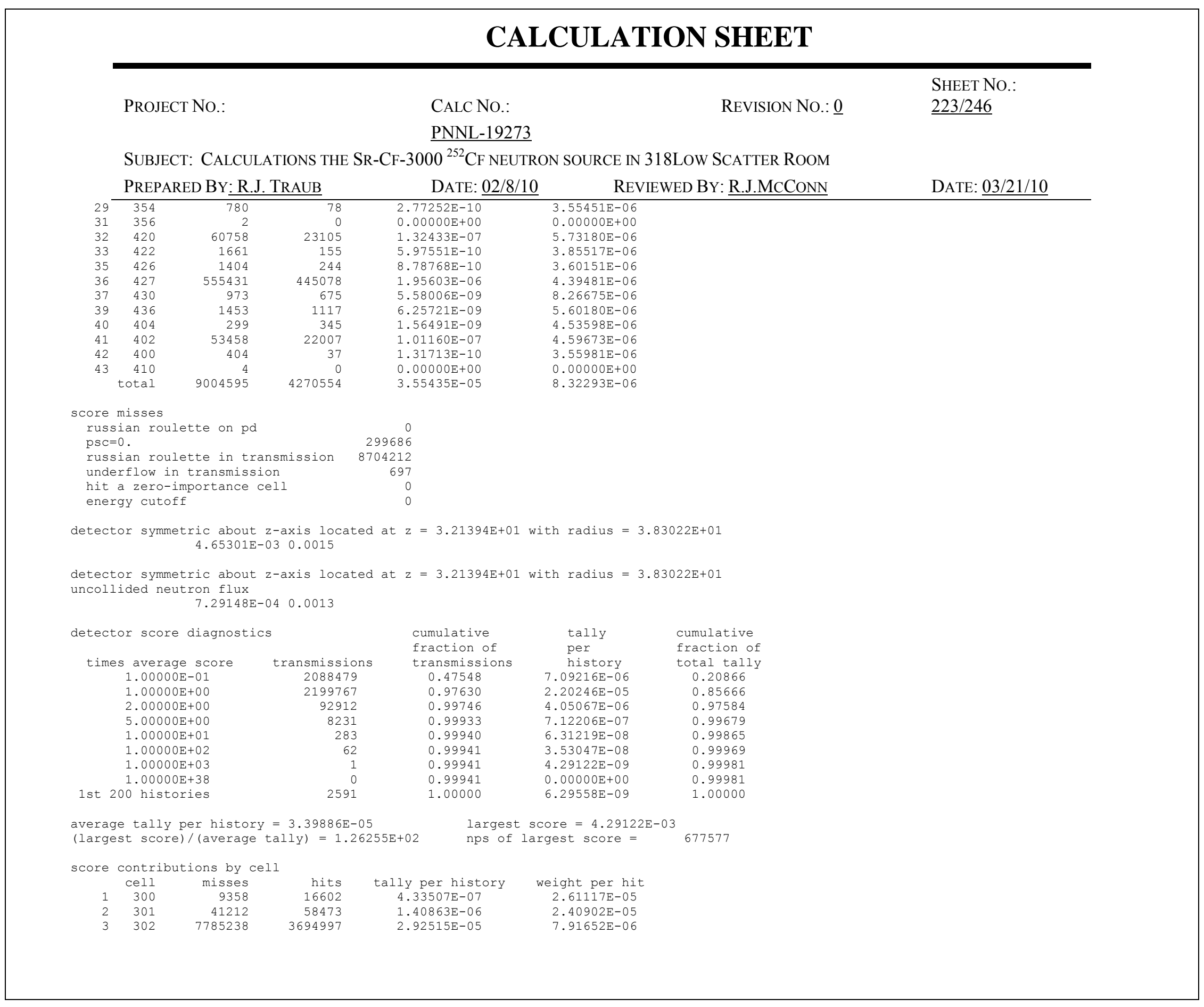




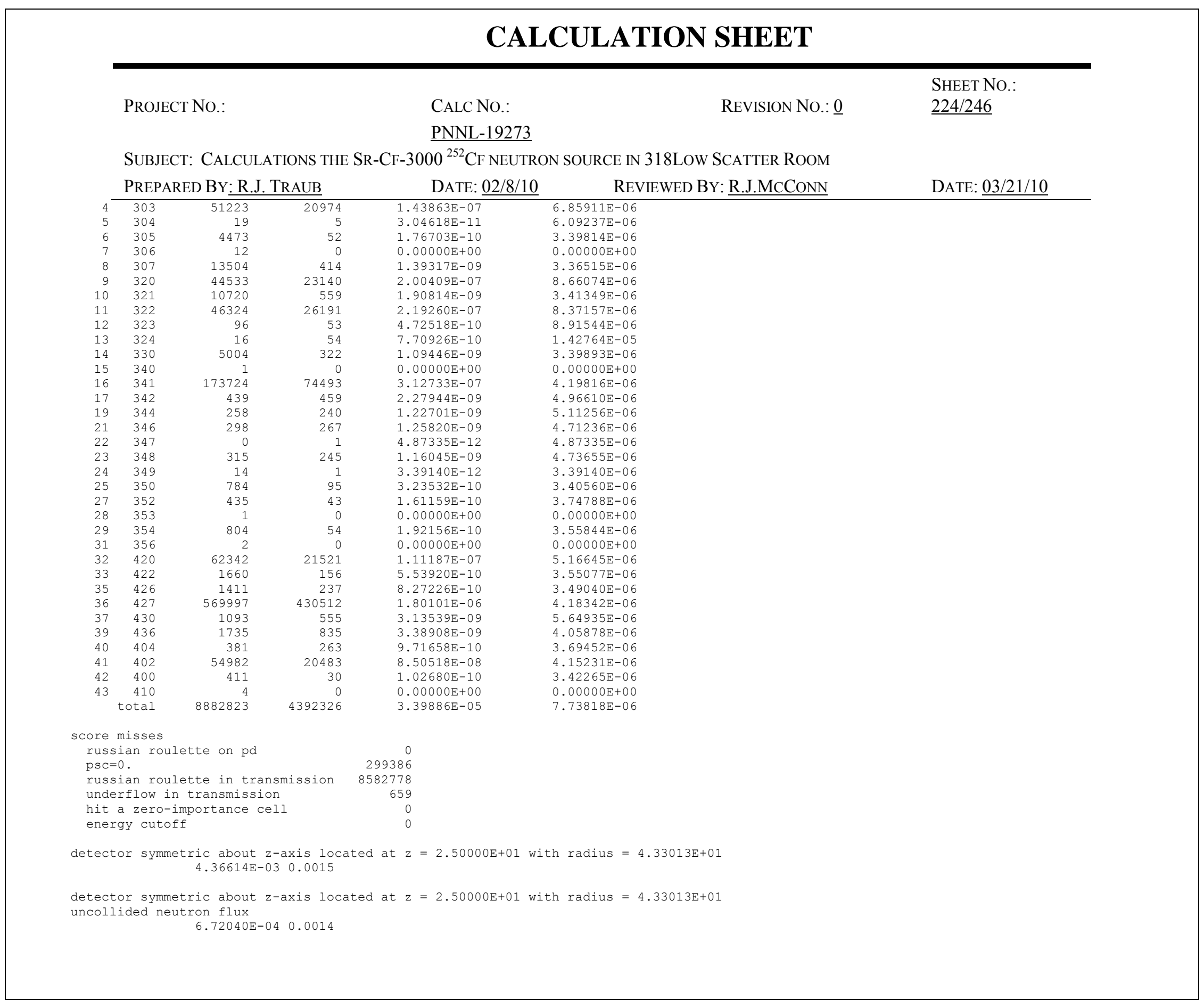




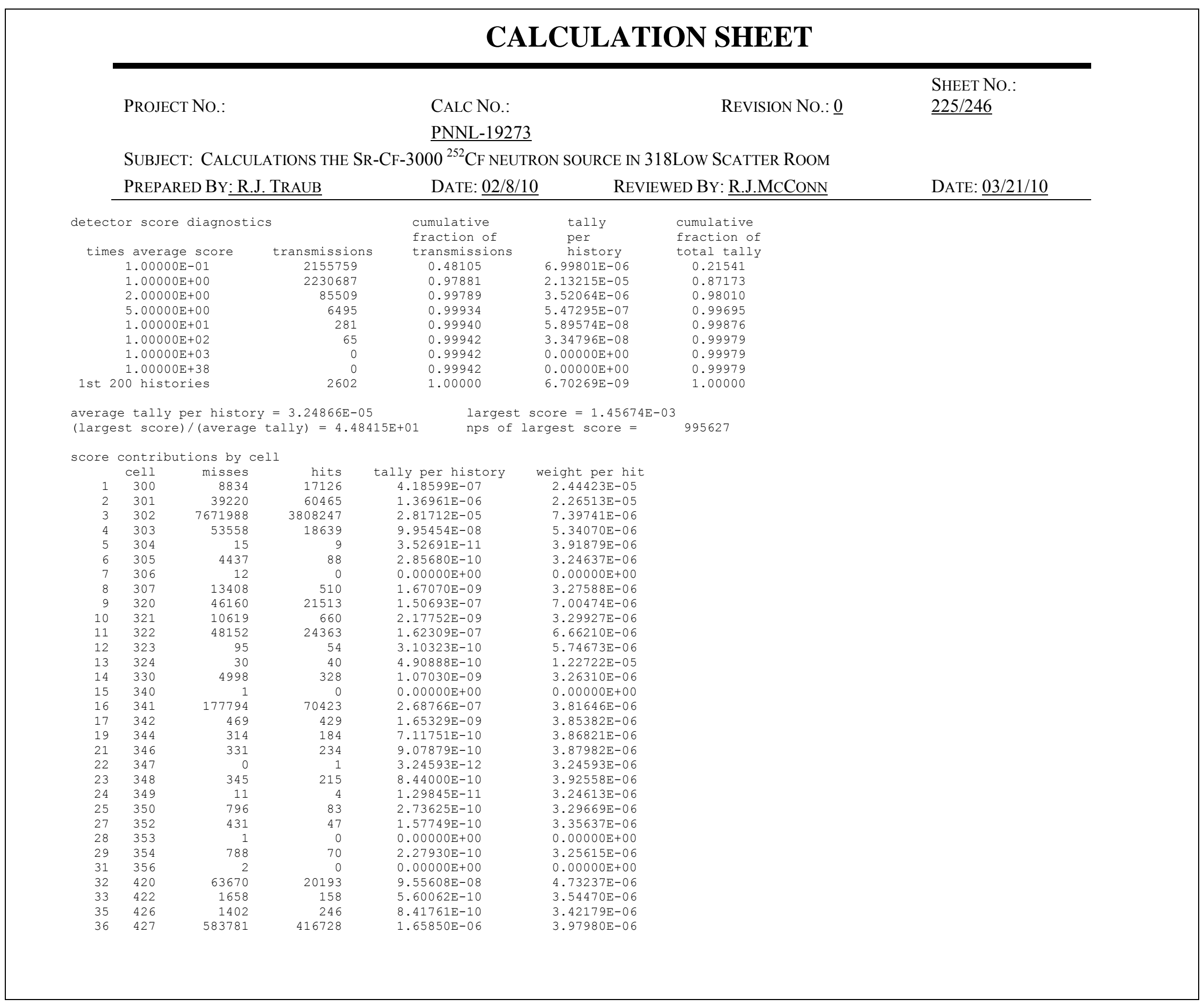




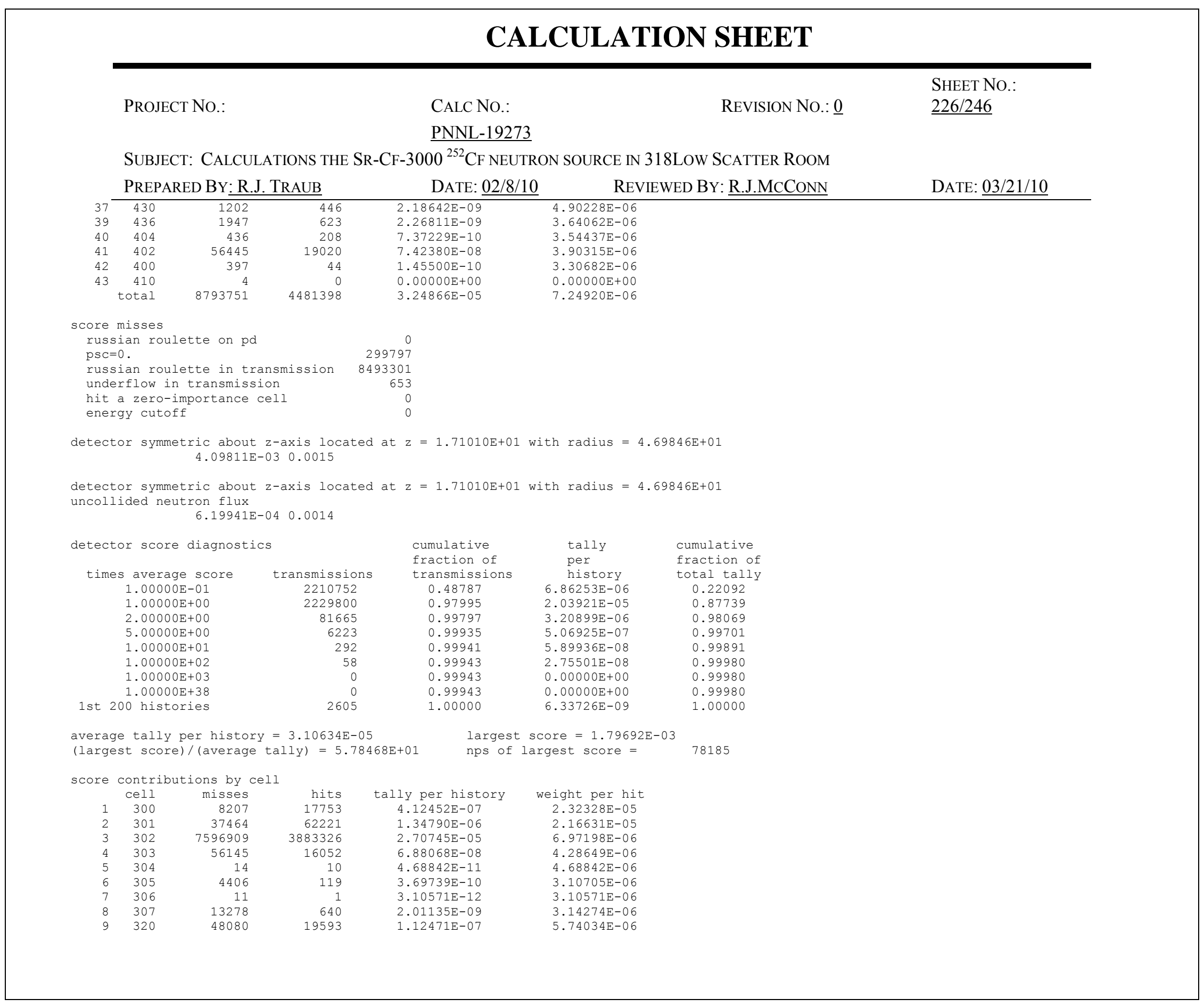




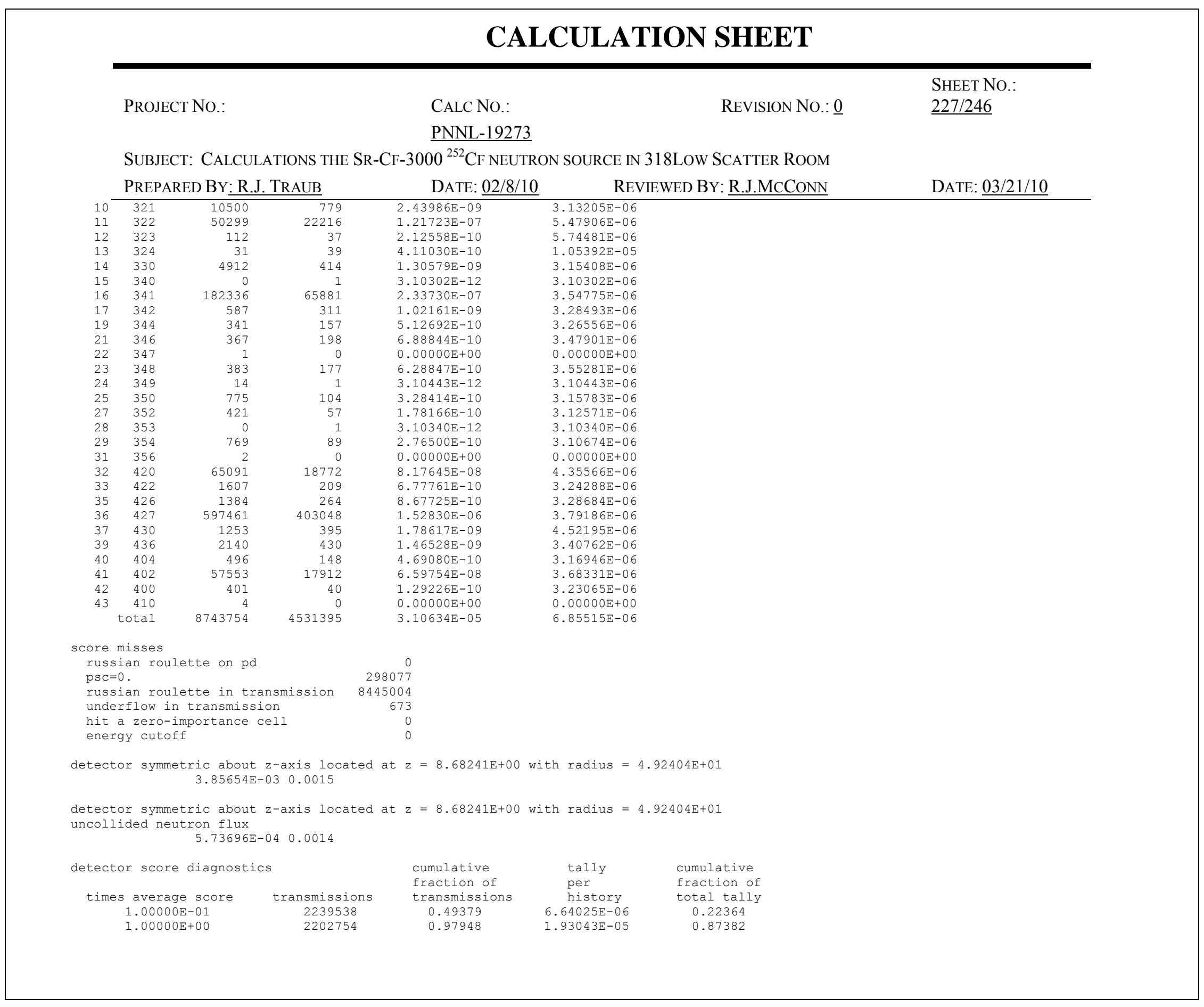




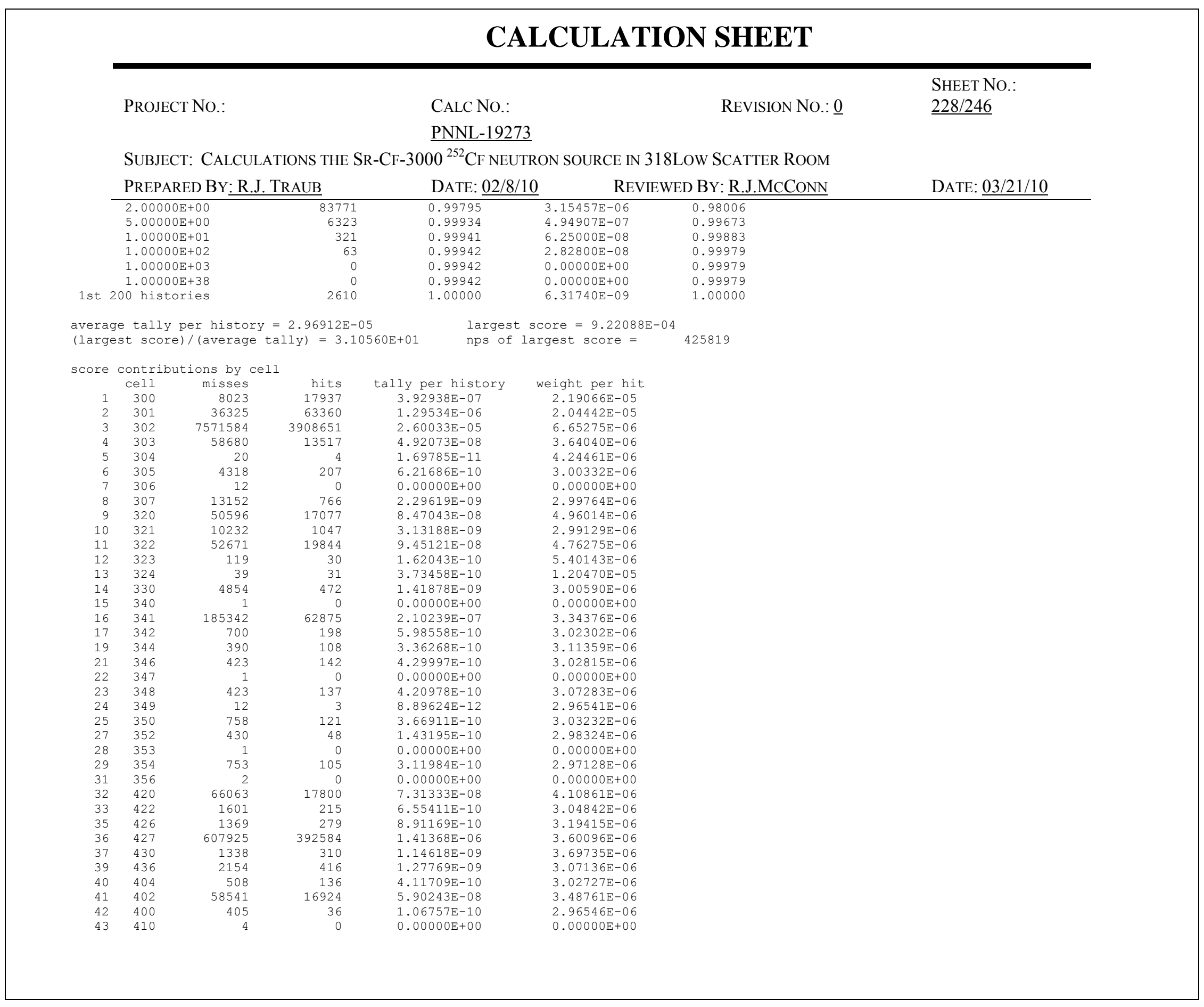




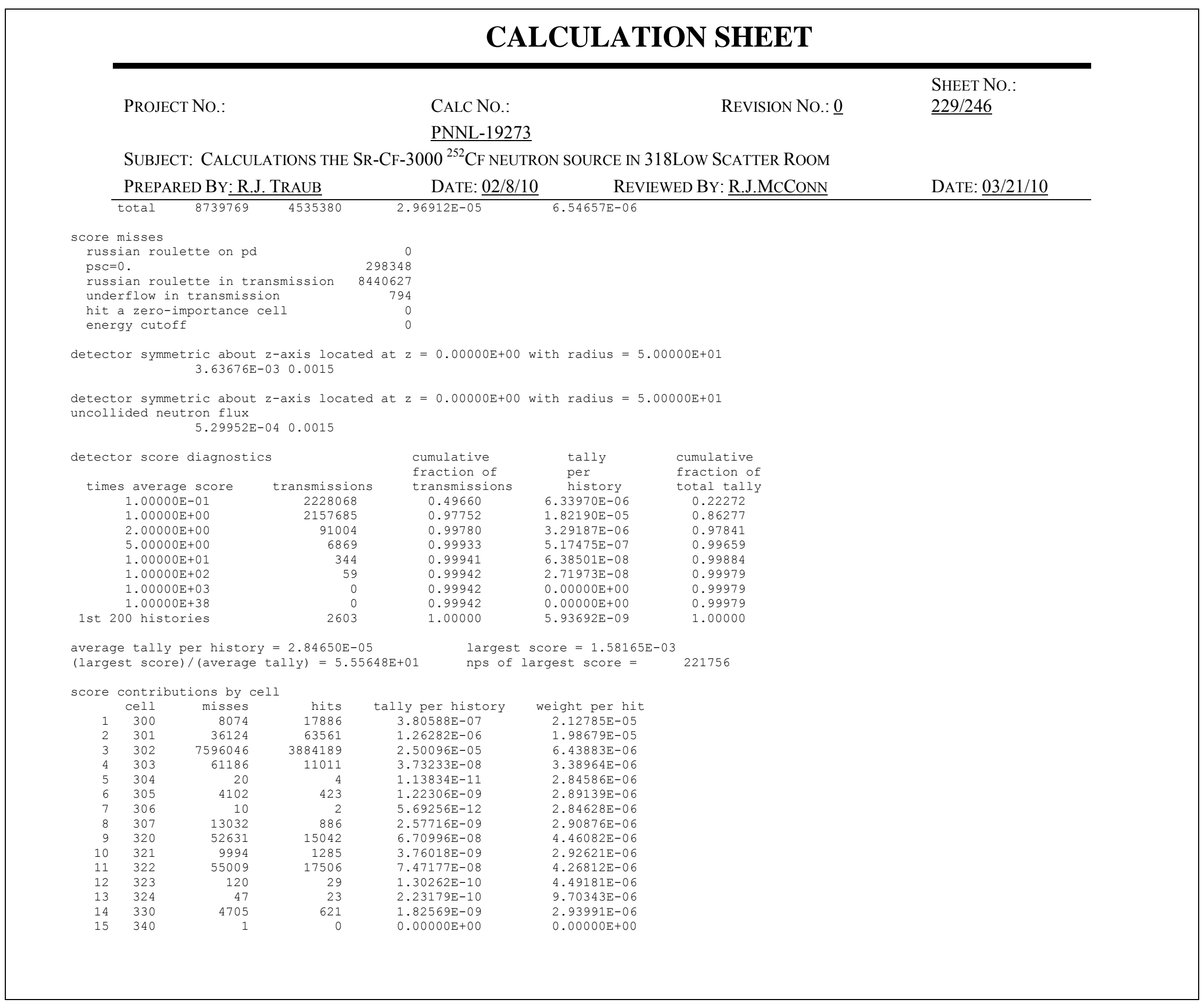




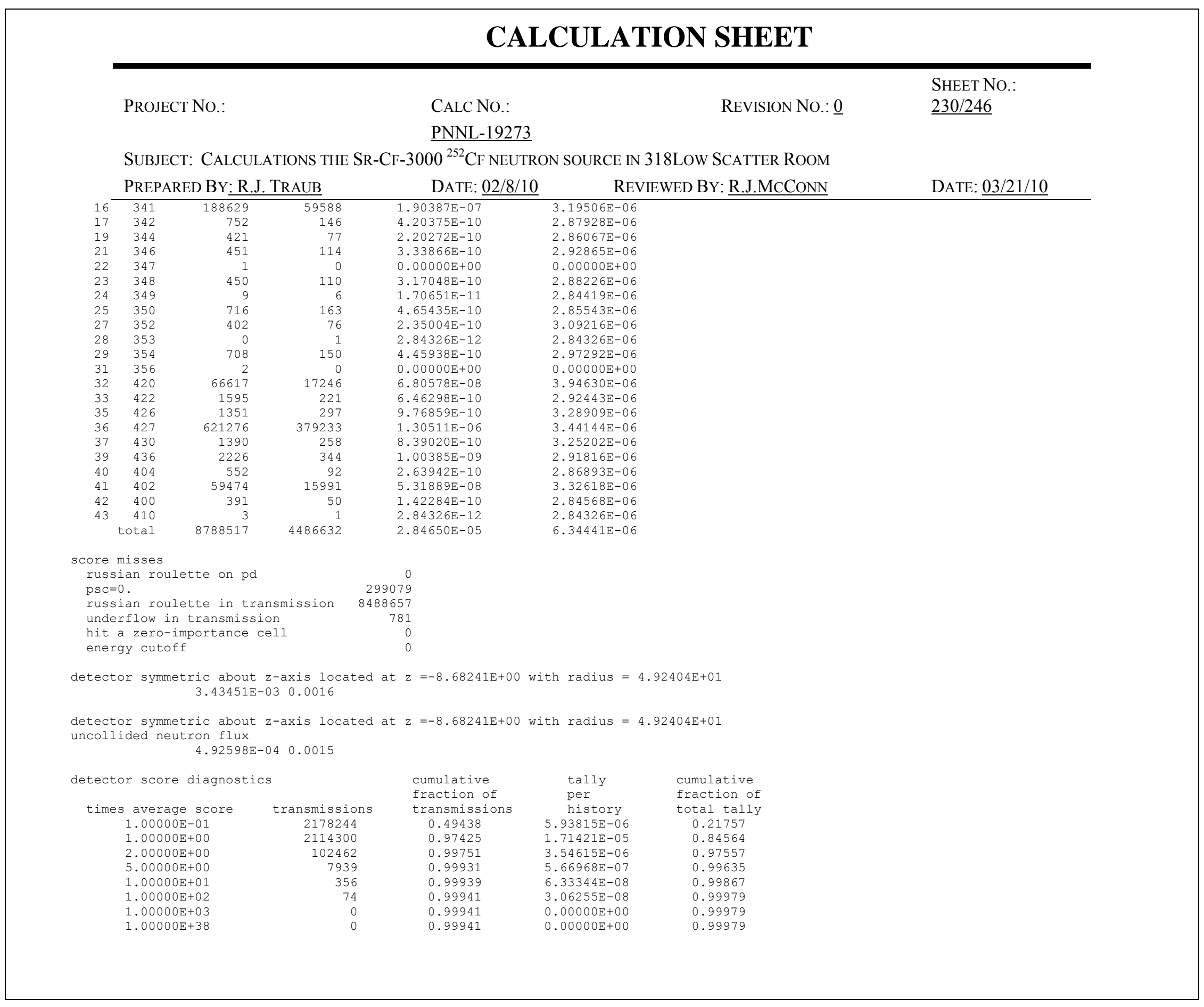




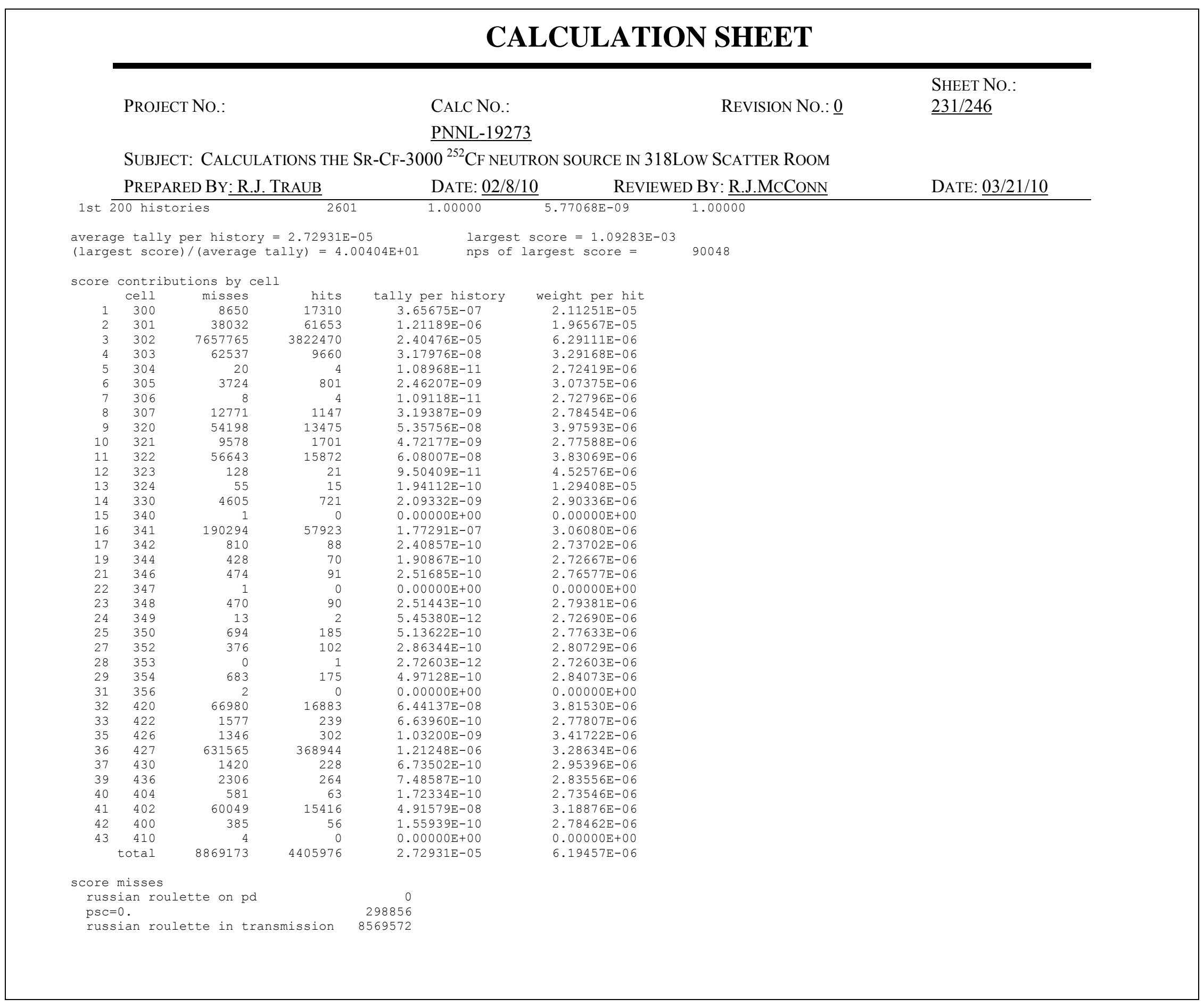




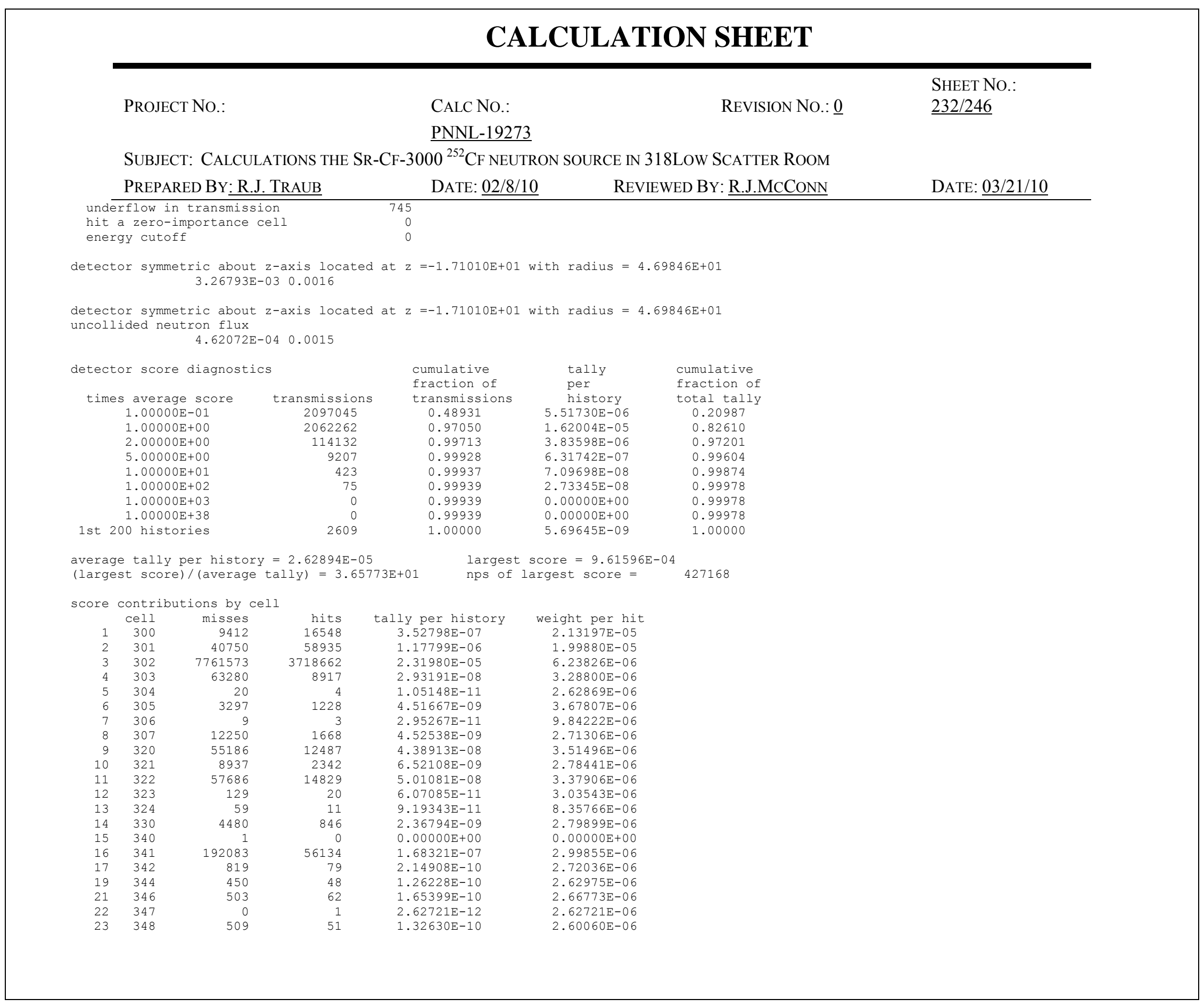




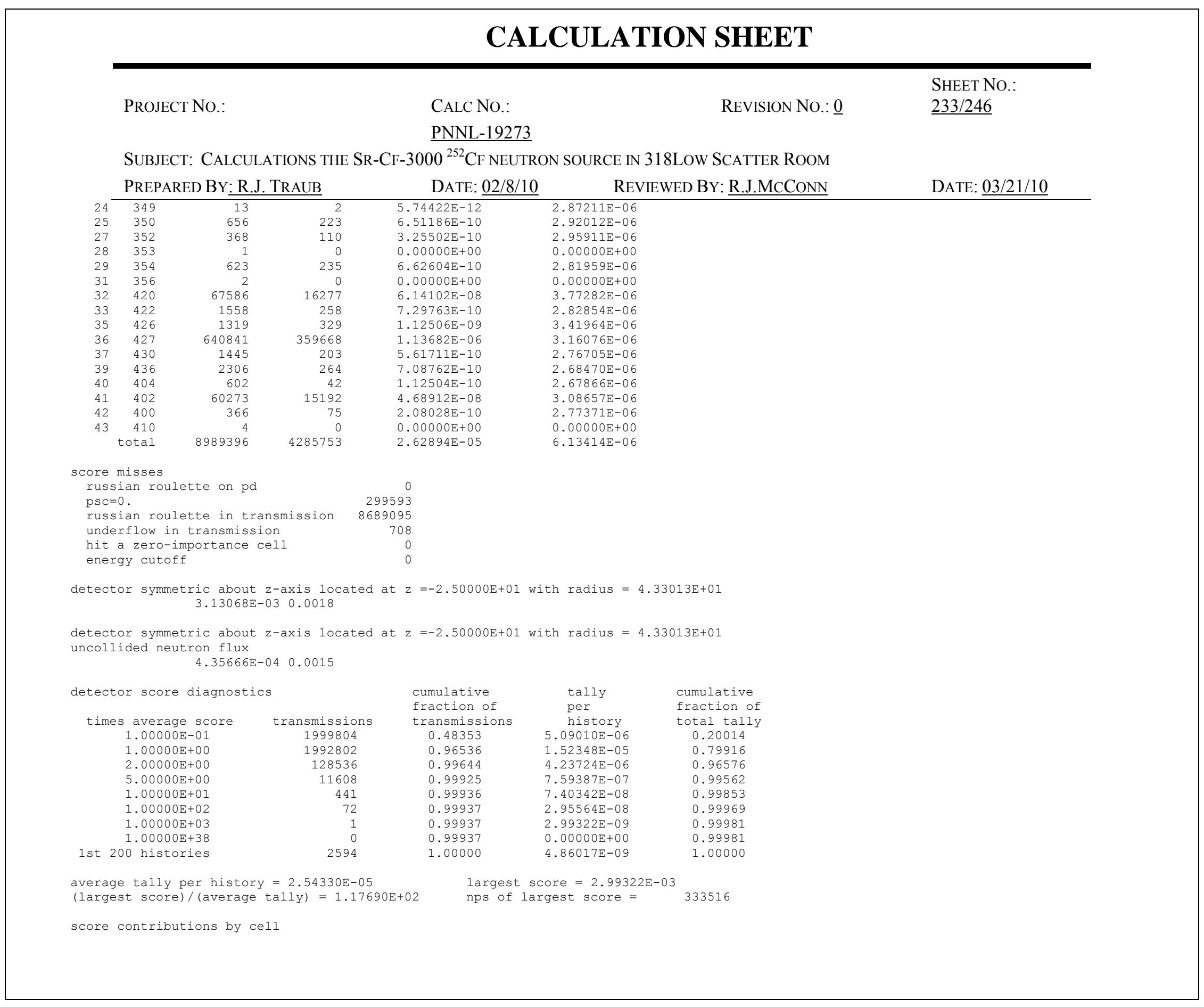




\begin{tabular}{|c|c|c|c|c|c|c|}
\hline \multicolumn{7}{|c|}{ CALCULATION SHEET } \\
\hline & PROJ & r No.: & & $\begin{array}{l}\text { CALC NO.: } \\
\text { PNNL-19273 }\end{array}$ & REVISION NO.: $\underline{0}$ & $\begin{array}{l}\text { SHEET NO.: } \\
\underline{234 / 246}\end{array}$ \\
\hline & \multicolumn{6}{|c|}{ 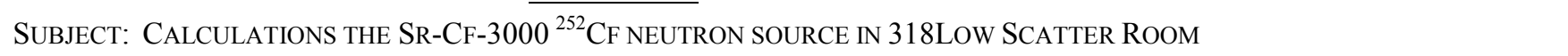 } \\
\hline & \multicolumn{3}{|c|}{ PREPARED By: R.J. TRAUB } & DATE: $\underline{02 / 8 / 10}$ & REVIEWED BY: R.J.MCCONN & \multirow[t]{2}{*}{ DATE: $\underline{03 / 21 / 10}$} \\
\hline & cell & misses & hits & tally per history & weight per hit & \\
\hline$\frac{1}{2}$ & 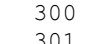 & 10477 & $\begin{array}{l}15483 \\
55568\end{array}$ & 3. $40224 \mathrm{E}-07$ & $2.19741 \mathrm{E}-05$ & \\
\hline $\begin{array}{l}2 \\
3\end{array}$ & $\begin{array}{l}301 \\
302\end{array}$ & 7898916 & $\begin{array}{r}55568 \\
3581319\end{array}$ & $\begin{array}{l}1.14455 \mathrm{E}-06 \\
2.24637 \mathrm{E}-05\end{array}$ & $\begin{array}{l}2.05973 \mathrm{E}-05 \\
6.27245 \mathrm{E}-06\end{array}$ & \\
\hline 4 & 303 & 63613 & 8584 & $2.81030 \mathrm{E}-08$ & $\begin{array}{l}6.27245 \mathrm{E}-06 \\
3.27388 \mathrm{E}-06\end{array}$ & \\
\hline 5 & 304 & 20 & 4 & $1.01709 \mathrm{E}-11$ & $2.54272 \mathrm{E}-06$ & \\
\hline 6 & 305 & 2733 & 1792 & 8.48170E-09 & $4.73309 \mathrm{E}-06$ & \\
\hline 7 & 306 & 5 & 7 & 3.32583E-11 & $4.75119 \mathrm{E}-06$ & \\
\hline 8 & 307 & $\begin{array}{l}11626 \\
55768\end{array}$ & 2292 & $6.37621 \mathrm{E}-09$ & $2.78194 \mathrm{E}-06$ & \\
\hline 9 & $\begin{array}{l}320 \\
321\end{array}$ & $\begin{array}{r}55688 \\
8294\end{array}$ & $\begin{array}{r}11995 \\
2985\end{array}$ & $\begin{array}{l}3.76171 \mathrm{E}-08 \\
8.5837 \mathrm{E}-09\end{array}$ & $3.15977 \mathrm{E}-06$ & \\
\hline 11 & $\begin{array}{l}321 \\
322\end{array}$ & $\begin{array}{r}8294 \\
58496\end{array}$ & $\begin{array}{r}2985 \\
14019\end{array}$ & $\begin{array}{l}8.54837 \mathrm{E}-09 \\
4.35566 \mathrm{E}-08\end{array}$ & $\begin{array}{l}2.86378 \mathrm{E}-06 \\
3.10697 \mathrm{E}-06\end{array}$ & \\
\hline 12 & 323 & 132 & 14017 & $\begin{array}{l}4.35566 \mathrm{E}-08 \\
4.33094 \mathrm{E}-11\end{array}$ & $3.0697 \mathrm{E}-06$ & \\
\hline 13 & 324 & 63 & 7 & $4.14731 \mathrm{E}-11$ & $\begin{array}{l}2.54161 \mathrm{E}-06 \\
5.92473 \mathrm{E}-06\end{array}$ & \\
\hline 14 & 330 & 4275 & 1051 & $2.90585 \mathrm{E}-09$ & $2.76484 \mathrm{E}-06$ & \\
\hline 15 & 340 & 1 & 0 & $0.00000 \mathrm{E}+00$ & $0.00000 \mathrm{E}+00$ & \\
\hline 16 & 341 & 192637 & 55580 & $1.65031 \mathrm{E}-07$ & $2.96925 \mathrm{E}-06$ & \\
\hline 17 & 342 & 844 & 54 & $1.38638 \mathrm{E}-10$ & $2.56737 \mathrm{E}-06$ & \\
\hline & 344 & & 45 & $1.14591 \mathrm{E}-10$ & & \\
\hline 21 & 346 & 517 & 48 & 1.24058E-10 & $2.58454 \mathrm{E}-06$ & \\
\hline 22 & 347 & $\begin{array}{r}1 \\
501\end{array}$ & $\begin{array}{r}0 \\
59\end{array}$ & $0.00000 \mathrm{E}+00$ & $0.00000 \mathrm{E}+00$ & \\
\hline $\begin{array}{l}23 \\
24\end{array}$ & $\begin{array}{l}348 \\
349\end{array}$ & $\begin{array}{r}501 \\
10\end{array}$ & $\begin{array}{r}59 \\
5\end{array}$ & $\begin{array}{l}1.47803 E-10 \\
1.5667 E-10\end{array}$ & $\begin{array}{l}2.50514 \mathrm{E}-06 \\
3.1933 \mathrm{~F}-06\end{array}$ & \\
\hline 24 & $\begin{array}{l}349 \\
350\end{array}$ & 619 & 260 & & $3.19333 \mathrm{E}-06$ & \\
\hline $\begin{array}{l}25 \\
27\end{array}$ & $\begin{array}{l}350 \\
352\end{array}$ & $\begin{array}{l}619 \\
319\end{array}$ & $\begin{array}{l}200 \\
159\end{array}$ & $\begin{array}{l}7.98046 \mathrm{E}-10 \\
4.75631 \mathrm{E}-10\end{array}$ & $3.06941 E-06$ & \\
\hline 28 & 353 & $\begin{array}{r}319 \\
0\end{array}$ & 1 & $\begin{array}{l}4.75631 \mathrm{E}-10 \\
2.54470 \mathrm{E}-12\end{array}$ & $\begin{array}{l}2.99139 E-06 \\
2.5470 E-06\end{array}$ & \\
\hline 29 & 354 & 582 & 276 & $\begin{array}{l}2.54410 \mathrm{E}-12 \\
8.34951 \mathrm{E}-10\end{array}$ & $\begin{array}{l}2.5440 \mathrm{E}-06 \\
3.02518 \mathrm{E}-06\end{array}$ & \\
\hline 31 & 356 & 1 & 1 & $2.54295 \mathrm{E}-12$ & $2.54295 \mathrm{E}-06$ & \\
\hline 32 & 420 & 67523 & 16340 & $6.05108 \mathrm{E}-08$ & $3.70323 \mathrm{E}-06$ & \\
\hline 33 & 422 & 1540 & 276 & $7.58494 \mathrm{E}-10$ & $2.74817 \mathrm{E}-06$ & \\
\hline 35 & 426 & 1280 & 368 & $1.47230 \mathrm{E}-09$ & $4.00081 \mathrm{E}-06$ & \\
\hline 36 & 427 & 648747 & 351762 & $1.07143 \mathrm{E}-06$ & $3.04590 \mathrm{E}-06$ & \\
\hline 37 & 430 & 1475 & 173 & $4.48936 \mathrm{E}-10$ & $2.59500 \mathrm{E}-06$ & \\
\hline 39 & & 2336 & 234 & $6.24781 \mathrm{E}-10$ & $2.67001 \mathrm{E}-06$ & \\
\hline 40 & 404 & 594 & 50 & $1.27214 \mathrm{E}-10$ & $2.54428 \mathrm{E}-06$ & \\
\hline 41 & 402 & 60424 & 15041 & $4.55105 \mathrm{E}-08$ & $3.02576 \mathrm{E}-06$ & \\
\hline \multirow{2}{*}{$\begin{array}{l}42 \\
43\end{array}$} & 400 & 348 & & $2.55042 \mathrm{E}-10$ & $2.74238 \mathrm{E}-06$ & \\
\hline & $\begin{array}{r}410 \\
\text { total }\end{array}$ & $\begin{array}{r}4 \\
9139291\end{array}$ & $\begin{array}{r}0 \\
4135858\end{array}$ & $\begin{array}{l}0.00000 \mathrm{E}+00 \\
2.54330 \mathrm{E}-05\end{array}$ & $\begin{array}{l}0.00000 \mathrm{E}+00 \\
6.14938 \mathrm{E}-06\end{array}$ & \\
\hline \multirow{2}{*}{\multicolumn{4}{|c|}{$\begin{array}{l}\text { score misses } \\
\text { russian roulette on pd }\end{array}$}} & & & \\
\hline & & & & 0 & & \\
\hline \multirow{2}{*}{\multicolumn{7}{|c|}{$\begin{array}{l}\text { PSC=0. } \\
\text { russian roulette in transmission } 8839189 \\
\text { underflow in transmission }\end{array}$}} \\
\hline & & & & & & \\
\hline \multirow{2}{*}{\multicolumn{7}{|c|}{$\begin{array}{l}\text { undertiow in transmission } \\
\text { hit a zero-importance cell }\end{array}$}} \\
\hline & & & & & & \\
\hline \multicolumn{7}{|c|}{$\begin{array}{c}\text { detector symmetric about } z \text {-axis located at } z=-3.21394 \mathrm{E}+01 \text { with radius }=3.83022 \mathrm{E}+01 \\
3.02356 \mathrm{E}-03 \quad 0.0019\end{array}$} \\
\hline
\end{tabular}




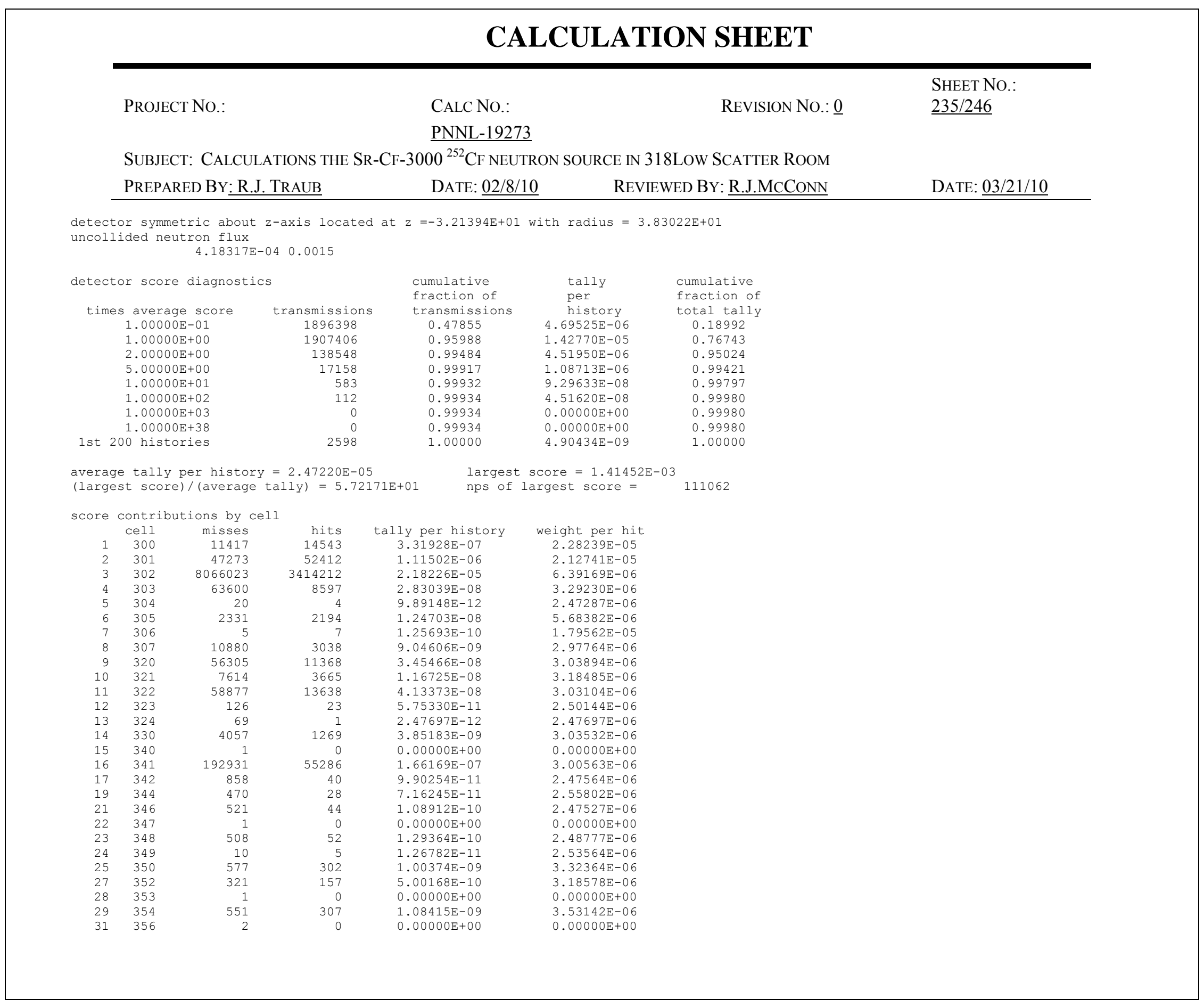




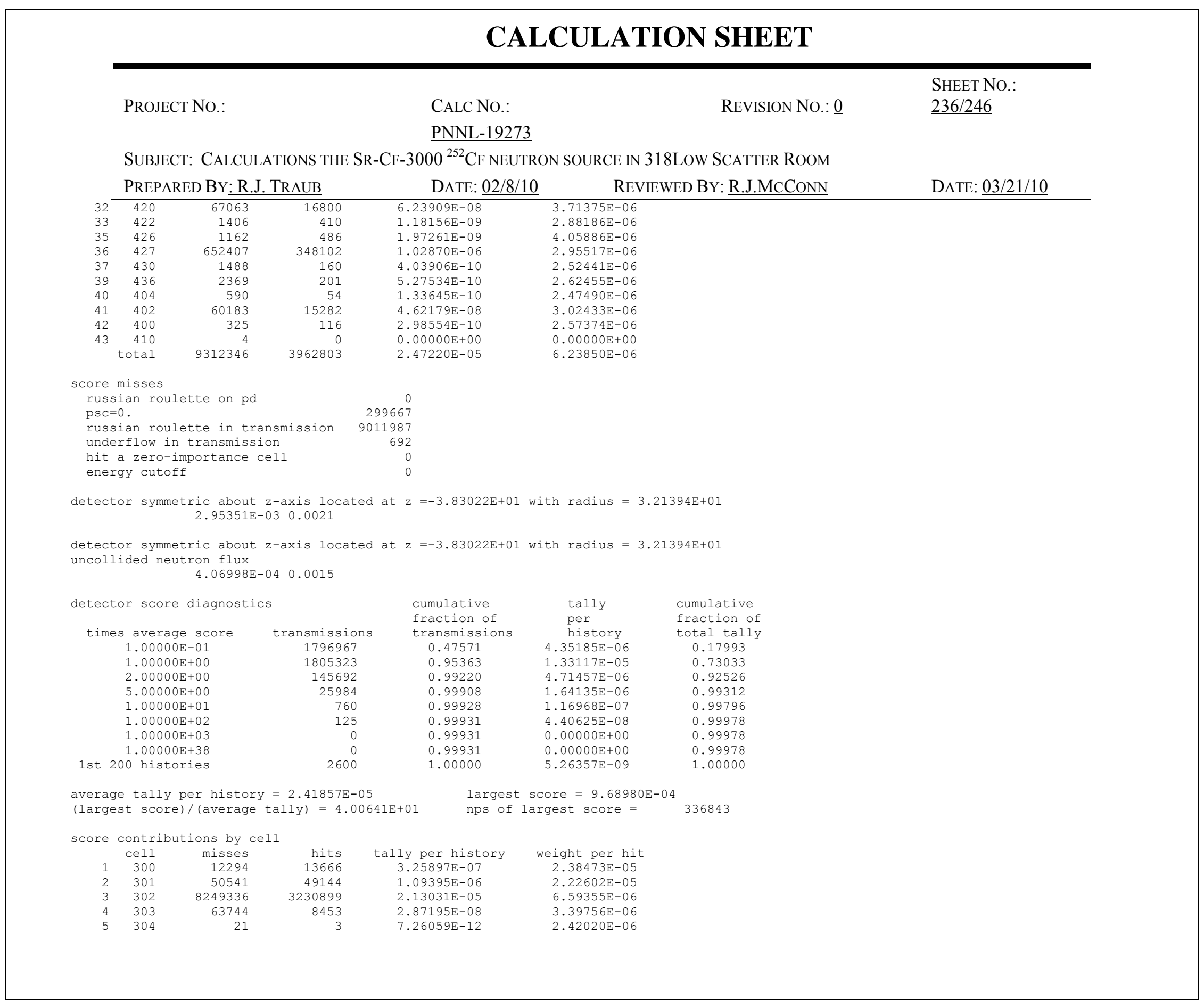




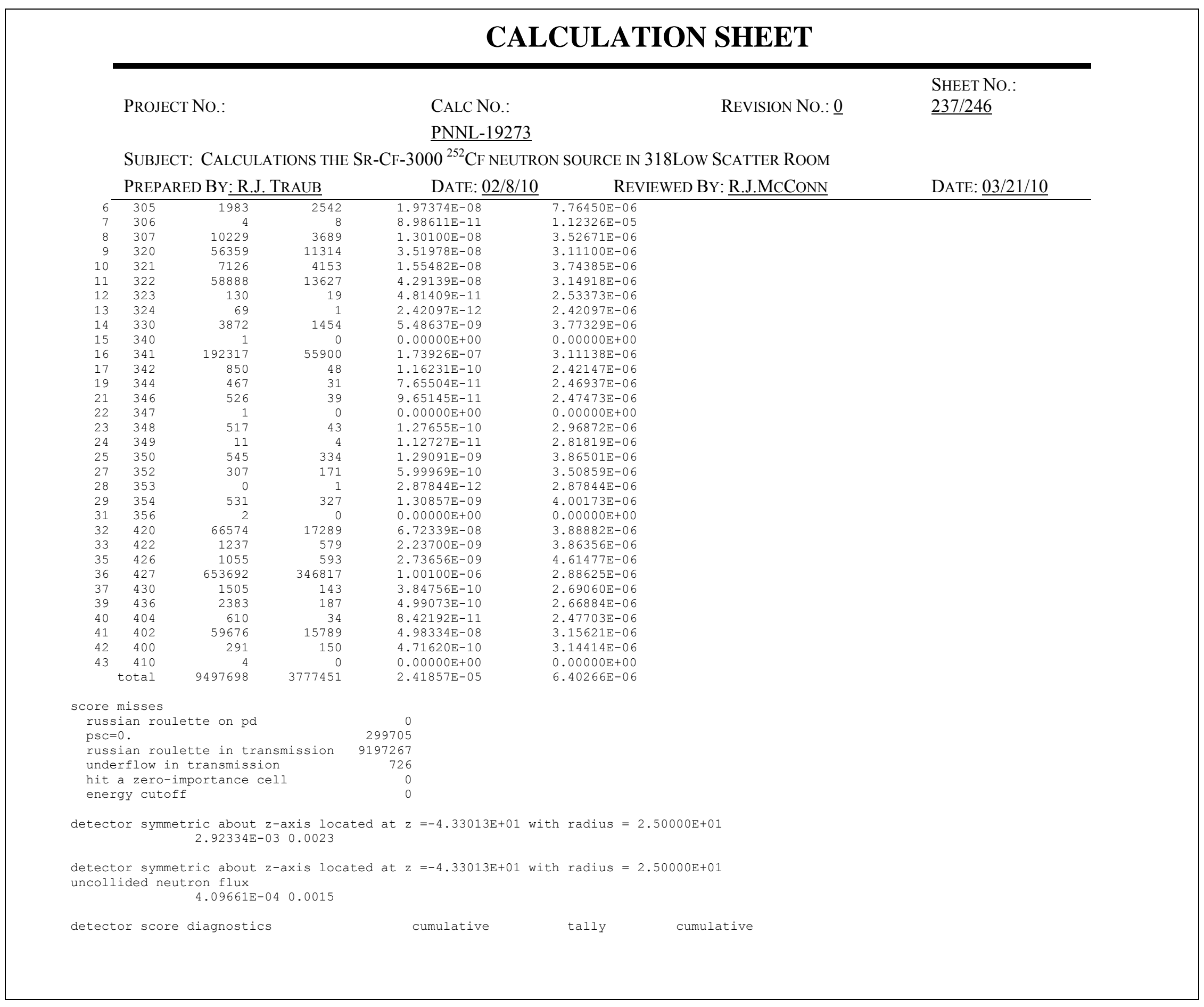




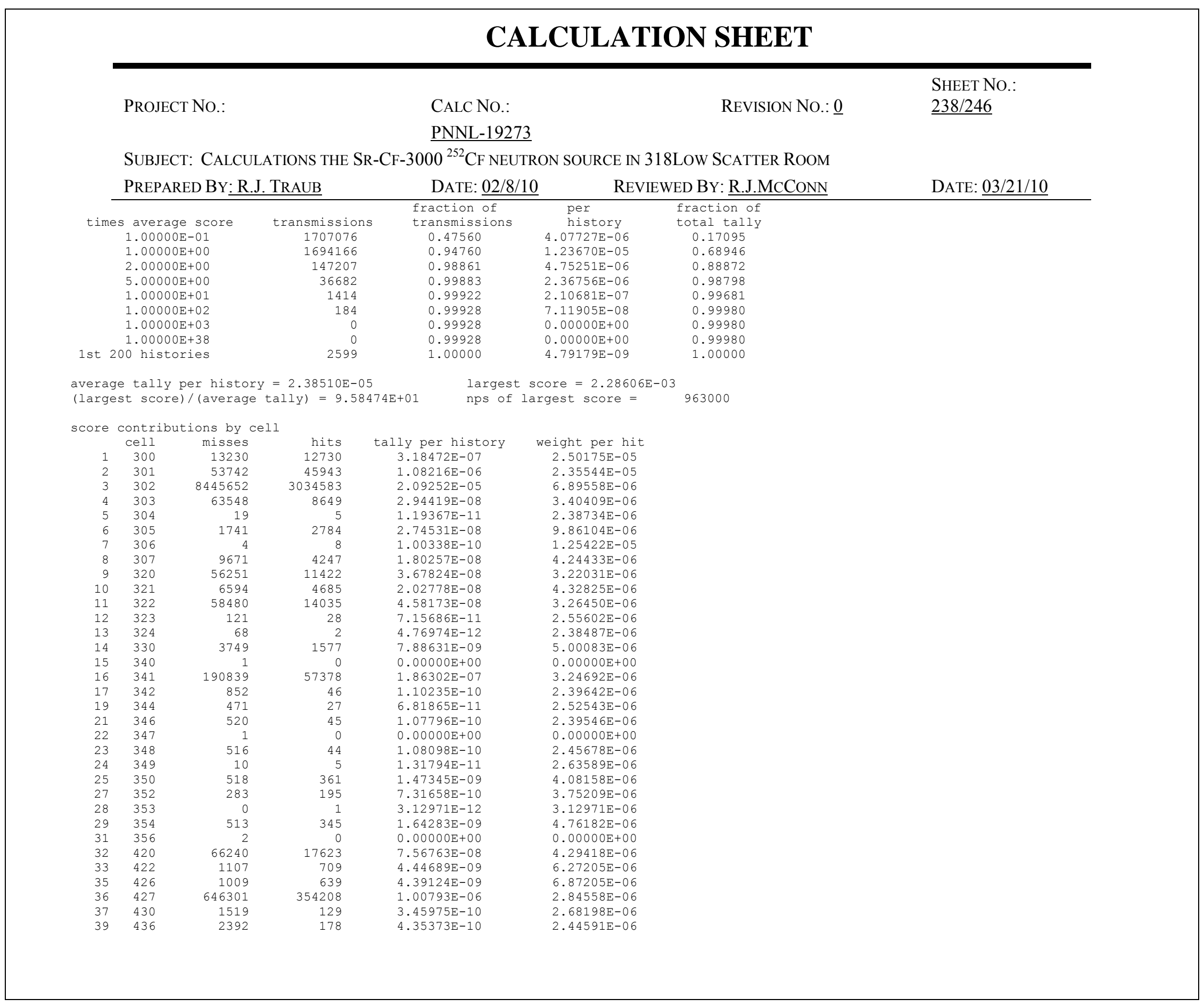




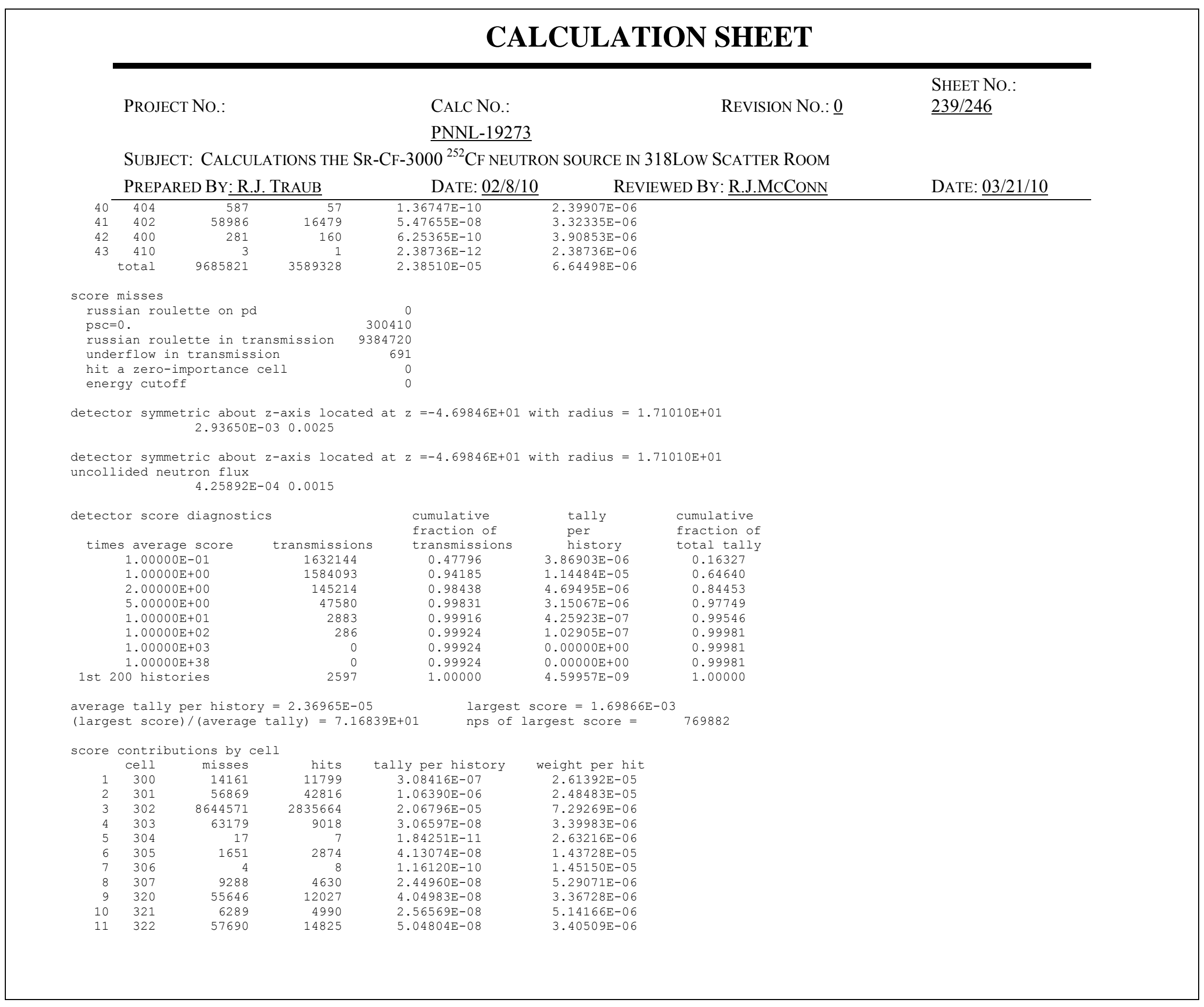




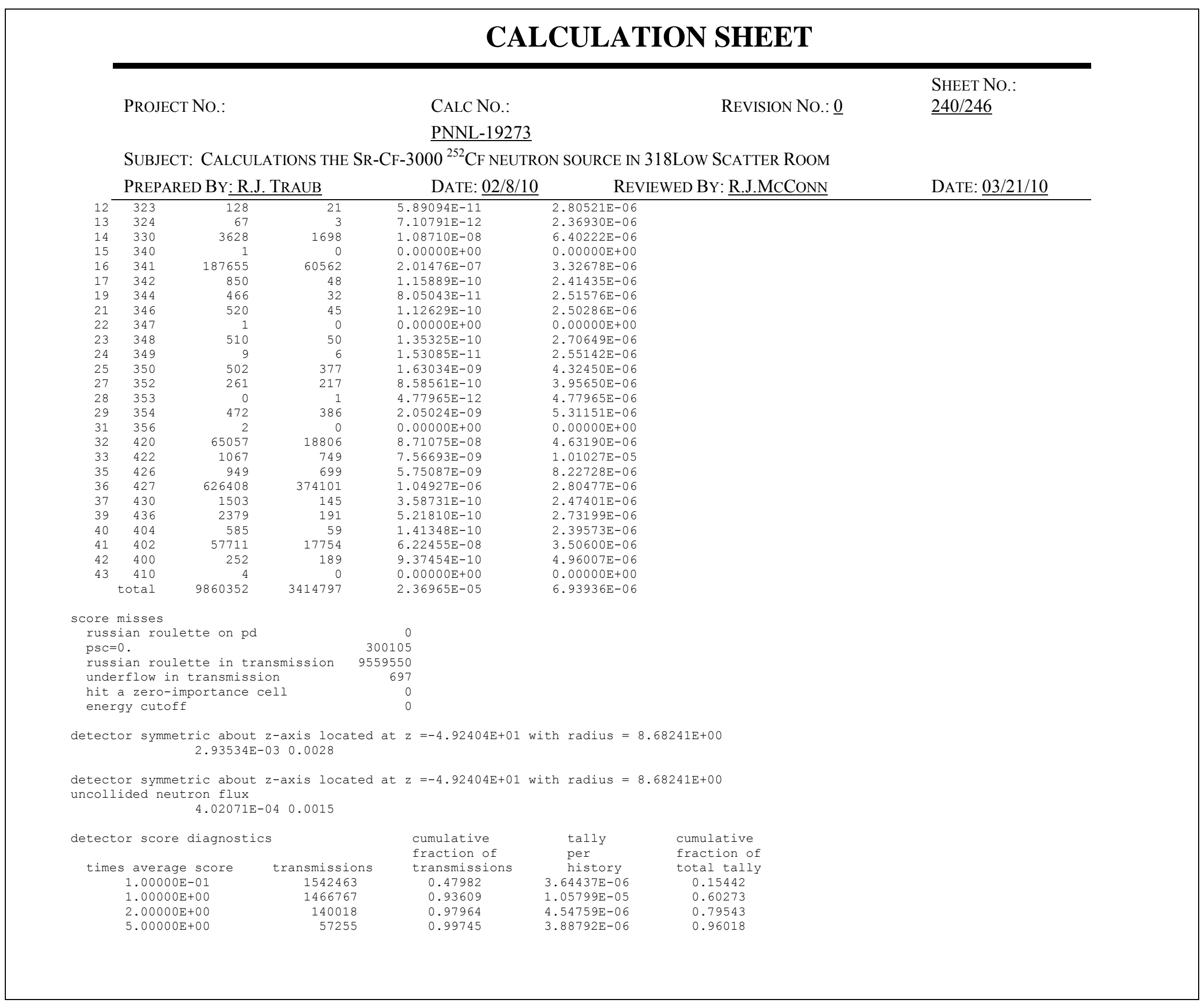




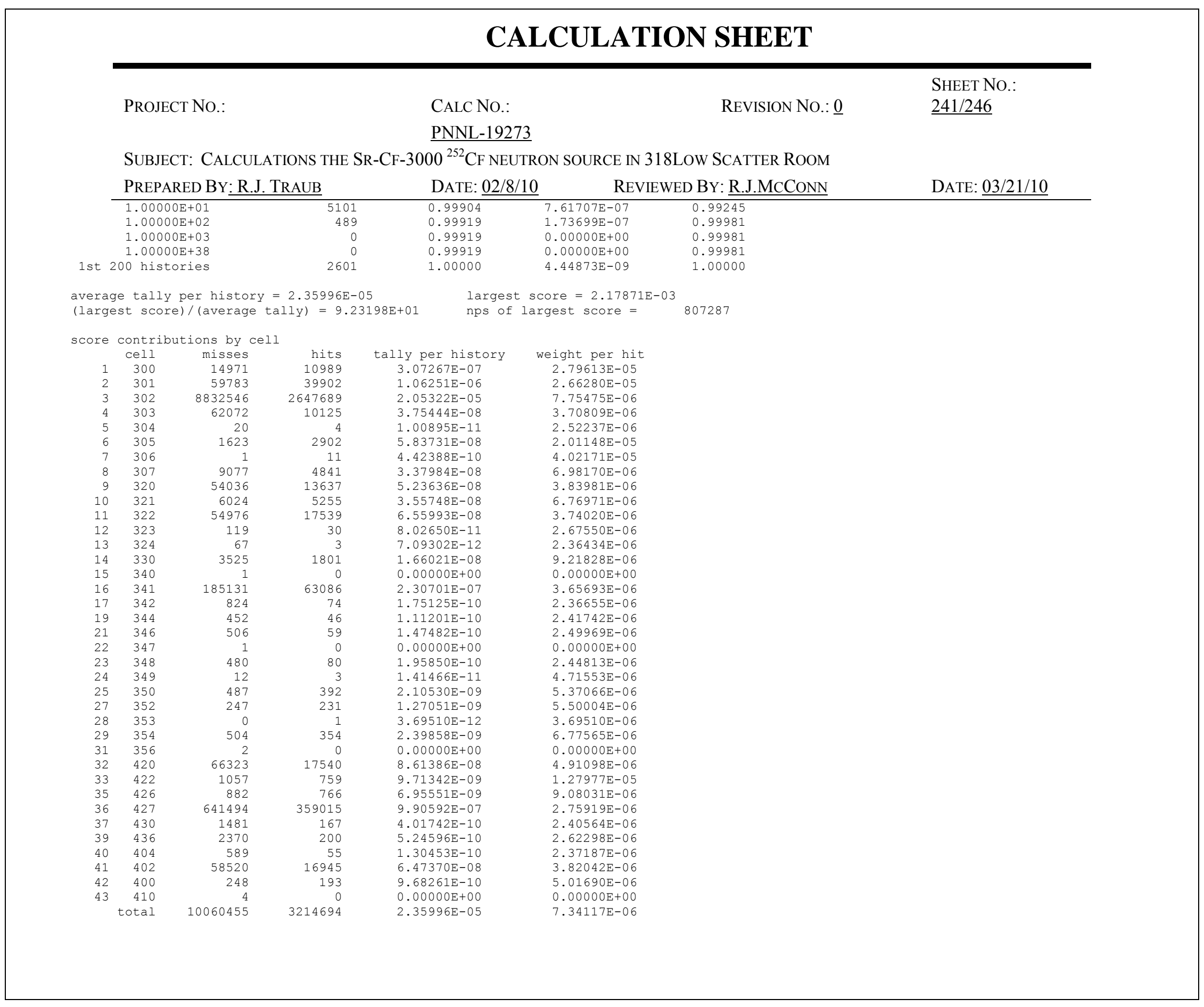




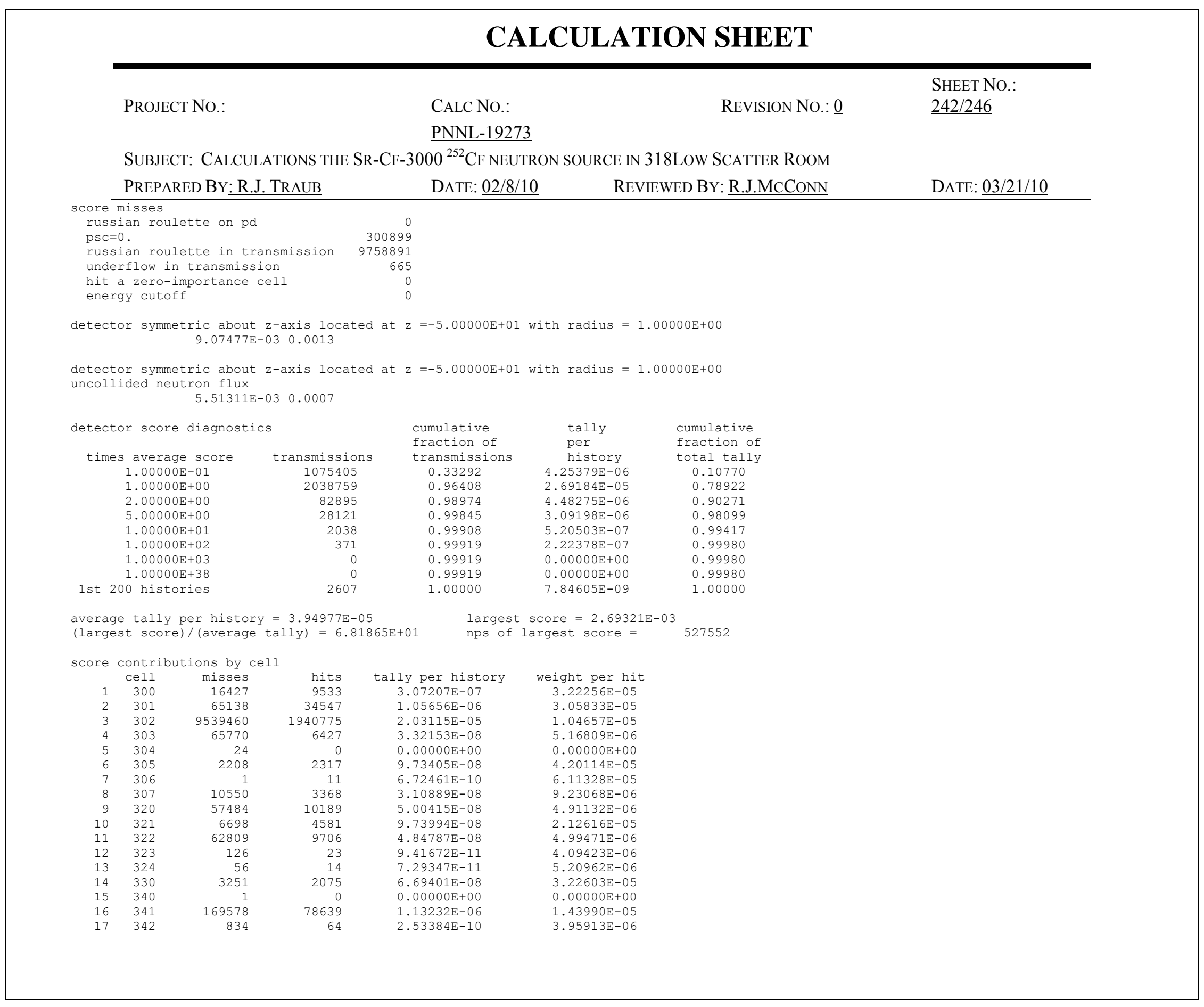




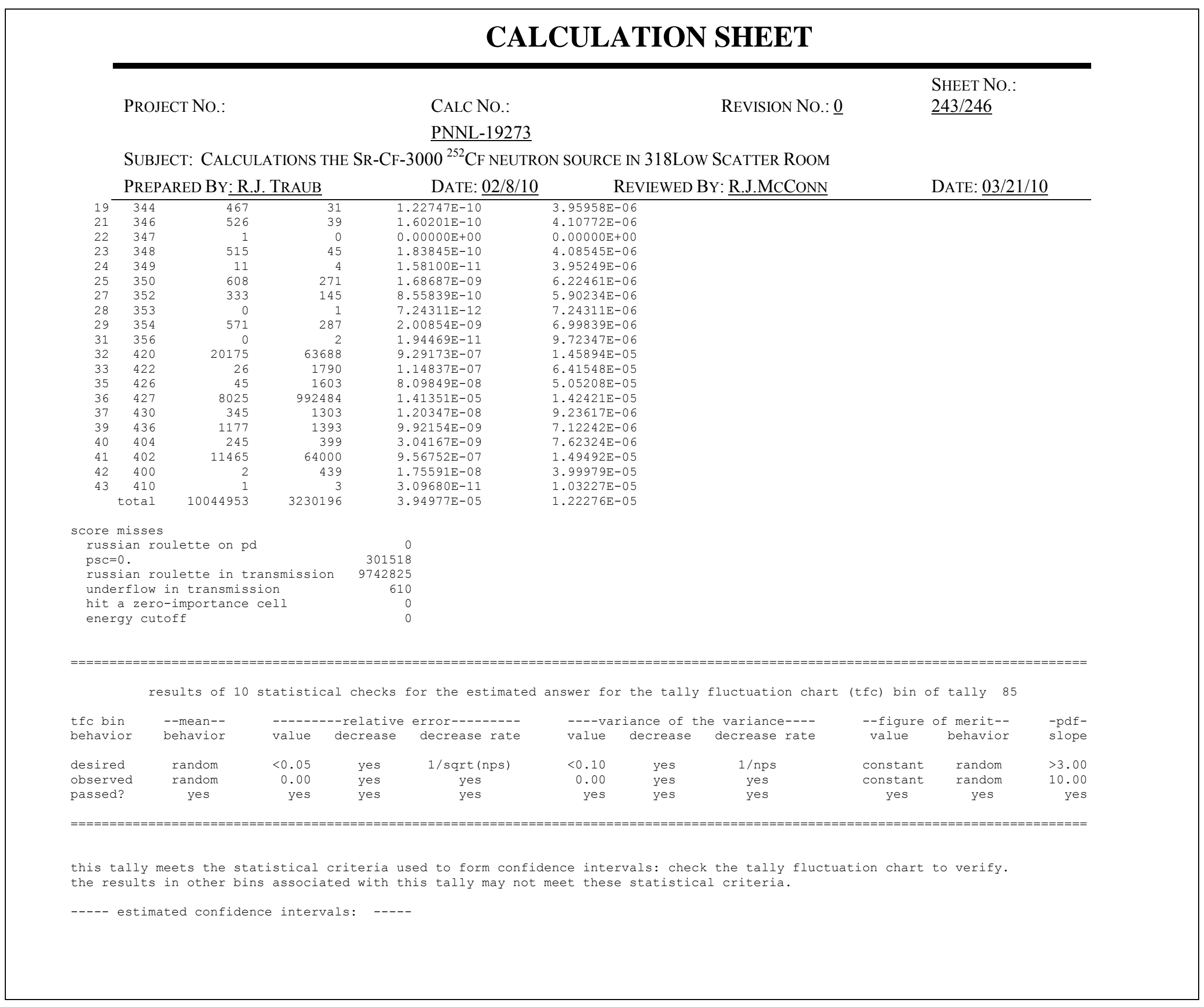




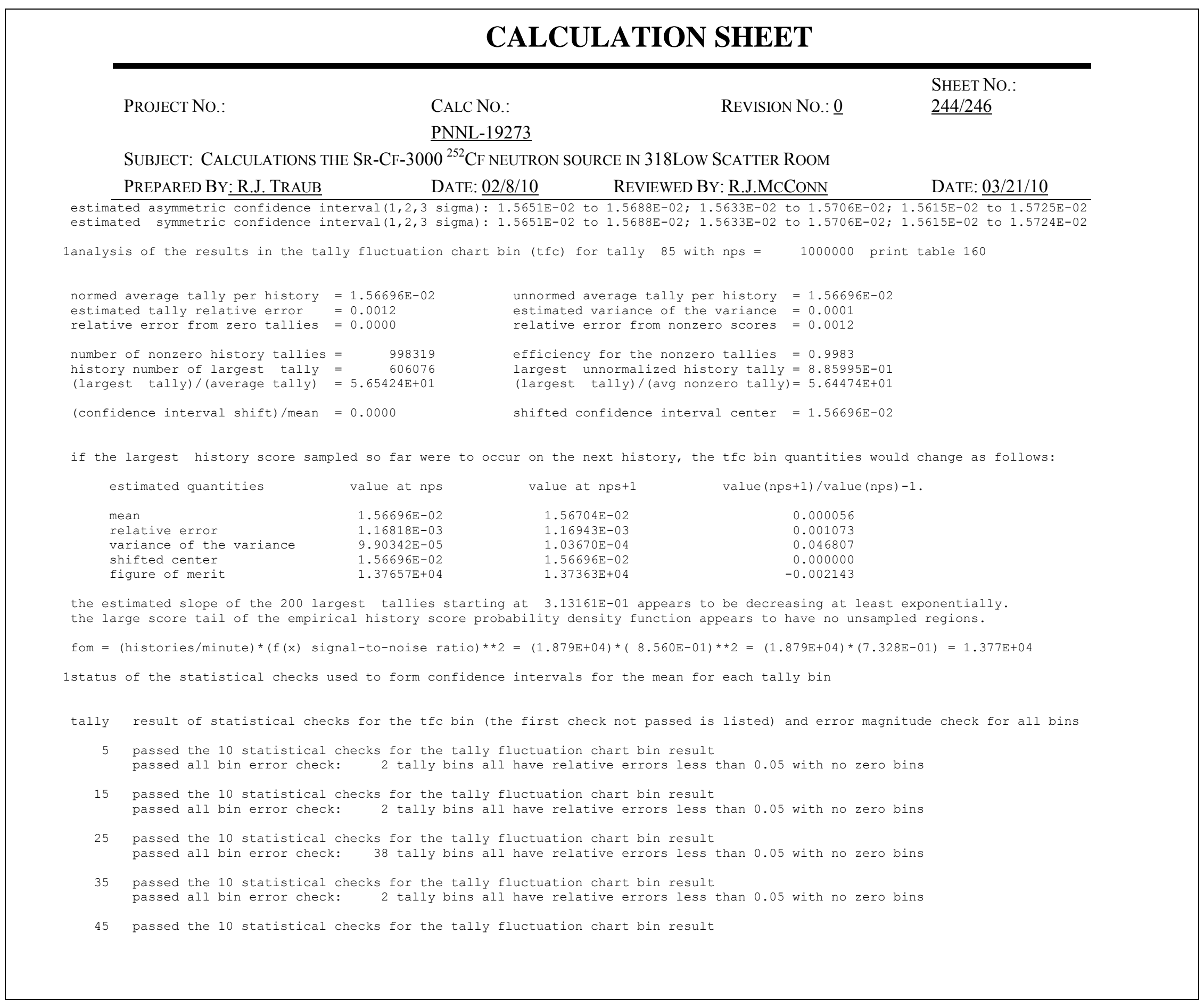




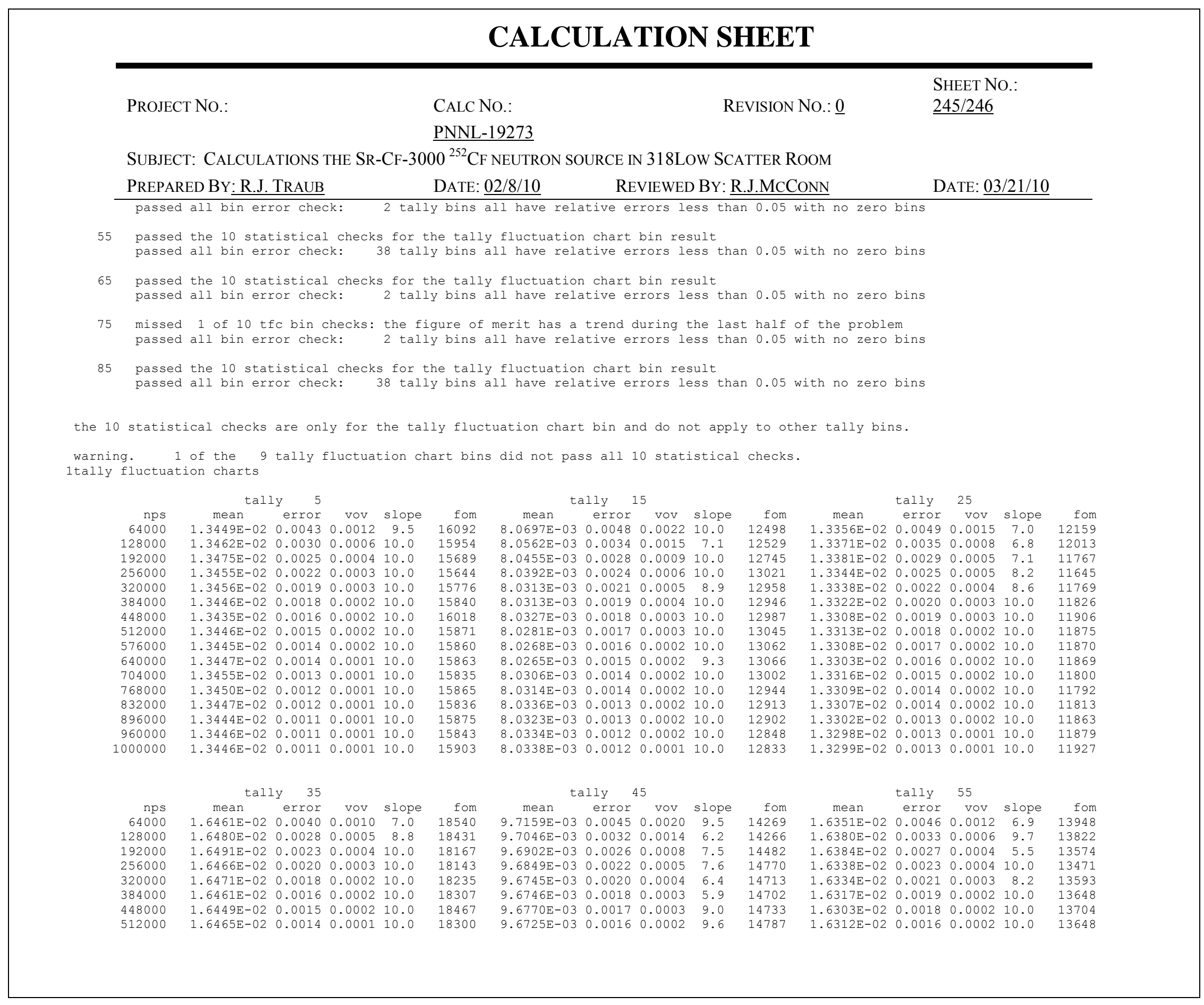




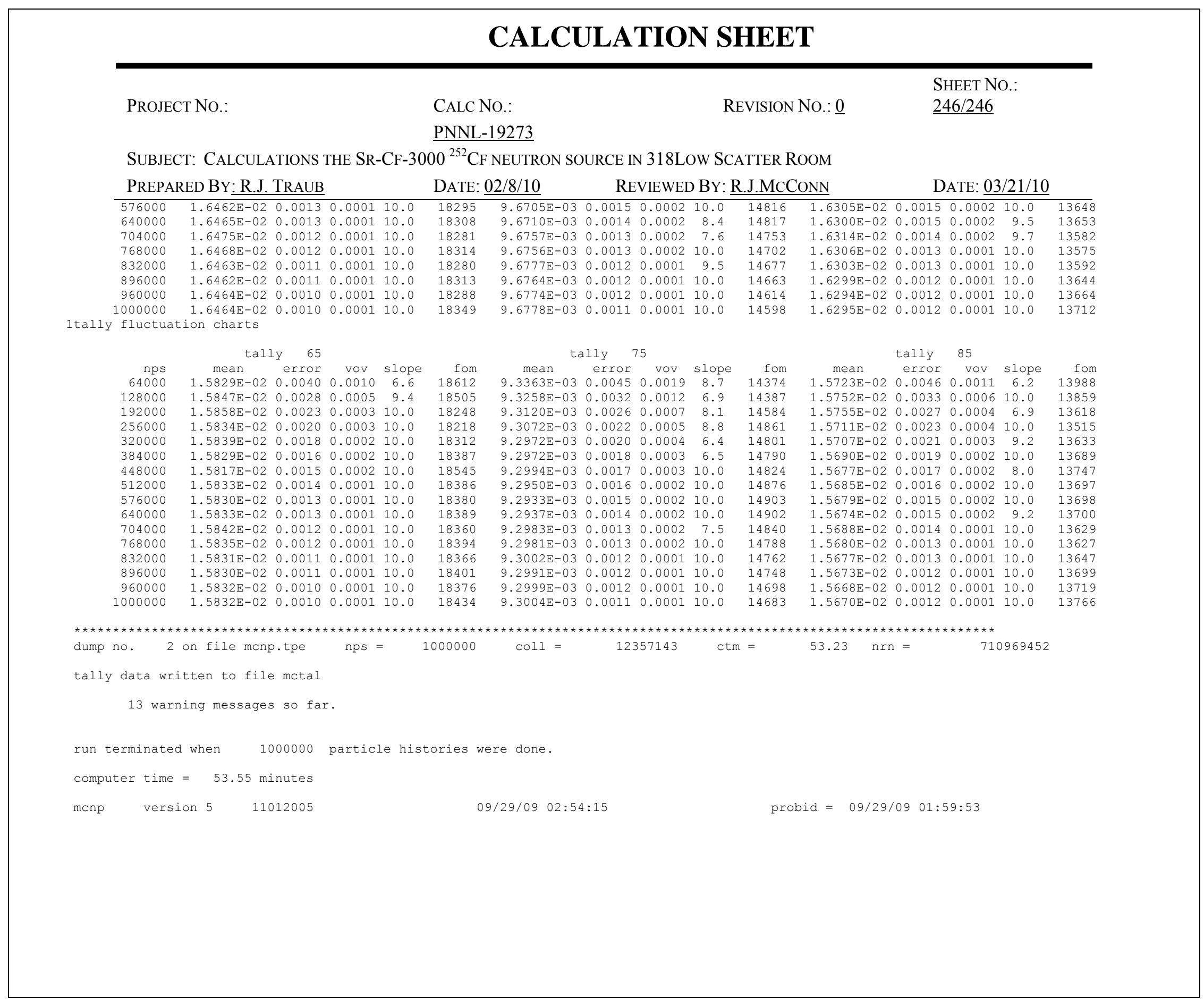




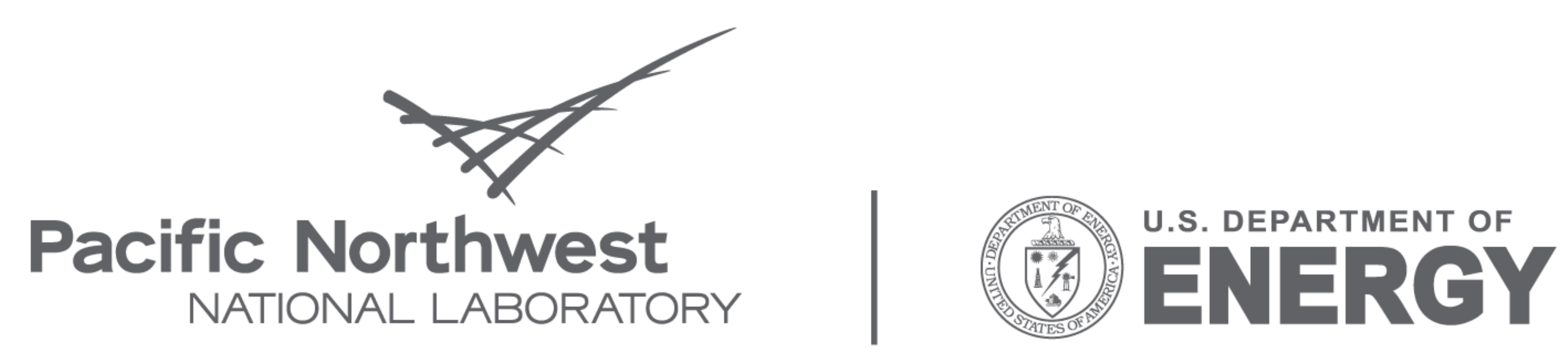

Proudly Operated by Battelle Since 1965

902 Battelle Boulevard

P.O. Box 999

Richland, WA 99352

1-888-375-PNNL (7665)

www.pnl.gov 